$\mathrm{N}^{\circ}$ D'ORDRE

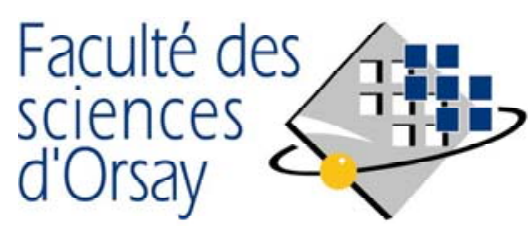

\begin{abstract}
THÈSE DE DOCTORAT
SPECIALITE : PHYSIQUE

Ecole Doctorale « Sciences et Technologies de l'Information des Télécommunications et des Systèmes "
\end{abstract}

Présentée par :

\title{
Ming SHI
}

\section{SIMULATION MONTE CARLO DE MOSFET A BASE DE MATERIAUX III-V POUR UNE ELECTRONIQUE HAUTE FREQUENCE ULTRA BASSE CONSOMMATION}

Soutenue le devant les membres du jury :

M .

$\mathrm{M}$

$\mathrm{M}$

M

M

M 


\section{Table des matières}

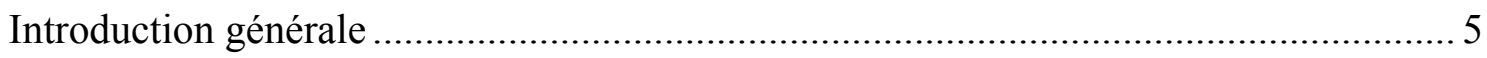

Chapitre I : Révision de la 'Roadmap' de l'ITRS et introduction de la technologie III-V

1. La ‘Roadmap' de l'ITRS des transistors MOS ................................................ 8

1-1 Tendance actuelle du dimensionnement ................................................ 8

1-2 Les innovations de la technologique du MOSFET .................................. 12

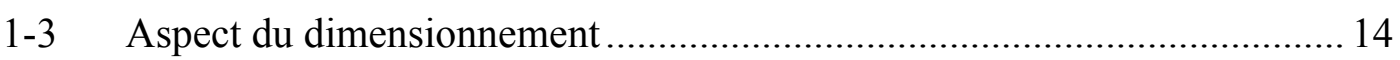

2 Pourquoi utiliser les matériaux III-V pour les futurs n-MOSFET .................... 16

3 Etats de l'art des FETs à base de III-V matériaux............................................. 17

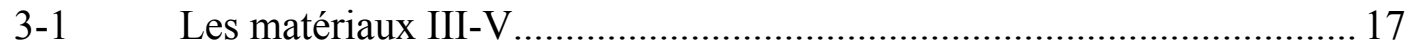

3-2 Transistor à haute mobilité électronique (HEMT) ................................... 19

3-3 Etat de 1'art des transistors hyperfréquences ........................................... 23

3-4 Evaluation des potentiels pour les applications logique ........................ 26

3-5 Limitation du dimensionnement des HEMT .............................................. 27

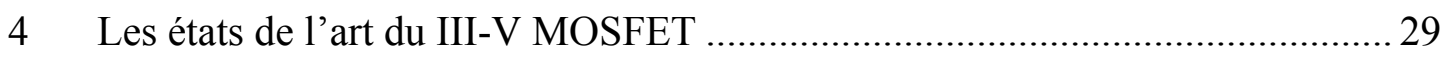

4-1 Système d'empilement diélectrique de grille (Métal/high-k/III-V) : Qualité de l'interface 31

4-2 Comparaison des différentes architectures de dispositifs ......................... 35

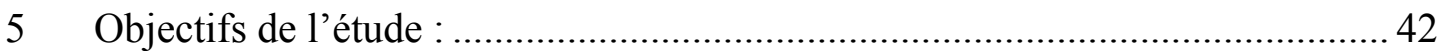

Chapitre II_Contrôle de charge et dimensionnement des structures MOS III-V par analyse Poisson-Schrödinger. 43

1. Présentation des structures étudiées et des modèles numériques .......................... 44

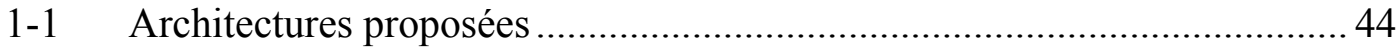

1-2 Dimensionnement préliminaire du dispositif......................................... 44

1-3 Mise en place d'un modèle pertinent ............................................................... 46

1-3-1 Modèle multi-vallées dans l'approximation de la masse effective pour les

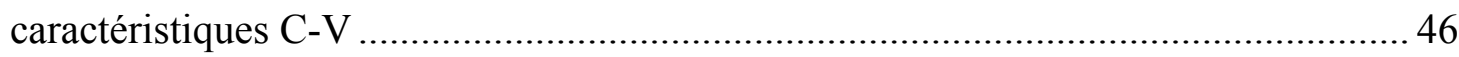

1-3-2 Correction de non-parabolicité ....................................................... 49

1-3-3 Comparaison entre résultats classiques avec les statistiques de MaxwellBoltzmann et de Fermi-Dirac 
1-3-4 Comparaison entre calculs classiques et quantiques ............................5 53

2. Contrôle de charge de la structure MOS III-V ...................................................... 55

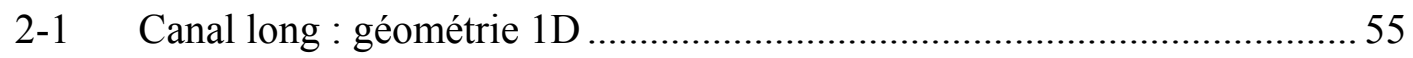

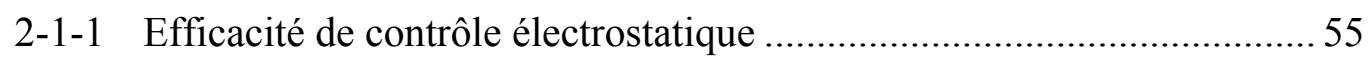

2-1-2 Modèle analytique pour estimer la tension de seuil .............................57

2-1-3 Modèle semi-analytique de la capacité quantique................................. 61

2-1-4 Particularités du contrôle de charge dans les structures alternatives...... 63

2-1-5 Plage de fonctionnement pour la tension de grille ............................... 65

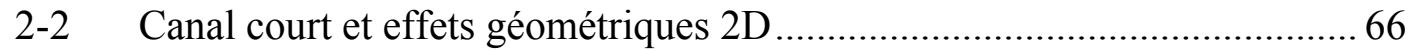

2-3 Prise en compte des états de pièges d'interface ........................................ 70

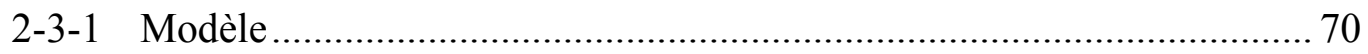

2-3-2 Efficacité du mouvement de niveau Fermi............................................. 72

2-3-3 Résultats et étude comparative avec des données expérimentales ......... 73

2-3-4 Dégradation de la performance en états OFF et $\mathrm{ON}$ due aux pièges...... 78

3. Performances des MOSFET III-V quasi-balistiques ........................................... 80

3-1 Modèle analytique pour le transport (quasi) balistique.............................. 80

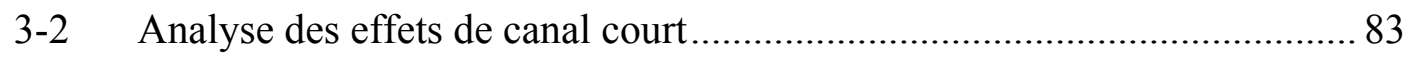

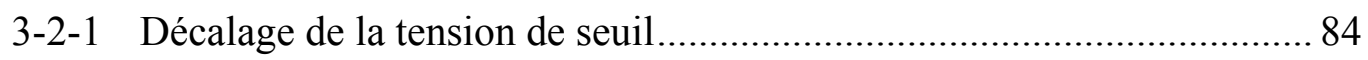

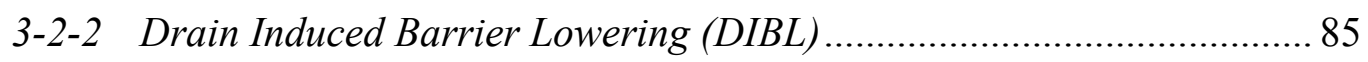

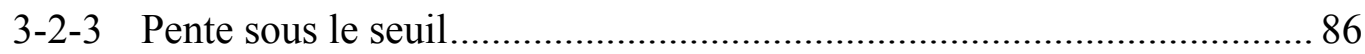

3-2-4 Comparaison avec le modèle analytique MASTAR et les spécifications $\begin{array}{lr}\text { ITRS } & 88\end{array}$

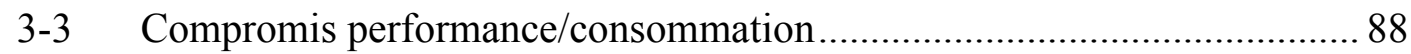

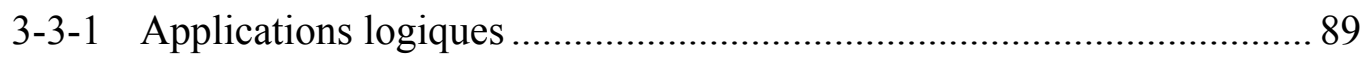

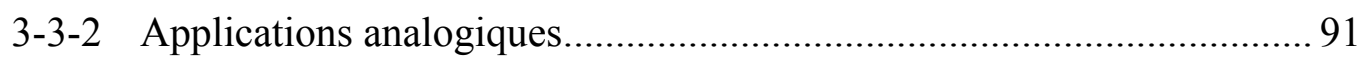

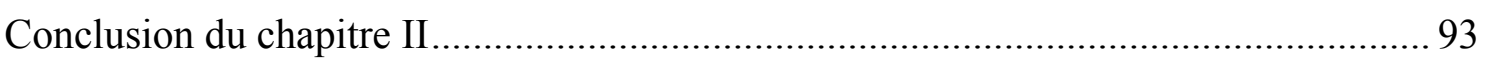

Chapitre III : Analyse semi-classique Monte Carlo des MOSFET III-V ....................... 94

1. Présentation de la simulation particulaire Monte Carlo semi-classique................. 94

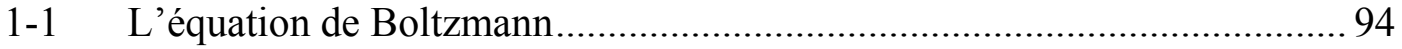

1-2 Simulateur particulaire MONACO : Principe et algorithme ......................95

1-3 Calcul de courant par la méthode de Ramo-Shockley ............................... 96

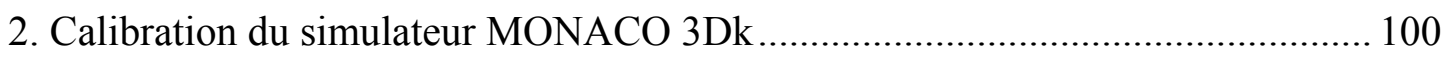

2-1 Structures de bandes et interactions prises en compte .......................... 100 
2-2 Calibration du modèle de transport pour un matériau massif à champ constant

2-3 Mobilité effective dans une couche d'inversion .................................... 103

2-4 Test sur un transistor de référence, résistances d'accès ........................... 106

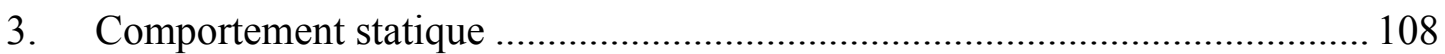

3-1 Caractéristiques I-V et microscopiques ............................................... 108

3-2 Analyse du comportement balistique des dispositifs ............................. 113

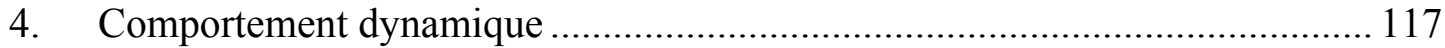

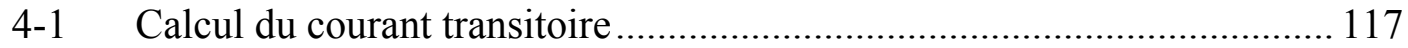

4-2 Extraction des paramètres d'admittance complexes intrinsèques............. 119

4-3 Extraction du circuit équivalent du modèle petit signal (SSEC) intrinsèque 122

4-4 Performance intrinsèque en fréquence ..................................................... 126

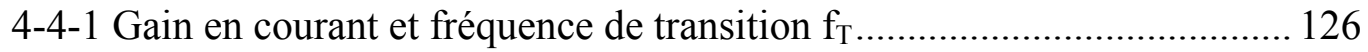

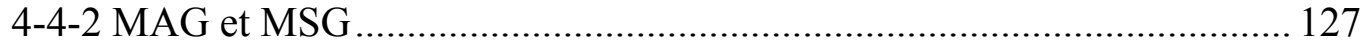

4-4-3 Gain unilatéral en puissance et fréquence $\mathrm{f}_{\mathrm{MAX}}$...................................... 128

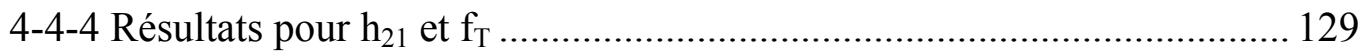

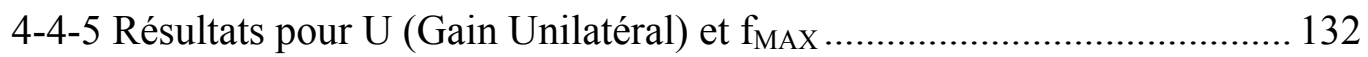

$5 \quad$ Performance RF vs. Dissipation de puissance .............................................. 137

$6 \quad$ Analyse de bruit intrinsèque en haute fréquence ............................................ 138

6-1 Source de bruit des fluctuations du courant .................................................. 139

6-2 Paramètres de bruit normalisés P, R et C.............................................. 142

6-3 Facteurs de mérite de bruit électronique des dispositifs .......................... 144

7 Comparaison des performances avec les MOSFET Si et HEMT III-V ............ 148

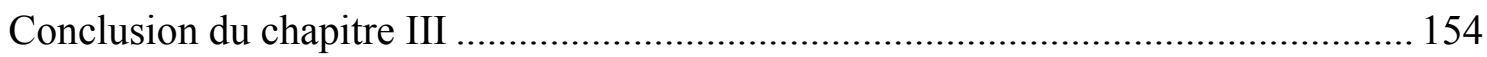

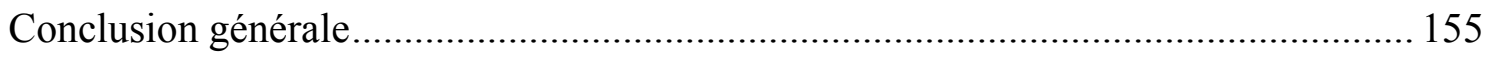

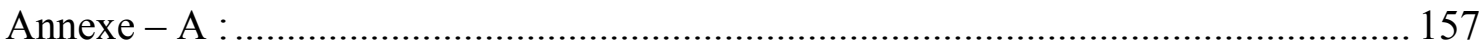

Modèle du Contrôle de charge : Poisson - Schrödinger solutions .............................. 157

A-a Mise en place d'un modèle pertinent .................................................... 157

A-b Correction de non-parabolicité ................................................................ 161

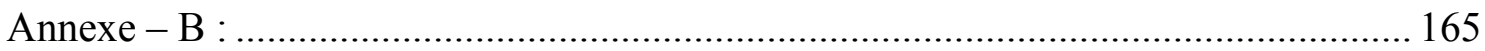

La méthode numérique et l'algorithme du calcul auto-cohérent des équations PoissonSchrödinger 165

Equation de Schrödinger.... 165 
Equation de Poisson.............................................................................................. 167

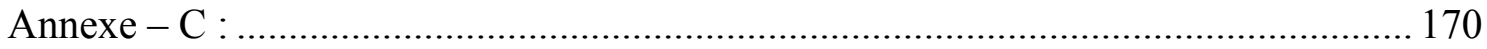

Présentation de la simulation particulaire Monte Carlo semi-classique ........................ 170

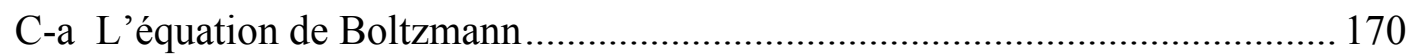

C-b Simulateur particulaire MONACO : Principe et algorithme ……………….... 172

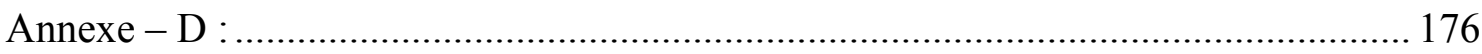

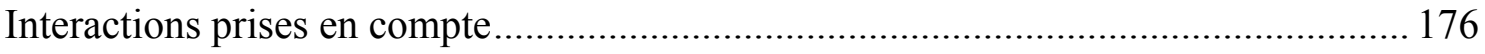

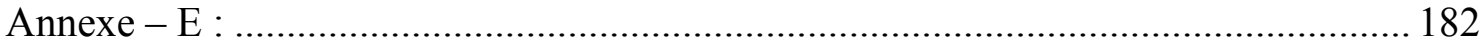

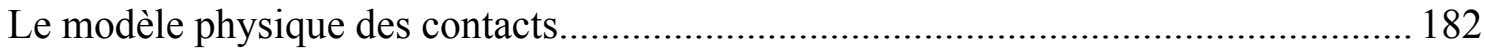

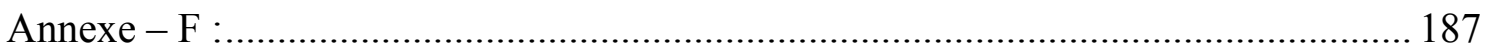

Calcul du gain unilatéral du composant actif linéaire à l'aide des éléments du modèle

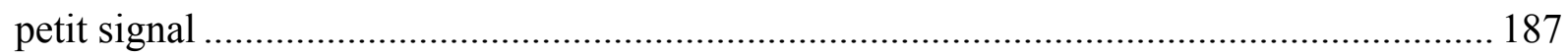

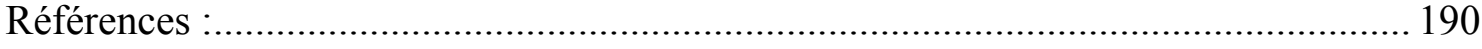

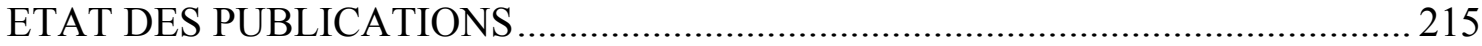

1. Articles dans des revues avec comité de lecture (acceptés)...................................215

2. Communications dans des conférences internationales avec actes (acceptées) ...215

3. Communications dans des conferences internationales sans actes .........................216

4. Communications nationales avec ou sans actes....................................................216

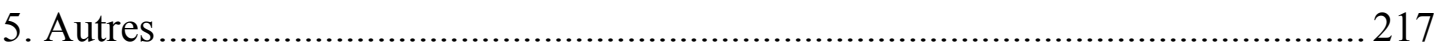




\section{Introduction générale}

L'évolution de la microélectronique a été principalement caractérisée par la loi de Moore dans les dernières décennies. L'esprit "le plus petit sera le mieux" de la miniaturisation a prédominé lors de la conception des composants. La plupart des industriels de la microélectronique focalisent leurs efforts sur la technologie CMOS (Complementary Metal Oxide Semiconductor) à base de transistors FET (Field Effect Transistor) en silicium. En effet, sa simplicité et sa faible consommation ont permis une très forte densité d'intégration des circuits. De plus ses facteurs de mérite obéissent à des lois d'échelle et la taille des transistors a ainsi été diminuée au cours des années selon une progression géométrique. Par exemple pour les opérations logiques, où un transistor se comporte comme un interrupteur commandé, la performance est caractérisée par la vitesse de commutation et par la consommation d'énergie associée.

Cependant, le dimensionnement des transistors a récemment été limité par la forte densité de puissance thermique dissipée par puce. Si la tendance exponentielle précédemment évoquée avait été poursuivie, la densité aurait atteint environ $200 \mathrm{~W} / \mathrm{cm}^{2}$ c'est à dire celle du cœur d'un réacteur de centrale nucléaire. Pour accroître encore le niveau d'intégration des transistors, il faut une réduction assez forte de la tension d'alimentation $\left(\mathrm{V}_{\mathrm{DD}}\right)$, mais cela dégrade également la performance. La mise à l'échelle des transistors n'est plus aussi simple...

Une solution possible est d'introduire un nouveau matériau de canal dans lequel les porteurs se déplacent à une vitesse beaucoup plus élevée que dans le silicium. Cela permettrait une réduction de la tension d'alimentation sans perte de performance. Les semi-conducteurs composés de matériaux des colonnes III et V sont des candidats prometteurs pour les canaux des transistors N-MOS (les porteurs sont des électrons). Grâce à leurs propriétés électroniques et optiques, les matériaux III-V comme GaAs, InAs et les alliages $\operatorname{In}_{1-\mathrm{x}} \mathrm{Ga}_{\mathrm{x}} \mathrm{As}$ ont été largement utilisés dans les domaines de la communication optique, de l'instrumentation et de la détection. Les transistors à base de ces matériaux constituent le cœur de nombreux systèmes électroniques dans le domaine de l'intelligence artificielle à haut-débit et à haute fréquence. En fait, l'industrie manufacturière front-end et back-end est assez mature pour les composants III-V et les circuits intégrés associés. Ils sont produits en grands volumes pour des applications aussi diverses que les téléphones intelligents, les stations de base cellulaires, les systèmes à fibres optiques, sans fil réseaux locaux, les communications par satellite, les 
radars, la radioastronomie et les systèmes de la défense. La brique de base de la technologie III-V pour les hautes fréquences est le HEMT (High Electron Mobility Transistor). Néanmoins, les HEMT auront bientôt atteint leurs limites en termes de lois d'échelle : longueur de grille $\mathrm{L}_{\mathrm{G}}$, épaisseur des hétérostructures. En effet, ils présentent une faible isolation de grille à cause de la faible hauteur de la barrière Schottky avec le semi-conducteur à grand gap d'épaisseur $\mathrm{e}_{\mathrm{GG}}$, ce qui autorise des courants de fuite par la grille élevés et donc une consommation statique élevée. En outre, l'architecture de HEMT III-V présente une large résistance série (200 à $300 \Omega . \mu \mathrm{m})$ par rapport au MOSFET Si (50 à $80 \Omega . \mu \mathrm{m}$ ), un faible rapport d'aspect $\mathrm{L}_{\mathrm{G}} / \mathrm{e}_{\mathrm{GG}}$ d'environ 5 (alors que $\mathrm{L}_{\mathrm{G}} / \mathrm{e}_{\mathrm{ox}}>25$ pour un MOSFET Si où l'épaisseur d'oxyde $e_{o x}$ est ajustée pour obtenir un bon contrôle de charge par la grille) et une large empreinte ("footprint") de l'ordre de 1 à $2 \mu \mathrm{m}$ (pour un MOSFET Si elle correspond au nœud technologique).

Grâce au développement des technologies utilisant des diélectriques de grille à forte permittivité ("high-k") compatible avec les matériaux III-V, l'architecture à grille isolée, c'està-dire le MOSFET III-V, devient envisageable pour améliorer les performances en particulier en termes de contrôle de grille et de footprint. Aujourd'hui, la technologie MOSFET III-V est un domaine de recherche très actif et a récemment été intégrée à la feuille de route de l'ITRS (International Technology Roadmap for Semiconductors).

Pour bénéficier des excellentes performances intrinsèques des matériaux III-V, plusieurs problèmes critiques doivent être résolus. En particulier la réalisation d'un bon empilement de grille, c'est-à-dire avec faible densité de pièges d'interface $\mathrm{D}_{\mathrm{it}}$ et rugosité réduite, constitue un obstacle sérieux. Pour améliorer la performance, on pourra étudier diverses topologies de transistors : à canal enterré, de type Thin-Body... En outre, si l'on privilégie la consommation, on doit travailler à de très faibles tensions d'alimentation, régime où le comportement fréquentiel et le bruit est mal connu.

Dans ce mémoire, tout d'abord, nous allons rappeler la feuille de route des transistors et les problèmes majeurs rencontrés dans le domaine de la microélectronique. Nous nous intéresserons à l'état de l'art des n-FET en technologie de silicium (MOSFET) et III-V (HEMT). La technologie MOSFET III-V sera ensuite introduite.

Dans le chapitre II, nous nous sommes intéressés au contrôle de charge dans une structure métal/oxyde high $\mathrm{k} / \mathrm{semiconducteur}$ III-V où l'isolant high $k$ à haute permittivité diélectrique est l'alumine. L'étude consiste à analyser théoriquement le fonctionnement d'une capacité MOS en résolvant de façon auto-cohérente les équations de Poisson et de Schrödinger (PS). Les résultats sont comparés à des résultats expérimentaux fournis par 
l'équipe de l'IEMN coordinatrice du projet ANR MOS35 qui a servi de cadre à cette thèse. On quantifiera les effets des états de pièges à l'interface high $\mathrm{k} / \mathrm{III}-\mathrm{V}$ sur les caractéristiques capacité -tension C-V. La simulation PS est ensuite étendue à une géométrie 2D pour prendre en compte les effets de canal court et les capacités parasites. Les performances des dispositifs FET sont une première fois estimées pour des applications logiques et analogiques à l'aide d'un modèle analytique de transport quasi-balistique.

Dans le chapitre III, nous avons ensuite étudié plus en détails les performances des MOSFET III-V en régimes statiques et dynamiques sous faible condition de polarisation, à l'aide du simulateur semi-classique de dispositifs Monte Carlo développé dans notre équipe à l'IEF. Les paramètres utilisés ont d'abord été validés par comparaison avec des données expérimentales, en particulier concernant une architecture de FET de type HEMT fabriquée par la société OMMIC partenaire du projet ANR MOS35. Puis, les caractéristiques de quatre topologies de MOSFET ont été quantitativement étudiées en termes de courants statiques et dynamique, de rendement fréquence/consommation et de bruit. Nous en tirons des conclusions sur l'optimisation des dispositifs. Enfin, l'étude comparative avec un FET à base de Si met en perspective le potentiel des MOSFET III-V pour des applications à haute fréquence et faible puissance de consommation. 


\section{Chapitre I :}

\section{Révision de la 'Roadmap' de l'ITRS et introduction de la technologie III-V}

\section{La 'Roadmap' de l'ITRS des transistors MOS}

\section{1-1 Tendance actuelle du dimensionnement}

La miniaturisation des transistors MOSFET vers le LSI (Large Scale Integration) reste toujours une méthode efficace pour atteindre les hautes performances et les faibles dissipations d'énergie. Ainsi, la loi de Moore, qui se traduit par un doublement du nombre de transistors par circuit intégré tous les 24 mois, a guidé l'industrie du semi-conducteur pendant plus de trente ans.

Idéalement, la miniaturisation doit être effectuée sans dégrader les caractéristiques du transistor. Alors, il faut adapter les différents paramètres en fonction de la longueur de grille selon des lois d'échelle. Ces paramètres sont des paramètres géométriques (épaisseur d'oxyde...), électriques (tension d'alimentation...) et physiques (dopages...). Ces lois ont été proposées initialement par R.H. Dennard [Dennard1974JSSC] et révisées par Y. Taur [Taur1998CUP]. Les lois d'échelle idéales permettent de conserver l'équation de Poisson invariante après miniaturisation. Les champs électriques présents dans un transistor court sont identiques à ceux apparaissant dans un transistor long. Un dispositif ainsi dimensionné bénéficie des bienfaits de la miniaturisation sans aucune dégradation de ses caractéristiques. Ces lois d'échelle sont détaillées dans le Tableau 1. Si toutes les dimensions et les tensions sont multipliées par un facteur $K(K<1)$ il faut augmenter les dopages d'un facteur $1 / K$. Cette méthode de dimensionnement dit "idéal" augmente bien la performance et la densité d'intégration mais implique une augmentation de la consommation par unité de surface. De plus, le coût de production par transistor se dégrade significativement avec la miniaturisation.

Dans les faits, depuis 1970, la longueur de grille, la profondeur de jonction et l'épaisseur d'oxyde de la grille ont diminué d'un facteur 100 fois, tandis que la tension d'alimentation a seulement été diminuée d'un facteur 10 (cf. Fig. 1 et tableau 2) [Iwai2002MR]. La fréquence d'horloge a ainsi été augmentée d'un facteur 1000 (seulement 10 espéré selon le dimensionnement idéal), mais la puissance consommée a été augmentée 
d'un facteur $10^{5}$ (1 pour le dimensionnement idéal). La puissance de consommation est donc le facteur limitant. Ainsi, les fréquences d'horloge et les surfaces des puces $\left(\mathrm{A}_{\text {chip }}\right)$ ne sont plus beaucoup augmentées dans les dernières générations de MOSFET.

\begin{tabular}{|c|c|c|c|}
\hline Lois d'échelle & Paramètres & Facteur & Commentaire \\
\hline Géométrie & $\mathrm{L}_{\mathrm{G}}, \mathrm{W}, \mathrm{T}_{\mathrm{OX}}$ & $\mathrm{K}$ & Paramètre du dimensionnement $(<1)$ \\
\hline Tension d'alimentation & $\mathrm{V}_{\mathrm{DD}}$ & $\mathrm{K}$ & Paramètre du dimensionnement $(<1)$ \\
\hline Courant de saturation & $\mathrm{I}_{\mathrm{d}}$ & $\mathrm{K}$ & $\begin{array}{c}\mathrm{I}_{\mathrm{d}}=\mathrm{V}_{\text {sat }} \mathrm{WC}_{\mathrm{G}}\left(\mathrm{V}_{\mathrm{G}}-\mathrm{V}_{\mathrm{T}}\right) \\
\rightarrow \mathrm{WT}_{\mathrm{OX}}{ }^{-1}\left(\mathrm{~V}_{\mathrm{G}}-\mathrm{V}_{\mathrm{T}}\right)=\mathrm{KK}^{-1} \mathrm{~K}=\mathrm{K}\end{array}$ \\
\hline $\mathrm{I}_{\mathrm{d}}$ par unité de $\mathrm{W}$ & $\mathrm{Id} / \mathrm{W}$ & 1 & $\mathrm{I}_{\mathrm{d}}$ par unité $\mathrm{W}=\mathrm{I}_{\mathrm{d}} / \mathrm{W}=1$ \\
\hline Capacité de grille & $\mathrm{C}_{\mathrm{G}}$ & $\mathrm{K}$ & $\mathrm{C}_{\mathrm{G}}=\mathrm{L}_{\mathrm{G}} \mathrm{W} / \mathrm{T}_{\mathrm{OX}} \rightarrow \mathrm{KK} / \mathrm{K}=\mathrm{K}$ \\
\hline Temps de commutation & $\mathrm{CV} / \mathrm{I}$ & $\mathrm{K}$ & $\tau=\mathrm{C}_{\mathrm{G}} \mathrm{V}_{\mathrm{DD}} / \mathrm{I}_{\mathrm{d}} \rightarrow \mathrm{KK} / \mathrm{K}=\mathrm{K}$ \\
\hline Fréquence d'horloge & $\mathrm{f}_{\text {clock }}$ & $1 / \mathrm{K}$ & $\mathrm{f}=1 / \tau=1 / \mathrm{K}$ \\
\hline Intégration & $\mathrm{N}$ & $1 / \mathrm{K}^{2}$ & $\mathrm{~N} \rightarrow 1 / \mathrm{K}^{2}$ \\
\hline Densité de puissance & $\mathrm{P}$ & 1 & $\mathrm{fNCV}^{2} / 2 \rightarrow \mathrm{K}^{-1} \mathrm{~K}^{-2} \mathrm{KK}^{2}=1$ \\
\hline
\end{tabular}

Tableau 1: Lois d'échelle idéales des transistors MOS [Dennard1974 et Taur1998]
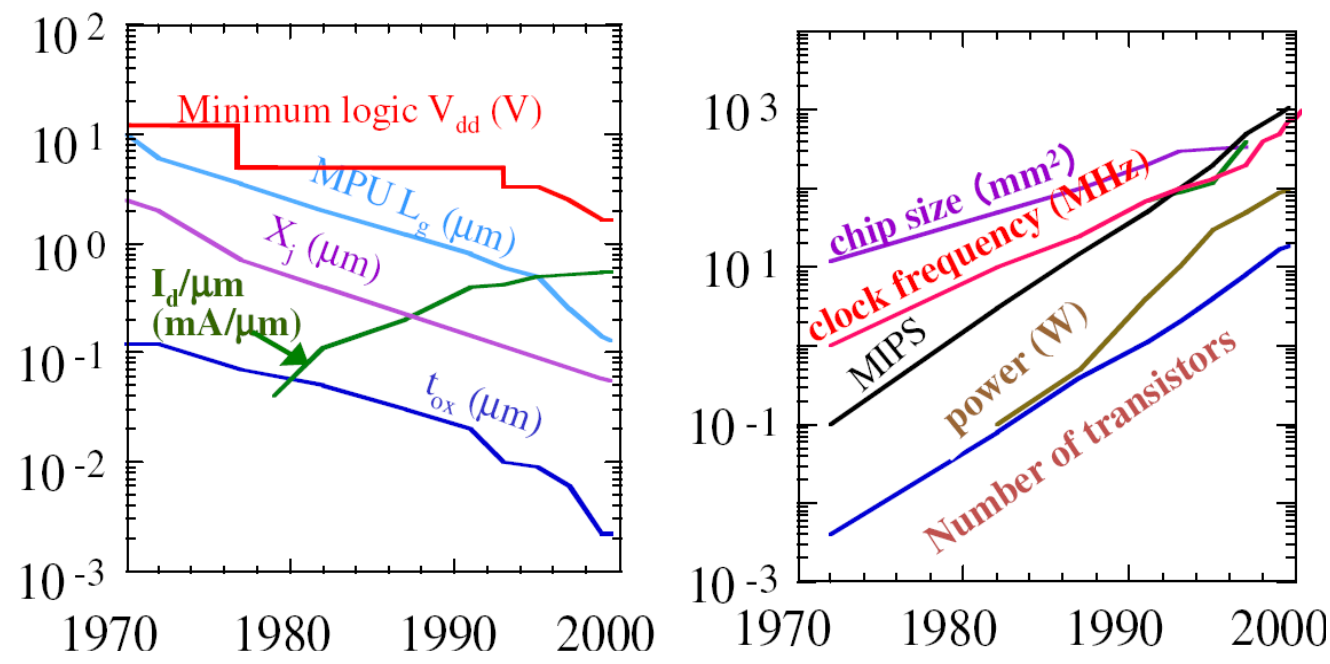

Figure 1 Tendance réelle du dimensionnement du transistor MOS de 1970 à 2000 [Iwai2002]

\begin{tabular}{|c|c|c|c|c|c|c|c|c|}
\hline & Idéal & Réel & & Idéal & Réel & & Idéal & Réel \\
\hline $\mathrm{L}_{\mathrm{G}}$ & $\mathrm{K}$ & $10^{-2}$ & $\mathrm{~A}_{\text {chip }}$ & $\alpha$ & $10^{1}$ & $\mathrm{~N}$ & $\alpha / \mathrm{K}^{2}\left(10^{5}\right)$ & $10^{4}$ \\
\hline $\mathrm{T}_{\mathrm{OX}}$ & $\mathrm{K}\left(10^{-2}\right)$ & $10^{-2}$ & $\mathrm{I}_{\mathrm{d}}$ & $\mathrm{K}\left(10^{-2}\right)$ & $10^{-1}$ & $\mathbf{f}$ & $\mathbf{1} / \mathrm{K}\left(\mathbf{1 0}^{2}\right)$ & $\mathbf{1 0}^{\mathbf{3}}$ \\
\hline $\mathrm{V}_{\mathrm{DD}}$ & $\mathrm{K}\left(10^{-2}\right)$ & $10^{-1}$ & $\mathrm{I}_{\mathrm{d}} / \mathrm{W}$ & 1 & $10^{1}$ & $\mathbf{P}$ & $\mathbf{1}$ & $\mathbf{1 0}^{\mathbf{5}}$ \\
\hline
\end{tabular}

Tableau 2 Comparaison du dimensionnement "idéal" et réel entre 1970 et 2000.

Néanmoins, les problèmes de dimensionnement des MOSFET devenus nanométriques sont devenus beaucoup plus difficiles à cause de la très forte augmentation du coût du développement des nouvelles technologies et de la forte variabilité des caractéristiques 
électrique. Dans la suite on va s'intéresser à la feuille de route de la technologie CMOS hautes performances pour les générations (ou nœuds technologiques) de $22 \mathrm{~nm}$ et en deçà [2008, 2009 et 2010. Edition International Technology Roadmap for semiconductors ITRS. Disponible sur www.itrs.net/reports].

\begin{tabular}{|c|c|c|c|c|c|}
\hline \multicolumn{2}{|c|}{ Technologie CMOS pour HP Logique } & & \multicolumn{3}{|c|}{ ITRS (2008-2010) } \\
\hline $\begin{array}{c}\text { Nœuds } \\
\text { technologiques (nm) }\end{array}$ & $\begin{array}{l}\text { Année de } \\
\text { départ }\end{array}$ & & Année & $\begin{array}{l}\text { Half pitch ( } 1^{\mathrm{er}} \\
\text { piste métal) (nm) }\end{array}$ & $\begin{array}{c}\mathrm{L}_{\mathrm{G}} \text { physique } \\
(\mathrm{nm})\end{array}$ \\
\hline \multirow[t]{2}{*}{45} & 2007 & $\leftrightarrow$ & 2007 & 68 & 32 \\
\hline & & & 2008 & 59 & 29 \\
\hline \multirow[t]{2}{*}{32} & 2009 & $\leftrightarrow$ & 2009 & 52 & 29 \\
\hline & & & 2010 & 45 & 27 \\
\hline \multirow[t]{2}{*}{22} & 2011-2012 & $\leftrightarrow$ & 2011 & 40 & 24 \\
\hline & & & 2012 & 36 & 22 \\
\hline \multirow[t]{2}{*}{16} & 2013-2014 & $\leftrightarrow$ & 2013 & 32 & 20 \\
\hline & & & 2014 & 28 & 18 \\
\hline
\end{tabular}

Tableau 3 Relation entre les nouds technologique CMOS et les valeurs des paramètres mentionnés dans le rapport de l'ITRS.

Le tableau 3 montre les valeurs des spécifications de l'ITRS mises à jour en 20082010. On doit noter que le nœud technologique se rapproche de la longueur physique de grille. Les nœuds technologiques ont différentes significations selon les époques. Historiquement $(8 \mu \mathrm{m}-0,5 \mu \mathrm{m})$, il représente la résolution de lithographie (half pitch of the lines), la longueur de grille minimale et le largueur de la piste métallique du premier niveau. Ce chiffre a diminué approximativement du fameux facteur 0,7 (loi de Moore) tous les 3 ans. A partir de la génération $0,35 \mu \mathrm{m}$, la technologique CMOS a introduit des longueurs de grille plus petites que la résolution de lithographie en introduisant une technique "resist-pattern-thinning" utilisant le plasma d'oxygène [Ono1995IEEE]. Cette longueur sert maintenant à définir le nœud technologique mais ne donne aucune indication sur la longueur physique de la grille et a un intérêt uniquement commercial (nœuds 45 et $32 \mathrm{~nm}$ ). A partir du nœud $22 \mathrm{~nm}$, ce chiffre est environ égal à la longueur physique de la grille, cette définition devrait être conservée pour la suite. On peut voir cette tendance technologique dans la version 2009 de l'ITRS (cf. Fig. 2). A partir du nœud $22 \mathrm{~nm}$, on peut voir le croisement et la superposition de ces allures pour les paramètres liés au nœud technologique. 


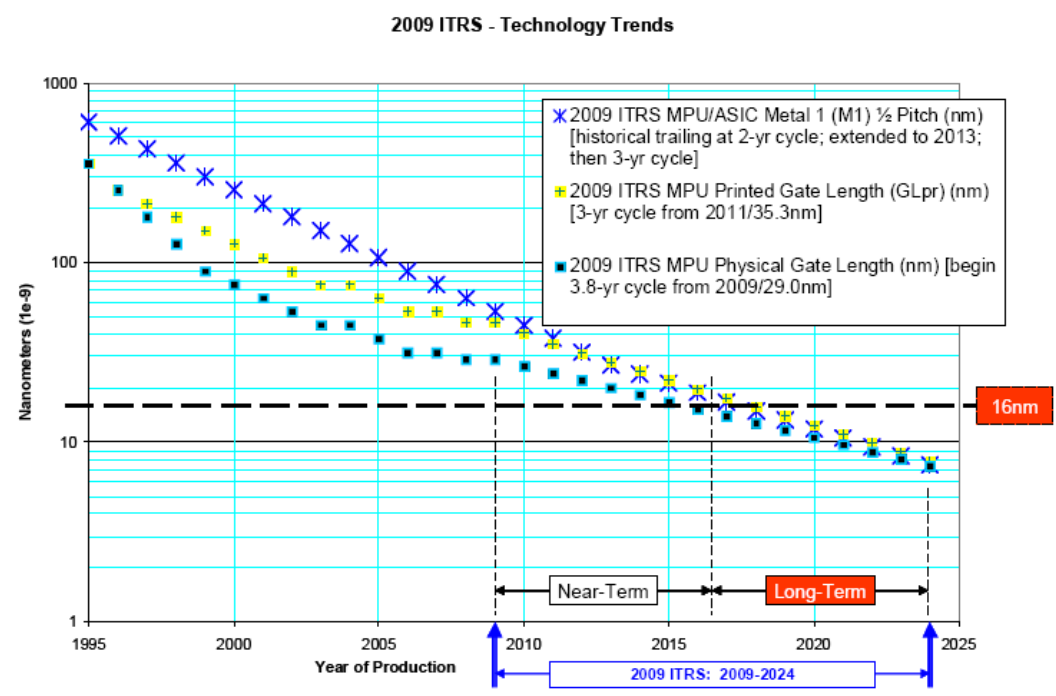

Figure 2 ITRS 2009 : La tendance technologique pour largueur de la piste métallique du premier niveau

M1, 1/2 Pitch et longueur physique et imprimée de grille en fonction de l'année de la production.

On doit noter que les versions de l'ITRS avant 2007 furent très agressives en termes d'objectif de réduction de la longueur de grille, et l'ITRS 2008 a donc été réajustée pour mieux suivre l'évolution réelle (cf. Fig. 3). Un délai de plus de 3 ans est mentionné entre 2 dimensionnements successifs de $\mathrm{L}_{\mathrm{G}}$.

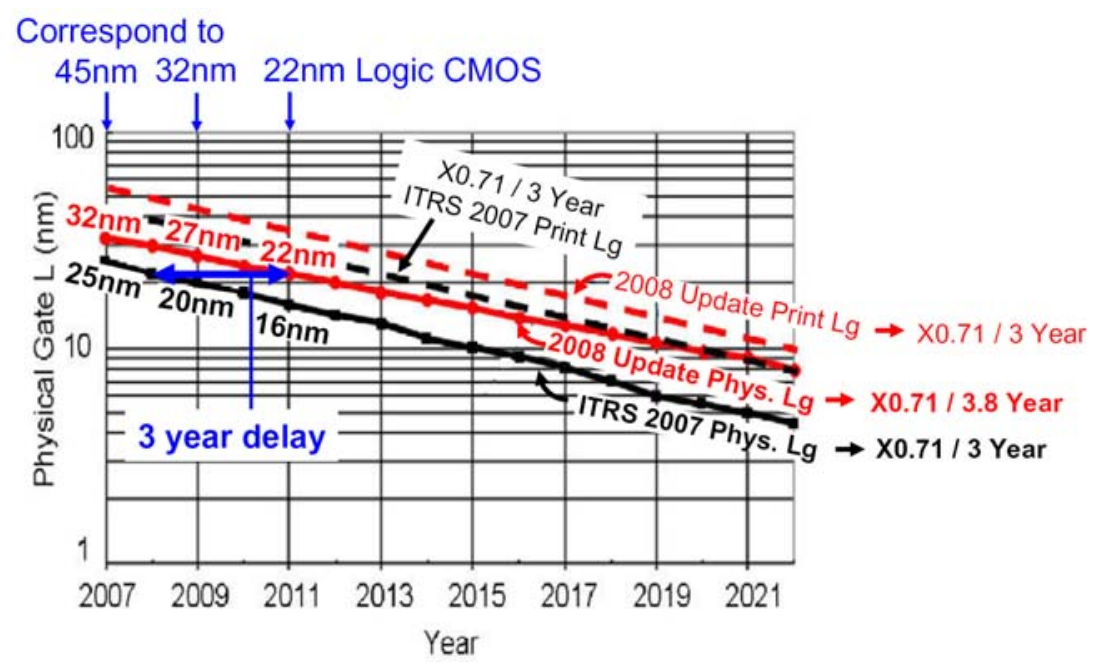

Figure 3 Comparaison des tendances prédites par les ITRS 2007 et 2008 pour les longueurs de grille physiques et imprimées.

De plus, à cause de la crise économique de ces dernières années, toutes les grandes compagnies du semi-conducteur sauf Intel ont réduit significativement leurs investissements en $R \& D$ technologique. Le dimensionnement de la longueur de grille devrait donc être affecté 
et également ceux de l'épaisseur équivalente d'oxyde (EOT) et de la profondeur de la jonction $\left(\mathrm{X}_{\mathrm{j}}\right)$ [Iwai2002IEDM].

\section{1-2 Les innovations de la technologique du MOSFET}

Dans cette section, nous allons rappeler les innovations technologiques principales du MOSFET (cf. Fig. 4) qui vont permettre à la loi de MOORE de continuer au moins dans la prochaine décennie. Dans le rapport d'ITRS, cela est réclamé comme une feuille de route technologique du dimensionnement équivalent "Equivalent Scaling Technology Roadmap".

La première grande innovation a concerné le design de l'empilement de la grille. Le poly-silicium du contact de grille a été remplacé par du métal, cela permet de réduire la résistance de grille et le "recul" de la fonction d'onde dit "dark space" côté de grille [Pacelli1999IEEE] et donc de diminuer l'épaisseur équivalente d'oxyde (EOT). De plus, la tension de seuil peut être ainsi ajustée par le travail de sortie du métal de grille.

Le diélectrique de grille historiquement en silice $\mathrm{SiO}_{2}$ a été remplacé par des oxydes de grande permittivité (high-k). Cela permet d'avoir une grande capacité d'oxyde avec une épaisseur d'oxyde physique plus grande qu'avec la silice et donc de limiter le courant de fuite par la grille.

Pour augmenter l'immunité contre les effets de canal court (ou SCE pour short channel effects), la structure traditionnelle comme de MOSFET sur substrat massif (MOS-Bulk) peut être remplacée par une architecture sur SOI (Silicon On Insulator). Le canal est alors un film mince, pris en sandwich entre 2 isolants (de grille et de substrat) et non intentionnellement dopé, la couche active peut être partiellement et complètement désertée (PDSOI et FDSOI). Cette structure reste planaire mais il existe aussi des structures 3D, ce sont notamment les FINFET avec double ou triple grille (cf. Fig. 4). Le contrôle de charge électrostatique par la grille est alors davantage renforcé.

Les propriétés du transport électronique dans la couche active ont aussi été améliorées. D'abord le canal en Si a été contraint. Le transport est amélioré car la dégénérescence des vallées $\Delta$ est partiellement levée et les interactions intervallées via les phonons deviennent moins probables. Mais dans l'avenir le transport pourrait être radicalement amélioré si le canal en Si était remplacé par des matériaux ayant une forte mobilité électronique tels que les composés III-V, comme InGaAs, InAs, InSb..., ou les dispositifs en carbone, à base de nanotubes ou de graphène. Les processus technologiques de type bottom-up à mettre en œuvre 
pour cette dernière classe de matériaux peuvent poser des problèmes fondamentaux [Chau2005CSIC].

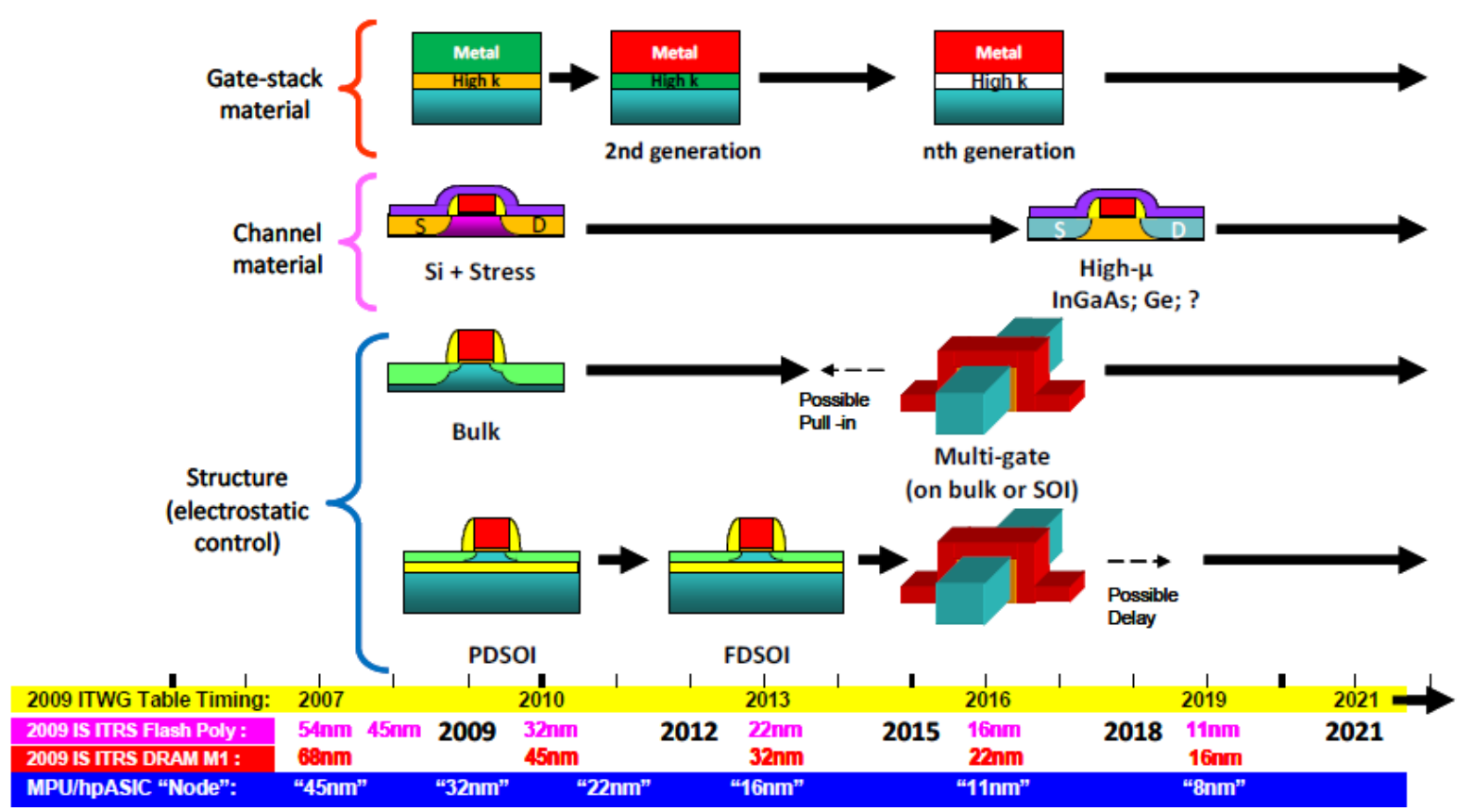

Figure 4 Innovations sur la structure du MOSFET dans le rapport de l'ITRS 2010, les nœuds technologiques pour application logique et l'année de la production

Cependant, l'introduction réelle de ces nouvelles technologies est plus lente que les anciennes prédictions de l'ITRS. Par exemple, l'introduction de la structure de type double grille (DG) ou FINFET a subi un retard d'environ 4 ans (cf. Fig. 5). Cependant, la génération $22 \mathrm{~nm}$ du CMOS prévue en production pour 2011-2012 utilise désormais la structure innovée MOS-SOI (FDSOI) planaire ou MOS-Multi-gate (FINFET) en 3D (cf. Fig. 4). Mais l'introduction de matériaux innovant (Ge, InGaAs et InAs) a été significativement retardée par rapport aux anciennes prédictions de l'ITRS 2008.

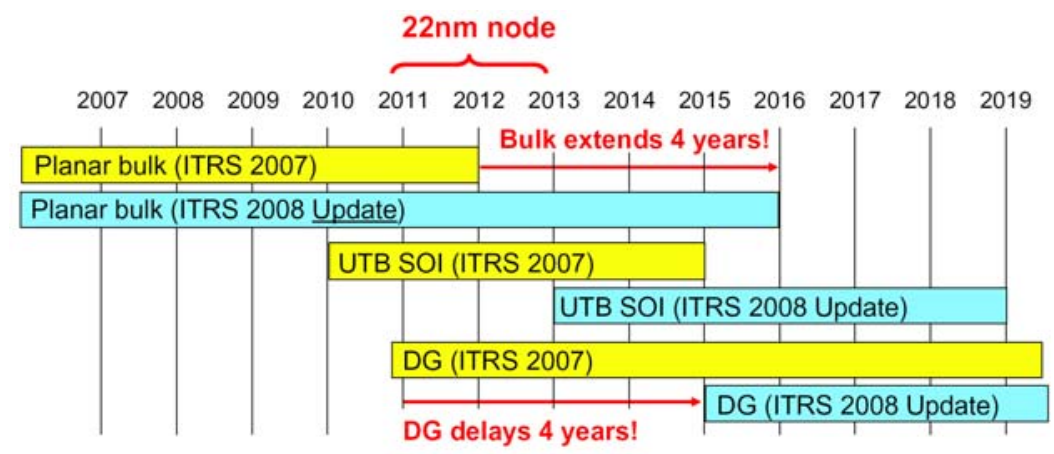

Figure 5 Retard dans l'introduction des structures innovantes de MOSFET d'après l'ITRS 2007-2008

[Iwai2009MicroEng] 


\section{1-3 Stratégies de dimensionnement}

La densité de puissance du CPU est limitée à $100 \mathrm{~W} / \mathrm{cm}^{2}$ [Pop2010NanoRes] et la rapidité d'horloge associée doit rester en dessous de 4 GHz. La Fig. 6 montre les allures de la densité de puissance et de la fréquence d'horloge des microprocesseurs en fonction des années. Diminuer la tension d'alimentation $\left(\mathrm{V}_{\mathrm{DD}}\right)$ est la méthode la plus efficace pour réduire la puissance dynamique consommée [Sakurai2003ISSCC] :

$$
\text { Puissance }=P(\text { active })+P(\text { passive }) \propto f \cdot C_{\text {load }} \cdot V_{D D}^{2} \cdot N+I_{O F F} \cdot V_{D D}
$$

où $\mathrm{f}$ est la fréquence d'opération et $\mathrm{N}$ le nombre de transistors. $\mathrm{C}_{\text {load }}$ est la capacité de charge.
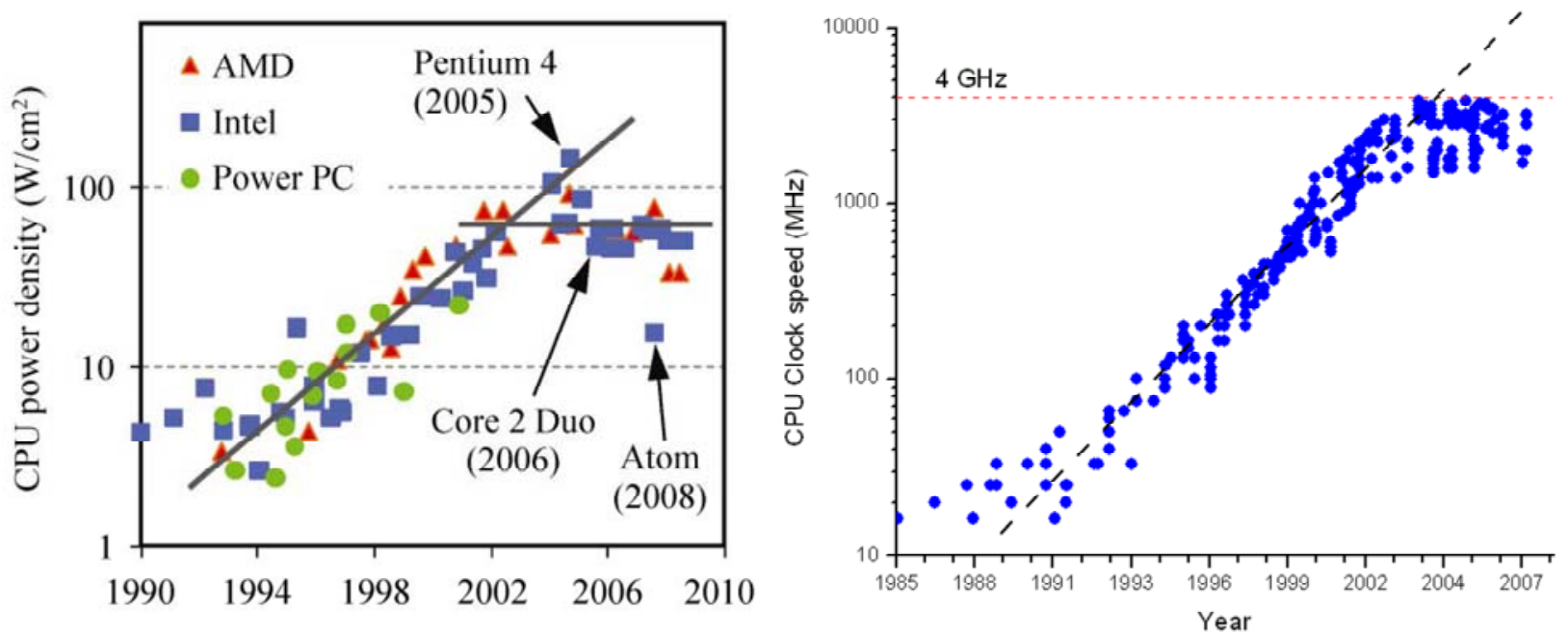

Figure 6 Gauche : Evolution de la densité de puissance du CPU [Pop2010NanoRes]. Droite : Evolution de la fréquence d'horloge du CPU [www.intel.com].

Cependant, afin de diminuer la tension d'alimentation $\left(\mathrm{V}_{\mathrm{DD}}\right)$, la tension de seuil $\left(\mathrm{V}_{\mathrm{T}}\right)$ devrait aussi être réduite. Or la tension de seuil et la tension d'alimentation sont très difficiles à ajuster simultanément. Par exemple (cf. Fig. 7 [Dewey2009IEDM]), si on ajuste $\mathrm{V}_{\mathrm{T}}$ à 0,1 V au lieu de $0,3 \mathrm{~V}$, en négligeant les effets de canal court et les états d'interface, la pente sous le seuil devrait atteindre sa valeur optimale de $60 \mathrm{mV} / \mathrm{dec}\left(\ln 10 \times \mathrm{k}_{\mathrm{B}} \mathrm{T} / \mathrm{q}\right)$ pour conserver un courant de fuite en état OFF $\mathrm{I}_{\mathrm{OFF}}$ acceptable ! Si on réduit simultanément la tension $\mathrm{V}_{\mathrm{DD}}$, le courant à l'état $\mathrm{ON} \mathrm{I}_{\mathrm{ON}}$ sera diminué, donc le rapport $\mathrm{I}_{\mathrm{ON}} / \mathrm{I}_{\mathrm{OFF}}$ sera fortement dégradé. Les valeurs de $V_{T}$ et $V_{D D}$ sont supposées restées en dessus de respectivement 0,1 et $0,9 \mathrm{~V}$ durant les prochaines années selon le rapport de l'ITRS 2008 (cf. Fig. 8-9) [Chen2009IEDM].

La réduction de $\mathrm{V}_{\mathrm{DD}}$ favorise les effets de canal court (SCE) qui peuvent être combattus par réduction de l'EOT. Mais cela génère un courant de fuite par la grille plus important. L'EOT sature autour de 0,5 nm à moyen terme (cf. Fig. 9). Donc la mise en œuvre des matériaux diélectrique d'oxyde avec high- $k$ est très importante. 

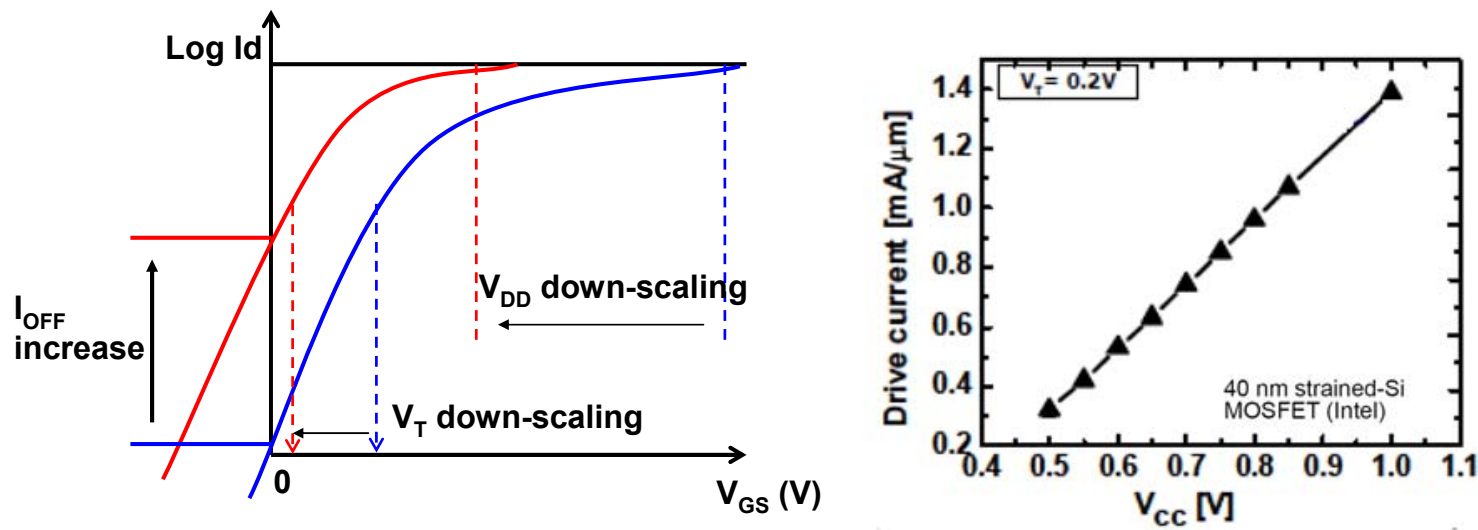

Figure 7 Gauche : Effet schématique de la réduction de $V_{D D}$ et $V_{T}$ sur $I_{O F F}$. Droite : Evolution de $I_{O N}$ en fonction de la tension d'alimentation $\left(V_{D D}\right.$ ou $\left.V_{C C}\right)$ pour un dispositif en Si [Dewey2009IEDM]

Chip Operating Voltage Range vs Scaling

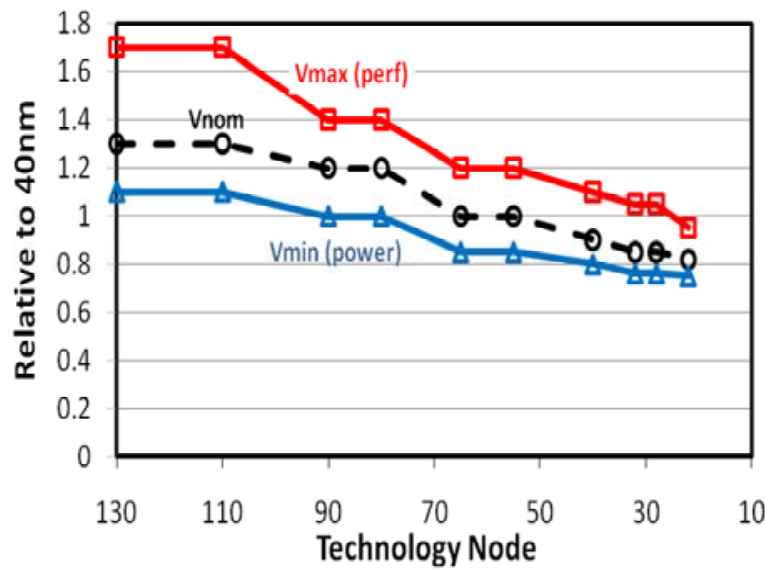

Vdd will stay higher in 2008 update

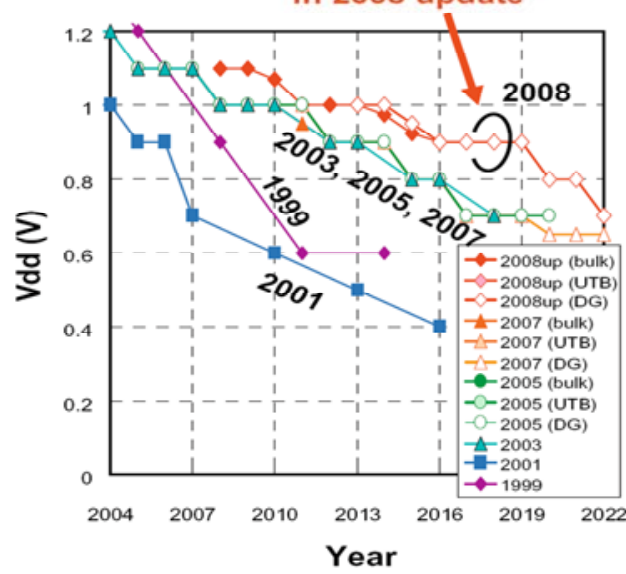

Figure 8 Gauche : Diminution du $V_{D D}$ en fonction des nœuds technologiques [Chen2009IEDM]. Droite : Evolution du $\mathbf{V}_{\text {DD }}$ selon les versions de l'ITRS [Iwai2009IEDST].

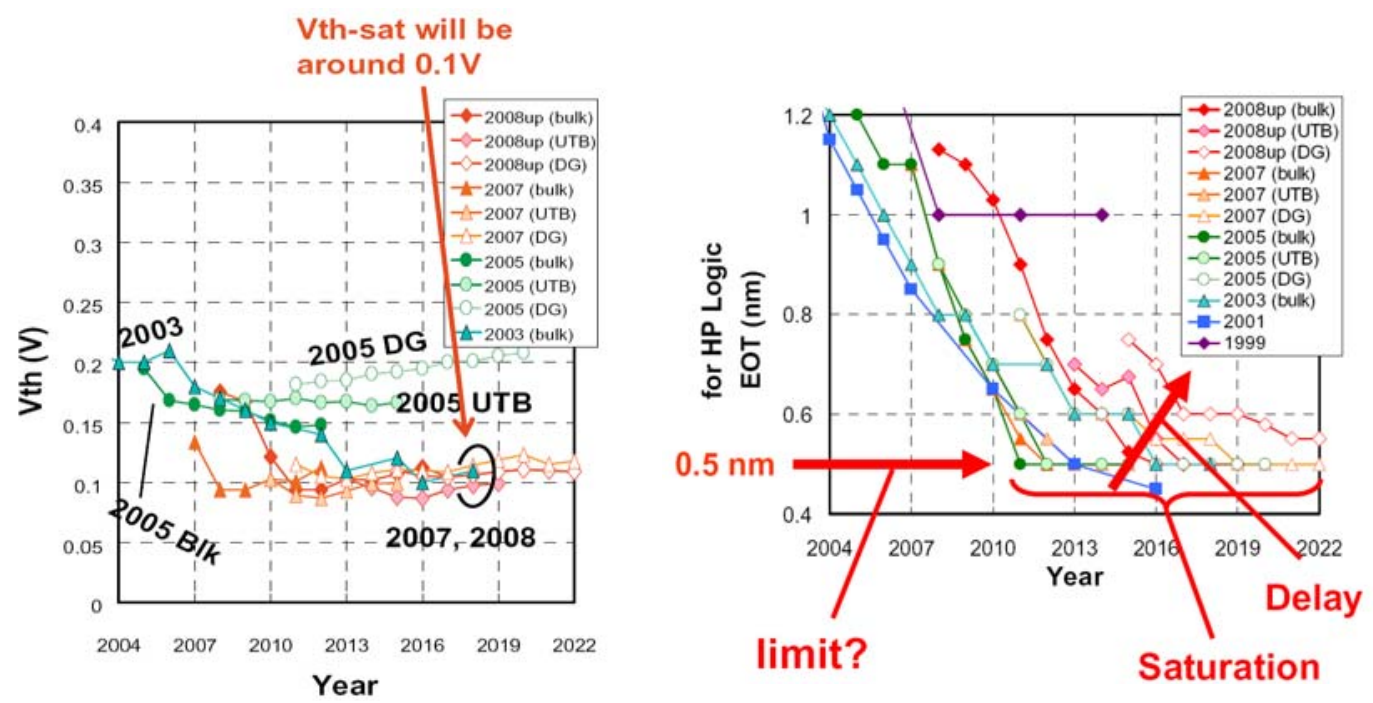

Figure 9 Prédictions de $\mathrm{V}_{\mathrm{T}}$ et EOT selon les différentes versions de l'ITRS [Iwai2009MicroEng]. 


\section{Pourquoi utiliser les matériaux III-V pour les futurs n-MOSFET}

L'innovation ne concerne pas uniquement le dimensionnement de plus en plus délicat des transistors, on cherche aussi des matériaux alternatifs au silicium. La Fig. 10 résume les évolutions technologiques en fonction des nœuds technologiques selon l'ITRS avec une projection vers les structures alternatives : transistors à effet de champ (FET) élaborés avec des nanotubes carbones (CNT) ou avec des alliages en matériaux III-V. Les processus technologiques et les synthèses chimiques d'assemblage de type bottom-up peuvent poser éventuellement des problèmes fondamentaux de variabilité des transistors en CNT. On ne s'intéressera uniquement par la suite qu'aux procédés top-down.

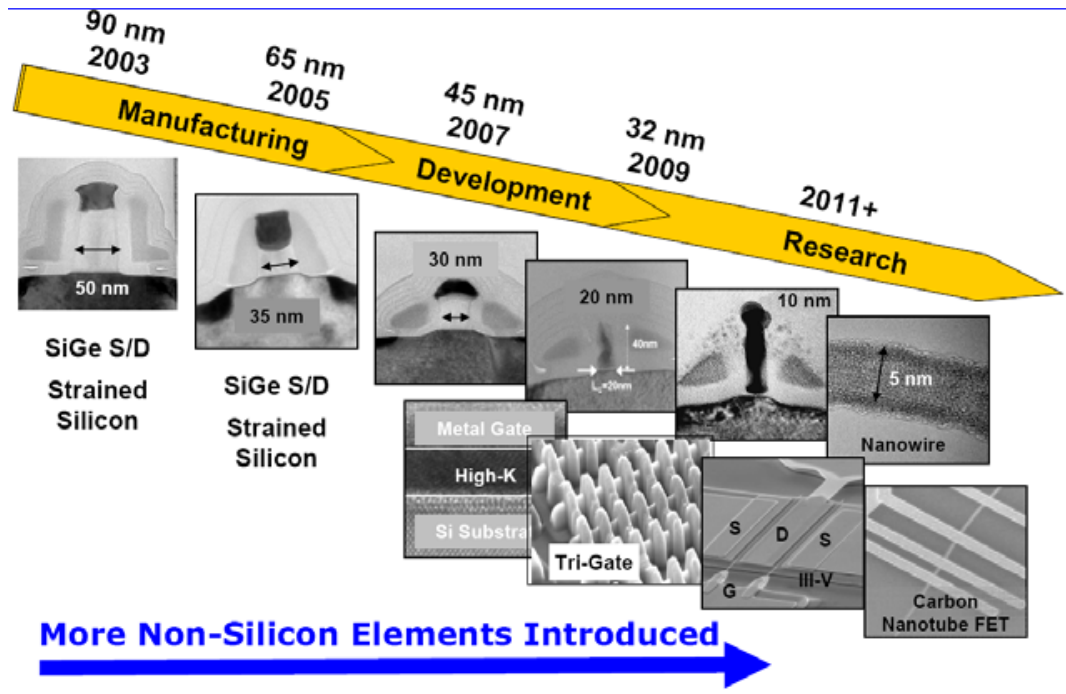

Figure 10 Les innovations de la conception du transistor MOS ainsi que les technologies émergeantes

\section{[Chau2005IEEE]}

On a déjà vu que le concept conventionnel de dimensionnement des dispositifs, en particulier pour la tension d'alimentation $\mathrm{V}_{\mathrm{DD}}$, la tension de seuil $\mathrm{V}_{\mathrm{T}}$ et l'épaisseur d'oxyde $\mathrm{T}_{\mathrm{OX}}$, touche ses limites en termes de compromis entre le courant $\mathrm{I}_{\mathrm{ON}}$, la consommation d'énergie liée au I $\mathrm{OFF}_{\mathrm{OF}}$ et l'immunité contre les effets de canal court [Shinichi2008IEEE].

En introduisant un matériau de canal avec une haute mobilité et une grande conductivité des porteurs (cf. Fig. 11), les nouvelles ingénieries de dispositifs ont pour objectifs d'atteindre simultanément la haute performance et la faible puissance de consommation tout en relâchant les contraintes de dimensionnement. On espère d'une part que certains matériaux III-V permettront d'obtenir, avec des épaisseurs d'oxyde $\mathrm{T}_{\mathrm{OX}}$ raisonnables, un compromis favorable au $\mathrm{I}_{\mathrm{ON}}$ entre la réduction de l'effet capacitif (par effet de capacité quantique lié à la faible densité d'états inhérente au matériau III-V) et l'augmentation de la vitesse d'injection au sommet de barrière du coté source (cf. Fig. 12). 
[Kim2009IEDM]. D'autre part, une meilleure immunité aux effets de canal court est rendue possible grâce aux structures de type UTB (ultra thin body) ou multi-grille.
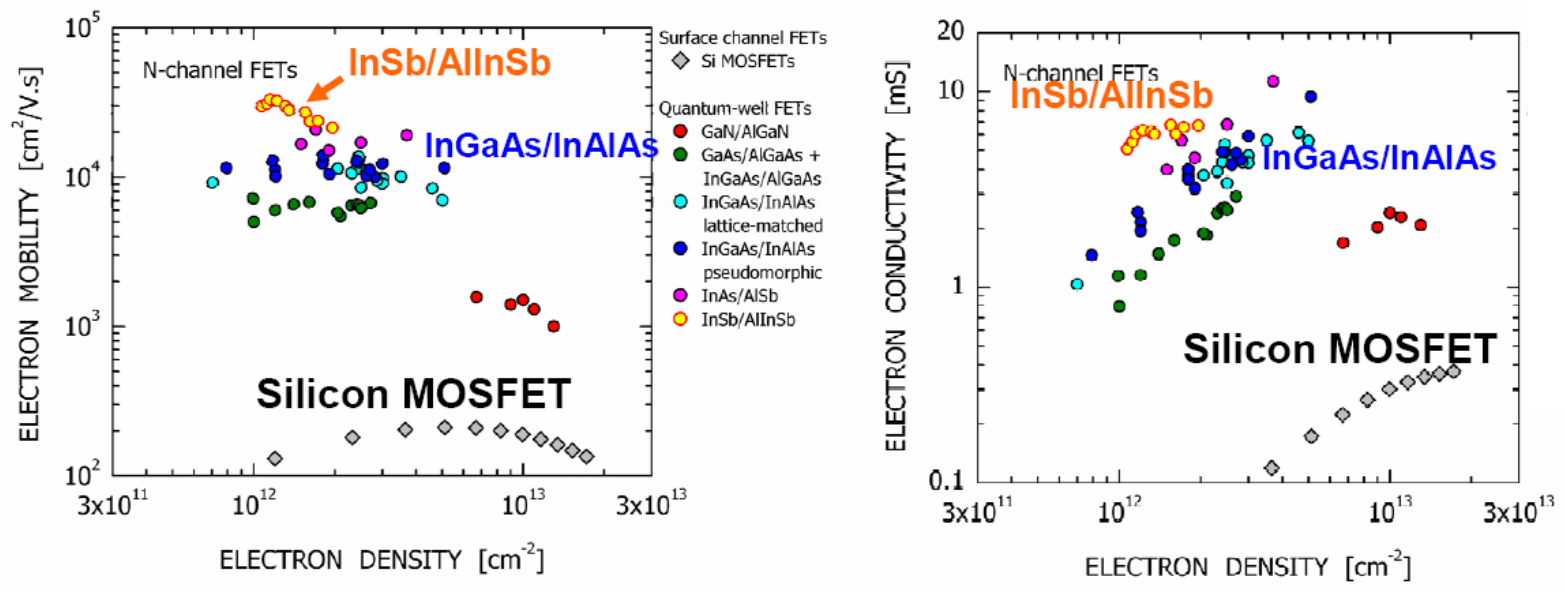

Figure 11 La mobilité électronique de Hall (Gauche) et la conductivité (Droite) en fonction de la densité de porteurs pour les hétéro-structures à base de matériaux III-V ou de silicium [Robert2005CSIC].
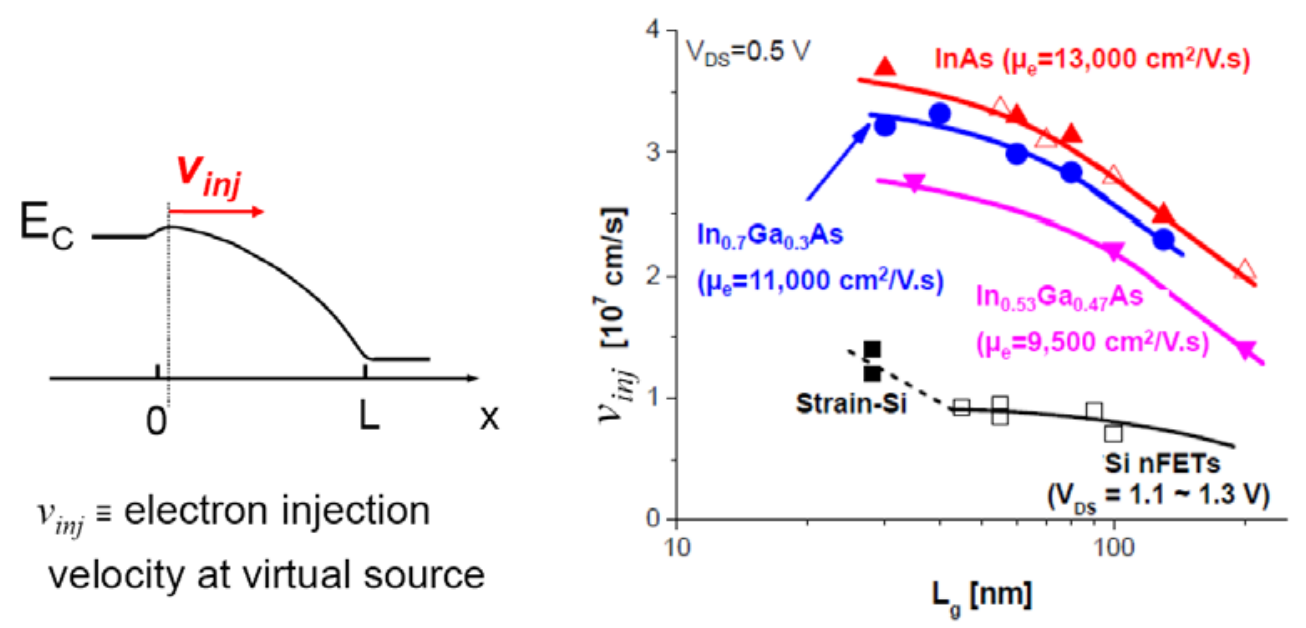

Figure 12 (Droite) Evolution de la vitesse d'injection au sommet de barrière d'injection (schéma à gauche) en fonction de la longueur de grille $\mathrm{L}_{\mathrm{G}}$ pour des transistors à base de III-V, Si contraint et non contraint. [Kim2009IEDM]

\section{Etats de l'art des FET à base de III-V matériaux}

\section{3-1 Les matériaux III-V}

Les matériaux III-V sont constitués des éléments des colonnes III et $\mathrm{V}$ de la classification périodique des éléments. Il existe des semi-conducteurs binaires comme l'arséniure de gallium (GaAs), l'antimoniure de gallium (GaSb), le phosphure d'indium (InP), 
l'arséniure d'indium (InAs), l'arséniure d'aluminium (AlAs). Il existe également les alliages ternaires et quaternaires qui en découlent. Ils présentent des propriétés très intéressantes pour les applications hyperfréquences. Ces alliages ternaires et quaternaires sont réalisés par substitution partielle de l'un des éléments par un élément de la même colonne. On sait, par exemple, obtenir des alliages des types $\mathrm{Ga}_{\mathrm{x}} \mathrm{Al}_{1-\mathrm{x}} \mathrm{As}$ ou $\mathrm{In}_{1-\mathrm{x}} \mathrm{Ga}_{\mathrm{x}} \mathrm{As}$. Ces alliages peuvent, comme dans le cas de 1 ' $\operatorname{In}_{0,53} \mathrm{Ga}_{0,47} \mathrm{As}$, donner des matériaux à très haute mobilité et alors constituer la zone active de dispositifs capables de fonctionner à très hautes fréquences.

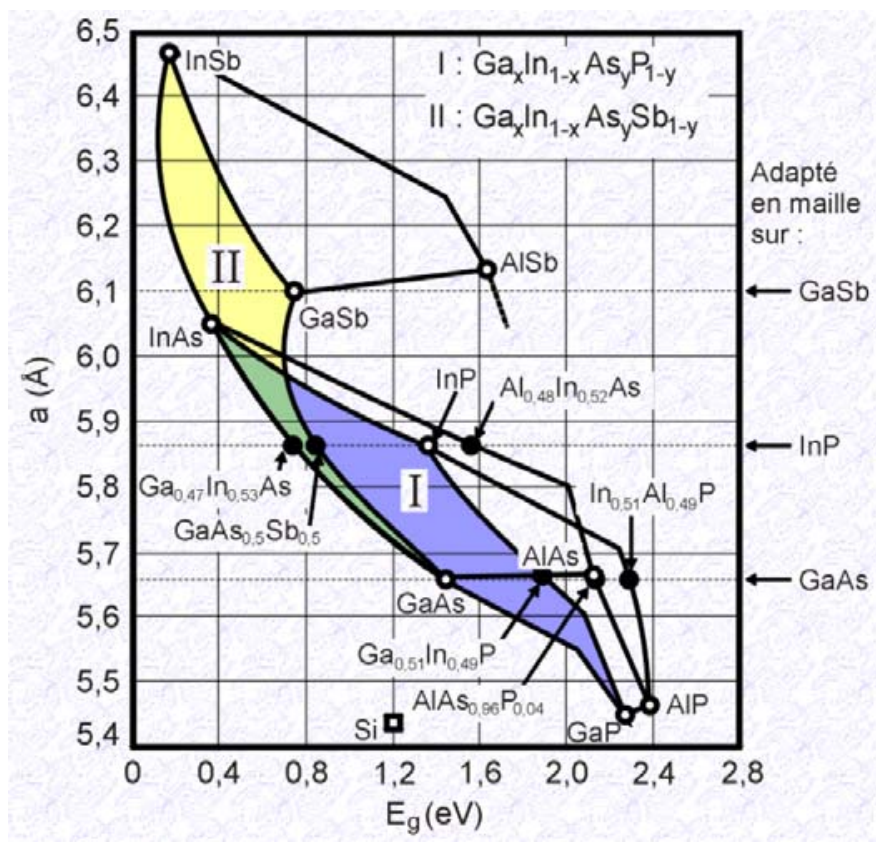

Figure 13 Bande interdite $E_{g}$ et paramètres cristallin des alliages de composés III-V [Chevalier1998thèse]

Le diagramme de la Fig. 13 représente les variations de la largeur de bande interdite $\mathrm{E}_{\mathrm{g}}$ en fonction du paramètre cristallin $a$ qui varie lui même avec la composition. Les points du graphe représentent la position des composés binaires stœchiométriques et les lignes représentent l'évolution continue du gap $\mathrm{E}_{\mathrm{g}}$ et du paramètre cristallin $a$ en fonction de la composition des alliages ternaires. Certaines lignes présentent un point anguleux qui dénote une transition entre un gap direct et un gap indirect. Ce diagramme est donc très important pour l'étude des alliages ternaires susceptibles d'être déposés en couche mince, par épitaxie, sur un substrat binaire comme GaAs ou InP. Les matériaux III-V offrent en fait une grande variété de compositions permettant de modifier leurs propriétés électroniques. On note que les recherches actuelles se focalisent sur les matériaux : InGaAs (avec des taux d'Indium de 53\%, $70 \%$ ou $80 \%$ ) et InAs. Ces deux types de matériaux à très haute mobilité appartiennent à deux filières différentes: InP et GaSb. Aujourd'hui, la technologie à base d'InP est plus mature pour les transistors HEMT à canal en InGaAs. 


\section{3-2 Transistor à haute mobilité électronique (HEMT)}

Le silicium est à la base de la plupart des composants à semi-conducteurs, mais des composants à base de matériaux III-V ont aussi attiré l'intérêt des chercheurs. Certains systèmes électroniques embarqués comme les récepteurs satellites, les radars et les téléphones portables, etc. utilisent déjà ces matériaux. Ils garantissent d'excellentes propriétés en termes de puissance à haute fréquence et de faible bruit. Les matériaux III-V couvrent une grande partie du spectre et ouvrent la voie à une multitude d'applications qui s'étendent des télécommunications à la technologie de la sécurité et du biomédical [Pavlidis2006].
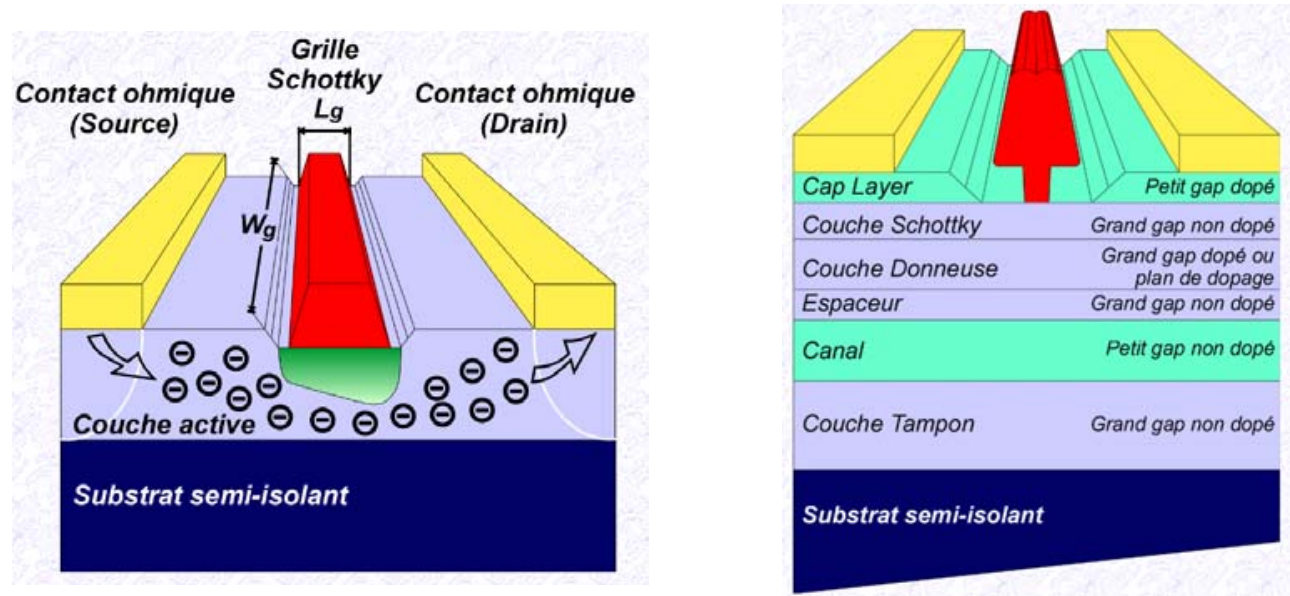

Figure 14 Structure d'un MESFET (gauche) et d'un HEMT (Droite)

La structure typique du transistor HEMT est schématisée sur la Fig. 14 [Chevalier1998thèse]. Dans cette structure, l'idée est d'utiliser une hétérojonction pour séparer spatialement les électrons et les impuretés ionisées, afin d'améliorer le transport électronique en réduisant l'interaction électrons-impuretés, ce qui favorise la montée en fréquence. La grande mobilité effective se trouve dans un canal non intentionnellement dopé. Cette structure est généralement appelée HEMT (High Electron Mobility Transistor) ou TEGFET (Two dimensional Electron Gas Field Effect Transistor) ou MODFET (Modulation Doped Field Effect Transistor) ou HFET (Heterojunction Field Effect Transistor). La topologie d'un HEMT semble très similaire à celle d'un MESFET (transistor à effet de champ à grille Schottky) conventionnel, mais dans le MESFET le transport a lieu dans une couche dopée et donc de faible mobilité.

Le HEMT est constitué essentiellement de trois matériaux différents : le substrat, un matériau à grand gap et un matériau à petit gap. On retrouve les électrodes de source, grille et drain, communes au transistor à effet de champ. La couche superficielle (appelée cap layer) est formée par un matériau de faible bande interdite pour permettre la réalisation des contacts 
ohmiques de source et de drain. Cette couche est généralement fortement dopée afin de diminuer la valeur des résistances de contact et donc celle des résistances d'accès. La couche à grand gap non dopée est destinée à la réalisation du contact Schottky de grille, qui est déposé après gravure du cap layer (fossé de grille ou recess). La couche de matériau à grand gap dopé a pour rôle de fournir les électrons libres à la structure, c'est la couche donneuse. Ce dopage, pouvant être volumique, est généralement réalisé par un plan de dopage au silicium. Vient ensuite l'espaceur (spacer), c'est une couche de matériau à grand gap non intentionnellement dopé (nid), permettant de séparer les atomes donneurs d'électrons de la couche active. Les interactions à courte distance électrons-impuretés sont ainsi fortement réduites. Plus cette couche sera épaisse, meilleure sera la mobilité des électrons dans le canal. A l'inverse, le transfert des électrons de la couche donneuse dans le canal est favorisé par un espaceur fin. Le canal est constitué d'un matériau à petit gap non intentionnellement dopé. Cette couche active reçoit le gaz bidimensionnel d'électrons. C'est elle qui déterminera les performances $\mathrm{du}$ transport des électrons dans le dispositif. Une couche tampon, communément appelée buffer, permet d'améliorer le confinement des électrons dans le canal en réduisant l'injection des porteurs vers le substrat. Cette couche permet également d'avoir un matériau de base de bonne qualité cristallographique nécessaire à la croissance des autres couches. Enfin, le substrat semi-isolant (SI) est un matériau binaire qui identifie la filière (GaAs, InP).

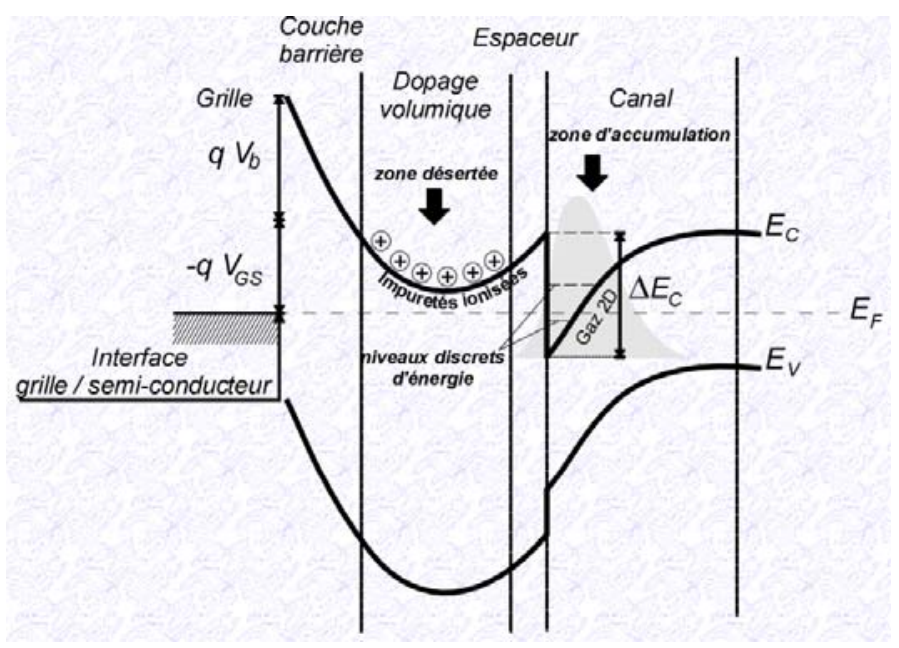

Figure 15 Structure de bande d'une hétérojonction en présence d'un potentiel de grille [http://www.polytech-lille.fr/cours-transistor-effet-champ/]

Dans le cas du HEMT, la juxtaposition d'un matériau à grand gap et d'un matériau à petit gap implique la création d'une discontinuité de bande de conduction à l'hétérointerface. Cette hétérojonction, illustrée par la Fig. 15, entraîne la formation d'un puits de potentiel dans le matériau à petit gap où transfèrent et s'accumulent les électrons provenant de la couche 
donneuse. L'hétérojonction est caractérisée par la discontinuité de bande de conduction $\Delta \mathrm{E}_{\mathrm{C}}$ entre les deux matériaux. Le transfert de charges génère dans la couche donneuse une zone désertée. Le profil électrique de charges détermine la courbure de bande de part et d'autre de l'hétérojonction, ce qui se traduit par la formation d'un puits de potentiel de forme triangulaire dans le canal. Pour une largeur de puits inférieure à la longueur d'onde de Broglie apparaissent les effets quantiques. Ces effets se traduisent par la quantification des niveaux d'énergie des électrons et par la restriction du mouvement des porteurs dans un plan parallèle à l'hétérojonction. On appelle gaz d'électrons bidimensionnel (2DEG : two Dimensional Electron Gas), l'accumulation des électrons dans ce puits. L'hétérojonction permet la séparation spatiale des atomes donneurs ionisés et des électrons libres. Ces électrons ne sont donc plus soumis aux interactions sur impuretés ionisées, et peuvent alors atteindre des mobilités importantes. Le HEMT bénéficie donc d'un transport électronique dans un gaz (quasi-bidimensionnel) bien supérieur à celui d'un matériau dopé. Notons que dans le cas de la Fig. 15, le canal du HEMT est situé entre deux matériaux de grand gap. La structure de bande n'est plus alors constituée d'une seule hétérojonction, comme sur la Fig. 15, mais d'une double hétérojonction, améliorant encore ainsi le confinement des électrons dans le canal. Les performances fréquentielles des HEMT sont liées au temps de transit entre la source et le drain. Monter en fréquence revient donc à réduire les dimensions du composant, mais également à utiliser des matériaux présentant des mobilités électroniques élevées. C'est cet aspect "matériaux" que nous allons aborder maintenant.

Quelques types principaux de HEMT ont été développés. Tels que :

\section{HEMT Conventionnel}

Sa couche active est constituée d'une hétérojonction GaAlAs $(\mathrm{N}+) / \mathrm{GaAs}$. Dès les premières réalisations en 1980, les performances en fréquence de transition, facteur de bruit et gain associé se sont montrées supérieures à celles du MESFET dont il reprend entièrement la technologie. Les performances du HEMT conventionnel sont limitées par la faible valeur de la discontinuité de bande de conduction $\Delta \mathrm{Ec}$, à l'interface de l'hétérojonction GaAlAs/GaAs $\left(\Delta \mathrm{E}_{\mathrm{C}} \approx 0,15\right.$ à $\left.0,2 \mathrm{eV}\right)$ et donc par la faible valeur de la densité de charges accumulées dans

GaAs $\left(\mathrm{N}_{\mathrm{S}} \approx 0,7\right.$ à $\left.0,8 \times 10^{12} \mathrm{~cm}^{-2}\right)$. Ses applications sont limitées à l'amplification faible signal bien que des structures à multiples hétérojonctions aient été proposées pour l'amplification de puissance. Cette structure de HEMT est pratiquement obsolète en technologie intégrée industrielle. 


\section{HEMT pseudomorphique}

Sa couche active est constituée d'une hétérojonction GaAlAs $(\mathrm{N}+) /$ GaInAs. Comme le matériau GaInAs n'est pas adapté en maille sur GaAs, seule la croissance d'une très faible épaisseur (10 à $20 \mathrm{~nm}$ ) est possible. Le matériau GaInAs est donc contraint ou pseudomorphique. Le transport s'effectue dans le matériau GaInAs non dopé. Les propriétés de transport de GaInAs contraint étant similaires à celles de GaAs, la supériorité du HEMT pseudomorphique sur le HEMT conventionnel réside uniquement dans une plus grande discontinuité $\Delta \mathrm{E}_{\mathrm{C}}$, à l'interface de l'hétérojonction GaAlAs/GaInAs $\left(\Delta \mathrm{E}_{\mathrm{C}} \approx 0,3 \mathrm{eV}\right)$, donc à une plus grande charge accumulée à l'interface $\left(\mathrm{N}_{\mathrm{S}} \approx 1.5 \times 10^{12} \mathrm{~cm}^{-2}\right)$ et à une meilleure efficacité de commande. Dans la mesure où sa technologie est identique à celle d'un HEMT conventionnel (ou d'un MESFET) et ses performances meilleures tant en faible signal qu'en puissance, il a remplacé le HEMT conventionnel (GaAlAs/GaAs) dans les technologies industrielles. Notons enfin que le caractère contraint du matériau GaInAs ne nuit pas à la fiabilité du dispositif.

\section{HEMT adapté en maille sur InP}

L'hétérojonction $\operatorname{InAlAs}(\mathrm{N}+) / \operatorname{InGaAs}$ adaptée en maille sur $\operatorname{InP}$ (pour une concentration d'indium de 53\%) est séduisante pour la réalisation de HEMT, car la discontinuité de bande de conduction est importante $\left(\Delta \mathrm{E}_{\mathrm{C}}=0,52 \mathrm{eV}\right)$ et les propriétés de transport de InGaAs sont excellentes (mobilité élevée $\mu \sim 10000 \mathrm{~cm}^{2} \mathrm{~V}^{-1} \mathrm{~s}^{-1}$, écart énergétique intervallée important $\left.\Delta \mathrm{E}_{\Gamma \mathrm{L}}=0,6 \mathrm{eV}\right)$. Avec des longueurs de grille de $100 \mathrm{~nm}$ voire moins (jusqu'à $25 \mathrm{~nm}$ ), cette filière a montré les meilleurs résultats en termes de fréquences de transition $\mathrm{f}_{\mathrm{T}}$. Elle présente aussi les plus faibles facteurs de bruit en gamme millimétrique. Des filières industrielles existent et les applications visées sont l'amplification faible bruit LNA (radar, radioastronomie, observations terrestres...) en bande W (75 à $110 \mathrm{GHz}$ ) et au-delà. Ces propriétés peuvent encore être améliorées par une couche active InGaAs en contrainte sur InP (en particulier pour une concentration d'indium supérieure à $53 \%$ en tendant vers le composé InAs). En contrepartie, ces composants ne peuvent travailler qu'à faible tension drain-source car le faible gap du matériau $\operatorname{In}_{0,53} \mathrm{Ga}_{0,47} \mathrm{As}\left(\mathrm{E}_{\mathrm{G}}=0,75 \mathrm{eV}\right)$ induit de l'ionisation par chocs dans le canal et limite fortement les applications de puissance.

\section{$\underline{\text { HEMT métamorphique sur GaAs }}$}


L'hétérojonction InAlAs $(\mathrm{N}+) / \mathrm{InGaAs}$ sur GaAs est complètement désadaptée mais une telle croissance en couche mince (10 à $20 \mathrm{~nm}$ ) est possible grâce une couche tampon InAlAs épaisse d'un micromètre environ et dont la composition en indium varie graduellement à partir d'une valeur faible côté substrat. Le matériau constituant le transistor est alors relaxé. Les taux d'indium peuvent être similaires à la filière $\mathrm{InP}$ ( $53 \%$ et plus), entraînant des performances fréquentielles comparables. L'avantage majeur est la plus grande disponibilité à plus faible coût du substrat de GaAs par rapport à l'InP. Ces filières sont aujourd'hui disponibles en technologie industrielle.

\section{$\underline{\text { HEMT de la filière GaN }}$}

Cette filière de composants redore aujourd'hui les activités autour des composants III-V. Ce matériau présente un niveau de bande interdite de $3,47 \mathrm{eV}$ et une mobilité électronique de l'ordre de $\mu \sim 2000 \mathrm{~cm}^{2} \mathrm{~V}^{-1} \mathrm{~s}^{-1}$. De telles caractéristiques permettent une alimentation continue $\mathrm{V}_{\mathrm{DD}}$ de grande valeur (quelques dizaines de volts), par ailleurs les bonnes caractéristiques de transport destinent cette filière de HEMT aux applications de forte puissance jusqu'aux ondes millimétriques. Des circuits intégrés de puissance sont d'ores et déjà commercialisés.

\section{3-3 Etat de l'art des transistors hyperfréquences}

La Fig. 16 représente les performances fréquentielles des transistors hyperfréquences, toutes filières confondues, au cours des 35 dernières années [Schwierz2001MR, 2003SSE et 2007SSE] et [Pavlidis1999]. Nous pouvons constater que les filières historiques silicium CMOS et bipolaires ont rattrapé depuis ces dernières années le domaine des $100 \mathrm{GHz}$ et au delà, domaine jusqu'alors réservé aux filières de transistors III-V.

L'état de l'art en $\mathrm{f}_{\mathrm{T}}$ et $\mathrm{f}_{\mathrm{MAX}}$ des transistors à effet de champ est donné aux Fig. 17. Ces fréquences caractéristiques atteignent environ $550 \mathrm{GHz}$ pour les filières HEMT sur InP et 250 $\mathrm{GHz}$ pour les MOSFET. Toutefois, nous devons noter que les filières HBT (transistor bipolaire à hétérojonctions) sur InP atteignent des fréquences limites de l'ordre de 500 à $600 \mathrm{GHz}$. Comme pour les bipolaires dans les FET l'optimisation simultanée de $\mathrm{f}_{\mathrm{T}}$ et $\mathrm{f}_{\mathrm{MAX}}$ n'est jamais obtenue. Pour les transistors de longueur de grille supérieure à $100 \mathrm{~nm}, \mathrm{f}_{\mathrm{MAX}}$ est supérieure ou égale à 2 fois $\mathrm{f}_{\mathrm{T}}$. Ce rapport entre ces deux fréquences diminue et tend vers l'unité pour les transistors les plus petits $\left(\mathrm{L}_{\mathrm{G}}<100 \mathrm{~nm}\right)$, cela est principalement dû à l'augmentation des effets de canal court (perte du contrôle électrostatique du canal par la 
grille) mais aussi à l'importance que prennent les éléments parasites (résistances, capacités) par rapport aux éléments intrinsèques. Les causes limitant la montée en fréquence (rapport $\mathrm{f}_{\mathrm{MAX}} / \mathrm{f}_{\mathrm{T}}$ ) sont les mêmes pour les MOSFET.

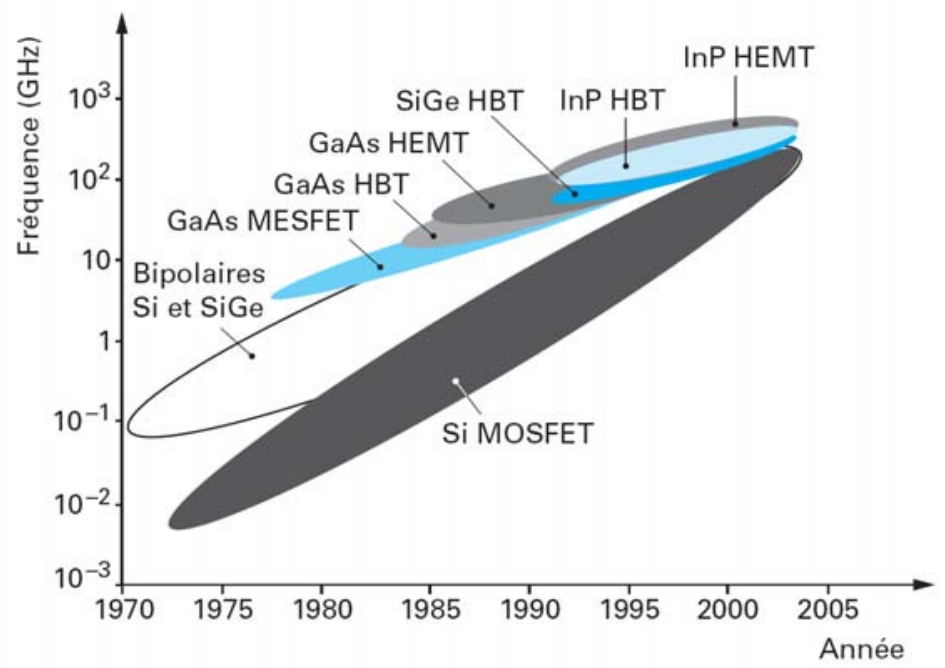

Figure 16 Performances fréquentielles des transistors hyperfréquences
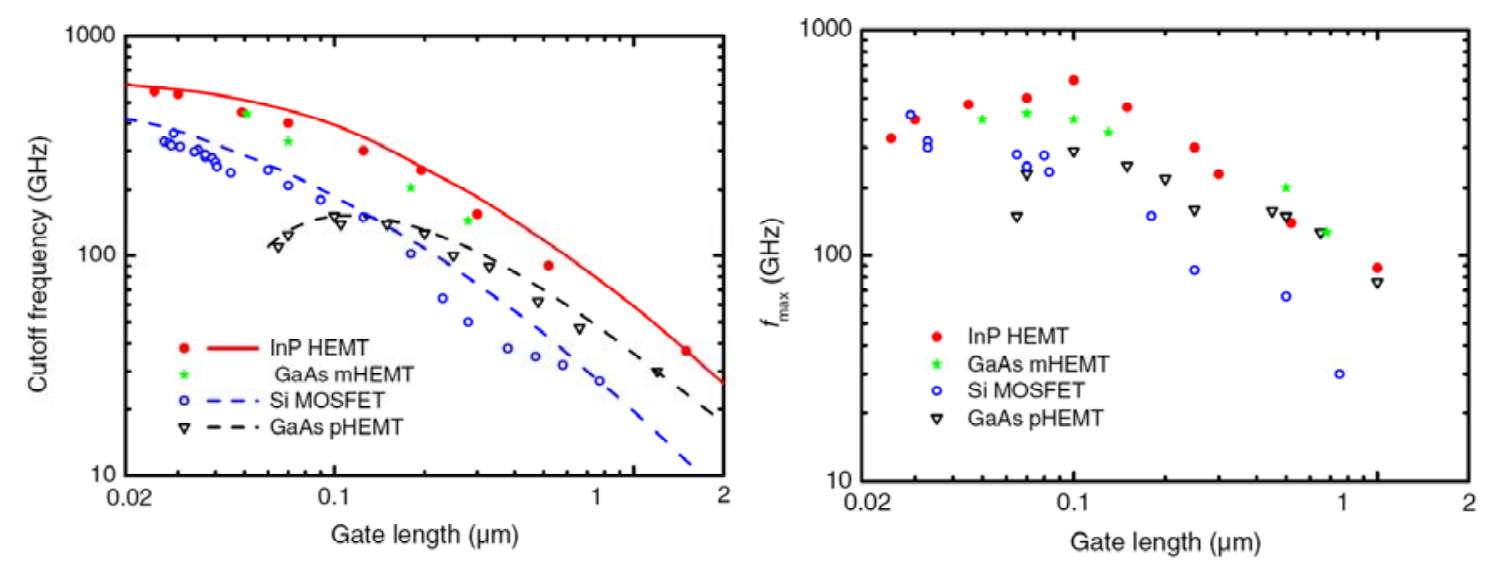

Figure 17 Gauche : fréquence de coupure $f_{T}$; droite : fréquence maximale d'oscillation $\mathbf{f}_{\text {MAX }}$ des HEMT

III-V et MOSFET Si en fonction de la longueur de grille $\mathbf{L}_{\mathrm{G}}$. Les lignes sont des guides pour l'œil

[Schwierz2001MR, 2003SSE, 2007SSE]

On peut citer deux exemples de HEMT pseudomorphiques à canal InAs [Kim2008IEDM] et $\operatorname{In}_{0,7} \mathrm{Ga}_{0,3}$ As [Kim2010IEDM] avec des longueurs de grille de respectivement $30 \mathrm{~nm}$ et $50 \mathrm{~nm}$. Ils présentent les meilleures performances à ce jour et sont illustrés sur la Fig. 18. Les deux HEMT fonctionnent en mode 'enrichissement', le rapport d'aspect est soigneusement choisi $\left(\mathrm{L}_{\mathrm{G}} \mathrm{sub}-50 \mathrm{~nm}\right)$ via un contrôle sur la distance $\mathrm{t}_{\mathrm{ins}}$ (distance entre le contact du métal de grille et la couche du canal). On peut noter que pour le HEMT à base de l'InAs, $f_{T}$ et $f_{M A X}$ sont supérieures à $600 \mathrm{GHz}$. Pour le HEMT à base d' $\mathrm{In}_{0,7} \mathrm{Ga}_{0,3} \mathrm{~A}, \mathrm{f}_{\mathrm{T}}=465 \mathrm{GHz}$ et $\mathrm{f}_{\mathrm{MAX}}$ sont supérieures à $1 \mathrm{THz}$. 

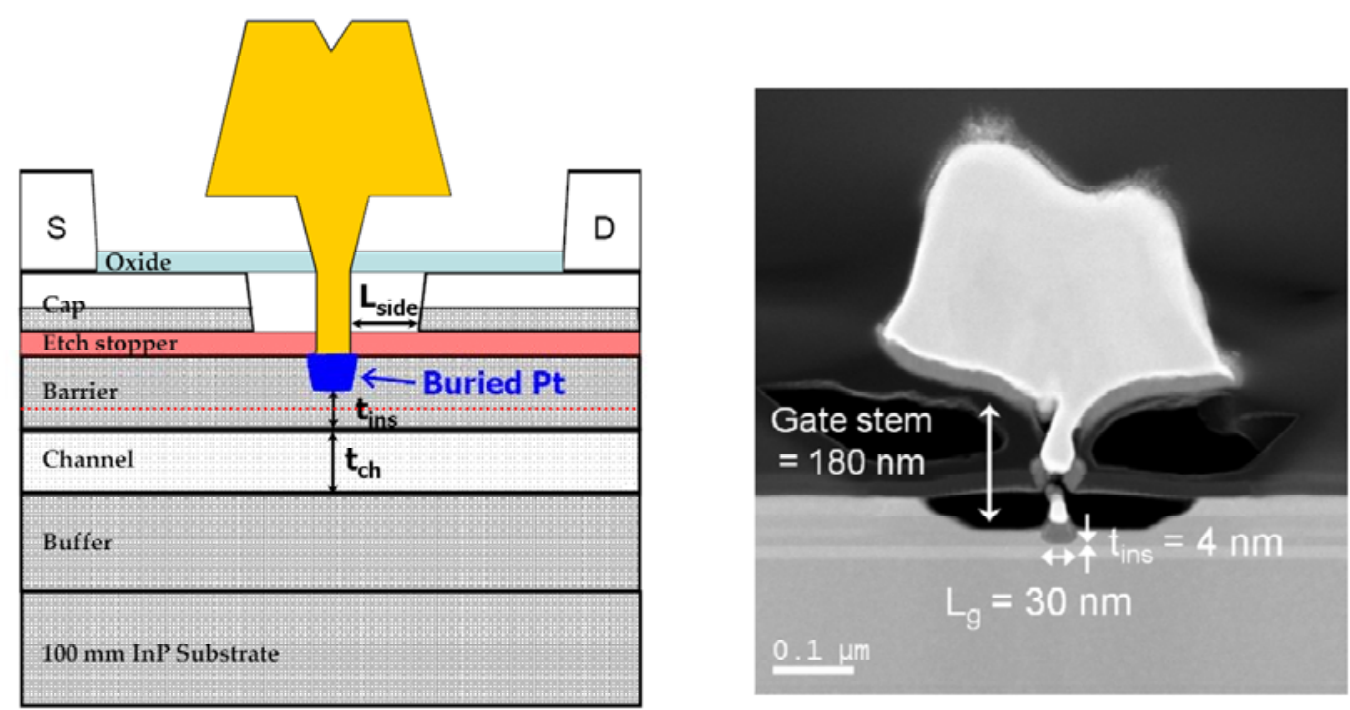

Figure 18 Structure et image au MEB de l'HEMT pseudomorphique à canal $\mathrm{In}_{0,7} \mathrm{Ga}_{0,3} \mathrm{As}\left(\mathrm{L}_{\mathrm{G}}=50 \mathrm{~nm}\right)$ et InAs $\left(L_{G}=30 \mathrm{~nm}\right)$ sur un substrat InP $100 \mathrm{~mm}$ où $t_{\text {ins }}=4 \mathrm{~nm}, t_{c h}=10 \mathrm{~nm}, L_{\text {side }}=150 \mathrm{~nm}$. Le dispositif est fabriqué avec une grille en Pt enterrée dans le grand gap InAIAs. La mobilité de Hall de l'InAs est 13000 $\mathrm{cm}^{2} \mathrm{~V}^{-1} \mathrm{~s}^{-1}$ et celle d' $\mathrm{In}_{0.7} \mathrm{Ga}_{0.3} \mathrm{As} 11000 \mathrm{~cm}^{2} \mathrm{~V}^{-1} \mathrm{~s}^{-1}$
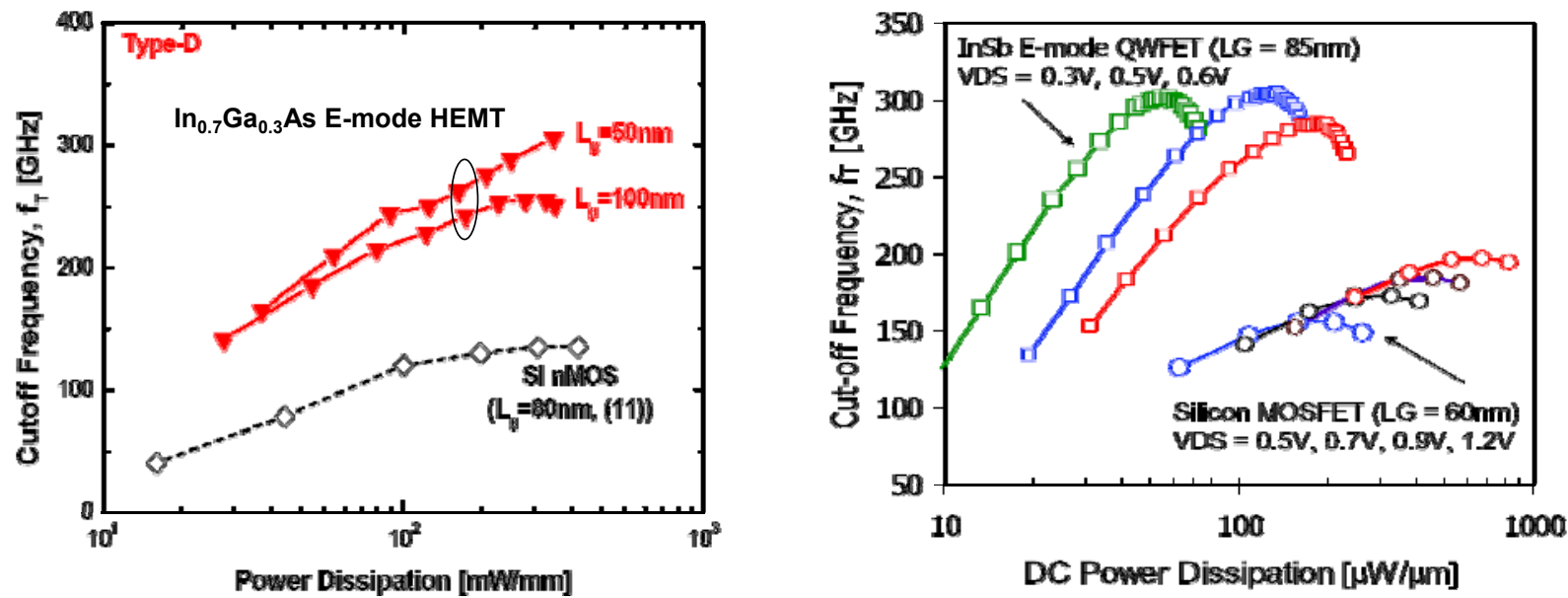

Figure 19 Fréquence de coupure $\mathrm{f}_{\mathrm{T}}$ en fonction de la puissance de consommation en DC régime. La comparaison de la performance est effectuée pour les structures de HEMT à canal de l'InGaAs et InSb et de MOSFET à canal Si. La longueur de grille $L_{G}$ et la condition de polarisation $V_{D S}$ sont aussi indiquées.

On peut observer (cf. Fig. 19 Kim2007IEEE, Datta2007ME) que les HEMT à base de matériaux III-V présentent un très bon compromis entre la performance fréquentielle et la consommation d'énergie par rapport au cas des dispositifs Si. Le fonctionnement du transistor de type HEMT sera donc bien assuré en domaine HF même sous faible tension d'alimentation $\mathrm{V}_{\mathrm{DD}}(\leq 0,5 \mathrm{~V})$ contrairement aux MOSFET Si (où $\mathrm{V}_{\mathrm{DD}} \sim 1 \mathrm{~V}$ pour assurer un fonctionnement correct). 


\section{3-4 Evaluation des potentiels pour les applications logique}

On va évaluer les applications logiques des HEMT III-V en les comparant avec les MOSFET à base de Si. Tout d'abord, on s'intéresse au contrôle électrostatique : pente sous le seuil SS et DIBL. Les résultats sont extraits des conférences IEDM2006-2008 et AVS 2010 par T. W. Kim et Jesús A. del Alamo (cf. Fig. 18).

Grâce au confinement du gaz 2D (2DEG) au sein du canal $\left(\mathrm{t}_{\mathrm{ch}} \sim 10 \mathrm{~nm}\right.$ en technologie courante), même si le canal est non intentionnellement dopé, les HEMT III-V sont moins affectés par les effets de canal court par rapport au MOSFET Si avec la même longueur de grille, si le rapport d'aspect $\left(\mathrm{L}_{\mathrm{G}} / \mathrm{t}_{\text {ins }}\right)$ est soigneusement choisi. Par ailleurs, le fort confinement géométrique pourra encore améliorer le contrôle électrostatique. Les résultats de T. W. Kim [Kim2010IPRM] montrent ces améliorations (cf. Fig. 20).
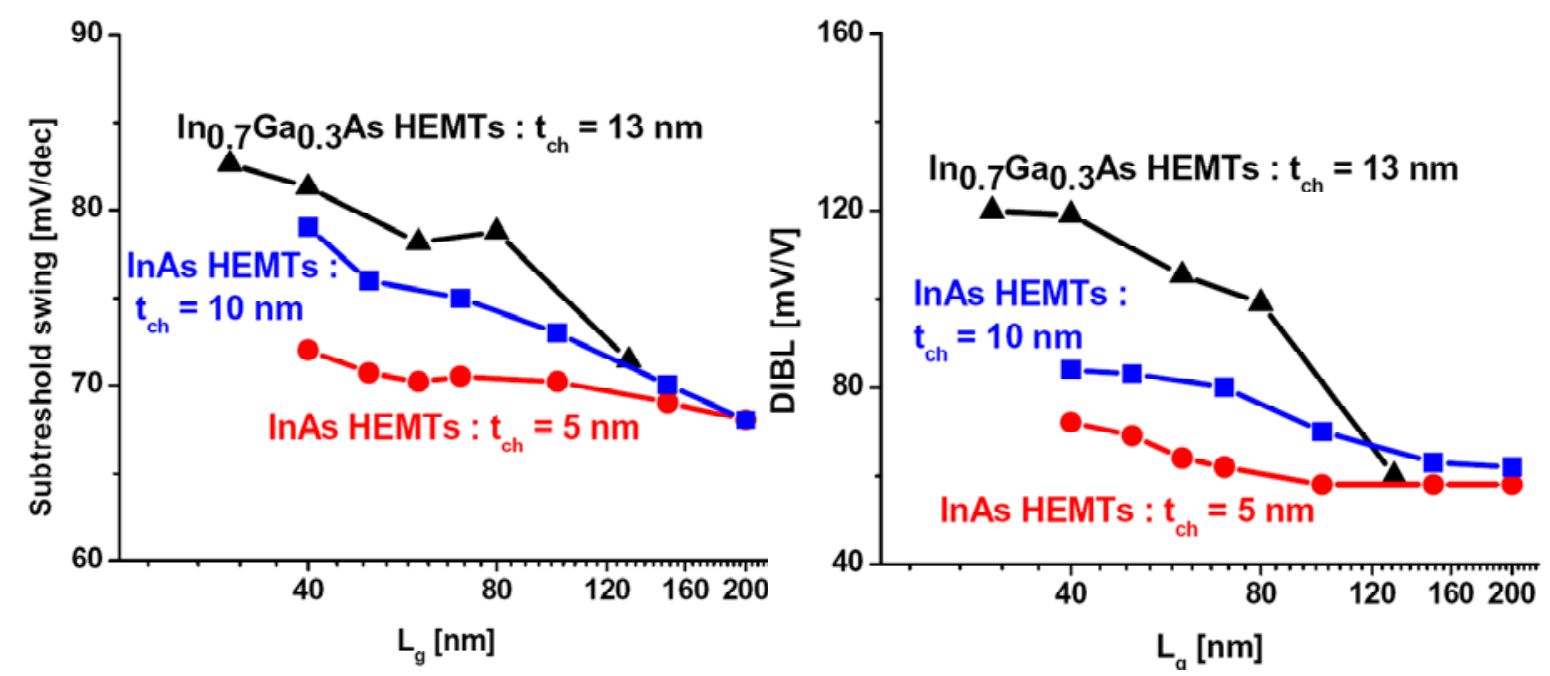

Figure 20 Gauche : pente sous le seuil (subthreshold swing) et droite : DIBL en fonction de la longueur de grille pour des HEMT à canal InAs et InGaAs avec différentes épaisseurs du canal $\left(t_{\mathrm{ch}}\right)$. [KimIPRM2010].

On peut comparer plus explicitement les performances des transistors HEMT et MOSFET pour les applications logiques. On utilise les facteurs mérites comme le délai intrinsèque de la grille $\left(\mathrm{C}_{\mathrm{G}} \mathrm{V}_{\mathrm{DD}} / \mathrm{I}_{\mathrm{ON}}\right)$ [Kim2008-2009IEDM, Del-Alamo2010AVS] et le produit énergie - délai (énergie de commutation: $\mathrm{C}_{\mathrm{G}} \mathrm{V}_{\mathrm{DD}} / \mathrm{I}_{\mathrm{ON}} \times \mathrm{C}_{\mathrm{G}} \mathrm{V}_{\mathrm{DD}}{ }^{2}$ ) [Chau2007Nature, Chau2005CSICS et Chau2008CS]. On note que les HEMT III-V possèdent un faible délai intrinsèque surtout à faible $\mathrm{V}_{\mathrm{DD}}$ par rapport à celui de $\mathrm{Si}$ (cf. Fig. 21). Cela permet une faible énergie de commutation (cf. Fig. 21). La meilleure performance est obtenue dans le HEMT à base des matériaux antimoniures. Les matériaux III-V (InGaAs, InAs et InSb) sont des candidats pour les applications logiques à haute performance et basse consommation. 

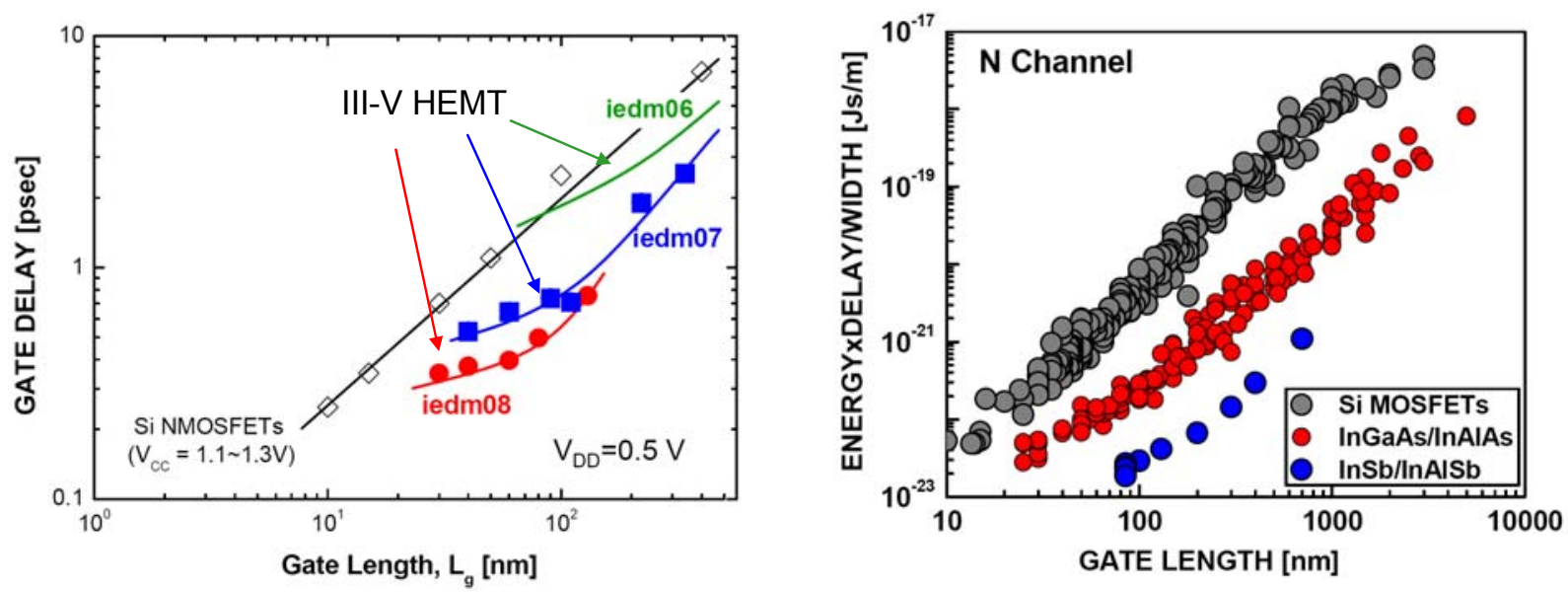

Figure 21 Gauche : délai intrinsèque de grille ; droite : produit énergie - délai pour les HEMT à canal IIIV (InGaAs, InAs et InSb) et les MOSFET à canal de Si.

\section{3-5 Limitation du dimensionnement des HEMT}

Les futurs dimensionnements des transistors de type HEMT seront toujours effectués en optimisaant également les couches de l'empilement afin de limiter les effets de canal court. La performance fréquentielle est très sensible à ces effets. Autrement dit, la miniaturisation est non seulement latérale (longueur de grille) mais aussi verticale (les couches d'épitaxie). Néanmoins, aujourd'hui ou à très court terme, ces architectures auront atteint leurs limites en termes de lois d'échelle car ils présentent une faible isolation de grille à cause de la faible hauteur de barrière Schottky (avec le semi-conducteur à grand gap) qui autorise des courants de fuite tunnel par la grille élevés et donc une consommation statique importante [Datta2007ME] (cf. Fig. 22, 25).

L'architecture HEMT actuelle possède également d'autres inconvénients.

i) Le rapport d'aspect (un rapport dimensionnel établi entre la distance verticale grille - canal et la longueur du canal) reste faible par rapport à celui des MOSFET. La meilleure valeur est limitée à environ 10 [Kim2008, 2010IEDM] à comparer à 25 voire plus [Schwierz2007] pour l'architecture MOSFET. Ce nombre limite directement le potentiel des lois d'échelle.

ii) Les empreintes (Footprint) associées aux HEMT restent très grandes par rapport à la topologie MOS (cf. Fig. 23), la densité d'intégration est considérablement plus faible. Pour l'instant, les HEMT sont utilisés pour des systèmes 'front-end' et les applications HF, sur lesquels la densité d'intégration n'est pas une préoccupation majeure. L'état de l'art des HEMT montre typiquement une séparation Source-Drain de l'ordre de 1,5 à $2 \mu \mathrm{m}$ très loin du standard VLSI 


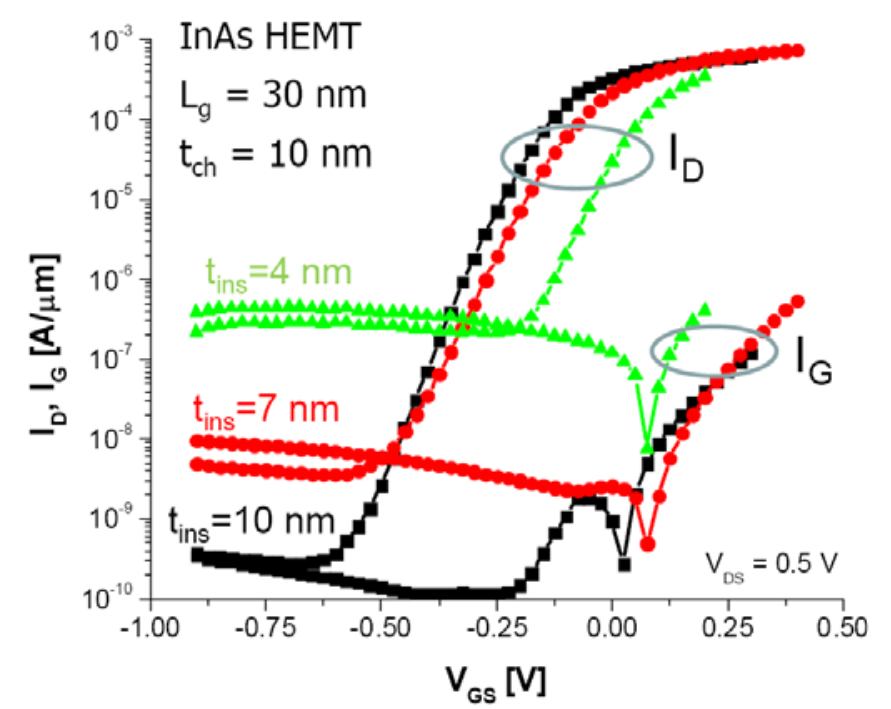

Figure 22 Augmentation du courant de fuite à cause de la diminution de l'épaisseur de la barrière grand gap $t_{\text {ins }}$ [Del-Alamo2010AVS].

iii) La réalisation de contacts auto alignés est difficile à effectuer par l'architecture HEMT [Waldron2007IEDM]. La technique d'auto-alignement la plus répandue pour le système InAlAs/InGaAs est d'utiliser une tête de grille en forme de T (cf. Fig. 23) comme un masque pour la déposition métallique de la source et du drain [Mishra1992IEDM] (la forme en T de la grille n'est pas adaptée pour le VLSI). Des excellents résultats ont été obtenus pour un dispositif de $50 \mathrm{~nm}$ en utilisant cette approche. Mais le degré d'auto-alignement est déterminé par la taille de la tête de la grille et l'espacement source-drain est toujours supérieur à $0,3 \mu \mathrm{m}$ [Moran2006IEEE]. Les capacités parasites de bord introduites par les chevauchements entre grille en $\mathrm{T}$ et les régions de source/drain poseraient toujours des inconvénients non négligeables. Les architectures actuelles sont conçues avec une conception multi-doigts pour réduire principalement les résistances de grille afin d'augmenter la performance fréquentielle (surtout en $\mathrm{f}_{\mathrm{MAX}}$ ), mais les capacités parasites grille/source et grille/drain sont aussi augmentées.

iv). Quand on réduit la longueur de la zone du contact, comme dans les travaux de [Waldron2010IEEE], la séparation entre la grille et les contacts ohmiques est dimensionnée en dessous de $30 \mathrm{~nm}$ (dimension 30 fois plus faible que dans les designs conventionnels). La résistance associée aux couches du grand gap domine la résistance de source. Si le dispositif est dimensionné vers une dimension réelle de VLSI, la résistance de source augmente considérablement (100 fois plus grande que la valeur ciblée par le nœud $22 \mathrm{~nm}$ ) si les technologies de la couche cap layer restent conventionnelles. L'auteur préconise le remplacement de la couche barrière par un diélectrique high- $k$ et le retraitement de la couche 
cap layer (augmenter le taux d'Indium voire un matériau purement binaire comme InAs), ce qui permet de diminuer la résistivité du contact et ainsi que la résistance de l'empilement du contact (cf. Fig. 24).
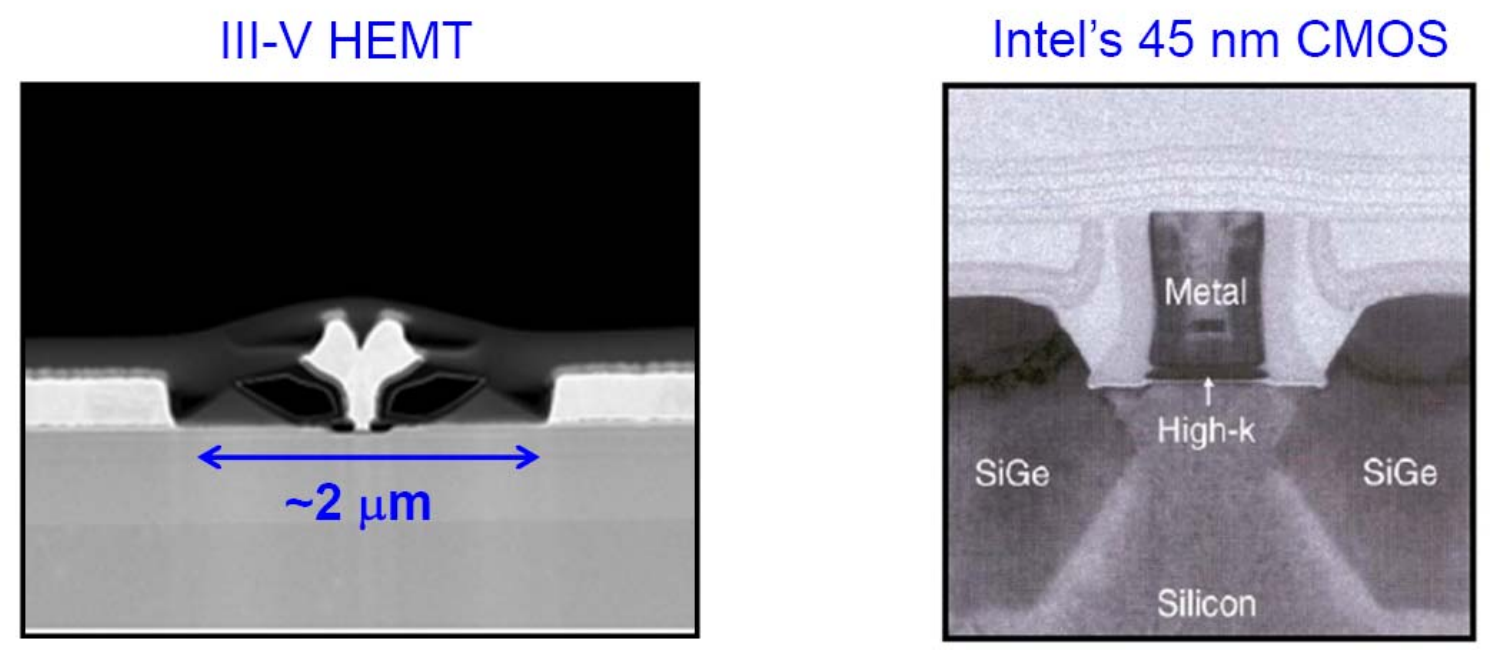

Figure 23 Images au MEB, d'une coupe de transistor à HEMT III-V standard (gauche) [DelAlamo2011EMRS] et d'un MOS-Bulk à Si contraint d'Intel en technologie 45 nm [MistryIEDM2007].

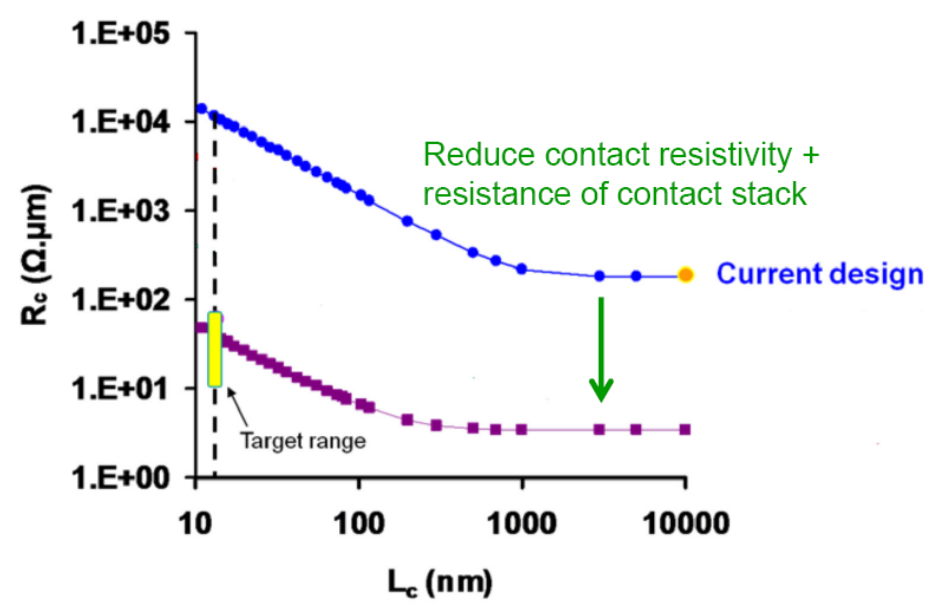

Figure 24 Effet du dimensionnement de la longueur de contact sur la résistance de contact pour un HEMT auto-aligné

Combiner l'utilisation des matériaux III-V avec la topologie MOS incluant un diélectrique high- $k$ semble une solution viable pour atteindre les critères spécifiés en termes de fréquence et de puissance de consommation.

\section{Etats de l'art des MOSFET III-V}


Les MOSFET III-V bénéficient des bonnes propriétés de transport, surtout sous faible condition de polarisation, des matériaux III-V. Le temps de transit des porteurs est faible et il assure donc de bonnes performances en fréquence. En parallèle, la topologie MOS à grille isolée permet de limiter les courants de fuite (cf. Fig. 25) tout en améliorant le rapport d'aspect ainsi que le potentiel en termes de loi d'échelle. Le design avec les contacts auto alignés permet de diminuer les éléments parasites.
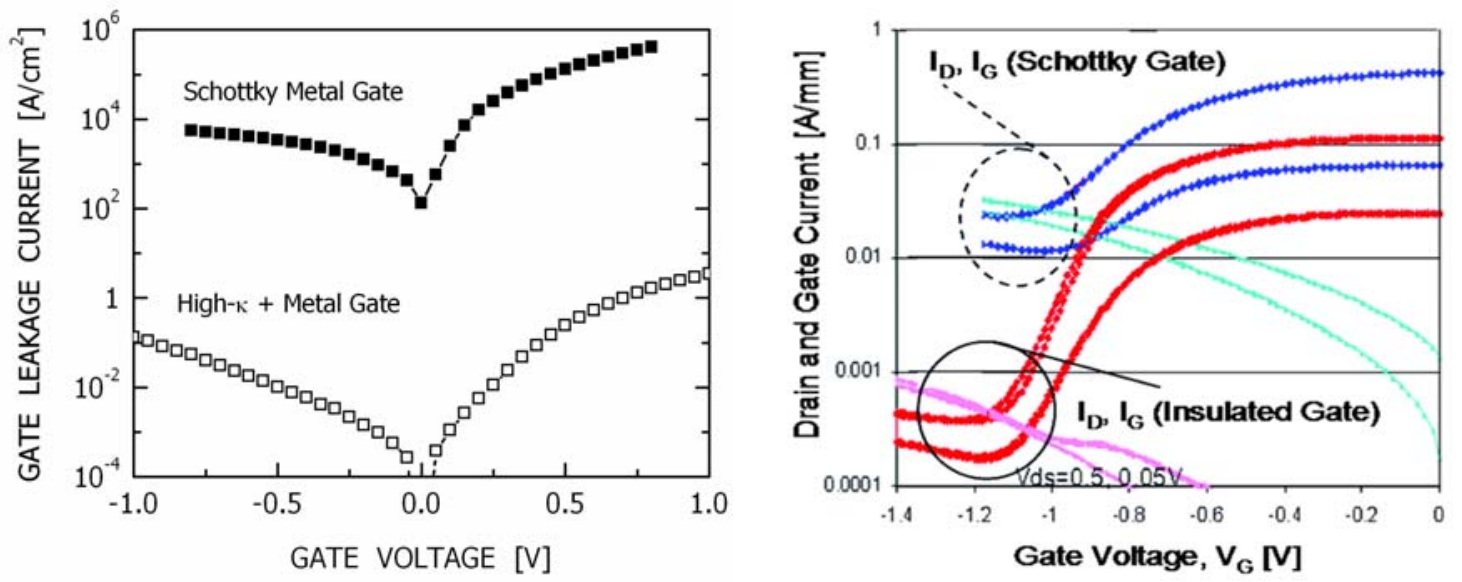

Figure 25 Gauche : courant de fuite en fonction de la tension de grille pour des empilements de grille Schottky métal/III-V et MOS métal/high-k/III-V. Droite : caractéristique de transfert de QWFET avec grille Schottky et grille isolée avec diélectrique $\mathrm{Al}_{2} \mathrm{O}_{3}$ [Datta2007ME].

Cependant, cette topologie doit franchir un certain nombre d'obstacles technologiques :

i) le faible gap peut générer non seulement un courant de fuite à état OFF par effet tunnel bande à bande, particulièrement sous forte polarisation de drain $V_{D S}$ [Fischetti2007IEEE], mais également une faible tension de claquage par ionisation par impact [Choo2004JAP].

ii) la densité des états (ou DOS pour density of states) intrinsèque des matériaux III-V à faible gap (InGaAs, InAs et InSb) est quasiment 100 fois (voire encore plus faible) plus faible que celle de Si [Sze1981]. Autrement dit, les places disponibles pour la population des porteurs sont 100 fois moindres que dans le Si. Donc l'effet capacitif de grille n'est pas autant efficace que dans le Si, c'est le "DOS bottleneck"[Fischetti2007IEDM]).

iii) les constantes diélectriques du matériau III-V à faible gap sont souvent plus grandes. Donc ils ont une plus faible immunité aux effets de canal court [Shinichi2008IEEE]. 
iv) la faible efficacité de l'implantation empêche la réalisation de bons contacts d'ohmiques. Les résistances séries parasites ont tendance à être élevées [Passlack2002IEEE, Thayne2009ECS et Waldron2010IEEE].

v) La qualité de l'interface high-k/III-V n'est pas idéale car la technologie n'est pas mature. Des pièges d'interfaces chargés existent, parfois en grand nombre, et l'interface peut être rugueuse. Cela dégrade les propriétés du transport et le contrôle électrostatique (ancrage du niveau de Fermi).

Il reste globalement beaucoup de travail pour maîtriser les processus technologiques, concevoir les architectures adaptées et également ajuster les modèles physiques. Dans la prochaine section, nous allons citer certains travaux remarquables.

\section{4-1 Système d'empilement diélectrique de grille (Métal/high-k/III-V) : qualité de l'interface}

Pour réaliser des MOSFET à canal III-V, un empilement diélectrique de grille adapté et/ou approprié doit être développé. Plus concrètement, on doit avoir :

i) une interface stable vis-à-vis du budget thermique du processus de fabrication du transistor,

ii) un diélectrique $h i g h-k$ (grande constante diélectrique) ayant une discontinuité de bande de conduction suffisante avec le matériau semi-conducteur III-V pour assurer une bonne densité électronique dans la couche active et aussi supprimer le courant de fuite par la grille,

iii) une interface propre avec une faible densité d'états de pièges d'interface $\left(D_{i t}\right)$ et avec une faible rugosité.

Alors que certains diélectriques $\left(\mathrm{Al}_{2} \mathrm{O}_{3}, \mathrm{HfO}_{2}, \mathrm{ZrO}_{2} \ldots\right)$ permettent de répondre au $1^{\mathrm{er}}$ et au $2^{\text {nd }}$ critères, le $3^{\text {ème }}$ critère reste extrêmement contraignant. La haute densité $D_{\text {it }}$ dégrade le contrôle du niveau de Fermi voire génère l'ancrage du niveau de Fermi à l'interface. Cela peut impliquer des problèmes majeurs en termes de contrôle de charge ce qui empêche de réaliser un bon transistor FET c'est-à-dire avec une faible pente sous le seuil et un grand courant à l'état $\mathrm{ON}$. Contrairement au $\mathrm{Si}$, les semi-conducteurs III-V ne possèdent pas d'oxyde natif de haute qualité et de bonne stabilité. De plus, la surface semi-conductrice III-V elle-même peut être sujette à des $D_{i t}$ élevés, tels que les lacunes, les défauts interstitiels ou dus à la dimérisation incomplète de la surface. Ce dernier effet peut générer des liaisons insaturées 
qui forment des pièges électriquement actifs [Robertson2011APL] qui peuvent être présents avant même la croissance du diélectrique $h i g h-k$ ou sont générés lors du dépôt.

Les méthodes expérimentales permettant de quantifier le $\mathrm{D}_{\mathrm{it}}$, la position du niveau d'énergie des pièges et l'efficacité du mouvement du niveau de Fermi à l'interface sont essentielles dans le développement d'interfaces high-k/III-V de haute qualité. Pour les composants III-V, en particulier, la caractérisation de la capacité pour une structure métaloxyde-semiconducteur (MOSCAP) est importante. Le processus de fabrication des transistors lui-même peut introduire des incertitudes supplémentaires qui doivent être traitées après l'optimisation initiale de l'interface high-k/III-V [Singisetti2009IEEE]. Les méthodes pour déterminer le $\mathrm{D}_{\text {it }}$ de l'interface diélectrique/semi-conducteur en utilisant des structures MOSCAP ont été développées pour l'empilement $\mathrm{SiO}_{2} / \mathrm{Si}$ depuis les années 1960 [Sze1981 et Nicollian1982]. A cause des plusieurs facteurs, l'interprétation de la réponse petit signal (en admittance) de la structure MOSCAP high-k/III-V en termes de $\mathrm{D}_{\text {it }}$ est plus difficile que celle de $\mathrm{Si}$ : faible DOS ena bande de conduction, temps de réponse des porteurs minoritaires difficile de déterminer [Ashkar2010IEEE, Enge-Herbert12010JAP]. Par ailleurs, la distribution en énergie du $\mathrm{D}_{\mathrm{it}}$ est différente de celle des interfaces dans les systèmes en $\mathrm{Si}$ [Spicer1980JVST].

Aujourd'hui, la passivation, le traitement et la caractérisation de la surface des MOSCAP III-V, sont les efforts principaux dans la réalisation de la technologie III-V MOS. Souvent, la qualité de surface est représentée par le profil de $\mathrm{D}_{\text {it }}$ dans le gap du matériau semiconducteur ou simplement par la valeur de $D_{\text {it }}$ au milieu du gap (mid-gap). Différentes méthodes existent pour déterminer ce profil :

i) l'interprétation du décalage de la courbe $\mathrm{C}-\mathrm{V}$ avec le travail de sortie $\varphi_{\mathrm{m}}$ du métal de grille [Kim2009JAP, Koveshnikov2008APL];

ii) la méthode de Castagné-Vapaille HF-LF (en combinant la capacité à haute et basse fréquence) [Castagne1971SS] ;

iii) la méthode de Terman (capacité à haute fréquence) [Goel2007APL, Chang2008APL, Lee2008APL] ;

iv) les analyses de Berglund (capacité quasi-statique et à basse fréquence) [Kim2009JAP, Kim2010APL, Ye2008JVST];

v) la méthode de conductance [Brammertz2009APL, Chiu2008APL, Lin2009Meng, Zhao2009APL, Goel2007APL, Hurley2009ECS, Connor2009APL, Hwang2010APL, Huang2009IEDM, Martens2008IEEE] ;

vi) la méthode de la pente sous le seuil [Varghese2008IEDM]; 
vii) la méthode du pompage de charge [Maneglia1996JAP];

viii) la méthode de l'efficacité du niveau de Fermi [Lin2009APL]. Récemment, l'université de Purdue a analysé la densité d'états d'interface dans une structure de $\mathrm{Al}_{2} \mathrm{O}_{3} / \mathrm{In}_{0,53} \mathrm{Ga}_{0,47} \mathrm{As}$ (type $\mathrm{p}$ avec $\mathrm{N}_{\mathrm{A}}=10^{17} \mathrm{~cm}^{-3}$ ) en utilisant ces principales techniques de métrologies [Varghese2008IEDM] (cf. tableau 4).

\begin{tabular}{|c|c|c|c|}
\hline \multirow{2}{*}{ Technique } & \multicolumn{3}{|c|}{$\mathrm{D}_{\text {it }}\left(\mathrm{cm}^{-2} \mathrm{eV}^{-1}\right)$} \\
\cline { 2 - 4 } & $\mathrm{BC}$ & mi-gap & $\mathrm{BV}$ \\
\hline Conductance & $2 \times 10^{12}$ & $3 \times 10^{12}$ & \\
\hline HF-LF & $6,7 \times 10^{12}$ & $8,4 \times 10^{12}$ & $1,6 \times 10^{13}$ \\
\hline Berglund (LF) & $5,6 \times 10^{12}$ & $1 \times 10^{13}$ & $1,5 \times 10^{13}$ \\
\hline Pente sous le seuil & & $1-2 \times 10^{13}$ & \\
\hline Pompage de charge & & $1,5-3 \times 10^{13}$ & \\
\hline
\end{tabular}

Tableau 4 Valeurs de $\mathrm{D}_{\text {it }}$ au sommet du bas de la bande de conduction (BC), du haut de la bande de valence (BV) et au mid-gap pour une structure MOSCAP $\mathrm{Al}_{2} \mathrm{O}_{3} / \mathrm{In}_{0,53} \mathrm{Ga}_{0,47} \mathrm{As}$ (dopé $\mathrm{P}$ à $10^{17} \mathrm{~cm}^{-3}$ )

Les particularités et la limitation de certaines méthodes sont discutées en détail dans la littérature [Nicollian1982, Sze1981, Spicer1980JVST et Enge-Herbert12010JAP]. Pour résumer et simplifier, les deux dernières méthodes ne permettent pas de capturer les profils de $\mathrm{D}_{\mathrm{it}}$ en fonction des niveaux d'énergie associés. La limitation de la méthode de conductance est principalement due à la forte asymétrie du pic de conductance en présence de $\mathrm{D}_{\mathrm{it}}$. Comme la distribution de $\mathrm{D}_{\mathrm{it}}$ de l'interface high-k/III-V est fortement non uniforme en énergie, la méthode de conductance conventionnelle est discutable. La méthode liée aux capacités HF et LF est limitée par les pièges à proximité du sommet de bande qui vont répondre beaucoup plus rapidement. La contribution AC des pièges réduit l'étirement apparent (stretch-out) de la courbe $\mathrm{C}-\mathrm{V}$ mesurée et le $\mathrm{D}_{\mathrm{it}}$ risque d'être sous-estimée en raison de l'augmentation du seuil de la courbe CV mesurée à haute fréquence. Enfin, la méthode de Berglund n'est peut être pas très adaptée aux les interfaces $h i g h-k / \mathrm{III}-\mathrm{V}$ en régime de forte inversion, en particulier pour ceux avec un petit EOT.

Le tableau 5 résume succinctement l'état de l'art de la technologie métal/high- $k /$ III-V. On peut observer que les $\mathrm{D}_{\text {it }}$ reportés différèrent par plusieurs ordres de grandeur et varient entre $10^{11} \mathrm{~cm}^{-2} \mathrm{eV}^{-1}$ et au-delà de $10^{13} \mathrm{~cm}^{-2} \mathrm{eV}^{-1}$. Ces mesures utilisent des méthodes différentes ce qui peut expliquer raisonnablement des écarts d'une moitié d'ordre de grandeur... 


\begin{tabular}{|c|c|c|c|c|}
\hline $\begin{array}{l}\text { Semi-conducteur } \\
\text { Dopage }\left(\mathrm{cm}^{-3}\right)\end{array}$ & $\begin{array}{c}\text { High-k } \\
\text { Dépôt méthode }\end{array}$ & $\begin{array}{l}\text { Analyse } \\
\text { méthode }\end{array}$ & $\begin{array}{c}\text { Dit }\left(\mathrm{cm}^{-2} \mathrm{eV}^{-1}\right) \text { et } / \mathrm{ou} \\
\Delta \psi_{\mathrm{s}}(\mathrm{eV})\end{array}$ & Ref \\
\hline \multirow[t]{2}{*}{$\mathrm{n} / \mathrm{p}$-type $2 \times 10^{17}$} & \multirow[t]{2}{*}{$\mathrm{Al}_{2} \mathrm{O}_{3}$ ALD } & Conductance & $\begin{array}{c}1 \times 10^{12}(\mathrm{BC}), 7 \times 10^{12}(\mathrm{MG}), \\
2 \times 10^{13}(\mathrm{BV}), 5 \times 10^{12}(\mathrm{MG}), \\
3 \times 10^{13}(\mathrm{BV})\end{array}$ & \multirow[t]{2}{*}{$\begin{array}{l}\text { Brammertz2009APL } \\
\text { Brammertz2009ECS }\end{array}$} \\
\hline & & Quasi-statique CV & $1.1 \mathrm{eV}$ & \\
\hline $\mathrm{n} / \mathrm{p}$-type $5 \times 10^{16}$ & $\mathrm{Al}_{2} \mathrm{O}_{3} \mathrm{ALD}$ & $\begin{array}{c}\text { Conductance } \\
\text { Pompage charge } \\
\text { BLQS }\end{array}$ & $\begin{array}{c}1-3 \times 10^{12} \text { (au dessus BG) } \\
3 \times 10^{11}(\text { au dessous BG) } \\
1.0 \mathrm{eV}\end{array}$ & Chiu2008APL \\
\hline $\mathrm{n} / \mathrm{p}$-type $1 \times 10^{17}$ & $\mathrm{Al}_{2} \mathrm{O}_{3} \mathrm{ALD}$ & $\begin{array}{c}\text { BLA }\left(5 \mathrm{kHz}, 110^{\circ} \mathrm{C}\right) \\
\text { Sensibilité } \varphi_{\mathrm{m}} \\
\end{array}$ & $\begin{array}{c}0.5-1.1 \mathrm{eV} \\
0.7 \mathrm{eV} \\
\end{array}$ & $\begin{array}{l}\text { Kim2009JAP } \\
\text { Kim2010APL }\end{array}$ \\
\hline $\mathrm{n} / \mathrm{p}$-type $2 \times 10^{17}$ & $\mathrm{Al}_{2} \mathrm{O}_{3} \mathrm{ALD}$ & Conductance & $\begin{array}{l}2 \times 10^{13}(\mathrm{MG}) \\
3 \times 10^{13}(\mathrm{BV}) \\
\end{array}$ & Lin2009MEng \\
\hline p-type $1 \times 10^{17}$ & $\mathrm{Al}_{2} \mathrm{O}_{3}$ ALD & HF-LF & $1,4 \times 10^{12}(\mathrm{MG})$ & Xuan2007IEEE \\
\hline n-type $5 \times 10^{16}$ & $\mathrm{Al}_{2} \mathrm{O}_{3}$ ALD & Conductance & $1 \times 10^{12}(\mathrm{MG})$ & Zhao2009APL \\
\hline n-type $3 \times 10^{18}$ & $\begin{array}{c}\mathrm{LaAlO}_{3} \mathrm{MBE}- \\
\mathrm{UHV}\end{array}$ & Conductance & $1,5 \times 10^{12}(\mathrm{MG})$ & Goel2007APL \\
\hline n-type $4 \times 10^{17}$ & $\mathrm{HfO}_{2}$ ALD & Conductance & $>1 \times 10^{13}$ & $\begin{array}{c}\text { Hurley2009ECS } \\
\text { O’Connor2009APL }\end{array}$ \\
\hline n-type $5 \times 10^{17}$ & $\mathrm{HfO}_{2}$ ALD & Terman & $\begin{array}{c}2 \times 10^{12}(\mathrm{BC}), 1 \times 10^{12}(\mathrm{MG}) \\
>1 \times 10^{13}(\mathrm{BV})\end{array}$ & Chang2008APL \\
\hline n-type $5 \times 10^{17}$ & $\mathrm{HfO}_{2}$ ALD & Terman & $2 \times 10^{12}(\mathrm{MG})$ & Lee2008APL \\
\hline $\mathrm{n} / \mathrm{p}$-type $1 \times 10^{17}$ & $\begin{array}{c}\mathrm{HfO}_{2} \text { MBE- } \\
\text { UHV }\end{array}$ & Conductance & $\begin{array}{c}5 \times 10^{11}(\mathrm{BC}), 1 \times 10^{12}(\mathrm{BV}) \\
>10^{13}(\mathrm{MG})\end{array}$ & Hwang2010APL \\
\hline n-type $1 \times 10^{17}$ & $\mathrm{ZrO}_{2} \mathrm{ALD}$ & $\begin{array}{c}\text { BLA }(50 \mathrm{kHz}, \\
\left.110^{\circ} \mathrm{C}\right)\end{array}$ & $>0.7 \mathrm{eV}$ & Engel2009APL \\
\hline $\mathrm{n} / \mathrm{p}$-type $3 \times 10^{17}$ & $\mathrm{ZrO}_{2}$ ALD & Sensibilité $\varphi_{\mathrm{m}}$ & $>10^{13}(\mathrm{MG}), 2 \times 10^{12}(150 \mathrm{~K})$ & Koveshnikov2008APL \\
\hline $\mathrm{n} / \mathrm{p}$-type $1 \times 10^{17}$ & $\mathrm{ZrO}_{2}$ ALD & Conductance & $1 \times 10^{12}(150 \mathrm{~K})$ & HuangIEDM2009 \\
\hline n-type $1,7 \times 10^{17}$ & Si ICL, Oxid & Terman & $3 \times 10^{12}(\mathrm{MG}) ; 0.65 \mathrm{eV}$ & Hasegawa1988JJAPP \\
\hline n-type $1 \times 10^{16}$ & Si ICL, Nitri & $\begin{array}{c}\text { Conductance, } \\
\text { BLQS }\end{array}$ & $\begin{array}{c}3 \times 10^{11}(\mathrm{MG}) \\
0.65 \mathrm{eV}\end{array}$ & Wang1992APL \\
\hline FlatBand Design & $\begin{array}{c}\mathrm{Ga}_{2} \mathrm{O} / \mathrm{GaGdO} \\
\mathrm{MBE}-\mathrm{UHV}\end{array}$ & HF-LF & $3 \times 10^{11}(\mathrm{MG})$ & $\begin{array}{c}\text { Passlack2008IEEE } \\
\text { Hill2007IEEE }\end{array}$ \\
\hline $\mathrm{n} / \mathrm{p}$-type $1 \times 10^{17}$ & $\begin{array}{c}\mathrm{Al}_{2} \mathrm{O}_{3 /} \\
\mathrm{Ga} 2 \mathrm{O} 3(\mathrm{Gd} 2 \mathrm{O} 3) \\
\text { MBE-UHV }\end{array}$ & $\begin{array}{c}\text { Conduction } \\
\text { Pompage de charge } \\
\text { HF-LF }\end{array}$ & $\begin{array}{c}2-3 \times 10^{12}(\mathrm{MG}) \\
2-4 \times 10^{11}(\mathrm{MG}) ; 1 \times 10^{12}(\mathrm{BC}) \\
1 \times 10^{11}(\mathrm{MG})\end{array}$ & $\begin{array}{c}\text { Hong2000APL } \\
\text { Shiu2008APL } \\
\text { Lin2009IPRM } \\
\text { Lin2010SSE }\end{array}$ \\
\hline
\end{tabular}

Tableau 5 Technologie et densité de pièges des états d'interface $D_{\text {it }}$ et plage de la variation du potentiel de surface $\Delta \psi_{\mathrm{s}}$ pour une structure MOS high-k/In ${ }_{0,53} \mathrm{GA}_{0,47} \mathrm{As}$ à $300 \mathrm{~K}$ (autres indications sinon) rapportées dans la littérature pour cette dernière décennie. Pour les acronymes : ALD : atomic layer deposition ; MBE : molecular beam epitaxy; UHV :ultrahigh vacuum ; BC : bande de conduction ; BV :bande de valence ; MG : mid-gap ; BG : band gap ; BLQS : analyse de Berglund appliquée sur CV quasi-statique ;

BLA : analyse de Berglund appliquée sur CV à basse fréquence. ICL : interface control layer.

Enfin, la structure de MOS fabriquée par National Tsing Hua University: $\mathrm{Al}_{2} \mathrm{O}_{3} / \mathrm{Ga}_{2} \mathrm{O}_{3}\left(\mathrm{Gd}_{2} \mathrm{O}_{3}\right) / \mathrm{In}_{0.53} \mathrm{Ga}_{0.47}$ As présente un $\mathrm{D}_{\mathrm{it}} \sim 10^{11} \mathrm{~cm}^{-2} \mathrm{eV}^{-1}$. Les auteurs affirment que d'après une analyse de Berglund, le niveau de Fermi peut traverser le gap entier $(0,75 \mathrm{eV}) \mathrm{de}$ $l^{\prime} \mathrm{In}_{0,53} \mathrm{Ga}_{0,47} \mathrm{As}$ avec une efficacité de $65 \%$ près de la région de mid-gap, proche donc de la 
valeur idéale (sans $\mathrm{D}_{\mathrm{it}}$ ) de $\sim 75 \%$ [Lin2009APL]. La plage de variation du potentiel de surface peut atteindre $1 \mathrm{eV}$ voire plus. Les auteurs définissent cette surface comme une surface sans effet d'ancrage du niveau de Fermi (unpinned high- $k /$ III-V interface). De plus, ils obtiennent également un faible courant de fuite associé $10^{-8} \mathrm{~A} / \mathrm{cm}^{2}$. Ces résultats prometteurs ont ouvert de nouvelles perspectives pour la technologie de MOS III-V.

\section{4-2 Comparaison des différentes architectures de dispositifs}

Diverses architectures de dispositifs MOSFET III-V sont représentées schématiquement sur la Fig. 26. Chacune possède son propre avantage à pondérer par ses inconvénients...

\section{$\underline{\text { L'architecture MOSFET massif standard en mode d'inversion : Fig. } 25 \text { - a) }}$}

Cette architecture a été proposée par différentes équipes comme l'University of Purdue [Xuan2007IEEE], National Tsing Hua University [Lin2010ECS], Intel [Goel2008IEDM et Radosavljevic2009IEDM] et University of Texas [Xue2011APL]. C'est celle d'un MOSFETBulk traditionnel. Les matériau de canal, du contact et du substrat sont des semi-conducteurs de type III-V. Le canal d'inversion est formé à l'interface entre la couche de semi-conducteur à haute mobilité et le diélectrique de grille. Les régions de source et drain sont formées par implantation d'ions. Les avantages de cette architecture sont qu'elle est bien connue en termes de processus de fabrication qui sont quasi identique à ceux réalisés sur silicium. Il devrait exister une loi d'échelles très semblable à celle des MOSFET massif à base de silicium. Les inconvénients sont que le canal est formé à l'interface semi-conducteur/diélectrique et que la grande mobilité des semi-conducteurs III-V peut donc être dégradée par l'interface rugueuse et aussi par les impuretés ionisées. En outre, les limitations de l'implantation ionique dans les matériaux III-V peuvent influencer le dimensionnement, notamment pour les régions de la source et du drain, ce qui peut provoquer une élévation des résistances d'accès. 

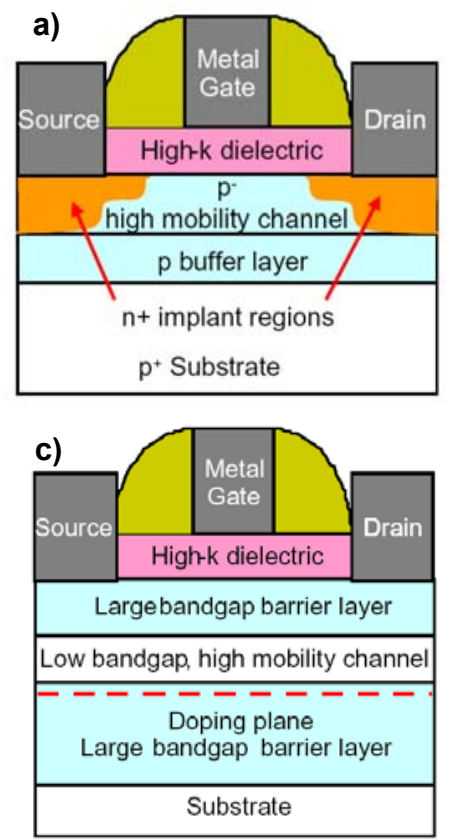
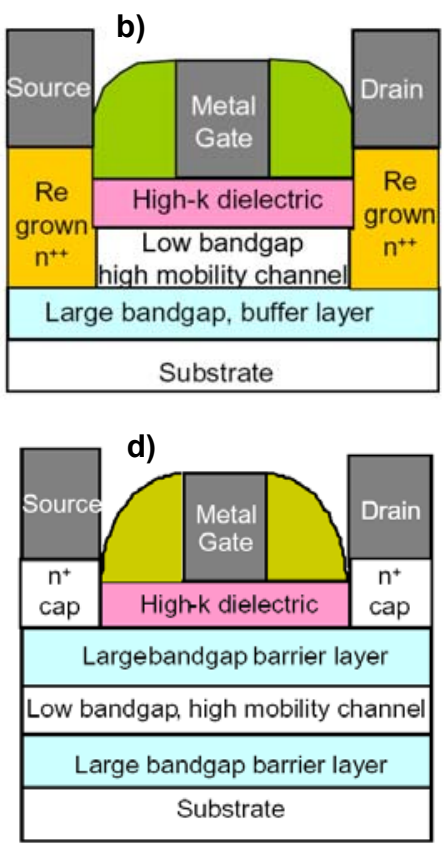

Figure 26 Différentes architectures de MOSFET III-V de simple grille (SG) à l'état de l'art [Thayne2009ECS].

\section{$\underline{\text { L'architecture avec source-drain surélevés : Fig. } 25-\text { b) }}$}

Cette architecture a été proposée par l'University of California Santa Barbara [Singisetti2009IEEE]. Par rapport à la précédente, les régions source et drain ont été surélevées pour réduire les résistances d'accès. En outre, cette structure comprend une couche tampon en matériau III-V à grand gap sous le canal pour confiner géométriquement les porteurs. Le dispositif est donc à zone active ultra-mince comme pour le SOI à utiliser pour les derniers nœuds technologiques. Cela doit atténuer les effets de canal court dans les dispositifs nanométriques tout en diminuant le niveau de dopage dans la couche active. Comme le canal de cette architecture est formé à l'interface diélectrique/semi-conducteur, les propriétés du transport sont toujours affectées par la rugosité d'interface.

\section{$\underline{\text { L'architecture du mode "Flaband" : Fig. } 25-\mathrm{c})}$}

Cette structure a été initialement développée par Motorola/Freescale [Passlack2002IEEE], puis en collaboration avec l'université de Glasgow [Passlack2006IEEE]. Elle reprend les originalités d'un transistor de type HEMT : le canal est formé dans une couche enterrée de matériau ayant une grande mobilité et une faible bande interdite. Les porteurs sont bien confinés par les hétérostructures. Cela permet d'obtenir un contrôle de 
charge comparable à celui d'un MOSFET SOI. Le canal est spatialement éloigné du diélectrique de grille donc l'interaction de la rugosité d'interface est susceptible d'être réduite par rapport au MOS standard. L'arrangement du dopage assure que les régions d'accès de chaque côté de la grille peuvent être préalablement peuplées par les porteurs (en mode normally $O N$ ), ce qui permet d'assurer une faible résistance d'accès et une haute vitesse d'injection. Quant à la région de canal, elle est désertée par le travail de sortie d'une grille métallique. Par rapport aux architectures à inversion (a) et (b), le dimensionnement ultime de cette structure peut être limité par la distance séparant le canal et la grille.

\section{$\underline{\text { L'architecture avec canal enterré et une grillé encastrée (recess gate) : Fig. } 25-\mathrm{d} \text { ) }}$}

Cette structure a été développée par IBM [Sun2008IEDM] et Intel [Goel2008IEDM]. Elle est similaire à la structure de la Fig. 25)-c, sauf en ce qui concerne la résistance d'accès. Elle est réduite en réalisant une couche cap layer fortement dopée entre les contacts de la source (ou du drain) et la grille. La grille est formée dans un "recess" gravé à travers la couche cap layer. Cette structure possède de nombreux autres avantages électrostatiques par rapport à l'architecture "flatband". La limitation du dimensionnement de cette structure peut aussi être due à la séparation entre le canal et la grille. Par ailleurs, du point de vue de la fabrication, cette architecture peut avoir un problème d'uniformité de la tension de seuil du fait des variations de profondeur d'encastrement gravé (recess region) comme dans la structure HEMT.

Les intérêts des conceptions alternatives sont leurs bonnes propriétés de transport électronique. La Fig. 27 montre les mobilités effectives d'électrons mesurées (par méthode Split $\mathrm{C}-\mathrm{V}$ ) à température ambiante dans les canaux des diverses architectures de type MOSFET de la Fig. 26 à base d' $\ln _{x} G_{1-x}$ As. Elles sont tracées soit en fonction du champ transverse effectif, soit de la concentration des porteurs dans canal. La courbe de mobilité universelle du silicium est également incluse dans la figure pour comparaison. 

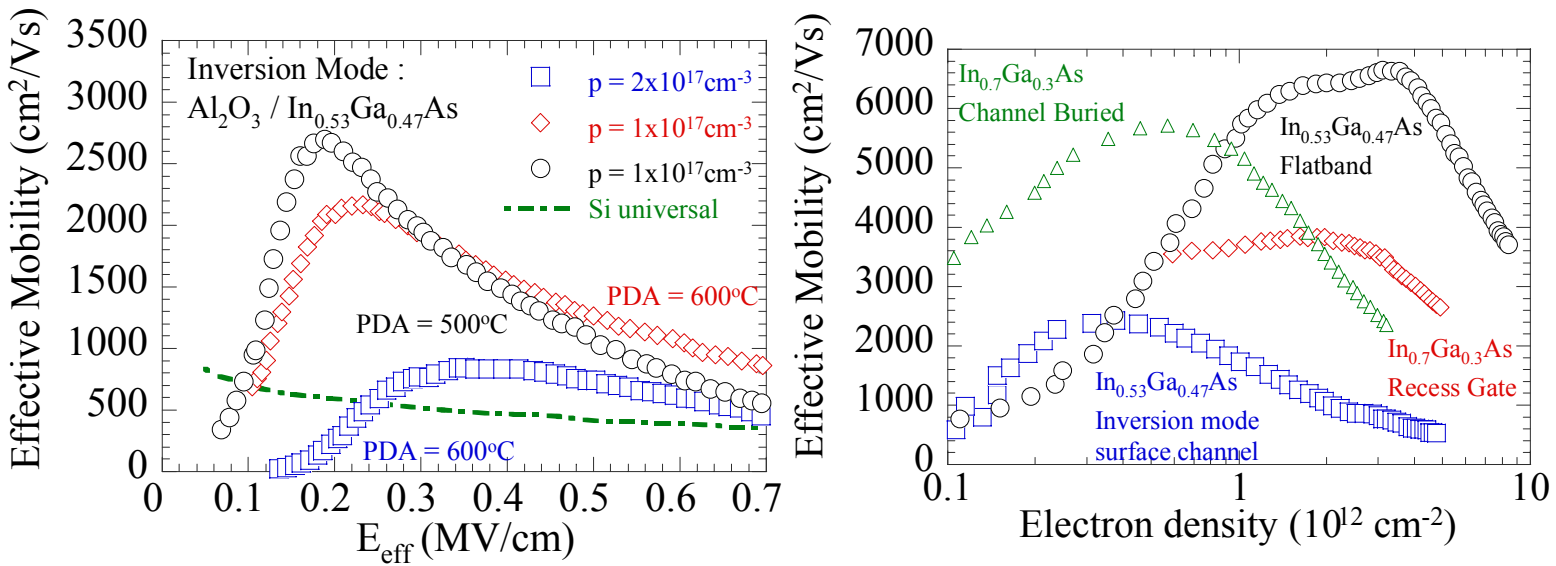

Figure 27 Gauche : mobilité effective dans la couche d'inversion $\mathrm{Al}_{2} \mathrm{O}_{3} / \mathrm{In}_{0,53} \mathrm{Ga}_{0,47} \mathrm{As}$ en fonction du champ effectif pour différents dopages et températures de recuit (PDA : postdeposition anneal [Xuan2007IEDM]. Droite : mobilité effective du canal dans les différentes architectures en fonction de la densité d'électrons [Goel2008IEDM] [Sun2008DRC] [Bentley2011IEEE] [Xue2011APL].

Ces figures révèlent certaines tendances très intéressantes. Tout d'abord, la mobilité extraite en mode d'inversion est significativement plus élevée que celle obtenue dans le silicium, particulièrement sous faible champ effectif c'est-à-dire pour de faibles concentrations d'électrons dans le canal. Mais en régime d'inversion forte $\left(E_{\text {eff }} \sim 1 \mathrm{MV} / \mathrm{cm}\right)$ pour une concentration du porteur supérieure à $5 \times 10^{12} \mathrm{~cm}^{-2}$, la mobilité du canal d'inversion III-V diminue vers des valeurs comparables avec celle de $\mathrm{Si}$. Ces observations peuvent éventuellement être dues à une forte rugosité d'interface ou aux interactions de nature polaire des III-V. En outre, les mobilités extraites en mode d'inversion dans les architectures alternatives dans lesquelles le canal est spatialement séparé de l'interface diélectrique/semiconducteur sont plus élevées. On note que l'architecture "flatband" avec 53\% de taux d'indium montre une meilleure mobilité effective que les architectures à canal enterré ou à "recess gate" même si le taux d'indium est plus grand (70\%). La dépendance en température de la mobilité pour ces types de structures permet d'étudier les mécanismes d'interaction dominants. Par ailleurs, ces mobilités vont être dégradées si l'on diminue l'épaisseur d'oxyde et/ou de barrière [Del-Alamo2011EMRS] pour augmenter la capacité totale de grille et renforcer l'immunité contre les SCE. Ces résultats sont récapitulés en Fig. 27.

Par ailleurs, on s'intéresse également aux caractéristiques électriques des transistors MOSFETs pour un type de technologie et de conception donné à base de 1 ' $\operatorname{In}_{\mathrm{x}} \mathrm{Ga}_{1-\mathrm{x}} \mathrm{As}$ et de $h i g h-k$ diélectrique. Le tableau 6 résume les caractéristiques électriques les plus significatives de ces dernières années [Lin2010SSE], [Sun2008DRC], [Bentley2011IEEE] et [Xue2011APL]. 


\begin{tabular}{|c|c|c|c|c|c|}
\hline Ref \& année & Groupe & Design/Canal & Oxyde (méthode dépôt $\& \mathrm{~T}_{\mathrm{OX}}$ ) & $\mathrm{L}_{\mathrm{G}}$ & Caractéristique \\
\hline Ren1998IEEE & Bell Labs & $\begin{array}{l}\text { Inversion mode } \\
\mathrm{In}_{0.53} \mathrm{Ga}_{0.47} \mathrm{As}\end{array}$ & $\begin{array}{c}\text { UHV E-beam } \\
\mathrm{Ga}_{2} \mathrm{O}_{3}\left(\mathrm{Gd}_{2} \mathrm{O}_{3}\right), \mathrm{T}_{\mathrm{OX}}=40 \mathrm{~nm}\end{array}$ & $\begin{array}{c}1 \mu \mathrm{m} \\
0.75 \mu \mathrm{m}\end{array}$ & $\mathrm{I}_{\mathrm{D}_{-} \max }=375 \mu \mathrm{A} / \mu \mathrm{m} ; \mathrm{gm}=190 \mu \mathrm{S} / \mu \mathrm{m}$ \\
\hline Wang1999 & Bell Labs & $\begin{array}{c}\text { Inversion mode } \\
\text { GaAs }\end{array}$ & $\begin{array}{c}\text { UHV E-beam } \\
\mathrm{Ga}_{2} \mathrm{O}_{3}\left(\mathrm{Gd}_{2} \mathrm{O}_{3}\right), \mathrm{T}_{\mathrm{OX}}=38-52 \mathrm{~nm}\end{array}$ & $1 \mu \mathrm{m}$ & $\mathrm{I}_{\mathrm{D}_{-} \max }=30 \mu \mathrm{A} / \mu \mathrm{m} ; \mathrm{gm}=4 \mu \mathrm{S} / \mu \mathrm{m}$ \\
\hline Xuan2007IEDM & Univ. Purdue & $\begin{array}{c}\text { Inversion mode } \\
\mathrm{In}_{0.53} \mathrm{Ga}_{0.47} \mathrm{As}\end{array}$ & $\begin{array}{c}\text { ALD } \\
\mathrm{Al}_{2} \mathrm{O}_{3}, \mathrm{~T}_{\mathrm{OX}}=8 \mathrm{~nm}\end{array}$ & $0.5 \mu \mathrm{m}$ & $\mathrm{I}_{\mathrm{D}_{-} \max }=430 \mu \mathrm{A} / \mu \mathrm{m} ; \mathrm{gm}=160 \mu \mathrm{S} / \mu \mathrm{m}$ \\
\hline Hill2007IEEE & $\begin{array}{c}\text { Freescale } \\
\text { Univ. Glasgow }\end{array}$ & $\begin{array}{c}\text { Flatband } \\
\mathrm{In}_{0.3} \mathrm{Ga}_{0.7} \mathrm{As}\end{array}$ & $\begin{array}{c}\text { UHV E-beam } \\
\mathrm{GaGdO} / \mathrm{Gd}_{2} \mathrm{O}_{3} \mathrm{~T}_{\mathrm{OX}}=10 \mathrm{~nm}\end{array}$ & $1 \mu \mathrm{m}$ & $\mathrm{I}_{\mathrm{D}_{-} \max }=407 \mu \mathrm{A} / \mu \mathrm{m} ; \mathrm{gm}=477 \mu \mathrm{S} / \mu \mathrm{m}$ \\
\hline Sun2007IEEE & IBM & $\begin{array}{l}\text { Canal enterré } \\
\mathrm{In}_{0.7} \mathrm{Ga}_{0.3} \mathrm{As}\end{array}$ & $\begin{array}{c}\text { ALD } \\
\mathrm{Al}_{2} \mathrm{O}_{3}, \mathrm{~T}_{\mathrm{OX}}=7 \mathrm{~nm}\end{array}$ & $0.26 \mu \mathrm{m}$ & $\mathrm{I}_{\mathrm{D}_{-} \max }=117 \mu \mathrm{A} / \mu \mathrm{m} ; \mathrm{gm}=157 \mu \mathrm{S} / \mu \mathrm{m}$ \\
\hline Xuan2008IEEE & Univ. Purdue & $\begin{array}{l}\text { Inversion mode } \\
\mathrm{In}_{0.65} \mathrm{Ga}_{0.35} \mathrm{As}\end{array}$ & $\begin{array}{c}\text { ALD } \\
\mathrm{Al}_{2} \mathrm{O}_{3}, \mathrm{~T}_{\mathrm{OX}}=10 \mathrm{~nm}\end{array}$ & $0.4 \mu \mathrm{m}$ & $\mathrm{I}_{\mathrm{D}_{-} \max }=1050 \mu \mathrm{A} / \mu \mathrm{m} ; \mathrm{gm}=350 \mu \mathrm{S} / \mu \mathrm{m}$ \\
\hline Lin2008APL & Univ. Tsin-Hua & $\begin{array}{l}\text { Inversion mode } \\
\mathrm{In}_{0.53} \mathrm{Ga}_{0.47} \mathrm{As}\end{array}$ & $\begin{array}{c}\text { UHV E-beam } \\
\mathrm{Al}_{2} \mathrm{O}_{3} / \mathrm{Ga}_{2} \mathrm{O}_{3}\left(\mathrm{Gd}_{2} \mathrm{O}_{3}\right) \\
\mathrm{T}_{\mathrm{OX}}=2 \mathrm{~nm} / 5 \mathrm{~nm}\end{array}$ & $1 \mu \mathrm{m}$ & $\mathrm{I}_{\mathrm{D} \_\max }=1050 \mu \mathrm{A} / \mu \mathrm{m} ; \mathrm{gm}=714 \mu \mathrm{S} / \mu \mathrm{m}$ \\
\hline Xuan2008IEDM & Univ. Purdue & $\begin{array}{l}\text { Inversion mode } \\
\mathrm{In}_{0.75} \mathrm{Ga}_{0.25} \mathrm{As}\end{array}$ & $\begin{array}{c}\text { ALD } \\
\mathrm{Al}_{2} \mathrm{O}_{3}, \mathrm{~T}_{\mathrm{OX}}=10 \mathrm{~nm}\end{array}$ & $0.75 \mu \mathrm{m}$ & $\mathrm{I}_{\mathrm{D}_{-} \max }=1000 \mu \mathrm{A} / \mu \mathrm{m} ; \mathrm{gm}=430 \mu \mathrm{S} / \mu \mathrm{m}$ \\
\hline Sun2008IEDM & IBM & $\begin{array}{c}\text { Canal enterré }+ \\
\text { gate recess } \\
\mathrm{In}_{0.7} \mathrm{Ga}_{0.3} \mathrm{As} \\
\end{array}$ & $\begin{array}{c}\mathrm{ALD} \mathrm{Al}{ }_{2} \mathrm{O}_{3}, \mathrm{~T}_{\mathrm{Ox}}=5.5 \mathrm{~nm} \\
\alpha-\mathrm{Si} \text { passivation, } \mathrm{T}_{\mathrm{Si}}<1.5 \mathrm{~nm}\end{array}$ & $0.08 \mu \mathrm{m}$ & $\mathrm{I}_{\mathrm{D} \_\max }=960 \mu \mathrm{A} / \mu \mathrm{m} ; \mathrm{gm}=793 \mu \mathrm{S} / \mu \mathrm{m}$ \\
\hline Sun2008DRC & IBM & $\begin{array}{c}\text { Canal enterré }+ \\
\text { gate recess } \\
\mathrm{In}_{0.7} \mathrm{Ga}_{0.3} \mathrm{As} \\
\end{array}$ & $\begin{array}{c}\mathrm{ALD} \mathrm{Al}_{2} \mathrm{O}_{3}, \mathrm{~T}_{\mathrm{OX}}=6 \mathrm{~nm} \\
\alpha-\mathrm{Si}, \mathrm{T}_{\mathrm{Si}}<1.5 \mathrm{~nm}\end{array}$ & $0.16 \mu \mathrm{m}$ & $\mathrm{I}_{\mathrm{D}_{-} \max }=825 \mu \mathrm{A} / \mu \mathrm{m} ; \mathrm{gm}=683 \mu \mathrm{S} / \mu \mathrm{m}$ \\
\hline Chiu2009DRC & Univ. Tsin-Hua & $\begin{array}{c}\text { Inversion mode } \\
\mathrm{In}_{0.53} \mathrm{Ga}_{0.47} \mathrm{As}\end{array}$ & $\begin{array}{c}\text { ALD } \\
\mathrm{Al}_{2} \mathrm{O}_{3}, \mathrm{~T}_{\mathrm{OX}}=6 \mathrm{~nm}\end{array}$ & $0.6 \mu \mathrm{m}$ & $\mathrm{I}_{\mathrm{D}_{-} \max }=678 \mu \mathrm{A} / \mu \mathrm{m} ; \mathrm{gm}=354 \mu \mathrm{S} / \mu \mathrm{m}$ \\
\hline Wu2009IEEE & Univ. Purdue & $\begin{array}{c}\text { Inversion mode } \\
\mathrm{In}_{0.75} \mathrm{Ga}_{0.25} \mathrm{As}\end{array}$ & $\begin{array}{c}\mathrm{ALD} \\
\mathrm{Al}_{2} \mathrm{O}_{3}, \mathrm{~T}_{\mathrm{OX}}=5 \mathrm{~nm}\end{array}$ & $0.13 \mu \mathrm{m}$ & $\mathrm{I}_{\mathrm{D}_{-} \max }=440 \mu \mathrm{A} / \mu \mathrm{m} ; \mathrm{gm}=705 \mu \mathrm{S} / \mu \mathrm{m}$ \\
\hline Chin2009IEEE & Univ. Singapore & $\begin{array}{c}\text { Inversion mode } \\
\mathrm{In}_{0.53} \mathrm{Ga}_{0.47} \mathrm{As}\end{array}$ & $\begin{array}{l}\text { MOCVD HfAlO } \\
, \mathrm{T}_{\mathrm{OX}}=\text { inconnu }\end{array}$ & $0.25 \mu \mathrm{m}$ & $\mathrm{I}_{\mathrm{D}_{-} \max }=420 \mu \mathrm{A} / \mu \mathrm{m} ; \mathrm{gm}=45 \mu \mathrm{S} / \mu \mathrm{m}$ \\
\hline Lin2010SSE & Univ. Tsin-Hua & $\begin{array}{l}\text { Inversion mode } \\
\mathrm{In}_{0.75} \mathrm{Ga}_{0.25} \mathrm{As}\end{array}$ & $\begin{array}{c}\text { UHV E-beam } \\
\mathrm{Al}_{2} \mathrm{O}_{3} / \mathrm{Ga}_{2} \mathrm{O}_{3}\left(\mathrm{Gd}_{2} \mathrm{O}_{3}\right), \\
\mathrm{T}_{\mathrm{OX}}=2 \mathrm{~nm} / 13 \mathrm{~nm}\end{array}$ & $1.2 \mu \mathrm{m}$ & $\mathrm{I}_{\mathrm{D}_{-} \max }=960 \mu \mathrm{A} / \mu \mathrm{m} ; \mathrm{gm}=410 \mu \mathrm{S} / \mu \mathrm{m}$ \\
\hline Lin2010SSE & Univ. Tsin-Hua & $\begin{array}{c}\text { Inversion mode } \\
\mathrm{In}_{0.75} \mathrm{Ga}_{0.25} \mathrm{As}\end{array}$ & $\begin{array}{c}\text { ALD } \\
\mathrm{Al}_{2} \mathrm{O}_{3}, \mathrm{~T}_{\mathrm{OX}}=6 \mathrm{~nm}\end{array}$ & $1 \mu \mathrm{m}$ & $\mathrm{I}_{\mathrm{D}_{-} \max }=740 \mu \mathrm{A} / \mu \mathrm{m} ; \mathrm{gm}=325 \mu \mathrm{S} / \mu \mathrm{m}$ \\
\hline Xue2011APL & Univ. Texas & $\begin{array}{l}\text { Canal enterré } \\
\mathrm{In}_{0.7} \mathrm{Ga}_{0.3} \mathrm{As}\end{array}$ & $\begin{array}{c}\text { ALD } \\
\mathrm{Al}_{2} \mathrm{O}_{3}, \mathrm{~T}_{\mathrm{OX}}=4.4 \mathrm{~nm}\end{array}$ & $20 \mu \mathrm{m}$ & $\mathrm{I}_{\mathrm{D}_{-} \max }=123 \mu \mathrm{A} / \mu \mathrm{m} ; \mathrm{gm}=55 \mu \mathrm{S} / \mu \mathrm{m}$ \\
\hline Bentley2011IEEE & $\begin{array}{c}\text { Freescale } \\
\text { Univ. Glasgow }\end{array}$ & $\begin{array}{c}\text { Flatband } \\
\mathrm{In}_{0.53} \mathrm{Ga}_{0.47} \mathrm{As}\end{array}$ & $\begin{array}{c}\text { ALD } \\
\mathrm{Al}_{2} \mathrm{O}_{3}, \mathrm{~T}_{\mathrm{OX}}=6 \mathrm{~nm}\end{array}$ & $20 \mu \mathrm{m}$ & $\mathrm{I}_{\mathrm{D}_{-} \max }=195 \mu \mathrm{A} / \mu \mathrm{m} ; \mathrm{gm}=7 \mu \mathrm{S} / \mu \mathrm{m}\left(\mathrm{V}_{\mathrm{ds}}=50 \mathrm{mV}\right)$ \\
\hline
\end{tabular}

Tableau 6 Récapitulation des performances en courant de drain $I_{d \_m a x}$ maximal et transconductance $g_{m}$ pour des MOSFETs à canal InGaAs en mode enrichissement sur cette dernière décennie. Les meilleures sont surlignées en vert.

D'après cette analyse récapitulative, on constate que les meilleures performances en termes de courant de drain et de transconductance sont atteintes par l'Université de Tsin Hua et IBM. L'équipe de Tsin Hua utilise un empilement diélectrique de grille comme $\mathrm{Al}_{2} \mathrm{O}_{3} / \mathrm{Ga}_{2} \mathrm{O}_{3}\left(\mathrm{Gd}_{2} \mathrm{O}_{3}\right)$. Grâce à leurs technologies de passivation et de traitement de surface, ils arrivent à réduire le niveau de $\mathrm{D}_{\mathrm{it}}$ autour de $10^{11} \mathrm{~cm}^{-2} \mathrm{eV}^{-1}$ au mid-gap. Pour un MOSFET bulk à canal $\operatorname{In}_{0,53} \mathrm{Ga}_{0,47} \mathrm{As}$ en mode d'inversion (cf. Fig. 26), les caractéristiques sont très prometteuses : un courant de drain supérieur à $1 \mathrm{~mA} / \mu \mathrm{m}$ et une transconductance supérieure à $700 \mu \mathrm{S} / \mu \mathrm{m}$. L'Université de Purdue a aussi obtenu une telle performance en courant en utilisant un empilement diélectrique de grille avec de $\mathrm{l}^{\prime} \mathrm{Al}_{2} \mathrm{O}_{3}$ et un canal en $\mathrm{In}_{0,65} \mathrm{Ga}_{0,35} \mathrm{As}$ mais avec une longueur de grille plus petite. Mais la transconductance reste relativement 
faible. IBM utilise également un empilement diélectrique de grille en $\mathrm{Al}_{2} \mathrm{O}_{3}$, mais la surface est passivée par une couche de contrôle à l'interface en silicium ( $\alpha$-Si ICL : interface control layer). La structure choisie est celle d'un MOSFET à canal enterré avec une grille encastrée (recess gate). Dans cette architecture alternative, le canal est spatialement séparé de l'interface diélectrique/semi-conducteur. L’Université de Glasgow utilise l'architecture "Flatband", malgré des résultats en régime DC peu convaincants, ils ont montré (pour la première fois à ma connaissance) des performances très prometteuses en domaine de radio fréquence (RF) à la conférence IEDM en 2007 [Passlack2007IEDM] (cf. Fig. 28).

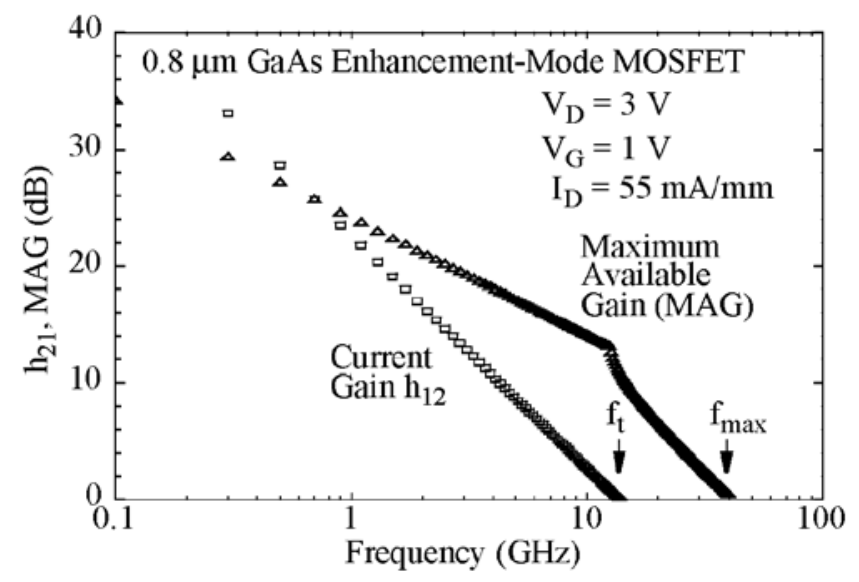

Figure 28 Le gain en courant $h_{21}$ et le gain maximal d'oscillation (MAG) en fonction de la fréquence pour un MOSFET en InGaAs avec une architecture "Flatband" et $\mathrm{L}_{G}=0,8 \mu \mathrm{m}$ à $\mathrm{I}_{D}=55 \mathrm{~mA} / \mathrm{mm}$.

[Passlack2007IEDM].

Nous avons récapitulé la grande progression des technologies MOS III-V. Mais comment se comparent-elles aux technologies matures telles que MOSFET sur Si et HEMT III-V ? Pour comparer l'efficacité de commutation, le groupe de Matthias Passlack [Doornbos2010IEEE] propose d'utiliser le ratio $\mathrm{Q}=\mathrm{g}_{\mathrm{m}} / \mathrm{S}$ (avec $\mathrm{g}_{\mathrm{m}}$ la transconductance et $\mathrm{S}$ la pente sous le seuil) comme facteur de mérite. Plus la valeur de Q est grande, plus la technologie est technologiquement mature. Les résultats sont montrés dans la figure 29 pour des FET à canal N [Doornbos2010IEEE]. Les lignes pointillées en gris illustrent les différents quotients de la valeur de Q. 


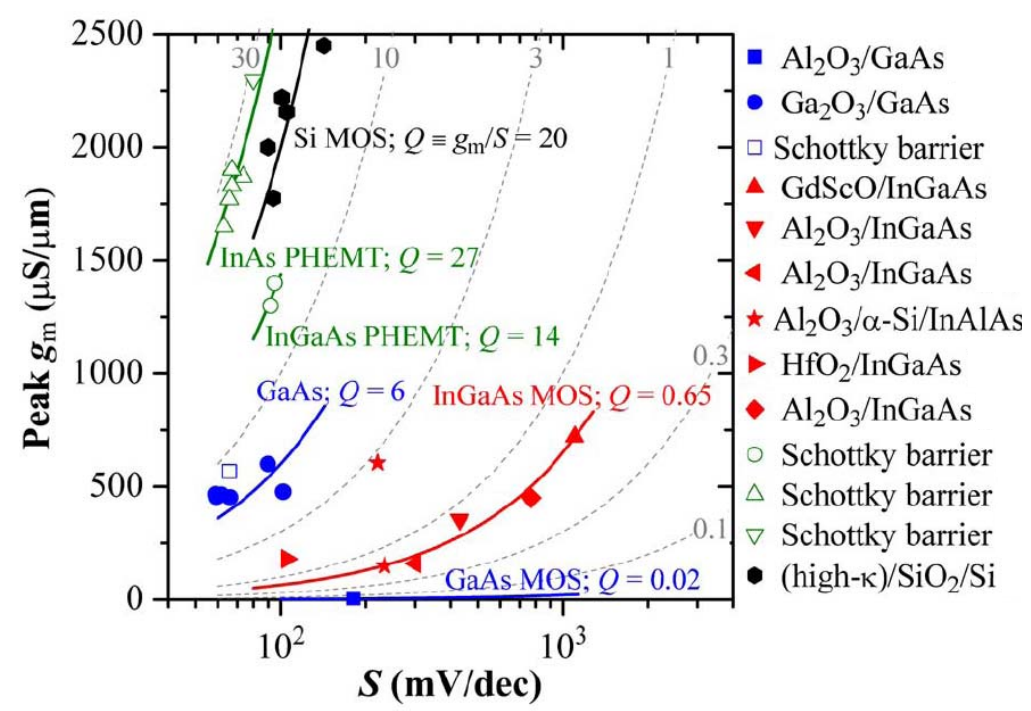

Figure 29 Le maximum de transconductance $\mathrm{g}_{\mathrm{m}}$ en fonction de la pente sous le seuil $\mathrm{S}$ associée pour des N-MOSFET en Si, GaAs et InGaAs (symboles pleins) et HEMT pseudomorphiques en GaAs, InGaAs et InAs (symboles vides). Les pointillés représentent $Q=g_{m} / S$ en $\mu S / \mu m . d e c / m V$. [Doornbos2010IEEE]

Visiblement le HEMT pseudomorphique à canal binaire d'InAs sur InP présente la meilleure valeur de $\mathrm{Q}(\mathrm{Q}=27 \mu \mathrm{S} / \mu \mathrm{m}$. dec/mV). La technologie avancée à canal de $\mathrm{Si}$ contraint permet d'atteindre $\mathrm{Q}=20$ jusqu'à la génération $32 \mathrm{~nm}$. Le HEMT pseudomorphique à canal ternaire d'InGaAs sur InP permet d'avoir $\mathrm{Q}=14 \mu \mathrm{S} / \mu \mathrm{m} . \mathrm{dec} / \mathrm{mV}$. En revanche avec un canal d'InGaAs, Q est réduit à seulement $0,65 \mu \mathrm{S} / \mu \mathrm{m}$.dec $/ \mathrm{mV}$. Pour l'instant, il n'y a qu'un seul résultat présentant un $\mathrm{Q}$ supérieur à $3 \mu \mathrm{S} / \mu \mathrm{m}$.dec $/ \mathrm{mV}$ parmi tous les MOSFET InGaAs. Il est fabriqué par IBM avec une architecture à canal enterré et un design de "recess gate" [Sun2008IEDM]. Une observation importante est que les données sur les MOSFET à base d'InGaAs semblent suivre une tendance universelle. Cela indique que le comportement en commutation est dominé par les $\mathrm{D}_{\text {it }}$ plutôt que par les effets de canal court comme dans le cas du Si. En outre, une forte progression peut être observée dans les MOSFET à base de GaAs, si on choisit $\mathrm{Ga}_{2} \mathrm{O}_{3}$ au lieu d' $\mathrm{Al}_{2} \mathrm{O}_{3}$ comme diélectrique de grille grâce à la réduction considérable de $\mathrm{D}_{\text {it, }} \mathrm{Q}$ atteint jusqu'à $6 \mu \mathrm{S} / \mu \mathrm{m} . \operatorname{dec} / \mathrm{mV}$ au lieu de $0,02 \mu \mathrm{S} / \mu \mathrm{m} \cdot \operatorname{dec} / \mathrm{mV}$. Clairement, la technologie MOSFET III-V est loin d'être mature, ses potentialités en termes d'applications logiques et/ou analogiques à haute performance et faible consommation est encore à démontrer. Si on peut limiter le niveau des pièges d'interface, si on confine bien les porteurs dans le canal ultra mince, si la topologie MOS permet d'augmenter le potentiel de dimensionnement jusqu'au tailles nanométriques, les N-MOSFET à base de III-V pourraient dépasser les performances des HEMT voire celles des MOS surSi sous faible polarisation $\left(\mathrm{V}_{\mathrm{GS}}-\mathrm{V}_{\mathrm{T}}\right.$ et $\left.\mathrm{V}_{\mathrm{DS}}\right)$. 


\section{Objectifs de l'étude :}

Cette première partie a mise en évidence l'intérêt de transistor FET de type $\mathrm{N}$ en matériaux III-V en termes de performance fréquentielle pour les applications logiques et analogiques. La combinaison de la technologie III-V et de la topologie MOS nécessite encore de relever de nombreux défis technologiques en particulier le contrôle la qualité de l'interface III-V/high- $k$. Dans ce contexte, ce travail a principalement évalué et exploré les potentialités de l'architecture MOSFET III-V sous faible polarisation pour des applications de basse puissance en utilisant des simulations numériques en régimes statiques et dynamiques.

Dans le chapitre II, nous nous intéresserons au contrôle de charge dans une structure métal/oxyde $h i g h-k /$ semi-conducteur III-V où l'isolant $h i g h-k$ à haute permittivité diélectrique est l'alumine. L'étude consiste à analyser théoriquement le fonctionnement d'une capacité MOS en résolvant de façon auto-cohérente les équations de Poisson et de Schrödinger (PS). Les résultats seront comparés à des résultats expérimentaux fournis par nos partenaires du projet ANR MOS35 qui a servi de cadre à cette thèse. Nous quantifierons les effets des états de pièges à l'interface $h i g h-k /$ III-V sur les caractéristiques C-V. La simulation PS sera ensuite étendue à une géométrie $2 \mathrm{D}$ pour prendre en compte les effets de canal court et les capacités parasites. Les performances des dispositifs FET seront enfin une première fois estimées pour des applications logiques et analogiques à l'aide d'un modèle analytique de transport quasibalistique.

Dans le chapitre III, nous étudierons ensuite plus en détails les performances des MOSFET III-V en régimes statiques et dynamiques sous faible condition de polarisation, à l'aide du simulateur semi-classique de dispositifs Monte Carlo développé dans l'équipe. Les paramètres utilisés ont d'abord été validés par comparaison avec des données expérimentales, en particulier concernant une architecture de FET de type HEMT fabriquée par la société OMMIC partenaire du projet ANR MOS35. Les caractéristiques de quatre topologies de MOSFET seront quantitativement étudiées en termes de courants statiques et dynamique, de rendement fréquence/consommation et de bruit. Nous en tirerons des conclusions sur l'optimisation des dispositifs. Enfin, l'étude comparative avec un FET à base de Si mettra en perspective le potentiel des MOSFET III-V pour des applications à haute fréquence et faible puissance de consommation. 


\section{Chapitre II}

\section{Contrôle de charge et dimensionnement des structures MOS III-V par analyse Poisson-Schrödinger}

Le contrôle de charge dans une capacité à base de silicium peut être traité de façon classique, sous l'hypothèse de non dégénérescence avec un modèle à une seule bande parabolique. La caractéristique capacité-tension $\mathrm{C}-\mathrm{V}$ est alors calculée en utilisant la densité de charge issue de l'équation de Poisson non linéaire, qui varie en fonction du potentiel de surface selon une statistique de Maxwell-Boltzmann. Ces approximations restent en général assez pertinentes pour $\mathrm{Si}$, caractérisé par une grande masse de densité d'états (ou DOS selon le sigle anglais), une faible non-parabolicité des relations de dispersion énergie (vecteur d'onde) et des vallées latérales à haute énergie très éloignées du bas de la bande de conduction. Mais l'utilisation d'un tel modèle devient très discutable pour des matériaux III-V de faible DOS et de forte non-parabolicité. Avec de telles caractéristiques pour les bandes électroniques, le centre de gravité des charges électriques, ou centroïde, se localise loin de l'interface oxyde/III-V du fait des effets de quantification et la dégénérescence est facilement atteinte. En outre, les vallées latérales à masse effective 'lourde' vont jouer un rôle de plus en plus important, d'autant plus dans le cas d'un fort confinement géométrique (film mince) ou d'un fort champ transverse (forte densité de dopants). Pour modéliser le contrôle de charge dans des capacités MOS high-K/III-V, on a donc besoin de modèles moins simplistes que ceux qui peuvent suffire dans le cas de $\mathrm{Si}$, avec la nécessaire prise en compte de la statistique de Fermi-Dirac, de la quantification des niveaux d'énergie, de la correction de non-parabolicité, et des aspects multi-vallées. La prise en compte auto-cohérente des états de pièges à l'interface au sein du contrôle de charge pourra être aussi étudiée. Le calcul auto-cohérent nous permet notamment de capturer l'effet d'ancrage du niveau de Fermi (pinning effect) ainsi que l'efficacité du mouvement de niveau Fermi à l'interface non seulement autour de la polarisation de bandes plates ou flat band mais également sur les régions de mid-gap et d'inversion. La validation du modèle global passe par une comparaison avec les données expérimentales. Par ailleurs, la caractéristique idéale $\mathrm{C}-\mathrm{V}$ est très utile pour extraire précisément les niveaux de pièges $\mathrm{D}_{\mathrm{it}}$ dans l'oxyde. Ce genre des études nous permettrait 
d'évaluer les effets extrinsèques dus aux défauts par rapport au cas idéal pour une technologie donnée.

\section{Présentation des structures étudiées et des modèles numériques}

\section{1-1 Architectures proposées}

Pendant ma thèse, j'ai choisi d'étudier les 4 architectures représentées sur la Fig. 30 de transistor de type MOS à inversion et à grille auto-alignée, conçues pour viser un fonctionnement à haute fréquence et à faible consommation. La structure de référence est celle sur substrat massif, ou MOS-Bulk avec canal en surface. Grâce à une forte concentration en dopants dans la zone active pour lutter contre les effets de canal court, on devrait avoir de bonnes performances à l'état OFF pour des structures déca-nanométriques. Cependant, le transport électronique est dégradé par les interactions de rugosité d'interface et les impuretés ionisées. Les structures alternatives de la Fig. 30 doivent permettre d'améliorer les propriétés de transport des porteurs dans le canal ou la qualité du contrôle par la grille. Le transistor qualifié de MOS-ThinBody, inspiré par la technologie SOI (Silicon on insulator) à couche active ultra-mince, permet de diminuer la résistivité du canal en conservant l'immunité contre les effets de canal court. L'oxyde enterré est remplacé par un semi-conducteur III-V ayant un grand gap en accord de maille sur le matériau du canal. La géométrie définie pour MOSHEMT permet d'éliminer les effets néfastes sur le courant des interfaces rugueuses oxyde/III-V. Le MOS-COMB est une combinaison des deux premières structures alternatives. Le matériau du canal est $\operatorname{In}_{0,53} \mathrm{Ga}_{0,47} \mathrm{As}$. Le matériau de l'espaceur entre oxyde et canal dans le MOS-HEMT et de la couche enterrée est $\operatorname{In}_{0,52} \mathrm{Al}_{0,48}$ As. Le substrat semi-isolant de la structure de famille ThinBody est InP. La longueur de grille $\left(\mathrm{L}_{\mathrm{G}}\right)$ et l'épaisseur d'oxyde $\left(\mathrm{T}_{\mathrm{OX}}\right)$ sont respectivement égales à $50 \mathrm{~nm}$ et $8 \mathrm{~nm}$. Le matériau high- $\kappa$ que l'on a choisi est $\mathrm{Al}_{2} \mathrm{O}_{3}$.

\section{1-2 Dimensionnement préliminaire du dispositif}

Tout d'abord, on doit définir quelques règles de dimensionnement pour contrer les effets de canal court. Pour réaliser cela, on a utilisé le modèle analytique compact MASTAR [Chen1996IEEE], [Skotnicki1998IEEE], [Skotnicki1994IEDM] et [Skotnicki2003] qui est largement utilisé pour la prédiction du dimensionnement de dispositifs à base de silicium spécifiée par l'ITRS sur plusieurs générations technologiques CMOS (de $1 \mu \mathrm{m}$ jusqu'à $0,1 \mu \mathrm{m}$ ) [ITRS2001]. Pour la structure MOS-Bulk, on calcule les trois facteurs de mérite de l'état 
OFF- SCE, DIBL et SS - en fonction de la concentration du dopant pour $\mathrm{L}_{\mathrm{G}}=50 \mathrm{~nm}$ et $\mathrm{T}_{\mathrm{OX}}=8 \mathrm{~nm}$ ( $c f$. Fig. 32, le choix de l'épaisseur d'oxyde sera justifié plus loin).

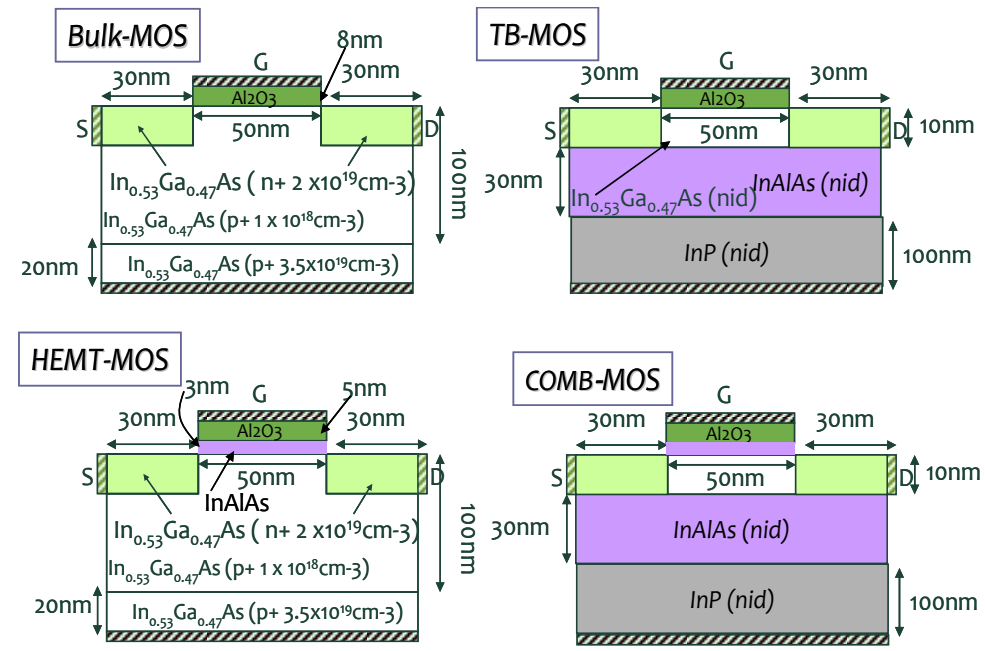

Figure 30 Les 4 types de transistors MOS en mode inversion étudiés avec un canal en $\mathrm{In}_{0,53} \mathrm{Ga}_{0,47} A s$. Le sigle nid désigne des zones intentionnellement non dopées. $\mathrm{L}_{\mathrm{G}}=50 \mathrm{~nm}$ et $T_{0 \mathrm{X}}=8 \mathrm{~nm}$

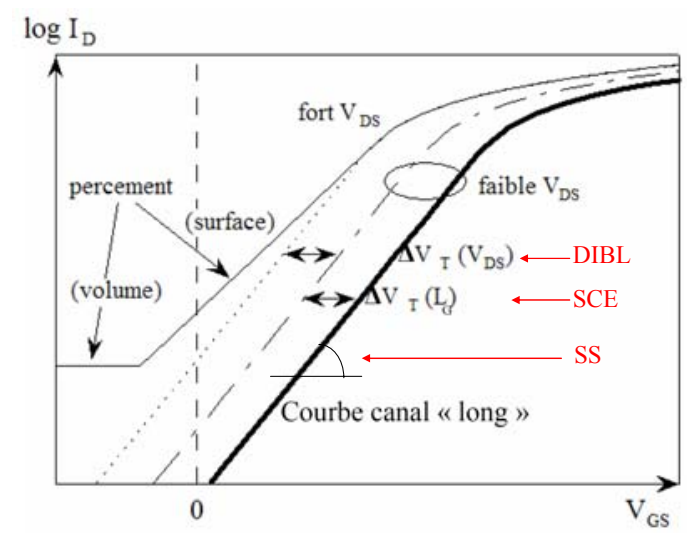

Figure 31 Caractéristique $I_{D S}\left(V_{G S}\right)$ sous le seuil d'un MOSFET à canal $N$ en tenant compte des diverses corrections dues à l'effet de canal court.

Comme décrit sur la Fig. 31, SCE (short channel effect) signifie le décalage de tension seuil dû à l'effet de canal court, DIBL (drain induced barrier lowering) et SS (subthreshold slope) sont respectivement l'abaissement supplémentaire de tension de seuil dû à la tension de drain et la pente sous le seuil. Pour la structure MOS-ThinBody, on calcule par résolution analytique de l'équation de Poisson une distance caractéristique $\lambda$ en fonction de l'épaisseur du body active $\mathrm{T}_{\text {body }}$ pour différentes épaisseurs d'oxyde [Yan1992IEEE] (cf. Fig. 32). Cette distance représente l'épaisseur des ZCE (zone de charge d'espace) des jonctions caissons/canal. Autrement dit, ce paramètre contrôle la diffusion du champ électrique dans la direction du transport. L'expression de $\lambda$ dépendra des conditions aux limites prises en compte. Ici on 
considère une structure simple grille. Pour se prémunir des effets de canal court, cette distance doit être très inférieure à $L_{G}$ et le facteur de forme $L_{G} / \lambda$ doit être au moins égal à 5 [SaintMartin2005Thèse] et [Bournel2006HDR].
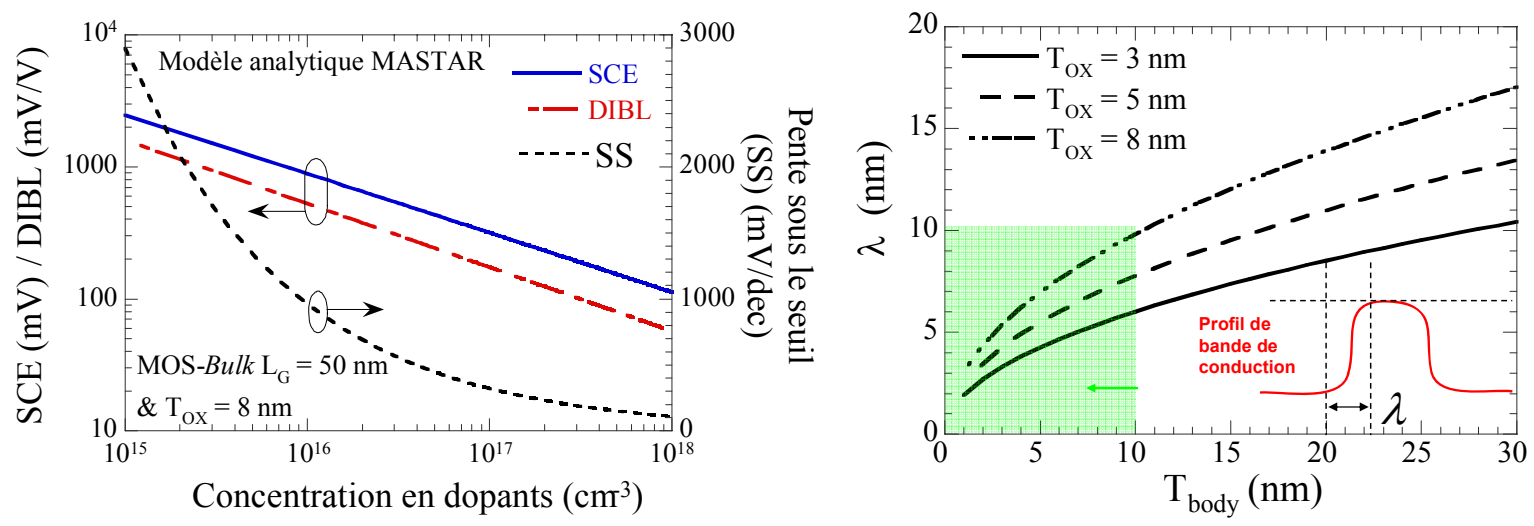

Figure 32 Gauche : Prédiction des facteurs de mérite de l'état OFF (SCE, DIBL et SS) en fonction de la concentration en dopants pour une structure MOS-Bulk. Droite : Longueur caractéristique $\lambda$ en fonction de l'épaisseur de couche active pour une structure MOS-ThinBody simple grille pour différentes épaisseurs d'oxyde

On peut constater que la structure MOS-Bulk doit avoir une concentration de dopage de $10^{18} \mathrm{~cm}^{-3}$ de type $\mathrm{P}$ afin d'avoir $\mathrm{SCE} \approx 120 \mathrm{mV}, \mathrm{DIBL} \approx 60 \mathrm{mV} / \mathrm{V}$ and $\mathrm{SS} \approx 110 \mathrm{mV} / \mathrm{dec}$ (cette dernière valeur restant assez loin de l'idéal à $60 \mathrm{mV} / \mathrm{dec}$ ). La structure MOS-ThinBody doit avoir une épaisseur de body d'au plus de $10 \mathrm{~nm}$ pour que $\lambda$ soit inférieure ou égale à $\mathrm{L}_{\mathrm{G}} / 5=10 \mathrm{~nm}$.

\section{1-3 Mise en place d'un modèle pertinent}

1-3-1 Modèle multi-vallées dans l'approximation de la masse effective pour les caractéristiques $\mathrm{C}-\mathrm{V}$

Les matériaux III-V comme InGaAs ont une faible masse effective ainsi qu'une faible densité d'états (DOS $\sim 10^{17} \mathrm{~cm}^{-3}$ à $300 \mathrm{~K}$ [Mathieu1998]) dans la bande de conduction. Le matériau high- $\kappa$ va introduire une large capacité électrostatique de géométrie $\mathrm{C}_{\mathrm{OX}}$. Le champ électrique appliqué au semi-conducteur devient rapidement grand quand la tension de grille appliquée augmente. Le niveau de Fermi peut alors se placer plus profondément dans la bande de conduction. La validité de la fonction de distribution de Boltzmann devient alors discutable. Par ailleurs, l'effet de la forte non-parabolicité de la bande de conduction de la vallée $\Gamma$ et le peuplement des vallées satellites ( $\mathrm{L}$ et $\mathrm{X}$ ) (ayant des énergies supérieures) doivent être soigneusement pris en compte. En particulier dans le cas où l'effet de la quantification des 
charges doit être également pris en compte (fort dopage ou film mince). Le modèle utilisé pour calculer la caractéristique $\mathrm{C}-\mathrm{V}$ théorique de la structure high-k/III-V est basé sur une approche multi-vallées dans l'approximation de la masse effective. La Fig. 33 montre un schéma de la structure de bande d' $\operatorname{In}_{0,53} \mathrm{Ga}_{0,47} \mathrm{As}$ massive à $300 \mathrm{~K}$ qui est utilisée dans le calcul de la caractéristique $\mathrm{C}-\mathrm{V}$.
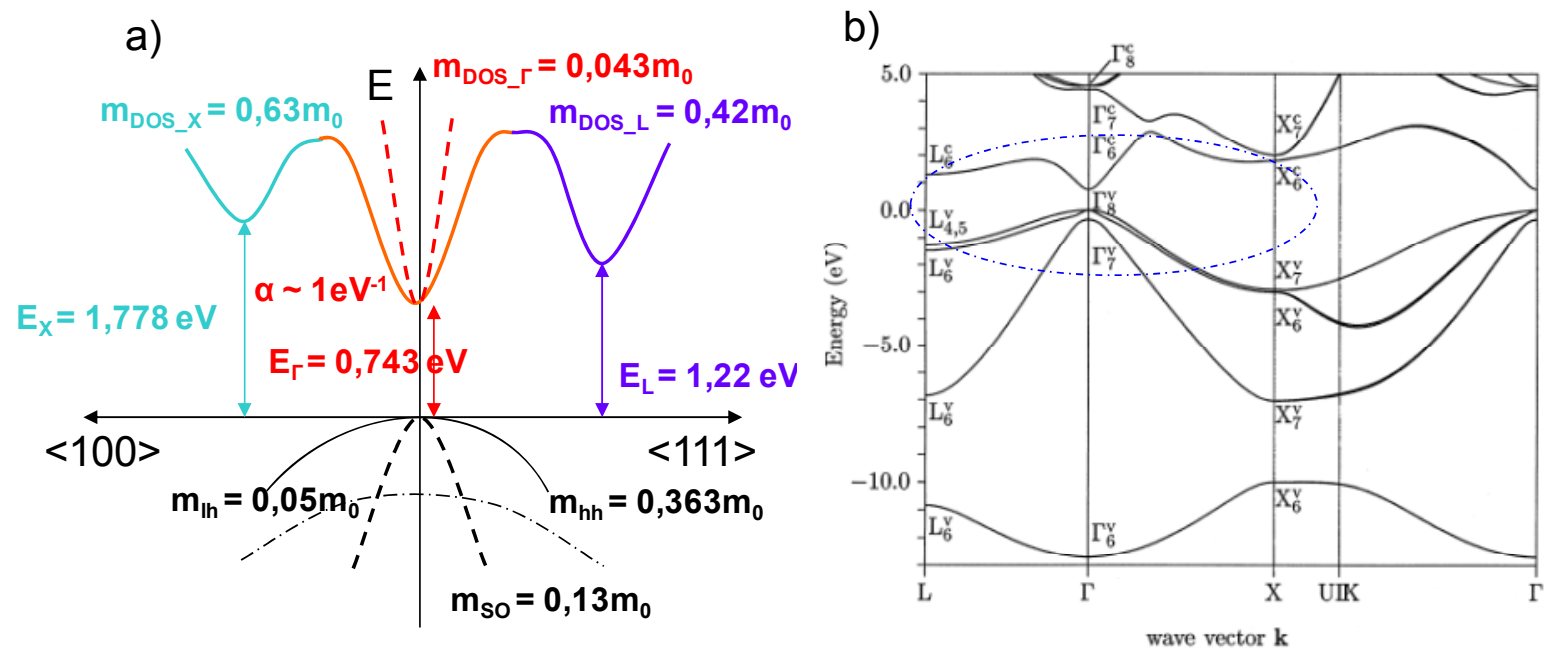

Figure 33 a) Un schéma représentatif de la structure de bandes de $\mathrm{In}_{0,53} \mathbf{G a}_{0,47} A s$ utilisant les paramètres provenant de la référence [Dittrich1999SSE] et [Vurgaftman2001JAP] pour T $=300 \mathrm{~K}$. b) La structure de bande de $\mathrm{In}_{0,53} \mathrm{Ga}_{0,47} \mathrm{As}$ est calculée par la méthode pseudo-potentiel empirique

[Dittrich1999SSE]

La capacité 1D idéale du semi-conducteur est modélisée par un empilement high-א/III-V semi-infini avec une concentration de dopage constante. Les conditions aux limites sont d'une part de nature Dirichlet du coté oxyde, c'est-à-dire un contact Schottky avec potentiel fixé, et d'autre part de nature Neumann du côté substrat, telle que la dérivée de potentiel est nulle, c'est-à-dire un contact Ohmique avec condition de champ nul. La tension totale de la grille est une somme de la tension de bande plate, de la chute de potentiel électrostatique dans l'oxyde et le potentiel de surface entre l'interface et la profondeur du substrat semi-conducteur (zone quasi-neutre). On suppose que la densité d'états dans la bande de conduction ne change pas sous l'influence du champ électrique. Le niveau de Fermi $\mathrm{E}_{\mathrm{F}} \mathrm{du}$ système est finalement fixé par la zone quasi-neutre qui se trouve après la zone de charge d'espace. Le potentiel électrostatique est relié à la densité de charge $\rho(z)$ par l'équation de Poisson :

$$
\frac{d^{2} \varphi(z)}{d z^{2}}=-\frac{\rho(z)}{\varepsilon_{s}}=-\frac{q\left(p(\varphi(z))-n(\varphi(z))+N_{D}-N_{A}\right)}{\varepsilon_{s}}
$$


La densité de charge $\rho(z)$ est une somme des différentes contributions des charges fixes et des porteurs libres. Les densités de donneurs $\mathrm{N}_{\mathrm{D}}$ et d'accepteurs $\mathrm{N}_{\mathrm{A}}$ sont supposées constantes dans l'approximation de complète ionisation. La densité des porteurs mobiles (électrons et trous) est une fonction du potentiel électrostatique. La densité d'électrons peut être obtenue à partir de l'intégration sur toute la plage des énergies supérieures à la bande de conduction utilisant une densité d'états $\mathrm{D}(\mathrm{E})$ multiplié par la fonction de distribution Fermi-Dirac comme :

$$
n=\int_{E_{C}}^{\infty} d E \frac{D(E)}{\exp \left(\frac{E-E_{F}}{k_{B} T}\right)+1}
$$

Le calcul classique de la distribution des charges mobiles est détaillé dans l'Annexe-A. Il est nécessaire pour avoir la solution "classique" de l'équation de Poisson non linéaire, c'est-à-dire le profil de potentiel initial.

Ensuite, la solution "quantique" pourra être obtenue par la résolution de l'équation de Schrödinger stationnaire 1D ( $c f$. Eq. II-3). L'énergie potentielle effective $E_{C v}$ dans l'équation de Schrödinger est supposée donnée par la solution de l'équation de Poisson (potentiel de déplétion et potentiel d'inversion). Le potentiel image et les effets d'échange et de corrélation sont négligés dans notre calcul.

$$
-\frac{\hbar^{2}}{2} \frac{\partial}{\partial z}\left(\frac{1}{m_{\text {conf_v }}(z)} \frac{\partial}{\partial z}+E_{C v}(z)\right) \xi_{\mu_{-} v}(z)=E_{\mu_{-} v} \xi_{\mu_{-} v}(z)
$$

Cette équation aux valeurs propres doit être résolue pour chaque vallée. Chacune des vallées peut avoir ses propres sous bande $E_{\mu_{-} v}$ (valeurs propres) et fonctions d'onde associées $\xi_{\mu_{-} v}(z)$ (vecteurs propres). $m_{c o n f_{-} v}$ est la masse de confinement pour chaque vallée selon la direction de quantification. Dans la fenêtre ondulatoire ou tranche Schrödinger (un intervalle $\left[\mathrm{z}_{1}, \mathrm{z}_{2}\right]$ où cette équation est résolue), les fonctions d'onde doivent être normalisées, c'est-à$\operatorname{dire} \int_{z_{1}}^{z_{2}}\left|\xi_{\mu_{-} v}(z)\right|^{2} d z=1$. Hors de cet intervalle, les fonctions d'onde sont considérées comme nulles. La pénétration des fonctions d'onde dans le diélectrique n'est pas prise en compte. Donc $\mathrm{z}_{1}$ est à l'interface oxyde/III-V et $\mathrm{z}_{2}$ est au-delà de la zone de charge d'espace.

Pour résoudre le problème en géométrie $2 \mathrm{D}$, nous avons utilisé 1'approche par modes (mode space) [Ren2001Thèse] et [SaintMartin2005Thèse]. Dans l'approximation de la masse effective, l'Hamiltonien associé à l'équation de Schrödinger stationnaire s'écrit comme : 


$$
-\frac{\hbar^{2}}{2 m_{x}} \frac{\partial^{2} \psi(x, z)}{\partial x^{2}}-\frac{\hbar^{2}}{2 m_{z}} \frac{\partial^{2} \psi(x, z)}{\partial z^{2}}+E_{C}(x, z) \psi(x, z)=E \times \psi(x, z)
$$

Ici, $E_{C}(x, z)$ est le profil de la bande de conduction issu de l'équation de Poisson 2D, c'est-àdire que ce potentiel électrostatique est résolu selon la direction de confinement $(\mathrm{z})$ pour chaque position selon la direction de transport (x). L'approche par modes permet de découpler l'équation de Schrödinger 2D en 2 équations 1D selon $\mathrm{x}$ et $\mathrm{z}$ sous certaines conditions. Les modèles physiques, la méthode et l'algorithme du calcul auto-cohérent des équations PoissonSchrödinger sont détaillés en Annexe - A et Annexe - B.

Enfin, la charge nette totale Q surfacique peut être obtenue en intégrant la densité volumique de charge $\rho(z)$ dans toute la structure. La capacité de grille peut être calculée par $C_{G} \approx d Q / d V_{G}$ et la capacité de couche d'inversion par $C_{I N V} \approx d Q / d \varphi_{S}$ où $\varphi_{S}$ est le potentiel de surface.

\section{1-3-2 Correction de non-parabolicité}

La structure de bandes des matériaux III-V se caractérise souvent par une grande nonparabolicité. La correction de non-parabolicité va influencer le contrôle de charges via la capacité de grille. Cette correction intervient non seulement dans le calcul classique (on ne résout que l'équation de Poisson) mais également dans le calcul quantique. Dans le cas classique, il faut que l'on recalcule la densité d'états dans l'espace réciproque 3D-k en prenant en compte l'effet de non-parabolicité [Ariel-Altschul1992IEEE] et [Islam2011SST]. L'Eq. II2 peut devenir :

$$
n_{v}(\varphi(x))=\frac{4}{\sqrt{\pi}}\left(\frac{2 \pi k_{B} T m_{v}^{*}}{h^{2}}\right)^{3 / 2} \times \int_{0}^{\infty} \frac{\sqrt{\eta_{v}}\left(1+\alpha \eta_{v}\right)\left(1+2 \alpha \eta_{v}\right)}{\exp \left(\eta_{v}-\eta_{F v}\right)+1} d \eta_{v}
$$

où $\alpha$ est le coefficient de non-parabolicité. Les notations sont inchangées par rapport à l'Eq. A-4 (Annexe - A). Cet effet de non-parabolicité va modifier la solution de l'équation Poisson non linéaire dans les calculs classiques.

La prise en compte de la correction non-parabolique n'est pas évidente pour des systèmes de gaz d'électrons de une dimension réduite (dans l'espace réciproque 2D-k, 1D-k voire 0D-k). En effet, la relation de dispersion dans le plan parallèle au transport et les niveaux d'énergie pour chaque sous bande doivent tous les deux contenir la correction nonparabolique. La prise en compte de la correction non-parabolique pour des systèmes de gaz d'électrons avec une dimension réduite est détaillée dans Annexe - A. Par conséquence, la 
densité d'états $\operatorname{DOS}_{\mu}(E)$ pour une sous bande $\mu$ donnée n'est plus une constante, elle doit dépendre de l'énergie et d'une densité de probabilité :

$$
\begin{gathered}
D O S_{\mu}(E)=\Theta\left(E-E_{\mu}\right) \frac{g_{\mu} m_{D O S_{-} \mu}}{\pi \hbar^{2}}\left\{1-2 \alpha\left(E-E_{\mu}\right)-2 \alpha \int_{0}^{\infty} d z\left(E_{\mu}^{(0)}-q \varphi(z)\right)\left|\xi_{\mu}^{(0)}(z)\right|^{2}\right\} \\
D_{O} S_{\mu}(E)=\operatorname{DOS}_{\mu}^{(0)}(E) \times N P\left(E-E_{\mu}\right)
\end{gathered}
$$

On constate que la densité d'états non paraboliques peut être exprimée par une multiplication entre une densité d'états paraboliques $\operatorname{DOS}_{\mu}^{(0)}(E)$ et un facteur de correction non parabolique $N P\left(E-E_{\mu}\right)$ pour une sous bande donnée. Ce facteur correctif peut également être utilisé pour les autres grandeurs physiques comme la vitesse de groupe etc... Finalement la densité totale d'électrons 2D-k dans la couche d'inversion en tenant compte de l'effet de nonparabolicité peut être calculée [Fischetti1993PRB] et [O'Regan2010JAP] :

$$
n_{2 D}(z)=\frac{k_{B} T}{\pi \hbar^{2}} \sum_{v} \sum_{\mu} g_{\mu} m_{D O S_{-} \mu, v}\left\{\left[1+2 \alpha\left(E_{\mu, v}-U_{\mu, v}\right)\right] \mathfrak{I}_{0}(\eta)+2 \alpha k_{B} T \mathfrak{I}_{1}(\eta)\right\}\left|\xi_{\mu}^{(0)}(z)\right|^{2}
$$

Ici, $\mathfrak{I}_{0}(\eta)$ et $\mathfrak{I}_{1}(\eta)$ sont les intégrales de Fermi-Dirac d'ordre zéro et un, avec $\eta=\left(E_{F}-E_{\mu, v}\right) / k_{B} T . m_{D O S_{-} \mu, v}$ est la masse effective de densité d'états de la sous bande $\mu$ de la vallée $v . U_{\mu, v}$ est la valeur de l'espérance de l'énergie de potentiel électrostatique vue par la sous bande $\mu$ de la vallée $v$.

Dans notre cas, d'un canal $\operatorname{In}_{0,53} \mathrm{Ga}_{0,47} \mathrm{As}$ avec une orientation (001), la vallée $\Gamma$ peut être modélisée par une approximation isotrope, c'est-à-dire $m_{c o n f}=m_{D O S}=m_{x, z}$. Une vallée $\mathrm{L}$ dont l'axe longitudinal est de type [111] est en revanche représentée par une approximation ellipsoïdale et le degré de dégénérescence vaut 4 . La masse effective résultante selon la direction de transport [100] peut être calculée comme [Himadri2008IEEE] :

$$
m_{\text {conf }}^{*}=\frac{3 m_{t} m_{l}}{m_{t}+2 m_{l}}, m_{D O S}^{*}=\sqrt{m_{t}\left(2 m_{l}+m_{t}\right) / 3}
$$

Le degré de dégénérescence de la vallée $X$ vaut 3, cette vallée est aussi modélisée par l'approximation ellipsoïdale. Le tableau 1 résume tous les paramètres physiques des matériaux (semi-conducteur, oxyde, métal) utilisés dans nos simulations Poisson-Schrödinger. 


\begin{tabular}{|c|c|c|c|c|c|}
\hline \multicolumn{2}{|c|}{$\begin{array}{c}\text { Semi } \\
\text { conducteur }\end{array}$} & $\ln _{0,53} \mathrm{Ga}_{0,47} \mathrm{As}$ & $\ln _{0,52} \mathrm{Al}_{0,48} \mathrm{As}$ & $\ln _{0,5} \mathrm{Ga}_{0,5} \mathrm{Sb}$ & $\mathrm{Si}$ \\
\hline \multicolumn{2}{|c|}{$\mathrm{Gap}(\mathrm{eV})$} & 0,74 & 1,45 & 0,43 & 1,12 \\
\hline \multicolumn{2}{|c|}{ Permittivité $\varepsilon_{\mathrm{r}}$} & 13,9 & 12,75 & 16,25 & 11,9 \\
\hline \multicolumn{2}{|c|}{$\mathrm{m}^{*}(\Gamma)\left(\mathrm{m}_{0}\right)$} & 0,041 & 0,083 & 0,025 & - \\
\hline \multirow{2}{*}{$\begin{array}{c}\text { masse } \\
(\mathrm{L})\end{array}$} & $\mathrm{m}_{\mathrm{t}}$ & 0,125 & 0,304 & 0,31 & - \\
\cline { 2 - 6 } & $\mathrm{m}_{\mathrm{y}}$ & 1,552 & 0,304 & 0,31 & - \\
\hline \multirow{2}{*}{$\begin{array}{c}\text { masse } \\
(\mathrm{X})\end{array}$} & $\mathrm{m}_{\mathrm{t}}$ & 0,25 & - & - & 0,19 \\
\cline { 2 - 6 } & $\mathrm{m}_{\mathrm{I}}$ & 2,26 & - & - & 0,916 \\
\hline Nonparabolicité & $1,0(\Gamma)$ & $0,543(\Gamma)$ & $2,63(\Gamma)$ & $0,5(\mathrm{X})$ \\
\hline \multicolumn{2}{|c|}{$\Delta \mathrm{E}_{\Gamma \mathrm{L}}(\mathrm{eV})$} & 0,61 & - & 0,22 & - \\
\hline \multicolumn{2}{|c|}{$\Delta \mathrm{Ec}(\mathrm{eV})$} & 0 & 0,51 & - & - \\
\hline \multicolumn{2}{|c|}{$\Delta \mathrm{EV}(\mathrm{eV})$} & - & 0,2 & 0 & - \\
\hline
\end{tabular}

\begin{tabular}{|c|c|}
\hline Oxyde & $\mathrm{Al}_{2} \mathrm{O}_{3}$ \\
\hline Gap $(\mathrm{eV})$ & 8,6 \\
\hline Permittivité $\varepsilon_{\mathrm{r}}$ & 11,5 \\
\hline $\mathrm{m}^{*}\left(\mathrm{~m}_{0}\right)$ & 0,28 \\
\hline$\Delta \mathrm{Ec}\left(\mathrm{In}_{0,53} \mathrm{Ga}_{0,47} \mathrm{As}\right)$ & 3,28 \\
\hline$\Delta \mathrm{Ev}\left(\mathrm{In}_{0,53} \mathrm{Ga}_{0,47} \mathrm{As}\right)$ & 4,58 \\
\hline$\Delta \mathrm{Ec}\left(\mathrm{In}_{0,52} \mathrm{Al}_{0,48} \mathrm{As}\right)$ & 2,77 \\
\hline$\Delta \mathrm{Ev}\left(\mathrm{In}_{0,52} \mathrm{Al}_{0,48} \mathrm{As}\right)$ & 4,38 \\
\hline$\Delta \mathrm{Ec}\left(\operatorname{In}_{0,5} \mathrm{Ga}_{0,5} \mathrm{Sb}\right)$ & 3,11 \\
\hline$\Delta \mathrm{Ec}(\mathrm{Si})$ & 2,6 \\
\hline $\mathrm{Mé}_{\mathrm{tal}}$ & $\mathrm{TaN}$ \\
\hline$\varphi_{\mathrm{M}}(\mathrm{eV})$ & 4,6 \\
\hline
\end{tabular}

Tableau 7 Paramètres physiques du matériau utilisés dans la simulation

\section{1-3-3 Comparaison entre résultats classiques avec les statistiques de Maxwell- Boltzmann et de Fermi-Dirac}

Tout d'abord, nous allons illustrer et discuter les résultats issus du calcul classique, c'est-à-dire que dans cette partie seule l'équation de Poisson est résolue. Considérons la structure 1D d'une capacité MOS TaN/ $\mathrm{Al}_{2} \mathrm{O}_{3} / \mathrm{In}_{0,53} \mathrm{Ga}_{0,47} \mathrm{As}$ avec une concentration $\mathrm{N}_{\mathrm{A}}=10^{18} \mathrm{~cm}^{-3}$ de type $\mathrm{P}$ et $\mathrm{C}_{\mathrm{OX}}=\varepsilon_{\mathrm{OX}} / \mathrm{T}_{\mathrm{OX}}=1,22 \mu \mathrm{F} . \mathrm{cm}^{-2}$. La caractéristique $\mathrm{C}_{\mathrm{G}}-\mathrm{V}_{\mathrm{G}}$ et le potentiel de surface théoriques sont calculés en utilisant différentes statistiques et structures de bandes. Les résultats sont montrés sur la Fig. 34 ci-dessous :

a)

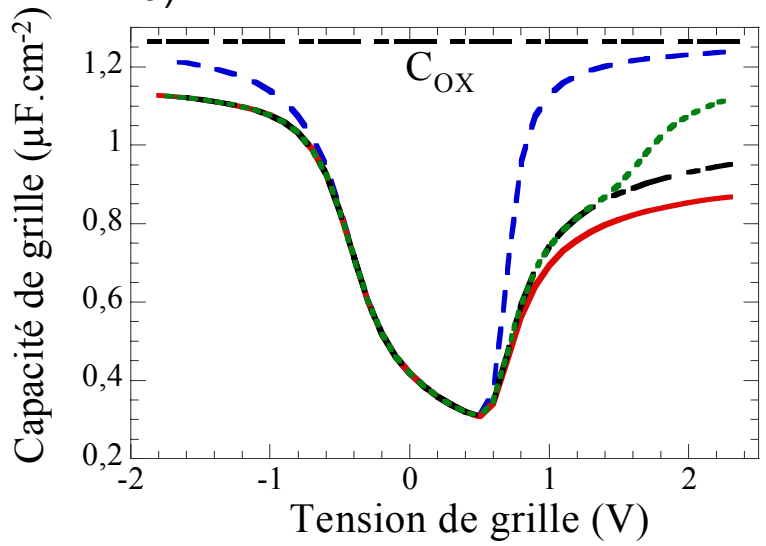

b)

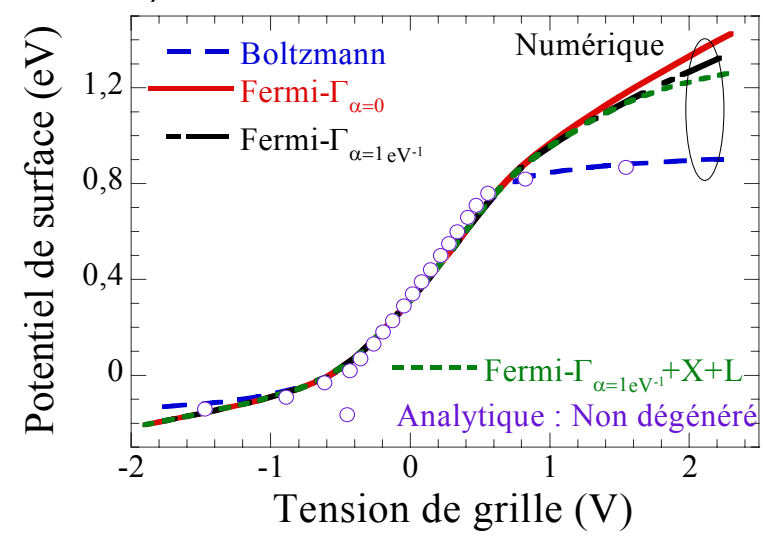

Figure 34 a) Caractéristique $C_{G}-V_{G}$ théorique et b) variation du potentiel de surface associée pour un empilement TaN/Al $\mathrm{A}_{2} \mathrm{O}_{3} / \mathrm{In}_{0,53} \mathrm{Ga}_{0,47} \mathrm{As}$ avec une concentration $\mathrm{N}_{\mathrm{A}}=10^{18} \mathrm{~cm}^{-3}$ de type $P$ et $\mathrm{C}_{\mathrm{OX}}=1,22 \mu \mathrm{F} . \mathrm{cm}^{-2}$, calculées avec différentes statistiques et structures de bandes.

On peut constater que la caractéristique $\mathrm{C}_{\mathrm{G}}-\mathrm{V}_{\mathrm{G}}$ calculée dans différents régimes (accumulation, déplétion et inversion), en utilisant la statistique de Boltzmann (tirets bleus), ne révèle pas la forme dissymétrique (entre régimes d'accumulation et d'inversion) due à la 
différence de DOS dans la bande de conduction et de valence. Le problème dû à la faible densité d'états dans la vallée $\Gamma$ ('DOS bottleneck') n'est pas mis en évidence. La capacité de grille $\mathrm{C}_{\mathrm{G}}$ augmente rapidement dans le régime de forte inversion et d'accumulation et s'approche de la valeur limite $\mathrm{C}_{\mathrm{OX}}$ de la capacité d'oxyde. Les électrons occupent très majoritairement les états de la vallée $\Gamma$ du bas de bande de conduction dans toute la plage de variation de la tension de grille ( $c f$. Fig. 36-37). Par comparaison avec les résultats obtenus avec Fermi-Dirac, on voit que la statistique de Boltzmann surestime la capacité de grille dans le régime d'accumulation et de forte inversion. La variation de potentiel de surface avec la tension de grille est sous-estimée dans ces deux derniers régimes. Elle suit une loi proportionnelle à $k_{B} T / q \ln \left(V_{G}\right)$ conformément à l'hypothèse de non-dégénérescence [Mathieu1998]. Sous cette hypothèse, on peut calculer analytiquement le potentiel de surface en fonction de la tension de grille. Ce calcul analytique permet de vérifier le résultat numérique en utilisant la statistique de Boltzmann. La relation entre le potentiel de surface et la tension de grille est déduite de l'évolution de la charge totale dans le semi-conducteur visà-vis d'une capacité d'oxyde : $V_{G}-\varphi_{S}=-Q_{S C} / C_{O X}$. La charge totale est calculée par le théorème de Gauss en utilisant la statistique de Boltzmann. En explicitant $Q_{S C}$ par le champ électrique à la surface du semi-conducteur, on a une relation analytique [Mathieu1998] :

$$
\begin{gathered}
V_{G}=\varphi_{S}+\beta \cdot \operatorname{sign}\left(\varphi_{S}\right) \cdot F\left(\varphi_{S}\right), \quad \beta=\varepsilon_{s c} k_{B} T / q C_{O X} L_{D} \\
F\left(\varphi_{S}\right)=\left\{e^{-e \varphi_{S} / k_{B} T}+\frac{e \varphi_{S}}{k_{B} T}+e^{e\left(\varphi_{S}-2 \phi_{F}\right) / k_{B} T}-1-\frac{n_{o}}{P_{o}}\left(\frac{e \varphi_{S}}{k_{B} T}+1\right)\right\}^{1 / 2}
\end{gathered}
$$

où $\phi_{F}$ est une distance énergétique entre le niveau de Fermi intrinsèque et le pseudo niveau de Fermi dû au dopage. $L_{D}$ est la longueur de Debye du semi-conducteur. $n_{o}$ et $p_{o}$ sont des densités d'électrons et de trous dans la région quasi neutre du semi-conducteur. Sur la Fig. 34$\mathrm{b}$, on peut observer un très bon accord entre le calcul analytique et numérique sous hypothèse de non-dégénérescence en prenant $\beta=0,051 \mathrm{~V}$.

Les différents modèles sont toutefois en très bon accord dans les régimes de déplétion et faible inversion. En effet, la distribution de charge résultante est alors dominée par les dopants ionisés qui ne devraient pas être influencés par la statistique de la population et la structure de bandes si l'hypothèse de complète ionisation est utilisée. La prise en compte de la statistique de Fermi-Dirac (lignes continues rouges) induit une grande modification sur la caractéristique $\mathrm{C}-\mathrm{V}$ en inversion. A tension de grille faible, la vallée $\Gamma$ de faible DOS est occupée et la capacité d'inversion est réduite. La limitation de $\mathrm{C}_{\mathrm{G}}$ provient de cette faible 
capacité de la couche d'inversion dans cette plage de tension. L'effet de la non-parabolicité (tirets-traits noirs) augmente $\mathrm{C}_{\mathrm{G}}$ de plus de $20 \%$ dans la vallée $\Gamma$ à cause de l'augmentation de la densité d'états. Le transfert vers les vallées satellites 'lourdes' L (voir les pointillés verts) est de plus en plus important quand on continue à augmenter la tension de grille $(>1,5 \mathrm{~V})$. Le contrôle de charge devient de plus en plus efficace mais l'augmentation de la masse moyenne des électrons est néfaste pour le transport dans le plan de la couche d'inversion.

\section{1-3-4 Comparaison entre calculs classiques et quantiques}

$\mathrm{Si}$ en outre on prend en compte la quantification en résolvant l'équation de Schrödinger de façon auto-cohérente avec Poisson, les résultats sont montrés dans la Fig. 35 ci-dessous (ligne continue rouge), et la comparaison avec le résultat classique (Poisson seul, tirets noirs) est aussi présentée. On constate que la tension de seuil est augmentée, la valeur de capacité est diminuée et la variation de potentiel de surface est plus importante surtout dans le régime de forte inversion. En effet le gap effectif augmente avec le confinement et le centroïde des charges se localise loin de l'interface, induisant ainsi une région de 'dark space' relativement large. Entre autres, les niveaux de sous bande se séparent par une grande distance énergétique pour des états quantiques à faible DOS de la vallée $\Gamma$. Pour moduler autant de densité de porteurs que dans le calcul classique, le potentiel de surface nécessaire devient plus grand. L'effet de la non-parabolicité (tirets-traits bleus) joue le même rôle que dans le cas classique : la capacité $\mathrm{C}_{\mathrm{G}}$ est augmentée d'environ $20 \%$ dans la vallée $\Gamma$.

a)

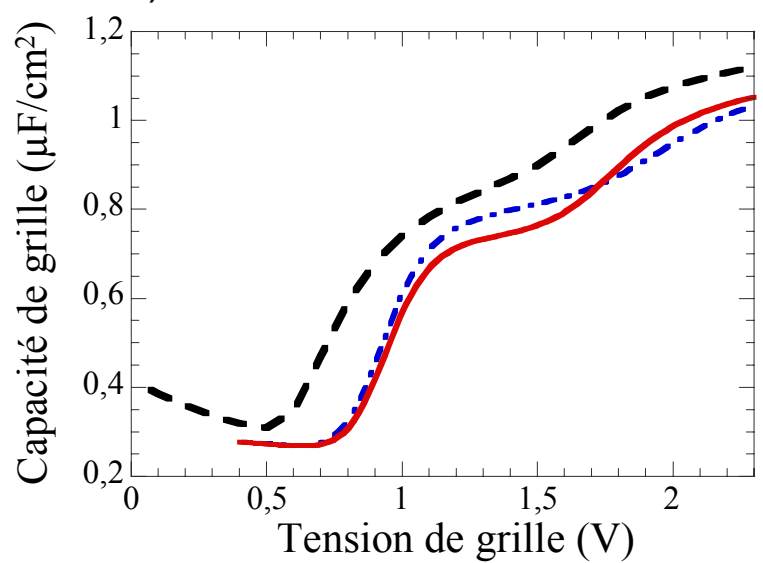

b)

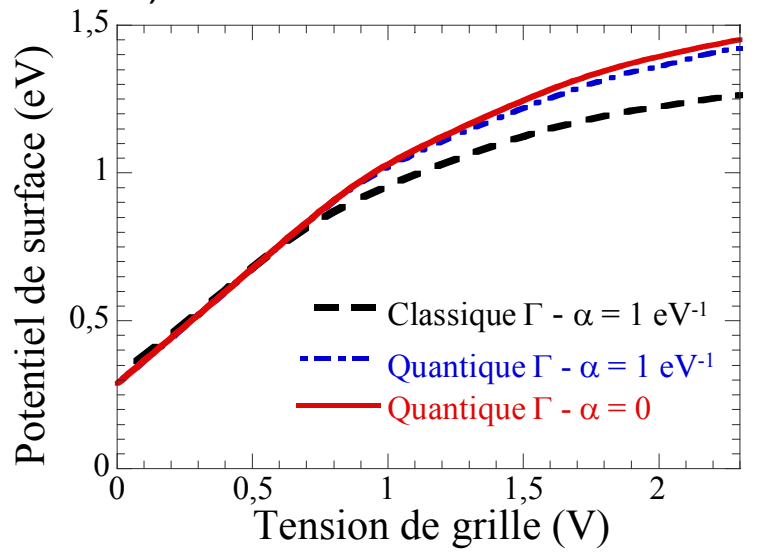

Figure 35 a) Caractéristique $C_{G}-V_{G}$ et b) variation du potentiel de surface dans le régime d'inversion pour la même structure de couche que Fig. 34, calculée avec la quantification de charge, la statistique de Fermi-Dirac et les multi-vallées, l'effet de non-parabolicité est pris en compte seulement dans la vallée $\Gamma$. La comparaison avec le calcul classique est aussi montrée. 
Pour obtenir de bonnes performances dans le domaine des radiofréquences (RF), il faut identifier une plage appropriée de variations de $V_{G}$ qui corresponde à une densité d'électrons 'rapides' dans la vallée $\Gamma$. La Fig. 36 peut donner une indication sur cette gamme de valeurs de tension de grille pour différents modèles. D'après les calculs classiques selon la statistique de Boltzmann, les porteurs occupent très majoritairement les états de la vallée $\Gamma$ dans toute la plage de variation de la tension de grille. En revanche, si le peuplement des états est régi par la statistique de Fermi Dirac, et quel que soit le formalisme de calcul (classique ou quantique, parabolique ou non-parabolique), le transfert vers les vallées satellites "lourdes" (ayant une masse effective plus grande) devient non négligeable pour de grandes $\mathrm{V}_{\mathrm{GS}}$ (ici $\mathrm{V}_{\mathrm{GS}}$ $>1,5 \mathrm{~V}$ pour une densité de dopage $\mathrm{N}_{\mathrm{A}}=10^{18} \mathrm{~cm}^{-3}$ ). La limitation de la condition de polarisation en $\mathrm{V}_{\mathrm{G}}$ ainsi que la tension d'alimentation requise pour un meilleur fonctionnement en RF seront discutées plus tard dans la section "Estimation de la plage de fonctionnement de la tension de grille".

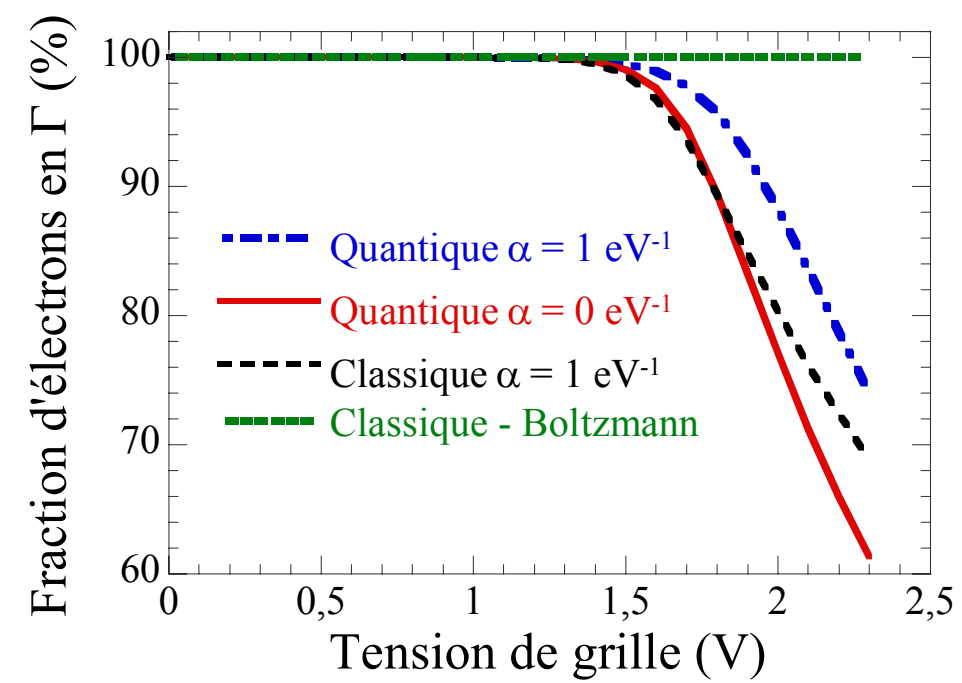

Figure 36 Distribution des électrons dans la vallée $\Gamma$ en fonction de la tension de grille pour différents modèles. La statistique utilisée est Fermi-Dirac sauf indication.

Dans cette première partie, nous avons mis en place les modèles pertinents qui permettent de calculer théoriquement la réponse statique $\mathrm{C}-\mathrm{V}$ et la variation du potentiel de surface pour une capacité métal/high-א/III-V. Dans la partie suivante, en utilisant ces modèles, nous allons étudier les aspects du contrôle de charge au sein de structures MOS III-V. Les modèles vont ensuite être adaptés aux géométries 2D. Les effets parasites induits par une géométrie du transistor en 2D pourront être analysés. La calibration de cette simulation avec les résultats expérimentaux nécessitera toutefois d'introduire les effets extrinsèques comme les états de pièges d'interface $D_{\text {it. }}$ Grâce à ces études, nous pourrons observer comment les $D_{\text {it }}$ dégradent les caractéristiques intrinsèques. 


\section{Contrôle de charge de la structure MOS III-V}

\section{2-1 Canal long : géométrie 1D}

\section{2-1-1 Efficacité de contrôle électrostatique}

La réduction de masse effective (cas des matériaux III-V à faible gap) dans le plan de transport permet d'améliorer la vitesse d'injection quel que soit le degré de dégénérescence du système et d'augmenter le temps de relaxation à faible champ (mobilité) pour ainsi diminuer le coefficient de rétrodiffusion [Shinichi2008IEEE] et [Lundstrom2002IEEE]. Cependant, une très faible masse effective pourrait dégrader l'efficacité du contrôle de charge. En effet, la densité d'états DOS en 2D-k de chaque sous bande dans la couche d'inversion sera donc réduite. Par conséquence, la capacité inhérente du semi-conducteur massif que l'on l'appelle capacité quantique (l'une des contributions à la capacité d'inversion) sera donc dégradée, ce qui reflète directement la conséquence de la faible DOS. On décrira en détails la modélisation de cette capacité de nature 'quantique' dans les paragraphes suivants.

Si on suppose que la zone de charge d'espace est d'épaisseur constante en régime de forte inversion, on peut négliger la capacité de déplétion en régime statique. La capacité d'inversion $C_{I N V}$ et la capacité d'oxyde $C_{O X}$ sont connectées en série, la capacité totale de grille est donc donnée par :

$$
\frac{C_{G}}{C_{O X}}=\frac{1}{1+\frac{C_{O X}}{C_{I N V}}}=\frac{1}{1+\left(\varepsilon_{O X} / T_{O X}\right) / C_{I N V}}
$$

Ici, $T_{O X}$ et $\varepsilon_{O X}$ sont respectivement l'épaisseur et la permittivité diélectrique de l'oxyde de grille. Dans le cas idéal, $\mathrm{C}_{\mathrm{G}} / \mathrm{C}_{\mathrm{OX}}$ vaut 1 mais ce rapport est dégradé pour une DOS faible par la diminution de $\mathrm{C}_{\mathrm{INV}}$. L'effet est d'autant plus important que $T_{O X}$ est petit. Il peut être représenté par une épaisseur d'oxyde effectivement plus grande que l'épaisseur réelle. On peut ainsi définir une épaisseur d'oxyde de Si équivalente ou effective (EOT selon le sigle en anglais) sous la forme :

$$
E O T=\frac{\varepsilon_{\mathrm{SiO}_{2}}}{C_{G}}
$$

D'après les Eq. II-11 et II-12, on a :

$$
\frac{1}{C_{G}}=\frac{1}{C_{O X}}+\frac{1}{C_{I N V}}=\frac{T_{O X}}{\varepsilon_{O X}}+\frac{1}{C_{I N V}}
$$

Et : 


$$
E O T=\frac{\varepsilon_{S i O_{2}}}{\varepsilon_{O X}} T_{O X}+\frac{\varepsilon_{S i O_{2}}}{C_{I N V}}
$$

Le premier terme ne fait que traduire l'épaisseur physique de high- $\kappa$ en épaisseur équivalente de $\mathrm{SiO}_{2}$ compte tenu de la différence de permittivité diélectrique. Le second correspond à l'augmentation $\triangle \mathrm{EOT}$ de l'EOT due à $C_{I N V}$, elle est caractéristique du semi-conducteur du canal et indépendante de $T_{O X}$. Dans le cas du Silicium, la densité d'états dans la bande de conduction est de l'ordre de $10^{19} \mathrm{~cm}^{-3}$ à $300 \mathrm{~K}$ et pour un oxyde relativement épais, on peut négliger le second terme. En revanche pour des matériaux III-V comme InGaAs, cette approximation devient discutable. Notre calcul Poisson-Schrödinger auto-cohérent peut fournir l'occupation des sous bandes dans chaque vallée $(\Gamma, \mathrm{L}$ et $\mathrm{X})$ selon la statistique de Fermi-Dirac afin d'estimer la capacité $C_{I N V}$. On pourra donc analyser cette dégradation de la capacité de grille vis-à-vis du problème de DOS 'bottleneck' dans la vallée $\Gamma$.

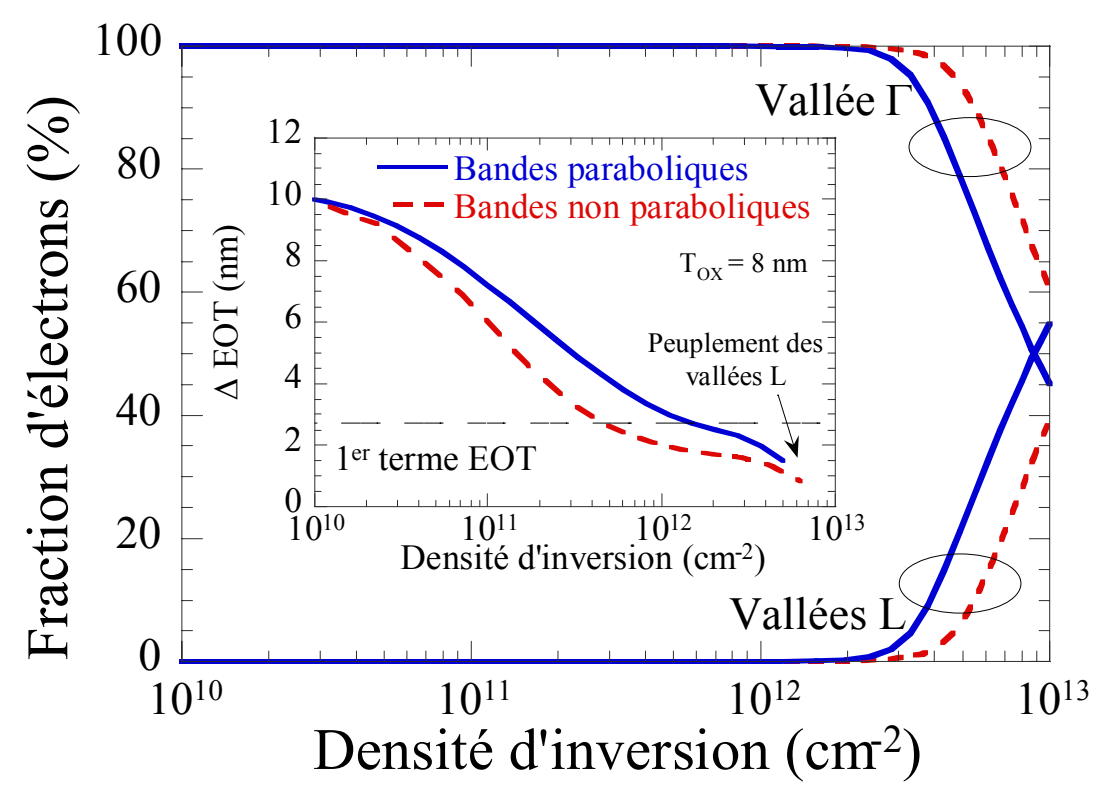

Figure 37 Distribution des électrons dans la vallée $\Gamma$ et $L$. Insert : variation du second terme de l'EOT dans l'Eq. II-16 en fonction de la densité des électrons d'inversion avec et sans effet de la non-parabolicité.

Les résultats sont illustrés sur la Fig. 37. L'augmentation d'épaisseur effective $\Delta \mathrm{EOT}$ est d'abord tracée en fonction de la densité de porteurs d'inversion. On peut constater que $\Delta$ EOT est importante dans la plage où la population des électrons est dans la vallée $\Gamma$. Elle d'autant plus importante que la densité est faible. En régime de forte inversion, elle est de l'ordre de 1,5 à $2,5 \mathrm{~nm}$ pour une densité d'électrons $\Gamma$ aux alentours de $3 \times 10^{12} \mathrm{~cm}^{-2}$. Elle devient de plus en plus faible quand les états électroniques des vallées L sont peuplés. L'effet 
de non-parabolicité augmente $C_{I N V}$ et ainsi diminue 1'EOT. Cette épaisseur effective sera donc surestimée si on ignore l'effet de non-parabolicité. L'augmentation de l'EOT par $\Delta \mathrm{EOT}$ va diminuer l'efficacité du contrôle de charge par la grille dans la plage où les électrons peuplent la vallée $\Gamma$, cet effet est d'autant plus important que l'épaisseur d'oxyde $T_{O X}$ est petite, mais conventionnellement on utilise souvent cette dernière méthode pour augmenter $C_{O X}$ ainsi que la capacité de grille. L'un des objectifs de cette étude est de trouver une épaisseur d'oxyde optimisée $T_{O X_{-} O p t}$ qui corresponde à un compromis entre l'efficacité du contrôle de charge et la dégradation de $C_{G}$ par rapport à $C_{O X}$. Pour cela, on trace $C_{G} / C_{O X}$ et $C_{G}$ en fonction de l'EOT sur le même graphe pour une tension $V_{G}$ donnée, dans laquelle la densité des électrons en vallée $\Gamma$ est quasiment 100 fois plus grande que la densité des électrons en vallée L. On choisit $1 \%<n_{s}(L) / n_{s}(\Gamma)<2 \%$. Les résultats sont montrés dans la Fig. 38 .
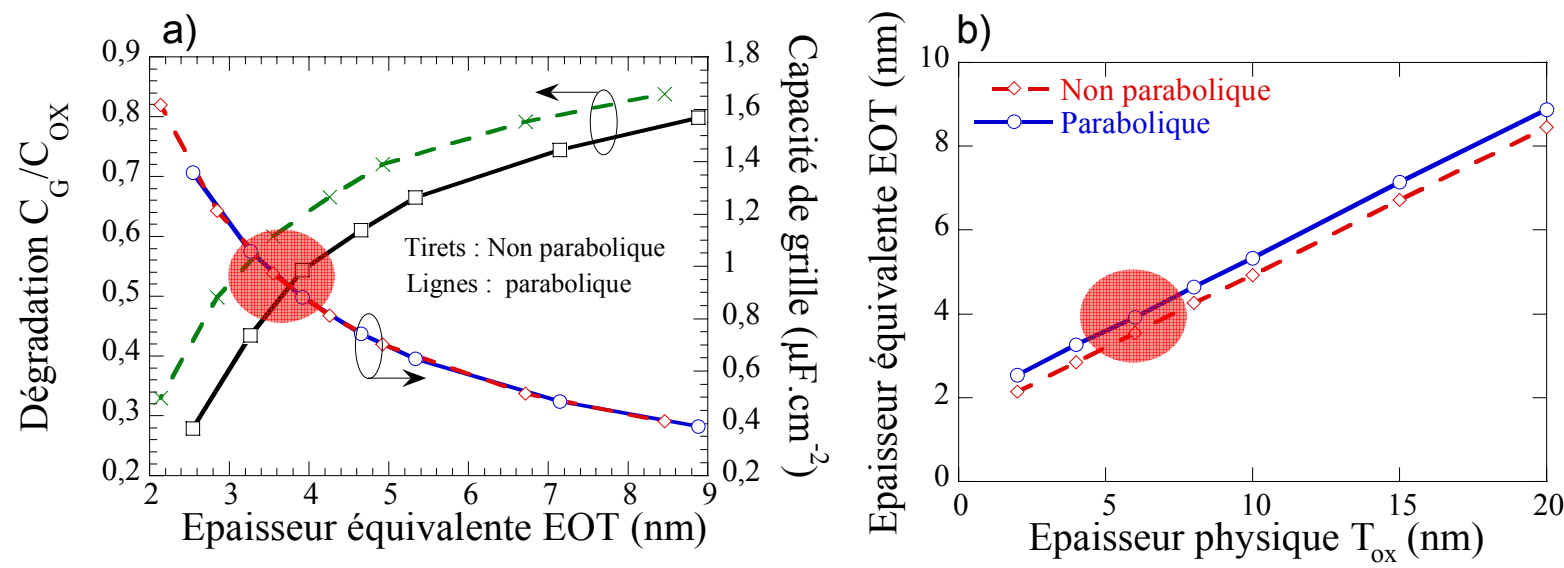

Figure 38 a) Dégradation de la capacité de grille $C_{G} / C_{O x}$ et $C_{G}$ en fonction de EOT. $C_{G}$ et EOT sont déterminées pour une $V_{G}$ dans laquelle la densité des électrons en vallée $\Gamma$ est quasiment 100 fois plus grande que la densité des électrons en vallée $L$. b) EOT en fonction de $T_{O x}$ à $V_{G}$ choisie. La région entourée en rouge montre la zone du compromis.

Pour limiter le courant de fuite par la grille, nous proposons une gamme d'épaisseur d'oxyde optimisée qui varie entre $5,5 \mathrm{~nm}$ et $8 \mathrm{~nm}$. Par la suite, nous choisirons une épaisseur d'oxyde de $8 \mathrm{~nm}$, qui correspond à un EOT d'environ 4,5 nm et à une capacité de grille de $57 \%$ (parabolique) à 69\% (non-parabolique) par rapport à la capacité d'oxyde.

\section{2-1-2 Modèle analytique pour estimer la tension de seuil}

La tension de grille $\mathrm{V}_{\mathrm{G}}$ dans la structure MOS peut s'écrire :

$$
V_{G}=V_{F B}+\psi_{S}+V_{O X}
$$


où $V_{F B}$ est la tension de bandes plates, dépendant de la différence $\phi_{m s}$ des travaux de sortie du semi-conducteur et de l'électrode de grille ainsi que de la présence de charges dans l'oxyde. Elle s'écrit : $V_{F B}=\phi_{m s}-Q_{i t} / C_{O X}-Q_{f i x e} / C_{O X}$. Les charges extrinsèques de défaut dans l'oxyde peuvent être de deux types : charges piégées à l'interface $\left(Q_{i t}\right)$ et charge fixes $\left(Q_{\text {fixe }}\right)$. De plus $\phi_{m s}=\phi_{m}-\chi_{s c}-E_{G} / 2-\psi_{F}$, où $\phi_{m}$ est le travail de sortie du métal et $\chi_{s c}$ est l'affinité du semi-conducteur. $E_{G}$ est le gap de la bande interdite. La quantité $\psi_{F}=k_{B} T / q \cdot \ln \left(N_{A} / n_{i}\right)$ représente dans le semi-conducteur, la distance du niveau de Fermi au niveau de Fermi intrinsèque. Pour une structure idéale, on néglige les contributions des charges issues de $Q_{i t}$ et $Q_{f i x e}$. On étudiera quantitativement les effets extrinsèques dans la section suivante. Par ailleurs, $\psi_{S}$ est le potentiel de surface dans le canal et $V_{O X}$ est la chute de potentiel dans l'oxyde $V_{O X}=-Q_{d e p} / C_{O X}$ avec $Q_{d e p}=q N_{A} T_{d e p}$, où $T_{d e p}=\sqrt{\varepsilon_{s c} / q N_{A}\left(\psi_{S}-V_{B u l k}\right)}$. Dans notre calcul, la condition aux limites est un champ nul dans la zone quasi-neutre du semiconducteur. Souvent la tension du substrat $V_{\text {Bulk }}$ permet d'ajuster la tension de seuil à postériori. Pour simplifier le calcul, la polarisation du substrat $V_{B u l k}$ est supposée d'être nulle dans la suite des calculs.

On définit la tension de seuil de la structure MOS comme la tension de grille nécessaire à l'établissement du régime de forte inversion, c'est-à-dire telle que la densité volumique de porteurs à la surface $n_{s}$ est égale à la concentration de dopant $\mathrm{N}_{\mathrm{A}}$. Cette valeur de tension de grille $\mathrm{V}_{\mathrm{TH}}$ pourra être déterminée à l'aide de la valeur de potentiel de surface qui s'exprime en fonction de $\psi_{F}$ selon le degré de dégénérescence.

$$
V_{T H}=V_{F B}+f\left(\psi_{F}\right)+\frac{\sqrt{2 q N_{a} \varepsilon_{s} f\left(\psi_{F}\right)}}{C_{o x}}
$$

Dans un premier temps il s'agit de calculer $n_{s}$ à la surface. En régime dégénéré on ne peut plus approcher la distribution de Fermi par une distribution de Boltzmann. Les densités de porteurs libres sont données par les expressions A-4 et A-5 (Annexe - A). Si on néglige l'effet de non-parabolicité (cet effet a très peu d'influence dans le régime sous le seuil), la densité d'électrons s'écrit $n=N_{C} \mathfrak{J}_{1 / 2}(\eta)$ avec :

$$
\mathfrak{I}_{1 / 2}(\eta)=\frac{2}{\sqrt{\pi}} \int_{0}^{\infty} \frac{\varepsilon^{1 / 2}}{1+e^{\varepsilon-\eta}} d \varepsilon
$$


où $\eta=\left(E_{F}-E_{C}\right) / k_{B} T$ et $\varepsilon=\left(E-E_{C}\right) / k_{B} T$. Cette fonction intégrale de Fermi-Dirac peut être approchée par des expressions analytiques selon la valeur de $\eta$ c'est-à-dire que leur expression dépend du degré de dégénérescence [Mathieu1998]. Si le semi-conducteur est non dégénéré, $\mathfrak{I}_{1 / 2}(\eta)=e^{\eta}$. Si le semi-conducteur n'est que faiblement dégénéré, c'est-à-dire pour $E_{F}<E_{C}+2 k_{B} T(\eta<2)$, on a: $\mathfrak{I}_{1 / 2}(\eta) \approx e^{\eta} /\left(1+e^{\eta} / 4\right)$. Si en revanche le semiconducteur est fortement dégénéré, c'est-à-dire $E_{F}>E_{C}+5 k_{B} T(\eta>5)$, il faut considérer : $\mathfrak{I}_{1 / 2}(\eta) \approx(4 / 3 \sqrt{\pi}) \eta^{3 / 2}$. Pour récapituler, la densité d'électrons s'écrit

$$
\begin{array}{ccc} 
& \eta<-2 \quad n \approx N_{C} \cdot e^{\eta} & \text { Boltzmann } \\
\eta<2 & n \approx N_{C} \cdot e^{\eta}\left(1 /\left(1+e^{\eta} / 4\right)\right) & \text { Boltzmann Corrigé 1 } \\
\eta>5 & n \approx N_{C} \cdot(4 / 3 \sqrt{\pi}) \eta^{3 / 2} & \text { Dégénéré Corrigé 2 }
\end{array}
$$

Ces différentes approximations sont comparées au résultat du calcul numérique sur la Fig. 39.

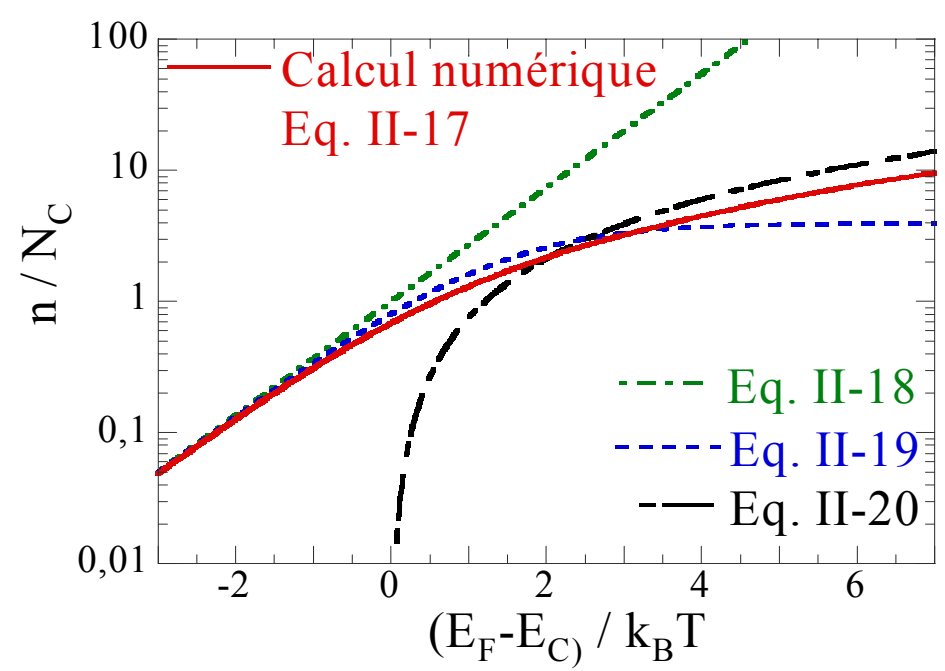

Figure 39 Calcul numérique de l'intégrale Fermi-Dirac et différentes approximations analytiques pour des semi-conducteurs présentant divers taux de dégénérescence

On calcule la densité de porteur volumique dans la direction de confinement en utilisant les approximations de l'intégrale de Fermi-Dirac (Eq. II-18-20). Si on est dans le régime faiblement dégénéré (cf. Eq. II-19), la densité de porteur peut s'exprimer par :

$$
n(z)=n_{i} \cdot e^{\frac{E_{F}-E_{i}(z)}{k_{B} T}} \cdot \frac{4}{4+\frac{n_{i}}{N_{C}} e^{\frac{E_{F}-E_{i}(z)}{k_{B} T}}}
$$

avec $n_{i}$ densité intrinsèque du semi-conducteur. Si on suppose que $\varphi(z)=E_{i}(z)-E_{F}$. La densité de porteur à l'interface est égale à $\left(\varphi(z=0)=\varphi_{s}\right)$ : 


$$
n_{s}=n(z=0)=n_{i} \cdot e^{-\frac{q \varphi_{s}}{k_{B} T}} \cdot \frac{4}{4+\frac{n_{i}}{N_{C}} e^{-\frac{q \varphi_{s}}{k_{B} T}}}
$$

avec $\varphi_{s}=\psi_{S}-\psi_{F}$. En outre, la concentration de dopants ionisés est égale à $N_{A}=p_{o}=n_{i} \cdot e^{\frac{q \psi_{F}}{k_{B} T}}$. Le seuil $\mathrm{V}_{\mathrm{TH}} \mathrm{du}$ régime de forte inversion correspond au potentiel de surface $\psi_{S}$ pour le quel $n_{s}=N_{A}$. On a finalement :

$$
\psi_{S}=f\left(\psi_{F}\right)=\psi_{F}-\frac{k T}{q} \ln \left[\frac{4 N_{c} \exp \left(\frac{-q \psi_{F}}{k T}\right)-n_{i}}{4 N_{c}}\right]
$$

On procède de la même façon pour les régimes non-dégénérés et fortement dégénéré, on a donc, respectivement :

$$
\begin{gathered}
\psi_{S}=f\left(\psi_{F}\right)=2 \psi_{F} \quad \text { pour } E_{F}<E_{C}-2 k_{B} T \\
\psi_{S}=f\left(\psi_{F}\right)=\frac{k T}{q}\left(\frac{n_{i} \cdot 3 \sqrt{\pi}}{4 N_{c}}\right)^{2 / 3} \exp \left(\frac{2 q \psi_{F}}{3 k T}\right)+\psi_{F}-\frac{E_{G}}{2 q} \quad \text { pour } E_{F}>E_{C}+5 k_{B} T
\end{gathered}
$$

En tenant compte de la quantification des niveaux, tout se passe comme si le gap du semiconducteur était augmenté de $E_{\text {fonda }}-E_{C}$. En première approximation la tension de seuil doit être corrigée de la valeur du premier niveau d'énergie du puits (cf. Eq. II-26), mais on a besoin des informations sur le premier sous bande. L'approximation analytique de la tension de seuil ne prend compte que l'effet de dégénérescence.

$$
V_{T H}^{2 D} \approx V_{T H}^{3 D}+\frac{E_{\text {fonda }}-E_{C}}{q}
$$

Les résultats d'estimation de cette tension de seuil sont illustrés sur la Fig. 40 cidessous, pour un travail de sortie du métal égal à $4,6 \mathrm{eV}$, une affinité électronique du semiconducteur $\left(\mathrm{In}_{0.53} \mathrm{Ga}_{0.47} \mathrm{As}\right)$ de $4,5 \mathrm{eV}$ et un oxyde $\mathrm{Al}_{2} \mathrm{O}_{3}$ avec une épaisseur de $8 \mathrm{~nm}$. On constate qu'il y a un très bon accord entre tous les modèles si la concentration du dopant est inférieure à la densité équivalente d'états $\left(10^{17} \mathrm{~cm}^{-3}\right.$ pour $\left.\operatorname{In}_{0,53} \mathrm{Ga}_{0,47} \mathrm{As}\right)$. Dans ce cas, les effets quantiques et de la dégénérescence sont très faibles et le modèle classique (Poisson seul + statistique de Boltzmann) est toujours valable. Mais au-delà de cette concentration, le modèle classique sous-estime la tension de seuil. L'effet de dégénérescence est pris en compte analytiquement en utilisant les fonctions d'approximation de l'intégrale de Fermi-Dirac, ce résultat est vérifié par les résultats de la simulation numérique. 


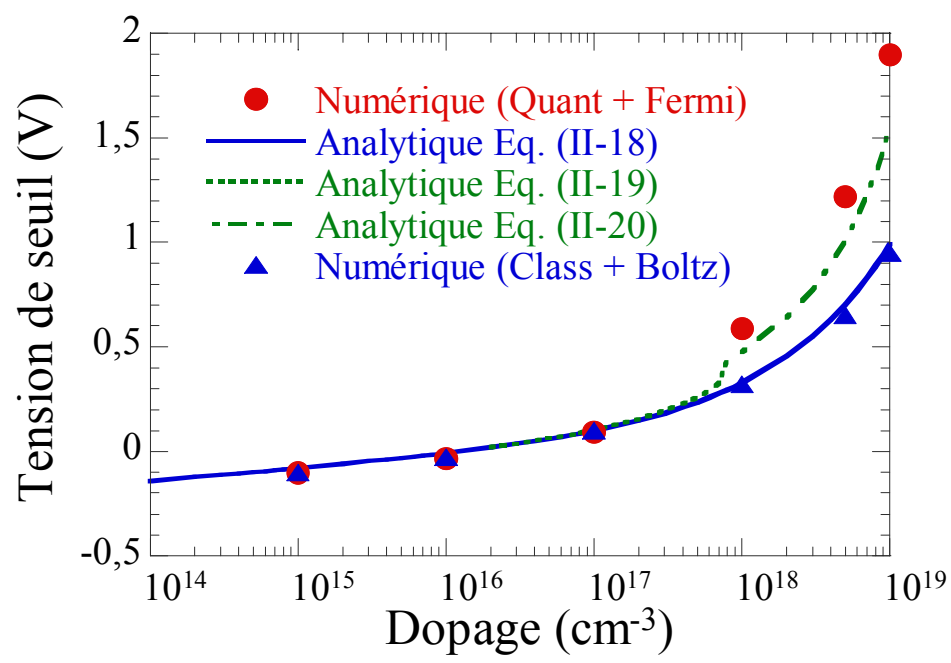

Figure 40 Evaluation de la tension de seuil en fonction de la concentration de dopants. Les symboles sont des résultats de simulation numérique (quantique $=$ Poisson+Schrödinger+Fermi-Dirac, classique $=$ Poisson+Boltzmann). Les traits sont des résultats de calculs analytiques approchés.

\section{2-1-3 Modèle semi-analytique de la capacité quantique}

Pour comprendre la dégradation de la capacité de grille due à la faible capacité d'inversion dans la vallée $\Gamma$ ayant une très faible masse effective, on propose un modèle semianalytique de la capacité de grille d'une structure 1D [Jin2009IEDM]. Il permet d'étudier quantitativement la capacité d'inversion. Ce modèle est calibré par des calculs numériques pour une structure MOS III-V.

a)

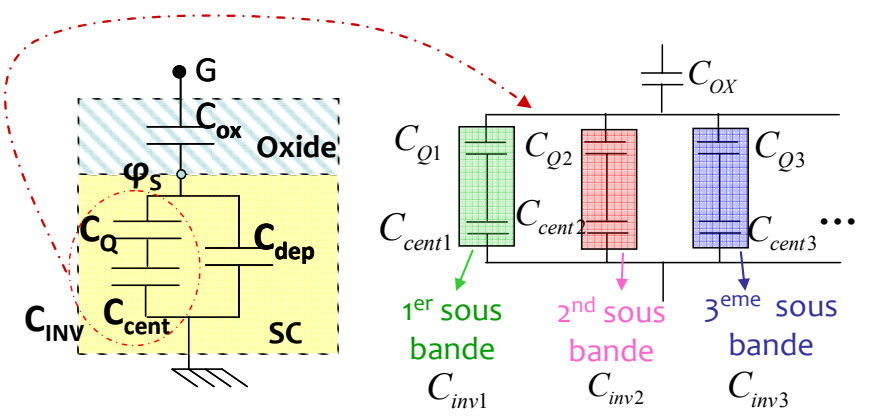

b)

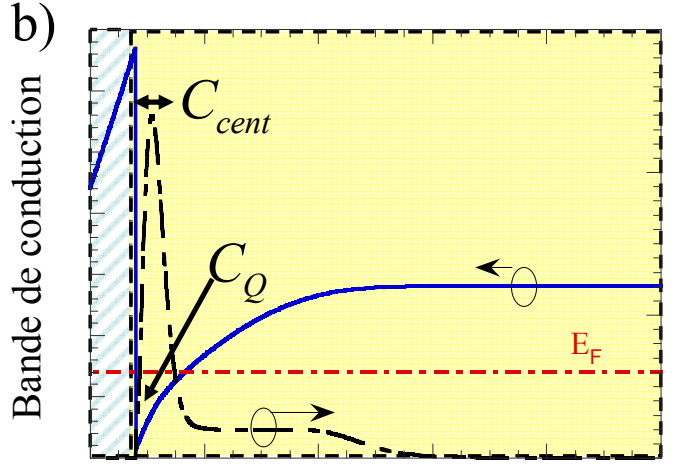

Distance selon $\mathrm{x}$

Figure 41 a) Modèle 1D semi-analytique pour la capacité de grille dans une structure MOS III-V.

b) La représentation physique des composantes de la capacité d'inversion

Ce modèle de capacité de grille totale est illustré sur la Fig. 41-a. La capacité équivalente dans le semi-conducteur est une connexion en parallèle entre une capacité de déplétion $C_{d e p}$ et de couche d'inversion $C_{I N V}$. La capacité de grille totale est une connexion en série entre la capacité équivalente dans le semi-conducteur et celle d'isolant. La capacité 
d'inversion a deux contributions principales: la capacité quantique $C_{Q}$ et la capacité de centroïde de charge $C_{c e n t}$. La première résulte de la DOS limitée dans les sous bandes d'un gaz d'électrons 2D dégénéré. La seconde est liée à la redistribution quantique spatiale de la charge dans la couche d'inversion. Ces deux contributions sont illustrées qualitativement dans la Fig. 41-b. Pour chaque sous bande $\mu$, la capacité d'inversion consiste en la capacité quantique $C_{Q_{-} \mu}$ et la capacité de centroïde $C_{\text {cent }{ }_{-} \mu}$. On va retrouver ces deux contributions à l'aide de la définition générale de $C_{I N V}$ [Himadri2008IEEE] et [Jin2009IEDM] :

$$
C_{I N V}=\frac{\partial\left(-Q_{S}\right)}{\partial \psi_{S}}=\frac{q \partial\left(-Q_{S}\right)}{\partial\left(E_{F}-E_{C}\right)}=\frac{q \partial\left(-\sum_{v} \sum_{\mu} Q_{\mu}\right)}{\partial\left(E_{F}-E_{C}\right)}
$$

et que,

$$
\frac{q \partial\left(-Q_{\mu}\right)^{-1}}{\partial\left(E_{F}-E_{C}\right)} \approx{\frac{q \partial\left(-Q_{\mu}\right)^{-1}}{\partial\left(E_{F}-E_{i}\right)}}^{-1}+\frac{q \partial\left(-Q_{\mu}\right)^{-1}}{\partial\left(E_{i}-E_{C}\right)}
$$

On peut donc écrire la capacité d'inversion analytiquement :

$$
C_{I N V}=\sum_{v} \sum_{\mu}\left(\frac{1}{C_{Q_{-} \mu}}+\frac{1}{C_{C_{e n t r_{-} \mu}}}\right)^{-1}
$$

avec

$$
C_{Q_{-} \mu}=\frac{m_{D O S_{-} \nu}^{*} q^{2} / \pi \hbar^{2}}{1+\exp \left(\frac{E_{\mu}-E_{F}}{k_{B} T}\right)} \quad \text { et } \quad C_{c e n t r_{-} \mu}=C_{Q_{-} \mu} \frac{\partial\left(E_{F}-E_{\mu}\right)}{\partial\left(E_{\mu}-E_{C}\right)}
$$

Ces formules analytiques nous permettent d'analyser précisément chaque contribution de sous bande sur les composantes de la capacité d'inversion, si la localisation des niveaux de sous bande et celle du niveau de Fermi par rapport au bas de bande de conduction sont connus. Notre simulation Poisson-Schrödinger nous permet d'obtenir des niveaux de sous bande en fonction de la tension de grille.

Pour l'épaisseur d'oxyde $T_{O X}=T_{O X_{-} O p t}=6 \mathrm{~nm}$, ce qui correspond à une EOT environ égale à 3,5 nm. Les résultats sont montrés sur la Fig. 42. On constate un très bon accord entre calcul numérique et modèle semi-analytique dans le cas d'électrons peuplant la vallée $\Gamma$. On note que les deux composantes de capacités d'inversion dans la première sous bande sont du même ordre de grandeur. Il faut donc prendre en compte à la fois la statistique de Fermi-Dirac et le confinement quantique pour modéliser le contrôle de charge dans une structure MOS pour un semi-conducteur caractérisé par une très faible masse effective. Ce modèle est aussi valable en prenant en compte les vallées satellites en sommant les contributions des sous 
bandes dans ces vallées latérales et les pondérant par leur taux d'occupation. Toutefois, ce modèle n'est pas purement analytique, en effet, la position du niveau Fermi par rapport aux niveaux de sous bande est issu de la résolution numérique des équations de PoissonSchrödinger.

a)

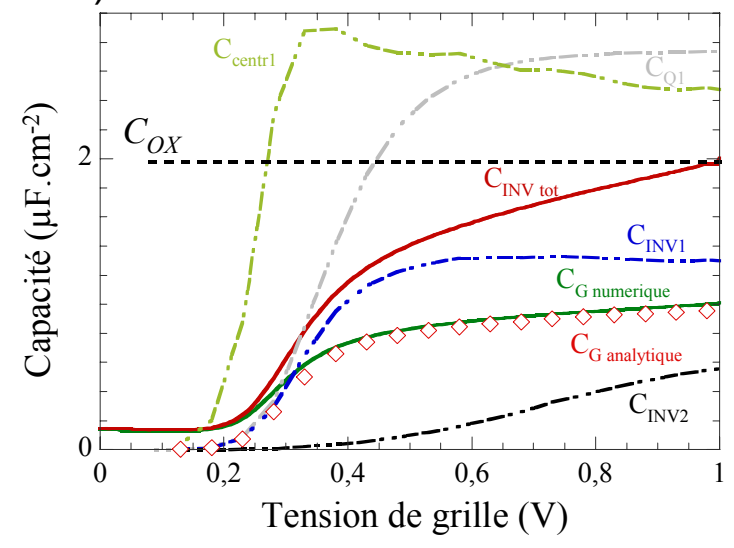

b)

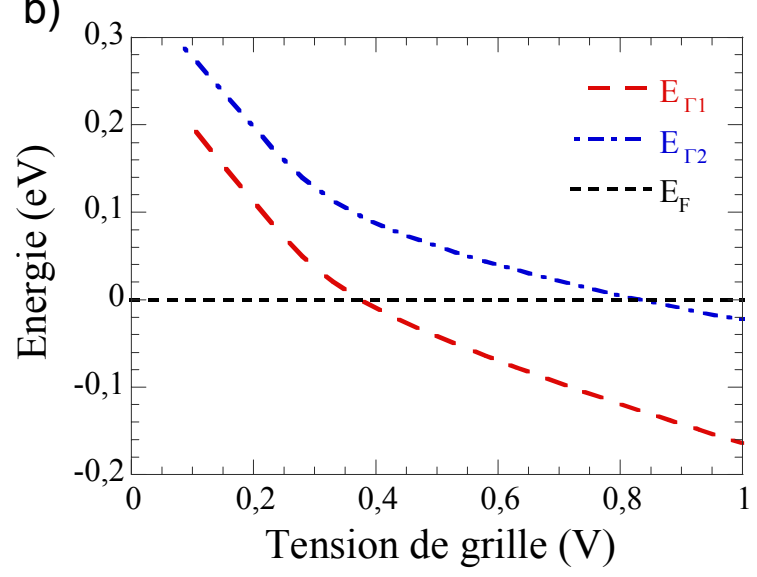

Figure 42 a) Variations des différentes composantes de la capacité d'inversion et de la capacité de grille, calculées numériquement et analytiquement pour $N_{A}=10^{17} \mathrm{~cm}^{-3}$ et $T_{O X}=6 \mathrm{~nm}$. b) L'évolution de la première et deuxième sous bande de la vallée $\Gamma$ par rapport au niveau de Fermi. Le transfert vers les vallées $L$ n'est pas pris en compte pour une faible densité de dopants.

\section{2-1-4 Particularités du contrôle de charge dans les structures alternatives}

Les performances de la structure conventionnelle MOS-Bulk sont a priori limitées par les mauvaises propriétés de transport électronique dans le canal (interactions avec la rugosité d'interface canal/diélectrique, coulombiennes avec les impuretés ionisées et les charges dues aux pièges d'interface...) et la faible immunité contre les effets de canal court (voir par exemple en Fig. 32 la relativement forte valeur de la pente sous le seuil pour un dopage déjà conséquent). Il est donc intéressant d'étudier les structures alternatives représentées sur la Fig. 30. Nous allons montrer quelques particularités en termes de contrôle de charge pour les structures MOS-ThinBody, MOS-HEMT et MOS-COMB.

D'après le dimensionnement préliminaire du premier paragraphe sur la structure ThinBody, nous notons que dans notre cas (épaisseur $\mathrm{d}^{\prime} \mathrm{Al}_{2} \mathrm{O}_{3}$ de $8 \mathrm{~nm}, \mathrm{~L}_{\mathrm{G}}=50 \mathrm{~nm}$ et une zone active (body) non dopée), l'épaisseur du body devrait être inférieure ou égale à $10 \mathrm{~nm}$ pour satisfaire à la condition de mise à l'échelle contre les effets de canal court. Généralement, nous pouvons améliorer le contrôle sous le seuil en diminuant l'épaisseur du film actif. Toutefois, l'effet de forte quantification et de la non-parabolicité des bandes devraient induire une forte modification d'occupation de la sous bande de différentes vallées [Liu2008IEEE_A] et ainsi augmenter la masse effective dans le plan de transport et dans le sens de confinement 
[Liu2008IEEE_B]. Sur la Fig. 43-a, on calcule ainsi la position de la première sous bande de différentes vallées par rapport au bas de bande de conduction à l'interface en fonction de l'épaisseur de la couche active $\left(\mathrm{T}_{\text {body }}\right)$. On illustre également l'effet de la non-parabolicité pour moduler la même densité d'électrons d'inversion $\left(3 \times 10^{12} \mathrm{~cm}^{-2}\right)$. Dans le cas parabolique, quand l'épaisseur de la couche d'InGaAs est réduite, le niveau d'énergie de la première sous bande dans la vallée $\Gamma$ monte rapidement en raison de la très faible masse effective dans le sens de confinement. En conséquence, le transfert vers les vallées L apparaît pour une faible tension de grille, l'essentiel de la charge d'inversion dans la couche active vient des vallées 'lourdes' L si $\mathrm{T}_{\text {body }}$ devient très petite. Ainsi, les vallées satellites sont peuplées plus facilement dans le MOS-ThinBody que dans la structure MOS-Bulk. Si l'on tient compte de l'effet de non-parabolicité, la situation est améliorée. L'accroissement de la sous- bande de la vallée de $\Gamma$ quand $\mathrm{T}_{\text {body }}$ diminue est plus lent. Cela assure la présence d'une densité significative d'électrons 'rapide' $\Gamma$, mais la tendance globale reste la même. Les effets de la quantification et de la non-parabolicité jouent donc un rôle très important dans la structure à film mince. Si nous choisissons $T_{\text {body }}=10 \mathrm{~nm}$, l'effet de la quantification dû à la géométrie est presque négligeable. De plus, les paramètres du matériau massif restent valables et dans le même temps, les SCE sont également limités par ce confinement modéré.

a)

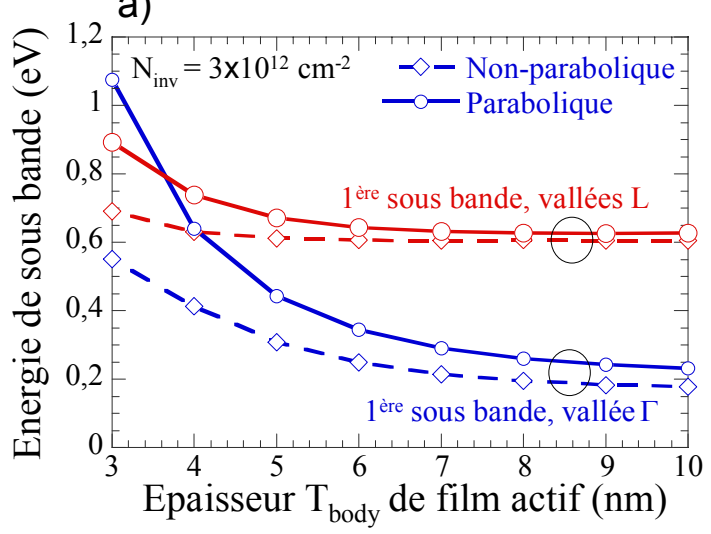

b)

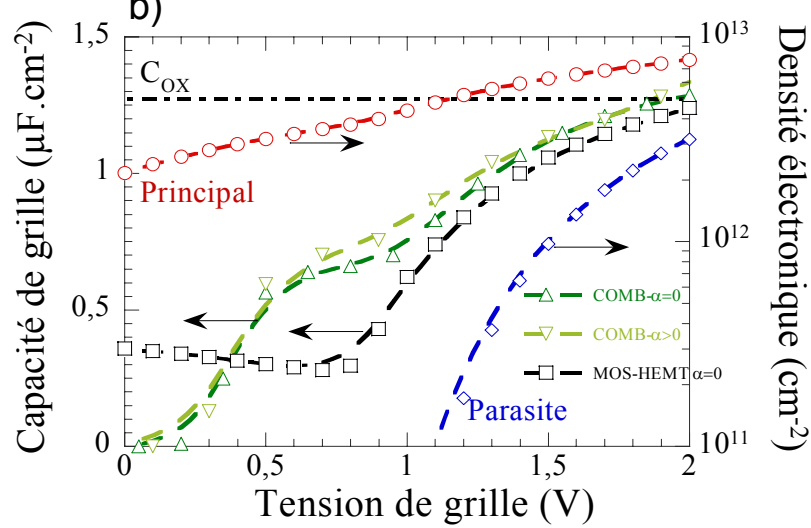

Figure 43 a) La variation de position de la première sous bande des vallées $\Gamma$ et $L$ en fonction de l'épaisseur de couche active pour une structure MOS-ThinBody. b) La capacité de grille totale dans les structures MOS-HEMT et MOS-COMB et les densités électroniques dans le canal principal et celui parasite dans la structure MOS-HEMT.

Pour limiter l'interaction de la rugosité à canal/diélectrique interface, nous avons introduit la structure MOS-HEMT. L'inconvénient de cette structure est le risque de transfert dans l'espace réel (par des électrons chauds et par contrôle électrostatique) vers l'espaceur de grand gap (canal parasite) des électrons du canal principal, ce qui est préjudiciable aux 
propriétés de transport. Cette situation peut se produire d'autant plus facilement que la masse effective du canal est faible et le niveau de dopage du substrat est élevé. En effet, plus la masse est faible et plus le niveau de Fermi doit varier rapidement pour moduler la même densité de porteurs dans le canal. De plus, une forte densité de dopage induit un fort confinement ce qui, combiné encore à la faible masse effective, tend à relever la position de la première sous bande. Cela facilite le peuplement du canal parasite qui peut se former dans l'espaceur. Sur la Fig. 43-b, on constate que la densité électronique dans l'espaceur devient non négligeable devant celle du canal pour une tension de grille supérieure à $1,4 \mathrm{~V}$ environ . Cela réduit la plage de $\mathrm{V}_{\mathrm{G}}$ pour un contrôle de charge efficace dans le canal principal. Pour notre structure avec $\mathrm{N}_{\mathrm{A}}=10^{18} \mathrm{~cm}^{-3}$, ce transfert a lieu avant le transfert inter-vallées, le 'plateau' dans la courbe de C-V dû aux vallées satellites a disparu.

Pour améliorer la plage de fonctionnement et le transport électronique du canal principal, nous devons réduire le niveau de dopage. Pour cette raison, nous introduisons la structure MOS-COMB, considérée comme une structure de compromis acceptable entre les propriétés de transport et le contrôle électrostatique. Nous pouvons noter que la population dans le canal parasite est nettement réduite, le 'plateau' de contrôle des électrons en vallée $\Gamma$ dans le canal devient même visible. Mais ce transfert n'est pas totalement limité, $\mathrm{C}_{\mathrm{G}}$ dépasse à $\mathrm{C}_{\mathrm{OX}}$ à valeur élevée de $\mathrm{V}_{\mathrm{G}}$. En outre, on peut une nouvelle fois remarquer que la nonparabolicité augmente $\mathrm{C}_{\mathrm{G}}$, en particulier dans la plage de contrôle en vallée $\Gamma$.

\section{2-1-5 Plage de fonctionnement pour la tension de grille}

Ces analyses permettent d'estimer une plage $\mathrm{du}$ fonctionnement de $\mathrm{V}_{\mathrm{G}}$ pour une structure de capacité MOS 1D. Dans cette gamme, on obtient une densité suffisante d'électrons 'rapides' en vallée $\Gamma$ pour assurer de bonnes performances RF. Cette plage étant déterminée, on peut alors estimer la limite maximale de la tension d'alimentation afin d'éviter le transfert inter-vallées. Pour fixer les idées et par référence à un choix standard en logique CMOS, on peut considérer $\mathrm{V}_{\mathrm{DDmax}}=3 / 2\left(\mathrm{~V}_{\mathrm{Gmax}}-\mathrm{V}_{\mathrm{T}}\right)$, la tension de seuil $\mathrm{V}_{\mathrm{T}}$ étant estimée à l'aide de la densité d'électrons d'inversion. Pour MOS-Bulk, la plage en $\mathrm{V}_{\mathrm{G}}$ est de $0,8 \mathrm{~V}$ à $1,6 \mathrm{~V}$ (valeur à laquelle on a plus de $5 \%$ d'électrons en vallées $\mathrm{L}$ ). Le seuil $\mathrm{V}_{\mathrm{T}}$ est entre 0,6 (pour $\left.n_{i n v}=10^{10} \mathrm{~cm}^{-2}\right)$ et $0,8 \mathrm{~V}\left(n_{i n v}=10^{11} \mathrm{~cm}^{-2}\right)$. Ainsi, la tension d'alimentation maximale peut varier entre 1,2 et 1,5 V. Pour les spécifications basse consommation, la tension d'alimentation à utiliser est en dessous de $0,6 \mathrm{~V}$, cela empêche alors les transferts intervallées dans les dispositifs étudiés. Pour la structure MOS-ThinBody, la plage de contrôle de $\mathrm{V}_{\mathrm{G}}$ est réduite en cas de forte quantification. $\mathrm{Si} \mathrm{T}_{\text {body }}$ vaut $10 \mathrm{~nm}$, la gamme de contrôle en $\mathrm{V}_{\mathrm{G}}$ et la 
limite maximale de $\mathrm{V}_{\mathrm{DD}}$ sont presque les mêmes que pour la structure MOS-Bulk. Pour le MOS-HEMT, la plage $\mathrm{V}_{\mathrm{G}}$ de contrôle de charge est fortement réduite par le canal parasite. Pour MOS-COMB, nous pouvons également estimer que l'on peut moduler des électrons $\Gamma$ dans le canal principal pour une tension de grille entre 0,4 et $1,0 \mathrm{~V}$. Le seuil $\mathrm{V}_{\mathrm{T}}$ est entre $0,1 \mathrm{~V}$ et $0 ., 2 \mathrm{~V}$. Ainsi, la tension d'alimentation maximale ne peut pas dépasser 1,2 V.

\section{2-2 Effets géométriques 2D et effets du canal court}

La détermination de $\mathrm{C}_{\mathrm{G}}$ qui vient d'être décrite ne prend en compte que la direction normale aux interfaces. L'utilisation d'un tel modèle 1D est discutable si on souhaite l'exploiter pour prévoir les performances de transistors de faible longueur de grille, ici $50 \mathrm{~nm}$. Les composants à base de III-V devraient en effet être affectés par les effets de canal court, à cause de la grande permittivité diélectrique de ces semi-conducteurs, et aussi par les effets de bord. Des analyses par résolution auto-cohérente 2D des équations de Poisson-Schrödinger sont donc nécessaires. Pour cela, nous avons procédé à des calculs où l'effet du drain est implémenté en différentiant deux pseudo-niveaux de Fermi dans les régions d'accès de source et de drain, avec la condition aux limites de Neumann (champ nul perpendiculairement aux bords du dispositif).
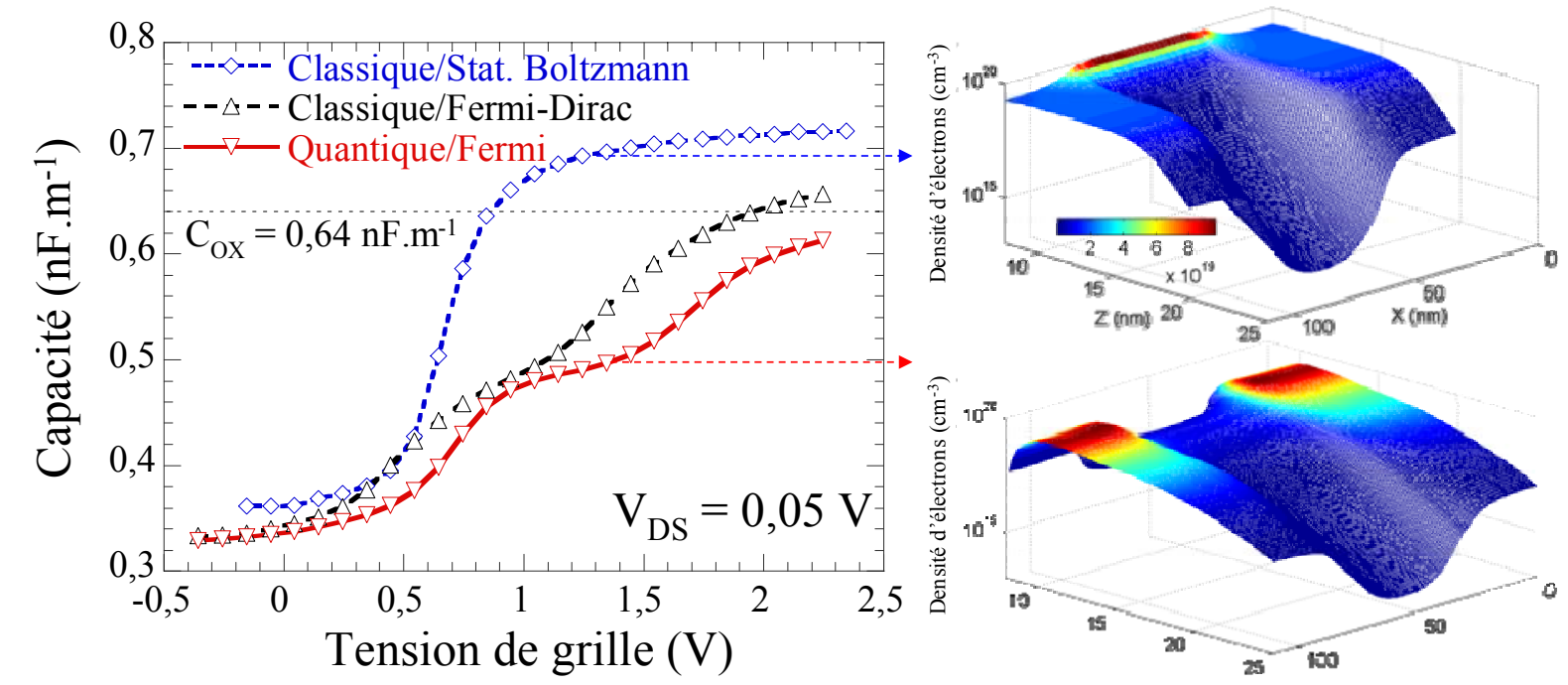

Figure 44 Capacité totale de grille pour une géométrie 2D de la structure MOS-Bulk (cf. Fig 30), calculée avec différents modèles. Cartographie dans deux cas de la densité d'électrons dans l'état $\mathrm{ON}$.

Tout d'abord, nous illustrons sur la Fig. 44 l'influence des modèles pris en compte (classique vs. quantique, choix de la statistique) sur les résultats. Les différences observées sont quasiment les mêmes qu'en 1D. Un calcul classique avec la statistique de Boltzmann surestime toujours la valeur de la capacité de grille sans permettre la prise en compte du 
transfert vers les vallées L. On montre également la cartographie de la densité d'électrons pour la même tension $\mathrm{V}_{\mathrm{G}}$ où les populations sont majoritairement en vallée $\Gamma$. Les résultats sont très différents entre les calculs classique et quantique. La densité d'électrons d'inversion dans le canal est surestimée dans le cas classique, elle est supérieure à celle des caissons et le maximum de densité de porteurs est au niveau de l'interface. Le calcul quantique conduit à une diminution des densités non seulement dans le canal mais aussi dans les caissons. Sur la Fig. 45, on montre quelques niveaux de sous bande, les densités surfaciques le long du dispositif, ainsi que le module au carré des fonctions d'onde associée pour des valeurs de $\mathrm{V}_{\mathrm{G}}$ et $\mathrm{V}_{\mathrm{DS}}$ données. On constate que la densité de probabilité de présence des électrons est plus étalée (la délocalisation est importante) dans la vallée $\Gamma$ par rapport à la vallée $L$ dans la direction du confinement à cause de sa faible masse effective.
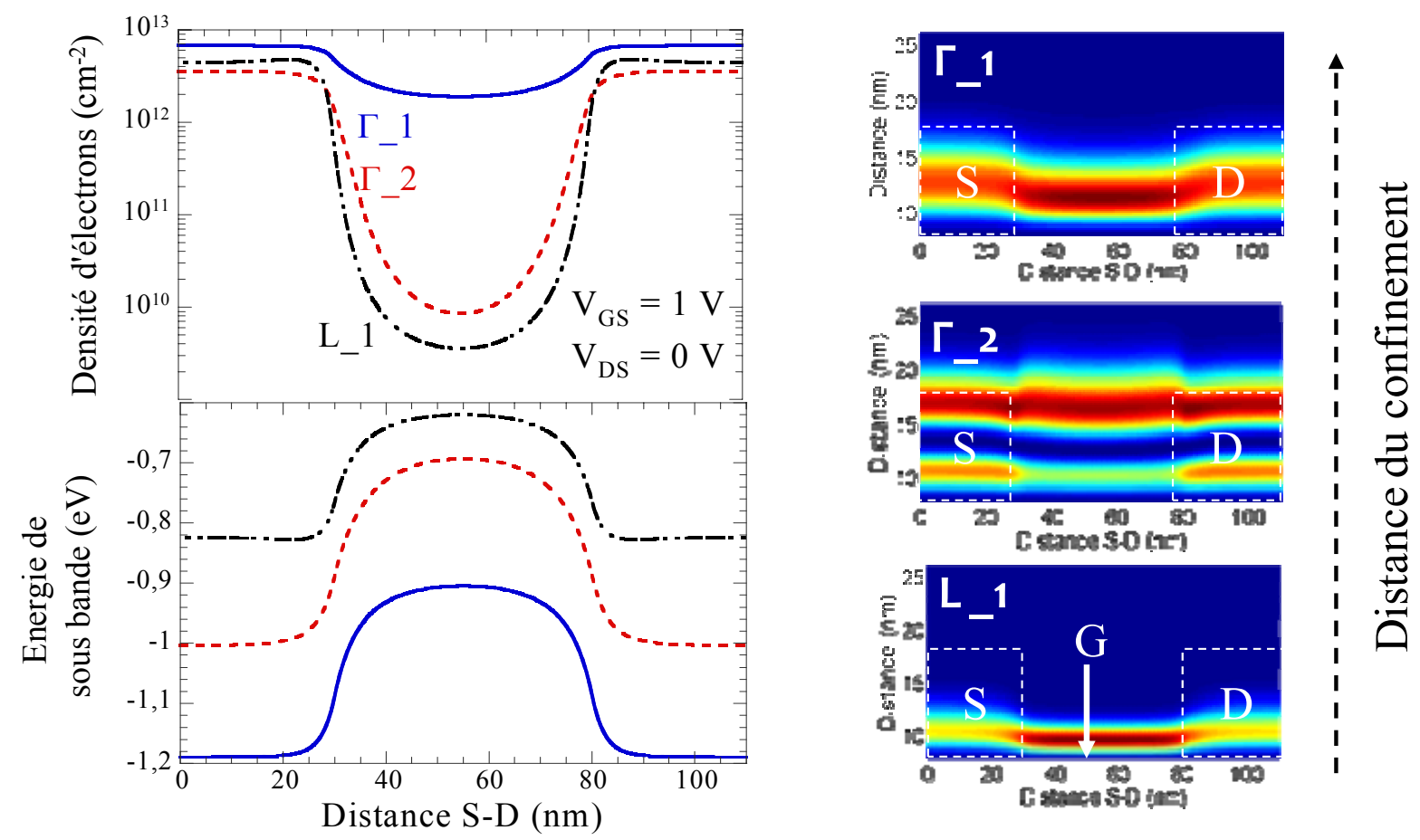

Figure 45 Densité d'électrons surfaciques, énergies de sous bande calculées et la densité de probabilité de présence associées le long du dispositif, pour deux niveaux en $\Gamma$ et un en $L$

Sur la Fig. 46, on observe des différences majeures entre capacités calculées par les modèle $1 \mathrm{D}$ et $2 \mathrm{D}$ : dans le régime sous le seuil : on observe bien de fortes concentrations au niveau des réservoirs de source et drain qui sont induites par la grille sur ces côtés par effet de bord. $\mathrm{C}_{\mathrm{G}}$ est en fait fortement augmenté en $2 \mathrm{D}$ par une capacité de bord $\mathrm{C}_{\mathrm{F}}$ (Fringing capacitance). Par ailleurs, $\mathrm{C}_{\mathrm{G}}$ devrait être influencé par les deux capacités $\mathrm{C}_{\mathrm{S}}$ et $\mathrm{C}_{\mathrm{D}}$ issues des modulations de charge par la grille dans les deux zones d'accès. Les capacités $C_{F}, C_{S}$ et $C_{D}$ associées doivent être incluses dans le réseau de capacités distribuées schématisé sur la Fig. 
47. On a essayé de quantifier ces composantes des capacités totales de la grille. Pour évaluer cela, des profils 2D de charges et de potentiel sont nécessaires pour décrire précisément la caractéristique capacité-tension en tenant compte des effets géométriques. Sur la cartographie de la différence de densités d'électrons entre deux états sous le seuil $\left(\mathrm{V}_{\mathrm{G}}=0\right.$ et $\left.0,1 \mathrm{~V}\right)$, on observe, dans les caissons, il y a un pic de variation de densité d'électrons de l'ordre de $10^{17}$ à $10^{18} \mathrm{~cm}^{-3}$ accumulés par les capacités de bord. La concentration en porteurs est en outre modulée de $10^{17} \mathrm{~cm}^{-3}$ dans les zones d'accès. Cela met en évidence le rôle des capacités séries $\mathrm{C}_{\mathrm{S}}$ et $\mathrm{C}_{\mathrm{D}}$ qui perturbent le contrôle de charge par ma grille par rapport au cas idéal 1D.
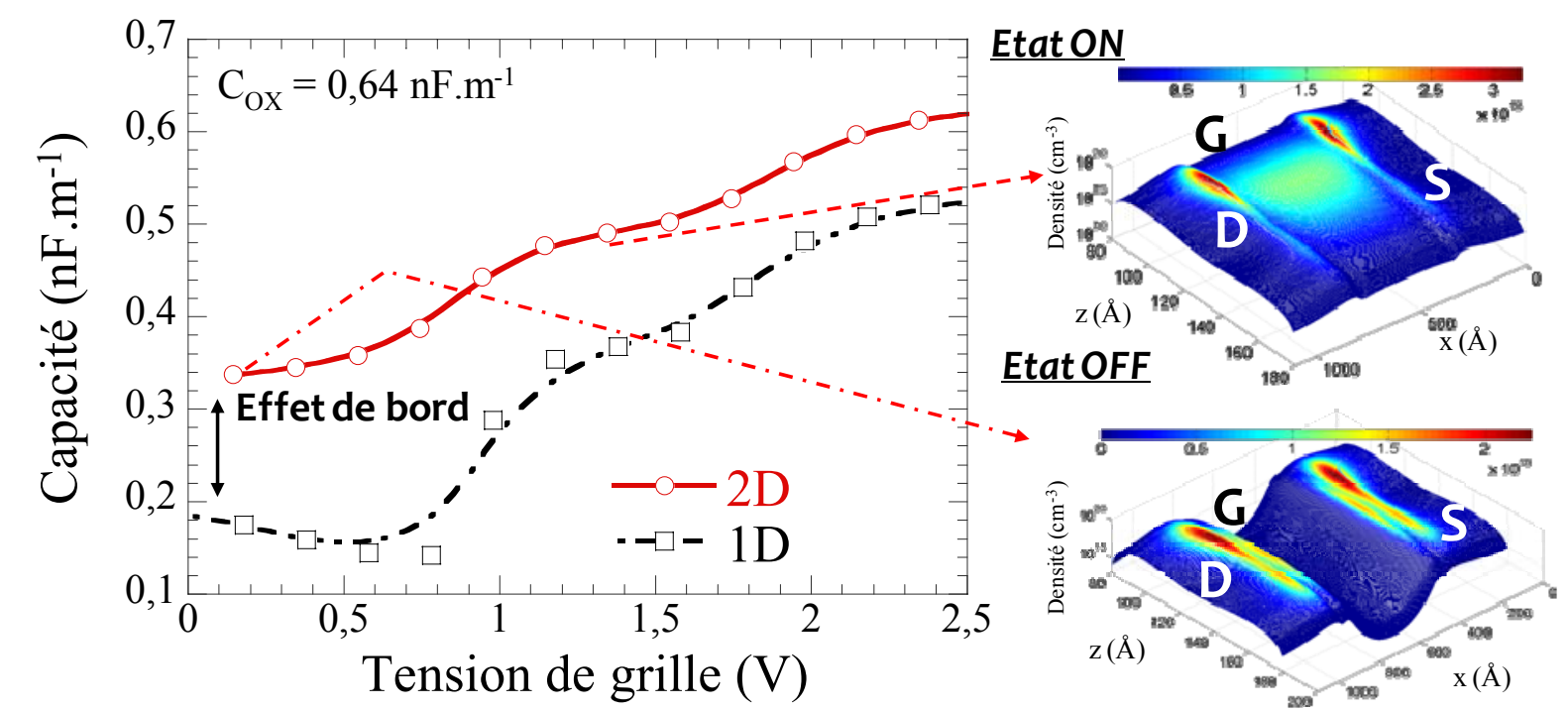

Figure 46 Comparaison des capacités de grille obtenues par des calculs 1D et 2D. Pour les points de polarisation $O N$ et $O F F$, la différence des densités des électrons entre deux valeurs voisines de $V_{G}$ proches de ces états illustre la localisation des charges due aux différentes capacités.

On peut évaluer ces composantes en fonction de la tension de grille à $V_{\mathrm{DS}}=0 \mathrm{~V} \cdot \mathrm{C}_{\mathrm{F}}$ est obtenue par une intégration des charges dans les zones de caisson. La capacité $\mathrm{C}_{\mathrm{G}}$ dite «pseudo 2D » est celle obtenue en étudiant un profil de charge dans une tranche qui se situe au sommet de barrière de potentiel, afin d'exclure les effets de bord et d'accès en gardant seulement l'effet latéral dû aux SCE. Pour $\mathrm{V}_{\mathrm{DS}}=0 \mathrm{~V}$, on suppose que $\mathrm{C}_{\mathrm{S}}=\mathrm{C}_{\mathrm{D}}$. La capacité $\mathrm{C}_{\mathrm{S}}$ d'accès du côté source peut être calculée par différentiation de la somme des charges dans les zones d'accès par le potentiel de surface pour deux $V_{G}$ proches, on désigne plus loin cette évaluation comme « $\mathrm{C}_{S}-$ calcul ». Cette capacité série peut également être déterminée par le schéma équivalent des capacités distribuées en prenant une capacité de bord $\mathrm{C}_{\mathrm{F}}$ constante, d'où la valeur dite « $\mathrm{C}_{\mathrm{S}}-$ schéma ». Les résultats ainsi obtenus sont illustrés sur la Fig. 48. 


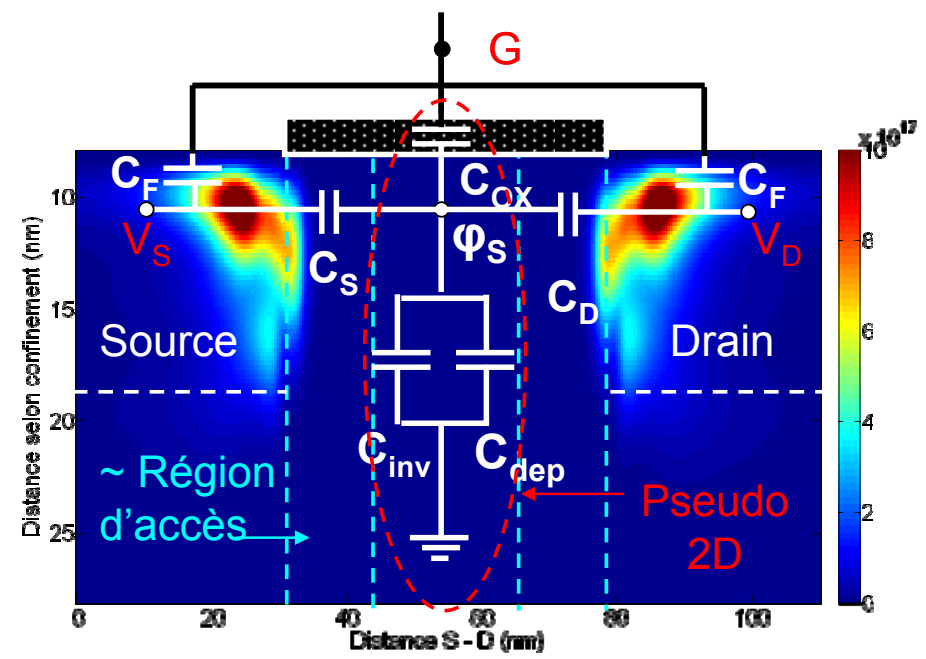

Figure 47 Schéma équivalent des capacités distribuées dans une géométrie 2D et cartographie de la différence de densités d'électrons entre deux états sous le seuil $\left(V_{G}=0\right.$ et $\left.0,1 \mathrm{~V}, V_{D S}=0 \mathrm{~V}\right)$. Pseudo 2D : correspond à une capacité équivalente obtenue par un profil de charge dans une tranche qui se situe au sommet de barrière, les effets du bord et de la zone d'accès sont a priori exclus. La différence majeure entre Pseudo 2D et 1D est que le modèle Pseudo 2D prend en compte l'effet latéral comme effet canal court.

a)

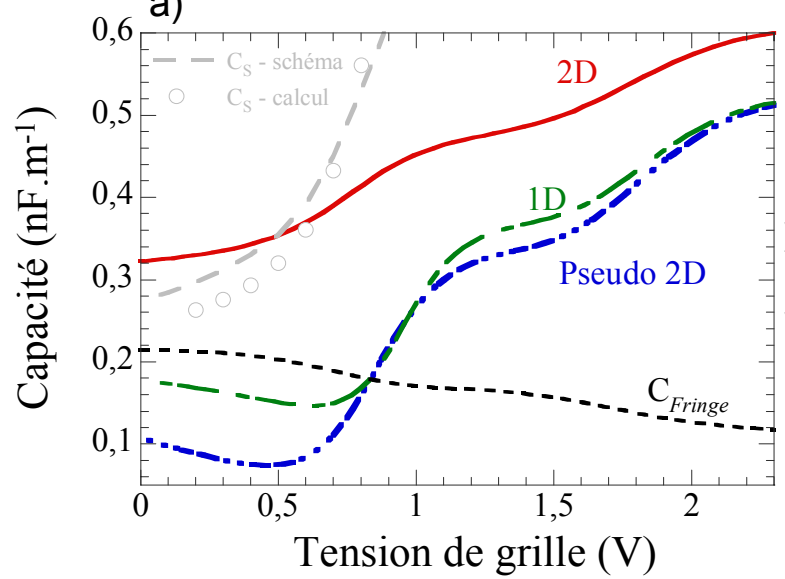

b)

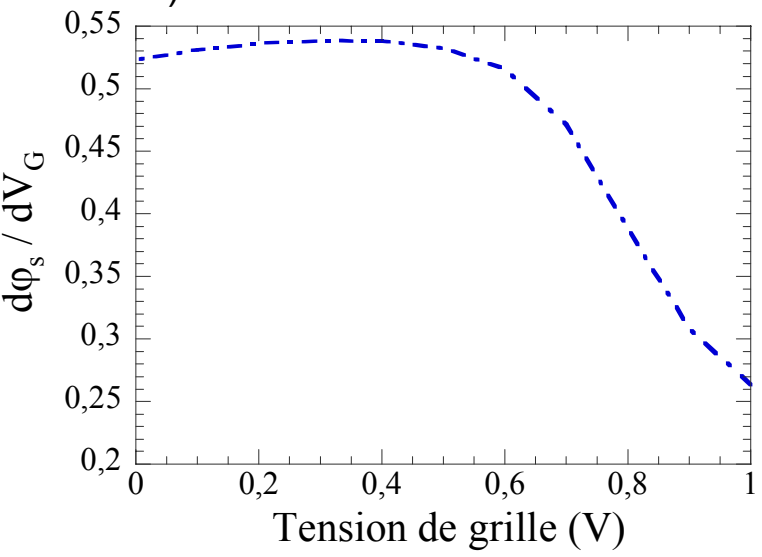

Figure 48 a) Composantes de la capacité totale de grille pour une géométrie en $2 \mathrm{D}$ à $\mathrm{V}_{\mathrm{DS}}=0 \mathrm{~V}$. $C_{O X}=0,64 \mathrm{nF} \cdot \mathrm{m}^{-1}$. Chaque capacité de bord $C_{F}$ est supposée être égale à $0,1 \mathrm{nF} \cdot \mathrm{m}^{-1}$ pour évaluer $C_{S}$ par le schéma équivalent $\left(C_{S}-\right.$ schéma). b) Dérivée du potentiel de surface par rapport à la variation de tension de grille en fonction de cette dernière. Cela est nécessaire pour calculer $C_{S}\left(C_{S}-\right.$ calcul).

La différence de capacité entre $1 \mathrm{D}$ et Pseudo 2D est due à l'effet de canal court qui diminue la capacité de grille surtout dans le régime de faible inversion et introduit un décalage de tension de seuil. Pour calculer la capacité d'accès $C_{S}$, on doit connaître la variation effective du potentiel de surface par rapport à celle de la tension de grille dans la plage de contrôle (cf. Fig. 48-b). Par différentiation de la somme des charges dans les zones d'accès par le potentiel de surface, on peut alors obtenir la valeur de $C_{S}$. On constate sur la Fig. 48-a 
un bon accord entre valeurs calculées $C_{S}-$ calcul et celles issues du schéma équivalent $C_{S}-$ schéma. L'approximation de la capacité de bord par une constante pour toute la plage de tension $\mathrm{V}_{\mathrm{G}}$ est discutable. Elle n'est effectivement valable que dans les régimes de déplétion ou de faible inversion.

\section{2-3 Prise en compte des états de pièges d'interface}

\section{2-3-1 Modèle}

Pour valider nos modèles sur le contrôle de charge, nous devons comparer nos résultats avec les caractéristiques $\mathrm{C}-\mathrm{V}$ expérimentales, ce qui nécessite également de prendre en compte l'effet des états d'interface.

Normalement les états des pièges d'interface $\left(D_{i t}\right)$ n'influencent principalement que les caractéristiques $\mathrm{C}-\mathrm{V}$ à basse fréquence, si la fréquence du signal $\mathrm{AC}$ appliqué est plus faible que la réponse fréquentielle des pièges. Dans nos études, nous nous focalisons sur l'influence de la densité $D_{i t}$ sur la caractéristique $\mathrm{C}-\mathrm{V}$ en régime statique et à l'équilibre thermodynamique. Cette influence peut se résumer en trois points : i) les pièges vont introduire une capacité $C_{i t}$ variable en fonction de la polarisation en parallèle avec la capacité d'inversion $C_{I N V}$. ii) il en résulte un autre composante de la tension de grille $V_{i t}$ qui dépend aussi la polarisation de grille elle-même. iii) Cette densité des pièges est très localisée à l'interface, elle peut ancrer violement le niveau de Fermi à l'interface par rapport au bas de bande de conduction. La charge totale des états d'interface est une fonction de la position du niveau Fermi à l'interface. Pour déterminer la charge associée aux pièges d'interface $Q_{i t}$, le profil de la densité des pièges d'interface $D_{i t}$ doit être intégré dans la plage d'énergie qui couvre la bande interdite, la bande de valence et la bande de conduction en pondérant par la probabilité d'occupation sous forme de la statistique Fermi-Dirac. On a utilisé la même représentation que pour l'interface $\mathrm{Si} / \mathrm{SiO}_{2}$ pour décrire le comportement des états d'interface pour une structure high-א/III-V [Brammertz2009APL] et [Xuan2007APL], c'est-à-dire que les états d'interface de type accepteur occupent la moitié supérieure da la bande interdite ( $>$ midgap) et les états de type donneur occupent la moitié inférieure de la bande interdite $(<$ midgap) [Schroder2003JAP]. Ces deux types de piège peuvent expérimentalement être interprétés par la distribution équivalente $D_{i t}$. En supposant que la transition entre les états de donneur et d'accepteur se produit au niveau d'énergie de neutralité de charge (CNL : Charge neutrality level) $E_{C N L}$ au sein de la bande interdite. A l'interface, si le niveau de Fermi $E_{F}$ est au dessus 
de $E_{C N L}$, l'état des pièges est dominé par celui d'accepteur, l'état sera négativement chargé. Si $E_{F}$ est au dessous $E_{C N L}$, l'état de piège est du type donneur et ainsi que l'état sera positivement chargé. Si on suppose que le niveau de l'énergie de stabilisation de $E_{F}$ se localise à $E_{C N L}$, la charge associée aux pièges d'interface $Q_{i t}$ peut être exprimée comme :

$$
Q_{i t}=-q \int_{E_{C N L}}^{+\infty} F_{A}(E) D_{i t}^{A}(E) d E+q \int_{-\infty}^{E_{C N L}}\left(1-F_{D}(E)\right) D_{i t}^{D}(E) d E
$$

Ici, la fonction de distribution des états de type donneur s'écrit :

$$
F_{D}(E)=\frac{1}{1+g \exp \left(\frac{E_{F}-E}{k_{B} T}\right)}
$$

quant à ceux accepteurs :

$$
F_{A}(E)=\frac{1}{1+\frac{1}{g} \exp \left(\frac{E-E_{F}}{k_{B} T}\right)}
$$

où $g$ est le degré de dégénérescence de l'état fondamental des pièges, il vaut 2 pour un donneur et 4 pour un accepteur. Le profil $D_{i t}$ en dehors de la bande interdite est aussi pris en compte. Dans notre calcul numérique, on suppose que l'énergie $E_{C N L}$ est égale à niveau intrinsèque $E_{i}$ au milieu du gap, c'est-à-dire $E_{B C}-E_{C N L} \sim 0,35 \mathrm{eV}(0,3 \mathrm{eV}$ dans la référence [Xuan2007APL]). Cette approximation est justifiée pour le silicium, mais peut ne pas être pas absolument vraie pour un semi-conducteur III-V. L'incertitude de l'estimation de $E_{C N L}$ n'induit qu'un décalage de $Q_{i t}$ sur la plage de $\mathrm{V}_{\mathrm{G}}$ sans changer leur forme. La discontinuité entre le champ dans le diélectrique et dans le semi-conducteur due à la présence de $Q_{i t}$ s'ajuste en considérant une chute de potentiel $V_{i t}=-Q_{i t} / C_{O X}$ qui dépend également la polarisation de grille. C'est-à-dire que pour avoir le même potentiel de surface que le cas idéal, la polarisation de grille va augmenter d'une quantité de $V_{i t}$.

Pour réévaluer le comportement de la capacité de la structure MOS en tenant compte des états d'interface ainsi que de l'effet de l'ancrage du niveau de Fermi (pinning effect), le calcul doit être auto-cohérent, c'est-à-dire que la quantité de charge issue des états d'interface doit participer à la boucle auto-cohérente de Poisson-Schrödinger jusqu'à l'atteinte de la convergence. La charge des pièges est localisée au niveau de l'interface, ce qui doit être implémenté dans l'équation de Poisson (Eq. 1). 


\section{2-3-2 Efficacité du mouvement de niveau Fermi}

L'efficacité du mouvement de niveau Fermi (FLE: Fermi Level Efficiency) à l'interface est donnée par [Lin2009APL] et [Sze1981] :

$$
\frac{d\left(E_{F}\left(V_{G}\right)-E_{C}\right)}{q \cdot d V_{G}}=\frac{d \psi_{S}\left(V_{G}\right)}{d V_{G}}
$$

Cela permet de caractériser la réponse du niveau de Fermi à l'interface vis-à-vis de la variation de la tension de grille appliquée. Pour une structure MOS idéale, elle peut être estimée en utilisant l'approximation de la somme des chutes de potentiel dans l'oxyde et le semi-conducteur [Sze1981] :

$$
V_{G}-V_{F B}=\psi_{S}+\frac{Q_{S C}}{C_{O X}}=\psi_{S}+\frac{q N_{A} w_{d e p}}{C_{O X}}=\psi_{S}+\frac{q N_{A}}{C_{O X}} \sqrt{\frac{2 \varepsilon_{s c} \psi_{S}}{q N_{A}}}
$$

où $Q_{S C}$ est la charge dans la zone de charge d'espace dans le régime déplétion. $w_{\text {dep }}$ est la largeur de cette zone. Cette efficacité pour une structure idéale, en supposant $V_{F B}$ indépendant de $\psi_{S}$, peut être calculée analytiquement :

$$
\frac{d\left(E_{F}\left(V_{G}\right)-E_{C}\right)}{q \cdot d V_{G}}=\frac{C_{O X}}{d Q_{S C} / d \psi_{S}}=\left(\frac{2 \psi_{S}}{q \cdot N_{a}}\right)^{1 / 2} C_{O X} / \sqrt{\varepsilon_{S C}}
$$

Pour maximiser cette valeur, on doit diminuer la concentration de dopant, augmenter la capacité d'oxyde et choisir un matériau de canal avec une faible permittivité diélectrique. Pour une structure de silicium de faible dopage $\left(10^{15} \mathrm{~cm}^{-3}\right)$ et forte capacité $\mathrm{C}_{\mathrm{OX}}\left(1 \mu \mathrm{F} . \mathrm{cm}^{-2}\right)$, le maximum d'efficacité est très proche de l'unité [Lin2009APL]. Pour un matériau III-V binaire comme GaAs, elle pourrait diminuer à $85 \%$ pour une concentration modérée $\left(10^{17} \mathrm{~cm}^{-3}\right)$ et avec le même $\mathrm{C}_{\mathrm{OXx}}$. Si on prend en compte l'effet des états de pièges d'interface, l'efficacité devient [Sze1981] :

$$
\frac{d\left(E_{F}\left(V_{G}\right)-E_{C}\right)}{q \cdot d V_{G}}=\frac{C_{O X}}{d Q_{S C}\left(\psi_{S}\right) / d \psi_{S}+d Q_{i t}\left(\psi_{S}\right) / d \psi_{S}}
$$

Notre calcul pourra estimer numériquement cette efficacité perturbée par des pièges. Expérimentalement, l'état de l'art se situerait toutefois à environ 20\% seulement à cause du 'pinning' du niveau de Fermi par les états d'interface. La valeur du FLE peut être considérée comme un 'benchmark' pour optimiser les designs de capacité MOS qui ont été affectés technologiquement par la forte densité de pièges d'interface. Par ailleurs, cette méthode de FLE permettrait de créer des possibilités d'évaluation des réponses de pièges d'interface non 
seulement dans le régime de déplétion mais aussi dans ceux d'inversion et d'accumulation. Toutefois, elle ne pourra pas être théoriquement décrite uniquement en termes de capacité et conductance, à cause des effets d'impédance série [Martens2007MicEng], [Lin2009APL] et [Engel-Herbert2010JAP]. En outre, la procédure d'évaluation de l'efficacité expérimentale reste entachée d'incertitude et il serait intéressant d'avoir un outil numérique fiable pour étudier plus précisément les phénomènes physiques mis en jeu. Cela nous permet d'expliquer la différence majeure entre les résultats expérimentaux et théoriques.

\section{2-3-3 Résultats et étude comparative avec des données expérimentales}

Nous allons recalculer la capacité de grille en tenant en compte les effets quantiques, de non-parabolicité, dégénérescence, multi-vallées et les états des pièges d'interface. En plus des états des pièges d'interface, on doit étudier l'efficacité du mouvement du niveau de Fermi par rapport au cas idéal (étude 1D ou canal long) en prenant en compte l'effet latéral de canal court (2D-SCE).

Tout d'abord, il faut extraire expérimentalement le profil de $D_{i t}$ dans la plage d'énergie considérée. Pour cela on utilise la méthode de la capacité totale à haute fréquence (HF) et à basse fréquence $(\mathrm{BF})$ selon la méthode décrite par Castagné et Vapaille [Castagne1971]). En haute fréquence, les pièges n'arrivent pas à suivre le signal alternatif appliqué sur les contacts, donc $C_{i t}=0$. A basse fréquence, la fréquence du signal doit être suffisamment faible pour que tous les pièges puissent suivre le signal et atteindre l'équilibre thermodynamique. La dépendance fréquentielle des pièges est liée au temps de réponse qui est donné par le taux de capture et d'émission selon la statistique de Shockley-Read-Hall [Shockley1952PR] et [Brammertz2007APL].

$$
\tau=\frac{\exp \left(\Delta E / k_{B} T\right)}{\sigma v_{t h} D O S}
$$

où $\Delta E$ est la différence d'énergie entre le niveau de piège et le sommet de la bande des porteurs majoritaires. $\sigma$ est la section de capture des pièges. $v_{t h}$ est la vitesse thermique moyenne des porteurs majoritaires. Les autres symboles ont leur sens conventionnel. Un signal continu est appliqué sur le métal de grille en plus de celui alternatif de faible amplitude $(\sim 25 \mathrm{mV})$ et avec une fréquence f typiquement entre $1 \mathrm{MHz}$ et $100 \mathrm{~Hz}$. On peut en déduire le profil de $D_{i t}$ par [Castagne1971] et [Engel-Herbert2010JAP] :

$$
D_{i t}\left(V_{G}\right)=\frac{C_{O X}}{q}\left(\frac{C_{t o t}^{B F}}{C_{O X}-C_{t o t}^{B F}}-\frac{C_{t o t}^{H F}}{C_{O X}-C_{t o t}^{H F}}\right)
$$


Pour déterminer les niveaux des pièges, la relation entre la tension de grille et le potentiel de surface sera nécessaire. Pour cela on utilise la méthode de l'intégrale de Berglund [Sze1981], [Kim2009JAP] et [Engel-Herbert2010JAP]:

$$
\psi_{S}=\psi_{S}^{0}+\int_{V_{G}^{0}}^{V_{G}}\left(1-\frac{C_{t o t}^{B F}\left(V_{G}\right)}{C_{O X}}\right) d V_{G}
$$

où $\psi_{S}^{0}$ correspond à la valeur de potentiel de surface pour $V_{G}=V_{G}^{0}$. Il est important de choisir cette valeur $\operatorname{de} V_{G}^{0}$ pour laquelle le potentiel de surface ne varie pas fortement. Ici, on a choisi la tension de flat band $V_{F B}$.

La capacité MOS est fabriquée par l'IEMN, coordinateur du projet ANR MOS35 (cf. Fig. 49). Ils nous fournissent le profil des pièges à l'interface en fonction de la tension de grille et les caractéristiques $\mathrm{C}-\mathrm{V}$ expérimentales pour une capacité MOS avec une forte densité de pièges d'interface.

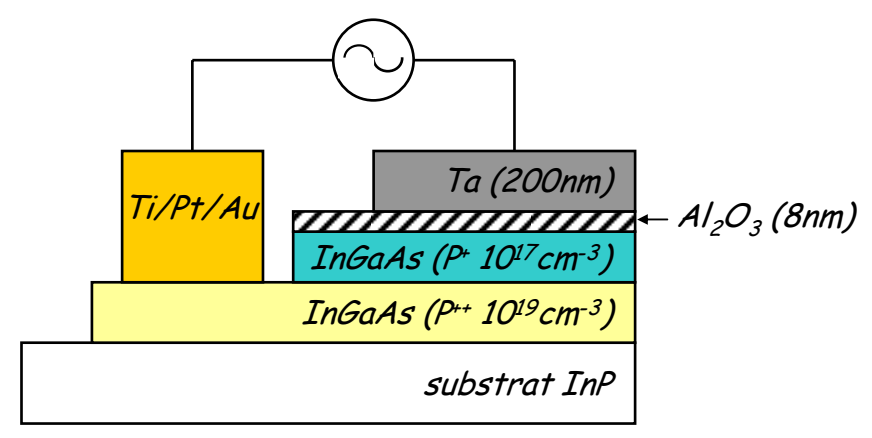

Figure 49 Structure de la capacité MOS de l'IEMN, préparée sous PDA (post deposition annealing) à $600^{\circ} \mathrm{C}$ pendant 1 minute, puis $\mathrm{Ta}^{+} \mathrm{Al}_{2} \mathrm{O}_{3}$ recuit à $400^{\circ} \mathrm{C}$ pendant 1 minute sous « forming gas » $\left(\mathrm{N}_{2} \mathrm{H}_{2}\right)$.

a)

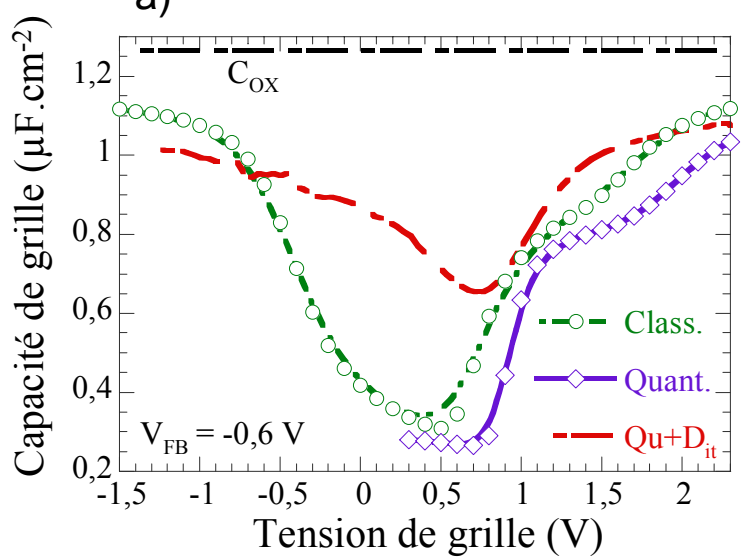

b)

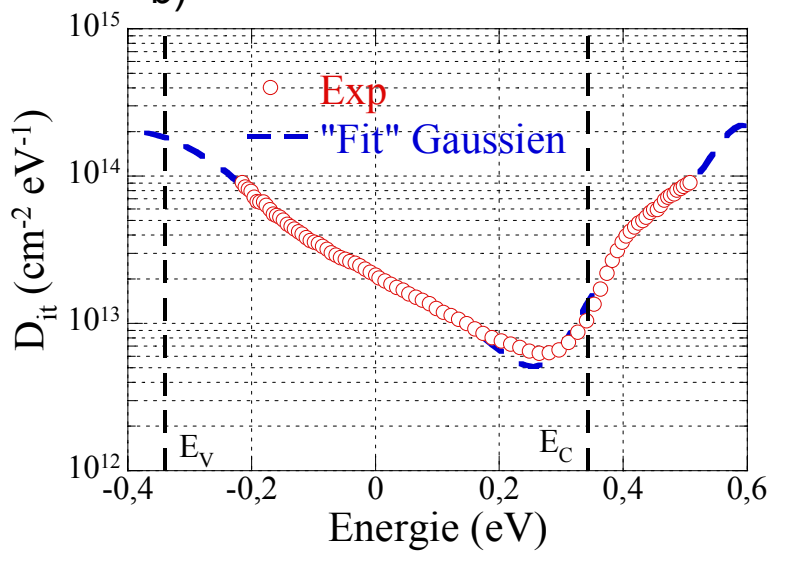

Figure 50 a) Capacité de grille totale calculée par différents modèles pour une structure MOS-Bulk avec $\mathrm{N}_{\mathrm{A}}=10^{18} \mathrm{~cm}^{-3}$ et $\mathrm{C}_{\mathrm{OX}}=1,27 \mu \mathrm{F} . \mathrm{cm}^{-2}$. La tension flat band $\mathrm{V}_{\mathrm{FB}}$ est égale à $-0,6 \mathrm{~V}$. La non-parabolicité et la statistique de Fermi-Dirac sont toujours prises en compte. Class. : calcul classique avec Poisson ; Quant. : résolution Poisson-Schrödinger; dans $Q u+D_{i t}$, on rajoute l'effet des états de pièges d'interface. b) 


\section{Symboles rouges : densité de pièges extraite des mesures (Eq. 56-57). Tirets bleus : fonction d'approximation utilisée dans la simulation (somme de Gaussiennes).}

D'après la Fig. 50 a), une fois l'effet des pièges d'interface pris en compte, on peut observer la disparition du changement de pente lié au transfert vers les vallées satellites. De plus, en régime de déplétion, la capacité augmente d'environ 0,4 à $0,6 \mu \mathrm{F} . \mathrm{cm}^{-2}$. La tension de seuil est modifiée, ce qui apparaît clairement sur le profil de la densité des porteurs tracé plus loin en Fig. 53. Comme nous allons le voir, ces différences peuvent s'expliquer via l'efficacité du mouvement de niveau Fermi (Figure 51) et les importances relatives des densités de charges de chaque contribution (charge d'inversion, charge de déplétion et des états de pièges d'interface, Fig. 52).

D’après les résultats reportés sur la Fig. 51, le maximum de cette efficacité se situe dans la région proche du régime mid-gap, c'est-à-dire à une valeur de $\mathrm{V}_{\mathrm{G}_{-} \mathrm{MG}}$ pour laquelle le niveau de Fermi atteint le milieu du gap à l'interface $\left(\mathrm{V}_{\mathrm{G} \_\mathrm{MG}}\right.$ est environ égale à $\mathrm{V}_{\mathrm{FB}}+0,8 \mathrm{~V}$ où $\mathrm{V}_{\mathrm{FB}}=-0,6 \mathrm{~V}$ ). FLE atteint alors environ $75 \%$ pour le cas idéal (canal long) et de $55 \%$ (efficacité dégradée par effet de canal court) pour le calcul $2 \mathrm{D}$ avec $\mathrm{L}_{\mathrm{G}}=50 \mathrm{~nm}$ sans piège. Elle est seulement de 20 à $25 \%$ quand on prend en compte les pièges d'interface. Cette valeur est en bon accord avec une estimation expérimentale $(23 \%$ à $500 \mathrm{~Hz})$ pour une capacité MOS avec forte densité de pièges d'interface [Lin2009APL].

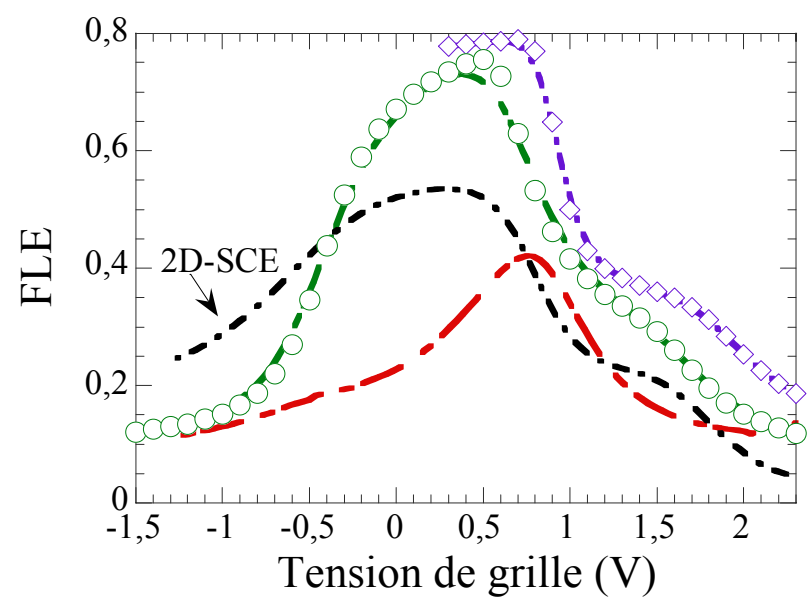

Figure 51 L'efficacité du mouvement de niveau Fermi (FLE : Fermi Level Efficiency) en fonction de la tension de grille. La légende est la même que pour la Fig. 23. 2D-SCE : cette courbe est obtenue en tenant compte de l'effet latéral en $2 \mathrm{D}$ c'est-à-dire de l'effet de canal court pour $L_{G}=50 \mathrm{~nm}$ et $N_{A}=10^{18} \mathrm{~cm}^{-3}$ et $T_{O X}=8 \mathrm{~nm}$. L'effet de $D_{\text {it }}$ n'est pas pris en compte dans le calcul $2 D$.

On peut par ailleurs estimer la pente sous le seuil en fin de régime de déplétion et en début de faible d'inversion par l'approximation $S S=k_{B} T / q \times \ln (10) \times\left(d \psi_{S} / d V_{G}\right)^{-1}$ [Bournel2006HDR]. Dans le cas idéal $1 \mathrm{D}$ on a $\mathrm{SS} \approx 77 \mathrm{mV} / \mathrm{dec}$, mais la pente augmente à 
environ $110 \mathrm{mV} / \mathrm{dec}$ par effet de canal court. Elle se dégrade encore plus fortement à cause des pièges avec SS de l'ordre de 240 à $300 \mathrm{mV} / \mathrm{dec}$.

Sur la Fig. 52, nous étudions plus quantitativement la perturbation du contrôle de charge par les états d'interface en distinguant les différentes contributions à la densité de charge.
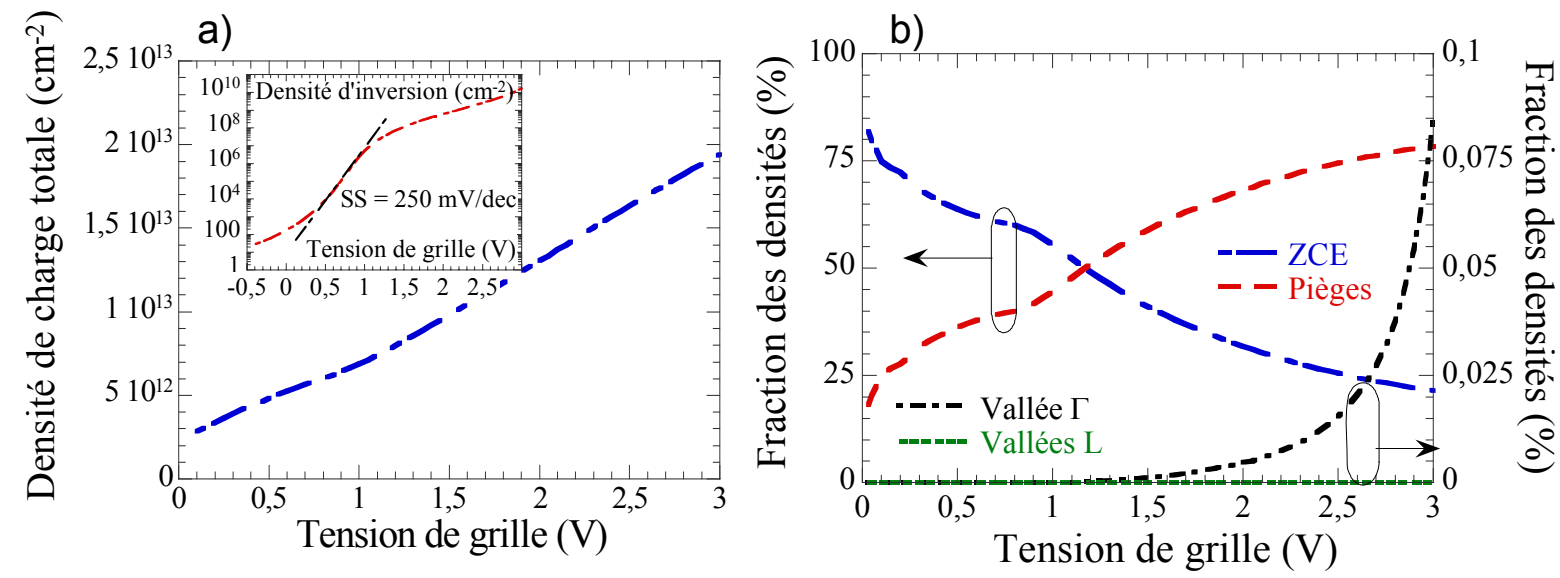

Figure 52 a) Densité de charges totale en fonction de la tension de grille. Insert : pente sous le seuil. b) Fraction des densités de charge pour chaque contribution : Vallées $\Gamma$ et $L=$ charges mobiles d'inversion $(<0,1 \%$, la contribution des vallées $\mathrm{L}$ est négligeable); ZCE : Charge de dopants ionisés dans la zone de charge d'espace ; pièges : charge due aux états d'interface.

A cause du fort effet de 'pinning' du niveau de Fermi imposé par les pièges d'interface et la densité de porteurs mobiles est fortement dégradée par rapport au cas d'une structure idéale. Cette perte de contrôle de la grille sur la formation du canal se manifeste non seulement dans régime sous le seuil mais également à l'état $\mathrm{ON}$, comme l'illustre la Fig. 53. L'effet de $\mathrm{D}_{\mathrm{it}}$ introduit aussi un décalage de tension de seuil par rapport au cas idéal. Pour avoir une même densité d'inversion, par exemple $10^{8} \mathrm{~cm}^{-2}$, ce décalage peut dépasser $1 \mathrm{~V}$.

a)

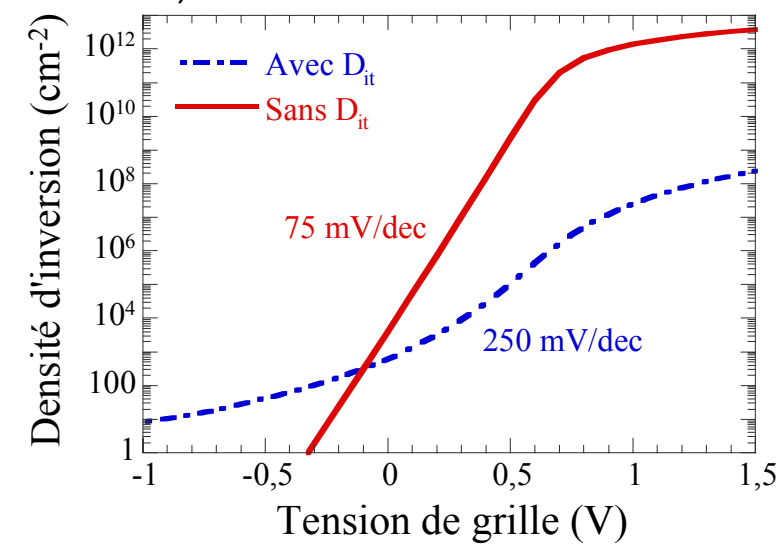

b)

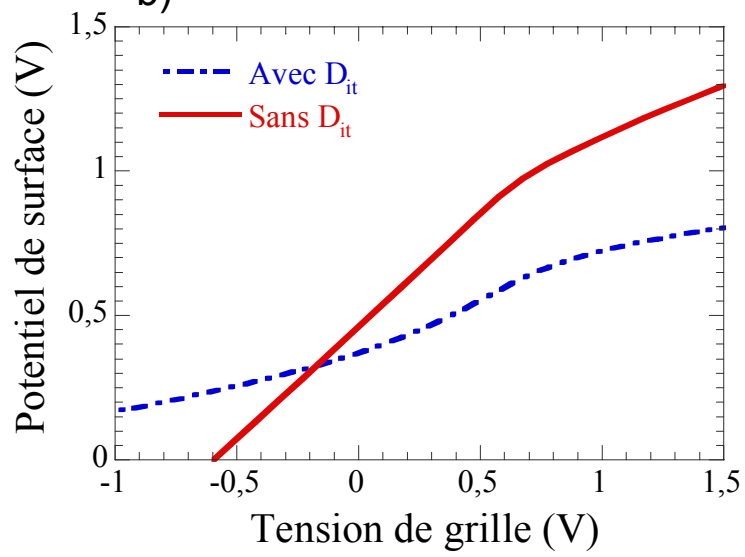

Figure 53 a) Densité de charges mobiles d'inversion et b) potentiel de surface en fonction de la tension de grille avec et sans $D_{i t}$. 
Le mouvement de $\mathrm{E}_{\mathrm{F}}$ à l'interface renforce l'occupation des pièges et non pas celle des états de la bande de conduction. Le profil de capacité totale de grille présenté sur la Fig. 54 illustre la large prédominance de la capacité de déplétion $C_{D E P}$ ou de celle de piège $C_{I T}$ suivant la valeur de la tension de grille. La contribution de la capacité d'inversion $C_{I N V}$ est presque totalement négligeable.

Enfin, comparons nos simulations avec des données expérimentales sur la Fig. 55. En pratique, la densité du dopage du substrat est $10^{17} \mathrm{~cm}^{-3}$. Les différents résultats théoriques sont montrés pour rappel. Le très bon accord entre expérience et simulation quand les pièges sont pris en compte confirme la pertinence de la simulation Poisson-Schrödinger mise en œuvre.

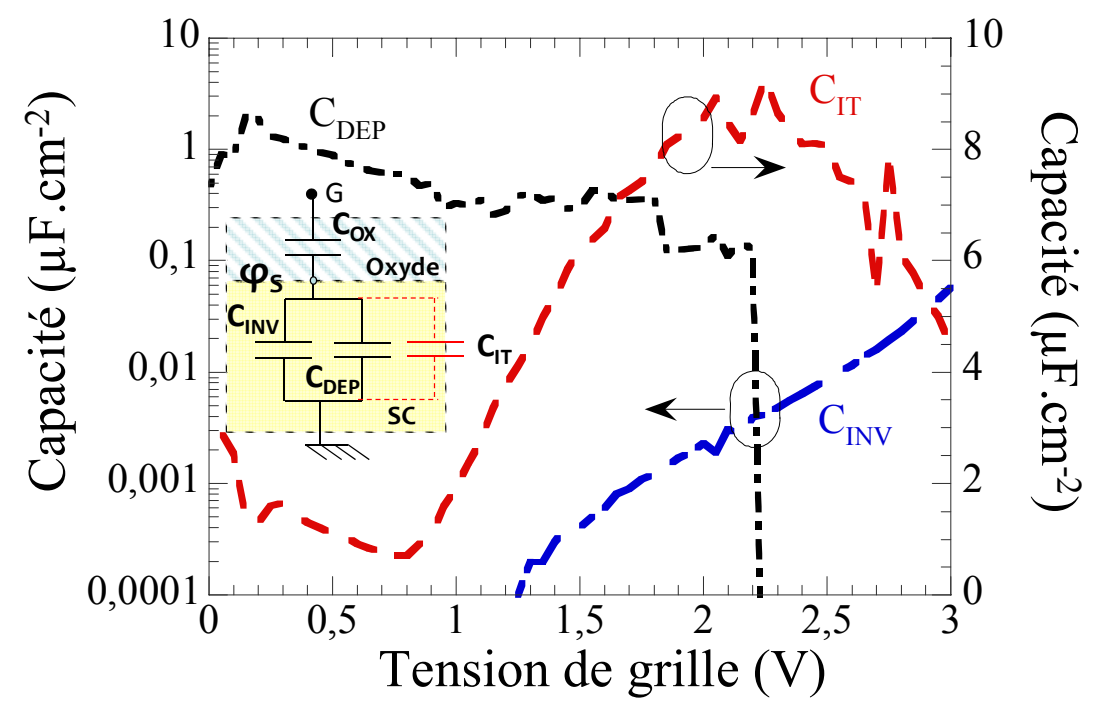

Figure 54 Les trois contributions de la capacité dans le matériau semi-conducteur. $C_{I N V}=q \partial N_{I N V} / \partial \varphi_{S}$,

$$
C_{D E P}=\varepsilon_{s c} / T_{D E P} \text { avec } T_{D E P} \approx \sqrt{\frac{\varepsilon_{s c}}{q N_{A}} \varphi_{S}} \text { et } C_{I T}=\partial Q_{i t} / \partial \varphi_{S}
$$




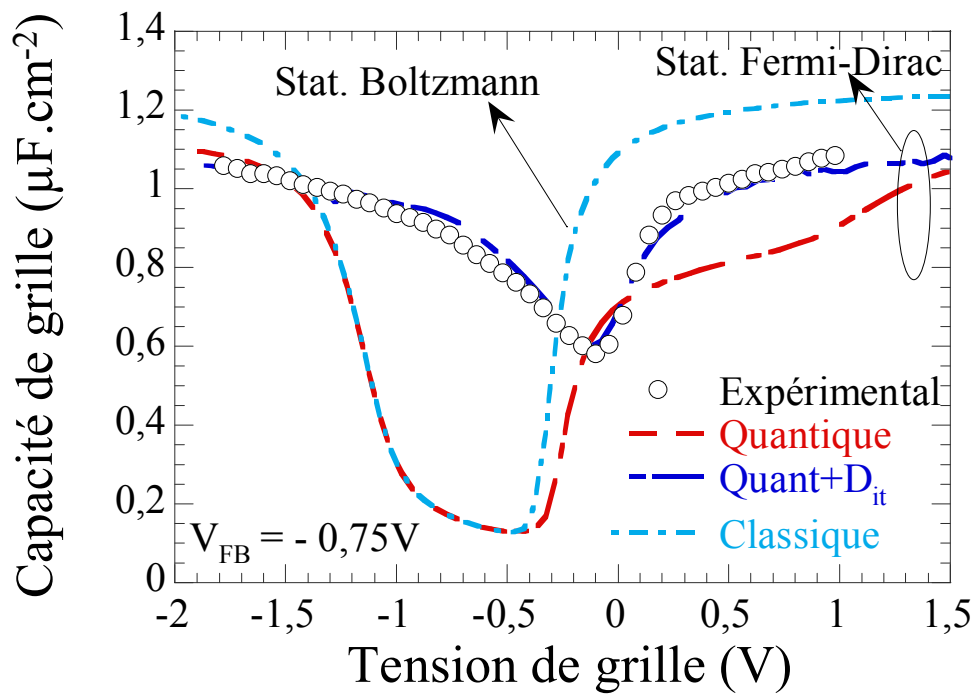

Figure 55 Comparaison des résultats entre simulation et expérience (à $210 \mathrm{~Hz}$ ). La tension de flat band $\mathrm{V}_{\mathrm{FB}}$ utilisée pour les calculs est ajustée à $\mathbf{- 0 , 7 5} \mathrm{V}$ pour la confrontation avec l'expérience.

\section{2-3-4 Dégradation des états $\mathrm{OFF}$ et $\mathrm{ON}$ due aux pièges}

Etudions quantitativement l'effet des pièges sur la performance d'un transistor. Elle peut être dégradée selon la densité des pièges à cause de l'effet de 'pinning' du niveau de Fermi à l'interface, non seulement au régime sous le seuil mais également à l'état $\mathrm{ON}$. Nous allons voir que la perte de contrôle de la grille due aux $\mathrm{D}_{\mathrm{it}}$ dépend aussi de la concentration du dopage du substrat $\mathrm{N}_{\mathrm{A}}$.

a)

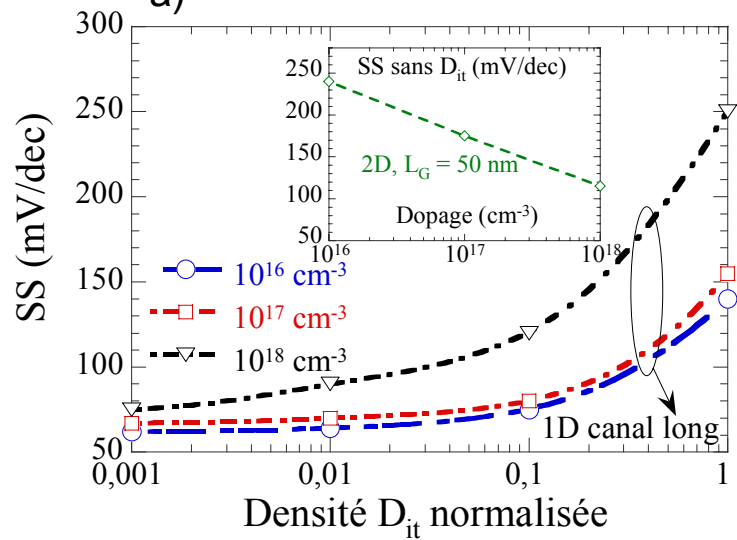

b)

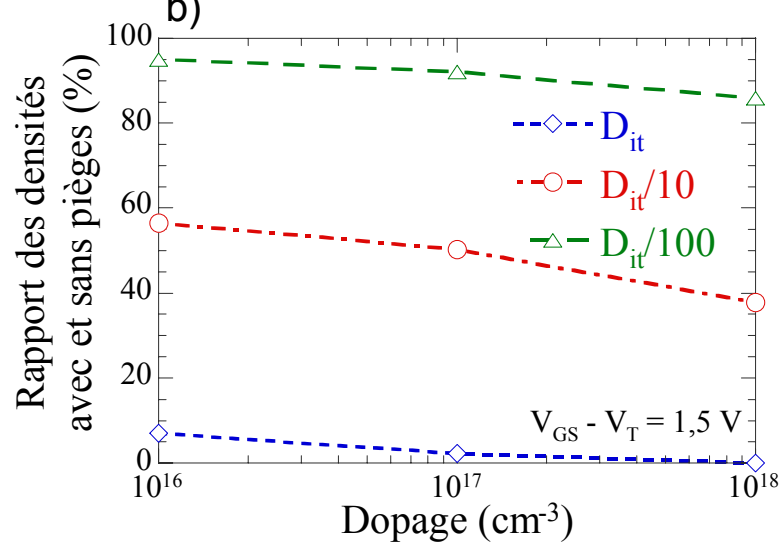

Figure 56 a) Pente sous le seuil SS pour différents dopages de substrat $\mathbf{N}_{\mathrm{A}}$ en fonction des densités de pièges. Ces profils de $D_{\text {it }}$ sont normalisés par rapport à celle expérimentale reportée sur la Fig. 50-b. Insert : pente sous le seuil SS intrinsèque (sans $D_{\text {it }}$ ) dans la structure MOS-Bulk avec une longueur de grille de $50 \mathrm{~nm}$. b) Dégradation de la densité de porteurs mobiles en régime de forte inversion en fonction de la concentration de dopant sous même condition de gate-overdrive $V_{\mathrm{GS}}-\mathrm{V}_{\mathrm{T}}=1,5 \mathrm{~V}$ par rapport au cas d'une structure idéale. 
Considérons le cas 1D, c'est-à-dire un dispositif avec un canal long (la prise en compte de $\mathrm{D}_{\text {it }}$ dans les calculs en 2D est en cours de développement). Sur la Fig. 56-a, on trace la pente sous le seuil SS pour différents dopages $\mathrm{N}_{\mathrm{A}}$ en fonction des densités de pièges $\mathrm{D}_{\text {it. }}$. Ces profils de $\mathrm{D}_{\mathrm{it}}$ sont normalisés par rapport à celle expérimentale reportée sur la Fig. 23-b. SS varie exponentiellement avec $D_{\text {it }}$ exprimée sur une échelle logarithmique, c'est-à-dire quasiment linéairement en fonction de la densité $\mathrm{D}_{\mathrm{it}}$. En outre, augmenter $\mathrm{N}_{\mathrm{A}}$ rend le contrôle de charge plus sensible aux pièges. A titre d'exemple, pour le profil expérimental reporté en Fig. 50-b), la pente sous le seuil SS est augmentée de quasiment $200 \%$ si la concentration de dopants passe de $10^{16} \mathrm{~cm}^{-3}$ à $10^{18} \mathrm{~cm}^{-3}$. Cette tendance se situe à l'opposée des lois d'échelle développées en technologie CMOS sur silicium où l'on augmente continûment la concentration en dopants pour accroître l'immunité contre l'effet de canal court, comme illustré par l'insert de la Fig. 56-a où est calculé la pente intrinsèque (sans $D_{i t}$ ) dans la structure MOS-Bulk avec une longueur de grille de $50 \mathrm{~nm}$.

Sur la Fig. 56-b, est reportée l'évolution au-dessus du seuil du rapport de la densité de porteurs mobiles avec et sans $D_{i t}$ à même $V_{G}-V_{T}=1,5 V$ en fonction de la concentration en dopants. Avec le profil expérimental de $\mathrm{D}_{\mathrm{it}}$ et $\mathrm{N}_{\mathrm{A}}=10^{18} \mathrm{~cm}^{-3}$, la densité de porteurs mobiles est très fortement réduite, on n'aura que $0,03 \%$ par rapport au cas idéal pour une même condition de polarisation. Si $\mathrm{N}_{\mathrm{A}}$ passe à $10^{17}$ ou $10^{16} \mathrm{~cm}^{-3}$, la situation est peu améliorée, on aura respectivement $2,5 \%$ et $7 \%$ de la densité électronique idéale. Avec une réduction d'un facteur 10 de la densité de pièges, on parviendrait à atteindre environ $50 \%$ de la charge électronique optimale. Avec un facteur 100 on se rapprocherait fortement du cas idéal.

Pour plus de détails à une concentration $\mathrm{N}_{\mathrm{A}}=10^{17} \mathrm{~cm}^{-3}$ donnée, nous étudions l'effet de pinning du niveau de Fermi via le FLE ainsi que les densités de porteurs mobiles dans chaque vallée sur la Fig. 57. Si le profil de $\mathrm{D}_{\text {it }}$ utilisé est celui expérimental de la Fig. 50-b $\left(\mathrm{D}_{\mathrm{it}}(\right.$ mid-gap $\left.) \sim 10^{13} \mathrm{eV}^{-1} \mathrm{~cm}^{-2}\right)$, le maximum du FLE est environ égale à $30 \%$ dans le régime sous le seuil, soit d'environ $-1 \mathrm{~V}$ à $-0,5 \mathrm{~V}$. Cette efficacité est bloquée vers $10 \%$ en régime de forte inversion. La contribution des vallées $\mathrm{L}$ peut dans ces conditions être totalement négligée.

Si la technologie est améliorée pour réduire $\mathrm{D}_{\text {it }}$ d'un facteur 10 , l'effet de pinning sera considérablement limité. Le FLE atteint alors $75 \%$ en régime de déplétion et $25 \%$ dans celui de forte inversion. Le contrôle par la grille sous le seuil et à l'état ON sera donc fortement améliorés, avec une contribution des vallées $\mathrm{L}$ inférieure à $1 \%$ par rapport celle de la vallée $\Gamma$ dans toute la plage de $\mathrm{V}_{\mathrm{G}}$. Pour les technologies actuellement à l'état de l'art, $\mathrm{D}_{\mathrm{it}}$ à mid-gap varie entre $10^{12}$ et $10^{13} \mathrm{eV}^{-1} \mathrm{~cm}^{-2}$. On n'observe alors que très rarement voire jamais de 
'plateau' dans les caractéristique $\mathrm{C}-\mathrm{V}$ dû aux transferts vers les vallées satellites. Pour une technologie améliorée, c'est-à-dire $\mathrm{D}_{\mathrm{it}}($ mid-gap $) \leq 1 \times 10^{12} \mathrm{eV}^{-1} \mathrm{~cm}^{-2}$ et l'effet de pinning est très fortement limité, le FLE tend vers sa valeur idéale : FLE d'environ 75\% en régime de déplétion (idéalement 90\%), une pente sous le seuil de $80 \mathrm{mV} / \mathrm{dec}$ (idéal : $67 \mathrm{mV} / \mathrm{dec}$ ) et une densité de porteurs à $51 \%$ de l'idéal pour $\mathrm{V}_{\mathrm{G}}-\mathrm{V}_{\mathrm{T}}=1,5 \mathrm{~V}$, avec $0,07 \%$ d'électrons en vallée $\mathrm{L}$.

a)

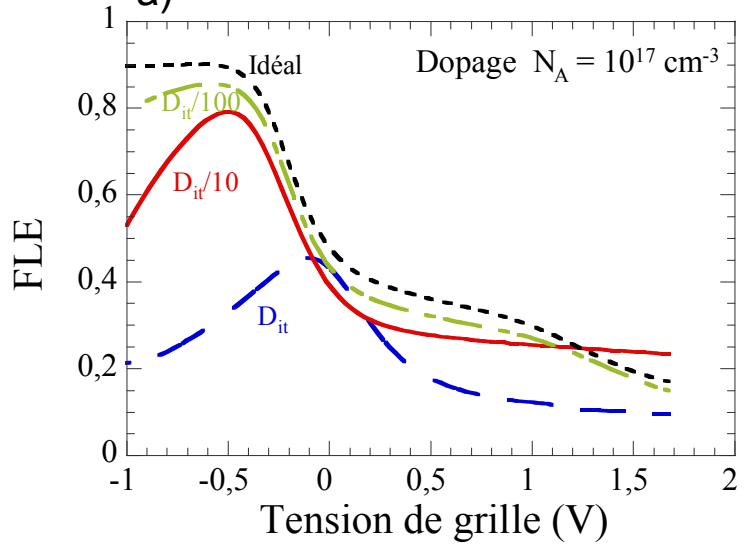

b)

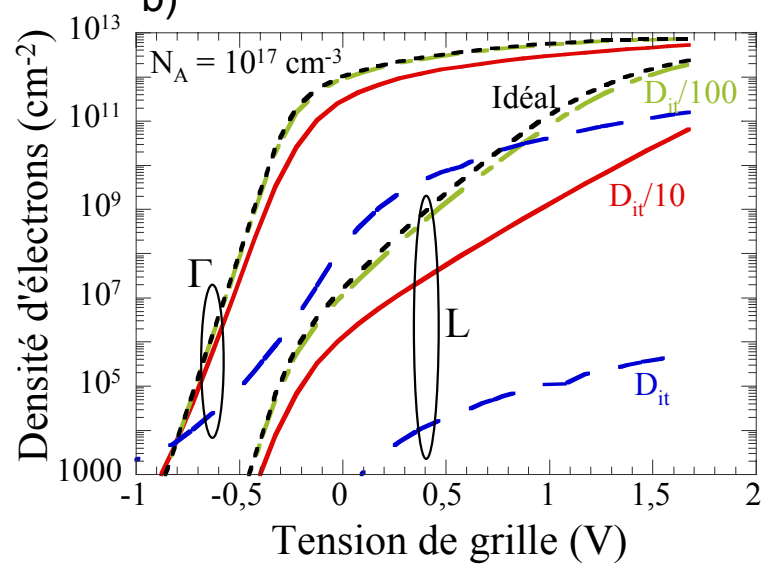

Figure 57 a) Fermi Level Efficiency (FLE) et b) densités de porteurs mobiles dans chaque vallée en fonction de la tension de grille pour différents niveaux de $D_{\text {it }}$ dans une capacité MOS-Bulk 1D avec $N_{A}=10^{17} \mathrm{~cm}^{-3}$ et $T_{O X}=8 \mathrm{~nm}$. Le profil $D_{\text {it }}$ est celui expérimental reporté sur la Fig. 50-b

\section{Performances des MOSFET III-V quasi-balistiques et sans $D_{i t}$}

Nous allons désormais ajouter aux résultats de simulation auto-cohérente PoissonSchrödinger un modèle de transport quasi-balistique pour une première estimation du courant de drain que peut délivrer un MOSFET III-V.

\section{3-1 Modèle analytique pour le transport (quasi) balistique}

Les modèles analytiques du courant balistique sont généralement issus du formalisme de Landauer [Natori1994JAP], [Datta1995]. Dans ce formalisme, le courant du drain $I_{D}$ est décomposé en une composante $\mathrm{I}^{+}$correspondant aux porteurs injectés depuis la source vers le drain, $\mathrm{I}^{-}$à ceux injectés par le drain. On a donc $\mathrm{I}_{\mathrm{D}}=\mathrm{I}^{+}-\mathrm{I}^{-}$. Comme schématisé sur la Fig. 58 pour un canal balistique, tous les porteur dans la source $\mathrm{s}$ avec une vitesse positive (selon l'axe source-drain) et une énergie $E$ supérieure à la barrière d'injection source/canal $E_{M A X_{-} \mu}$ pour une sous bande $\mu$, vont contribuer au courant $\mathrm{I}^{+}$. De même, tous les électrons du drain à vitesse négative et possédant une énergie $\mathrm{E}$ supérieure à $E_{M A X_{-} \mu}+q V_{D S}$ vont contribuer 
au courant $\mathrm{I}^{-}$. La condition sur l'énergie peut s'exprimer en termes de vitesse si on utilise une relation de dispersion parabolique : $v_{\mu}=\sqrt{2\left(E-E_{M A X_{-} \mu}\right) / m^{*}}$.

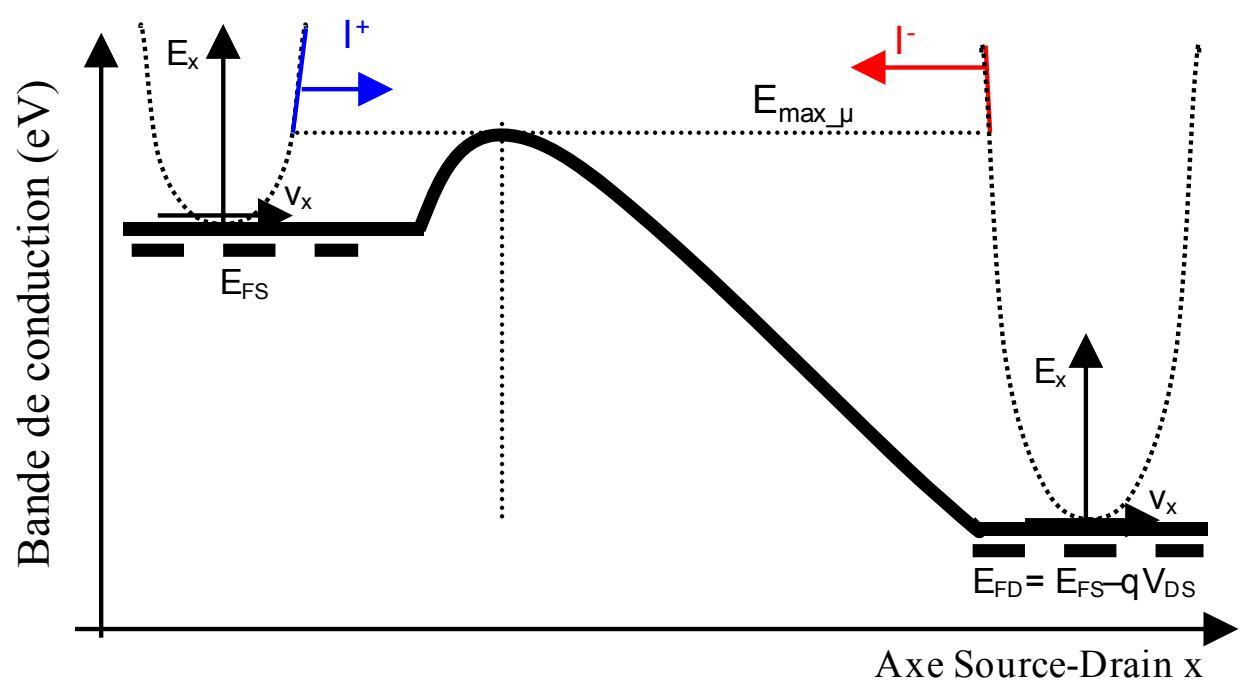

Figure 58 Evolution typique de la bande de conduction le long de l'axe source-drain dans un transistor balistique en train continu. Les tirets en gras symbolisent les niveaux de Fermi $\mathbf{E}_{\mathrm{FS}}$ et $\mathrm{E}_{\mathrm{FD}}$ respectivement dans les caissons de Source et de Drain. Les relations de dispersion paraboliques $\mathbf{E}_{\mathbf{x}}\left(\mathrm{v}_{\mathbf{x}}\right)$ sont indiquées en tirets fins. Les états de la courbe $E_{x}\left(v_{x}\right)$ intervenant dans le calcul des courants $I^{+}$et $I^{-}$ sont surlignés.

Dans notre cas, le profil du potentiel ainsi que la barrière d'injection associée à chaque sous bande est issue de la résolution auto-cohérente de l'équation Poisson-Schrödinger 2D. Le niveau de Fermi dans la région de source $E_{F S}$ est choisi comme référence pour les niveaux de sous bandes dans la source. Si l'on applique la loi de conservation du courant au sommet de la barrière d'injection source-drain pour les différentes populations d'électrons, on $\mathrm{a}$ : $I^{+}=q \cdot n_{S} \cdot v_{S}$ et $I^{-}=q \cdot n_{D} \cdot v_{D}$ où les densités et vitesses sont calculées chaque sous niveau comme suit. Pour une sous bande $\mu$ donnée, les densités d'électrons du côté source $n_{S}$ et du côté drain $n_{D}$ sont données par:

$$
\begin{aligned}
& n_{S}=\frac{m^{*}}{2 \pi \hbar^{2}} k_{B} T \mathfrak{I}_{0}\left(\frac{E_{F S}-E_{M A X \_\mu}}{k_{B} T}\right) \\
& n_{D}=\frac{m^{*}}{2 \pi \hbar^{2}} k_{B} T \mathfrak{I}_{0}\left(\frac{E_{F S}-E_{M A X \_\mu}-q V_{D S}}{k_{B} T}\right)
\end{aligned}
$$

Les vitesses peuvent s'écrire comme (avec l'approximation parabolique) : 


$$
\begin{aligned}
& v_{S}=\sqrt{\frac{2 k_{B} T}{\pi m^{*}} \frac{\mathfrak{I}_{1 / 2}\left(\frac{E_{F S}-E_{M A X_{-} \mu}}{k_{B} T}\right)}{\mathfrak{I}_{0}\left(\frac{\left.E_{F S}-E_{M A X_{-} \mu}\right)}{k_{B} T}\right)}} \\
& v_{D}=\sqrt{\frac{2 k_{B} T}{\pi m^{*}}} \frac{\mathfrak{I}_{1 / 2}\left(\frac{E_{F S}-E_{M A X_{-} \mu}-q V_{D S}}{k_{B} T}\right)}{\mathfrak{I}_{0}\left(\frac{E_{F S}-E_{M A X_{-} \mu}-q V_{D S}}{k_{B} T}\right)}
\end{aligned}
$$

Le premier terme de ces vitesses d'injection $\sqrt{2 k_{B} T / \pi m^{*}}$ est indépendante de la tension de grille. C'est la vitesse thermique d'injection dans la limite de non-dégénérescence. Dans le cas général, la vitesse d'injection dépend du niveau de Fermi ainsi que de la densité de porteurs locale et de la tension de grille.

Le courant total pour un canal balistique peut être évalué par l'expression :

$$
I_{D_{-} B a l} / W=\sum_{v} \sum_{\mu} q \cdot g_{\mu} \cdot\left(n_{S_{-} \mu_{-} v} \times v_{S_{-} \mu_{-} v}-n_{D_{-} \mu_{-} v} \times v_{D_{-} \mu_{-} v}\right)
$$

Le courant quasi-balistique s'écrit simplement comme $I_{D}=B_{\text {eff }} \times I_{D_{-} B a l}$, avec $B_{\text {eff }}$ le coefficient de balisticité effective [Saint-Martin2004IEEE], qui correspond au rapport de courant $B_{e f f}=I_{O N} / I_{O N_{-} b a l}$. $I_{O N_{-} b a l}$ est le courant à l'état passant dans un transistor virtuel parfaitement identique mais ayant un canal balistique. Pour estimer ce coefficient, nous utilisons des résultats microscopiques obtenus par notre simulateur de transport particulaire Monte Carlo (MC) sur l'autre paramètre qui décrit plus microscopique le transport. D'après les travaux de thèse de J. Saint Martin et al on peut accéder à une valeur de $B_{\text {eff }}$ approximée en connaissant la 'balisticité intrinsèque' $B_{\text {int }}$ qui correspond au pourcentage d'électrons parfaitement balistique en fin de canal [Saint-Martin2004IEEE]. Cette dernière fraction peut être directement déduite des résultats de simulation de dispositif par notre code Monte Carlo MONACO. En estimant ainsi la valeur de $B_{\text {eff }}$, nos résultats de courant quasi-balistique dans les structures MOS-Bulk et MOS-ThinBody sont comparés en Fig. 59-a) et 59-b) par rapport à des calculs issus de simulations Monte Carlo semi-classiques. Le courant quasi-balistique est calculé en utilisant les deux premières sous bandes en vallée $\Gamma$. 
a)

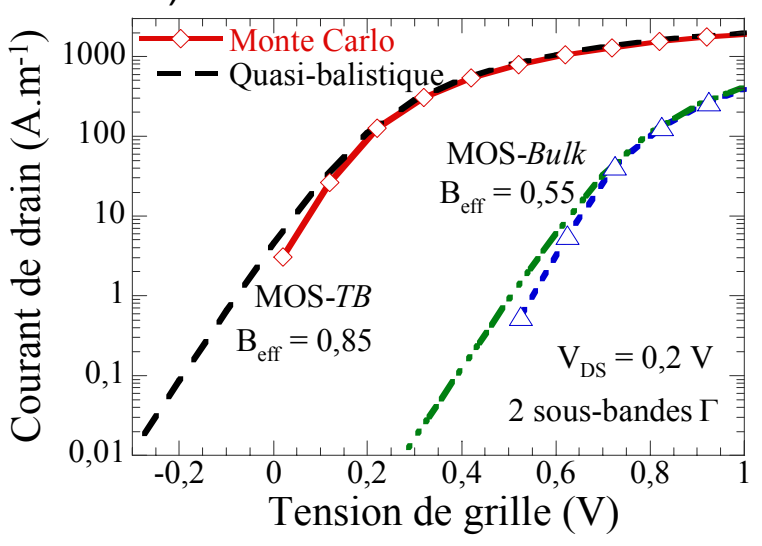

b)

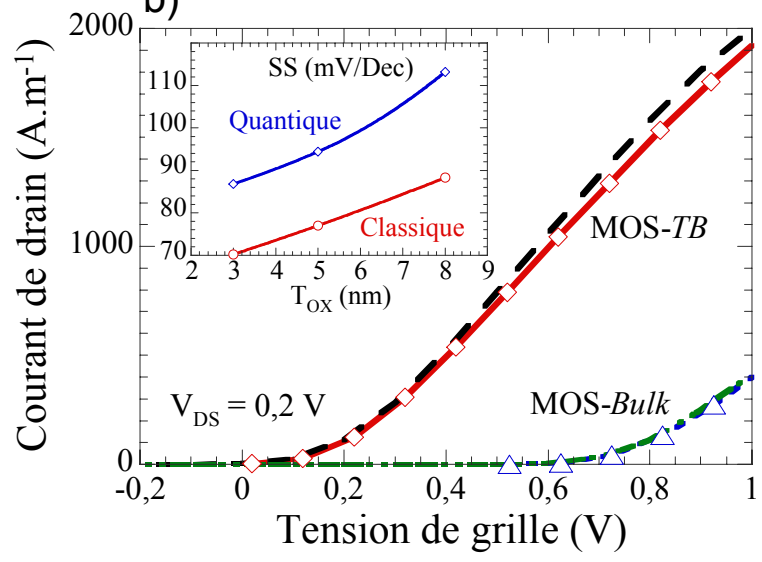

Figure 59 a) Comparaison du courant de drain par calcul quasi-balistique et par la simulation Monte Carlo semi-classique (sans correction quantique) pour les dispositifs MOS-Bulk et MOS-ThinBody avec $L_{G}=50 \mathrm{~nm}$ et $T_{O X}=8 \mathrm{~nm}$. Le courant quasi-balistique est calculé en utilisant les deux premières sous bandes en vallée $\Gamma$. $B_{\text {eff }}$ est respectivement égale à 0,85 et 0,55 pour MOS-Bulk et MOS-ThinBody. b) Même variation en échelle linéaire. Insert : La pente sous seuil de la structure MOS-Bulk calculée via les variations de courant quasi-balistique dans les cas quantique et classique (on résout seulement l'équation de Poisson) en fonction de l'épaisseur d'oxyde pour une longueur de grille 50 nm.

En ajustant la tension de seuil, un bon accord est obtenu entre le modèle quasibalistique et les résultats Monte Carlo en prenant $B_{\text {eff }}=0,55$ pour MOS-Bulk et 0,85 pour MOS-ThinBody à l'état bloqué et à l'état passant. Le courant sous le seuil est un peu plus grand en calcul quasi-balistique qu'en MC. Cette dégradation de la performance sous le seuil, visible en insert de la Fig. 59-b), est due à l'effet de darkspace qui accroît l'épaisseur effective d'isolant et qui n'est pas pris en compte en Monte Carlo (pas de correction quantique).

\section{3-2 Analyse des effets de canal court}

Si on fait varier la longueur de grille, on n'a pas des informations complètes sur le coefficient de balisticité effective pour toutes les longueurs de grille. Dans ce cas là, on calcule le courant purement balistique. Les résultats du modèle de courant balistique couplé à la résolution 2D Poisson-Schrödinger permettent d'évaluer l'influence des effets de canal court dans les dispositifs aux états $\mathrm{ON}$ et $\mathrm{OFF}$. Sur les caractéristiques $\mathrm{I}_{\mathrm{D}}\left(\mathrm{V}_{\mathrm{GS}}\right)$ de la Fig. 60 dans le cas de l'architecture MOS-Bulk, on constate ainsi que la tension de seuil décroît significativement quand $\mathrm{L}_{\mathrm{G}}$ diminue en dessous de $100 \mathrm{~nm}$, que la pente sous le seuil augmente dans les mêmes conditions de même que le décalage en tension de seuil à $\mathrm{L}_{\mathrm{G}}$ donné quand $V_{D s}$ augmente, du fait de l'effet DIBL. Nous allons analyser en détails les évolutions de la variation $\Delta \mathrm{V}_{\mathrm{T}}$ de tension de seuil entre canaux longs et courts, du DIBL et de la pente 
sous le seul SS pour les structures MOS-Bulk et MOS-ThinBody, mais aussi différents matériaux de canal : $\operatorname{In}_{0,53} \mathrm{Ga}_{0,47} \mathrm{As}$ (désigné comme InGaAs dans les paragraphes suivants), $\mathrm{Si}$ et $\operatorname{In}_{0,5} \mathrm{Ga}_{0,5} \mathrm{Sb}$ (InGaSb ci-après).

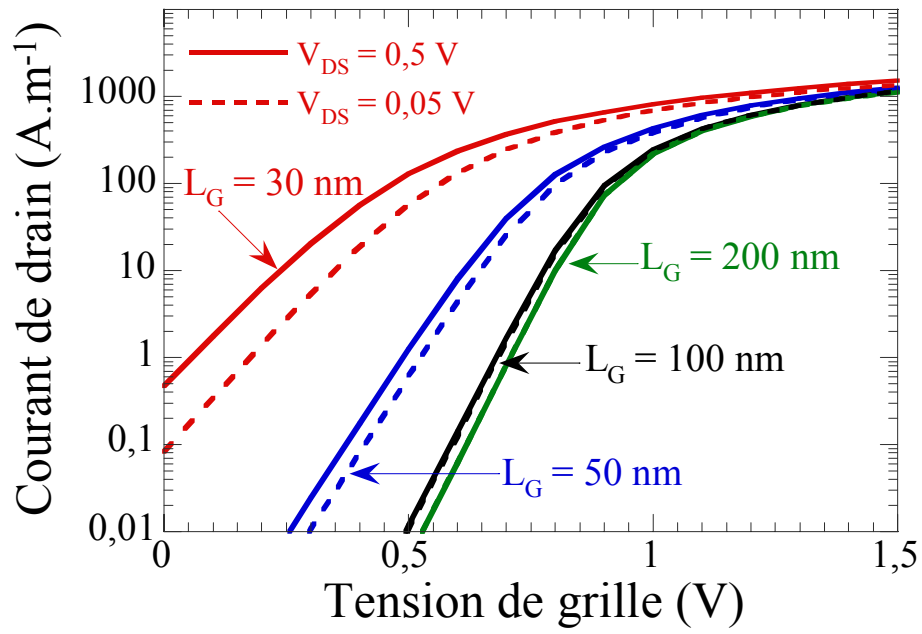

Figure 60 Caractéristiques du courant balistique en fonction de la tension de grille pour une structure MOS-Bulk avec différents $L_{G}$ et $V_{D S}$ pour un canal en $\operatorname{In}_{0,53} G_{a_{0,47}} A s$ et $T_{O X}=8 \mathrm{~nm}$.

\section{3-2-1 Décalage de la tension de seuil}

Sur la Figure 61-a), on constate comme attendu que le décalage $\Delta \mathrm{V}_{\mathrm{T}}$ de tension de seuil dû au SCE est plus important dans les structures à base de matériaux III-V que dans le cas du silicium sous mêmes conditions de polarisation de drain, d'épaisseur d'oxyde et de niveau de dopage. Les résultats pour InGaAs et InGaSb sont quasiment confondus. L'insert montre également que dans le cas d'une structure de MOS-Bulk à base d'InGaAs un calcul classique est assez satisfaisant pour un canal long $(>100 \mathrm{~nm})$. Mais il sous-estime $\Delta \mathrm{V}_{\mathrm{T}}$ pour les dispositifs déca-nanométriques $(<50 \mathrm{~nm})$. Pour $\mathrm{L}_{\mathrm{G}}=50 \mathrm{~nm}$ et $\mathrm{T}_{\mathrm{OX}}=8 \mathrm{~nm}, \Delta \mathrm{V}_{\mathrm{T}}$ vaut $130 \mathrm{mV}$ d'après la prédiction classique contre $245 \mathrm{mV}$ en quantique. Sur la Figure 61-b), le décalage est étudié dans la structure MOS-ThinBody pour plusieurs épaisseurs de couche active $\mathrm{T}_{\text {body }}$ en InGaAs non dopé. Le confinement géométrique améliore le contrôle électrostatique et ainsi augmente l'immunité contre les SCE. Mais comme on l'a vu précédemment une très forte quantification réduit la largeur de la plage de contrôle d'électrons rapides en vallée $\Gamma$. $T_{\text {body }}=10 \mathrm{~nm}$ semble constituer un bon compromis entre cet effet et la tenue aux SCE : la structure MOS-ThinBody avec cette épaisseur de zone active non dopée présente une même performance en termes de décalage de tension de seuil que la structure MOS-Bulk fortement dopée $\left(\mathrm{N}_{\mathrm{A}}=10^{18} \mathrm{~cm}^{-3}\right)$. 
a)

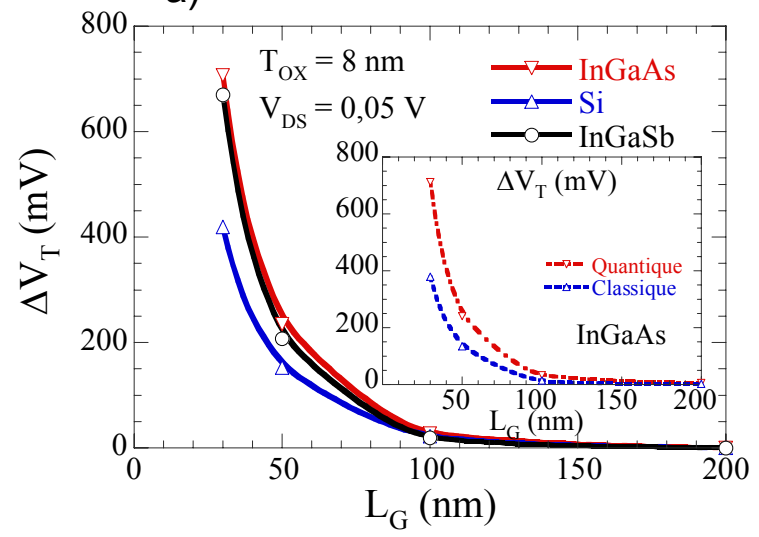

b)

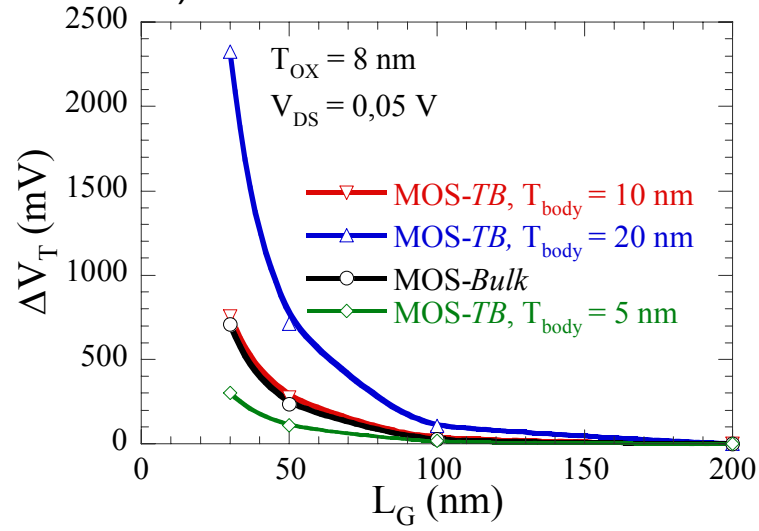

Figure 61 a) Décalage de tension seuil $\left(\Delta V_{T}\right)$ en fonction de la longueur de grille $L_{G}$ pour une structure MOS-Bulk avec $T_{O X}=8 \mathrm{~nm}$ et différents matériaux de canal. Insert : Comparaison avec un calcul classique pour InGaAs. b) Même facteur de mérite calculé pour une structure MOS-ThinBody à base d'InGaAs pour différentes épaisseurs $T_{\text {body }}$ de couche active (la courbe pour MOS-Bulk est également reportée).

\section{3-2-2 Drain Induced Barrier Lowering (DIBL)}

Considérons désormais l'influence de la tension de drain. Elle a pour effet d'abaisser la barrière de potentiel entre source et canal quand les deux réservoirs sont trop proches. Sur la Fig. 62, on compare l'effet DIBL (variation de la tension de seuil normalisée par rapport à celle de $\mathrm{V}_{\mathrm{DS}}$ ) en fonction de longueur de grille et de l'épaisseur d'oxyde pour les différents matériaux et structures. Les commentaires généraux faits à propos des évolutions de $\Delta \mathrm{V}_{\mathrm{T}}$ restent valables pour le DIBL. L'écart entre prédictions classiques et quantiques est toutefois plus faible: respectivement est $35 \mathrm{mV} / \mathrm{V}$ et $75 \mathrm{mV} / \mathrm{V}$ pour un MOS-Bulk de $50 \mathrm{~nm}$ de longueur en InGaAs. Le DIBL peut également être très fortement réduit en diminuant l'épaisseur d'oxyde d'après la Fig. 62-b et -d). 
a)

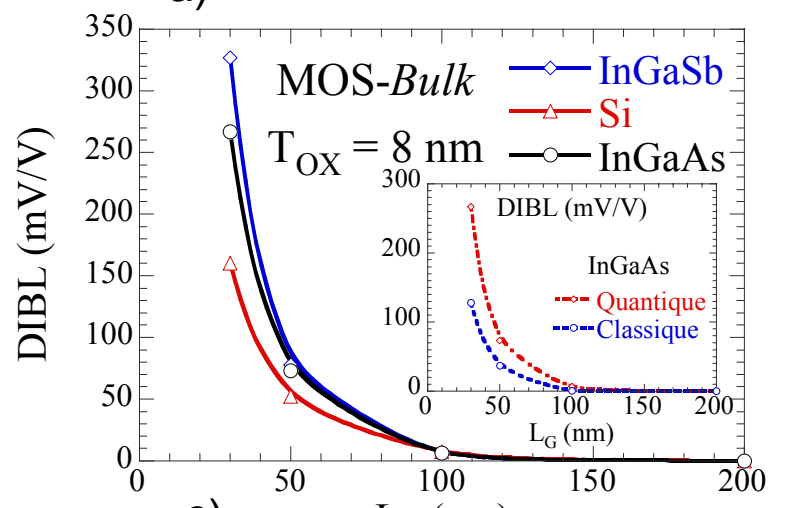

c) $\quad \mathrm{L}_{\mathrm{G}}(\mathrm{nm})$

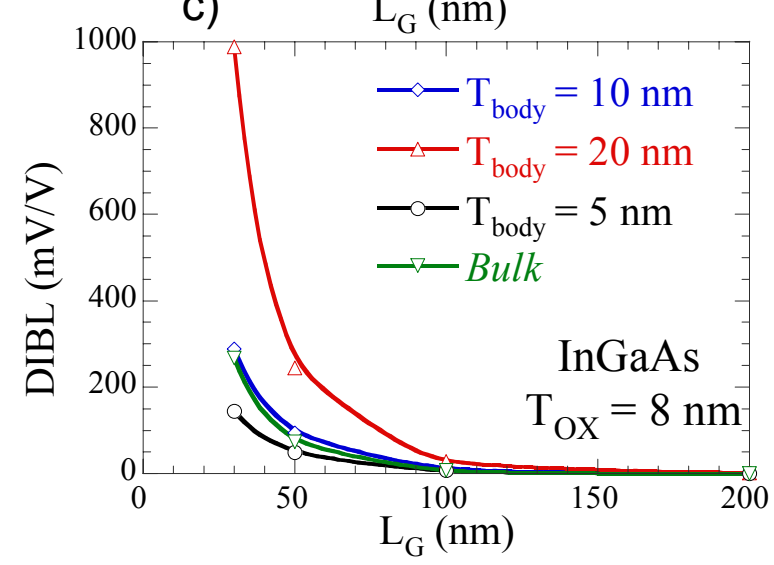

b)
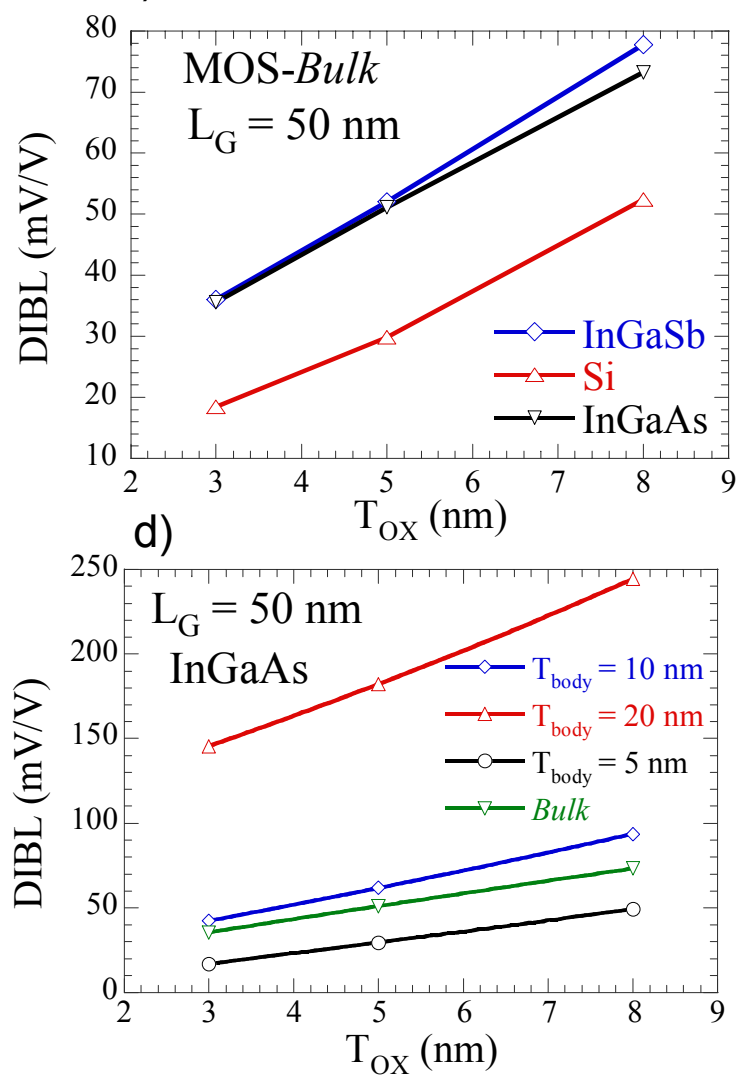

Figure 62 a) DIBL en fonction de la longueur de grille $L_{G}$ pour une structure MOS-Bulk avec $T_{O X}=8 \mathbf{n m}$ et différents matériaux de canal. Insert : comparaison classique/quantique pour InGaAs. b) DIBL en fonction de l'épaisseur d'oxyde pour la même structure avec $L_{G}=50 \mathrm{~nm}$. c) DIBL en fonction de $L_{G}$ pour des structures MOS-ThinBody à base d'InGaAs pour différentes épaisseurs de couche active $\mathbf{T}_{\text {body }}$. d) DIBL en fonction de l'épaisseur d'oxyde pour MOS-ThinBody avec $\mathrm{L}_{\mathrm{G}}=\mathbf{5 0} \mathrm{nm}$.

\section{3-2-3 Pente sous le seuil}

Dans ce paragraphe, nous nous intéressons à une structure idéale (sans piège d'interface) de MOSFET en 2D, dont le fonctionnement est affecté seulement par les SCE.

La pente sous le seuil caractérise l'efficacité à $\mathrm{V}_{\mathrm{GS}}<\mathrm{V}_{\mathrm{T}}$ du contrôle de la grille sur le potentiel de canal $\psi_{S}$ (potentiel de surface) par la capacité de l'oxyde de grille. Ce couplage capacitif entre grille et canal est perturbé par deux capacités en série, celle $\mathrm{C}_{\text {dép }}$ liée à la zone de déplétion et $\mathrm{C}_{\mathrm{IT}}$ aux états d'interface). Dans le paragraphe précédent, nous avons déjà discuté de façon quantitative l'effet des états d'interface sur le contrôle de charge. Les résultats dépendent très fortement des processus de fabrication et les défauts extrinsèques sont susceptibles de dégrader énormément l'efficacité du contrôle de charge. 

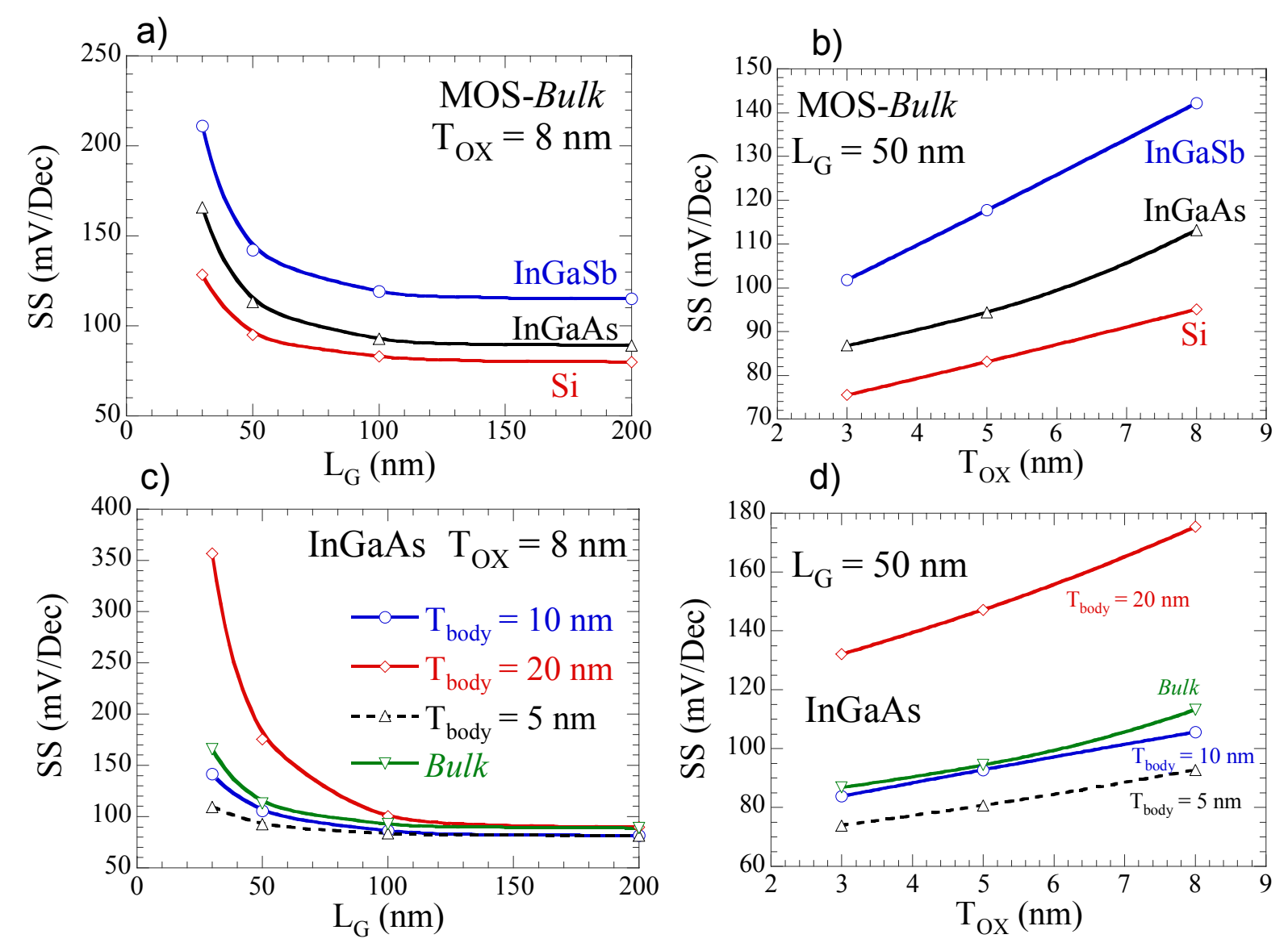

Figure 63 a) Pente sous le seuil $S S$ en fonction de la longueur de grille $L_{G}$ pour une structure MOS-Bulk avec $T_{O X}=8 \mathrm{~nm}$ et différents matériaux de canal à $V_{D S}=0 \mathrm{~V}$. b) $S S$ en fonction de l'épaisseur d'oxyde $T_{0 x}$ pour la même architecture et $\mathrm{L}_{G}=50 \mathrm{~nm}$. c) $\mathrm{SS}\left(\mathrm{L}_{\mathrm{G}}\right)$ pour des MOS-ThinBody à base d'InGaAs avec différents $T_{\text {body. }}$ d) SS(TOX) pour des MOS-ThinBody pour $L_{G}=50 \mathrm{~nm}$.

Sur la Fig. 63, sont tracées des variations de la pente sous le seuil en fonction de la longueur de grille et de l'épaisseur d'oxyde pour différentes matériaux et structures. La pente SS intrinsèque est dégradée quand on passe de Si à InGaAs. C'est encore plus le cas avec InGaSb. Le contrôle sous le seuil est naturellement amélioré en diminuant l'épaisseur d'oxyde pour les trois matériaux. Pour les structures à canal non intentionnellement dopé, le confinement géométrique améliore également la pente sous le seuil qui est légèrement meilleur dans un MOS-ThinBody avec $\mathrm{T}_{\text {body }}=10 \mathrm{~nm}$ que dans le MOS-Bulk étudié. 


\section{3-2-4 Comparaison avec le modèle analytique MASTAR et les spécifications ITRS}

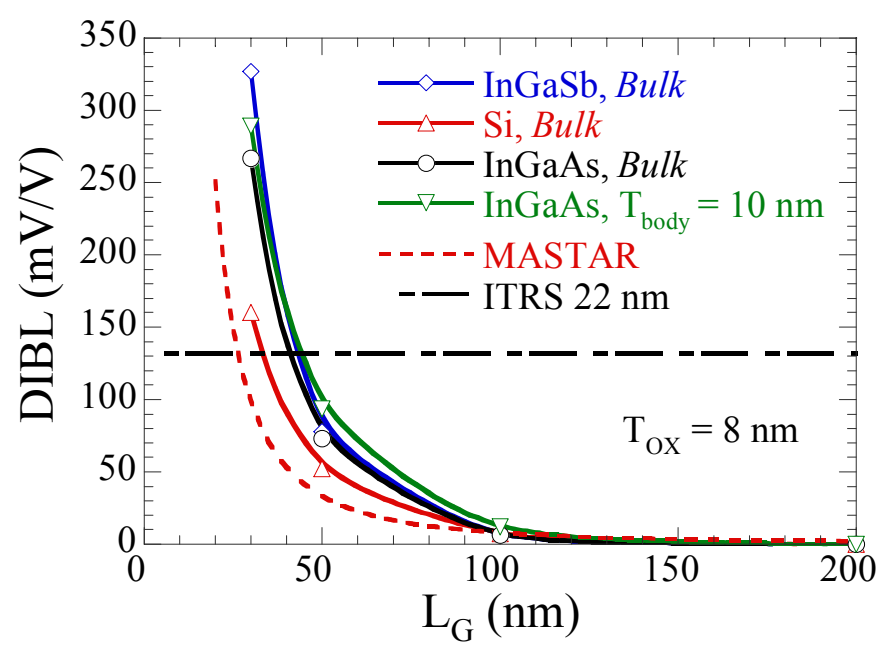

Figure 64 Comparaison entre les prédictions de DIBL pour les différentes structures étudiées et celle fournie par le modèle analytique MASTAR [Skotnicki2003]. La spécification de l'ITRS 2009 pour le nœud technologique $22 \mathrm{~nm}$ (pour lequel le MOSFET à canal de l'InGaAs est l'un des candidats) est également donnée [ITRS2009].

Sur la Fig. 64, nous comparons nos résultats de DIBL sur les dispositifs étudiés (les courbes seraient similaires pour $\Delta \mathrm{V}_{\mathrm{T}}$ et SS) et ceux fournis par le modèle analytique MASTAR [Skotnicki2003]. Ce modèle inclut l'effet de géométrie 2D via une transformation tension - dopage (VDT : Voltage Doping Transformation) [Skotnicki1994IEDM]. Il permet de prendre en compte l'impact de l'épaisseur d'oxyde $\mathrm{T}_{\mathrm{OX}}$, de la densité de dopage $\mathrm{N}_{\mathrm{A}}$ ainsi que de la profondeur de la jonction $\mathrm{X}_{\mathrm{j}}$ sur les effets de canal court. Si les valeurs de DIBL sont très similaires à grande longueur de grille, MASTAR donne des valeurs plus optimistes en dessous de $100 \mathrm{~nm}$. Pour une structure MOS-Bulk à base de l'InGaAs avec $\mathrm{L}_{\mathrm{G}}=50 \mathrm{~nm}$ et $\mathrm{T}_{\mathrm{OX}}=8 \mathrm{~nm}$, le modèle numérique prédit un DIBL de $75 \mathrm{mV} / \mathrm{V}$ contre seulement $30 \mathrm{mV} / \mathrm{V}$ d'après MASTAR. Ces valeurs sont plus faibles que la spécification de l'ITRS 2009 pour la génération $22 \mathrm{~nm}(132 \mathrm{mV} / \mathrm{V})$ [ITRS2009]. Comme attendu, le MOSFET à canal de l'InGaAs s'avère bien l'un des candidats pour atteindre les spécifications de l'ITRS au-delà de la génération $22 \mathrm{~nm}$

\section{3-3 Compromis performance/consommation}

Il nous reste désormais à déduire du modèle balistique et des simulations 2D PoissonSchrödinger des options de dimensionnement qui permettraient de remplir les spécifications de faible puissance et haute vitesse, pour des applications dans les domaines logiques ou analogiques. 


\section{3-3-1 Applications logiques}

a)

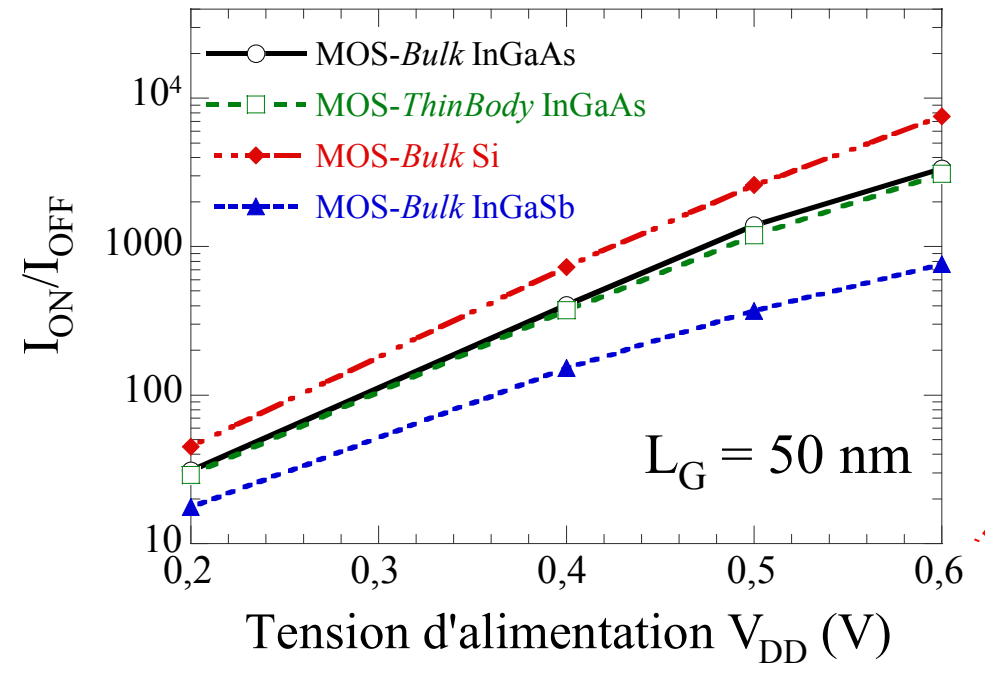

b)

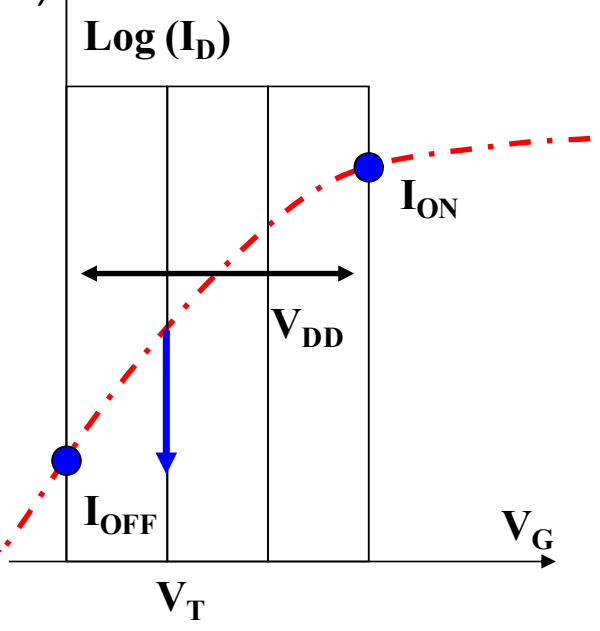

Figure 65 a) : Ratio entre courants quasi-balistiques $I_{\mathrm{ON}} / \mathrm{I}_{\mathrm{OFF}}$ pour les structures $\operatorname{MOS}-B$ ulk $\left(\mathrm{L}_{\mathrm{G}}=50 \mathrm{~nm}\right.$, $T_{O X}=8 \mathrm{~nm}$ et $\left.N_{A}=10^{18} \mathrm{~cm}^{-3}\right)$ et MOS-ThinBody $\left(L_{G}=50 \mathrm{~nm}, T_{O X}=8 \mathrm{~nm}\right.$ et $\left.T_{b o d y}=10 \mathrm{~nm}\right)$ avec différents matériaux en fonction de la tension d'alimentation $\mathrm{V}_{\mathrm{DD}} \cdot \mathrm{b}$ ) : Illustration du choix du critère d'ajustement de la tension de seuil $V_{T}$, au tiers de la tension $V_{D D}$.

La consommation de circuits numériques en technologie CMOS est due pour une bonne part à la consommation statique (DC power) ou en veille (standby power) due au courant $\mathrm{I}_{\mathrm{OFF}}$. Quant à la rapidité de commutation des portes, elle est conditionnée par le courant $\mathrm{I}_{\mathrm{ON}}$. Le délai intrinsèque $\mathrm{C}_{\mathrm{G}} \mathrm{V}_{\mathrm{DD}} / \mathrm{I}_{\mathrm{ON}}$ est un paramètre généralement employé pour faciliter la comparaison entre des architectures qui peuvent être très différentes et/ou sous des tensions d'alimentation variables. Or une faible valeur de $V_{T}$ est néfaste à $\mathrm{I}_{\mathrm{OFF}}$ même si elle permet d'augmenter $\mathrm{I}_{\mathrm{ON}}$ donc de diminuer $\mathrm{C}_{\mathrm{G}} \mathrm{V}_{\mathrm{DD}} / \mathrm{I}_{\mathrm{ON}}$. La définition de $\mathrm{V}_{\mathrm{T}}$ résulte donc généralement d'un compromis, par exemple entre vitesse et puissance. $\mathrm{I}_{\mathrm{ON}}$ varie par rapport à $\mathrm{V}_{\mathrm{T}}$ selon une loi de puissance tandis qu' $\mathrm{I}_{\mathrm{OFF}}$ dépend exponentiellement de la tension de seuil. Un bon compromis vitesse/puissance est souvent obtenu en fixant $\mathrm{V}_{\mathrm{T}}$ au $1 / 3$ de la tension d'alimentation comme illustré sur la partie droite de la Fig. 65-b. Nous avons adopté ce critère par la suite. Dans notre cas, $\mathrm{V}_{\mathrm{T}}$ est extrait dans le régime sous le seuil pour une densité d'inversion $n_{i n v}=10^{10} \mathrm{~cm}^{-2}$ pour tous les dispositifs sous investigation et les valeurs de $\mathrm{I}_{\mathrm{OFF}}$ et $\mathrm{I}_{\mathrm{ON}}$ sont respectivement obtenues à une tension $\mathrm{V}_{\mathrm{GS}}$ à $\mathrm{V}_{\mathrm{DD}} / 3$ sous le seuil et $2 \mathrm{~V}_{\mathrm{DD}} / 3$ au-dessus de $\mathrm{V}_{\mathrm{T}}$, avec dans les deux cas $\mathrm{V}_{\mathrm{DS}}=\mathrm{V}_{\mathrm{DD}}$.

Sur la Fig. 65-a, sont d'abord tracées les variations du rapport des courants $\mathrm{I}_{\mathrm{ON}} / \mathrm{I}_{\mathrm{OFF}}$ pour MOS-Bulk et MOS-ThinBody, différents matériaux de canal et en fixant la longueur de grille à $\mathrm{L}_{\mathrm{G}}=50 \mathrm{~nm}$. Les deux architectures (MOS-Bulk et MOS-ThinBody) présentent des 
performances pratiquement identiques. Sur la Fig. 66, on observe que pour un dispositif avec un canal long, on peut diminuer $\mathrm{V}_{\mathrm{DD}}$ jusqu'à $0,4 \mathrm{~V}$ en maintenant $\mathrm{I}_{\mathrm{ON}} / \mathrm{I}_{\mathrm{OFF}}$ au-dessus de 1000 tandis que le dispositif avec $\mathrm{L}_{\mathrm{G}}$ de $50 \mathrm{~nm}$ (résultats non représentés) doit être alimenté sous $0,5 \mathrm{~V}$ pour le même ratio de courant. Une grille aussi courte que $30 \mathrm{~nm}$ ne permet pas d'atteindre ce critère dans cette gamme de $\mathrm{V}_{\mathrm{DD}}$.

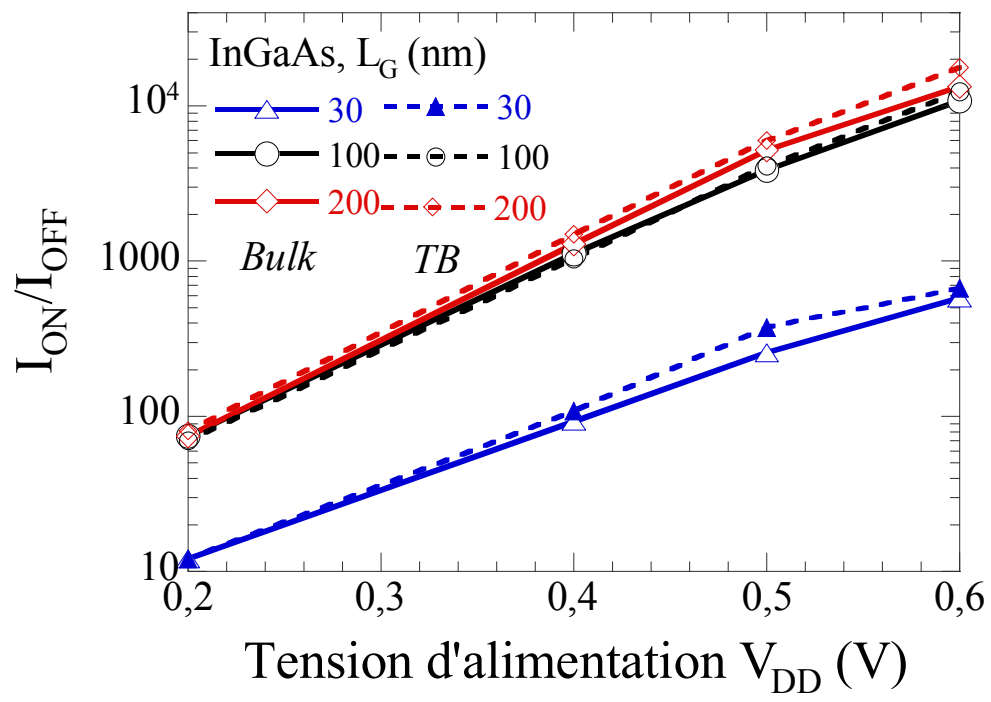

Figure 66 Ratio du courant balistique $I_{O N} / I_{O F F}$ pour les structures MOS-Bulk $\left(T_{O X}=8\right.$ nm et $\left.\mathrm{N}_{\mathrm{A}}=10^{18} \mathrm{~cm}^{-3}\right)$ et MOS-ThinBody $\left(T_{\mathrm{OX}}=8 \mathrm{~nm}\right.$ et $\left.T_{\text {body }}=10 \mathrm{~nm}\right)$. La longueur de grille $L_{G}$ est égale à 30, 100 et $200 \mathrm{~nm}$.

En ce qui concerne l'influence des matériaux, le rapport $\mathrm{I}_{\mathrm{ON}} / \mathrm{I}_{\mathrm{OFF}}$ est plus grand dans la structure à base de Si par rapport aux canaux en III-V sous même condition de $\mathrm{V}_{\mathrm{DD}}$. La plus mauvaise performance est observée dans la structure MOS-Bulk à base de InGaSb à cause de leur très faible DOS dans la vallée $\Gamma$ et à l'écart faible en énergie avec les vallées $L$ $\Delta \varepsilon_{\Gamma L} \sim 0,25 \mathrm{eV}$ [Robertsona2006JAP] (ce qui limite la plage de contrôle de grille pour des électrons majoritairement en $\Gamma$ ). La Fig. 67 montre les variations du délai intrinsèque $\mathrm{C}_{\mathrm{G}} \mathrm{V}_{\mathrm{DD}} / \mathrm{I}_{\mathrm{ON}}$ pour des dispositifs avec $\mathrm{L}_{\mathrm{G}}=50 \mathrm{~nm}$ et $\mathrm{T}_{\mathrm{OX}}=8 \mathrm{~nm}$ en fonction de $\mathrm{I}_{\mathrm{ON}} / \mathrm{I}_{\mathrm{OFF}}$ pour différentes valeurs de $\mathrm{V}_{\mathrm{DD}}(0,2 \mathrm{~V}, 0,4 \mathrm{~V}, 0,5 \mathrm{~V}$ et $0,6 \mathrm{~V})$. La valeur de capacité utilisée dans le calcul du délai comprend les éléments parasites dus aux effets de bord et d'accès. La balisticité effective $B_{\text {eff }}$ est considérée égale à 0,3 pour Si et 0,55 pour InGaSb comme pour InGaAs. Le domaine de fonctionnement intéressant à viser est indiqué par la zone hachurée en vert : $\mathrm{I}_{\mathrm{ON}} / \mathrm{I}_{\mathrm{OFF}}>1000$ et $\mathrm{C}_{\mathrm{G}} \mathrm{V}_{\mathrm{DD}} / \mathrm{I}_{\mathrm{ON}}<1$ ps. Un canal en $\mathrm{InGaAs}$ est la seule possibilité permettant d'atteindre ces objectifs avec la géométrie considérée, la structure MOS-ThinBody s'avérant meilleure en termes de délai. Néanmoins, la tension d'alimentation doit dépasser $0,5 \mathrm{~V}$ ce qui n'était pas souhaité. 


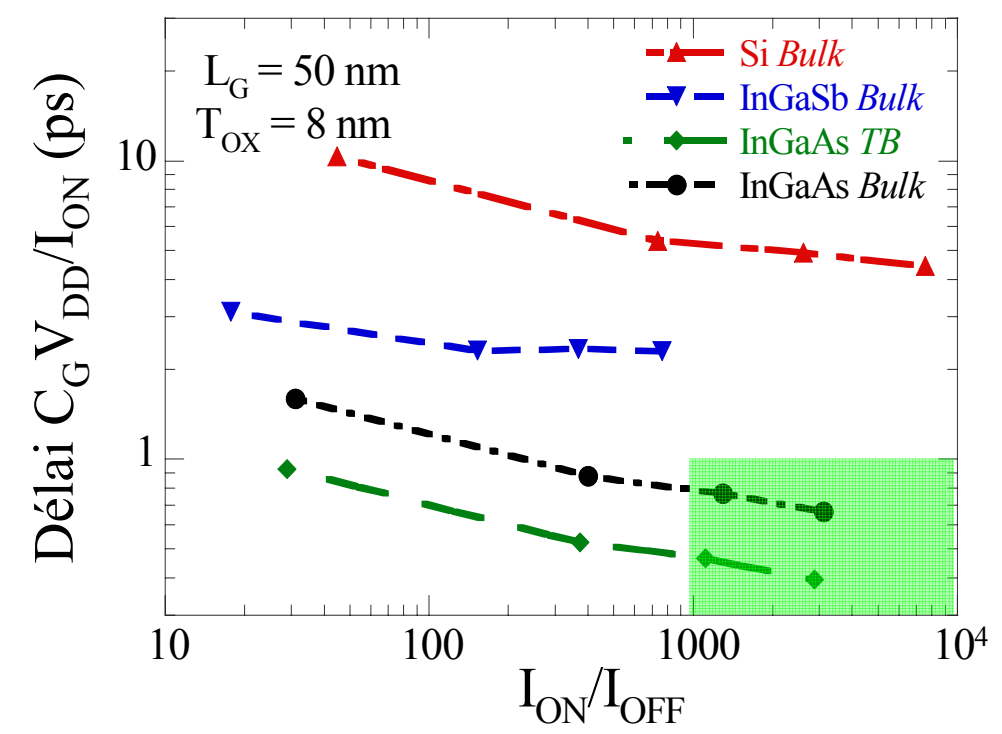

Figure 67 Evaluation de $\mathbf{C}_{\mathrm{G}} \mathbf{V}_{\mathrm{DD}} / \mathbf{I}_{\mathrm{ON}}$ en fonction de $\mathbf{I}_{\mathrm{ON}} / \mathbf{I}_{\text {OFF }}$ pour les structures MOS-Bulk et MOS-ThinBody avec différents matériaux de canal et valeurs de $V_{D D}(0,2,0,4,0,5$ et $0,6 \mathrm{~V})$. Pour tous les dispositifs, $L_{G}=50 \mathrm{~nm}$ et $T_{O X}=8 \mathrm{~nm}$. La zone sur fond vert est celle correspondant aux limites intéressantes à viser, avec $I_{O N} / I_{O F F}>1000$ et $C_{G} V_{D D} / I_{O N}<1$ ps.

\section{3-3-2 Applications analogiques}

La fréquence de transition $\mathrm{f}_{\mathrm{T}}$, qui correspond à un gain en courant unitaire entre drain et grille, est un facteur de mérite pour les applications analogiques qui peut être estimé par la formule quasi-statique $G_{m} / 2 \pi C_{G}$. Dans notre cas, $G_{m}$ est calculé à partir de courants quasi balistiques et $C_{G}$ est extraite des simulations Poisson-Schrödinger 2D. Des exemples de résultats sont donnés sur la Fig. 68-a). Nous avons utilisé le même critère pour l'ajustement de la tension de seuil que précédemment pour les applications numériques. La fréquence $\mathrm{f}_{\mathrm{T}}$ est ensuite tracée sur la Fig. 68-b) en fonction de la dissipation de puissance active. La capacité de grille totale contient les différentes composantes comme la capacité d'oxyde, d'inversion, de déplétion et les capacités de bord et des zones d'accès). La capacité des pièges d'interface $\mathrm{C}_{\mathrm{IT}}$ n'a pas prise en compte dans ces analyses en $2 \mathrm{D}$. 
a)

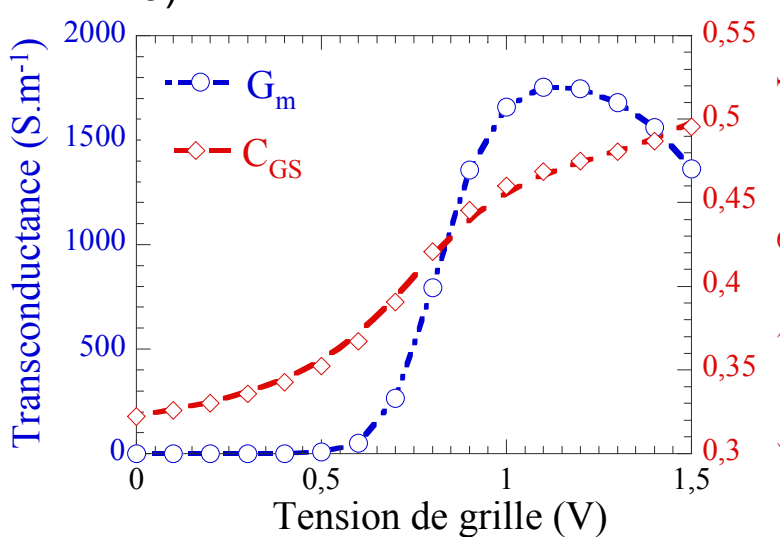

b)

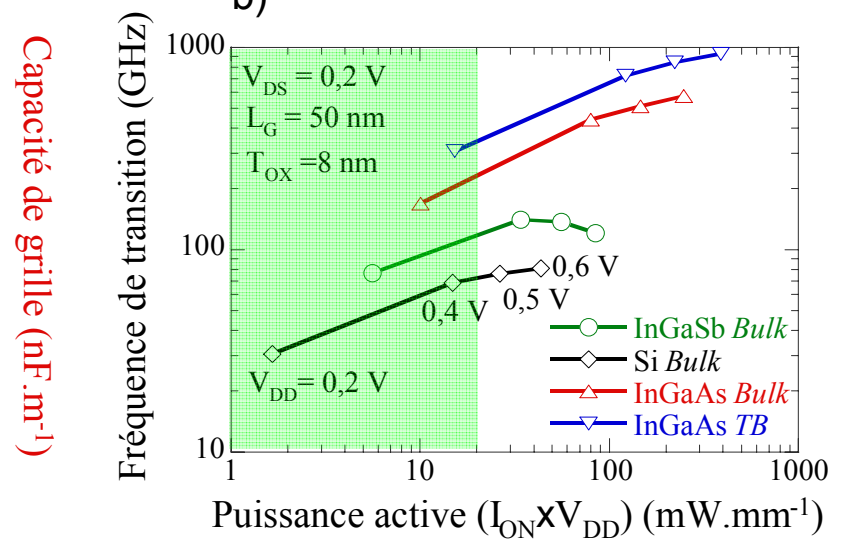

Figure 68 a) Transconductance « quasi-balistique » et capacité de grille en fonction de $\mathbf{V}_{G S}$ à $V_{D S}=0,2 \mathrm{~V}$ pour la structure MOS-Bulk à base d'InGaAs. b) Evaluation de la fréquence de transition $f_{T}$ en fonction de puissance active statique $\left(I_{O N} \times V_{D D}\right)$ pour différents $V_{D D}(0,2,0,4,0,5$ et $0,6 \mathrm{~V})$ à $V_{D S}=0,2 \mathrm{~V}$ pour les structures MOS-Bulk et MOS-ThinBody avec $\mathrm{L}_{\mathrm{G}}=50 \mathrm{~nm}, \mathrm{~T}_{\mathrm{OX}}=8 \mathrm{~nm}$ et différents matériaux.

Sur la Fig. 68, nous traçons la fréquence de transition en fonction de la dissipation de puissance statique $\left(\mathrm{I}_{\mathrm{ON}} \times \mathrm{V}_{\mathrm{DD}}\right)$ pour différents matériaux de canal et architectures, avec dans tous les cas $\mathrm{L}_{\mathrm{G}}=50 \mathrm{~nm}, \mathrm{~T}_{\mathrm{OX}}=8 \mathrm{~nm}$ et $\mathrm{V}_{\mathrm{DS}}=0,2 \mathrm{~V}$, pour différentes valeurs de $\mathrm{V}_{\mathrm{DD}}$ (et donc de $V_{G S}-V_{T}$ ). Les meilleures performances sont obtenues dans la structure MOS-ThinBody à base d'InGaAs. Ce dispositif peut fonctionner avec une large autonomie en énergie, c'est-àdire une dissipation de puissance inférieure à $20 \mathrm{~mW} \cdot \mathrm{mm}^{-1}$ [Aarts2003], tout en gardant une fréquence $\mathrm{f}_{\mathrm{T}}$ à plus d'une centaine de $\mathrm{GHz}$. 


\section{Conclusion du chapitre II}

Dans ce chapitre, nous avons étudié théoriquement le contrôle de charge dans un système MOS du type métal/high- $/$ /III-V. Le modèle conventionnel utilisé dans le cas de Si n'est pas transposable au cas des systèmes à base de matériaux III-V, il doit être amélioré en ajoutant les différents phénomènes physiques suivants: statistique de Fermi-Dirac, quantification des niveaux d'énergie, correction de non-parabolicité, évaluation multi-vallées ainsi que charges supplémentaires dues aux pièges localisées à l'interface diélectrique/semiconducteur.

La faible densité d'états des matériaux III-V envisagés tend à réduire la capacité d'inversion des structures MOS. L'étude physique que nous avons réalisée aboutit à une épaisseur d'oxyde optimisée qui correspond un compromis entre l'efficacité du contrôle de charge et la dégradation de la capacité de grille par rapport celle d'oxyde. Les particularités du contrôle de charge dans les structures alternatives, à film actif mince (Thin Body) et/ou, canal enterré, ont aussi été évaluées.

La commande de charge par la grille au sein du semi-conducteur III-V résulte d'une compétition entre les charges mobiles issues de la densité d'états intrinsèque du semiconducteur et des charges localisées dues aux pièges d'interface. Ces deux contributions pouvant s'influencer entre elles, un calcul auto-cohérent est nécessaire. Avec un niveau de $\mathrm{D}_{\text {it }}$ réduit à $10^{11} \mathrm{eV}^{-1} \mathrm{~cm}^{-2}$ (mid-gap), on retrouverait les performances d'une structure MOS intrinsèque.

La simulation est ensuite étendue au cas d'une géométrie 2D pour prendre en compte les effets de canal court et les capacités parasites. Nous avons ajouté aux résultats de simulation auto-cohérente Poisson-Schrödinger un modèle de transport quasi-balistique pour une première estimation du courant de drain que peut délivrer intrinsèquement un MOSFET III-V. Les meilleures performances sont obtenues dans la structure MOS-ThinBody à base d'InGaAs. Le transport quasi-balistique, les caractéristiques électriques en statique et dynamique ainsi que le comportement du bruit à haute fréquence seront quantitativement étudiés dans le chapitre suivant. 


\section{Chapitre III : Analyse semi-classique Monte Carlo des MOSFET III-V}

Dans ce chapitre, on s'intéresse au transport électronique dans les dispositifs. On suppose que le transport est semi-classique, homogène dans les espaces réciproques et géométriques et qu'il peut être décrit dans l'approximation de la masse effective. Dans ce contexte simplifié, il est possible d'étudier l'évolution temporelle de la fonction de distribution des porteurs de charge sous l'influence des forces externes, des gradients spatiaux et des interactions.

Nous commencerons ce chapitre par des rappels succincts sur la théorie de base du transport semi-classique et le principe et algorithme du simulateur particulaire Monte Carlo (MC). Ensuite, nous verrons comment le modèle particulaire MC développé dans l'équipe est calibré pour la simulation de MOSFET III-V. Puis, les propriétés de transport quasi-balistique, les caractéristiques électriques statiques et dynamiques ainsi que le comportement du bruit à haute fréquence seront quantitativement étudiés. Trois architectures alternatives par rapport à la structure en bulk sont ainsi évaluées et des conclusions tirées en termes de rendement fréquence/consommation et d'adaptation de bruit. L'étude comparative avec les dispositifs à base de Si démontre enfin clairement le potentiel des MOSFET III-V submicronique pour les applications à haute fréquence et faible puissance de consommation.

\section{Présentation de la simulation particulaire Monte Carlo semi-} classique

\section{1-1 L’équation de Boltzmann}

Dans ce paragraphe, nous allons réviser rapidement les grands principes de l'équation de Boltzmann du transport électronique. Les points essentiels sont détaillés dans l'Annexe - C. En physique classique statistique, les quantités caractérisant un système de particules sont déterminées par la fonction de distribution $f(\vec{r}, \vec{p}, t)$ qui donne la probabilité de trouver des particules à la position $\vec{r}$, avec l'impulsion $\vec{p}$ et au temps t. L'étude du transport consiste à déterminer l'évolution de cette fonction de distribution hors d'équilibre, c'est-à-dire à résoudre l'équation de Boltzmann [Jacoboni1989] en présence des collisions que subissent les porteurs avec le milieu de propagation : 


$$
\frac{\partial f}{\partial t}+\nabla_{\vec{r}} f \cdot \frac{\hbar \vec{k}}{m}-\frac{e}{\hbar} \nabla_{\vec{k}} f \cdot E(\vec{r})=S_{\text {coll }} f
$$

où le terme de collision $S_{\text {coll }} f(\vec{r}, \vec{p}, t)$ est écrit comme un opérateur sur la fonction de distribution. Il peut être développé sous la forme suivante si les particules perdent toutes les corrélations avec les autres particules [Ferry1997] :

$$
\begin{aligned}
S_{\text {coll }} f & =\sum_{\vec{k}^{\prime}} S\left(\vec{k}, \vec{k}^{\prime}\right) \cdot f(\vec{r}, \vec{k}, t) \cdot\left[1-f\left(\vec{r}, \vec{k}^{\prime}, t\right)\right] \\
& -\sum_{\vec{k}} S\left(\vec{k}^{\prime}, \vec{k}\right) \cdot f\left(\vec{r}, \vec{k}^{\prime}, t\right) \cdot[1-f(\vec{r}, \vec{k}, t)]
\end{aligned}
$$

Le premier terme décrit l'augmentation de $f(\vec{r}, \vec{k}, t)$ due à la transition de l'état initial $\vec{k}$ vers l'état final $\vec{k}^{\prime}$, pour laquelle la densité de probabilité par unité de temps est donnée $\operatorname{par} S\left(\vec{k}, \vec{k}^{\prime}\right)$. Réciproquement pour le second terme. Le principe d'exclusion de Pauli est pris en compte par le facteur $1-f$ qui limite les transitions aux états d'arrivée non occupés. Dans le traitement quantique des interactions, les densités de probabilité de transition $S\left(\vec{k}, \vec{k}^{\prime}\right)$ sont évaluées en utilisant la règle d'or de Fermi [Mark2000]. Les études sur les propriétés de transport d'un point de vue semi-classique consistent à résoudre cette équation de Boltzmann. De façon générale, la résolution de l'équation de Boltzmann se divise en trois types: résolution déterministe directe (ex: expansion des harmoniques sphériques) [Vecchi1998IEEE], résolution simplifiée (ex: modèle dérive-diffusion et modèle hydrodynamique) [Nekovee1992PRB] et résolution statistique (ex: Monte Carlo). Cette dernière est très bien adaptée à la simulation de dispositifs de taille nanométrique en régime statique (DC) [Dollfus1993JAP] et également en régime transitoire (AC) [Dollfus1992EL] reproduisant de façon naturelle les effets de transport fortement hors équilibre comme le régime balistique.

\section{1-2 Simulateur particulaire MONACO : principe et algorithme}

Dans ce paragraphe, nous allons décrire les principes généraux de la méthode Monte Carlo (MC) sur laquelle est basé le simulateur particulaire MONACO, développé au sein de notre équipe depuis une bonne vingtaine d'années [Hesto1984Thèse], [Galdin1992Thèse], [Brisset1994Thèse], [Dollfus1999HDR], [Barraud2001Thèse], [SaintMartin2005Thèse], [Bournel2006HDR], [Huet2008Thèse] et [Querlioz2008Thèse]. Nous en rappelons brièvement le principe et algorithme. Les points essentiels sont détaillés dans l'Annexe - C. 
Dans un matériau ou un dispositif semi-conducteur soumis à un champ électrique, les porteurs sont d'une part accélérés par la force électromotrice résultante et d'autre part conditionnés par les différentes collisions (interactions) qu'ils subissent dans leur environnement. La trajectoire des particules est une succession de vols libres entrecoupés d'interactions de différentes natures [Jacoboni1989]. Le principe de base de cette méthode consiste en un tirage au sort de variables aléatoires visant à déterminer : i) la valeur du temps de vol libre; ii) l'interaction subie à la fin du vol libre ; iii) l'effet de l'interaction sélectionnée (déviation du vecteur d'onde, modification éventuelle de l'énergie du porteur). Pour décrire les lois de probabilité afin d'effectuer les tirages au sort et le mouvement sous le champ de force extérieur durant chaque vol libre, on a aussi besoin de modèles physiques décrivant : i) la relation de dispersion énergie-vecteur d'onde; ii) l'équation du mouvement; iii) les fréquences d'interaction.

Le champ électrique qui agit sur les électrons dépend lui-même de la fonction de répartition des électrons dans le dispositif par intermédiaire de l'équation de Poisson. On découple temporellement le mouvement des électrons et le calcul du champ dans un intervalle de temps $\Delta t$. L'équation de Poisson est résolue tous les $\Delta t$ par une méthode de différences finies. Ce pas de temps doit être choisi suffisamment petit pour que l'on puisse considérer le champ calculé comme constant durant ce pas de temps. Durant $\Delta t$ on calcule la dynamique de chaque électron qui subit une succession de vols libres sous l'effet du champ et d'interactions supposées instantanées. Le temps de vol libre entre deux interactions et la nature des interactions subies sont déterminés par tirage au sort. La distribution de charges dans le dispositif est calculée par moyennes spatio-temporelles. A la fin d'un pas sur le temps, on résout l'équation de Poisson et on calcule le champ électrique qui sera utilisé pour le pas $\Delta t$ suivant.

\section{1-3 Calcul de courant par la méthode de Ramo-Shockley}

Le calcul du courant traversant les électrodes au sein du dispositif est le point crucial pour obtenir une analyse correcte des caractéristiques électroniques macroscopiques, tant dans le régime permanent (caractéristique courant - tension) qu'en transitoire (paramètres $\mathrm{Z}, \mathrm{Y}$ et $\mathrm{S}$, circuit équivalent petit signal, bruit).

Pour réduire le bruit numérique sur les courants aux contacts, il suffit a priori d'améliorer la statistiquement par l'augmentation du nombre de particules implantés et d'itérations de résolution de l'équation de Poisson. Cette méthode est généralement bien appropriée pour la détermination des caractéristiques statiques du dispositif. Pour ce qui est 
des études en transitoire, la méthode de Ramo-Shockley a été implémentée dans notre simulateur Monte Carlo. Elle permet de déterminer les courants instantanés aux contacts en utilisant la contribution du mouvement de toutes les charges mobiles dans le composant. Cette approche peut fournir une valeur du courant instantané avec moins de fluctuations, en diminuant le bruit numérique.

Le théorème de Ramo-Shockley était originalement prévu pour le calcul des courants instantanés induits par le mouvement d'électrons dans un tube à vide [Shockley1938JAP] et [Ramo1939IRE]. De Visschere [De Visschere1990SSE] a ensuite démontré que ce théorème est également valable si tient compte des contributions de charges spatiales fixes, dans un cas statique tel que les électrodes sont localisées dans un milieu avec une permittivité diélectrique uniforme et maintenues à une polarisation ou des potentiels constants. Puis Kim l'a étendu en régime dynamique pour un milieu diélectrique non-homogène avec une distribution de charges mobiles et fixe arbitraire et sous potentiels variables aux électrodes [Kim1991SSE]. Nous utilisons l'approximation de Kim, le courant de chaque électrode est calculé via le théorème de Ramo-Shockley généralisé pour un dispositif de type MOSFET. Nous allons décrire désormais les détails de cette méthode.

Supposons un système comportant $M$ électrodes de formes arbitraires localisées dans un milieu de permittivité diélectrique non-homogène et $\mathrm{N}$ particules chargées se mouvant dans l'espace. Les $\mathrm{M}$ électrodes sont maintenus aux tensions $V_{1}(t) \ldots, V_{M}(t)$. Les positions, vitesse et charge de la i-ème particule sont $r_{i}(t), v_{i}(t)$ et $q_{i}(t)$ à un instant $t$ respectivement, et la densité de charge $\rho(r, t)$ peut s'exprimer par :

$$
\rho(r, t)=\sum_{i=1}^{N} q_{i}(t) \delta\left(r-r_{i}\right)
$$

où $\delta\left(r-r_{i}\right)$ est la fonction de Dirac. La quantité $\rho(r, t)$ inclut les charges spatiales libres et fixes. Le potentiel à un temps donné $\mathrm{t}$ et en un point $r$ du dispositif peut se décomposer suivant le principe de superposition en deux contributions :

$$
\varphi(r, t)=\varphi^{\prime}(r, t)+\varphi^{\prime \prime}(r, t)
$$

avec $\varphi^{\prime}(r, t)$ le potentiel lié à $\rho$ quand les $\mathrm{M}$ électrodes sont connectées à la masse et $\varphi^{\prime \prime}(r, t)$ celui lié aux tensions appliquées quand il n'y a pas de charges dans le dispositif (exemple sur la Fig. 69). Ces deux composantes du potentiel vérifient respectivement les équations de Poisson et de Laplace : 


$$
\begin{array}{lll}
-\nabla\left[\varepsilon(r) \nabla \varphi^{\prime}(r, t)\right]=\rho(r, t) & \text { avec } & \varphi_{1}^{\prime}(t)=0, \ldots, \varphi_{M}^{\prime}(t)=0 \\
-\nabla\left[\varepsilon(r) \nabla \varphi^{\prime \prime}(r, t)\right]=0 & \text { avec } & \varphi_{1}^{\prime \prime}(t)=V_{1}(t), \ldots, \varphi_{M}^{\prime \prime}(t)=V_{M}(t)
\end{array}
$$

En appliquant le théorème de Gauss pour les milieux non-homogènes [Pellegrini1986PRB], il est possible de calculer la charge associée à la j-ème électrode, $Q_{j}(t)$, qui se divise en deux contributions, $Q_{j}^{\prime}(t)$ et $Q_{j}^{\prime \prime}(t)$, avec $Q_{j}^{\prime}(t)$ la charge induite à la j-ème électrode par les $\mathrm{N}$ particules chargées :

$$
Q_{j}^{\prime}(t)=-\sum_{i=1}^{N} q_{i}(t) f_{j}\left(r_{i}\right)
$$

où $f_{j}\left(r_{i}\right)$ est une distribution du potentiel électrique en position $r_{i}$ quand une tension d'1 V est appliquée sur la j-ème électrode tandis que les autres électrodes sont court-circuitées à la masse. Autrement dit, $f_{j}(r)$ est la solution de l'équation de Laplace $\nabla\left[\varepsilon(r) \nabla f_{j}(r)\right]=0$. Cette partie de charge $Q_{j}^{\prime}(t)$ peut être considérée comme un facteur "géométrique », indépendant du temps $t$ et de $\rho$.

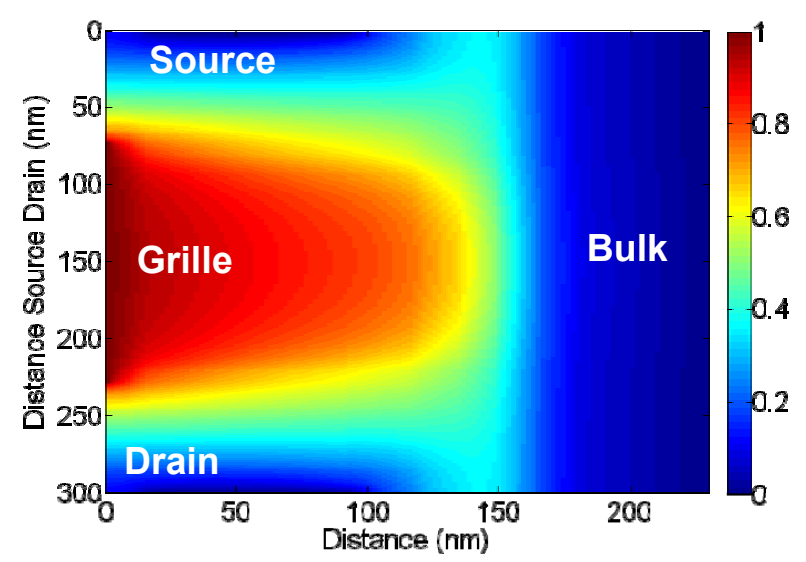

Figure 69 Solution de l'équation de Laplace quand l'électrode de grille est maintenue à la tension de $1 \mathrm{~V}$ tandis que les autres sont reliées à la masse, pour un dispositif de MOS-Bulk avec $L_{G}=200 \mathbf{n m}$ et $\mathbf{T}_{\mathbf{O X}}=\mathbf{8} \mathbf{n m}$

Quant à l'autre contribution, $Q_{j}^{\prime \prime}(t)$ représente la charge induite dans la j-ème électrode par une tension externe $V_{1}(t), \ldots, V_{j}(t), \ldots, V_{M}(t)$ appliquée sur chaque électrode grâce à un effet capacitif, cette partie peut aussi être obtenue par le théorème de Gauss comme :

$$
Q_{j}^{\prime \prime}(t)=\int_{S_{j}} \varepsilon\left(r_{S}\right) \nabla \varphi^{\prime \prime}\left(r_{S}, t\right) d S
$$


où $S_{j}$ est la surface de la j-ème électrode, $d S$ est le vecteur sortant normal à la surface et $r_{S}$ est le vecteur position repéré par rapport à la surface.
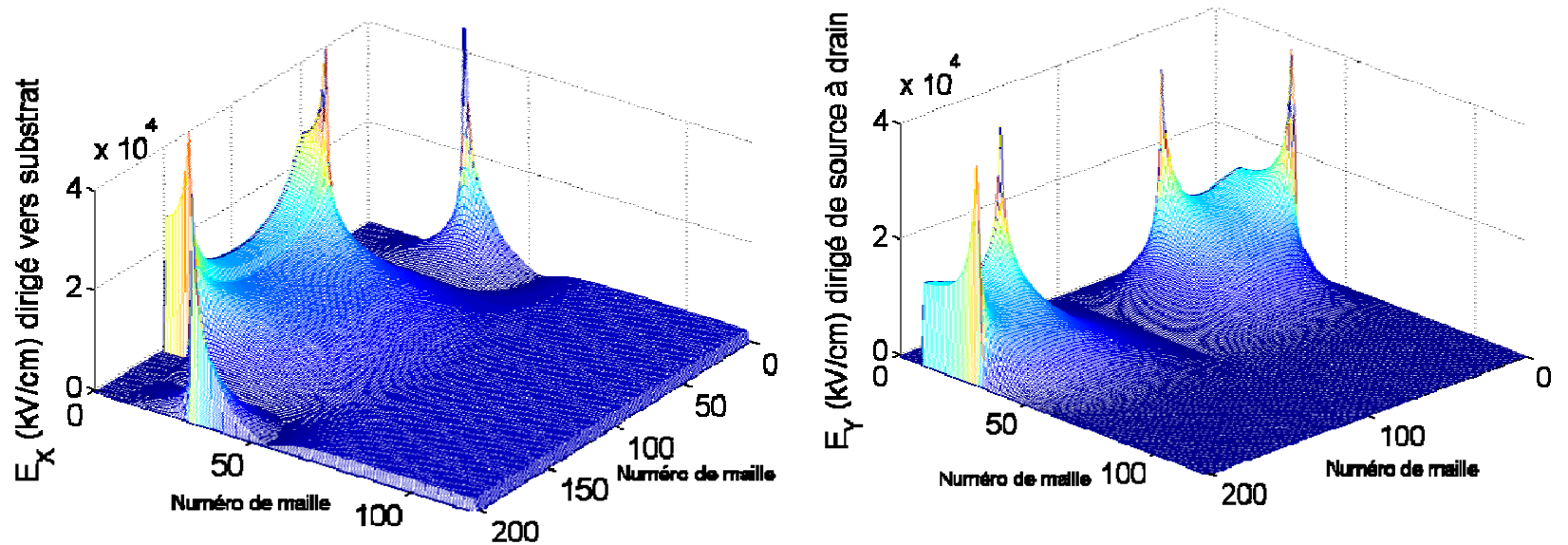

Figure 70 Valeur absolue de la solution $\nabla \varphi^{\prime \prime}\left(r_{S}, t\right)$ en de l'équation (III-7) (champ électrique) selon $\mathrm{X}$ et $\mathrm{Y}$ pour $\varphi_{j}^{\prime \prime}=V_{\text {Grille }}$ et les autres termes associés aux électrodes de Source, Drain et Bulk sont nuls. Ces solutions sont nécessaires pour calculer $Q_{j}^{\prime \prime}(t)$ ainsi que la capacité du couplage $C_{i j}$

Pour calculer le courant total qui traverse l'électrode $j$, nous dérivons l'expression de la charge par rapport au temps, évidemment le courant comporte deux contributions associées respectivement à $Q_{j}^{\prime}(t)$ et à $Q_{j}^{\prime \prime}(t)$, que l'on notera $I_{j}^{\prime}(t)$ et $I_{j}^{\prime \prime}(t)$. La contribution $I_{j}^{\prime}(t)$ est due à $\mathrm{N}$ particules chargées mobiles tandis que les électrodes restent à potentiel constant dans le temps. $I_{j}^{\prime \prime}(t)$ est le courant dû aux potentiels variables dans le temps des électrodes induits au travers des capacité couplées entre électrodes [Nonner2004BJ]. Ce théorème permet de calculer de manière directe le courant de l'électrode $j, I_{j}^{\prime}(t)$, en utilisant les contributions de chaque particules au sein de la structure, à chaque instant par la relation [Kim1991SSE] :

$$
I_{j}^{\prime}(t)=-\sum_{i=1}^{N} q_{i} \cdot v_{i}(t) \nabla f_{i}\left(r_{i}\right)
$$

La simulation Monte Carlo permet de fournir les valeurs de la vitesse et de la position de chaque particule à chaque instant, cette partie de courant peut aisément être évaluée. Toutes les particules au sein du dispositif contribuent au calcul de $I_{j}^{\prime}(t)$, d'où l'augmentation de la précision.

La seconde composante du courant $I_{j}^{\prime \prime}(t)$ n'est pas nulle seulement lorsqu'il existe un couplage entre électrodes quand l'un des potentiels appliqués est modifié brutalement dans le 
temps. $I_{j}^{\prime \prime}(t)$ peut être calculé à partir des composantes de la matrice de capacité de couplage $C_{i j}$ associée aux électrodes du dispositif [Babiker1998IEEE]. Pour cela, on résout initialement l'équation de Laplace en fixant le potentiel de chaque électrode à $0 \mathrm{~V}$ et, ensuite, on résout le potentiel de chaque électrode en incrémentant son potentiel par la tension $\Delta V_{i}$, la capacité du coulage est donnée par [Babiker1998IEEE]:

$$
C_{i j}=\frac{\Delta Q_{j}^{\prime \prime}}{\Delta V_{i}}
$$

Quand on applique un signal échelon de potentiel $\Delta V_{i}$ sur la i-ème électrode, le courant $I_{j}^{\prime \prime}(t)$ de nature capacitif circule dans la j-ème électrode seulement durant le premier pas de temps $\Delta t$ juste après l'application d'un signal [Babiker1998IEEE] :

$$
I_{j}^{\prime \prime}(t)=C_{i j} \frac{\Delta V_{i}(t)}{\Delta t}
$$

Durant le reste du temps, le courant de déplacement est relié aux charges induites de l'électrode par les particules mobiles et est inclus dans le courant $I_{j}^{\prime}(t)$ donné par l'équation (III-8).

\section{Calibration du simulateur MONACO 3Dk}

\section{2-1 Structures de bandes et interactions prises en compte}

Dans MONACO, nous décrivons la bande de conduction des matériaux III-V comme $\mathrm{In}_{0,53} \mathrm{Ga}_{0,47} \mathrm{As}$ ou $\mathrm{In}_{0,52} \mathrm{Al}_{0,48} \mathrm{As}$ par un modèle à trois types de vallées : (i) la vallée $\Gamma$ avec un minimum $\vec{k}=0$; (ii) 4 vallées L équivalentes avec des minima aux extrémités de la première zone de Brillouin dans les directions [111]; (iii) 3 vallées $\mathrm{X}$ équivalentes avec des minima aux extrémités de la première zone de Brillouin dans les directions [100]. Ces vallées sont décrites dans l'approximation de la masse effective comme sphériques et non-paraboliques, $\Gamma$ est celle la plus basse en énergie. Dans un premier temps, le principe d'exclusion de Pauli n'est pas pris en compte.

Les imperfections du cristal semi-conducteur massif ou de la géométrie du dispositif, telles que les vibrations des atomes, la présence d'impuretés et la rugosité d'interface, perturbent la trajectoire des porteurs. Ces perturbations, appelées interactions, modifient l'état du porteur qui les subit. Dans une approche MC, le calcul des probabilités d'interaction est crucial, puisque c'est grâce à ces dernières qu'il nous est possible de décrire la physique des phénomènes microscopiques qui régissent le transport électronique. Les mécanismes 
d'interaction inclus dans MONACO sont: les collisions avec les impuretés ionisées, les transitions dues à l'absorption et à l'émission des phonons optiques polaires et non polaires, les collisions avec des phonons acoustiques, les interactions inter-vallées, le désordre d'alliage et la rugosité de surface. Les fréquences d'interaction associées dans le cas d'un gaz d'électrons à 3 dimensions dans l'espace réciproque sont données par la règle d'or de Fermi [Cohen-Tannoudji1973] (des illustrations quantitatives sont données en annexe D pour $\left.\mathrm{In}_{0,53} \mathrm{Ga}_{0,47} \mathrm{As}\right)$.

\section{2-2 Calibration du modèle de transport pour un matériau massif à champ constant}

Les paramètres utilisés pour décrire le modèle microscopique de transport électronique doivent être calibrées pour reproduire les données expérimentales disponibles. Dans un premier temps, nous avons effectué ce travail pour le matériau massif utilisé pour le canal, avec des valeurs de mobilité en fonction de la concentration d'impuretés ionisées en régime statique à $300 \mathrm{~K}$. La densité d'impuretés ionisées est prise égale à la densité électronique. En simulation, on applique un champ électrique fixe selon une direction sur un barreau d' $\operatorname{In}_{0,53} \mathrm{Ga}_{0,47} \mathrm{As}$, et on s'intéresse à la vitesse moyenne des porteurs une fois le régime d'équilibre atteint.

A l'équilibre thermodynamique, les électrons se trouvent dans la vallée $\Gamma$, c'est-à-dire au niveau d'énergie minimal. Quand on applique un faible champ électrique, les porteurs restent dans la vallée $\Gamma$. Si l'on augmente la valeur du champ électrique, un certain nombre de porteurs gagne assez d'énergie pour passer dans les vallées supérieures ( $\mathrm{L}$ et $\mathrm{X}$ ) ayant une grande masse effective. La variation de vitesse commence à diminuer. La masse effective résultante augmente jusqu'à rejoindre la valeur de la masse effective de la vallée L. A très faible champ électrique, la vitesse croit linéairement, on est dans le régime ohmique ou de mobilité. Les porteurs restent alors dans la même vallée avec une vitesse de dérive $v=\mu_{0} E$, où $\mu_{0}$ est la mobilité ohmique dans la région linéaire. En augmentant le champ, la vitesse devient non-linéaire, $v(E)=\mu(E) E$, c'est-à-dire que la mobilité dépend aussi du champ électrique. Dans ce régime, la mobilité différentielle $d v / d E$ passe par des valeurs négatives quand les vallées L se remplissent majoritairement. Puis à très fort champ la vitesse sature quand tout gain supplémentaire d'énergie cinétique pour les porteurs est quasi immédiatement perdu par émission de phonons optiques polaires. Sur la Fig. 71, les résultats $v(E)$ dans $\mathrm{In}_{0,53} \mathrm{Ga}_{0,47} \mathrm{As}$ à température ambiante et pour différentes concentrations d'impuretés ionisées 
sont comparés à plusieurs données (expérimentales et issues d'autres simulations) de la littérature.

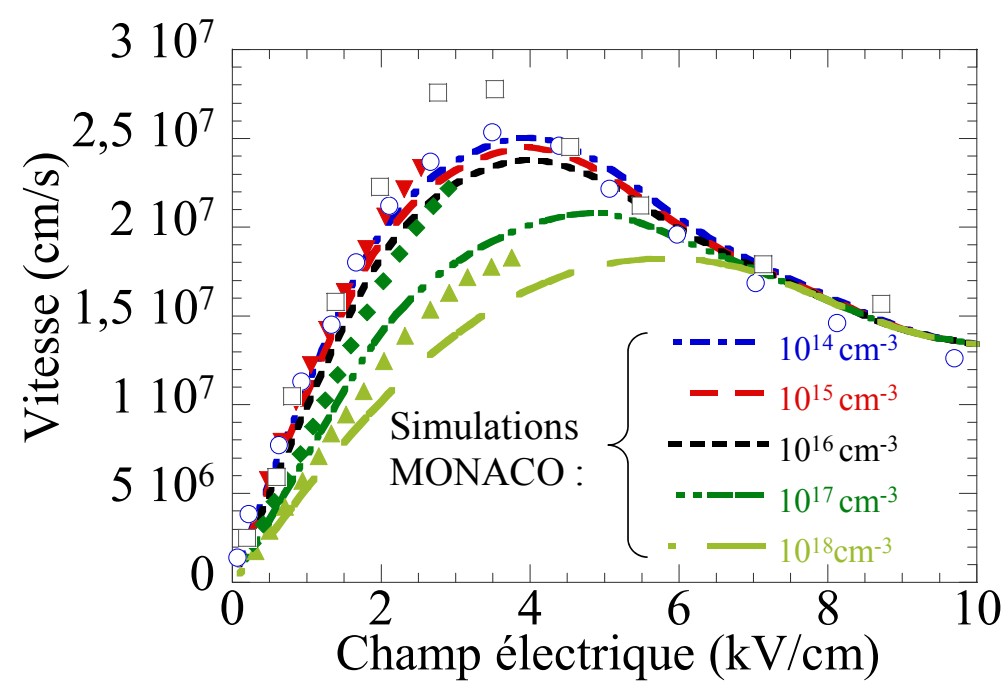

Figure 71 Vitesse de dérive en fonction du champ électrique dans $\operatorname{In}_{0,53} \mathrm{Ga}_{0,47} \mathrm{As}$ à $300 \mathrm{~K}$ pour diverses concentrations $N_{D}$ en impuretés donneuses : $10^{14}, 10^{15}, 10^{16}, 10^{17}, 10^{18} \mathrm{~cm}^{-3}$. Les traits représentent nos résultats de simulation MC. Les symboles pleins représentent les valeurs expérimentales obtenues par [Hasse1999SSE] pour $N_{D}=10^{15}, 10^{17}, 10^{18} \mathrm{~cm}^{-3}$. Les ronds creux en bleu correspondent à des résultats de simulations MC avec $N_{D}=10^{14} \mathrm{~cm}^{-3}$ faite par [Thobel1990APL]. Les carrés creux en noir sont des données expérimentales pour $N_{D}=10^{14} \mathrm{~cm}^{-3}$ rapportées par Balynas [Balynas1990APL].

Les valeurs de vitesse pour différents $\mathrm{N}_{D}$ issues de MONACO sont en bon accord avec les mesures et d'autres simulations MC, surtout en régime ohmique. La transition liée au transfert inter-vallée n'est pas encore parfaitement modélisée, mais cela ne nous empêche pas d'estimer la valeur de mobilité à faible champ en régime ohmique ( $c f$. Fig. 72). Nous pouvons noter que nos résultats sont très proches des valeurs expérimentales publiées par Chin et al. [Chin1995MJ] pour un faible niveau de dopage $\left(\leq 10^{17} \mathrm{~cm}^{-3}\right)$. Il y a également un bon accord par rapport aux calculs de Pearsall [Pearsall1982] pour un niveau de dopage jusqu'à à $10^{18} \mathrm{~cm}^{-3}$. La simulation Monte Carlo mise en œuvre semble bien pertinente pour reproduire les propriétés de transport électronique dans $\operatorname{In}_{0,53} \mathrm{Ga}_{0,47} \mathrm{As}$ massif. 


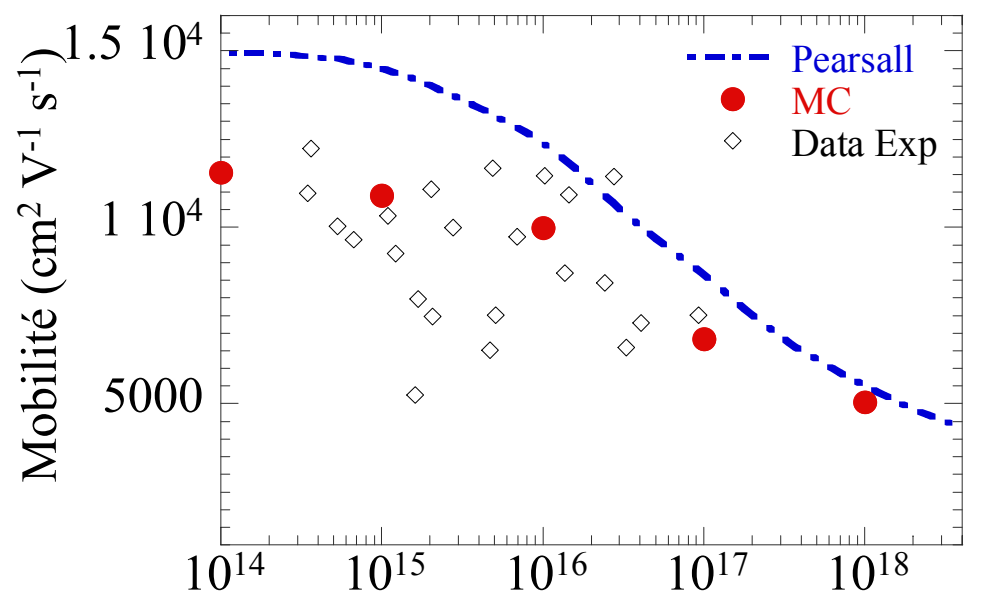

Concentration des impuretés ionisées $\left(\mathrm{cm}^{3}\right)$

Figure 72 Mobilité à faible champ en fonction de la concentration en impuretés donneuses pour $\mathrm{In}_{0,53} \mathrm{Ga}_{0,47} \mathrm{As}$ à $300 \mathrm{~K}$. Les losanges creux en noir et le trait en bleu représentent plusieurs résultats expérimentaux [Chin1995MJ] et de calculs de la littérature [Pearsall1982].

\section{2-3 Mobilité effective dans une couche d'inversion}

Il existe certains travaux très remarqués consacrés à l'étude expérimentale du transport dans une couche d'inversion d' $\operatorname{In}_{0.53} \mathrm{Ga}_{0.47}$ As pour un canal en surface et enterré par plusieurs groupes de recherche académique et industrielle: Univ. Purdue [Xuan2007IEDM], Univ. Texas [Xue2011APL], Univ. Glasgow [Bentley2011IEEE] et IBM [Sun2008DRC] et Intel [Goel2008IEDM]. Mais des questions restent encore ouvertes y compris dans l'étude théorique la plus sophistiquée [O'Regan2010JAP]. D'après les travaux de Sabnis et Clemens [Sabnis1979IEDM], ce type de transport est généralement caractérisé par une analyse de la mobilité des porteurs en fonction de la moyenne du champ électrique perpendiculaire pondéré par la concentration de porteurs, que l'on appelle champ effectif [Saint-Martin2005thèse] :

$$
E_{\text {eff }}=\int_{\substack{\text { couche } \\ \text { inversion }}} n(z) \cdot E_{z}(z) d z / \int_{\substack{\text { couche } \\ \text { inversion }}} n(z) d z
$$

où $E_{z}$ est la composante du champ électrique perpendiculaire au canal. Par résolution de l'équation de Poisson dans la couche d'inversion $\left(0<z<z_{i}\right)$, on a [Sonnet2011APL] :

$$
E_{e f f}(z)=\frac{q N_{D E P}}{\varepsilon_{\text {InGaAs }}}+\frac{q}{\varepsilon_{\text {InGaAs }}} \int_{z}^{z_{i}} n_{I N V}(u) d u
$$

où $n_{I N V}$ et $N_{D E P}$ est respectivement la densité de charges d'inversion et de déplétion par unité de volume et de surface. $\varepsilon_{\text {InGaAs }}$ est la constante diélectrique d'InGaAs. Le champ électrique 
moyen qui régit le transport de la charge d'inversion totale $N_{I N V}$ (par unité de surface) peut être décrit par [Sabnis1979IEDM] et [Cristoloveanu2010IEEE]:

$$
E_{e f f}=\frac{q \cdot\left(N_{D E P}+\eta N_{I N V}\right)}{\varepsilon_{I n G a A s}}
$$

où $\eta$ est un paramètre empirique qui dépend seulement de la structure de bande du semiconducteur et de la distribution de porteur dans l'espace géométrique et en énergie [Cristoloveanu2010IEEE]. A priori, il ne dépend pas de la concentration du dopage ni de la polarisation du substrat, ni encore du diélectrique qui sépare le canal de la grille. Pour des MOSFET massifs sur $\mathrm{Si}, \eta$ est en général égal à 0,5 . Avec cette valeur, la mobilité électronique suit en fonction du champ effectif une loi « universelle », indépendante du niveau de dopage du substrat [Sun1980IEEE] et [Takagi1994IEEE]. Pour des canaux en $\mathrm{In}_{0,53} \mathrm{Ga}_{0,47} \mathrm{As}$, on doit plutôt considérer $\eta=0,25 \pm 0,05$ [Sonnet2011APL]. Cette faible valeur de $\eta$ par rapport au cas du Si est liée à la large distribution de charge d'inversion dans $\mathrm{In}_{0,53} \mathrm{Ga}_{0,47} \mathrm{As}$.

Pour calculer la mobilité effective en Monte Carlo, le couplage avec l'équation de Poisson est désactivé. L'équation de Poisson 1D est résolue dans la capacité MOS pour une polarisation de la grille donnée avant simulation MC du transport, fournissant ainsi le profil de champ vertical, la distribution de la charge d'inversion ainsi que le champ effectif. La vitesse moyenne de porteur est ensuite évaluée par MC en fonction d'un champ uniforme dans le plan de l'interface. Cette étude est faite en 3Dk, c'est-à-dire dans l'espace réciproque 3D sans effet de quantification. En principe, le taux d'interaction avec les impuretés ionisées varie en fonction du champ effectif, l'effet d'écrantage dépendant de la densité locale des porteurs [Aubry-Fortuna2005SSE]. La rugosité de surface est traitée par une technique largement utilisée pour les gaz d'électrons 3D, c'est-à-dire par une combinaison empirique de diffusions et de réflexions spéculaires à l'interface du canal avec l'oxyde. Ici, la fraction de réflexions spéculaires est fixée à $18 \%$ selon le choix explicité auparavant. Les résultats que nous avons obtenus sont reportés sur la Fig. 73 : 


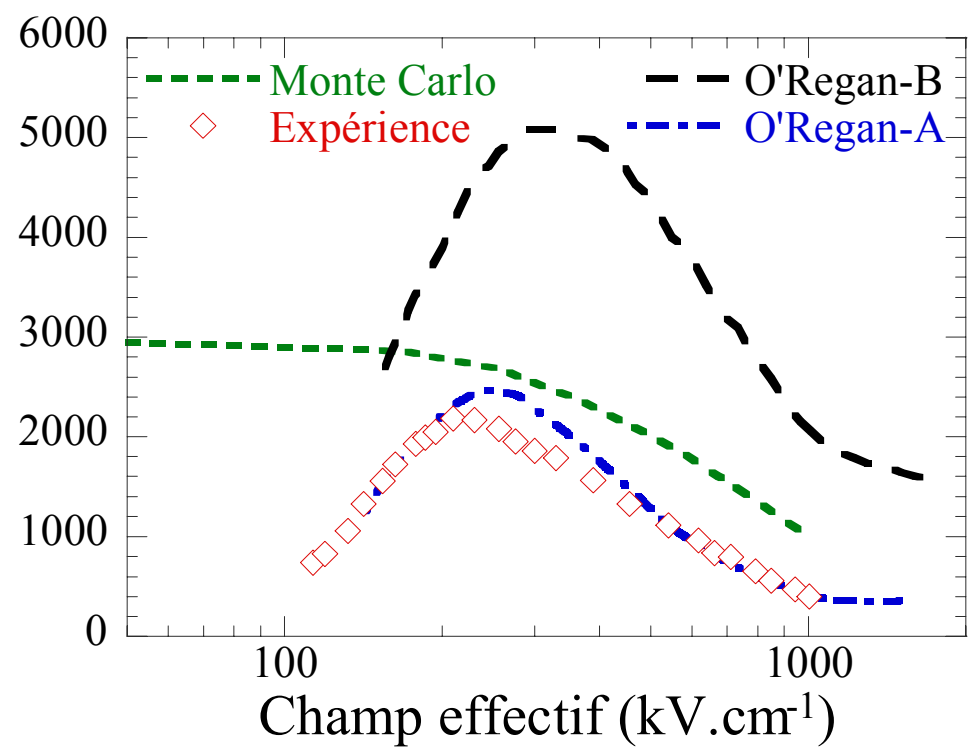

Figure 73 Comparaison des mobilités calculées par MC avec des données expérimentales [Xuan2007IEDM], pour une structure bulk $\mathrm{Al}_{2} \mathrm{O}_{3} / \mathrm{In}_{0.53} \mathrm{Ga}_{0.47} \mathrm{As}$ avec un dopage accepteur $\mathrm{N}_{\mathrm{A}}=10^{17} \mathrm{~cm}^{-3}$. Des résultats de calculs en 2Dk sont également présentés [O'Regan2010JAP]

Les simulations MC sont effectuées pour une structure parfaite, sans prise en compte des pièges d'interface. Le modèle d'écrantage variable n'est pas incorporé. Cela explique l'écart entre les résultats $\mathrm{MC}$ et les tendances expérimentales en régime de faible champ effectif. L'accord est en revanche bon sous champ effectif modéré ou fort, zones dans lesquelles les phonons et la rugosité d'interface prennent des rôles de plus en plus importants. Des calculs en gaz 2D de l'équipe de M. V. Fischetti par la méthode de Kubo-Greenwood sont également présentés [O'Regan2010JAP]. Ils prennent en compte les effets liés aux défauts d'interface tels que (i) les interactions coulombiennes avec les charges des pièges d'interface qui écrantent diélectriquement les porteurs [Fischetti1993PRB] et (ii) la rugosité d'interface caractérisée par une longueur d'auto-corrélation $\Lambda$ et une amplitude $\Delta$ [Ando1982RMP] et [Jin2007IEEE]. Les résultats 'O'Regan-B' (resp. 'O'Regan-A') sont calculés pour une densité de pièges de $5 \times 10^{12} \mathrm{~cm}^{-2}, \Lambda=1,2 \mathrm{~nm}$ et $\Delta=0,48 \mathrm{~nm}$ (resp. $10^{13} \mathrm{~cm}^{-2}, \Lambda=1,2 \mathrm{~nm}$ et $\Delta=1,2 \mathrm{~nm}$ ). Ils permettent de mettre en évidence l'importance des défauts extrinsèques dans les technologies à base de III-V, vis-à-vis des phénomènes intrinsèques au matériau (phonons, impuretés, effets d'alliage et piézoélectriques). A faible champ effectif, le transport est dominé par l'interaction coulombienne avec les charges d'interface de forte densité $\left(\geq 10^{13} \mathrm{~cm}^{-2}\right)$ tandis qu'en fort champ la mobilité est dégradée par une rugosité avec une grande longueur d'auto-corrélation et une amplitude significative ( $\geq$ 
$1 \mathrm{~nm}$ ). Notre étude $\mathrm{MC}$ en 3Dk indiquera donc les performances intrinsèques que l'on peut attendre de dispositifs quasi-idéaux, c'est-à-dire avec une technologie parvenue au niveau de maturité du système $\mathrm{SiO}_{2} / \mathrm{Si}$.

\section{2-4 Test sur un transistor de référence, résistances d'accès}

Nous considérons d'abord une structure de référence, un transistor de type HEMT avec une grille en forme de T. Ce dispositif est fabriqué par le partenaire industriel OMMIC du projet MOS35. L'hétérostructure mise en œuvre, schématisée sur la Fig. 74, est basée sur un canal pseudomorphique en InGaAs à fort taux d'indium $(80 \%)$ sur InP. En partant du substrat InP semi-isolant, l'empilement épitaxié comprend une couche tampon de $100 \mathrm{~nm}$ en $\mathrm{In}_{0.52} \mathrm{Al}_{0.48} \mathrm{As}$, un sous canal de $7,5 \mathrm{~nm}$ en $\operatorname{In}_{0,53} \mathrm{Ga}_{0,47} \mathrm{As}$, un canal principal de $7,5 \mathrm{~nm}$ en $\mathrm{In}_{0.8} \mathrm{Ga}_{0.2} \mathrm{As}$, un espaceur de $3 \mathrm{~nm}$ en $\mathrm{In}_{0,52} \mathrm{Al}_{0,48} \mathrm{As}$ non intentionnellement dopé (nid), un plan de dopage $\delta$ en silicium de $3 \mathrm{~nm}$ avec une densité surfacique de $10^{13} \mathrm{~cm}^{-2}$, une barrière Schottky en $\operatorname{In}_{0,52} \mathrm{Al}_{0,48} \mathrm{As}$ (nid) de $10 \mathrm{~nm}$ et une couche de contact (cap layer) en InGaAs fortement dopée de $26 \mathrm{~nm}$. On note également la présence de la passivation à l'intérieur de la zone de « recess 》 de grille.

Le dispositif simulé correspond à la zone encadrée par le rectangle en tirets rouges. Les contacts ohmiques de source et drain sont positionnés verticalement aux bords de la structure. Les résistances séries seront ainsi exclues de la zone de simulation. Elles sont incluses séparément en cours de simulation dans une phase de traitement externe [Babiker1996IEEE]. L'effet extrinsèque dû aux résistances séries peut être présenté comme une correction à l'ensemble original des données de simulation Monte Carlo. On peut définir d'après la correction une nouvelle série des données I-V du dispositif comme : $\left(V_{D}^{\prime}, V_{G}^{\prime}, I_{D}\right)$, où $V_{D}^{\prime}$ et $V_{G}^{\prime}$ sont les tensions nécessaires pour maintenir le même courant de drain $I_{D}$ quand les résistances séries de la source $R_{S}$ et du drain $R_{D}$ sont prises en compte. Les relations avec les tensions originales $V_{D}$ et $V_{G}$ sont donc données par:

$$
\begin{aligned}
& V_{D}^{\prime}=V_{D}+I_{D} \times\left(R_{S}+R_{D}\right) \\
& V_{G}^{\prime}=V_{G}+I_{D} \times R_{S}
\end{aligned}
$$

Ces résistances incluent les résistances de contact et celles associées aux équipements des mesures, a priori indépendantes des conditions de polarisation. La caractéristique I-V simulée en prenant compte les résistances séries externes [Bournel2007SSE] peut être comparée avec celle expérimentale du dispositif réellement fabriqué. 


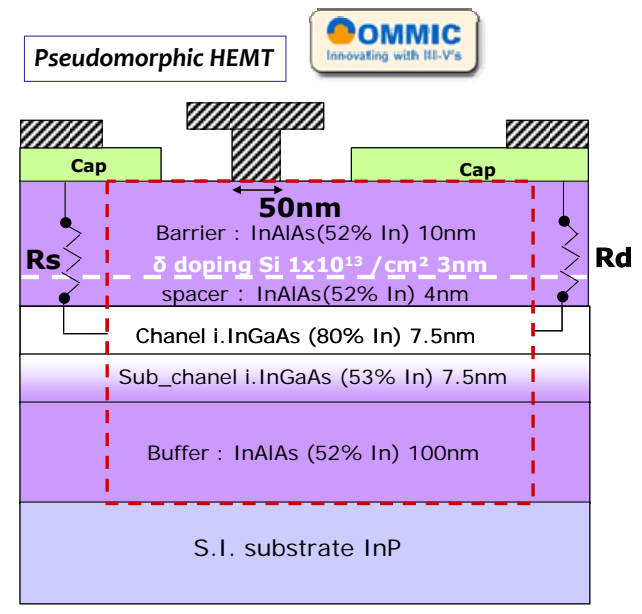

Figure 74 Structure de référence HEMT pseudomorphique fabriquée par OMMIC. La longueur de grille est $50 \mathrm{~nm}$. La zone encadrée par le rectangle en tirets rouges est celle effectivement simulée. L'effet de résistances série $\mathbf{R}_{S D}$ est ensuite ajouté [Babiker1996IEEE] et [Bournel2007SSE].

Pour résoudre l'équation de Poisson toutes les $0,1 \mathrm{fs}$, on définit des mailles de dimension variant entre 5 et $0,5 \mathrm{~nm}$, en fonction de la densité de charges prévue et des possibles fortes variations de potentiel. Les calculs sont faits à $300 \mathrm{~K}$, avec 50000 à 100000 particules simulées. Aux contacts, le potentiel est fixé par rapport à celui appliqué (conditions aux limites de type Dirichlet) tandis qu'il reste flottant sur les autres bords du dispositif où l'on considère les conditions de Neumann (composante normale du champ électrique nulle). Les électrodes de source et drain sont supposées ohmiques, elles absorbent le flux des électrons collectées et injectent des porteurs selon une distribution Maxwellienne de vitesse (de forme gaussienne déplacée, une interprétation explicite se trouve dans l'annexe) pour maintenir la neutralité électrique dans les mailles adjacentes du contact. Le contact de grille est de type Schottky, absorbant les particules, mais sans en injecter. La résistance du contact de Schottky est supposée d'être infiniment grande et le courant statique de la grille est ignoré. Le calcul du courant sera normalisé par rapport à la profondeur $\mathrm{W}$ du dispositif qui est supposée suffisamment grande. Enfin, la chute de tension due aux résistances séries est mise à jour à chaque pas sur le temps durant la simulation.

Les caractéristiques obtenues sont présentées sur la Fig. 75. Les résistances séries du côté source $R_{S}$ et drain $R_{D}$ sont respectivement ajustées à $0,05 \Omega . m m$ et $0,13 \Omega . m m$ et la barrière Schottky de grille de $1,08 \mathrm{eV}$ par confrontation par rapport à l'expérience. Il est à noter que l'ionisation par impact n'est pas prise en compte dans cette version du simulateur, aucun effet kink n'étant observée aux faibles valeurs de $\mathrm{V}_{\mathrm{DS}}$ considérées $(\leq 0,6 \mathrm{~V})$. 

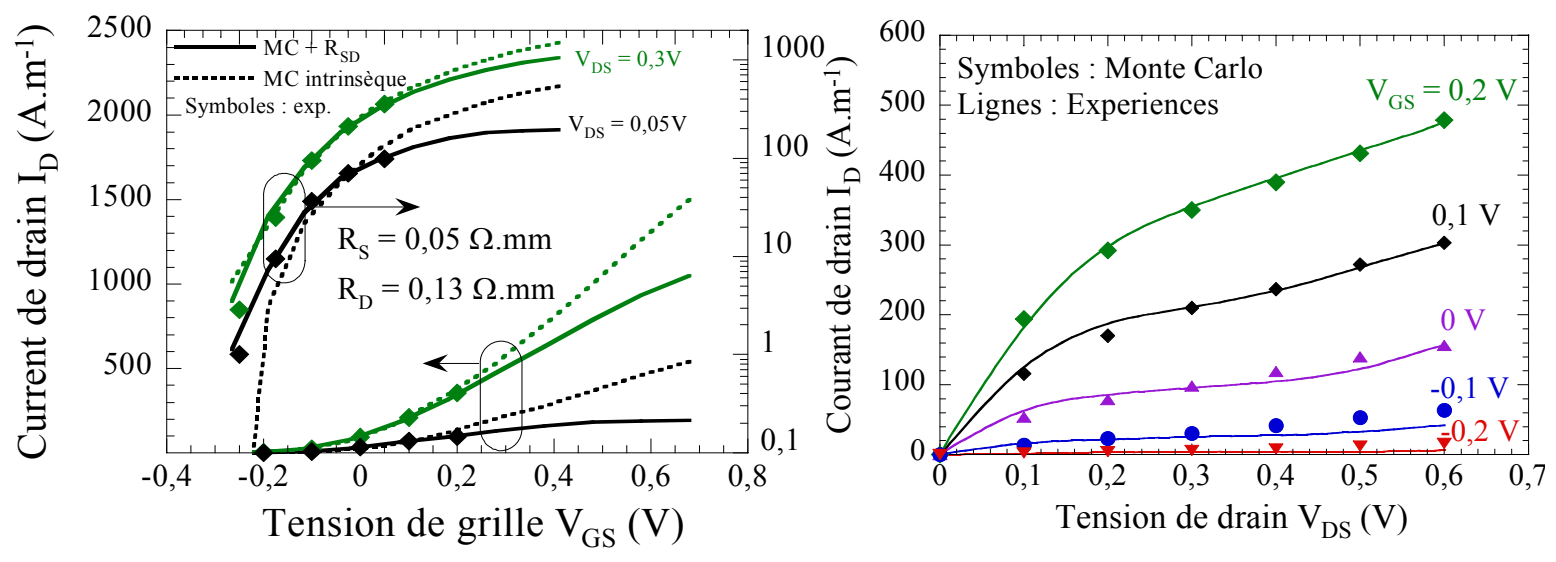

Figure 75 Gauche : caractéristiques de transfert $I_{D}-V_{G S}$ pour deux valeurs de $V_{D S}$ avec et sans résistances séries, comparaison avec les données expérimentales d'un HEMT de $70 \mathrm{~nm}$. Droite : caractéristiques de sortie $I_{D}-V_{D S}$ pour différents $V_{G S}$ avec prise en compte des résistances séries, comparaison avec les données expérimentales.

\section{Comportement statique}

\section{3-1 Caractéristiques I-V et microscopiques}

D'après les procédures de calibration sur les matériaux massifs, sur une couche d'inversion et sur un dispositif réellement fabriqué, nous pouvons effectuer une étude électrique en régime statique pour les quatre structures de type MOSFET définies au chapitre II (schémas en Fig. 30) : structure MOS-Bulk de référence, MOS-ThinBody conçue pour réduire les effets de canal court tout en améliorant la propriété du transport, MOS-HEMT pour réduire l'impact de la rugosité d'interface et MOS-COMB qui combine les deux précédentes. Sur la Fig. 76 sont tracées les caractéristiques de transfert $\mathrm{I}_{\mathrm{D}}-\mathrm{V}_{\mathrm{GS}}$ intrinsèques des dispositifs et les transconductances associées pour $\mathrm{V}_{\mathrm{DS}}=0,2 \mathrm{~V}$. Nous constatons que les courants de drain et les transconductances sont augmentés pour les trois structures alternatives par rapport à ceux du MOS-Bulk, grâce à une amélioration de la mobilité effective dans le canal. Comme nous le verrons dans le paragraphe suivant, cette augmentation est bien corrélée à la proportion d'électrons balistiques traversant le canal, elle permet d'atteindre de grandes valeurs de transconductance $G_{m}$ même pour une faible tension $\mathrm{V}_{\mathrm{DS}}$. Parmi ces structures alternatives, la performance du MOS-ThinBody est comme attendu dégradée par l'interaction de rugosité d'interface oxyde/canal $\left(G_{m_{-} M A X}=2,5 \mathrm{mS} / \mu \mathrm{m} \mathrm{V}_{\mathrm{DS}}=0,2 \mathrm{~V}\right.$ et $\mathrm{I}_{\mathrm{D}}=$ $480 \mathrm{~mA} / \mathrm{mm}$ à $\left.\mathrm{V}_{\mathrm{DS}}=\mathrm{V}_{\mathrm{G}}-\mathrm{V}_{\mathrm{T}}=0,2 \mathrm{~V}\right)$. Cette interaction est rendue négligeable dans le MOSHEMT par l'insertion d'un espaceur. Mais les propriétés de transport sont dégradées par 
l'interaction avec les impuretés ionisées du canal $\left(G_{m_{-} M A X}=3 \mathrm{mS} / \mu \mathrm{m}\right.$ à $\mathrm{V}_{\mathrm{DS}}=0,2 \mathrm{~V}$ et $\mathrm{I}_{\mathrm{D}}=595 \mathrm{~mA} / \mathrm{mm}$ à $\left.\mathrm{V}_{\mathrm{DS}}=\mathrm{V}_{\mathrm{G}}-\mathrm{V}_{\mathrm{T}}=0,2 \mathrm{~V}\right)$.
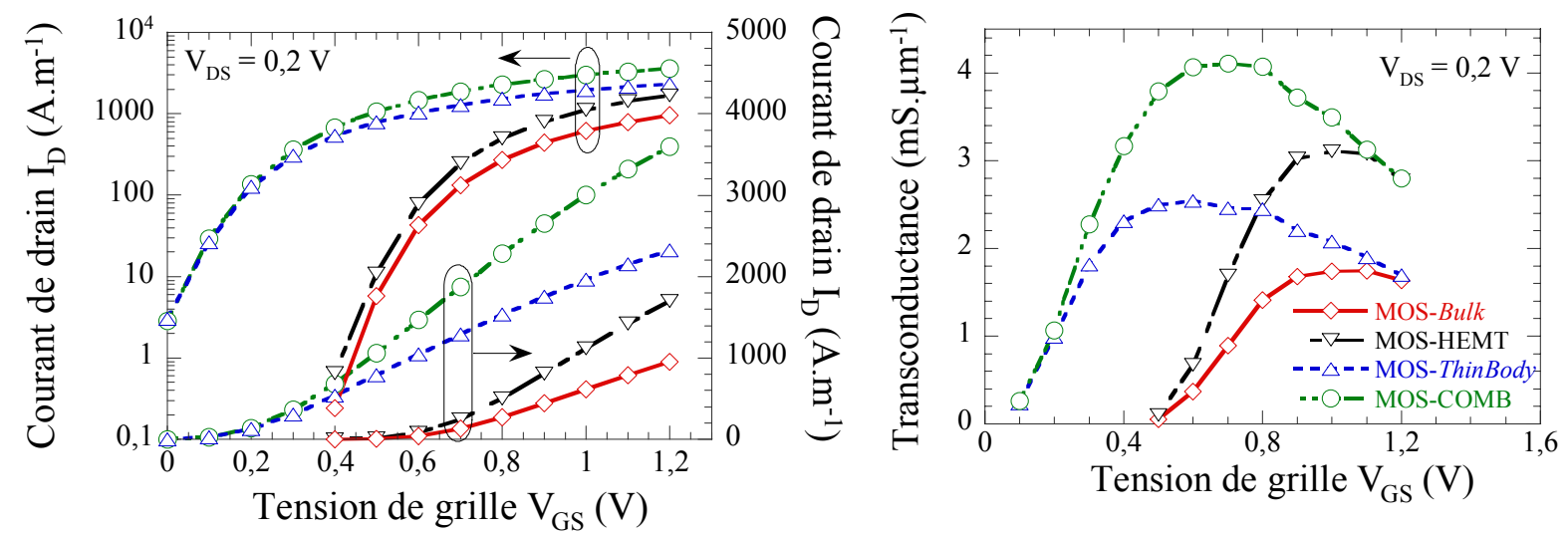

Figure 76 Gauche : caractéristiques de transfert $I_{D}-V_{G S}$ intrinsèques à $V_{D S}=0,2 \mathrm{~V}$ des quatre structures proposées de type MOSFET à base d' $\mathbf{I n}_{0.53} \mathbf{G a}_{0.47}$ As. Droite : transconductances associées
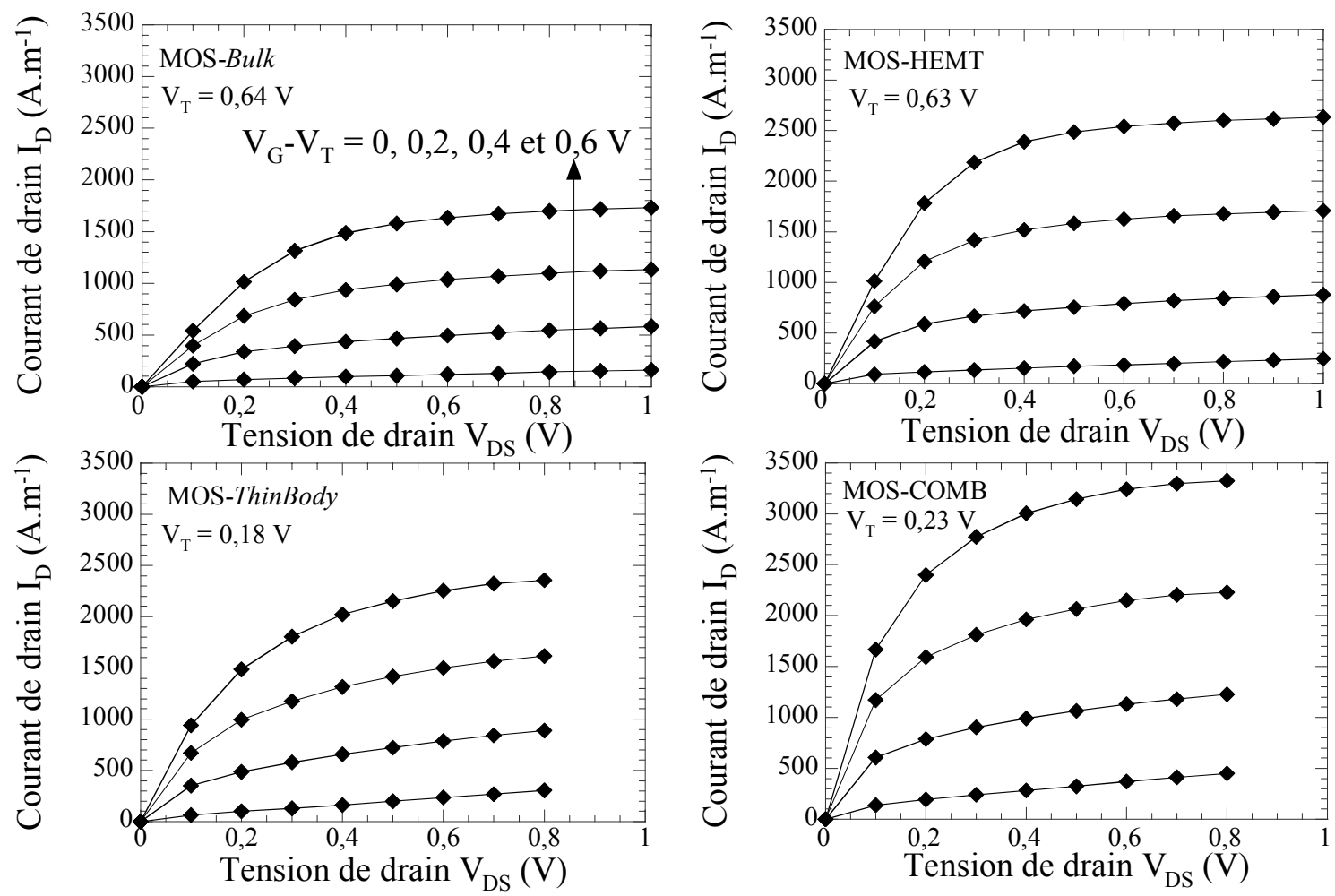

Figure 77 Caractéristiques de sortie $I_{D}-V_{D S}$ intrinsèques pour différents $V_{G s}-V_{T}\left(V_{T}\right.$ est déterminé par une méthode d'extrapolation sur les caractéristiques $\left.I_{D}-V_{G S}\right)$.

Les meilleures performances en courant et en transconductance sont obtenues dans la structure MOS-COMB $\left(G_{m_{-} M A X}=4,1 \mathrm{mS} / \mu \mathrm{m}\right.$ à $\mathrm{V}_{\mathrm{DS}}=0,2 \mathrm{~V}$ et $\mathrm{I}_{\mathrm{D}}=778 \mathrm{~mA} / \mathrm{mm}$ à $\mathrm{V}_{\mathrm{DS}}=\mathrm{V}_{\mathrm{G}^{-}}$ $\mathrm{V}_{\mathrm{T}}=0,2 \mathrm{~V}$ ), grâce à la combinaison des idées précédentes. Sur les caractéristiques de sortie 
$\mathrm{I}_{\mathrm{D}}-\mathrm{V}_{\mathrm{DS}}$ de la Fig. 77, on constate toutefois que la conductance $G_{d}$ est augmentée dans les structures alternatives en régime de source de courant, la résistance ohmique du canal $R_{\text {on_lin }}$ a été diminuée dans les structures alternatives en régime linéaire. Souvent une augmentation de la tension de grille conduit le dispositif d'un régime saturé vers un régime linéaire. Ici, pour une tension $\mathrm{V}_{\mathrm{GS}}-\mathrm{V}_{\mathrm{T}}$ allant jusqu'à $0,6 \mathrm{~V}$, la tension de drain en régime saturé $V_{D S \_S a t}$ est environ égale à $0,4-0,5 \mathrm{~V}$. En outre, au régime ohmique (faible $\mathrm{V}_{\mathrm{DS}} \leq 0,2 \mathrm{~V}$ ), la conductance équivalente dite 'petits signaux', donnée par $\partial I_{D} / \partial V_{D S}$ [Skotnicki2003], risque d'être amplifiée dans les structures alternatives surtout dans la structure MOS-COMB, cela dégrade le gain intrinsèque en tension $G_{m} / G_{d}$ voire celui en puissance, qui conditionne les performances en fréquence. Dans ce cas, l'augmentation de $G_{m}$ doit être suffisamment grande pour permettre de compenser celle de la conductance de sortie. Nous étudierons quantitativement ces dernières dans le paragraphe suivant sur la réponse indicielle des composants. On note que comme les effets quantiques et les interactions inter-sous bande ne sont pas pris en compte, la survitesse ( $c f$. Fig. 79) et la transconductance ( $c f$. Fig. 76) peuvent être surestimées d'environ 15\% [Babiker1998IEEE].

Pour ce qui est des spécifications de faible consommation, on cherche une faible plage de tension de grille avec une tension de seuil $\mathrm{V}_{\mathrm{T}}$ optimisée par rapport à celle d'alimentation $\mathrm{V}_{\mathrm{DD}}$ [Chau2005IEEE], telle que $\left(V_{G}-V_{T}\right)_{\text {Optimisé }}=\alpha \cdot V_{D D}$, où $\alpha$ est souvent supérieur à 0,5 pour assurer une valeur satisfaisante du courant $\mathrm{I}_{\mathrm{ON}}$ à l'état $\mathrm{ON}$. Un métal de grille approprié doit être choisi de manière à ajuster $\mathrm{V}_{\mathrm{T}}$ proche de zéro. Le maximum de transconductance doit être plus grand possible pour assurer une bonne performance fréquentielle à une faible tension $\mathrm{V}_{\mathrm{DS}}$ donnée. Sur l'autre partie de la plage de tension, c'est-à-dire $(1-\alpha) \cdot V_{D D}$, il s'agit d'optimiser le contrôle de charge pour assurer l'immunité contre l'effet de canal court. Sur la Fig. 76, on observe que $\mathrm{V}_{\mathrm{T}}$ est augmenté en raison du dopage de la zone active pour les structures MOS-Bulk et MOS-HEMT par rapport à MOS-ThinBody et MOS-COMB. A $\mathrm{V}_{\mathrm{DS}}=0,2 \mathrm{~V}$, on obtient une pente $\mathrm{SS}$ de $80 \mathrm{mV} /$ décade dans les deux premières architectures contre $105 \mathrm{mV} /$ décade dans les deux autres structures à canal nid (nid: non intentionnellement dopé). Notons que le confinement quantique n'est pas pris en compte dans ces simulations $\mathrm{MC}$, son influence devrait être faible pour les structures à canal nid, mais pas pour les niveaux de dopage plus grand. La quantification de charge risque de dégrader la valeur de SS par rapport au calcul classique, comme démontré par nos analyses Poisson/Schrödinger du chapitre II. 


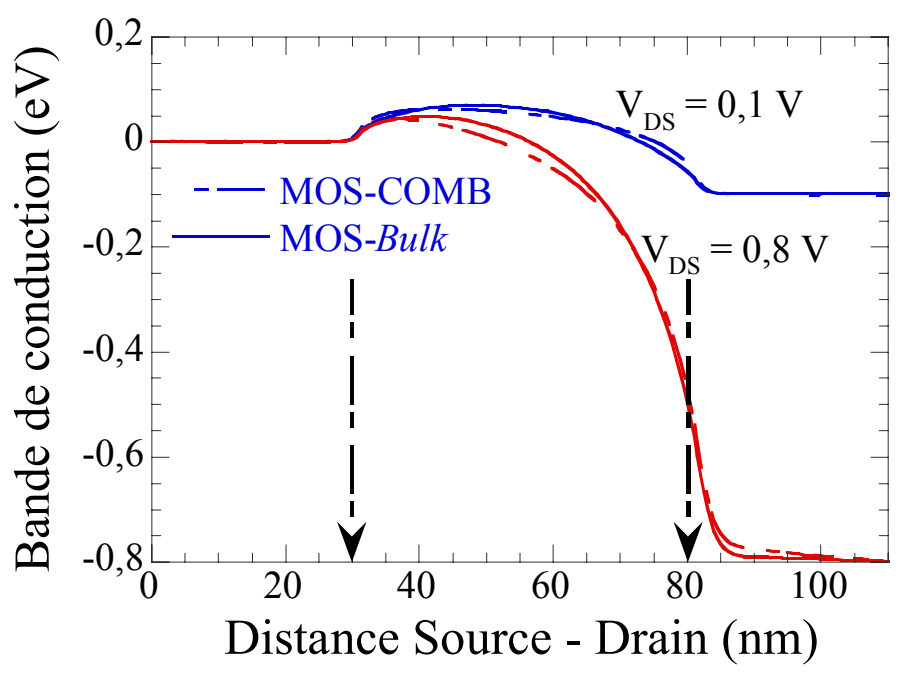

Figure 78 Profil de la bande de conduction (à $1 \mathbf{~ n m}$ au-dessous de l'oxyde) en fonction de la distance le long de l'axe source drain à $V_{G S}-V_{T}=0,6 \mathrm{~V}$ (canal dopé) et $0,2 \mathrm{~V}$ (canal nid), $V_{D S}=0,1 \mathrm{~V}$ et $0,8 \mathrm{~V}$ pour les MOS-Bulk et MOS-COMB. Les flèches verticales indiquent les limites du canal. MOS-HEMT et MOSThinBody présentent des profils en énergie très similaires aux cas respectifs de MOS-Bulk et MOS-COMB.

Le comportement face aux effets de canal court peut également être observé sur les profils de bande de conduction à $\mathrm{V}_{\mathrm{GS}}-\mathrm{V}_{\mathrm{T}}$ fixé pour différentes valeur de $\mathrm{V}_{\mathrm{DS}}$. La Fig. 78 montre que le dopage du canal $\left(\mathrm{N}_{\mathrm{A}}=10^{18} \mathrm{~cm}^{-3}\right)$ induit une augmentation de la hauteur de barrière source-canal à même condition de polarisation par rapport à une structure à canal nid, et ainsi réduit l'effet DIBL. Ce comportement est observé dans le calcul semi-classique (sans confinement quantique). Dans le calcul quantique, cette bonne immunité contre les effets de canal court dans la structure à canal fortement dopé devrait être atténuée voire disparaître ( $c f$. Chapitre II). Les structures à canal nid, comme MOS-COMB et MOS-ThinBody, sont a priori très prometteuses du point de vue du transport. Mais il faudrait réduire encore l'épaisseur de la zone active $(\sim 5 \mathrm{~nm})$, pour permettre le renforcement de l'immunité aux effets de canal court ( $c f$. Chapitre II). Dans ce cas-là, les données sur les masses effectives du matériau massif ne sont toutefois plus valables, il faut les prédéterminer via des calculs de structure de bandes [Liu2008IEEE_A]. Une correction de non-parabolicité sera alors nécessaire pour calculer l'occupation des électrons en vallée $\Gamma$ ( $c f$. chapitre II).

Une augmentation rapide de la vitesse moyenne des électrons le long du canal peut être observée pour les quatre dispositifs sur la Fig. 79 pour $\mathrm{V}_{\mathrm{DS}}=0,2 \mathrm{~V}$ et $\mathrm{V}_{\mathrm{GS}}-\mathrm{V}_{\mathrm{T}} \sim 0,2 \mathrm{~V}$. On constate que pour MOS-COMB, le régime de mobilité ne se manifeste pas : la vitesse augmente très rapidement dès l'entrée dans le canal, elle est même supérieure à la vitesse de saturation stationnaire et à la survitesse dans $\operatorname{In}_{0,53} \mathrm{Ga}_{0,47} \mathrm{As}$ massif (respectivement égales à $10^{7} \mathrm{~cm} / \mathrm{s}$ et $3 \times 10^{7} \mathrm{~cm} / \mathrm{s}$ [Pearsall1982]). En revanche, nous observons pour MOS-Bulk une 
évolution « lente » de la vitesse là où le champ est relativement faible, comme dans le régime de mobilité. La vitesse dépasse ensuite rapidement sa valeur de saturation mais reste inférieure à la survitesse du matériau massif. Le comportement est donc plutôt « diffusif ». Une chute brutale de la vitesse peut être observée quand les électrons atteignent la zone d'accès de drain où la concentration en dopants est grande. Comme l'effet de dégénérescence n'est pas pris en compte, l'énergie cinétique dans la zone d'accès est environ égale à l'énergie thermique $3 \mathrm{k}_{\mathrm{B}} \mathrm{T} / 2$ à l'équilibre, indépendamment du niveau de Fermi ainsi et des concentrations locales de charge. La température des électrons devient rapidement bien supérieure à celle du réseau cristallin, surtout en fin du canal, le transport étant fortement hors équilibre.
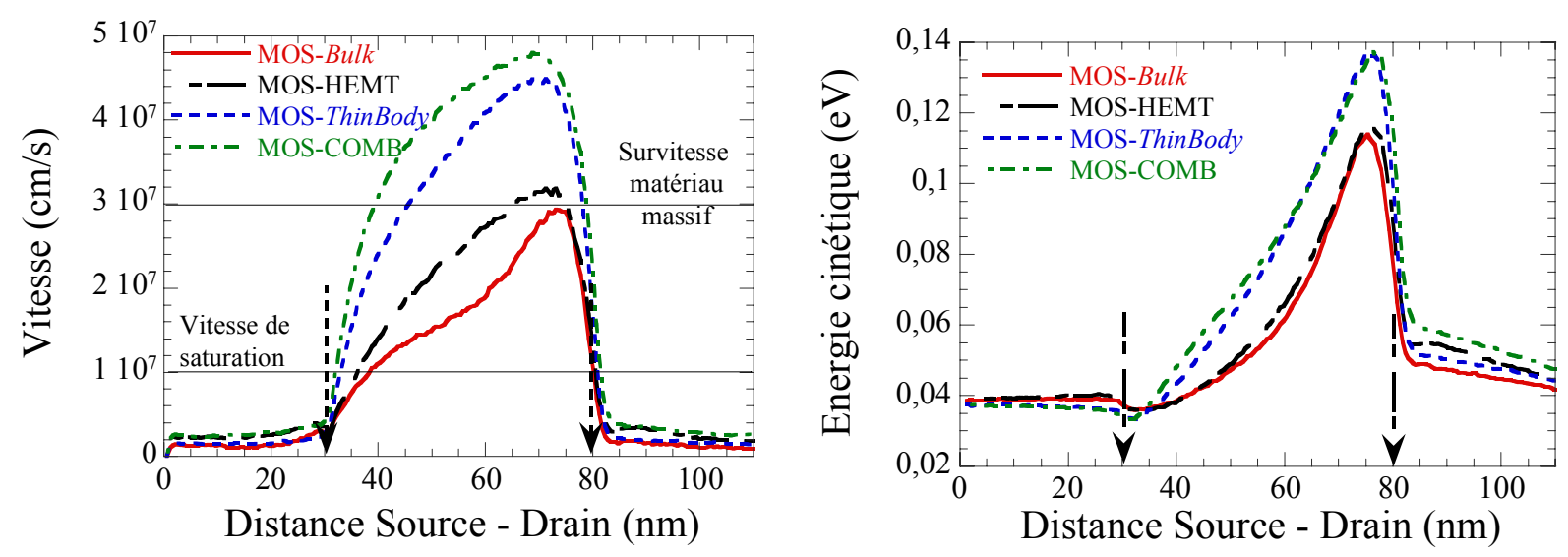

Figure 79 Gauche : vitesse moyenne des électrons. Droite : énergie cinétique moyenne des électrons en fonction de la distance le long de l'axe source-drain à $V_{G S}-V_{T}=0,2 \mathrm{~V}$ et $V_{D S}=0,2 \mathrm{~V}$ pour les quatre MOSFET étudiés. Les flèches indiquent les limites du canal.

Dans l'hypothèse quasi-statique, on peut estimer la fréquence de transition intrinsèque (gain en courant unitaire entre drain et grille) : $\mathrm{f}_{\mathrm{T}}=\mathrm{G}_{\mathrm{m}} /\left(2 \pi\left(\mathrm{C}_{\mathrm{GS}}+\mathrm{C}_{\mathrm{GD}}\right)\right.$ ), où $\mathrm{C}_{\mathrm{GS}}+\mathrm{C}_{\mathrm{GD}}=\mathrm{d}\left(\mathrm{Q}_{\mathrm{tot}}\right) / \mathrm{dV}_{\mathrm{GS}}$ pour une tension $\mathrm{V}_{\mathrm{DS}}$ donnée (ici 0,2 V). Q $\mathrm{Qtot}_{\text {to }}$ est la charge totale moyenne au sein du dispositif dans la simulation MC. La Fig. 80 présente la variation de $\mathrm{f}_{\mathrm{T}}$ en fonction de la tension de grille pour différentes structures, non seulement pour les MOSFET de $50 \mathrm{~nm}$, mais également pour des HEMT de même longueur. Intrinsèquement, le HEMT atteint une performance supérieure aux MOS-Bulk et MOS-ThinBody et est comparable au MOS-HEMT. La meilleure valeur de $\mathrm{f}_{\mathrm{T}}$ est atteinte pour MOS-COMB grâce à une très forte transconductance intrinsèque. Par ailleurs, l'influence de résistances de contact de source et drain $R_{D S}$ est également présentée. La fréquence $f_{T}$ baisse fortement lorsque des éléments parasites passifs réalistes sont inclus. 


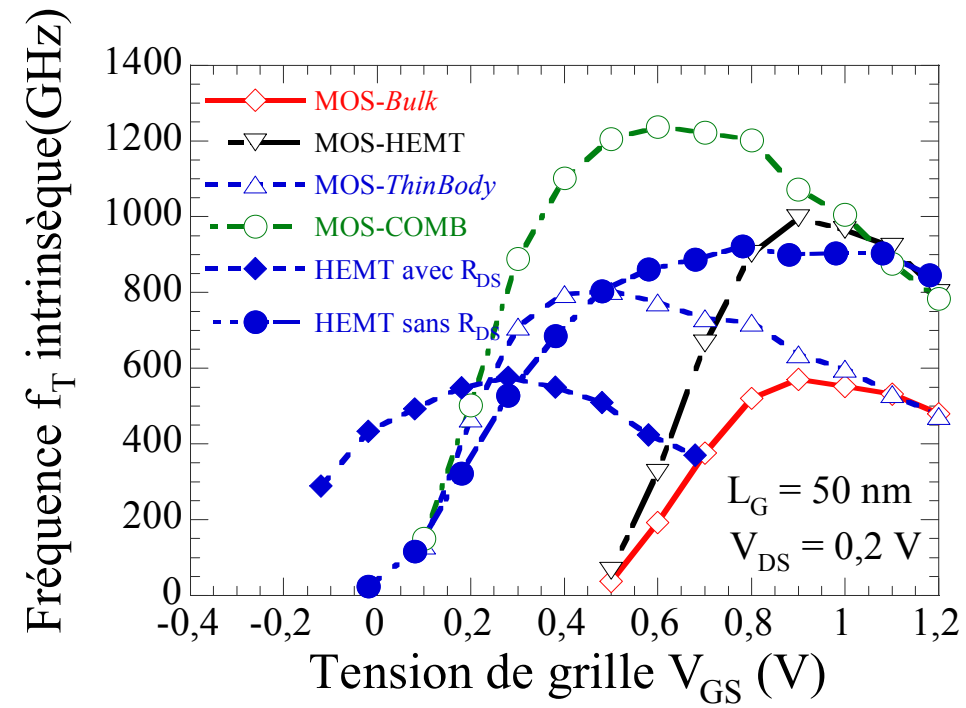

Figure 80 Fréquence de transition $f_{T}$ estimée par l'approche quasi-statique. $R_{D S}$ : résistance extrinsèque de contact de source et drain (même valeur que pour la calibration du transistor HEMT de longueur $\left.\mathrm{L}_{\mathrm{G}}=70 \mathrm{~nm}\right)$.

\section{3-2 Analyse du comportement balistique des dispositifs}

Quand la longueur de grille devient comparable au libre parcours moyen inélastique des porteurs (environ $100 \mathrm{~nm}$ à température ambiante pour $\operatorname{In}_{0,53} \mathrm{Ga}_{0,47} \mathrm{As}$ nid [Mateos2003IEEE]), les électrons traversent la région active avec une forte probabilité d'être (quasi) balistique. Cela induit un transport fortement hors équilibre ainsi que des survitesses importantes, même à de faibles valeurs de $\mathrm{V}_{\mathrm{DS}}$. Pour quantifier les propriétés balistiques, on peut étudier les spectres en vitesse et le coefficient de "balisticité" intrinsèque [SaintMartin2004IEEE] qui représente le pourcentage d'électrons qui n'ont subi aucune interaction à la traversée du canal entre source et drain. Cela nous permet d'évaluer plus en détails le transport électronique à l'échelle déca-nanométrique.

Un spectre en vitesse présente la distribution en vitesse d'une population d'électrons. Afin d'étudier plus finement ces spectres, on a "marqué" les électrons qui sont injectés dans la zone active depuis la source. On peut ainsi sélectionner les populations d'électrons et calculer des spectres en ne comptant compte que des électrons venant de la source. La même peut être appliquée aux électrons venant du drain. Les spectres en vitesse pour l'ensemble des électrons à $1 \mathrm{~nm}$ sous l'oxyde sont tracés en Fig. 81. La polarisation de grille correspond à la transconductance maximale à $\mathrm{V}_{\mathrm{DS}}=0,2 \mathrm{~V}$. La forme gaussienne centrée sur zéro est due aux électrons injectés depuis le drain dans la zone active. 


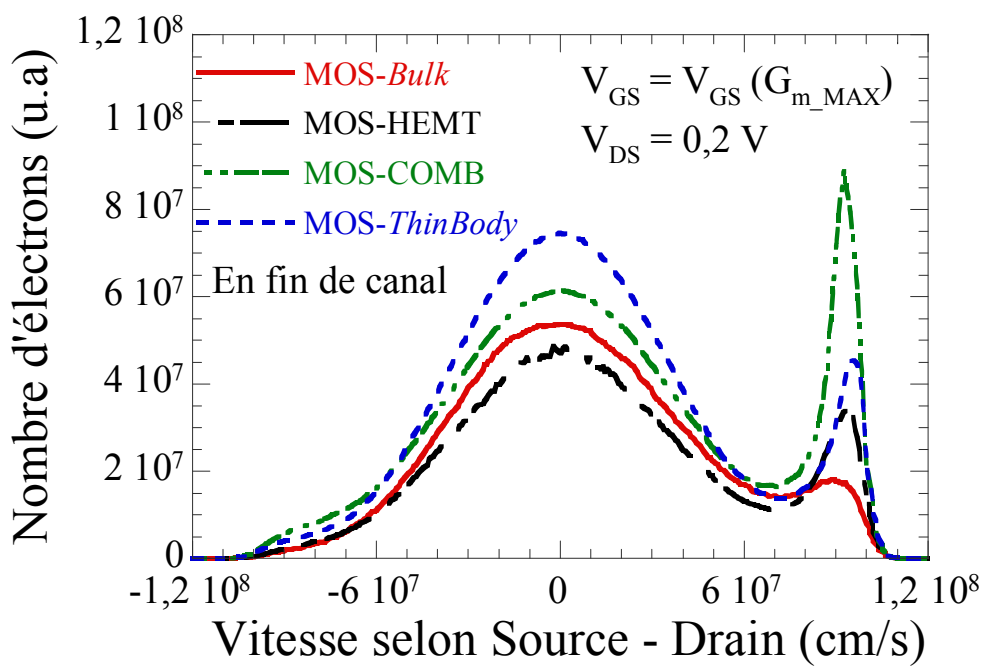

Figure 81 Spectres en vitesse calculés en fin de canal à $1 \mathbf{n m}$ sous l'oxyde dans les quatre MOSFET étudiés. La valeur de $V_{G S}$ correspond au maximum de transconductance à $V_{D S}=0,2 \mathrm{~V}$.

a)

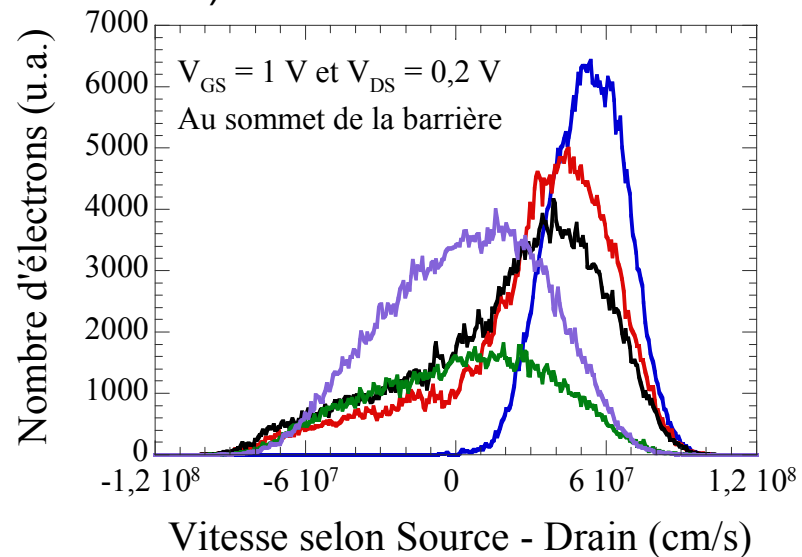

b)

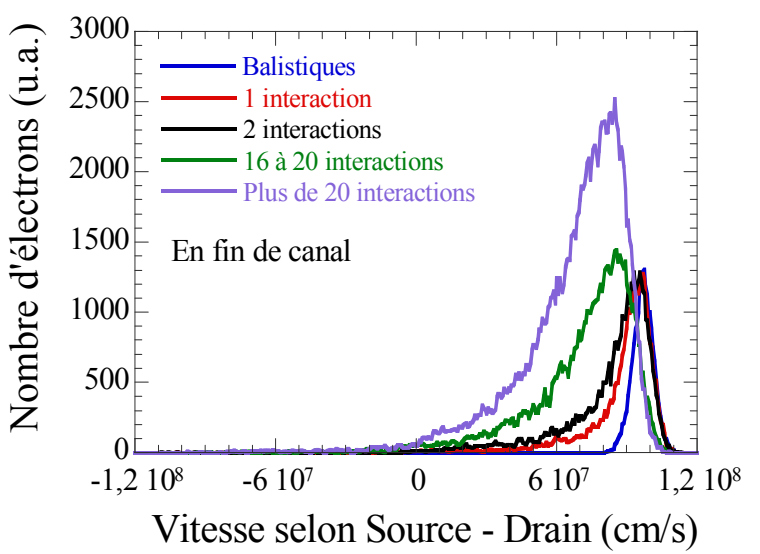

Figure 82 Spectres en vitesse calculés a) au sommet de la barrière et b) en fin de canal dans la structure MOS-Bulk de longueur $L_{G}=50 \mathrm{~nm}$ en fonction du nombre d'interactions subies durant la traversée du canal de la source jusqu'au drain. $V_{\mathrm{GS}}=1 \mathrm{~V}$ (correspond à la transconductance maximale) et

$$
\mathrm{V}_{\mathrm{DS}}=0,2 \mathrm{~V} \text {. }
$$

La forme est très différente pour la distribution en vitesse des électrons injectés depuis la source et qui traversent le canal. Elle est constituée d'un pic qui se situe à environ $9,5 \times 10^{7} \mathrm{~cm} / \mathrm{s}$ pour les quatre structures. Son apparition peut s'analyser grâce aux spectres distinguant les électrons en fonction du nombre d'interactions subies. Les Fig. 82-a et 82-b représentent ce type de spectres. L'entrée de la zone de comptage est prise à la jonction métallurgique source - canal. Ces spectres sont relevés sur une surface verticale par rapport au sens du transport sur une épaisseur de $30 \mathrm{~nm}$ sous l'oxyde, respectivement à la jonction métallurgique source - canal, au sommet de la barrière et en fin du canal dans le transistor MOS-Bulk. La Fig. 82-b montre que le pic est dû aux électrons balistiques et à ceux ayant 
subi de 1 à 2 interactions. Le transport au sein de MOS-Bulk est toutefois dominé par les interactions, non seulement au sommet de barrière mais également en fin de canal. La présence des balistiques est beaucoup plus marquée dans les structures alternatives, au sommet de la barrière d'injection comme en fin de canal, comme on peut le voir sur la Fig. 83 .

a)

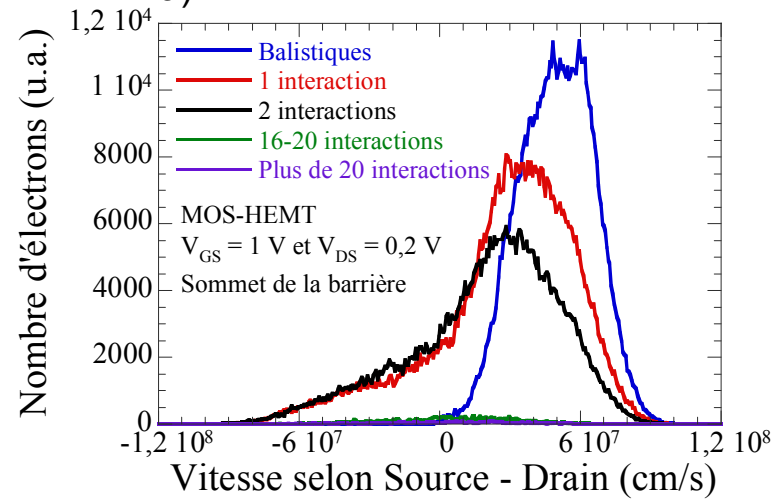

c)

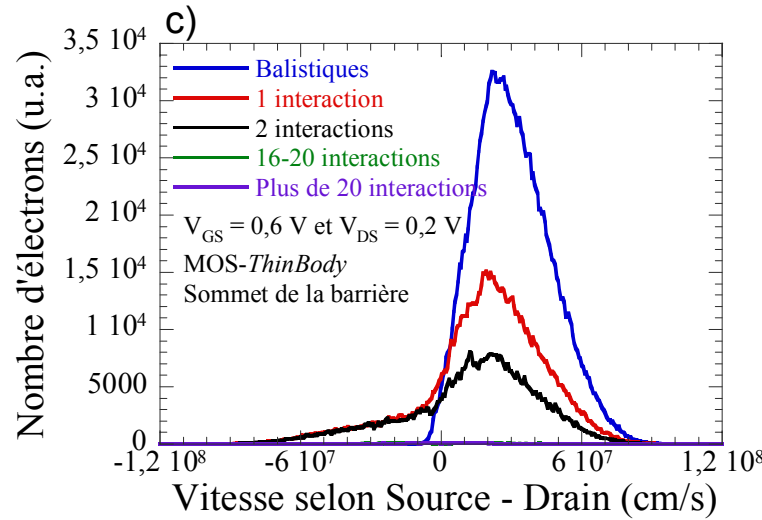

Selon Source - Drain $(\mathrm{cm} / \mathrm{s})$

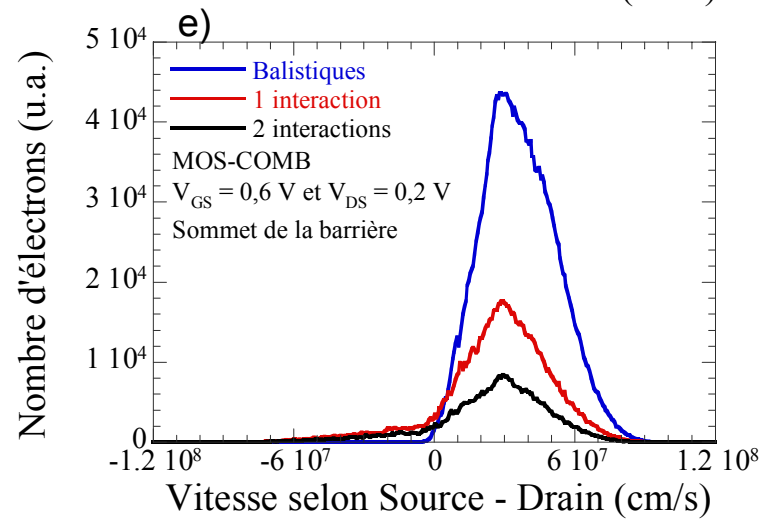

b)

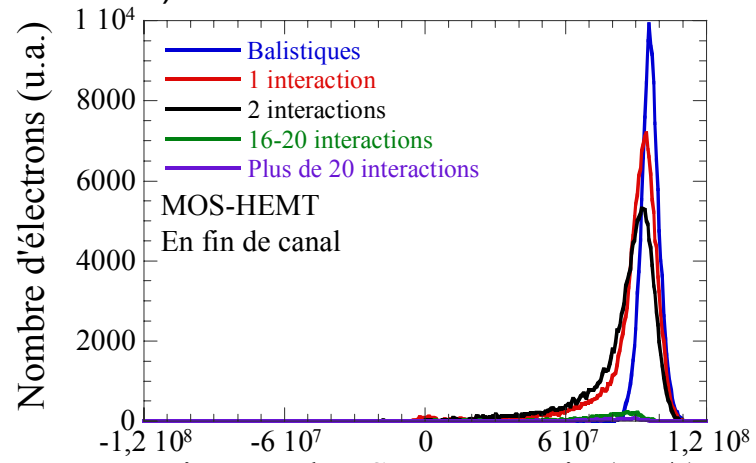

Vitesse selon Source - Drain $(\mathrm{cm} / \mathrm{s})$

d)

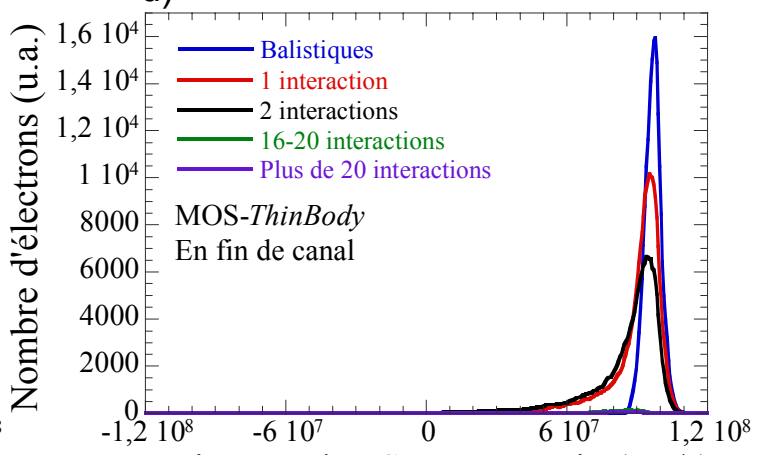

Vitesse selon Source - Drain $(\mathrm{cm} / \mathrm{s})$

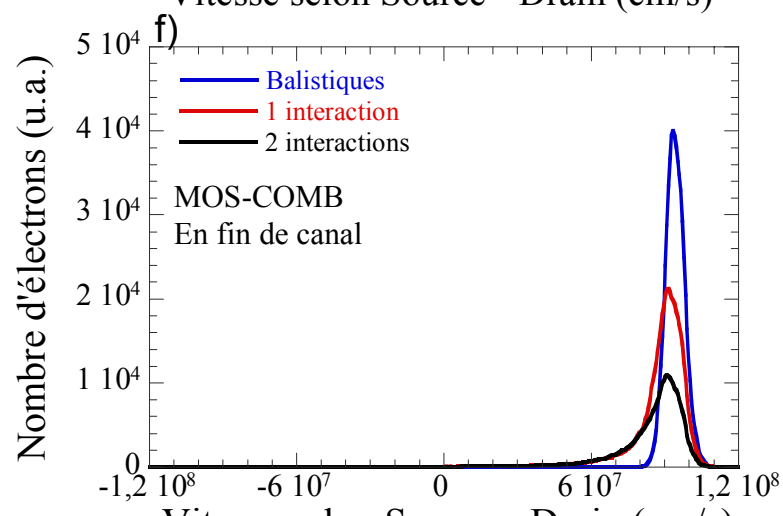

Vitesse selon Source - Drain $(\mathrm{cm} / \mathrm{s})$

Figure 83 Spectres en vitesse calculés au sommet à $V_{D S}=0,2 \mathrm{~V}$ de la barrière et en fin de canal, respectivement dans les structures $(a+b)$ MOS-HEMT à $V_{G S}=1 \mathrm{~V},(c+d) M O S-T B$ à $V_{G S}=0,6 \mathrm{~V}$ et $(e+f)$

MOS-COMB à $V_{G S}=0,6 \mathrm{~V}$. Ces tensions $V_{G S}$ correspondent au maximum de transconductance.

Ces évolutions des spectres en vitesse dans les structures alternatives (MOS-ThinBody, MOS-HEMT et MOS-COMB) sont très similaires à celles obtenues dans un canal purement balistique présentées dans la référence [Rahman2003IEEE]. Cependant, contrairement au cas 
purement balistique, les pics en fin de canal ont une queue de distribution non nulle, ce qui est dû aux électrons non balistiques. Au sommet de la barrière, la population d'électrons à vitesse négative est celle injectée par la source et rétrodiffusée par le canal. Le transport n'est donc ni purement balistique, ni diffusif dans ces structures alternatives pour $\mathrm{L}_{\mathrm{G}}=50 \mathrm{~nm}$. Il est quasibalistique.

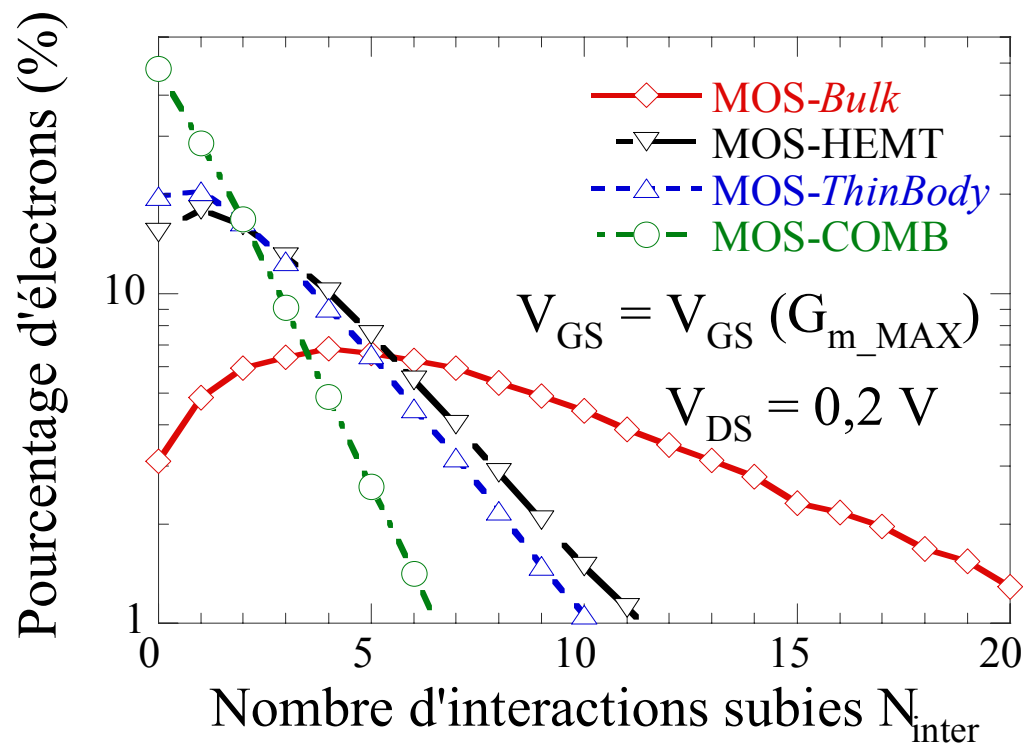

Figure 84 Spectres d'interactions en fin de canal, soit le pourcentage d'électrons en fonction du nombre d'interactions qu'ils ont subies pendant la traversée du canal de la source jusqu'au drain. $V_{G S}$ correspond au maximum de transconductance à $V_{D S}=0,2 \mathrm{~V}$.

Pour décrire plus quantitativement le transport quasi-balistique, on définit un paramètre appelé "balisticité intrinsèque" $B_{\text {int }}$. Ce paramètre permet de caractériser le pourcentage en termes de flux d'électrons purement balistiques en fin de canal [SaintMartin2005Thèse]. La Fig. 84 présente les spectres d'interactions à la fin du canal dans les quatre transistors simulés pour $\mathrm{V}_{\mathrm{GS}}$ donnant le maximum de transconductance à $\mathrm{V}_{\mathrm{DS}}=0,2 \mathrm{~V}$. Ils correspondent au pourcentage d'électrons en fin de canal en fonction du nombre d'interactions $\mathrm{N}_{\text {inter }}$ subies lors de la traversée du canal. $\mathrm{B}_{\text {int }}$ vaut $8 \%$ pour MOS-Bulk, $37 \%$ pour MOS-ThinBody, 22\% pour MOS-HEMT et 55\% pour MOS-COMB. Pour MOSBulk, le spectre d'interaction est en forme de cloche - plutôt caractéristique d'un transport « quasi-stationnaire » - et son maximum est centré sur 5 interactions. On peut aussi estimer le libre parcours moyen qui vaut environ $1 \mathrm{pm} \approx 50 / 5=10 \mathrm{~nm}$. Cela est presque équivalent au cas du Si non dopé (transistor sur SOI par exemple). Le transport dans les dispositifs MOSThinBody et MOS-HEMT est fortement hors d'équilibre, tandis que dans MOS-COMB il est clairement quasi-balistique (décroissance exponentielle du spectre en fonction de $\mathrm{N}_{\text {inter }}$ ) avec 
une grande valeur de $\mathrm{B}_{\text {int. }}$ Grâce au transport fortement hors d'équilibre, voire quasi-balistique, ces structures alternatives pourraient présenter de hautes performances en termes de rapidité, même à faible $\mathrm{V}_{\mathrm{DS}}(0,2 \mathrm{~V})$ pour avoir avec une faible consommation de puissance.

\section{Comportement dynamique}

\section{4-1 Calcul du courant transitoire}

Les analyses transitoires par simulation $\mathrm{MC}$ de la réponse indicielle ou impulsionnelle de dispositifs permettent d'investiguer de façon plus quantitative leurs performances fréquentielles. Ces analyses radiofréquences (RF) sont principalement basées sur une représentation matricielle des quadripôles constitués par les transistors. Les paramètres les plus utilisés sont ceux d'impédance $Z_{\mathrm{ij}}$, d'admittances $Y_{\mathrm{ij}}$ et de chaîne $\mathrm{C}_{\mathrm{ij}}$, où les indices 'ij' repèrent les électrodes des quadripôles. Dans notre cas, les paramètres d'admittance complexes $\mathrm{Y}_{\mathrm{ij}}$ sont utilisés pour caractériser les dispositifs en très haute fréquence, ils peuvent être directement et précisément extraits des simulations MC pour une large plage de fréquence, au-delà du THz, à l'aide du courant transitoire. Le courant instantané à chaque contact est calculé par le théorème de Ramo-Shockley décrit dans la section "Calcul de courant par la méthode de Ramo-Shockley" du premier paragraphe. Le courant 'Ramo' permet de déduire la densité spectrale de puissance de fluctuation du courant par une décomposition dans l'espace de Fourier. L'évolution de la densité en fonction de la fréquence se caractérise par un pic d'oscillation plasma autour de $20 \mathrm{THz}$ pour un canal d' $\mathrm{In}_{0,53} \mathrm{Ga}_{0,47} \mathrm{As}$ avec une densité des porteurs de l'ordre de $10^{12} \mathrm{~cm}^{-2}$, ce qui correspond bien aux résultats de la littérature [Millithaler2008APL]. Nous en reparlerons dans la section "Analyse de bruit intrinsèque en haute fréquence" de ce chapitre.

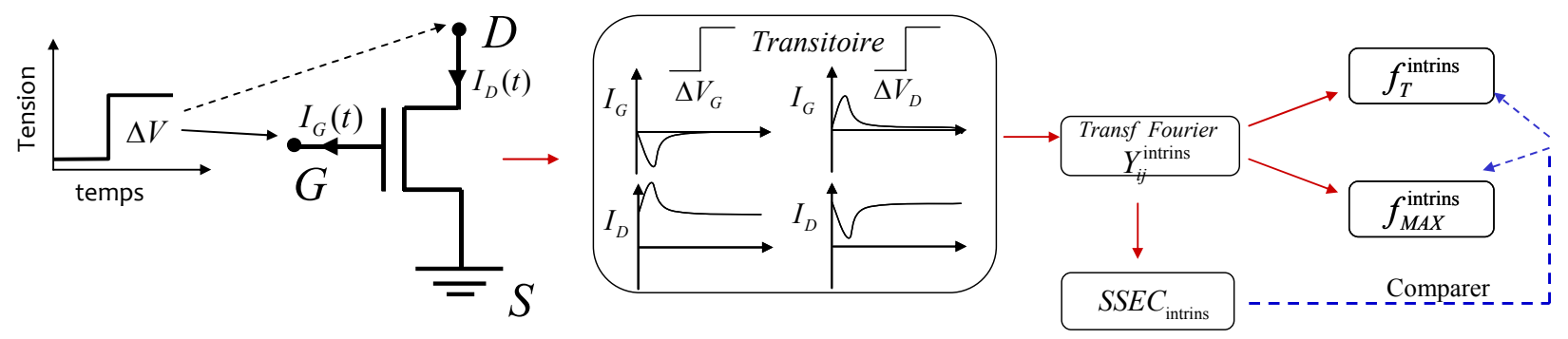

Figure 85 Les étapes des analyses RF : i) calcul du courant transitoire de réponse indicielle sous une perturbation en échelon de tension, ii) extraction des paramètres d'admittance, iii) extraction des éléments du modèle petit signal (small signal equivalent circuit ou SSEC), iv) détermination des facteurs de mérite

fréquentiels en utilisant les résultats numériques et les résultats calculés par le modèle SSEC (pour identifier le domaine de validité du schéma équivalent). 
Dans cette analyse RF, le point de fonctionnement statique (DC) $\mathrm{V}_{\mathrm{GS}}$ est choisi pour atteindre le maximum de $\mathrm{G}_{\mathrm{m}}$ à $\mathrm{V}_{\mathrm{DS}}$ donné (ici $0,2 \mathrm{~V}$, dans le régime plutôt linéaire, $c f$. Fig. 77). Les étapes de la procédure sont résumées en Fig. 85. Pour un état statique donné, préliminairement établi par une simulation MC suffisamment longue pour atteindre des courants stationnaires aux électrodes, une perturbation de tension en forme d'échelon est appliquée, consécutivement à l'électrode de drain et à celle de grille. Pour estimer précisément le courant transitoire immédiatement après la perturbation en limitant des bruits numériques, la fréquence d'échantillonnage $f_{\text {sample }}=1 / \Delta t$ doit être soigneusement choisie, par rapport à la fréquence plasma, au temps de relaxation diélectrique et éventuellement en fonction de l'oscillation due à l'effet Gunn et à la fréquence maximale du fonctionnement du transistor. $\mathrm{f}_{\text {sample }}$ doit être beaucoup plus grande que les fréquences caractéristiques associées à ces phénomènes intrinsèque au sein du dispositif. Par ailleurs, l'amplitude du courant de déplacement et celle du courant capacitif inter-électrode immédiatement après perturbation sont toutes les deux proportionnelles à $\mathrm{f}_{\text {sample }}(c f$. paragraphe : 1-3). Nous avons choisi en conséquence $\mathrm{f}_{\text {sample }}=10^{4} \mathrm{THz}$. Si on veut que la limite du côté des basses fréquences soit inférieure ou égale à $10 \mathrm{GHz}$, on doit simuler un courant transitoire qui dure au moins $100 \mathrm{ps}$,

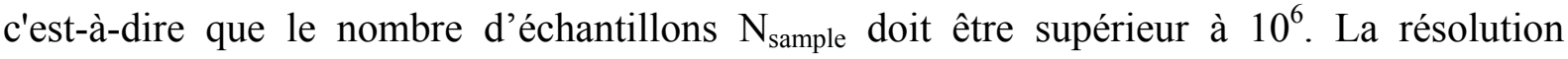
fréquentielle est de plus égale à $f_{\text {sample }} / N_{\text {sample }}$. En outre, nous avons considéré un grand nombre de particules simulées - environ 100000 - afin d'améliorer la statistique pour le calcul du courant transitoire aux électrodes [Gonzalez1995IEEE-A]. Enfin, l'amplitude du signal en échelon doit être soigneusement choisie. Il faut qu'elle soit suffisamment importante pour permettre la résolution correcte en régime dynamique vis-à-vis de la nature stochastique des simulations MC [Babiker1998IEEE]. Mais elle doit aussi être assez faible pour éviter la génération d'harmoniques pendant le transitoire [Laux1985IEEE]. Dans notre calcul, l'amplitude de signal échelon est de $0,1 \mathrm{~V}$ pour l'électrode de grille et $0,2 \mathrm{~V}$ pour l'électrode de drain.

Avant d'extraire les paramètres d'admittance, le courant transitoire issu de la simulation MC est post-traité. Nous avons utilisé des procédures de filtrage comme la moyenne locale temporaire et un filtre numérique à réponse impulsionnelle infinie de type Butterworth pour réduire les bruits numériques parasites dans la plage de très haute fréquence ( $>500 \mathrm{THz}$ ), afin de diminuer les erreurs numériques dans le calcul des intégrales nécessaires à la déduction des paramètres Y. Des exemples de résultats sont montrés sur la Fig. 86. Le courant capacitif associé aux matrices de capacité Ramo inter-électrode durant le 
premier pas de temps au moment de l'échelon est très significatif. Les variations dans le reste de temps peuvent être dues à l'oscillation plasma dans les régions de fort dopage ou forte densité de porteurs et éventuellement à l'effet Gunn [Pérez2008JAP].
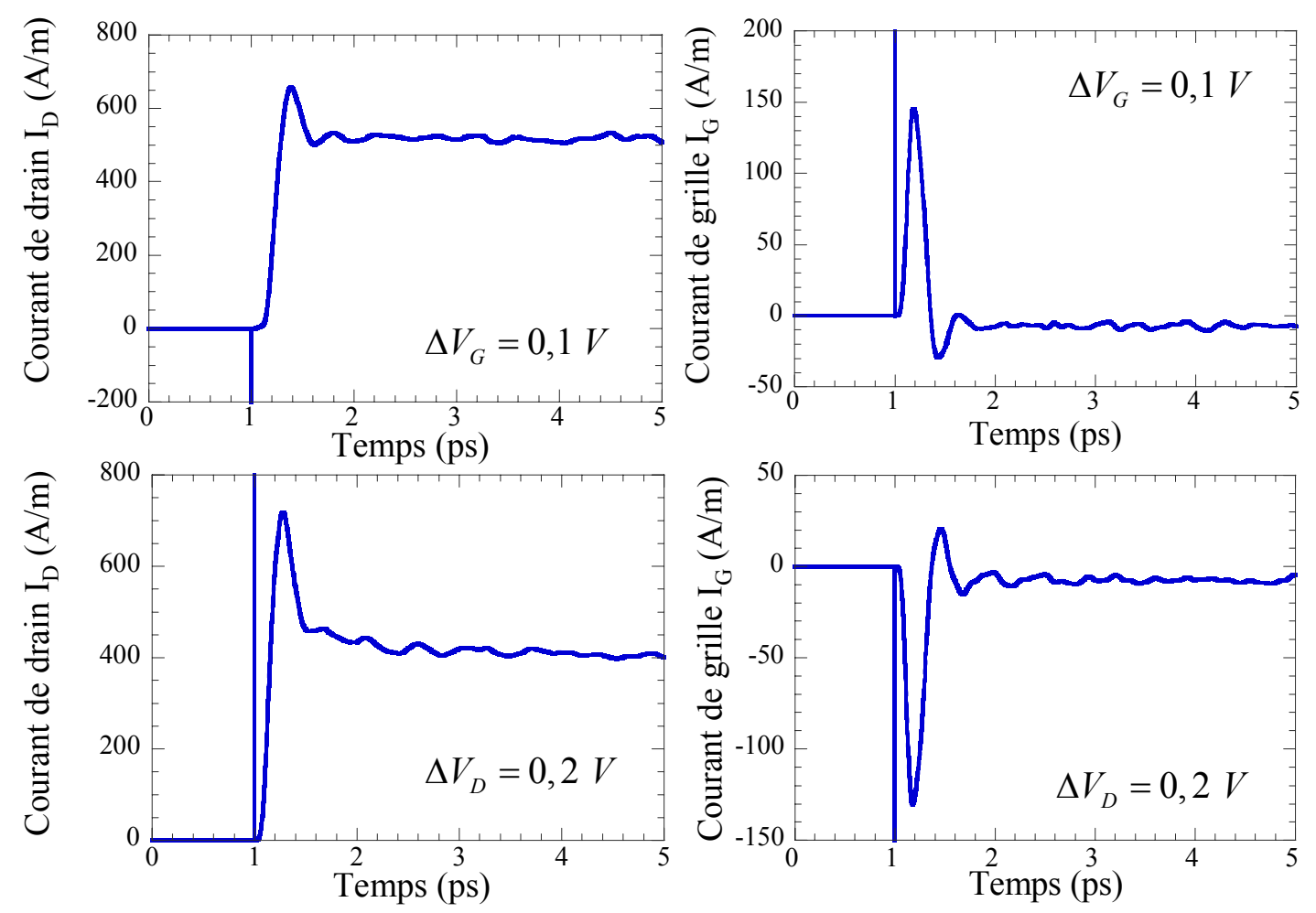

Figure 86 Courants transitoires pendant les premiers 4 ps pour MOS-Bulk après échelon en tension $\left(\Delta V_{G}\right.$ ou $\left.\Delta V_{D}\right)$ appliqué sur l'électrode de grille ou de drain.

\section{4-2 Extraction des paramètres d'admittance complexes intrinsèques}

Nous devons effectuer l'extraction des paramètres $\mathrm{Y}$ complexes sur 2 ports (quadripôle avec une configuration en source commune) du composant MOSFET III-V sous investigation en fonction de la fréquence. Ils sont calculés par transformée de Fourier de la réponse en courant à un échelon en tension sur les terminaux du transistor. Les variations de ces petits signaux peuvent être exprimées sous la forme :

$$
\left(\begin{array}{l}
\Delta I_{G} \\
\Delta I_{D}
\end{array}\right)=\left(\begin{array}{ll}
Y_{11} & Y_{12} \\
Y_{21} & Y_{22}
\end{array}\right)\left(\begin{array}{l}
\Delta V_{G} \\
\Delta V_{D}
\end{array}\right)
$$

où les indices $\mathrm{G}$ et $\mathrm{D}$ se rapportent respectivement eux électrodes de grille et de drain. La transformation de Fourrier d'un signal d'échelon $\Delta V_{j}$ en tension dans le domaine fréquentiel est $\Delta V_{j} / j \omega$. Alors, l'équation III-40 devient :

$$
\Delta I_{i}(j \omega)=Y_{i j}(j \omega) \frac{\Delta V_{j}}{j \omega}
$$


où $\Delta I_{i}(j \omega)$ est la transformation de Fourier des courants transitoires dans le domaine fréquentiel. Les paramètres Y complexes s'écrivent comme :

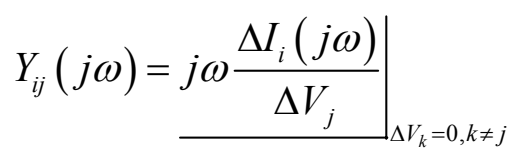

Ils peuvent se décomposer en deux termes, un relatif au régime de très basse fréquence et l'autre à celui de haute fréquence [Laux1985IEEE] :

$$
Y_{i j}(j \omega)=\frac{I_{i}(\infty)-I_{i}(0)}{\Delta V_{j}}+\frac{j \omega}{\Delta V_{j}} \mathbb{T F}\left\{I_{i}(t)-I_{i}(\infty)\right\}
$$

Ainsi, la partie réelle et la partie imaginaire du paramètre $\mathrm{Y}$ peuvent explicitement s'écrire sous la forme [Babiker1998IEEE]:

$$
\begin{gathered}
\operatorname{Re}\left(Y_{i j}(\omega)\right)=\frac{I_{i}(\infty)-I_{i}(0)}{\Delta V_{j}}+\frac{\omega}{\Delta V_{j}} \int_{0}^{T}\left[I_{i}(t)-I_{i}(\infty)\right] \cdot \sin (\omega t) d t \\
\operatorname{Im}\left(Y_{i j}(\omega)\right)=\frac{\omega}{\Delta V_{j}} \int_{0}^{T}\left[I_{i}(t)-I_{i}(\infty)\right] \cdot \cos (\omega t) d t
\end{gathered}
$$

où $I_{i}(0)$ et $I_{i}(\infty)$ sont les courant $\mathrm{DC}$ en régime stationnaire aux électrodes de grille et de drain respectivement avant et après l'échelon de tension. $I_{i}(t)$ est le courant transitoire calculé par la méthode Ramo-Shockley. $T$ est le temps d'acquisition du régime transitoire,

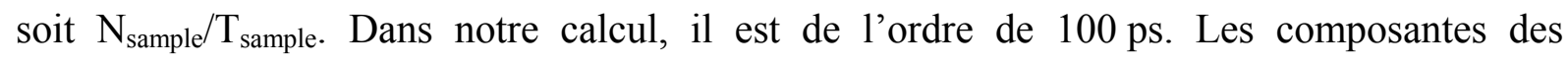
paramètres Y s'interprètent physiquement comme la matrice des conductances et celle des capacités du composant, on peut alors retrouver ses formulations en régime de très basse fréquence d'après les équations III-19 et 20 [Laux1985IEEE] :

$$
\begin{gathered}
G_{i j}(\omega \rightarrow 0)=\frac{I_{i}(\infty)-I_{i}(0)}{\Delta V_{j}} \\
C_{i j}(\omega \rightarrow 0)=\frac{1}{\Delta V_{j}} \int_{0}^{T}\left[I_{i}(t)-I_{i}(\infty)\right] \cdot d t
\end{gathered}
$$



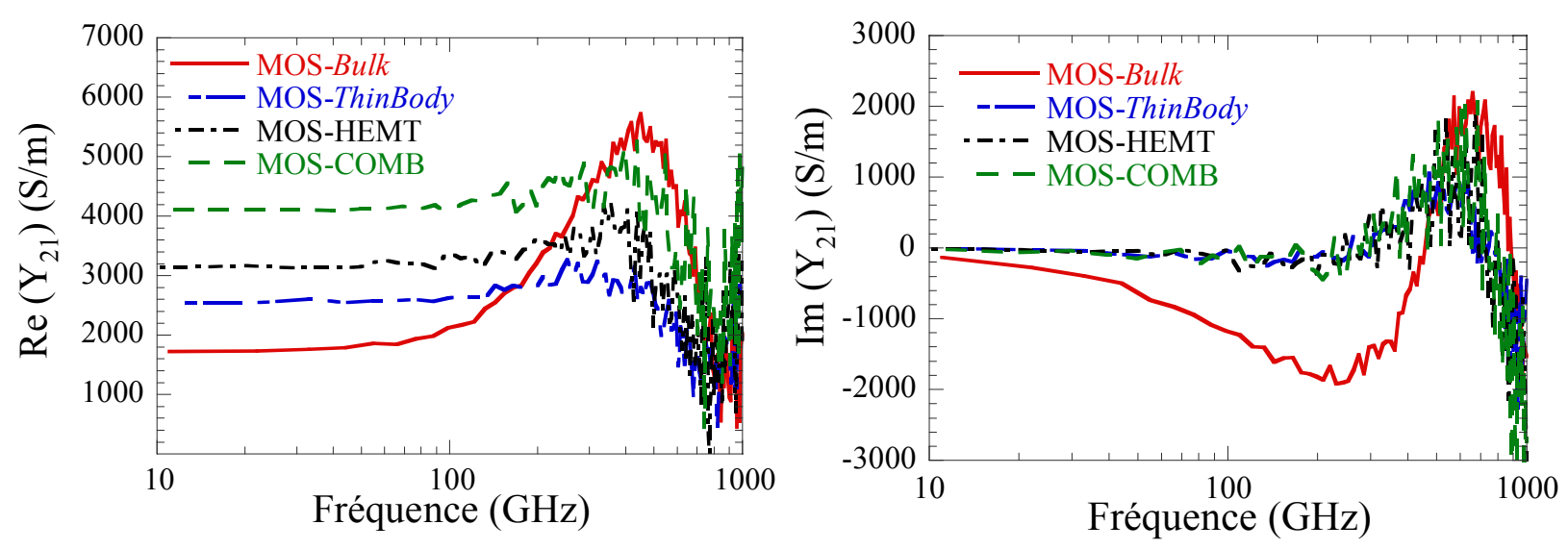

Figure 87 Parties réelle et imaginaire du paramètre $Y_{21}$ pour les 4 MOSFET
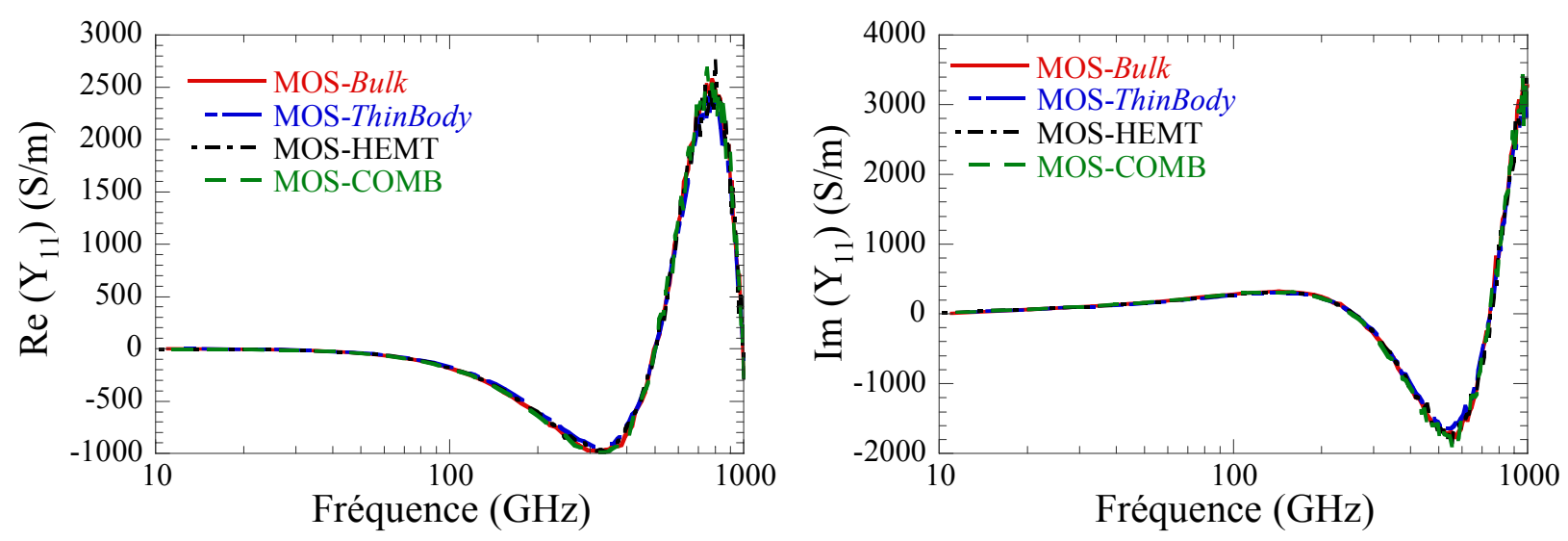

Figure 88 Parties réelle et imaginaire du paramètre $Y_{11}$ pour les 4 MOSFET
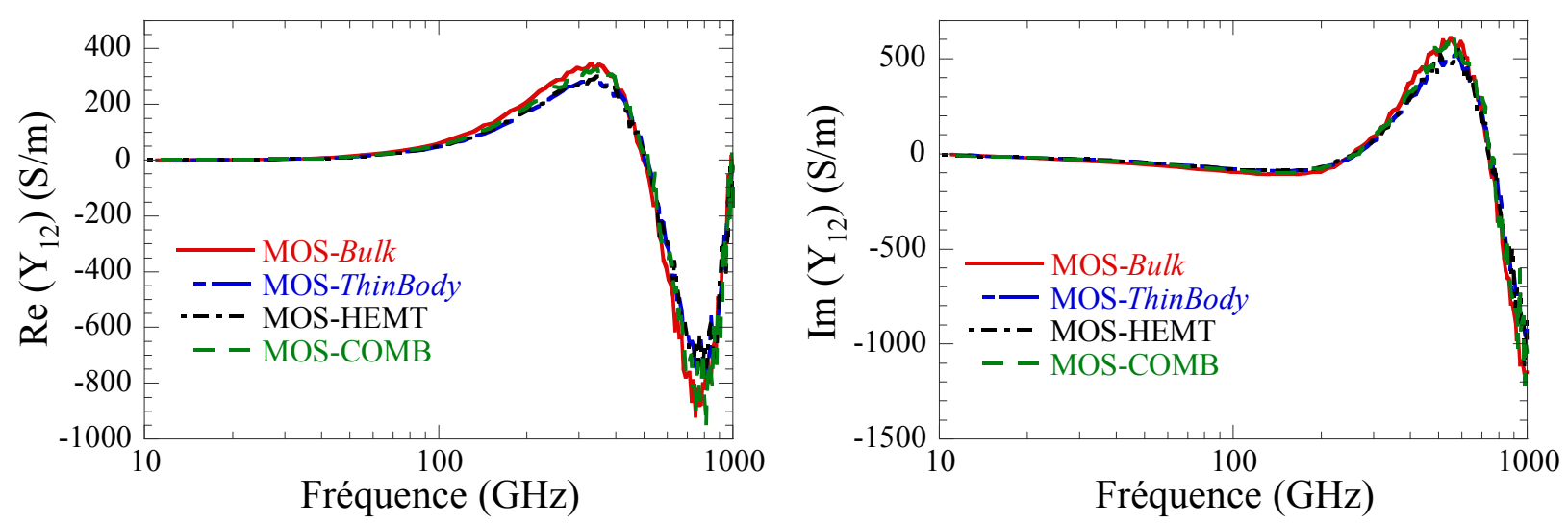

Figure 89 Parties réelle et imaginaire du paramètre $Y_{12}$ pour les 4 MOSFET

Les évolutions des $\mathrm{Y}_{\mathrm{ij}}$ en fonction de la fréquence pour une configuration en source commune sont tracées sur les Fig. 87 à 90. On peut constater que les valeurs des paramètres $\mathrm{Y}_{11}$ et $\mathrm{Y}_{12}$ sont quasiment identiques pour les quatre structures de MOSFET étudiées. Les différences majeures se trouvent sur les paramètres $Y_{21}$ et $Y_{22}$. Physiquement, $Y_{11}$ et $Y_{12}$ représentent le comportement dynamique plutôt lié à la réactance du système tandis que les paramètres $Y_{21}$ et $Y_{22}$ représentent celui de la conductance. Les caractérisations plus 
fonctionnelles dans le domaine fréquentiel seront effectuées par l'extraction des éléments du modèle petit signal (SSEC).
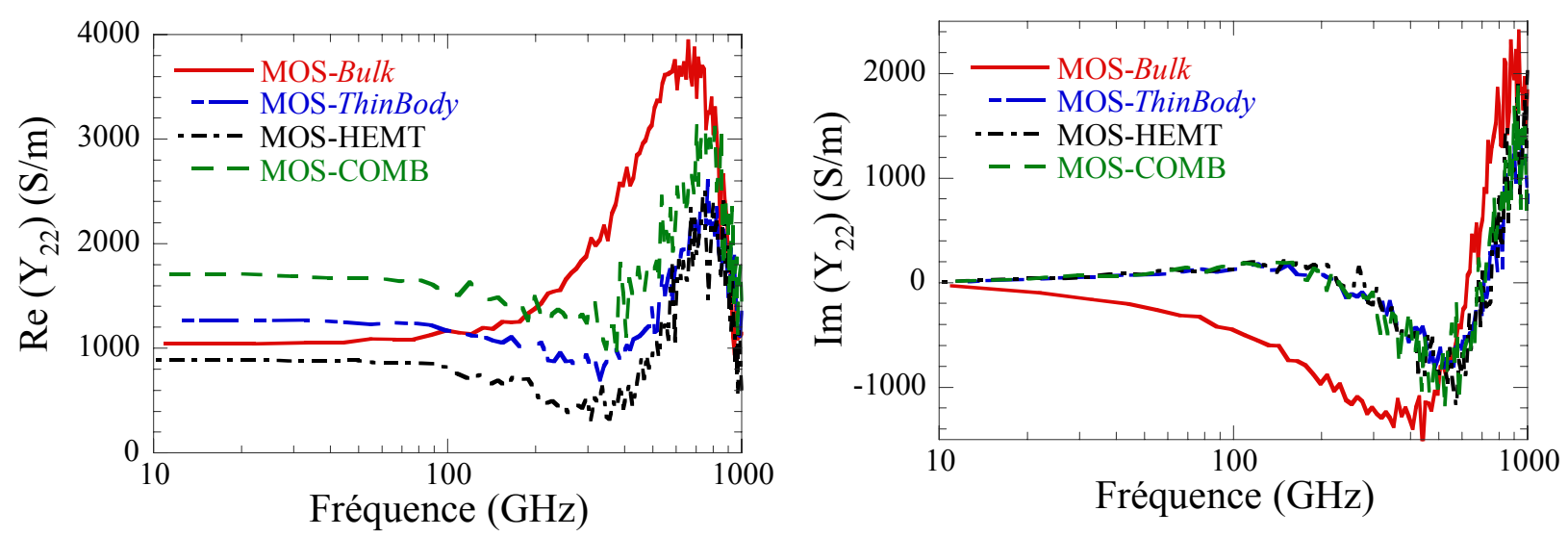

Figure 90 Parties réelle et imaginaire du paramètre $Y_{22}$ pour les 4 MOSFET

\section{4-3 Extraction du circuit équivalent du modèle petit signal (SSEC) intrinsèque}

En conception de circuits micro-ondes, la connaissance précise du circuit équivalent en petit signal (SSEC) d'un transistor est un outil très utile pour caractériser le comportement dynamique du dispositif. Par exemple, il permet de calculer les différents gains associés en fonction de la fréquence. En outre, il rend possible l'analyse en termes de bruit du composant pour une gamme de fréquence de l'ordre de GHz. Le point crucial pour l'utilisation d'un modèle SSEC est la détermination de sa plage de fréquence de validité. Habituellement, les éléments du SSEC d'un FET sont définis pour représenter globalement certains aspects physiques du composant. Le modèle SSEC qui est largement accepté comporte seize éléments a priori indépendants de la fréquence dans le domaine de validité : huit d'entre eux correspondent à des effets parasites externes et ils sont normalement indépendants de la condition de polarisation; les huit autres décrivent le comportement intrinsèque du FET et ils dépendent des conditions de polarisation.

Les quatre paramètres $\mathrm{Y}_{\mathrm{ij}}$ calculés par simulation Monte Carlo peuvent décrire complètement la réponse en petit signal du composant intrinsèque. Une fois les éléments extérieurs parasites mis de côté, les éléments SSEC du FET se réduisent à une topologie présentée sur la Fig. 91. $C_{D S}, C_{G S}$ et $C_{G S}$ correspondent respectivement aux capacités distribuées entre les régions drain-source, grille-source et grille-drain. $R_{i}$ est la résistance ohmique dans le canal entre la source et la grille. $G_{m}^{0}$ représente la transconductance dans le régime stationnaire et $\tau$ est le temps de retard du transistor, c'est-à-dire le temps nécessaire 
pour qu'un signal appliqué à la grille se propage jusqu'à drain. $G_{d}$ est la conductance de drain. $C_{D C}$ est une transcapacité dans la région canal-drain, elle est associée à la création de dipôles à haute fréquence dans la région où le champ électrique est grand [Mateos2004IEEE-A]. Cette réactance permet de prendre en compte l'effet non quasi-stationnaire, elle provoque une contre-réaction positive dans le dispositif qui modifie fortement le gain en puissance du transistor à haute fréquence [Mateos2004IEEE-A]. L'effet de cette transcapacité sera étudié quantitativement dans la section suivante.

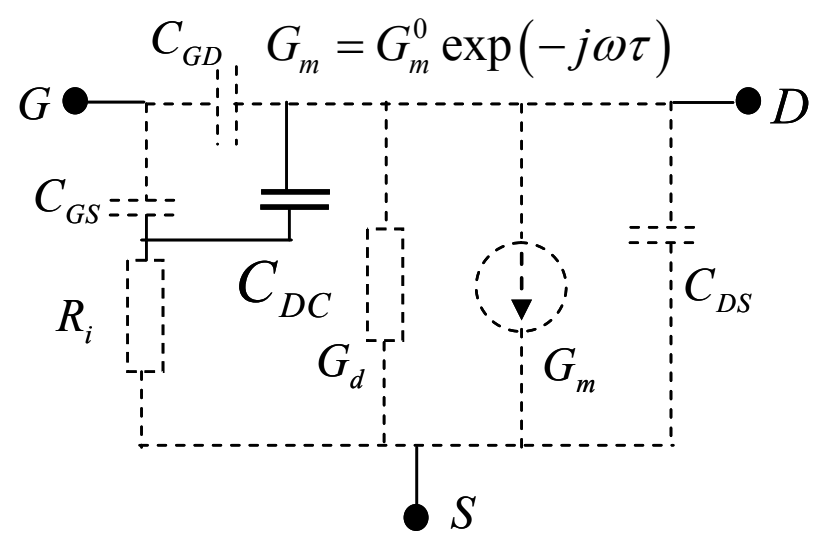

Figure 91 Circuit équivalent petit signal d'un FET intrinsèque (configuration en source commune)

Les éléments du circuit équivalent intrinsèque peuvent être obtenus pour une condition de polarisation donnée par les parties réelles $\mathfrak{R}$ et imaginaires $\mathfrak{I}$ des paramètres $\mathrm{Y}_{\mathrm{ij}}$ intrinsèques [Gonzalez1995IEEE-B], [Dambrine1988IEEE] et [Pailloncy2004SSE] :

$$
\begin{aligned}
& C_{G D}=-\frac{\mathfrak{I}\left(Y_{12}\right)}{2 \cdot \omega} \cdot\left(1+\left(\frac{\mathfrak{R}\left(Y_{12}\right)}{\mathfrak{I}\left(Y_{12}\right)}\right)^{2}\right) \\
& C_{G S}=\frac{\mathfrak{I}\left(Y_{11}\right)+\mathfrak{I}\left(Y_{12}\right)}{2 \cdot \omega}\left(1+\left(\frac{\mathfrak{R}\left(Y_{11}\right)+\mathfrak{R}\left(Y_{12}\right)}{\mathfrak{I}\left(Y_{11}\right)+\mathfrak{I}\left(Y_{12}\right)}\right)^{2}\right) \\
& C_{D S}=\frac{\mathfrak{I}\left(Y_{22}\right)+\mathfrak{I}\left(Y_{12}\right)}{2 \cdot \omega} \\
& G_{m}^{0}=\left|\left(Y_{21}-Y_{12}\right) \cdot\left(1+j \frac{\mathfrak{R}\left(Y_{11}\right)+\mathfrak{R}\left(Y_{12}\right)}{\mathfrak{I}\left(Y_{11}\right)+\mathfrak{I}\left(Y_{12}\right)}\right)\right| \\
& G_{d}=\mathfrak{R}\left(Y_{22}\right)+\mathfrak{R}\left(Y_{12}\right) \\
& R_{i}=\frac{1}{2 \cdot \omega} \cdot \frac{\mathfrak{R}\left(Y_{11}\right)+\mathfrak{R}\left(Y_{12}\right)}{\mathfrak{I}\left(Y_{11}\right)+\mathfrak{I}\left(Y_{12}\right)}
\end{aligned}
$$




$$
\tau=-\frac{1}{2 \cdot \omega} \arg \left(\left(Y_{21}-Y_{12}\right) \cdot\left(1+j \frac{\mathfrak{R}\left(Y_{11}\right)+\mathfrak{R}\left(Y_{12}\right)}{\mathfrak{I}\left(Y_{11}\right)+\mathfrak{I}\left(Y_{12}\right)}\right)\right)
$$
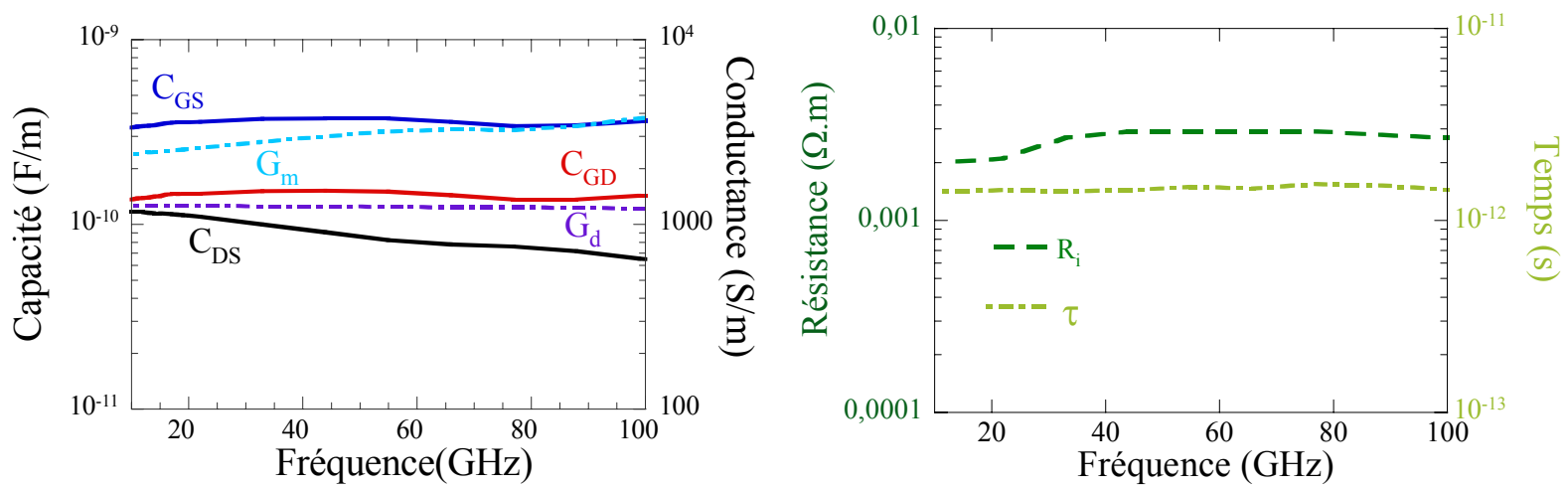

Figure 92 Les différents éléments du schéma petit signal en fonction de la fréquence dans la plage 10 à $100 \mathrm{GHz}$ pour le MOS-ThinBody. $\mathrm{V}_{\mathrm{GS}}=0,6 \mathrm{~V}$ et $\mathrm{V}_{\mathrm{DS}}=0,2 \mathrm{~V}$.

Pour valider le circuit équivalent proposé, il faut examiner la dépendance en fréquence de chaque élément. Un exemple des résultats est montré sur la Fig. 92 où sont tracées les évolutions avec la fréquence des différents éléments du modèle SSEC dans la plage 10 à $100 \mathrm{GHz}$ pour le transistor MOS-ThinBody. Les éléments sont quasiment indépendants de la fréquence jusqu'à $100 \mathrm{GHz}$, sauf $C_{D S}$ qui diminue faiblement quand la fréquence est augmentée. Cette dépendance a également été constatée expérimentalement dans les travaux de l'IEMN. On peut vérifier plus précisément la validité du SSEC en comparant dans la même gamme de fréquence les paramètres $\mathrm{Y}_{\mathrm{ij}}$ aux valeurs déduites du circuit équivalent. Le résultat est tracé sur la Fig. 93.

Si le modèle SSEC valide de 10 à $100 \mathrm{GHz}$, son utilisation devient discutable pour des fréquences supérieures à $200 \mathrm{GHz}$. Le tableau 8 ci-dessous résume les éléments des modèles SSEC extraits à $20 \mathrm{GHz}$ pour les quatre MOSFET étudiés. De même EOT, les dispositifs ont des capacités de grille $\mathrm{C}_{\mathrm{GG}}=\mathrm{C}_{\mathrm{GS}}+\mathrm{C}_{\mathrm{GD}}$ quasiment identiques. Par ailleurs, la capacité du couplage entre la source et le drain $\mathrm{C}_{\mathrm{DS}}$ est plus importante dans les structures à fort niveau du dopage. Les grandes valeurs de $\mathrm{G}_{\mathrm{d}}$ liées à la faible valeur de $\mathrm{V}_{\mathrm{DS}}$ sont probablement plus problématiques pour ce qui est des performances fréquentielles. La situation s'aggrave dans les structures alternatives à faible niveau de dopage dans le canal. Les effets négatifs sur la performance RF peuvent être les suivants :

(i) Une forte conductance $G_{d}$ diminue intrinsèquement le gain en tension $A_{v}=G_{m} / G_{d}$ en circuit ouvert. Ce ratio est déterminant pour la valeur du gain unilatéral à basse fréquence à 
capacité $\mathrm{C}_{\mathrm{GG}}$ fixée, et donc pour celle de la fréquence d'oscillation maximale $\mathrm{f}_{\mathrm{MAX}}$. La transconductance doit être fortement améliorée pour compenser les effets néfastes liés à $G_{d}$, ce qui est l'objectif des structures alternatives. Le ratio $G_{m} / G_{d}$ est effectivement maximisé dans la structure MOS-HEMT $(3,5)$.

(ii) Extrinsèquement, une grande valeur de $G_{d}$ risque d'augmenter la sensibilité $d u$ composant aux éléments parasites, en particulier aux résistances de contact $R_{S}, R_{D}$ et $R_{G}$.

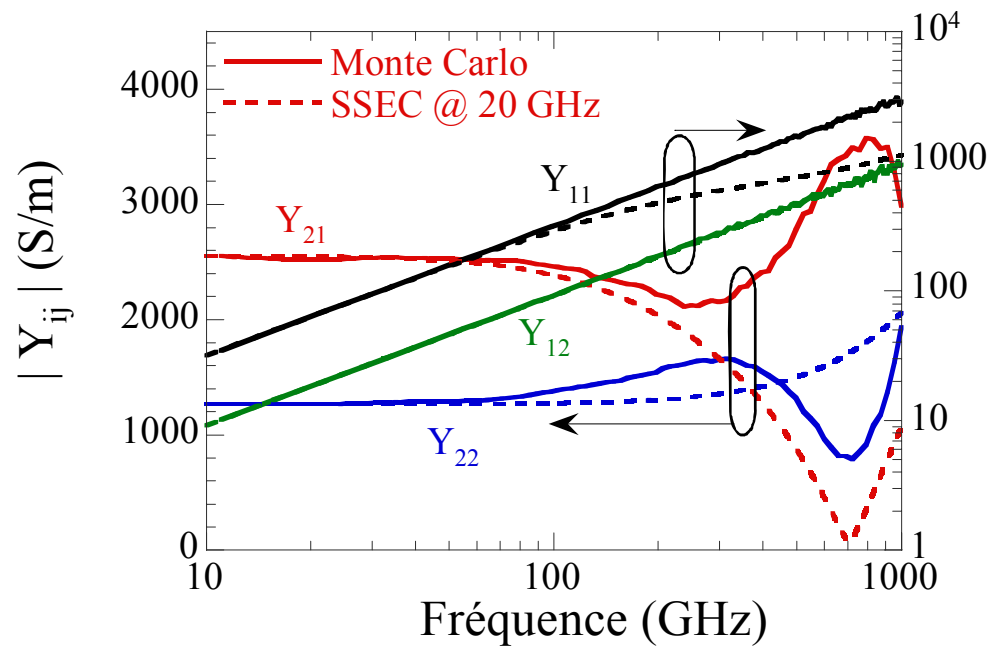

Figure 93 Comparaison des paramètres $Y_{\mathrm{ij}}$ calculés par le modèle SSEC extrait à $20 \mathrm{GHz}$ et des valeurs directement issues de la simulation Monte Carlo dans une plage de fréquence 10-100 GHz pour le MOS-ThinBody. $\mathrm{V}_{\mathrm{GS}}=0,6 \mathrm{~V}$ et $\mathrm{V}_{\mathrm{DS}}=0,2 \mathrm{~V}$

\begin{tabular}{|c|c|c|c|c|}
\hline $\begin{array}{c}\text { SSEC } \\
@ 20 \mathrm{GHz}\end{array}$ & $\begin{array}{c}\text { MOS- } \\
\text { Bulk }\end{array}$ & $\begin{array}{c}\text { MOS- } \\
\text { ThinBody }\end{array}$ & $\begin{array}{c}\text { MOS- } \\
\text { HEMT }\end{array}$ & $\begin{array}{c}\text { MOS- } \\
\text { COMB }\end{array}$ \\
\hline $\mathrm{C}_{\mathrm{GS}}(\mathrm{pF} / \mathrm{m})$ & 360 & 360 & 380 & 380 \\
\hline $\mathrm{C}_{\mathrm{GD}}(\mathrm{pF} / \mathrm{m})$ & 180 & 150 & 150 & 160 \\
\hline $\mathrm{C}_{\mathrm{GC}}(\mathrm{pF} / \mathrm{m})$ & 540 & 510 & 530 & 540 \\
\hline $\mathrm{C}_{\mathrm{DS}}(\mathrm{pF} / \mathrm{m})$ & 160 & 120 & 170 & 120 \\
\hline $\mathrm{G}_{\mathrm{m}}(\mathrm{S} / \mathrm{m})$ & 1740 & 2560 & 3180 & 4120 \\
\hline $\mathrm{G}_{\mathrm{d}}(\mathrm{S} / \mathrm{m})$ & 960 & 1265 & 900 & 1710 \\
\hline $\mathrm{R}_{\mathrm{i}}(\Omega \cdot \mathrm{m})$ & 0,0022 & 0,0021 & 0,0018 & 0,0014 \\
\hline$\tau(\mathrm{ps})$ & 1,5 & 1,4 & 1,15 & 0,85 \\
\hline
\end{tabular}

Tableau 8 Eléments du modèle SSEC pour les quatre MOSFET étudiés. L'extraction est faite à $20 \mathrm{GHz}$ pour un point de fonctionnement en DC tel que $V_{G S}$ corresponde au maximum de transconductance à $\mathrm{V}_{\mathrm{DS}}=0,2 \mathrm{~V}$.

Enfin, on note que le canal est moins résistif et le retard du transistor diminué dans les MOS-HEMT et surtout MOS-COMB, grâce à un transport qui tend de plus en plus vers le 
régime balistique. Cela est très approprié pour obtenir une faible consommation dynamique de puissance.

\section{4-4 Performance intrinsèque en fréquence}

Traditionnellement, un transistor RF est caractérisé par des facteurs de mérite tels que la fréquence $\mathrm{f}_{\mathrm{T}}$ de transition (ou de coupure) du gain en courant et la fréquence maximale $f_{\text {MAX }}$ d'oscillation du gain en puissance unilatéralisé. Nous allons rappeler brièvement les principaux gains des transistors RF en définissant ces deux critères en fréquence.

\section{4-4-1 Gain en courant et fréquence de transition $f_{T}$}

Le courant et la tension d'un quadripôle sont définis dans la Fig. 94-a. Si on considère une configuration en source commune (Fig. 94-b) :

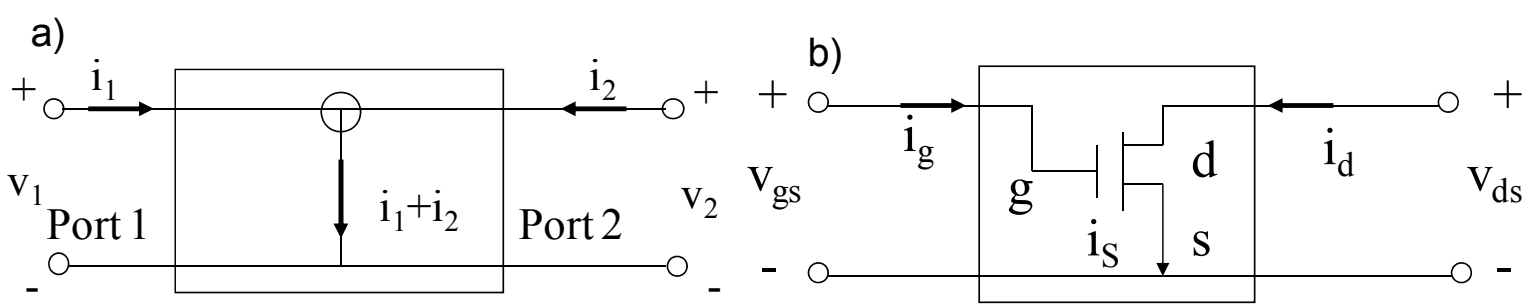

Quadripôle deux ports

Figure 94 Représentations petit signal du réseau (network) du quadripôle à deux ports. En (a), transistor avec trois terminaux et en (b) transistor sous une configuration source commune.

Les paramètres Y permettent d'écrire en petit signal :

$$
\begin{aligned}
& i_{g}=y_{g g}(\omega) v_{g s}+y_{g d}(\omega) v_{d s} \\
& i_{d}=y_{d g}(\omega) v_{g s}+y_{d d}(\omega) v_{d s}
\end{aligned}
$$

La fréquence de transition du gain en courant $\mathrm{f}_{\mathrm{T}}\left(\omega_{T} / 2 \pi\right)$ est définie comme celle pour laquelle le gain en courant avec sortie en court-circuit est égale à l'unité.

$$
h_{21}\left(\omega_{T}\right)=\frac{\left|y_{21}\left(\omega_{T}\right)\right|}{\left|y_{11}\left(\omega_{T}\right)\right|}=\frac{\left|y_{d g}\left(\omega_{T}\right)\right|}{\left|y_{g g}\left(\omega_{T}\right)\right|}=1
$$

Dans cette configuration, le gain en courant peut être approché à basse fréquence par :

$$
\frac{\left|y_{21}(\omega)\right|}{\left|y_{11}(\omega)\right|}=\frac{\left|y_{d g}(\omega)\right|}{\left|y_{g g}(\omega)\right|} \propto \frac{G_{m}}{\omega \cdot C_{G G}}
$$

Ce gain diminue en fonction de la fréquence avec une pente de $-20 \mathrm{~dB} / \mathrm{dec}$, la fréquence $\mathrm{f}_{\mathrm{T}}$ peut être aisément déterminée par extrapolation par rapport à cette tendance ou l'expression dite "quasi - statique". Pour un canal long, c'est-à-dire $L_{G}>\left(V_{G}-V_{T}\right) / F_{C}$, où $F_{C}$ est le champ 
critique sous lequel les porteurs peuvent atteindre leur vitesse effective de saturation. Le maximum de $G_{m}$ peut être exprimé en région du pincement (pinch region) comme :

$$
G_{m_{-} \max }(\text { Pinch }) \approx \frac{\mu C_{G G} W_{G}}{L_{G}}\left(V_{G}-V_{T}\right)
$$

La fréquence de transition du gain en courant $\mathrm{f}_{\mathrm{T}}$ est approchée par la relation :

$$
\omega_{T}=2 \pi f_{T} \approx \frac{\mu}{L_{G}^{2}}\left(V_{G}-V_{T}\right)
$$

c'est-à-dire que $\mathrm{f}_{\mathrm{T}}$ varie en $1 / L_{G}^{2}$. Pour un canal court, c'est-à-dire $\mathrm{L}_{\mathrm{G}}<\left(\mathrm{V}_{\mathrm{G}}-\mathrm{V}_{\mathrm{T}}\right) / \mathrm{F}_{\mathrm{C}}$, le maximum de $G_{m}$ sature à :

$$
G_{m_{-} \max }(\text { Saturation }) \approx v_{S} C_{G G} W_{G}
$$

Le maximum de $G_{m}$ peut être exprimé par la vitesse effective de saturation. Cette fois, la fréquence $\mathrm{f}_{\mathrm{T}}$ est approchée par :

$$
\omega_{T}=2 \pi f_{T} \approx \frac{v_{S}}{L_{G}}
$$

et donc $\mathrm{f}_{\mathrm{T}}$ varie en $1 / L_{G}$. On peut noter que, dans tous les cas, la fréquence de transition du gain en courant devrait être contrôlée par la longueur de grille. Pour un canal long, elle dépendra aussi de la mobilité effective du canal, mais pour un canal court, elle dépendra de la vitesse effective de saturation.

\section{4-4-2 MAG et MSG}

En RF, on utilise également le gain transducique maximum en puissance, qui peut correspondre soit au «maximum available power gain» (MAG), soit au «maximum stable gain» (MSG). Le gain transducique en puissance $G_{T}$ est quant à lui défini comme le rapport entre la puissance délivrée à la charge $P_{L}$ et la puissance disponible par la source $P_{S}: G_{T}=P_{L} / P_{S}$, dans la situation définie sur la Fig. 95.

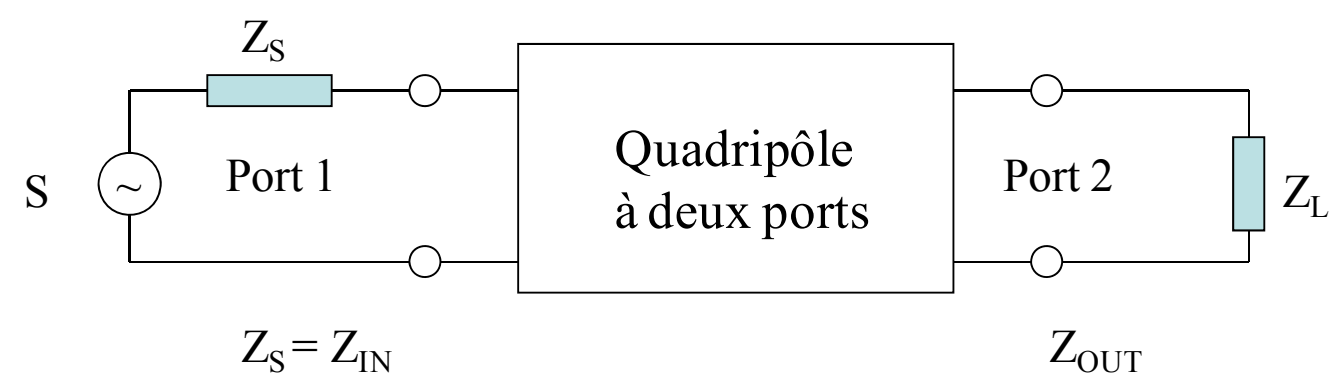

Figure 95 Quadripôle à deux ports avec les impédances de source et de charge. 
Le maximum du gain en puissance est obtenu (s'il existe) par une adaptation simultanée entre entrée et en sortie pour obtenir une adaptation conjuguée. Physiquement, cette adaptation signifie que l'impédance de la source $Z_{S}$ et l'impédance de la charge $Z_{L}$ satisfont simultanément les conditions telles que $Z_{S}=Z_{I N}^{*}$ et $Z_{L}=Z_{\text {OUT }}^{*}$, où $Z_{I N}$ est l'impédance d'entrée mesurée au port $1 \mathrm{du}$ quadripôle avec l'impédance de charge $Z_{L}$ connectée au port 2. $Z_{\text {OUT }}$ est l'impédance de sortie mesurée au port 2 du quadripôle avec l'impédance de source $Z_{S}$ connectée au port 1. Dans ces conditions, le maximum du gain transducique en puissance $\left(\mathrm{MAG}\right.$ ou $\left.G_{T, M A X}\right)$ peut s'écrire [Rollett1962IRE] :

$$
M A G=\frac{\left|y_{21}\right|^{2}}{2 \mathfrak{R}\left(y_{11}\right) \mathfrak{R}\left(y_{22}\right)-\mathfrak{R}\left(y_{12} y_{21}\right)+\left\{\left[2 \mathfrak{R}\left(y_{11}\right) \mathfrak{R}\left(y_{22}\right)-\mathfrak{R}\left(y_{12} y_{21}\right)\right]^{2}-\left|y_{12} y_{21}\right|^{2}\right\}^{1 / 2}}
$$

Il peut être réécrit comme :

$$
M A G=\frac{|y 21|}{|y 12|}\left[K-\left(K^{2}-1\right)^{1 / 2}\right]
$$

où K est le facteur de stabilité de "Rollet" :

$$
K=\frac{2 \mathfrak{R}\left(y_{11}\right) \mathfrak{R}\left(y_{22}\right)-\mathfrak{R}\left(y_{12} y_{21}\right)}{\left|y_{21} y_{12}\right|}
$$

Le maximum du gain transducique en puissance (MAG) existe si le facteur de stabilité K est supérieur à 1. Quand le facteur de stabilité est inférieur à 1 , le fonctionnement du transistor est instable. Pour le stabiliser, on peut utiliser par exemple une charge résistive (résistance série ou parallèle sur le port d'entrée ou de sortie) ou une charge de contre-réaction [Gupta1992IEEE]. Le Maximum Stable Gain (MSG) est défini comme le MAG mais avec $\mathrm{K}=1: M S G=\left|y_{21}\right| /\left|y_{12}\right|$.

\section{4-4-3 Gain unilatéral en puissance et fréquence $f_{\text {MAX }}$}

Le gain unilatéral en puissance U (dérivé par S. J. Mason [Mason1954IRE]) du transistor est également souvent utilisé pour caractériser les performances à haute fréquence.

$$
U=\frac{\left|y_{21}-y_{12}\right|^{2}}{4 \cdot\left[\mathfrak{R}\left(y_{11}\right) \mathfrak{R}\left(y_{22}\right)-\mathfrak{R}\left(y_{12}\right) \mathfrak{R}\left(y_{21}\right)\right]}
$$

Il représente le maximum du gain transducique en puissance (MAG) quand le transistor est unilatéralisé $\left(\mathrm{y}_{12}=\mathrm{z}_{12}=\mathrm{s}_{12}=0\right)$ en utilisant la technique de contre-réaction sans perte (lossless 
feedback technique). La contre-réaction d'un circuit pour unilatéraliser le dispositif avec trois terminaux a été proposée par Mason et améliorée par Gupta [Gupta1992IEEE]. A faible fréquence, $\mathfrak{R}\left(y_{11}\right) \propto \omega^{2}, \mathfrak{R}\left(y_{12}\right) \propto-\omega^{2}, y_{22}=G_{d}$ et $Y_{21}=G_{m}$, le gain unilatéral $\mathrm{U}$ doit satisfaire $U(\omega) \propto 1 / \omega^{2}$. La fréquence maximale d'oscillation $\mathrm{f}_{\mathrm{MAX}}$ est définie comme celle pour laquelle U est égal à l'unité. On note que le gain MAG est aussi égal à l'unité pour cette fréquence. On peut donc trouver ce facteur de mérite via le gain U ou le MAG si le transistor est inconditionnellement stable [Gupta1992IEEE].

\section{4-4-4 Résultats pour $h_{21}$ et $f_{T}$}

Nous allons comparer les gains en court-circuit en courant $\mathrm{h}_{21}$ calculés directement par la simulation $\mathrm{MC}$ et par le modèle $\mathrm{SSEC}$ extrait à $20 \mathrm{GHz}$, puis les fréquences $\mathrm{f}_{\mathrm{T}}$ estimées par différentes méthodes: simulation $\mathrm{MC}$, modèle $\mathrm{SSEC}$, extrapolation à $-20 \mathrm{~dB} / \mathrm{dec}$ et approximation quasi-statique (QS, donnée par $G_{m} / 2 \pi \cdot\left(C_{G S}+C_{G D}\right)$ ). Un exemple des résultats est donné sur la Fig. 96 pour le transistor MOS-Bulk.

Les évolutions de $\mathrm{h}_{21}$ en fonction de la fréquence données par $\mathrm{MC}$, le SSEC et l'extrapolation de la pente à $-20 \mathrm{~dB} / \mathrm{dec}$ sont en très bon accord si $\mathrm{f}<200 \mathrm{GHz}$. Les différences au delà montrent que les effets de réactance vont jouer un rôle de plus en plus important à haute fréquence et cet effet non quasi-statique n'est pas reproduit par les éléments du modèle SSEC. La détermination de $\mathrm{f}_{\mathrm{T}}$ via le modèle SSEC, l'approximation quasi-statique ou l'extrapolation à $-20 \mathrm{~dB} /$ déc devient discutable. Le tableau 9 ci-dessous résume les différentes valeurs de $\mathrm{f}_{\mathrm{T}}$ issues des méthodes évoquées pour le MOS-Bulk. On note que les fréquences $\mathrm{f}_{\mathrm{T}}$ obtenues par l'approximation quasi-statique et par l'extrapolation à $-20 \mathrm{~dB} / \mathrm{déc}$ sont très similaires, mais un peu sous-estimées par rapport au calcul direct par MC. Le modèle SSEC donne une valeur encore plus faible. 


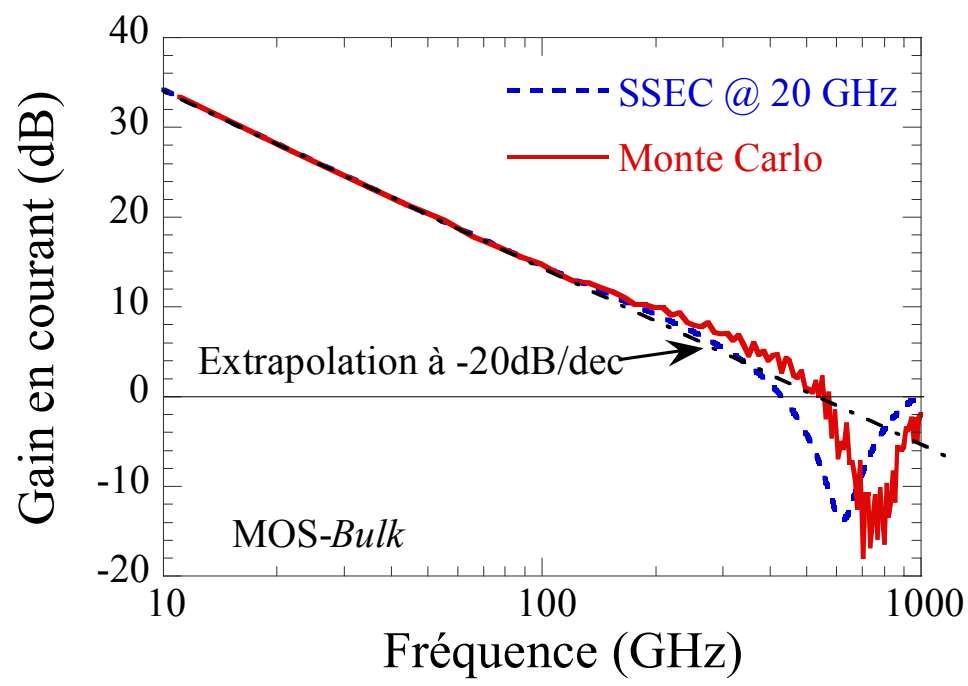

Figure 96 Gain en courant $h_{21}$ pour une sortie en court circuit en fonction de la fréquence pour MOS-Bulk en utilisant le calcul direct par simulation Monte Carlo et le modèle SSEC extrait à 20 GHz.

L'extrapolation de la pente à $-20 \mathrm{~dB} /$ décade est aussi tracée (pointillés-tirets noirs)

\begin{tabular}{|c|c|c|c|c|}
\hline Méthode & Monte Carlo & Modèle SSEC & Quasi-statique & Extrapolation \\
\hline $\mathrm{f}_{\mathrm{T}}(\mathrm{GHz})$ & 560 & 430 & 510 & 520 \\
\hline
\end{tabular}

Tableau 9 Fréquence de transition $\mathbf{f}_{\mathrm{T}}$ obtenue par différentes méthodes pour le MOS-Bulk.

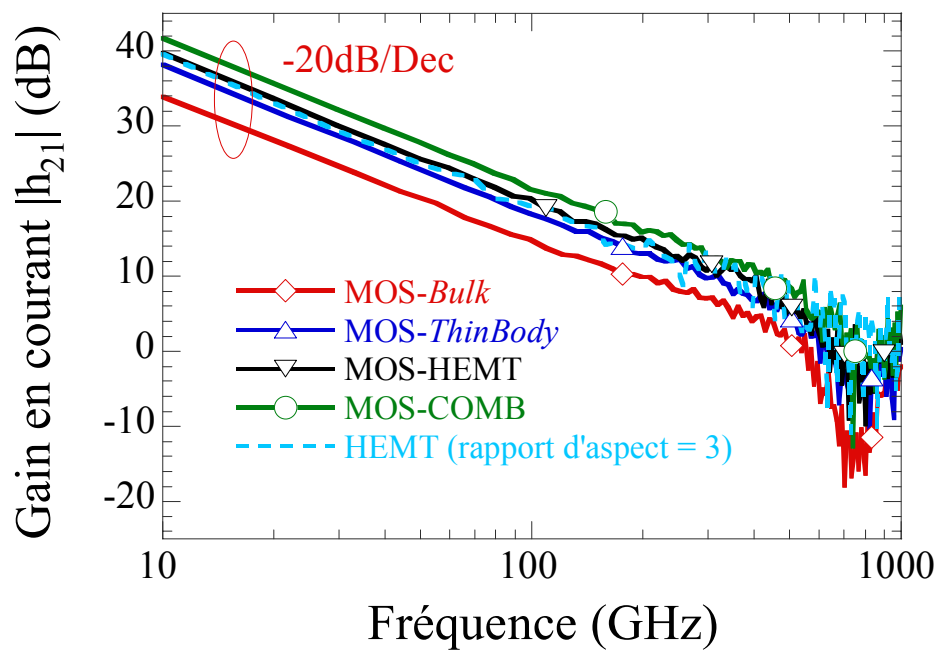

Figure 97 Gain en courant de court-circuit $h_{21}$ en fonction de la fréquence pour des structures MOSFET et HEMT (rapport d'aspect = 3) de longueur de grille égale à $50 \mathrm{~nm}$. Résultats de simulation Monte Carlo.

Comparons désormais l'ensemble des dispositifs simulés avec une longueur de grille de $50 \mathrm{~nm}$. La structure de référence est un HEMT de $50 \mathrm{~nm}$ avec exactement la même structure de couche que celui de $70 \mathrm{~nm}$ décrit dans la section de calibration, avec fort taux d'indium - 80\% - dans le canal. Pour les structures de type HEMT, le paramètre physique à optimiser est le rapport d'aspect, c'est-à-dire que le ratio entre la distance séparant la grille et 
le canal (somme des épaisseurs de barrière Schottky, de la couche de $\delta$ dopage et de l'espaceur) et la longueur effective de la grille. Pour la structure de référence, ce paramètre est fixé à 3 , nous l'avons fait varier entre 3 et 5 pour améliorer les performances. Les résultats sur $\mathrm{h}_{21}$ ou $\mathrm{f}_{\mathrm{T}}$ sont globalement montrés sur les Fig. 97 et 98 :

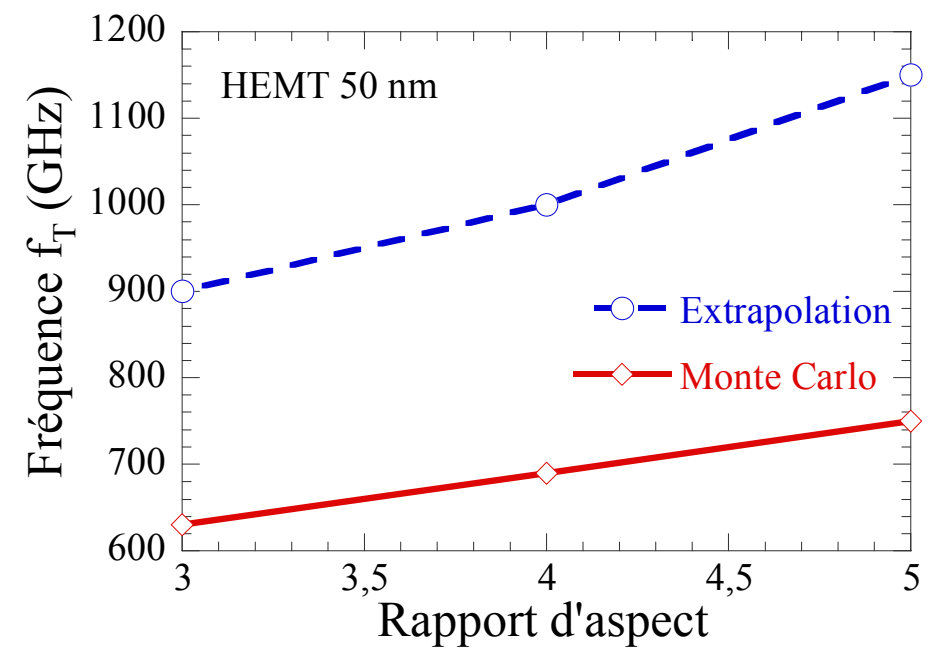

Figure 98 Fréquence de transition $f_{T}$ en fonction du rapport d'aspect pour la structure de type HEMT $\operatorname{avec} \mathrm{L}_{\mathrm{G}}=\mathbf{5 0} \mathrm{nm}$.

Le HEMT de référence avec rapport d'aspect 3 est plus performant que MOS-Bulk, mais de fortes améliorations sont obtenues dans les MOSFET alternatifs. Comme prévu et présenté sur la Fig. 98, la valeur de $\mathrm{f}_{\mathrm{T}}$ pour le HEMT peut être améliorée par augmentation du rapport d'aspect. Le gain est toutefois moins important sur la valeur directement issue de MC que sur celle correspondant à l'extrapolation par rapport à la tendance à $-20 \mathrm{~dB} / \mathrm{déc}$. Le tableau 10 ci-dessous résume ce benchmark de la performance fréquentielle pour nos structures FET.

\begin{tabular}{|c|c|c|}
\hline $\mathbf{L}_{\mathbf{G}}=50 \mathrm{~nm}$ & $\begin{array}{c}\mathbf{f}_{\mathrm{T}} \text { extrap } \\
(\mathrm{GHz})\end{array}$ & $\begin{array}{c}\mathbf{f}_{\mathrm{T}} \mathrm{MC} \\
(\mathrm{GHz})\end{array}$ \\
\hline MOS-Bulk & 530 & 520 \\
\hline MOS-ThinBody & 800 & 600 \\
\hline MOS-HEMT & 990 & 690 \\
\hline MOS-COMB & 1230 & 750 \\
\hline HEMT (rap $=3$ ) & 900 & 630 \\
\hline HEMT (rap $=4$ ) & 1000 & 690 \\
\hline HEMT (rap $=5$ ) & 1150 & 750 \\
\hline
\end{tabular}

Tableau 10 Fréquences de transition obtenues par extrapolation et calculs directs MC pour les HEMT et MOSFET étudiés. Ces dispositifs ont la même longueur de grille $L_{G}=50 \mathrm{~nm}$. 
La meilleure performance est atteinte avec MOS-COMB, elle est comparable voire audelà des caractéristiques d'un HEMT de même longueur de grille avec grand rapport d'aspect et à plus fort taux d'indium. Nous avons évalué pour cette structure l'impact de la longueur de grille $\mathrm{L}_{\mathrm{G}}$ sur $\mathrm{f}_{\mathrm{T}}$, comme reporté sur la Fig. 99.
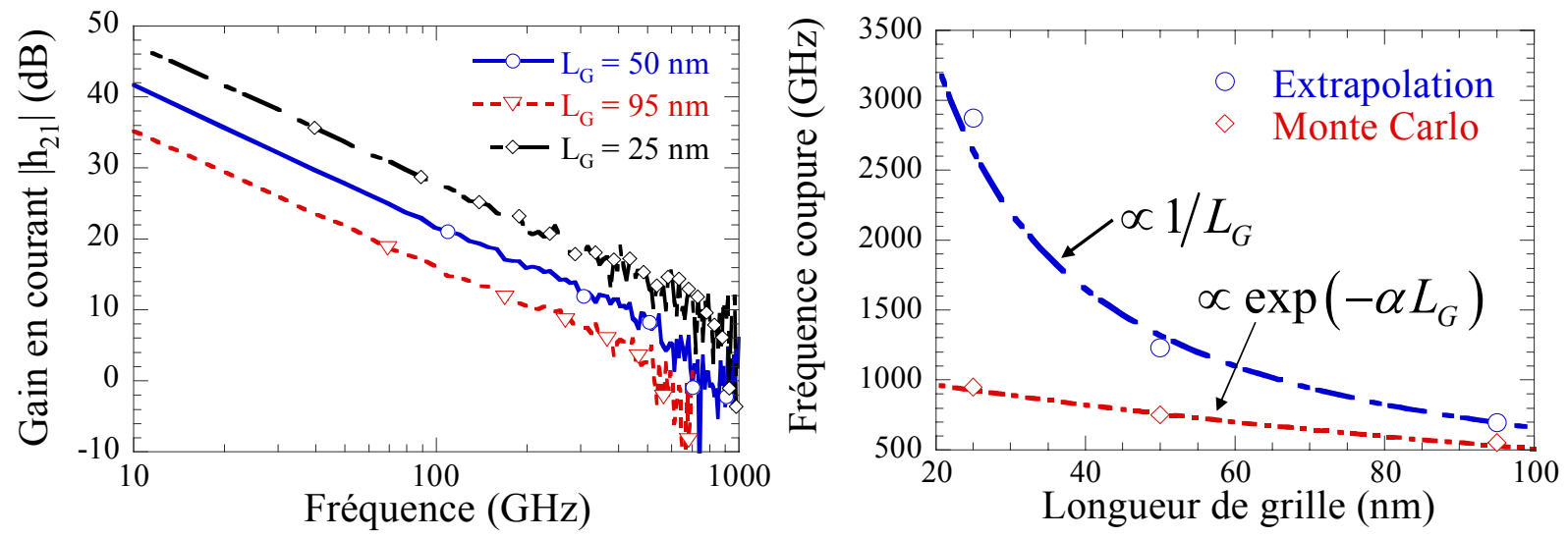

Figure 99 Gauche : Gain de court circuit en courant $h_{21}$ en fonction de la fréquence pour la structure MOS-COMB. Droite : Fréquence de transition $\mathrm{f}_{\mathrm{T}}$ obtenue par calcul direct MC et extrapolation en fonction de $L_{G}$.

En approximation quasi statique, le maximum de la fréquence de transition pour une faible $\mathrm{L}_{\mathrm{G}}$ est proportionnel à $\omega_{T}=2 \pi f_{T} \propto v_{S} / L_{G}$ où $v_{\mathrm{S}}$ est la vitesse limite atteinte par les électrons en régime de saturation. Cette dépendance en $1 / L_{G}$ est observée si on détermine $\mathrm{f}_{\mathrm{T}}$ par extrapolation par rapport à la pente à $-20 \mathrm{~dB} /$ décade. Cependant, les effets non quasi statiques sont prépondérants en très haute fréquence. La fréquence $\mathrm{f}_{\mathrm{T}}$ déterminée directement par la simulation MC ne suit pas du tout cette prédiction et varie comme une fonction en $\exp \left(-\alpha \mathrm{L}_{\mathrm{G}}\right)$ avec un très faible coefficient $\alpha$, qui peut donc être donc approchée par une fonction linéaire de $-\mathrm{L}_{\mathrm{G}}$.

\section{4-4-5 Résultats pour $U$ (Gain Unilatéral) et $f_{\text {MAX }}$}

Dans cette section, nous allons étudier le comportement du gain unilatéral en fonction de la fréquence ainsi que la fréquence maximale d'oscillation $\mathrm{f}_{\mathrm{MAX}}$.

Dans un premier temps, nous allons nous intéresser au domaine de validité du modèle SSEC. Comme déjà vu, quand la fréquence augmente au-delà de $100 \mathrm{GHz}$, le circuit équivalent déterminé à plus basse fréquence ne peut plus être utilisé, c'est-à-dire que les éléments du modèle SSEC dépendent considérablement de la fréquence. A haute fréquence, les effets intrinsèques non quasi-statiques deviennent de plus en plus importants, cela oblige à prendre en compte la transcapacité $C_{D C}$ [Steer1986IEEE] et [Mateos2004IEEE-A]. La création 
du domaine dipolaire à l'origine de $C_{D C}$ avait été déjà observée par simulation MC pour une structure d'HEMT déca-nanométrique dans le régime de saturation du courant. Elle résultait de la présence à la fois d'une zone de charge d'espace en fin de canal et d'une accumulation d'électrons lents ayant transféré vers les vallées latérales [Dollfus1993JAP]. Cet effet a une forte influence sur le gain du transistor et pourrait opposer une limite fondamentale à la montée en fréquence. La prédiction traditionnelle basée sur une extrapolation du comportement de basse fréquence à $-20 \mathrm{~dB} /$ décade qui néglige ces phénomènes est alors tout à fait discutable.

Nous calculons les paramètres $\mathrm{Y}$ du modèle en utilisant le schéma électrique équivalent $\mathrm{du}$ transistor intrinsèque proposé en Fig. 23, pour en déduire une formule analytique du gain unilatéral $U$ en fonction de la fréquence et des éléments du SSEC. Pour les fréquences telles que $f<\left[2 \pi R_{i}\left(C_{G S}+C_{D C}\right)\right]^{-1}$ et avec $\tau \gg R_{i} C_{D C}$ et $\tau \gg R_{i} C_{G S}$ où $\tau$ est le temps de retard du transistor, U peut s'écrire comme [Steer1986IEEE] (les calculs détaillés sont fournis en Annexe F) :

$$
U \approx\left[\frac{G_{m}^{0^{2}} / G_{d}}{4 C_{G S} R_{i}\left(C_{G S}-C_{D C} G_{m}^{0} / G_{d}\right)}\right]\left[\frac{1}{\omega^{2}\left(1-p_{d}^{2} \omega^{2}\right)}\right]
$$

avec :

$$
p_{d}^{2}=\frac{\left(R_{i}^{2} C_{G S}\right)\left(C_{D C}+C_{G S}\right)^{2}+C_{D C} G_{m}^{0} \tau^{2} / 2 G_{d}}{\left(C_{D C} G_{m}^{0} / G_{d}-C_{G S}\right)}
$$

En négligeant $C_{D C}, \mathrm{U}$ devient à basse fréquence (soit $f<\left[2 \pi R_{i} C_{G S}\right]^{-1}$ ) :

$$
U \approx \frac{G_{m}^{0^{2}} / G_{d}}{4 \omega^{2} C_{G S}^{2} R_{i}}
$$

Le comportement en basse fréquence (-20dB/décade) est dû au terme en $1 / \omega^{2}$. Si $C_{D C}$ est prise en compte, l'ordre du système est augmenté à cause du terme $1 /\left(1-p_{d}^{2} \omega^{2}\right)$, le comportement de décroissance deviendrait donc de $-40 \mathrm{~dB} /$ décade, c'est-à-dire qu'on ajoute un autre pôle complexe conjugué à une fréquence $f_{p}$ :

$$
f_{p}=\frac{1}{2 \pi\left|p_{d}\right|}
$$

Pour les transistors en RF, cette fréquence du pôle est typiquement plus faible que $\mathrm{f}_{\mathrm{MAX}}$, donc elle peut influencer fortement le comportement du gain $U$. 

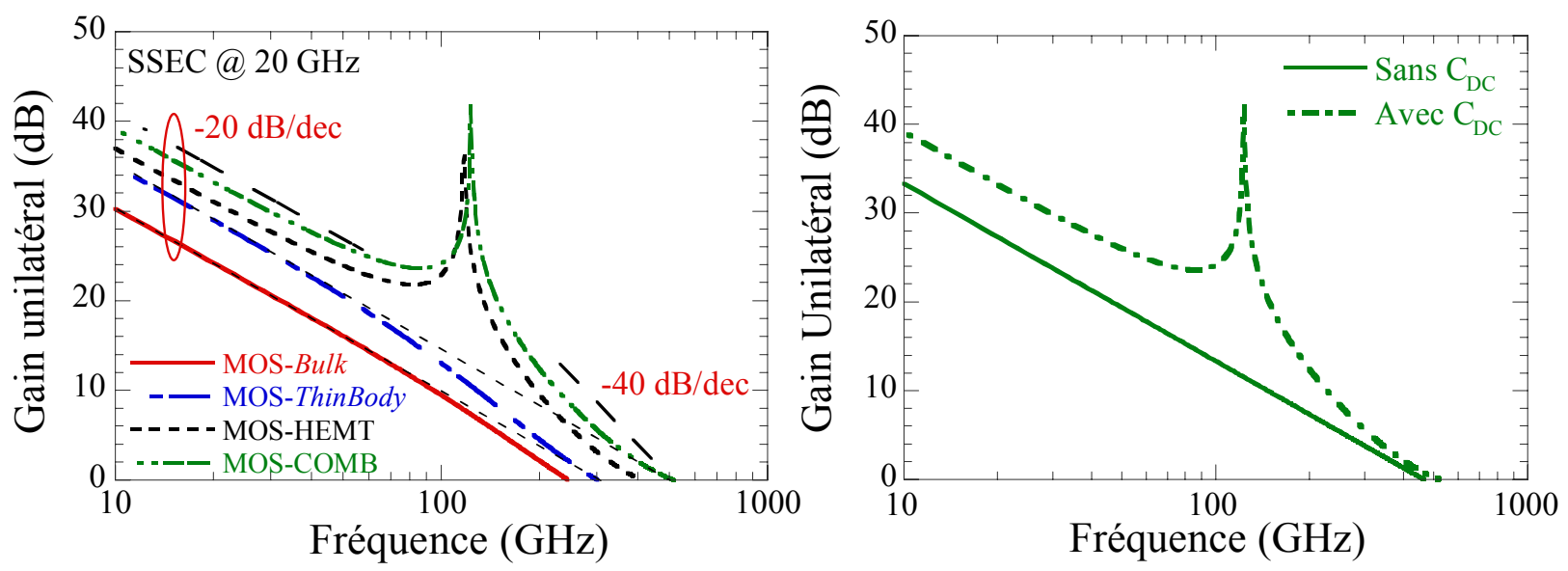

Figure 100 Gauche : Le gain unilatéral $U$ calculé par le modèle SSEC extrait à $20 \mathrm{GHz}$ pour les MOSFET étudiés. Droite : $\mathrm{U}$ dans la structure MOS-COMB sans et avec $C_{D C}=200 \mathrm{pF} . \mathrm{m}^{-1}$.

Les résultats des analyses sur le modèle SSEC sont montrés sur la Fig. 100. Les valeurs de $C_{D C}$ sont extraites à l'aide des paramètres $\mathrm{Y}$ calculés directement par la simulation MC [Tang2010SSE] :

$$
C_{D C} \approx \frac{\mathfrak{I}\left(y_{12}\right)-\mathfrak{I}\left(y_{21}\right)}{\omega}
$$

On observe que dans la structure MOS-COMB, $C_{D C}$ augmente le gain dans la gamme de basse fréquence, mais il ne modifie quasiment pas la valeur de $f_{\mathrm{MAX}}$. A faible fréquence, l'effet de cette contre-réaction interne apportée par $C_{D C}$ est représenté par le dénominateur de l'équation III-41 : $\left(C_{G S}-C_{D C} G_{m}^{0} / G_{d}\right)$. Il peut être positif ou négatif. Le gain à la fréquence inférieure à $f_{p}$ a une valeur finie parce que la boucle de transmission est complexe et que $\mathrm{U}$ décroît selon $-20 \mathrm{~dB} /$ décade comme il est dominé par le pôle $1 / \omega^{2}$. Si la fréquence est égale à $f_{p}$, la fonction de transfert de la boucle de transmission devient majoritairement réelle et $U$ devient très grand. Au-delà de $f_{p}$ la boucle de transmission redevient complexe et $\mathrm{U}$ décroît maintenant selon $-40 \mathrm{~dB} /$ décade à cause du pôle $1 /\left(1-p_{d}^{2} \omega^{2}\right)$. Par ailleurs, $\mathrm{U}$ montre un pic de résonance à la fréquence de $125 \mathrm{GHz}\left(f_{p}=119 \mathrm{GHz}\right.$ dans cette structure) quand l'effet de la contre-réaction qui rend le dénominateur de l'équation III-41 inférieur à zéro, soit $C_{G S} \leq\left(G_{m}^{0} / G_{d}\right) C_{D C}$. Pour que cette condition soit satisfaite, il faut que (i) les éléments parasites soient négligeables, c'est-à-dire qu'il ne peut être observé que pour le composant intrinsèque; (ii) le composant représente simultanément un fort gain de circuit ouvert en tension $G_{m}^{0} / G_{d}$ et une transcapacité $C_{D C}$ non négligeable devant $C_{G S}$. Visiblement cette 
condition est vérifiée dans les transistors MOS-COMB et MOS-HEMT. Dans ce cas là et comme montré sur le tableau 11, la différence est très grande entre la valeur 'exacte' de $f_{\text {MAX }}$ (juste pour le modèle SSEC) et la valeur extrapolée.

\begin{tabular}{|c|c|c|}
\hline $\begin{array}{c}\mathbf{L}_{\mathbf{G}}=\mathbf{5 0} \mathbf{n m} \\
\text { Modèle SSEC }\end{array}$ & $\begin{array}{c}\mathbf{f}_{\text {MAX }} \text { MC } \\
(\mathrm{GHz})\end{array}$ & $\begin{array}{c}\mathbf{f}_{\text {MAX }} \text { extrap } \\
(\mathrm{GHz})\end{array}$ \\
\hline MOS-Bulk & 220 & 310 \\
\hline MOS-ThinBody & 300 & 500 \\
\hline MOS-HEMT & 420 & $\mathbf{8 2 0}$ \\
\hline MOS-COMB & 520 & 1100 \\
\hline
\end{tabular}

Tableau 11 Fréquences maximales d'oscillation $\mathbf{f}_{\text {MAX }}$ exactes et extrapolées par rapport à la tendance en -20 dB/déc pour les MOSFET étudiés. Calculs effectués à partir des modèles SSEC extraits à $20 \mathrm{GHz}$. Cependant, le gain $U$ estimé en utilisant les paramètres $Y$ directement calculés par la simulation Monte Carlo donne un écart par rapport aux résultats issus du modèle SSEC. Les résultats obtenus par MC sont montrés sur la Fig. 101 :

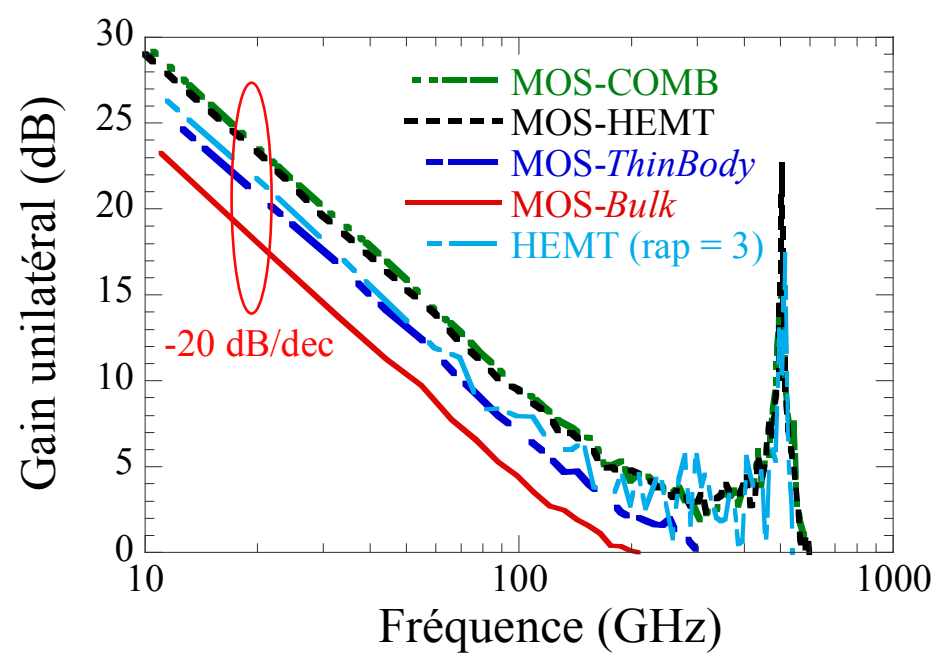

Figure 101 Gain unilatéral $U$ calculé en utilisant les paramètres $Y$ directement issus de la simulation MC pour les MOSFET et HEMT étudiés avec $\mathrm{L}_{\mathrm{G}}=\mathbf{5 0} \mathrm{nm}$.

Le pic de résonance se trouve cette fois aux alentours de $500 \mathrm{GHz}$ pour les dispositifs MOSCOMB, MOS-HEMT et HEMT, alors que le modèle SSEC prévoyait $120 \mathrm{GHz}$ ). Le comportement à faible fréquence est correspond bien à une décroissance en $-20 \mathrm{~dB} /$ décade. Le tableau 12 permet de résumer les performances fréquentielles pour toutes les structures envisagées. Cette fois, les fréquences extrapolées sous-estiment $f_{\text {MAX }}$ par rapport aux calculs directs. Visiblement, le schéma électrique équivalent du transistor intrinsèque proposé en Fig. 91 ne permet pas de rendre compte de tous les effets non quasi-statiques. Par ailleurs, il est intéressant d'étudier des fréquences $\mathrm{f}_{\mathrm{p}}$ et $\mathrm{f}_{\mathrm{MAX}}$ en fonction de la longueur de grille. Les résultats pour MOS-COMB sont montrés sur la Fig. 102. 


\begin{tabular}{|c|c|c|}
\hline $\begin{array}{c}\mathbf{L}_{\mathbf{G}}=\mathbf{5 0} \mathbf{n m} \\
\text { Monte Carlo }\end{array}$ & $\begin{array}{c}\mathbf{f}_{\text {MAX }} \text { MC } \\
(\mathbf{G H z})\end{array}$ & $\begin{array}{c}\mathbf{f}_{\text {MAX }} \text { extrap } \\
(\mathbf{G H z})\end{array}$ \\
\hline MOS-Bulk & $\mathbf{2 0 0}$ & $\mathbf{1 5 5}$ \\
\hline MOS-ThinBody & $\mathbf{3 0 0}$ & $\mathbf{2 2 0}$ \\
\hline MOS-HEMT & $\mathbf{5 5 0}$ & $\mathbf{2 9 0}$ \\
\hline MOS-COMB & $\mathbf{6 0 0}$ & $\mathbf{3 1 5}$ \\
\hline HEMT (rap = 3 ) & $\mathbf{5 2 0}$ & $\mathbf{2 4 0}$ \\
\hline HEMT (rap $=\mathbf{4}$ ) & $\mathbf{5 9 0}$ & $\mathbf{2 7 5}$ \\
\hline HEMT (rap $=5$ ) & $\mathbf{6 2 0}$ & $\mathbf{3 3 0}$ \\
\hline
\end{tabular}

Tableau 12 Fréquences maximales d'oscillation obtenues par extrapolation à -20dB/décade et issues des calculs directs MC pour les dispositifs HEMT et MOSFET étudiés.
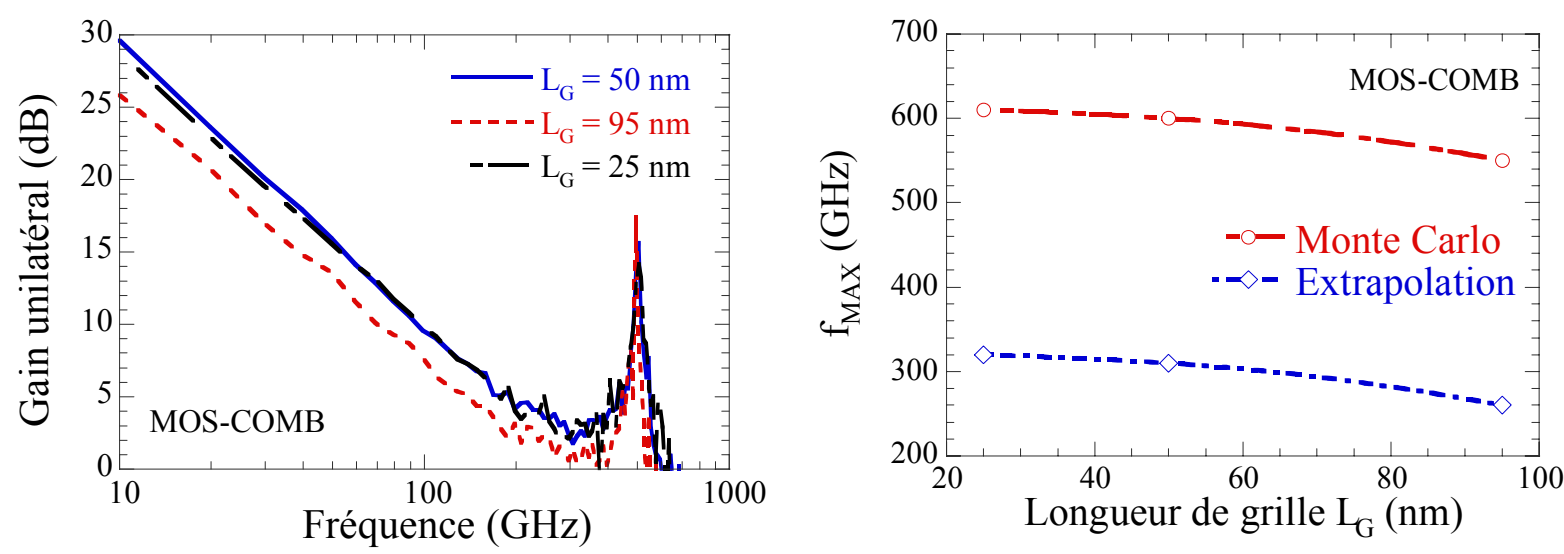

Figure 102 Structure MOS-COMB. Gauche : Gain unilatéral obtenu par MC en fonction de la fréquence pour trois longueurs de grille. Droite : Fréquence $f_{M A X}$ exacte et extrapolée en fonction de la longueur de grille $\mathbf{L}_{\mathbf{G}}$.

On observe que la fréquence de la résonance ne dépend quasiment pas de la longueur de grille. Cette fréquence $f_{p}$ ne semble dépendre ni de la géométrie, ni de la topologie du dispositif, elle pourrait être liée à une propriété intrinsèque du matériau de canal. Or, la formation du moment dipolaire de charges est liée à la mobilité différentielle négative des semiconducteurs III-V comme InGaAs. Elle dépend fortement de la fréquence et du champ appliqué, son effet va tendre vers zéro à très haute fréquence et sous fort champ électrique. La transcapacité $C_{D C}$ devrait donc être fortement atténuée à très haute fréquence. Le groupe de Luca Varani à l'université Montpellier 2 étudie le comportement de la mobilité différentielle d'InGaAs et d'InAs à haute fréquence par un modèle hydrodynamique. Ces études sont basées principalement sur les réponses dynamiques en vitesse et son énergie suite à une perturbation harmonique dépendant du temps [Sabatini2009Thèse]. Les résultats montrent que le tenseur de la mobilité différentielle d'InGaAs est complexe dans la représentation fréquentielle, sa partie réelle représente traditionnellement la dépendance linéaire entre la 
vitesse et le champ appliqué. A faible champ électrique, la partie réelle décrit une fonction Lorentzienne en fréquence, mais pour de forts champs, elle prend une forme gaussienne. On a en fait apparition d'un pic en fréquence avant la coupure correspondant à l'annulation de la partie réelle. Pour une plage de champ de 5 à $10 \mathrm{kV} / \mathrm{cm}$, ce pic se situe aux alentours de $500 \mathrm{GHz}$ pour une concentration de dopage de $10^{16} \mathrm{~cm}^{-3}$. La partie imaginaire traduit quant à elle le déphasage entre le champ et la vitesse qui est de plus en plus important au fur et à mesure que nous nous rapprochons de la fréquence de coupure. Ce déphasage limite l'efficacité du champ électrique sur la dynamique des porteurs qui répondent moins rapidement à la perturbation. Une forte valeur correspond de fait à une mobilité effective moins importante. Dans la plage de champ de 5 à $10 \mathrm{kV} / \mathrm{cm}$, elle tend vers zéro. Le pic de mobilité différentielle à environ $500 \mathrm{GHz}$ permet d'introduire une forte contre-réaction interne au sein du dispositif, en augmentant fortement la transcapacité liée au domaine dipolaire de charge. Cette explication est qualitative et préliminaire, les analyses plus profondes sur le champ et sur la densité de charge seront nécessaires dans un futur travail. Enfin, on peut observer une tendance à la saturation de $\mathrm{f}_{\mathrm{MAX}}$ autour de $600 \mathrm{GHz}$ quand on diminue la longueur de grille, de MOS-COMB.

\section{$5 \quad$ Performance $R F$ vs. dissipation de puissance}

Si nous connaissons les performances $\mathrm{RF}$ sur toute la plage de $\mathrm{V}_{\mathrm{GS}}$ à une faible valeur de $V_{\text {DS }}$ donnée, on peut estimer le meilleur compromis entre fréquence et dissipation de puissance sous mêmes conditions de tension d'alimentation $V_{D D}$ et d'état $O F F$. On fixe pour cela le courant $\mathrm{I}_{\mathrm{OFF}}$ pour une tension $\mathrm{V}_{\mathrm{DS}}$ donnée, puis on en déduit les informations en $\mathrm{DC}$ et en $\mathrm{AC}, \mathrm{I}_{\mathrm{ON}}$ et fréquences de mérite. Les résultats de ces analyses pour différents FET à base d'InGaAs sont illustrés sur la Fig. 103.

Le rendement entre rapidité et puissance dissipée à faible $V_{D S}$ d'une structure HEMT (avec rapport d'aspect 3 et $\operatorname{In}_{0,8} \mathrm{Ga}_{0,2} \mathrm{As}$ en canal) est augmenté par la topologie MOS (avec un canal de $\left.\mathrm{In}_{0,53} \mathrm{Ga},{ }_{47} \mathrm{As}\right)$, en particulier pour de très faibles consommations. Cette amélioration devrait être encore plus marquée si on augmentait le taux d'indium dans le canal des MOS. En effet, la structure HEMT a besoin d'un grand décalage en tension par rapport à $V_{T}\left(V_{G S}-V_{T}\right.$ $>0,6 \mathrm{~V})$ pour assurer de bonnes performances RF, ce qui est directement lié à la consommation. Les performances sont encore accrues avec les structures MOS alternatives. L'architecture MOS-COMB correspond au dispositif le plus rapide. Dans l'approximation quasi-statique, la fréquence $\mathrm{f}_{\mathrm{T}}$ intrinsèque est au-delà de $1 \mathrm{THz}$, ce qui correspond à la spécification RF de la feuille de route internationale des semi-conducteurs ITRS 2009 pour 
une longueur de grille de $10 \mathrm{~nm}$. Entre autres, ce transistor optimisé permet d'atteindre une fréquence de transition intrinsèque de $400 \mathrm{GHz}$ tout en maintenant une puissance dissipée inférieure à $20 \mathrm{~mW} / \mathrm{mm}$, ce qui est nécessaire pour un fonctionnement dans un système embarqué avec une autonomie raisonnable en énergie [Aarts2003DATE].

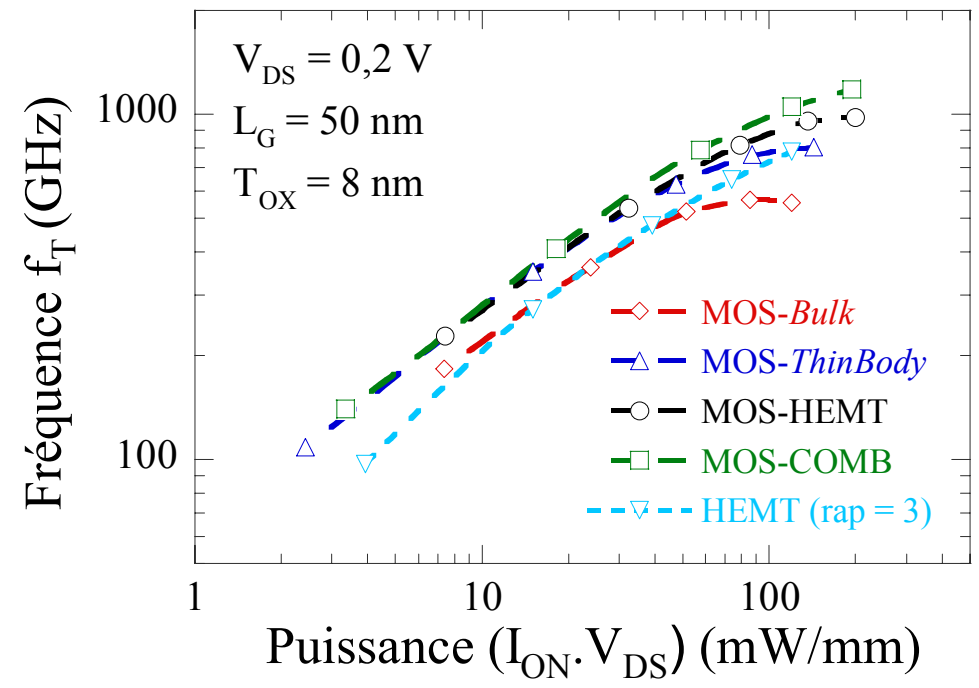

Figure 103 Fréquence de transition $f_{T}$ en fonction de la dissipation de puissance pour $V_{D D}$ de 0,2 à $0,6 \mathrm{~V}$ pour différentes architectures de FET III-V avec la même longueur de grille. $I_{O F F}$ est fixé à $0,2 \mathrm{~mA} / \mathrm{mm}$.

Le canal de la structure HEMT est à plus fort taux d'indium (80\%) que les MOSFET (53\%).

\section{Analyse de bruit intrinsèque en haute fréquence}

Pour compléter notre travail sur l'analyse fréquentielle du dispositif, nous allons étudier nos structures de type MOSFET à base de InGaAs du point de vue de leurs caractéristiques de bruit. En effet, ces nano-dispositifs doivent pouvoir fonctionner sous une puissance d'alimentation très limitée. Ils demandent une très large bande passante, d'où une forte transconductance qui amplifie la source du bruit thermique ou de diffusion [VanderZiel1986] à très faible $\mathrm{V}_{\mathrm{DS}}$ (dans le régime ohmique). L'étude du comportement de bruit et de ses causes possibles devient très importante puisqu'il ne faut pas que le signal RF du fonctionnement en régime ohmique soit noyé dans le bruit intrinsèque. Dans le cadre de cette analyse, basée sur des simulations MC, le bruit est simulé de façon naturelle, ce qui permet l'observation des fluctuations du courant en régime stationnaire.

La caractéristique du bruit pour un dispositif intrinsèque avec une configuration source commune pourrait être représentée par un dispositif idéal sans bruit avec deux générateurs corrélés de bruit en courant, l'un se situe à l'entrée (grille) et l'autre se situe à la sortie (drain) du dispositif (cf. Fig. 104) [Gonzalez1995IEEE-A]. Le dispositif sans bruit est caractérisé par les paramètres d'admittance $\mathrm{Y}$ calculés dans la section précédente en régime transitoire. La 
méthode MC permet en outre de calculer les sources de bruit équivalentes ainsi que leurs corrélations à travers l'analyse des fluctuations des courants de grille et de drain sans faire aucune hypothèse sur leur origine physique [Gonzalez1995IEEE-A]. Par ailleurs, elle fournit une compréhension microscopique des sources de bruit au moyen de l'étude des mécanismes internes concernant le contrôle de charge et le transport électronique au sein du dispositif.

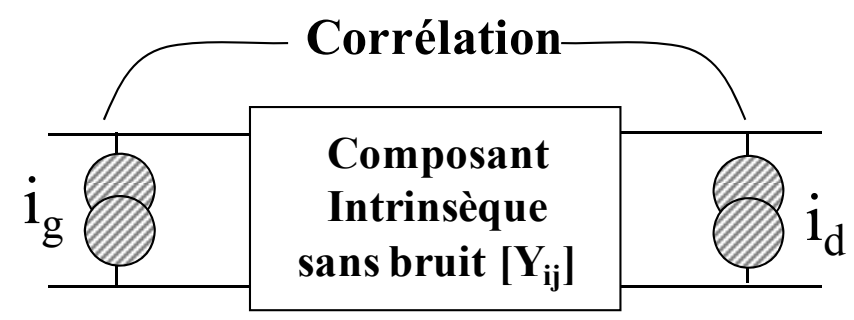

Figure 104 Circuit équivalent du bruit intrinsèque en une représentation courant-courant

\section{6-1 Source de bruit des fluctuations du courant}

D'après le théorème de Wiener-Khintchine, la source du bruit intrinsèque est directement fournie par la simulation MC via une analyse de Fourier de la fonction de corrélation des fluctuations du courant instantané durant un régime stationnaire. Le temps de simulation dans nos calculs est de $250 \mathrm{ps}$ avec une période d'échantillonnage $T_{\text {sample }}=0,1 \mathrm{fs}$. Les sources de bruit sont alors caractérisées par la densité spectrale de puissance (DSP) des fluctuations du courant de drain $\left(\mathrm{S}_{\mathrm{d}}\right)$ et de la grille $\left(\mathrm{S}_{\mathrm{g}}\right)$ ainsi que leur corrélation croisée $\left(\mathrm{S}_{\mathrm{dg}}\right)$. [Rengel2011IEEE]

$$
\begin{aligned}
& S_{d}(f)=\int_{-\infty}^{+\infty}\left(\lim _{T \rightarrow \infty} \int_{0}^{T} \delta J_{d}\left(t^{\prime}\right) \delta J_{d}\left(t^{\prime}+t\right) d t^{\prime}\right) \cdot e^{j 2 \pi f t} d t \\
& S_{g}(f)=\int_{-\infty}^{+\infty}\left(\lim _{T \rightarrow \infty} \int_{0}^{T} \delta J_{g}\left(t^{\prime}\right) \delta J_{g}\left(t^{\prime}+t\right) d t^{\prime}\right) \cdot e^{j 2 \pi f t} d t \\
& S_{d g}(f)=\int_{-\infty}^{+\infty}\left(\lim _{T \rightarrow \infty} \int_{0}^{T} \delta J_{g}\left(t^{\prime}\right) \delta J_{d}\left(t^{\prime}+t\right) d t^{\prime}\right) \cdot e^{j 2 \pi f t} d t
\end{aligned}
$$

où $\delta J_{d}$ et $\delta J_{g}$ est la fluctuation du courant instantané. Les expressions dans les parenthèses des trois équations précédentes représentent les fonctions d'auto-corrélation et de corrélation croisée des fluctuations de courant.

Ces expressions sont valables pour un calcul où l'espace de temps est continu, mais dans notre cas, les signaux sont traités en temps discrets, on peut alors les réécrire de la façon 
suivante. La fonction d'auto-corrélation de la fluctuation de courant de l'électrode i (grille ou drain) peut être calculée par les valeurs discrètes échantillonnées du courant instantané (à chaque pas du temps $m T_{\text {sample }}$ où l'indice $\mathrm{m}$ varie entre 0 et $\mathrm{n}, \mathrm{n}$ est le nombre total d'échantillons) :

$$
C_{J_{i}}\left(l T_{\text {sample }}\right)=\frac{1}{n-k} \sum_{m=k+1}^{n} \delta J_{i}\left((m-l) T_{\text {sample }}\right) \delta J_{i}\left(m T_{\text {sample }}\right)
$$

où l'indice $l$ varie entre 0 et $k$, en supposant que la fonction d'auto-corrélation est nulle pour des temps plus longs que $k T_{\text {sample }}$. La fonction de corrélation croisée des fluctuations de courant de l'électrode $\mathrm{i}$ et $\mathrm{j}$ est donnée par :

$$
C_{J_{i} J_{j}}\left(l T_{\text {sample }}\right)=\frac{1}{n-k} \sum_{m=k+1}^{n} \delta J_{i}\left((m-l) T_{\text {sample }}\right) \delta J_{j}\left(m T_{\text {sample }}\right)
$$

où l'indice $l$ varie entre $-k$ et $k$, en supposant que la fonction de corrélation croisée est nulle pour $l T_{\text {sample }}<-k T_{\text {sample }}$ et $l T_{\text {sample }}>k T_{\text {sample }}$. Finalement la densité spectrale de puissance de la fluctuation de courant de l'électrode i $\left(D S P_{J_{i}}\right)$ est donnée par :

$$
D S P_{J_{i}}=\frac{1}{f_{\text {sample }}} \sum_{l=0}^{+k} C_{J_{i}}\left(l T_{\text {sample }}\right) \cdot \cos \left(2 \pi l f / f_{\text {sample }}\right)
$$

et la densité spectrale de la corrélation croisée de la fluctuation de courant de l'électrode i et j $\left(D S P_{J_{i} J_{j}}\right)$ est donnée par :

$$
D S P_{J_{i} J_{j}}=\frac{1}{f_{\text {sample }}} \sum_{l=-k}^{+k} C_{J_{i} J_{j}}\left(l T_{\text {sample }}\right) \cdot \exp \left(j 2 \pi l f / f_{\text {sample }}\right)
$$

Des exemples de résultats pour MOS-Bulk sont donnés sur les Fig. 105 et 106. Rappelons que les points de fonctionnement statique (valeurs de $\mathrm{V}_{\mathrm{GS}}$ en $\mathrm{DC}$ ) sont choisis pour atteindre le maximum de $\mathrm{G}_{\mathrm{m}}$ à $\mathrm{V}_{\mathrm{DS}}=0,2 \mathrm{~V}$.

Pour cette condition de polarisation, on a au sommet de barrière d'injection (Virtual Source region) une densité surfacique d'électrons d'inversion de $3,95 \times 10^{12} \mathrm{~cm}^{-2}$. Sur les DSP se manifeste un pic d'oscillation plasma autour de $20 \mathrm{THz}$, avec un lobe secondaire à environ $40 \mathrm{THz}$. Cette valeur est en bon accord avec les travaux de la J.-F. Millithaler et al. [Millithaler2008APL] qui étudient les différents modes (3D et 2D) d'oscillation plasma dans un canal en $\operatorname{In}_{0,53} \mathrm{Ga}_{0,47} \mathrm{As}$ avec une longueur de canal inférieure à $100 \mathrm{~nm}$, une largeur de $1 \mathrm{~nm}$ et une densité surfacique d'électrons libres de l'ordre de $10^{12} \mathrm{~cm}^{-2}$. Au-delà de cette fréquence, les DSP s'atténuent avec des pentes semblables, on estime que ce comportement pourrait être dû à la prédominance du bruit numérique en régime de très haute fréquence, soit 
au-delà de $100 \mathrm{THz}$. Les DSP de fluctuation des courants de drain $\left(\mathrm{S}_{\mathrm{d}}\right)$ et de grille $\left(\mathrm{S}_{\mathrm{g}}\right)$ sont réelles tandis que celle de la corrélation croisée $\left(\mathrm{S}_{\mathrm{dg}}\right)$ est complexe. Elle est en fait dominée par sa partie imaginaire dans toute la plage de fréquence étudiée. Ce résultat est cohérent avec le modèle proposé par Van der Ziel [VanderZiel1986].
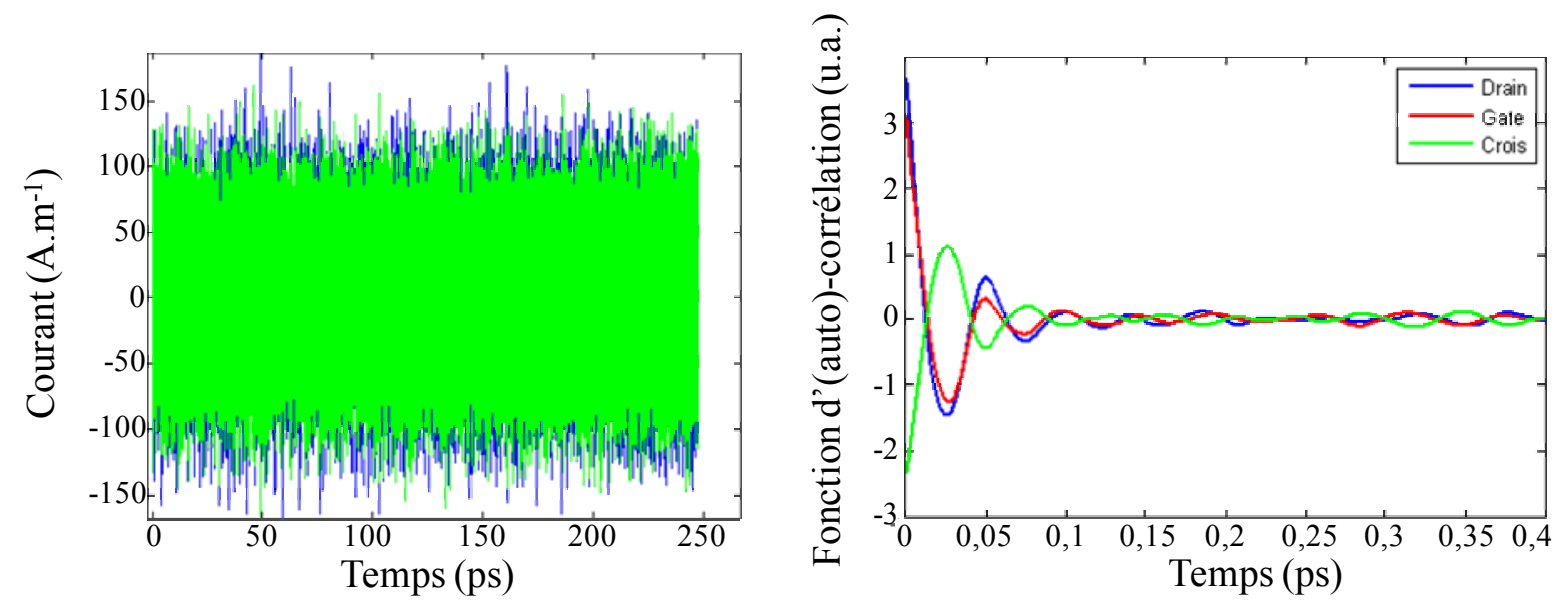

Figure 105 Gauche : Fluctuations des courants instantanés de drain (bleu) et de grille (vert) pour MOS-

Bulk. Droite : Fonctions d'auto-corrélation associées et de corrélation croisée.
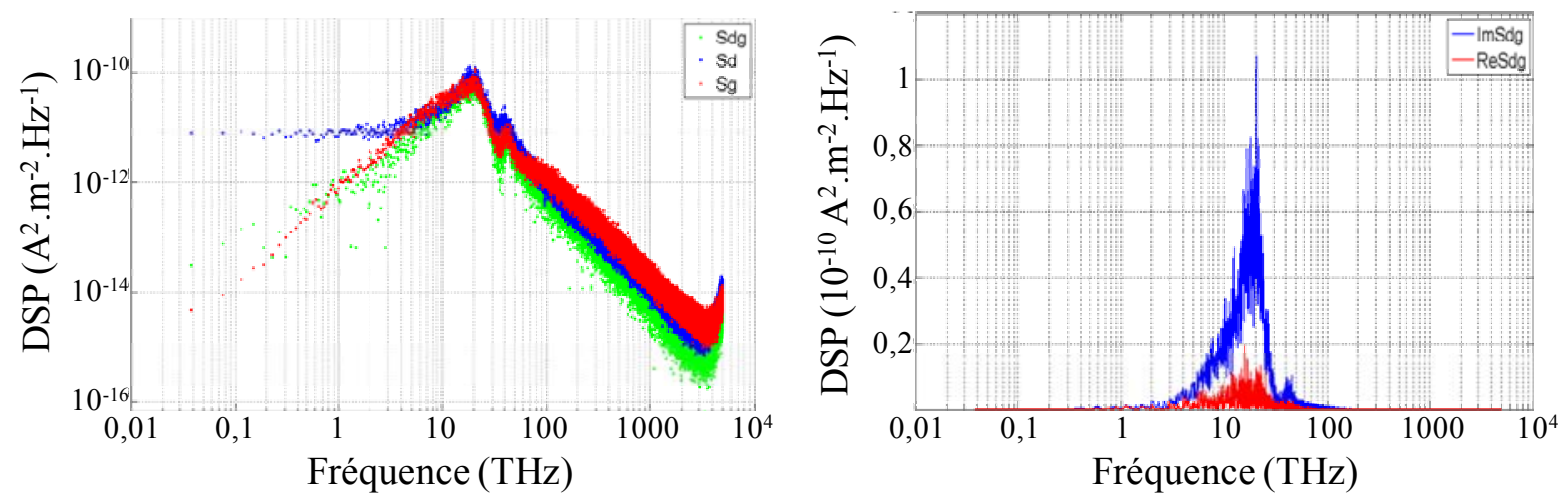

Figure 106 Gauche : Densités spectrales de puissance (DSP) des fluctuations du courant de drain $\left(S_{d}\right)$ et de grille $\left(S_{g}\right)$ ainsi que leur corrélation croisée $\left(S_{d g}\right)$ en fonction de la fréquence pour MOS-Bulk. Droite : Parties réelle et imaginaire du DSP de la corrélation croisée. La fréquence de résonance de l'oscillation plasma se trouve autour de $20 \mathrm{THz}$.

La Fig. 107 montre les DSP $S_{d}, S_{g}$ et $S_{d g}$ en fonction de la fréquence dans la gamme $10 \mathrm{GHz}-$ $1 \mathrm{THz}$ à $\mathrm{V}_{\mathrm{DS}}=0,2 \mathrm{~V}$ pour les quatre MOSFET étudiés. $\mathrm{S}_{\mathrm{d}}$ est quasiment indépendant de la fréquence, ce qui correspond à une source de bruit "blanc" de nature thermique ou de diffusion, il est en effet dû à la fluctuation de vitesse des porteurs dans le canal. La source du bruit dans la grille, $\mathrm{S}_{\mathrm{g}}$, peut être associée au couplage capacitif de grille qui génère des fluctuations de la densité de charge dans le canal. A haute fréquence et à faible $\mathrm{V}_{\mathrm{DS}}$, le 
MOSFET peut être considéré comme un réseau RC distribué ; le couplage capacitif avec la grille représente la capacité distribuée et le canal représente la résistance distribuée [VanderZiel1986]. Les deux sources de bruit $S_{d}$ et $S_{g}$ sont ainsi corrélées $\left(S_{g d}\right)$. Dans la gamme de fréquence étudiée, $\mathrm{S}_{\mathrm{g}}$ varie pratiquement selon une fonction quadratique en $f^{2}$ tandis que $\mathrm{S}_{\mathrm{dg}}$ évolue selon une loi linéaire en $f$. Ces résultats sont qualitativement en accord avec d'autres résultats Monte Carlo sur le bruit HF des composants à effet de champ (MESFET [Gonzalez1995IEEE-A], HEMT [Mateos2000IEEE] et MOSFET à base de Si [Rengel2001SST]) effectués à l'université de Salamanque.

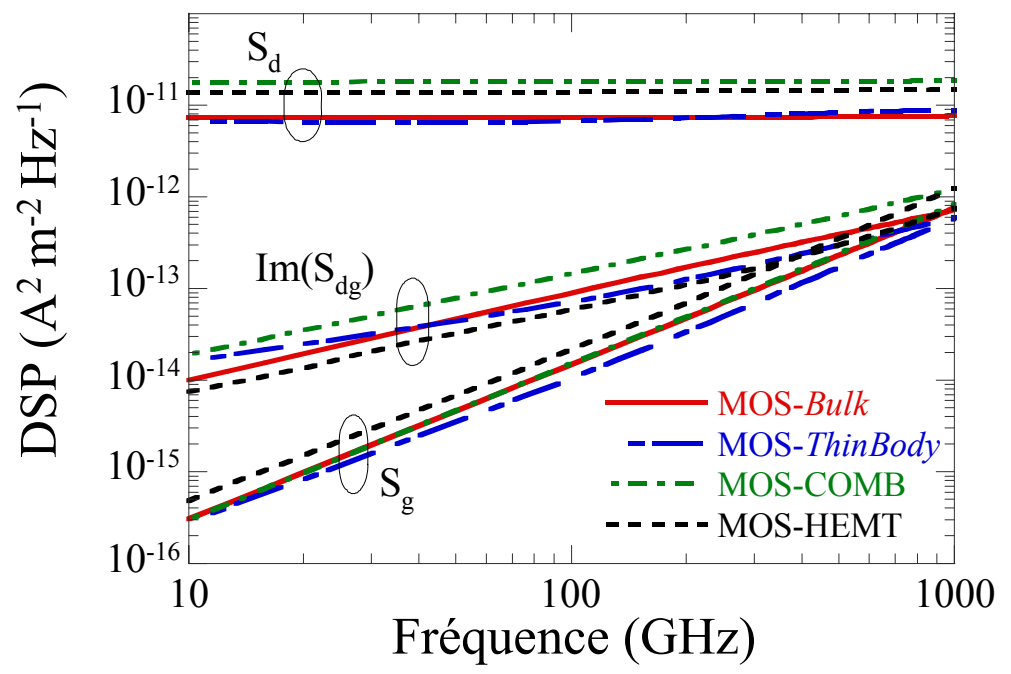

Figure 107 Densité spectrale de puissance (DSP) des fluctuations des courants de drain $S_{d}$ et de grille $S_{g}$, ainsi que la partie imaginaire de leur corrélation croisée $S_{\mathrm{d} g}$, en fonction de la fréquence pour les

MOSFET étudiés.

Les quatre types de MOSFET possédant quasiment la même capacité de grille $C_{G S}+C_{G D}$ à la polarisation étudiée, c'est le transistor MOS-COMB, de fortes balisticité et transconductance, qui présente la DSP de bruit en courant de drain $\mathrm{S}_{\mathrm{d}}$ la plus importante. En outre, on observe que $S_{g}$ est plus importante dans les transistors avec un espaceur InAlAs entre oxyde et canal par rapport aux structures sans espaceur, avec le même EOT. Cela d'autant plus que le niveau de dopage est élevé. Le MOS-HEMT présente en fait la DSP $\mathrm{S}_{\mathrm{g}}$ la plus importante. Par ailleurs, la plus grande valeur de $S_{d g}$ est obtenue dans MOS-COMB. Cela est éventuellement lié au caractère quasi-balistique du transport.

\section{6-2 Paramètres de bruit normalisés $P$, $R$ et $C$}

Les paramètres de bruit normalisés [VanderZiel1986] sont souvent utilisés pour une comparaison quantitative directe des comportements en bruit intrinsèque de différents dispositifs. En général, ils sont nommés comme $\mathrm{P}, \mathrm{R}$ et $\mathrm{C}$ pour un FET et peuvent donner des 
informations significatives sur l'origine physique du bruit vis-à-vis des réponses dynamiques $\mathrm{du}$ transistor [Pucel1975AEEP] et [Danneville1994IEEE]. P permet de mesurer le bruit généré par le drain, $\mathrm{R}$ est une jauge associée pour le bruit induit par la grille et $\mathrm{C}$ caractérise la source équivalente de bruit due à la corrélation croisée entre drain et grille. Ces paramètres peuvent être calculés à travers les expressions suivantes [Danneville1994IEEE] :

$$
\begin{gathered}
P=\frac{S_{d}}{4 k_{B} T\left|Y_{21}\right|} \\
R=\frac{S_{g}\left|Y_{21}\right|}{4 k_{B} T\left|Y_{11}\right|^{2}} \\
C=\frac{\Im\left(S_{d g}\right)}{\sqrt{S_{d} \cdot S_{g}}}
\end{gathered}
$$

Sur la Fig. 108, sont tracés les paramètres de bruit normalisés $\mathrm{P}, \mathrm{R}$ et $\mathrm{C}$ en fonction de la fréquence pour les quatre MOSFET sous la condition de polarisation $V_{G S}=V_{G S}\left(G_{m \_M A X}\right)$ et $\mathrm{V}_{\mathrm{DS}}=0,2 \mathrm{~V}$. Comme la transconductance correspond au module de $\mathrm{Y}_{21}$ et $S_{d} \approx 2 / 3 \cdot 4 k_{B} T \cdot G_{m}$, la valeur théorique de $\mathrm{P}$ pour un MOSFET en Si à canal long doit être d'environ 2/3. Dans les MOSFET III-V à canal court étudiés, les valeurs de $\mathrm{P}$ sont beaucoup plus élevées à cause du bruit excessif du courant drain. Comme la source du bruit est pondérée par la réponse dynamique, le bruit issu du drain est plus important dans les composants où la rugosité d'interface est négligeable, c'est-à-dire MOS-HEMT et MOS-COMB. Quant au paramètre R, sa valeur théorique pour un canal long est d'environ 0,2 à 0,3 [VanderZiel1986]. Le résultat pour MOS-Bulk correspond bien à cette prédiction, alors que nous avons vu que le transport dans le canal est majoritairement diffusif. Van der Ziel a prédit que R était augmenté par la présence de porteurs chauds sous un régime très fortement hors équilibre voire quasibalistique. R est effectivement plus important dans les MOSFET alternatifs par rapport MOSBulk. En outre, ce type de bruit induit par la grille risque d'être amplifié en introduisant un espaceur en InAlAs, ce qui explique qu'il soit en effet plus important dans le dispositif MOSHEMT. 

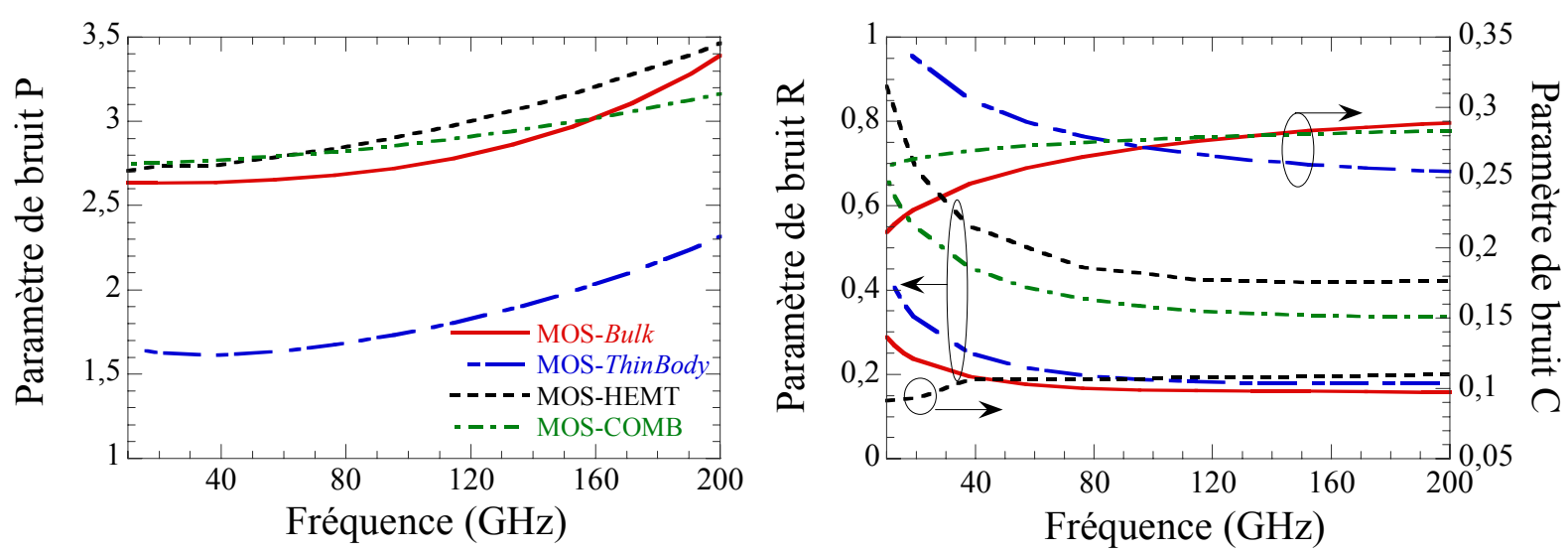

Figure 108 Paramètres de bruit normalisés $\mathrm{P}, \mathrm{R}$ et $\mathrm{C}$ des MOSFET étudiés en fonction de la fréquence

$$
\text { pour la polarisation } V_{G S}=V_{G S}\left(G_{m_{-} M A X}\right) \text { et } V_{D S}=0,2 \mathrm{~V}
$$

Enfin, la valeur théorique du paramètre de corrélation $C$ est d'environ 0,395 en régime de saturation pour un MOSFET à canal long sur Si [VanderZiel1986]. Ce paramètre s'avère plus faible dans les MOSFET III-V étudiés à faible $\mathrm{V}_{\mathrm{DS}}$, dans le régime de transition entre la zone ohmique et celle de saturation à $\mathrm{V}_{\mathrm{GS}}$ assez grand $\left(\mathrm{V}_{\mathrm{GS}}-\mathrm{V}_{\mathrm{T}}=0,4\right.$ à $0,5 \mathrm{~V}$, cf. la caractéristique $\mathrm{I}_{\mathrm{D}}-\mathrm{V}_{\mathrm{DS}}$ en Fig. 77) même si le transport est quasi-balistique. Les résultats obtenus pour des MOSFET à base de Si dans les travaux de $\mathrm{R}$. Rengel et al. montrent que le transport quasibalistique dans le régime de saturation permet d'avoir une forte corrélation $(>0,7)$ entre les terminaux [Rengel2001SST]. Mais dans notre cas, la corrélation a le même ordre de grandeur que l'on soit en transport diffusif et quasi-balistique. Ce bruit induit par la corrélation $\mathrm{C}$ est très faible dans la structure MOS-HEMT à cause des fortes sources de bruit issues des terminaux individuels $S_{d}$ et $S_{g}$ et également à cause de la faible corrélation croisée interélectrode $\mathrm{S}_{\mathrm{dg}}$. Il faut noter qu'une faible corrélation peut conduire à un fort facteur de bruit minimum $\mathrm{NF}_{\min }$ dans la plage fréquentielle de fonctionnement, ce qui très problématique même pour un dispositif aux bonnes performances RF par ailleurs. Nous allons examiner quantitativement ce point dans la section suivante.

\section{6-3 Facteurs de mérite de bruit électronique des dispositifs}

Bien que les paramètres de bruit normalisés de Pucel $\mathrm{P}, \mathrm{R}$ et $\mathrm{C}$ puissent fournir des informations utiles sur le comportement du bruit, le facteur de mérite le plus typique pour une caractérisation expérimentale globale est le facteur de bruit minimum $\mathrm{NF}_{\min }$. Les méthodes d'extraction ou de calcul du $\mathrm{NF}_{\min }$ sont détaillées dans les travaux de Greaves, S. D. and Unwin, R. T. [Greaves1993MOTL] et Mateos, J. [Mateos1997SSE]. Ils sont basés sur ceux de Rothe, H. and Dahlke, W. [Rothe1956IRE]. L'expression de $\mathrm{NF}_{\min }$ est :

$$
N F_{\text {min }}=1+2 \cdot R_{n} \cdot\left(\mathfrak{R}\left(Y_{\text {cor }}\right)+\mathfrak{R}\left(Y_{\text {opt }}\right)\right)
$$


où $R_{n}$ est la résistance de bruit, $Y_{c o r}$ est l'admittance de corrélation, $Y_{o p t}$ est l'admittance d'entrée optimisée avec

$$
\begin{gathered}
R_{n}=\frac{S_{d}}{4 k_{B} T\left|Y_{21}\right|^{2}} \\
\mathfrak{R}\left(Y_{c o r}\right)=\mathfrak{R}\left(Y_{11}-Y_{21} \frac{S_{d g}^{*}}{S_{d}}\right) \\
\mathfrak{R}\left(Y_{o p t}\right)=\sqrt{\left|Y_{21}\right|^{2} \frac{S_{g}}{S_{d}}+\left|Y_{11}\right|^{2}-2 \mathfrak{R}\left(Y_{11} Y_{21}^{*} \frac{S_{d g}}{S_{d}}\right)-\left(\mathfrak{I}\left(Y_{11}-Y_{21} \frac{S_{d g}^{*}}{S_{d}}\right)\right)^{2}}
\end{gathered}
$$

$N_{\text {min }}, Y_{\text {opt }}$ et $R_{n}$ peuvent également s'exprimer en utilisant les paramètres de Pucel $P, R$ et C [Danneville1994IEEE] et [Lim2008IEEE]. Les travaux de Rengel, R. et al. ont déjà vérifié que les équations III-60 et III-56 donnent le même résultat sur $\mathrm{NF}_{\text {min }}$ intrinsèque [Rengel2001SST] :

$$
\begin{gathered}
N F_{\min }=1+2 \cdot \frac{f}{f_{T}} \sqrt{P \cdot R\left(1-C^{2}\right)} \\
Y_{o p t}=G_{o p t}+j \cdot B_{o p t} \\
=\omega\left(C_{G S}+C_{G D}\right)(C \sqrt{R / P}-1)+j \cdot \omega\left(C_{G S}+C_{G D}\right) \sqrt{R / P} \sqrt{1-C^{2}} \\
R_{n}=P / G_{m}
\end{gathered}
$$

où $\omega=2 \pi f . Y_{\text {opt }}$ correspond à l'admittance que l'on doit connecter en parallèle à l'entrée du transistor pour obtenir une condition d'adaptation permettant d'atteindre le niveau de bruit minimum, comme schématisé sur la Fig. 109. Elle donne aussi la représentation équivalente vue de la sortie.

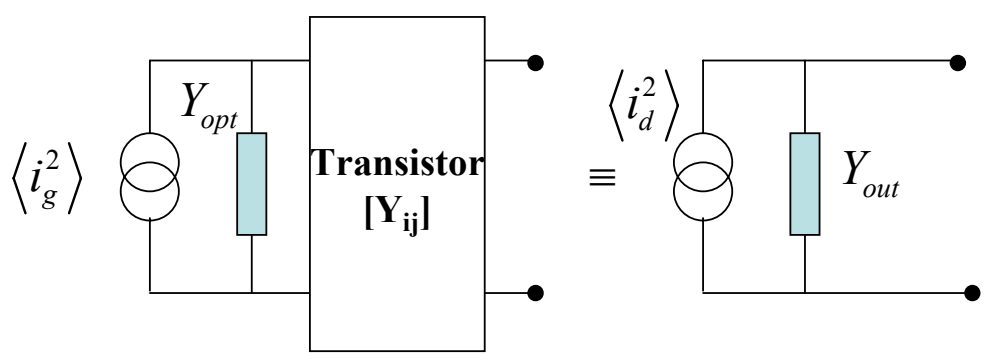

Figure 109 Schéma de configuration de la régulation du bruit en entrée et son équivalent vu de la sortie du transistor [Mateos2000IEEE].

Le gain transducique disponible de la puissance associé à cette condition de bruit minimum $G_{\text {ass }}$ peut aussi être calculée par [Mateos2000IEEE] : 


$$
G_{\text {ass }}=\frac{P_{o u t}}{P_{\text {in }}}=\frac{\mathfrak{R}\left(Y_{o p t}\right)}{\mathfrak{R}\left(Y_{22}-\frac{Y_{21} Y_{12}}{Y_{o p t}+Y_{11}}\right)}\left|\frac{Y_{21}}{Y_{o p t}+Y_{11}}\right|^{2}
$$

Habituellement, la grandeur mesurable n'est pas $Y_{\text {opt }}$, souvent on utilise le coefficient complexe de réflexion optimale $\Gamma_{o p t}$ :

$$
\Gamma_{o p t}=\frac{1-Z_{o} Y_{o p t}}{1+Z_{o} Y_{o p t}}
$$

où $Z_{o}$ est l'impédance caractéristique, dans notre cas $50 \Omega$. Les analyses complètes de la performance en bruit consistent à déterminer ces quatre paramètres de bruit : $\mathrm{NF}_{\min }, \mathrm{R}_{\mathrm{n}}, \Gamma_{\mathrm{opt}}$ et $\mathrm{G}_{\text {ass }}$ en fonction de la fréquence. Souvent ces grandeurs mesurables du bruit ne sont pas normalisables par rapport à la largeur $\mathrm{W}$ du dispositif, comme les paramètres S. Dans notre calcul, la largeur $\mathrm{W}$ est supposée égale à $10 \mu \mathrm{m}$. Les grandeurs précédentes sont reliées au facteur du bruit total NF par [Dambrine1999IEEE] :

$$
N F\left(\Gamma_{S}\right)=N F_{\min }+\frac{4 \frac{R_{n}}{Z_{o}}}{\left|1+\Gamma_{o p t}\right|^{2}} \frac{\left|\Gamma_{S}-\Gamma_{o p t}\right|^{2}}{1-\left|\Gamma_{S}\right|^{2}}
$$

NF quantifie l'impact du bruit ajouté par le dispositif sur le signal qui est en cours d'amplification, il est donc minimisé $\left(\mathrm{NF}_{\min }\right)$ lorsque le coefficient de réflexion en entrée $\Gamma_{\mathrm{S}}$ est égal à $\Gamma_{\mathrm{opt}}$. $\mathrm{R}_{\mathrm{n}}$ est une jauge de la sensibilité de NF aux changements de l'admittance d'entrée par rapport à sa valeur optimale. Sur l'évolution de $\mathrm{G}_{\mathrm{ass}}$ en fonction de la fréquence on peut mesurer la bande passante du gain disponible de puissance quand le transistor est adapté pour atteindre la condition du bruit minimum $\mathrm{NF}_{\min }$. Les résultats sont dans les Fig. 110 et 111 en supposant la largeur du composant $\mathrm{W}=10 \mu \mathrm{m}$ :
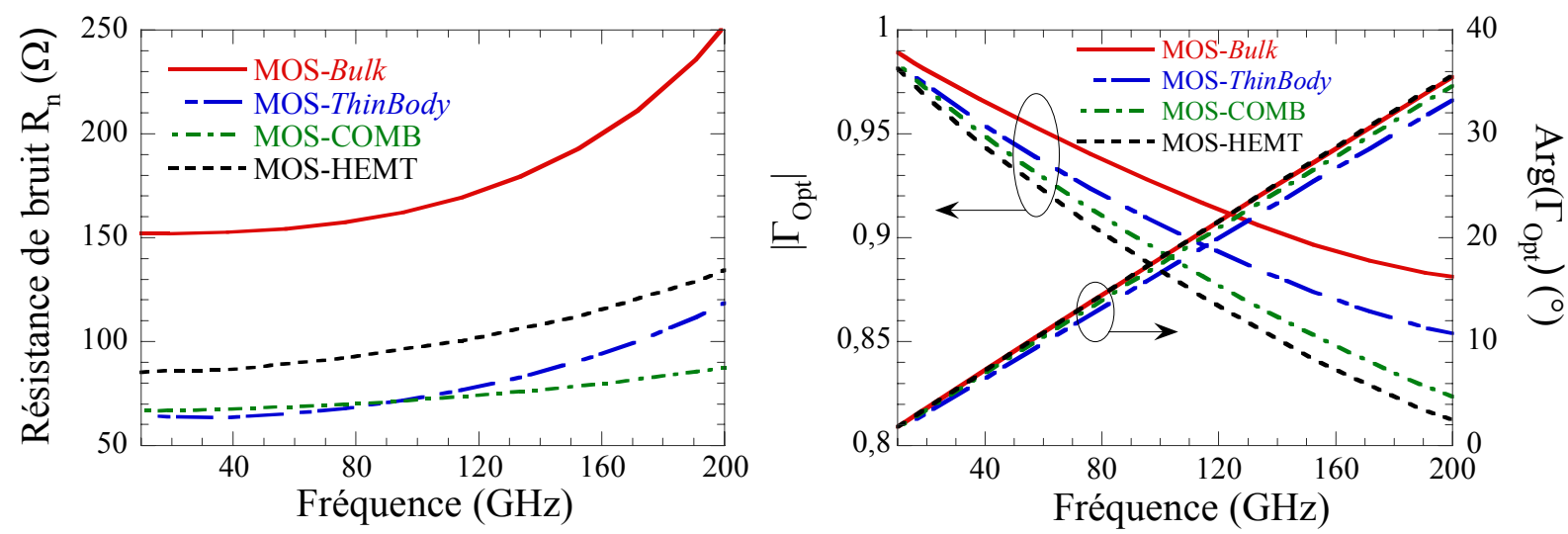

Figure 110 Résistance équivalente de bruit $R_{n}$ (gauche) et coefficient de réflexion $\Gamma_{\text {opt }}$ en module et en phase (droite) en fonction de la fréquence pour les MOSFET considérés à $V_{D S}=0,2 \mathrm{~V}$ et $\mathrm{W}=10 \mu \mathrm{m}$. 
A faible $V_{D S}$ et pour des fréquences inférieures à environ $50 \mathrm{GHz}, \mathrm{R}_{\mathrm{n}}$ ne dépend quasiment pas de la fréquence. La valeur la plus importante est obtenue dans MOS-Bulk où $\mathrm{R}_{\mathrm{n}}$ vaut environ $155 \Omega$ à $20 \mathrm{GHz}$, soit le même ordre de grandeur qu'une résistance de bruit mesurée dans un transistor SOI [Dambrine1999IEEE]. Cela est principalement dû à la forte source de bruit du drain et à la relativement faible transconductance intrinsèque. Le facteur de bruit est alors très sensible à l'admittance et à l'impédance d'entrée. La réduction de $\mathrm{R}_{\mathrm{n}}$ sera l'une des préoccupations majeures pour effectuer le plus efficacement possible la condition d'adaptation du bruit minimum. Cette valeur est fortement réduite dans les structures alternatives, surtout dans les structures avec un canal nid. Les structures MOS-ThinBody et MOS-COMB ont une résistance de bruit de $55 \Omega$ à $20 \mathrm{GHz}$ (3 fois plus faible). Cette valeur est comparable avec celle d'un HEMT à base de InGaAs comme étudié par J. Mateos et al. [Mateos2004IEEE-B]. La Fig. 110 montre aussi la variation du module et de l'argument du coefficient de réflexion optimale $\Gamma_{\text {opt }}$ en fonction de la fréquence. Pour $\mathrm{f}<50 \mathrm{GHz},\left|\Gamma_{\text {opt }}\right|$ et $\operatorname{Arg}\left(\Gamma_{\text {opt }}\right)$ sont proches respectivement de l'unité et de $0^{\circ}$. Ils varient quasi linéairement avec la fréquence, ce qui correspond bien au modèle proposé par M.W. Pospieszalski et al. [Pospieszalski1989IEEE] dans une plage de fréquence de quelques dizaines de GHz. Quand le module $\left|\Gamma_{\text {opt }}\right|$ est proche de l'unité, la condition d'adaptation est difficile à accomplir à faible perte [Dambrine1999IEEE]. Les structures alternatives sont également prometteuses de ce point de vue. Les coefficients de réflexion sont relativement plus petits dans les structures alternatives. On peut expliquer qualitativement ces comportements à l'aide du modèle compact proposé par M.W. Pospieszalski :

$$
Y_{o p t} \propto \frac{f}{f_{T}}\left(\sqrt{\frac{G_{d}}{R_{i}}} \sqrt{\frac{T_{d}}{T_{g}}}+j \frac{G_{d}}{G_{m} R_{i}} \frac{T_{d}}{T_{g}}\right)
$$

Dans son modèle, il a introduit deux températures de bruit équivalentes pour les électrodes de drain et de grille $T_{d}$ et $T_{g}$, elles sont proportionnelles aux sources du bruit, à la conductance et à la bande de fréquence considérée. Les résultats expérimentaux [Dambrine1998IEEE] et de modélisation [Danneville1994IEEE] montrent que $T_{g}$ est très proche de la température du réseau et quasi indépendante du niveau de courant. Mais $T_{d}\left(T_{d} \propto S_{d} / 4 k_{B} G_{d}\right)$ dépendra strictement du courant et peut atteindre 1000 à $2000 \mathrm{~K}$ au sein de GaAs et InGaAs. Cela est dû à l'effet des électrons chauds. Dans les structures alternatives, les fréquences de coupure $\mathrm{f}_{\mathrm{T}}$ ne sont pas très différentes (100 à $150 \mathrm{GHz}$ de différence), le terme $f / f_{T}$ dans l'équation III66 n'est pas a priori prédominant. En outre, pour les structures éliminant la rugosité 
d'interface, le transport est très fortement hors équilibre en fin de canal, coté drain où ils sont chauffés. Dans la structure MOS-HEMT qui présente une forte source de bruit, une conductance relativement faible et une transconductance modérée (par rapport aux autres structures envisagées), elle atteint donc la valeur la plus grande de $Y_{o p t} / Y_{0}\left(Y_{0}=20 \mathrm{mS}\right)$ ainsi qu'une faible valeur de coefficients de réflexion.
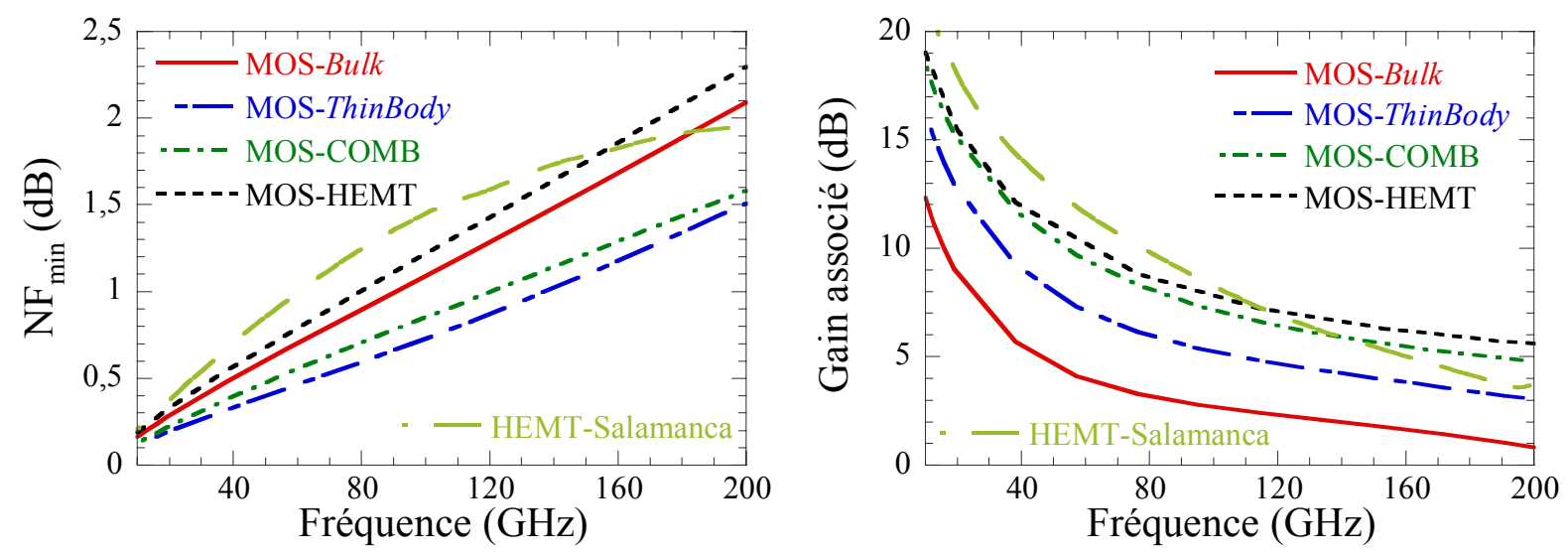

Figure 111 Facteur de bruit minimum intrinsèque $\mathbf{N F}_{\min }$ (gauche) et gain transducique disponible associé $G_{\text {ass }}$ (droite) en fonction de la fréquence pour les MOSFET étudiés à $V_{D S}=0,2 \mathrm{~V}$ (même EOT, $L_{G}=50 \mathrm{~nm}$ et $\mathrm{W}=\mathbf{1 0} \mu \mathrm{m})$. Les calculs Monte Carlo semi-classiques de l'université de Salamanque [Mateos2000IEEE] pour une structure HEMT "lattice matched" avec $\mathrm{L}_{\mathrm{G}}=100 \mathrm{~nm}$ et rapport d'aspect de 5 à $\mathrm{V}_{\mathrm{DS}}=0,8 \mathrm{~V}$ sont également tracés pour comparaison.

La Fig. 111 récapitule les performances en bruit des MOSFET à base d' $\operatorname{In}_{0.53} \mathrm{Ga}_{0.47} \mathrm{As}$ étudiés. Une comparaison est également effectuée avec un HEMT de même matériau de canal [Mateos2000IEEE]. Le compromis entre facteur de bruit, coefficient de réflexion d'entrée, résistance de bruit et gain associé disponible est plus facile à obtenir dans une structure ayant un Thin body comme couche active, c'est-à-dire MOS-ThinBody et MOS-COMB. La meilleure performance est obtenue pour le premier des deux transistors, avec $\mathrm{NF}_{\min }=0,2 \mathrm{~dB}$ et $\mathrm{G}_{\mathrm{ass}}=13 \mathrm{~dB}$ à $20 \mathrm{GHz}$ pour un courant intrinsèque du drain de $700 \mathrm{~mA} \cdot \mathrm{mm}^{-1}$ en DC. On peut ajouter que les MOSFET avec $\mathrm{L}_{\mathrm{G}}=50 \mathrm{~nm}$ présentent à $\mathrm{V}_{\mathrm{DS}}=0,2 \mathrm{~V}$ un faible $\mathrm{NF}_{\min }$ en gardant un gain associé comparable voire encore plus large bande par rapport au HEMT de longueur de grille effective de $100 \mathrm{~nm}$ à $\mathrm{V}_{\mathrm{DS}}=0,8 \mathrm{~V}\left(\mathrm{NF}_{\min }=0,4 \mathrm{~dB}\right.$ pour $\mathrm{G}_{\mathrm{ass}}=18 \mathrm{~dB}$ à $20 \mathrm{GHz}$ pour avec un courant extrinsèque de drain d'environ $60 \mathrm{~mA} \cdot \mathrm{mm}^{-1}$ en DC).

\section{Comparaison des performances avec les MOSFET Si et HEMT}

\section{III-V}

Il est intéressant de comparer les différentes performances intrinsèques des MOSFET III-V étudiés sous faible $\mathrm{V}_{\mathrm{DS}}$ avec celles d'un transistor sur Si dans les mêmes conditions. 

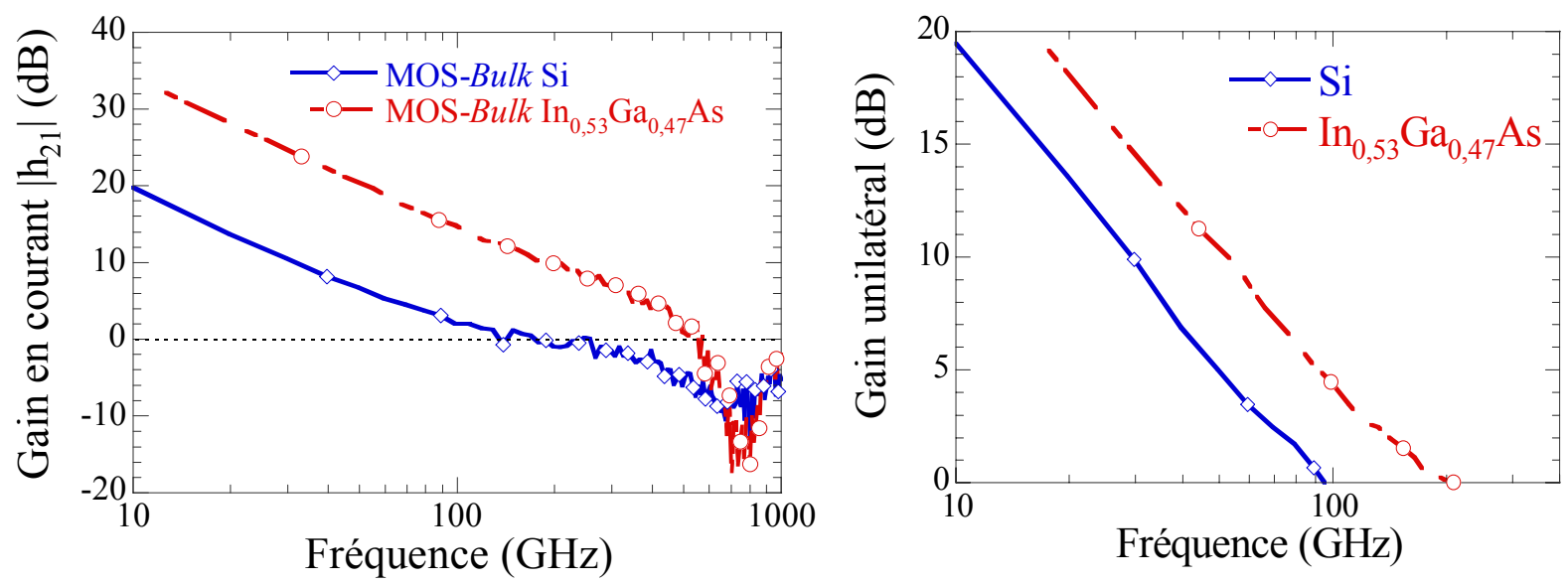

Figure 112 Gain de court-circuit en courant $h_{21}$ intrinsèque (gauche) et gain unilatéral $U$ intrinsèque (droite) en fonction de la fréquence pour des MOSFET sur substrat massif à base de Si et d' $\mathrm{In}_{0,53} \mathrm{Ga}_{0,47} \mathrm{As}$ de même géométrie ( $\mathrm{L}_{\mathrm{G}}$, EOT, dopages) et sous des conditions de polarisation équivalentes : $V_{G S}=V_{G S}\left(G_{m_{-}}\right.$MAX $)$et $V_{D S}=0,2 \mathrm{~V}$. Les courants de drain DC sont respectivement égaux à 75 mA.mm ${ }^{-1}$ (Si) et $440 \mathrm{~mA} \cdot \mathrm{mm}^{-1}$ (InGaAs).

Grâce à l'amélioration de la mobilité effective du transport électronique dans InGaAs par rapport au $\mathrm{Si}$ et à quasiment même $\mathrm{C}_{\mathrm{GG}}$, on obtient une forte augmentation de la transconductance $\mathrm{G}_{\mathrm{m}}(\times 4,9)$ qui détermine le gain $\mathrm{h}_{21}$ à faible fréquence. Le terme $G_{m}^{2} / G_{d}$ déduit des caractéristiques dynamiques à $20 \mathrm{GHz}$ est également amélioré $(\times 2,8)$, ce qui permet d'avoir une augmentation du gain unilatéral à faible fréquence. Comme on peut le voir sur la Figue. 112, la fréquence de transition $\mathrm{f}_{\mathrm{T}}$ et la fréquence d'oscillation maximale $\mathrm{f}_{\mathrm{MAX}}$ ont considérablement été améliorées : $520 \mathrm{GHz}$ au lieu de $120 \mathrm{GHz}(\times 4,3)$ pour $\mathrm{f}_{\mathrm{T}}$ et $200 \mathrm{GHz}$ au lieu de $95 \mathrm{GHz}(\times 2.1)$ pour $\mathrm{f}_{\mathrm{MAX}}$. Cette amélioration de la performance $\mathrm{RF}$ intrinsèque se produit dans toute la plage de contrôle en $\mathrm{V}_{\mathrm{GS}}$, comme illustré sur la Fig. 113 où l'on trace $\mathrm{f}_{\mathrm{T}}$ pour les différents points de fonctionnement en $\mathrm{DC}$ à $\mathrm{V}_{\mathrm{DS}}=0,2 \mathrm{~V}$, ainsi qu'en fonction de la dissipation de puissance sous mêmes conditions de tension d'alimentation et pour un même état OFF.

Le MOSFET sur Si présente un mauvais rendement rapidité/puissance par rapport aux FET à base de III-V. De ce point de vue, les composants à canal en $\operatorname{In}_{0,53} \mathrm{Ga}_{0,47} \mathrm{As}$ et de topologie MOS-COMB ont l'avantage sur le HEMT. 

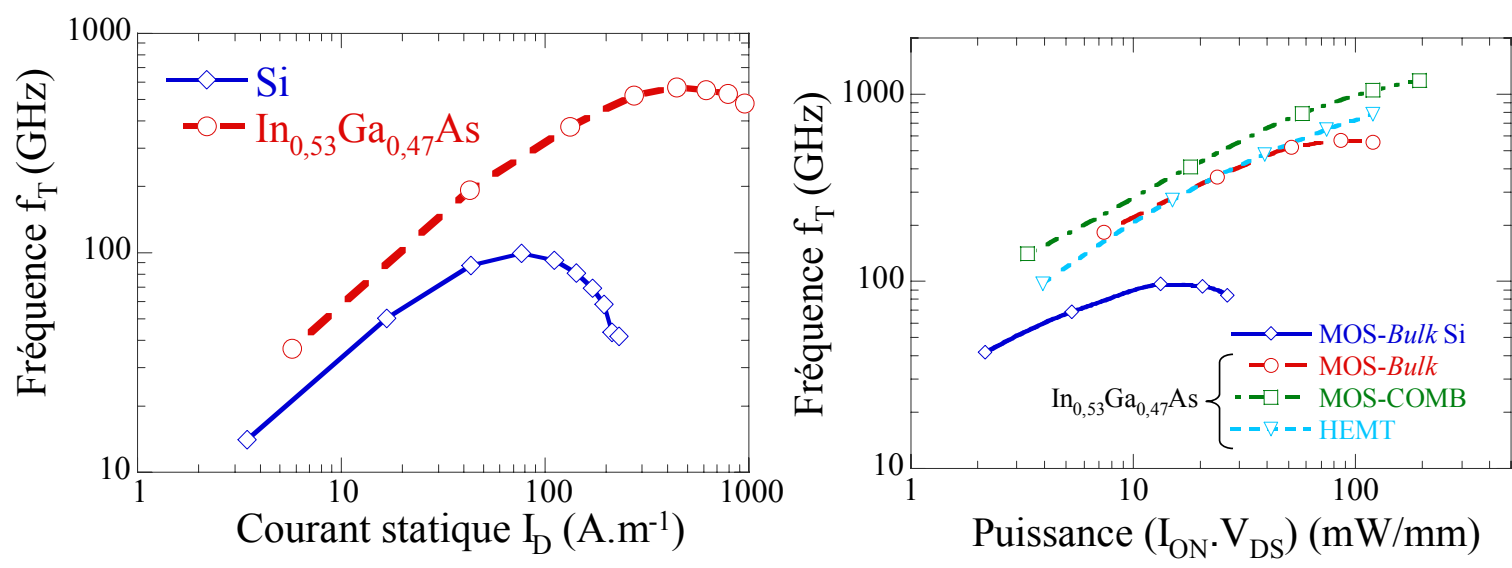

Figure 113 Gauche : Fréquence de transition $\mathrm{f}_{\mathrm{T}}$ pour différents points de fonctionnement en $\mathrm{DC}$ à $V_{D S}=0,2 \mathrm{~V}$ pour MOS-Bulk à base de Si ou d' $\mathrm{In}_{0,53} \mathbf{G a}_{0,47} A s$. Droite $: \mathbf{f}_{\mathrm{T}}$ en fonction de la dissipation de puissance pour $\mathrm{V}_{\mathrm{DD}}$ de 0,2 à $0,6 \mathrm{~V}$ pour différents transistors. $\mathrm{I}_{\mathrm{OFF}}$ est fixé à $0,2 \mathrm{~mA} . \mathrm{mm}^{-1}$. La longueur de grille $\mathrm{L}_{\mathrm{G}}$ est égale à $50 \mathrm{~nm}$ dans tous les composants étudiés.

Pour ce qui est du bruit, la Fig. 114 présente les évolutions des DSP et paramètres Pucel (P, R et $\mathrm{C}$ ) pour les MOS-Bulk à base de Si ou d'InGaAs. La DSP $\mathrm{S}_{\mathrm{d}}$ est moins importante avec Si que dans InGaAs, du fait d'une moindre proportion d'électrons chauds à la fin du canal. Une augmentation de $\mathrm{S}_{\mathrm{d}}$ peut être observée à haute fréquence dans le cas du $\mathrm{Si}$, cela à cause du pic de l'oscillation de plasma qui se trouve entre 6 et $7 \mathrm{THz}$, contre $20 \mathrm{THz}$ pour InGaAs. En revanche, $S_{g}$ est plus grande pour Si dont la DOS en bande de conduction est environ 150 fois plus importante que dans le cas d'InGaAs [O'Regan2011APL], ce qui génère des fluctuations plus fortes de la densité de charge dans le canal. Enfin, la comparaison entre les deux matériaux quant au bruit de corrélation croisée entre drain et grille semble dépendre de la fréquence d'opération. Afin de faciliter l'évaluation, intéressons-nous désormais aux paramètres de Pucel de bruit normalisés. Le paramètre $\mathrm{P}$ est plus important dans le dispositif Si malgré la valeur inférieure $S_{d}$ et ce à cause de la faible transconductance $G_{m}$. La courbe $P(f)$ présente un minimum $100 \mathrm{GHz}$ dans le cas de $\mathrm{Si}$ alors que dans le transistor en $\operatorname{In}_{0,53} \mathrm{Ga}_{0,47} \mathrm{As}$ il augmente continûment avec $\mathrm{f}$ croissant dans la plage étudiée. $\mathrm{R}$ est aussi plus grand dans le cas de $\mathrm{Si}$, à cause du couplage capacitif renforcé qui génère le fort $\mathrm{S}_{\mathrm{g}}$. En revanche, la corrélation C est plus faible dans la structure en Si dans la gamme de fréquence d'opération.

En Fig. 115 et 116 sont tracées les évolutions fréquentielles des facteurs de mérite liés au bruit $\mathrm{R}_{\mathrm{n}}, \mathrm{G}_{\mathrm{opt}}, \mathrm{NF}_{\text {min }}$ et $\mathrm{G}_{\mathrm{ass}}$. Le MOS-Bulk à base de Si possède une très forte résistance du bruit $R_{n}$, c'est-à-dire que le facteur du bruit est très sensible à l'admittance ou l'impédance d'entrée, autrement dit, si le coefficient de réflexion diffère légèrement de sa valeur optimale (faible désadaptation de l'impédance), le facteur du bruit du transistor va fortement augmenter. $\mathrm{R}_{\mathrm{n}}$ atteint comme $\mathrm{P}$ un minimum autour de $100 \mathrm{GHz}$ dans le cas du Si. Pour ce qui est de $\Gamma_{\mathrm{opt}}$ 
et $\mathrm{NF}_{\text {min }}$, tous deux varient avec $\mathrm{f} / \mathrm{f}_{\mathrm{T}}$ (il devrait être le terme prédominant cette fois pour $\left.Y_{o p t} / Y_{0}\right)$ et un compromis est à établir sur la valeur de la fréquence de transition pour optimiser leurs valeurs (augmenter $\mathrm{f}_{\mathrm{T}}$ réduit $\mathrm{NF}_{\text {min }}$ mais augmente $\left.\left|\Gamma_{\text {out }}\right|\right)$.
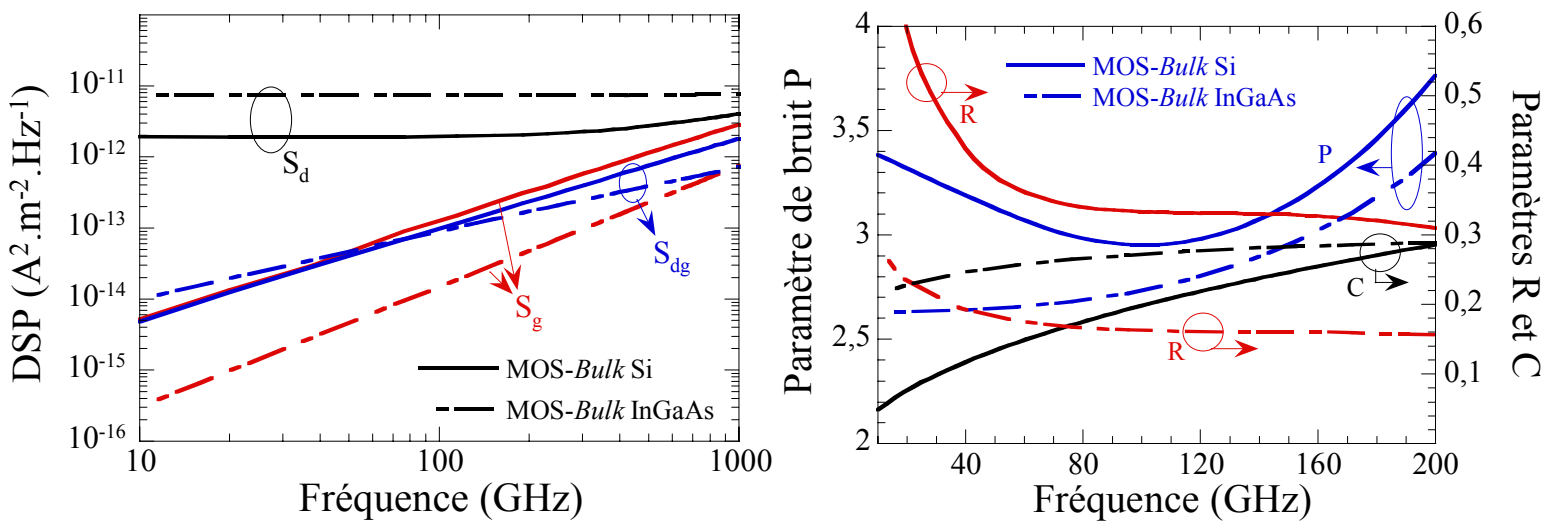

Figure 114 Densité spectrale de puissance DSP (gauche) et paramètres de Pucel de bruit normalisés (droite) en fonction de la fréquence pour les structures MOS-Bulk à base de Si et $\operatorname{In}_{0,53} \mathbf{G a}_{0,47} \mathrm{As}$
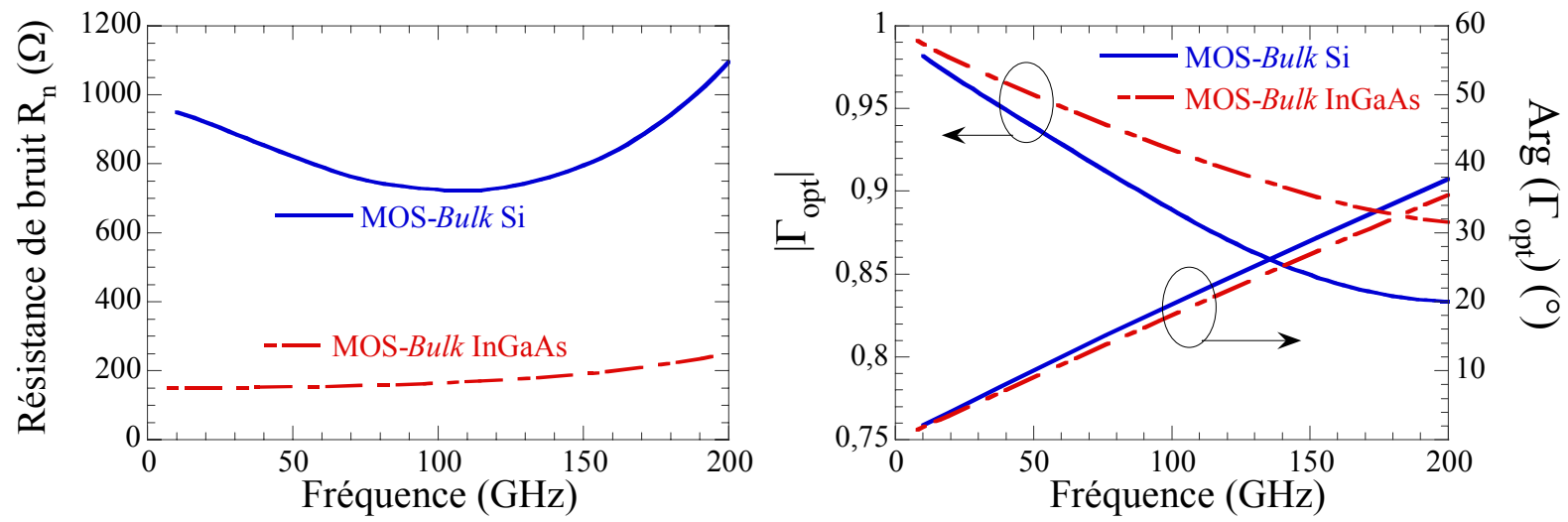

Figure 115 Résistance de bruit $R_{n}$ (gauche) et coefficient de réflexion optimale (en module et en phase, droite) en fonction de la fréquence pour les structures MOS-Bulk à base de Si ou $\operatorname{In}_{0,53} \mathbf{G a}_{0,47} \mathrm{As}$
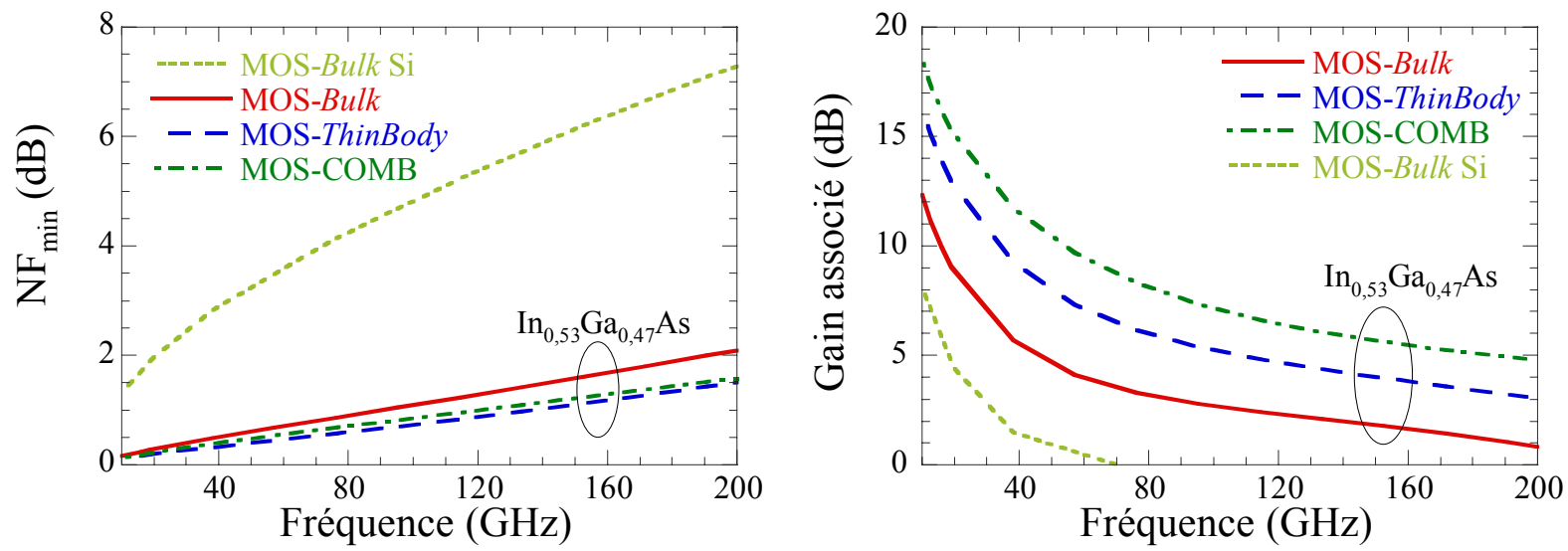

Figure 116 Facteur de bruit minimum (gauche) et gain transducique associé (droite) en fonction de la fréquence pour différents transistors à base de $\mathrm{Si}$ ou $\mathrm{In}_{0,53} \mathrm{Ga}_{0,47} \mathrm{As}$ 
Mais ce compromis est difficile à obtenir dans le transistor sur Si : il présente un très grand facteur du bruit minimum par rapport au MOS-Bulk en $\operatorname{In}_{0.53} \mathrm{Ga}_{0.47} \mathrm{As}$ dans la gamme de fréquence étudiée. Par ailleurs, la bande passante associée au gain transducique de la puissance est réduite fortement à la condition d'adaptation.

\begin{tabular}{|c|c|c|c|c|c|c|c|c|}
\hline FET & $\begin{array}{l}\mathbf{L}_{\mathbf{G}} \\
(\mathbf{n m})\end{array}$ & $\begin{array}{c}\mathbf{f}_{\mathbf{T}} \\
(\mathbf{G H z})\end{array}$ & $\begin{array}{c}\mathbf{f}_{\mathrm{MAX}} \\
(\mathbf{G H z})\end{array}$ & $\mathbf{P}$ & $\mathbf{R}$ & $\mathbf{C}$ & $\begin{array}{l}\mathrm{NF}_{\min } \\
(\mathrm{dB})\end{array}$ & Référence \\
\hline $\begin{array}{l}\text { MOSFET- } \\
\text { Si Bulk }\end{array}$ & 300 & 65 & - & 2,6 & 2,2 & 0,5 & $\begin{array}{c}3 @ \\
12 \mathrm{GHz}\end{array}$ & Rengel2001SST \\
\hline $\begin{array}{l}\text { MOSFET- } \\
\text { Si Bulk }\end{array}$ & $\begin{array}{c}55 \& \\
65\end{array}$ & $\begin{array}{c}178 \\
(55 \mathrm{~nm})\end{array}$ & $\begin{array}{c}127 \\
(55 \mathrm{~nm})\end{array}$ & $\begin{array}{c}2,01 \\
(65 \mathrm{~nm})\end{array}$ & 0,22 & 0,3 & $\begin{array}{c}0,8 @ 12 \\
\mathrm{GHz}\end{array}$ & $\begin{array}{l}\text { Dambrine2005 \& } \\
\text { Kakushi2010SST }\end{array}$ \\
\hline $\begin{array}{l}\text { MOSFET- } \\
\text { Si LAC* }\end{array}$ & 100 & 110 & - & 1 & 0,4 & 0,8 & $\begin{array}{l}0,38 @ \\
12 \mathrm{GHz}\end{array}$ & $\begin{array}{l}\text { Lim2008IEEE \& } \\
\text { Rengel2011IEEE }\end{array}$ \\
\hline PDSOI & 600 & 23 & - & - & - & - & $\begin{array}{l}1,8 @ \\
2 \mathrm{GHz}\end{array}$ & $\begin{array}{c}\text { Dambrine1999IE } \\
\text { EE }\end{array}$ \\
\hline FDSOI & 250 & - & - & 1,75 & 0,5 & 0,3 & $\begin{array}{c}2,2 @ \\
16 \mathrm{GHz}\end{array}$ & Rengel2006IEEE \\
\hline $\begin{array}{l}\text { MOSFET- } \\
\text { Si Bulk }\end{array}$ & 50 & 120 & 95 & 3,3 & 0,6 & 0.07 & $\begin{array}{c}1,5 @ \\
12 \mathrm{GHz}\end{array}$ & Ce travail \\
\hline $\begin{array}{l}\text { pHEMT- } \\
\text { GaAs }\end{array}$ & 130 & 82 & 75 & 1 & 0,5 & 0,9 & $\begin{array}{l}0,31 @ \\
12 \mathrm{GHz}\end{array}$ & Lee1995IEEE \\
\hline $\begin{array}{l}\text { pHEMT- } \\
\text { InGaAs }\end{array}$ & 50 & 630 & 520 & - & - & - & - & Ce travail \\
\hline $\begin{array}{l}\text { pHEMT- } \\
\text { InGaAs }\end{array}$ & $\begin{array}{c}50 \& \\
100\end{array}$ & $\begin{array}{c}550 \\
(50 \mathrm{~nm})\end{array}$ & $\begin{array}{c}500 \\
(50 \mathrm{~nm})\end{array}$ & $\begin{array}{c}2 \\
(100 \mathrm{~nm})\end{array}$ & 0,55 & 0,3 & $\begin{array}{l}0,35 @ \\
12 \mathrm{GHz}\end{array}$ & $\begin{array}{l}\text { Mateos2000IEEE } \\
\text { Mateos2004IEEE }\end{array}$ \\
\hline $\begin{array}{c}\text { MOS- } \\
\text { Bulk }\end{array}$ & 50 & 520 & 200 & 2,6 & 0,2 & 0,23 & $\begin{array}{l}0,28 @ \\
12 \mathrm{GHz}\end{array}$ & Ce travail \\
\hline $\begin{array}{l}\text { MOS- } \\
\text { ThinBody }\end{array}$ & 50 & 600 & 300 & 1,6 & 0,35 & 0,33 & $\begin{array}{l}0,19 @ \\
12 \mathrm{GHz} \\
\end{array}$ & Ce travail \\
\hline $\begin{array}{l}\text { MOS- } \\
\text { HEMT }\end{array}$ & 50 & 690 & 550 & 2,7 & 0,7 & 0.1 & $\begin{array}{l}0,35 @ \\
12 \mathrm{GHz}\end{array}$ & Ce travail \\
\hline $\begin{array}{l}\text { MOS- } \\
\text { COMB }\end{array}$ & 50 & 750 & 600 & 2,75 & 0,55 & 0,26 & $\begin{array}{l}0,21 @ \\
12 \mathrm{GHz}\end{array}$ & Ce travail \\
\hline
\end{tabular}

Tableau 13 Comparaison des performances intrinsèques en RF et en bruit pour différents FET à base de Si et InGaAs. LAC : Laterally Asymmetric Channel.

Le tableau 13 ci-dessus récapitule les caractéristiques des structures de type MOSFET envisagées. Nous comparons également les performances à celles reportées dans la littérature (données expérimentales et en simulation pour les dispositifs intrinsèques, les éléments parasites sont exclus) pour différents transistors à effet de champ FET à base de Si et InGaAs. Nos calculs sur la performance RF et en bruit sont cohérents avec les résultats reportés dans la 
littérature pour des MOSFET à base de Si et pour des HEMT à base d'InGaAs. On constate que la plus mauvaise performance en bruit est obtenue dans la structure en MOS-Bulk à base de Si. Les facteurs de mérite restent relativement bas et le facteur de bruit assez élevé, surtout pour les dispositifs à canal long. Les performances semblent être améliorées dans les structures sur SOI (FDSOI et PDSOI). La meilleure performance en bruit pour la technologie Si sera obtenue dans le dispositif avec un canal latéralement asymétrique (LAC), où le canal est graduellement dopé. Par ailleurs, la performance RF et celle en bruit sont toutes les deux améliorées en introduisant la technologie III-V. Pour une longueur de grille modérée de l'ordre de $100 \mathrm{~nm}$, l'architecture HEMT à base d'InGaAs permet d'accroître nettement $(\sim \times 3$ à 5) la performance RF en maintenant un niveau de bruit similaire à celui du transistor LAC à base de Si. Pour nos nano-transistors proposés avec un fort coefficient de balisticité comme les MOSFET III-V - en particulier MOS-ThinBody et MOS-COMB - la performance à haute fréquence est encore améliorée. Ils semblent constituer une solution viable pour améliorer la performance en RF, le rendement rapidité/dissipation ainsi que la condition d'adaptation de bruit minimum à faible $\mathrm{V}_{\mathrm{DS}}$ tout en évitant le courant de fuite par la grille (grillé isolée). 


\section{Conclusion du chapitre III}

Le transport électronique a été étudié dans ce chapitre par la méthode particulaire Monte Carlo semi-classique,. Les performances de MOSFET III-V en régimes statique et dynamique ont ainsi été évaluées à faible condition de polarisation. Nous nous sommes enfin intéressés au comportement du bruit à haute fréquence de dispositifs intrinsèques. Nous avons proposé trois architectures alternatives par rapport à la structure bulk. La meilleure conception correspond à la structure MOS-COMB avec un canal non dopé enterré, une zone active mince et un espaceur intercalé entre l'oxyde de grille et le canal principal. Ce genre de dispositif (avec $\mathrm{L}_{\mathrm{G}}=50 \mathrm{~nm}$ ) peut atteindre une forte balisticité intrinsèque $(55 \%)$. Grâce au transport quasi-balistique, le transistor présente de hautes performances en termes de rapidité, même à faible $\mathrm{V}_{\mathrm{DS}}$, et donc avec une faible consommation de puissance. Si on vise une application analogique comme un LNA (Low Noise Amplifier), MOS-COMB atteint une performance dynamique intrinsèque très prometteuse : $\mathrm{f}_{\mathrm{T}}=750 \mathrm{GHz}, \mathrm{f}_{\mathrm{MAX}}=600 \mathrm{GHz}$ et NFmin $=0,5 \mathrm{~dB}$ à $50 \mathrm{GHz}$ pour $\mathrm{W}=10 \mu \mathrm{m}$ ainsi qu'un rendement fréquence-consommation remarquable : 400 $\mathrm{GHz}$ de fréquence d'opération pour $20 \mathrm{~mW} / \mathrm{mm}$ de puissance à $\mathrm{V}_{\mathrm{DS}}=0,2 \mathrm{~V}$. Par ailleurs, la performance en bruit pourrait être améliorée dans une structure à canal non dopé et sans espaceur comme MOS-ThinBody, mais la performance fréquentielle sera alors dégradée à cause de la diminution de la balisticité intrinsèque (25\%) ainsi que de la transconductance. Enfin, l'étude comparative (sous même condition physique : $\mathrm{L}_{\mathrm{G}}$, EOT, $\mathrm{L}_{\text {acc }}$ et $\mathrm{V}_{\mathrm{DS}}$ ) avec les dispositifs à base de Si d'architecture conventionnelle permet de démontrer clairement le potentiel de l'architectures de III-V MOSFET submicronique à haute fréquence pour les applications analogique à petit signal de faible puissance de consommation : dans le cas de $\mathrm{Si}$, $\mathrm{f}_{\mathrm{T}}=120 \mathrm{GHz}, \mathrm{f}_{\mathrm{MAX}}=95 \mathrm{GHz}$ et $\mathrm{NFmin}=3,5 \mathrm{~dB}$ à $50 \mathrm{GHz}$ pour $\mathrm{W}=10 \mu \mathrm{m}$ avec comme rendement fréquence/ consommation $90 \mathrm{GHz} / 20 \mathrm{~mW} / \mathrm{mm}$. 


\section{Conclusion générale}

Parmi les semi-conducteurs standard, les matériaux III-V à masse effective de conduction sont des candidats de choix pour obtenir les meilleures performances fréquentielles à partir de transistors n-FET destinés à des applications analogiques voire numériques. Mais introduire la topologie MOS - qui a par ailleurs fait le succès de la filière $\mathrm{Si}$ - dans les technologies III-V pose de nombreux problèmes en particulier en termes de contrôle de la qualité de l'interface III-V/diélectrique. Dans ce contexte, ce travail a principalement exploré et évalué par simulations numériques en régime statique ou dynamique les différentes potentialités des architectures de MOSFET III-V sous faible tension de polarisation pour des applications à faible consommation.

Dans le chapitre II, nous avons étudié théoriquement le contrôle de charge dans un système MOS de type métal/high-k/III-V. Le modèle conventionnel utilisé dans le cas du Si n'est pas transposable directement et a dû être amélioré en ajoutant les différents phénomènes physiques suivants: statistique de Fermi-Dirac, quantification des niveaux d'énergie, correction de la non-parabolicité et pièges d'interface. L'étude physique que nous avons réalisée aboutit à une épaisseur d'oxyde correspondant à un compromis entre l'efficacité du contrôle de charge et la dégradation de la capacité de grille due à la faible densité d'états des matériaux envisagés. Les particularités du contrôle de charge dans des structures alternatives, à film actif mince (Thin Body) et/ou, canal enterré, ont aussi été évaluées. Un point très important est que la commande de charge par la grille au sein du semi-conducteur III-V peut être influencée par des pièges d'interface réduisant alors l'efficacité du mouvement du niveau de Fermi et donc dégradant la pente sous le seuil.

La simulation a ensuite été étendue au cas d'une géométrie 2D pour prendre en compte les effets de canal court et les capacités parasites. Nous avons appliqué aux résultats de Poisson-Schrödinger un modèle de transport quasi-balistique pour effectuer une première série d'estimations du courant de drain délivré par un MOSFET III-V. Les meilleures performances ont été obtenues dans la structure MOS-ThinBody à base d'InGaAs. Une dissipation de puissance inférieure à $20 \mathrm{~mW} / \mathrm{mm}$ à $\mathrm{V}_{\mathrm{DS}}=0,2 \mathrm{~V}$ a été obtenue tout en gardant une fréquence de transition $\mathrm{f}_{\mathrm{T}}$ supérieure à quelques $100 \mathrm{GHz}$. 
Dans le cadre d'une approche semi-classique, le transport électronique a été étudié grâce à la méthode particulaire Monte Carlo, en régimes statiques et dynamiques sous faible polarisation. Le transport quasi-balistique, les caractéristiques électriques statiques et dynamiques ainsi que le bruit en haute fréquence ont été quantitativement étudiés. En outre, nous avons comparé trois architectures alternatives à la structure "bulk" standard. Les analyses MC de ces dispositifs ont fourni des lignes directrices pour la conception des dispositifs III-V. Parmi les 4 structures étudiées, la meilleure, même en termes de bruit, est la structure MOS-COMB à canal mince, enterré et non dopé. Finalement, par comparaison avec des dispositifs sur Si, ces études montrent clairement l'intérêt des architectures de MOSFET III-V pour les applications à haute fréquence et à faible consommation.

Mes travaux pourraient se poursuivre par une étude très large concernant la modélisation des dispositifs. La description de la dynamique d'un gaz d'électrons dans les MOS III-V doit être raffinée grâce à des valeurs expérimentales de mobilité. Cela permettrait aussi d'inclure les effets des phonons optiques 'soft' d'interface et des effets Coulombien dus aux pièges d'interface. La densité de porteurs est souvent grande en comparaison de la densité d'états effective (pour InGaAs $\sim 10^{17} \mathrm{~cm}^{-3}$ ) particulièrement dans les zones d'accès. Le principe d'exclusion de Pauli devrait donc être implémenté dans la simulation particulaire. Les phénomènes de confinement quantique devraient aussi être pris en compte, par exemple dans une approche par mode ou 'mode space'. Tout cela permettrait de réévaluer plus finement les performances statiques, dynamiques. Enfin l'utilisation de la contrainte bi-axiale pourrait être une solution pour améliorer les performances de ce type de dispositifs. 


\section{Annexe - A :}

\section{Modèle du contrôle de charge : résolution Poisson - Schrödinger}

\section{A-a Mise en place d'un modèle pertinent}

Le potentiel électrostatique est relié à la densité de charge $\rho(z)$ par l'équation de Poisson :

$$
\frac{d^{2} \varphi(z)}{d z^{2}}=-\frac{\rho(z)}{\varepsilon_{s}}=-\frac{q\left(p(\varphi(z))-n(\varphi(z))+N_{D}-N_{A}\right)}{\varepsilon_{s}}
$$

La densité de charge $\rho(\mathrm{z})$ est une somme des différentes contributions des charges fixes et des porteurs libres. Les densités de donneurs $\mathrm{N}_{D}$ et accepteurs $\mathrm{N}_{\mathrm{A}}$ sont supposées constantes dans l'approximation de complète ionisation. La densité des porteurs mobiles (électrons et trous) est une fonction du potentiel électrostatique. La densité d'électrons peut être obtenue à partir de l'intégration sur toute la plage des énergies supérieures à la bande de conduction utilisant une densité d'états $\mathrm{D}(\mathrm{E})$ multiplié par la fonction de distribution Fermi-Dirac comme :

$$
n=\int_{E_{C}}^{\infty} d E \frac{D(E)}{\exp \left(\frac{E-E_{F}}{k_{B} T}\right)+1}
$$

On peut procéder de la même façon par rapport aux énergies de la bande de valence pour calculer la densité de trous. Pour une densité d'états dans l'espace réciproque 3D-k et en négligeant l'effet de non-parabolicité, on a donc :

$$
n=\frac{1}{2 \pi^{2}}\left(\frac{2 m_{e_{-} D O S}}{\hbar^{2}}\right)^{3 / 2} \int_{E_{C}}^{\infty} d E \frac{\sqrt{E-E_{C}}}{\exp \left(\frac{E-E_{F}}{k_{B} T}\right)+1}
$$

On peut utiliser le changement de variable suivant :

$$
\begin{gathered}
n_{v}(\varphi(z))=\frac{4}{\sqrt{\pi}}\left(\frac{2 \pi k_{B} T m_{D O S_{-} v}^{*}}{h^{2}}\right)^{3 / 2} \times \int_{0}^{\infty} \frac{\sqrt{\eta_{v}}}{\exp \left(\eta_{v}-\eta_{F v}\right)+1} d \eta_{v} \\
\text { Avec } \eta_{v}=\frac{E-E_{C v}(z)}{k_{B} T}, \quad \eta_{F v}=\frac{E_{F}-E_{C v}(z)}{k_{B} T}, E_{C v}(z)=-q \varphi(z)-\Delta E_{B C}
\end{gathered}
$$

L'indice de vallée v vaut $\Gamma, \mathrm{L}$ ou $\mathrm{X} . \Delta \mathrm{E}_{\mathrm{BC}}$ est la discontinuité de la bande de conduction par rapport au matériau de référence. En faisant apparaitre la densité équivalente d'états $N_{C_{-} v}$ et l'intégrale de Fermi-Dirac, on obtient : 


$$
\begin{gathered}
n_{v}(\varphi(z))=N_{c_{-} v} \times \mathfrak{J}_{1 / 2}\left(\frac{E_{F}-E_{C v}(z)}{k_{B} T}\right) \\
\text { avec } N_{c_{-} v}=2\left(\frac{2 \pi m_{D S_{-} v}^{*} k_{B} T}{h^{2}}\right)^{3 / 2} \mathfrak{J}_{1 / 2}\left(\eta_{F v}\right)=\frac{2}{\sqrt{\pi}} \int_{0}^{\infty} \frac{\sqrt{\eta_{v}}}{\exp \left(\eta_{v}-\eta_{F v}\right)+1} d \eta_{v}
\end{gathered}
$$

La densité d'électrons est obtenue par somme sur ces trois vallées $(\Gamma, \mathrm{L}$ et $\mathrm{X})$. Le calcul de la densité des trous est très similaire que celui des électrons. Leur densité résulte de la somme sur deux vallées : trous lourds hh et trous légers lh (la contribution de la bande split-off ou SO, très éloignée des précédentes, est ignorée). On a pour chaque vallée :

$$
p_{v}(\varphi(z))=N_{v} \times \mathfrak{J}_{1 / 2}\left(\frac{E_{V v}(z)-E_{F}}{k_{B} T}\right) \text { avec } E_{V v}(z)=-q \varphi(z)+\Delta E_{B V}
$$

$\Delta \mathrm{E}_{\mathrm{BV}}$ est la discontinuité de la bande de valence par rapport au matériau de référence. On introduit les Eq. A-5 et A-6 dans 1'Eq. A-1, on obtient une équation de Poisson non linéaire à résoudre avec comme inconnue le potentiel $\varphi(\mathrm{z})$. On utilise la méthode de différence finie, puis un algorithme itératif de type Newton-Raphson [Nougier2001]. Cette première étape de calcul peut nous fournir la solution 'classique' concernant le potentiel initial ainsi que la distribution des charges.

Ensuite, la solution 'quantique' pourra être obtenue par résolution de l'équation de Schrödinger stationnaire 1D. L'énergie potentielle effective $E_{C v}$ dans l'équation de Schrödinger est supposée donnée par la solution de l'équation de Poisson (potentiel de déplétion et potentiel d'inversion). Le potentiel image et les effets d'échange et corrélation sont négligés dans notre calcul.

$$
-\frac{\hbar^{2}}{2} \frac{\partial}{\partial z}\left(\frac{1}{m_{\text {conf_v }}(z)} \frac{\partial}{\partial z}+E_{C v}(z)\right) \xi_{\mu_{-} v}(z)=E_{\mu_{-} v_{\mu_{-} v}} \xi(z)
$$

Cette équation aux valeurs propres doit être résolue pour chaque vallée. Chacune des vallées a ses propres sous bande $E_{\mu_{-} v}$ (valeurs propres) et fonctions d'onde associées $\xi_{\mu_{-} v}(z)$ (vecteur propre). $m_{\text {conf } \_}$est la masse de confinement pour chaque vallée selon la direction de quantification. Dans la fenêtre ondulatoire ou tranche Schrödinger (un intervalle $\left[\mathrm{z}_{1}, \mathrm{z}_{2}\right]$ où cette équation est résolue), les fonctions d'onde doivent être normalisées, c'est-à-dire $\int_{z_{1}}^{z_{2}}\left|\xi_{\mu_{-} v}(z)\right|^{2} d z=1$. Hors de cet intervalle, les fonctions d'onde sont considérées comme nulles. La pénétration des fonctions d'onde dans le diélectrique n'est pas prise en compte, du fait que 
la discontinuité en bande de conduction est supérieure à $3 \mathrm{eV}$. Donc $\mathrm{z}_{1}$ est à l'interface oxyde/III-V et $\mathrm{z}_{2}$ est au-delà de la zone de charge d'espace.

Le schéma numérique est obtenu en approximant les opérateurs par les méthodes classiques de différences finies pour les opérations différentielles. La recherche des valeurs et des vecteurs propres s'effectue numériquement grâce à une méthode d'Arnoldi [Lehoucq1998] sur l'équation matricielle obtenue par discrétisation dans la tranche Schrödinger. La densité totale d'électrons en l'espace réciproque 2D-k dans la couche d'inversion est donnée par :

$$
\begin{aligned}
& n_{2 D}(z)=\sum_{v} \sum_{\mu} g_{v} n_{\mu, v}\left|\xi_{\mu}(z)\right|^{2} \\
& \text { où } n_{\mu, v}=\frac{m_{v_{-} D O S}}{\pi \hbar^{2}} \int_{E_{\mu, v}}^{\infty} \frac{d E}{1+\exp \left(\frac{E-E_{F}}{k_{B} T}\right)}=\frac{k_{B} T m_{v_{-} D O S}}{\pi \hbar^{2}} \operatorname{Ln}\left(1+\exp \left(\frac{E_{F}-E_{\mu, v}}{k_{B} T}\right)\right)
\end{aligned}
$$

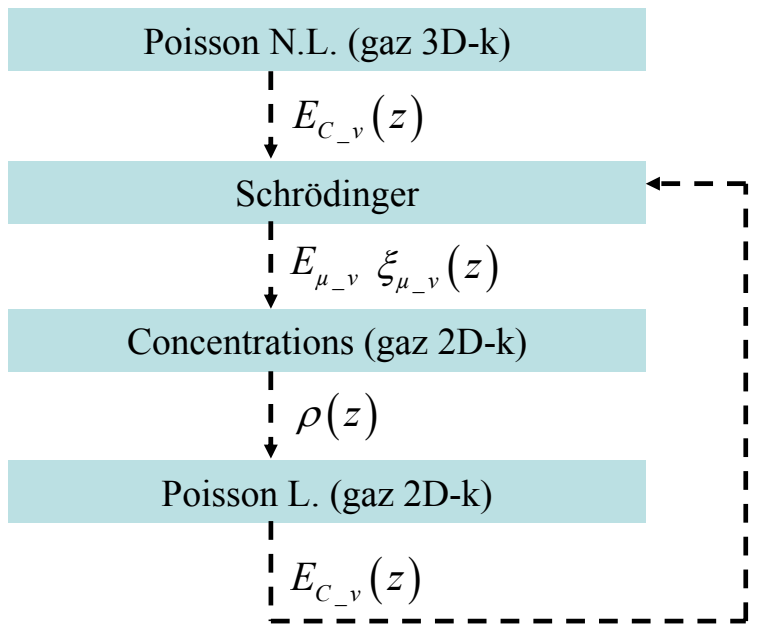

Figure A-1 Les séquences de la résolution couplée des équations de Schrödinger et de Poisson. N.L. signifie que l'équation est non linéaire et 'L.' qu'elle est linéaire [SaintMartin2005Thèse]

Cette densité est obtenue en sommant toute les contributions de chaque vallées ' $\mathrm{v}$ ' et de chaque niveau d'énergie de sous bande ' $\mu$ '. $g_{v}$ est du degré de dégénérescence de vallée. Ces contributions sont pondérées par la densité de probabilité de présence $\left|\xi_{\mu}(z)\right|^{2}$ associée. Les trous sont traités de façon très similaire si leurs états sont considérés comme quantifiés, sinon on utilise l'Eq. A-6. La densité volumique totale de charge $\rho(z)$ s'obtient en sommant les différentes contributions : $\rho(z)=q \times\left(N_{D}(z)-N_{A}(z)+p_{2 D}(z)-n_{2 D}(z)\right)$. Ensuite on résout l'équation linéaire de Poisson pour raffiner l'énergie potentielle effective $E_{C v}(z)$. Puis ces valeurs de $E_{C v}(z)$ sont injectées dans l'équation de Schrödinger pour recalculer l'énergie des sous bandes $E_{\mu_{-} v}$ et les fonctions d'onde associées $\xi_{\mu_{-} v}(z)$. On reconsidère alors l'équation 
linéaire de Poisson en introduisant la densité totale de charge en 2D-k. Finalement on aura un nouveau profil de $E_{C v}(z)$. On résout ainsi le système d'équations de manière auto-cohérente par un processus itératif avec un schéma d'algorithme 'prédiction-correction' : on choisit un potentiel d'essai, on obtient alors les fonctions d'ondes électroniques à partir desquelles on modifie le potentiel d'essai et on recommence le calcul. La solution numérique du problème est obtenue quand le potentiel calculé est suffisamment proche du potentiel d'essai. La méthode la plus intuitive consiste à prendre comme potentiel d'essai pour résoudre la $(n+1)^{\text {ème }}$ itération, une combinaison linéaire du potentiel d'essai et du potentiel calculé de l'itération précédente :

$$
E_{C v_{-} e s s}^{n+1}(z)=E_{C v_{-} e s s}^{n}(z)+k \cdot\left(E_{C v_{-} \text {cal }}^{n}(z)-E_{C v_{-} e s s}^{n}(z)\right)
$$

Il existe différentes méthodes empiriques ou systématiques, permettant de choisir le coefficient k. La méthode et l'algorithme du calcul auto-cohérent des équations PoissonSchrödinger sont détaillés en Annexe B. Les séquences sont effectuées en boucle jusqu'à ce que la convergence soit atteinte (cf. Fig. A-1). Cette convergence est obtenue quand le potentiel calculé est suffisamment proche du potentiel d'essai en chaque nœud entre les itérations. Cette méthode permet de corriger le potentiel électrostatique non seulement en fonction de la densité de charges mais également de la distribution spatiale de ces charges.

Enfin, la charge nette totale Q surfacique peut être obtenue en intégrant la densité volumique de charge $\rho(z)$ dans toute la structure. La capacité de grille est calculée par $C_{G} \approx d Q / d V_{G}$ et la capacité de couche d'inversion par $C_{I N V} \approx d Q / d \varphi_{S}$ où $\varphi_{S}$ est le potentiel de surface.

Pour résoudre le problème en géométrie $2 \mathrm{D}$, nous avons utilisé 1'approche par modes (mode space) [Ren2001Thèse] et [SaintMartin2005Thèse]. Dans l'approximation de la masse effective, l'Hamiltonien associé à l'équation de Schrödinger stationnaire s'écrit comme :

$$
-\frac{\hbar^{2}}{2 m_{x}} \frac{\partial^{2} \psi(x, z)}{\partial x^{2}}-\frac{\hbar^{2}}{2 m_{z}} \frac{\partial^{2} \psi(x, z)}{\partial z^{2}}+E_{C}(x, z) \psi(x, z)=E \times \psi(x, z)
$$

Ici, $E_{C}(x, z)$ est le profil de la bande de conduction issu de l'équation de Poisson 2D, c'est-àdire que ce potentiel électrostatique est résolu selon la direction de confinement (z) pour chaque position selon la direction de transport (x). L'approche par modes permet de découpler sous certaines conditions l'équation de Schrödinger 2D en 2 équations 1D selon x et z. Dans cette approximation, les fonctions d'onde sont mises sous la forme $\psi(x, z)=\sum_{n} \varphi_{n}(x) \times \xi_{n}(x, z)$ avec $\xi_{n}(x, z)$ fonction d'onde enveloppe associée à une 
sous bande, ou mode, avec une énergie propre $E_{n}(x)$ pour une position de coordonnées x. $\left|\varphi_{n}(x)\right|^{2}$ représente la probabilité de trouver un électron sur le mode 'n' à la position $\mathrm{x}$ dans la tranche Schrödinger. L'équation 2D (A-10) peut se découpler en deux systèmes d'équations 1D :

$$
\begin{aligned}
& -\frac{\hbar^{2}}{2 m_{z}} \frac{\partial^{2} \xi_{n}\left(x_{0}, z\right)}{\partial z^{2}}+E_{C}\left(x_{0}, z\right) \xi_{n}\left(x_{0}, z\right)=E_{n}\left(x_{0}\right) \times \xi_{n}\left(x_{0}, z\right), \text { pour tout } \mathrm{x}_{0} \\
& -\frac{\hbar^{2}}{2 m_{x}} \frac{\partial^{2} \varphi_{n}(x)}{\partial x^{2}}+E_{n}(x) \varphi_{n}(x)=E \times \varphi_{n}(x)
\end{aligned}
$$

si les couplages entre les modes $\int \xi_{n}^{*}\left(x_{0}, z\right) \frac{\partial \xi_{m}\left(x_{0}, z\right)}{\partial x} d z$ et $\int \xi_{n}^{*}\left(x_{0}, z\right) \frac{\partial^{2} \xi_{m}\left(x_{0}, z\right)}{\partial x^{2}} d z$ sont négligeables devant $\int \xi_{n}^{*}\left(x_{0}, z\right) \xi_{m}\left(x_{0}, z\right) d z$. La justification est détaillée dans la référence [Ren2001Thèse] et [SaintMartin2005Thèse]. Notre calcul s'effectue de façon auto-cohérente entre l'équation de Poisson 2D et celle de la première équation dans A-11. La solution 2D nous fournit l'évolution des énergies des modes $E_{n}(x)$ dans le sens du transport ainsi que les fonctions d'enveloppe $\xi_{n}(x, z)$ dans le sens du transport et de confinement. Cette approche est d'autant plus valide que les fonctions $\xi_{n}(x, z)$ n'évoluent pas fortement le long du dispositif [SaintMartin2005Thèse].

\section{A-b Correction de non-parabolicité}

La structure de bandes des matériaux III-V se caractérise souvent par une grande nonparabolicité. La correction de non-parabolicité va influencer le contrôle de charges via la capacité de grille. Cette correction intervient non seulement dans le calcul classique (on ne résout que l'équation de Poisson) mais également dans le calcul quantique. Dans le cas classique, il faut que l'on recalcule la densité d'états dans l'espace réciproque 3D-k en prenant en compte l'effet de non-parabolicité [Ariel-Altschul1992IEEE] et [Islam2011SST]. L'Eq. A4 peut devenir :

$$
n_{v}(\varphi(x))=\frac{4}{\sqrt{\pi}}\left(\frac{2 \pi k_{B} T m_{v}^{*}}{h^{2}}\right)^{3 / 2} \times \int_{0}^{\infty} \frac{\sqrt{\eta_{v}}\left(1+\alpha \eta_{v}\right)\left(1+2 \alpha \eta_{v}\right)}{\exp \left(\eta_{v}-\eta_{F v}\right)+1} d \eta_{v}
$$

où $\alpha$ est le coefficient de non-parabolicité. Les notations sont inchangées par rapport à l'Eq. A-4. Cet effet de non-parabolicité va modifier la solution de l'équation Poisson non linéaire dans les calculs classiques. 
La prise en compte de la correction non-parabolique n'est pas évidente pour des systèmes de gaz d'électrons avec une dimension réduite (dans l'espace réciproque 2D-k, 1D-k voire 0D-k). En effet, la relation de dispersion dans le plan parallèle du transport et les niveaux d'énergie pour chaque sous bande doivent tous les deux contenir la correction nonparabolique. Dans cette section, nous révisons cette correction non-parabolique pour un gaz d'électrons dans l'espace réciproque 2D-k (2DEG), suivant une approche largement inspirée de [Fischetti1993PRB], [Jin2007JAP] et [O'Regan2010JAP]. Nous renvoyons à ces références pour plus de détails.

Dans le semi-conducteur massif, la non-parabolicité est généralement prise en compte par l'expression de la relation de dispersion énergie - vecteur d'onde au voisinage de la minimale de la bande de conduction [Jacoboni1983RMP] :

$$
E_{K}=\frac{-1+\sqrt{1+4 \alpha \gamma_{K}}}{2 \alpha}
$$

avec

$$
\gamma_{K}=\frac{\hbar^{2} k_{x}^{2}}{2 m_{x}}+\frac{\hbar^{2} k_{y}^{2}}{2 m_{y}}+\frac{\hbar^{2} k_{z}^{2}}{2 m_{z}}
$$

où $m_{x}, m_{y}$ et $m_{z}$ sont des masses effectives a priori anisotropes. $K=\left(\vec{k}, k_{z}\right)$ est le vecteur d'onde dans l'espace réciproque $3 \mathrm{D}, \vec{k}=\left(k_{x}, k_{y}\right)$ est le vecteur d'onde dans le plan $(x, y)$. $E_{K}$ est l'énergie des électrons mesurée à partir du minimum de la bande de conduction. Quand le confinement est par exemple selon la direction $\mathrm{z}$, les équations correspondantes comme l'équation de Luttinger-Kohn [Luttinger1955PR] et de Schrödinger modifiée [LópezVillanueva1993PRB] deviennent plus compliquées. En effet, $E_{K}$ ne peut plus être décomposée en énergies cinétiques dans les directions parallèles et perpendiculaires au confinement. Pour résoudre ces équations, il est préférable de réécrire celle dans l'espace réciproque en utilisant des fonctions de base planes ou sinusoïdales et recalculer l'énergie de valeurs propres pour chaque composante transversale de vecteur d'onde [Fischetti1993PRB] et [Esseni2005PRB]. Pour éviter ces difficultés de calcul, deux approches simplifiées ont été proposées, basées sur les niveaux d'énergie de sous bande $E_{\mu}^{(0)}$ et les fonctions d'onde associées $\xi_{\mu}^{(0)}(z)$ obtenus par résolution de l'équation Schrödinger dans l'approximation des bandes paraboliques. La première méthode est proposée par Jungemann [Jungemann1993SSE], qui prend en compte la correction de la bande parabolique seulement dans la direction du transport : 


$$
\begin{aligned}
& E_{t o t} \approx E_{\mu}^{(0)}+\frac{-1+\sqrt{1+4 \alpha \gamma_{\vec{k}}}}{2 \alpha} \\
& \gamma_{\vec{k}}=\frac{\hbar^{2} k_{x}^{2}}{2 m_{x}}+\frac{\hbar^{2} k_{y}^{2}}{2 m_{y}}
\end{aligned}
$$

Dans ce modèle, la non-parabolicité est complètement négligée dans la direction du confinement, le minimum de la sous bande $E_{\mu}^{(0)}$ n'est donc pas touché par cette correction. Ce modèle est largement utilisé dans les cas 2D-k [Lundstrom2000] et 1D-k [Ramayya2007IEEE] grâce à sa simplicité. La deuxième méthode est proposée par Fischetti et Laux [Fischetti1993PRB], ils utilisent des informations supplémentaires contenues dans la fonction d'onde $\xi_{\mu}^{(0)}(z)$. Les énergies propres seront estimées par le traitement sur les termes qui sont proportionnels à $\alpha$, considérés comme des perturbations. Les niveaux minima des sous bandes $E_{\mu}$ et l'énergie totale $E_{\text {tot }}$ seront corrigés par le théorème de perturbation stationnaire d'ordre un de la mécanique quantique :

$$
\begin{gathered}
E_{t o t} \approx E_{\mu}+\gamma_{\vec{k}}\left(1-\alpha \gamma_{\vec{k}}-2 \alpha \int_{0}^{\infty} d z\left(E_{\mu}^{(0)}-q \varphi(z)\right)\left|\xi_{\mu}^{(0)}(z)\right|^{2}\right) \\
E_{\mu} \approx E_{\mu}^{(0)}-\alpha \int_{0}^{\infty} d z\left(E_{\mu}^{(0)}-q \varphi(z)\right)^{2}\left|\xi_{\mu}^{(0)}(z)\right|^{2}
\end{gathered}
$$

où $q \varphi(z)$ est l'énergie de potentiel électrostatique. Les termes en intégrale représentent les valeurs de l'espérance pour une densité de probabilité $\left|\xi_{\mu}^{(0)}(z)\right|^{2}$. Cette méthode a pris en compte la correction non-parabolique dans la direction du confinement et dans le plan du transport. L'Eq. A-16 est obtenue par un développement de l'Eq. A-13 pour $\gamma_{K}$ tendant vers zéro :

$$
E_{K}=\gamma_{K}\left(1-\alpha \gamma_{K}\right)
$$

Ce modèle n'est toutefois pas bien approprié aux cas de figure comme les structures à base d'UTBSOI (ultra thin body silicon on insulator) ou les MOSFET III-V. En effet l'Eq. A-16 devient discutable quand les niveaux d'énergie des sous bandes parabolique sont supérieurs à $0,5 \mathrm{eV}$ par rapport au bas de la bande de conduction $\left(E_{\mu}^{(0)}-E_{C}>0.5 \mathrm{eV}\right)$ [Jin2007JAP]. Cette situation peut être s'observée sous forte quantification (dans un puits quantique très mince formé dans un matériau qui possède une très faible masse effective selon la direction de confinement) et forte non-parabolicité (coefficient supérieur à $0,5 \mathrm{eV}^{-1}$ ) [O'Regan2010JAP]. Ce problème est résolu phénoménologiquement en corrigeant l'énergie de sous bande 
[Jin2007JAP]. On substitue alors l'opérateur de l'énergie cinétique parabolique selon la direction du confinement par la valeur de l'espérance (selon une densité de probabilité $\left.\left|\xi_{\mu}^{(0)}(z)\right|^{2}\right)$ dans l'Eq. A-13. En réglant $\gamma_{\vec{k}}=0$, on aura le niveau minimum de la sous bande :

$$
E_{\mu}=U_{\mu}+\frac{-1+\sqrt{1+4 \alpha\left(E_{\mu}^{(0)}-U_{\mu}\right)}}{2 \alpha}
$$

où la valeur de l'espérance de l'énergie de potentiel électrostatique $U_{\mu}$ est donnée par :

$$
U_{\mu}=\int q d z \varphi(z)\left|\xi_{\mu}^{(0)}(z)\right|^{2}
$$

Le terme secondaire de l'Eq. A-19 peut être considéré comme la valeur de l'espérance de l'énergie cinétique dans la direction du confinement. Par ailleurs, les quantités physiques pertinentes du système doivent être également modifiées après la correction non parabolique, comme les vecteurs d'onde, les vitesses de groupe et la densité des états dans chaque sous bande $\mu$. Par exemple, la densité d'états $D O S_{\mu}(E)$ pour une sous bande $\mu$ donnée n'est plus une constante, elle doit dépendre de l'énergie et d'une densité de probabilité :

$$
\begin{gathered}
D_{O}(E)=\Theta\left(E-E_{\mu}\right) \frac{g_{\mu} m_{D O S_{-} \mu}}{\pi \hbar^{2}}\left\{1-2 \alpha\left(E-E_{\mu}\right)-2 \alpha \int_{0}^{\infty} d z\left(E_{\mu}^{(0)}-q \varphi(z)\right)\left|\xi_{\mu}^{(0)}(z)\right|^{2}\right\} \\
D_{0}(E)=D_{\mu}\left(S_{\mu}^{(0)}(E) \times N P\left(E-E_{\mu}\right)\right.
\end{gathered}
$$

On constate que la densité des états non paraboliques peut être exprimée par une multiplication entre une densité d'états parabolique $\operatorname{DOS}_{\mu}^{(0)}(E)$ et un facteur de correction non parabolique $N P\left(E-E_{\mu}\right)$ pour une sous bande donnée. Ce facteur correctif peut également être utilisé pour les autres grandeurs physiques comme la vitesse de groupe. Finalement la densité totale d'électrons 2D-k dans la couche d'inversion en tenant compte de l'effet de non-parabolicité peut être calculée [Fischetti1993PRB] et [O'Regan2010JAP] :

$$
n_{2 D}(z)=\frac{k_{B} T}{\pi \hbar^{2}} \sum_{v} \sum_{\mu} g_{\mu} m_{D O S_{-} \mu, v}\left\{\left[1+2 \alpha\left(E_{\mu, v}-U_{\mu, v}\right)\right] \mathfrak{I}_{0}(\eta)+2 \alpha k_{B} T \mathfrak{I}_{1}(\eta)\right\}\left|\xi_{\mu}^{(0)}(z)\right|^{2}
$$

Ici, $\mathfrak{I}_{0}(\eta)$ et $\mathfrak{J}_{1}(\eta)$ sont les intégrales de Fermi-Dirac d'ordre zéro et un, avec $\eta=\left(E_{F}-E_{\mu, v}\right) / k_{B} T . m_{D O S_{-} \mu, v}$ est la masse effective de densité d'états de la sous bande $\mu$ de la vallée $v$. 
Annexe - B :

\section{La méthode numérique et l'algorithme du calcul auto-cohérent des équations Poisson-Schrödinger}

\section{Equation de Schrödinger}

Afin de résoudre numériquement l'équation de Schrödinger stationnaire en 1D :

$$
-\frac{\hbar^{2}}{2} \frac{d}{d x}\left(\frac{1}{m^{*}(x)} \frac{d}{d x}\right) \psi(x)+V(x) \psi(x)=E \psi(x)
$$

En utilisant la méthode des différences finies, on discrétise cette équation différentielle à l'aide de trois points consécutifs :

$$
-\frac{\hbar^{2}}{2}\left(\frac{2\left(\psi_{i+1}-\psi_{i}\right)}{m_{i+1 / 2} h_{i}\left(h_{i}+h_{i-1}\right)}-\frac{2\left(\psi_{i}-\psi_{i-1}\right)}{m_{i-1 / 2} h_{i-1}\left(h_{i}+h_{i-1}\right)}\right)=\lambda \psi_{i}
$$

Cette relation pourra être mise sous la forme d'une équation matricielle :

$$
\sum_{j=1}^{n} A_{i j} \psi_{j}=\lambda \psi_{i}
$$

avec

$$
A_{i j}=\left\{\begin{array}{ll}
-\frac{\hbar^{2}}{2}\left(\frac{2}{m_{i+1 / 2}} \frac{1}{h_{i+1}\left(h_{i}+h_{i-1}\right)}\right) & \text { si } j=i+1 \\
-\frac{\hbar^{2}}{2}\left(\frac{2}{m_{i-1 / 2}} \frac{1}{h_{i+1}\left(h_{i}+h_{i-1}\right)}\right) & \text { si } j=i-1 \\
-A_{i i+1}-A_{i i-1}+V_{i} & \text { si } j=i \\
0 & \text { sinon }
\end{array}\right\}
$$

où l'indice i peut identifier les points du maillage, $h_{i}$ est la taille du maillage entre deux points adjacents $x_{i}$ et $x_{i+1}$, son indice est référé par $i \pm 1 / 2$. Cette matrice $A_{i j}$ est tridiagonale, elle est en outre symétrique si et seulement si la taille du maillage $h_{i}$ est uniforme dans toute la structure. Dans nos simulations, on a utilisé des maillages non-uniformes selon la variation du potentiel local, ce qui rend le calcul plus compliqué. On définit un paramètre comme :

$$
L_{i}^{2}=\left(h_{i}+h_{i-1}\right) / 2
$$

L’équation B-4 devient : 


$$
A_{i j}=\left\{\begin{array}{ll}
-\frac{\hbar^{2}}{2}\left(\frac{2}{m_{i+1 / 2}} \frac{1}{h_{i}}\right) \frac{1}{\mathrm{~L}_{\mathrm{i}}^{2}} & \text { si } j=i+1 \\
-\frac{\hbar^{2}}{2}\left(\frac{2}{m_{i-1 / 2}} \frac{1}{h_{i-1}}\right) \frac{1}{\mathrm{~L}_{\mathrm{i}}^{2}} & \text { si } j=i-1 \\
-A_{i i+1}-A_{i i-1}+V_{i} & \text { si } j=i \\
0 & \text { sinon }
\end{array}\right\}
$$

Si on pose : $B_{i j}=L_{i}^{2} A_{i j}$, on peut s'écrire : $B=M A$. Ici $M$ est une matrice diagonale avec ses éléments $L_{i}^{2}$. La matrice $B$ est considérée comme un produit de deux matrices, l'une est diagonale et l'autre est tridiagonale, La matrice $B$ est donc tridiagonale. D'après les équations A-5 et A-6, la matrice $B$ est également symétrique, c'est-à-dire $B_{i i+1}=B_{i+1 i}$. Cela permet de résoudre l'équation suivante :

$$
B \psi=M A \psi=\lambda M \psi
$$

La matrice diagonale M sera obtenue par la méthode des différences finies, on peut l'exprimer comme : $M=L L$, où $L$ est une matrice diagonale avec des éléments $L_{i}$. D'après l'équation A-7, on a donc :

$$
L^{-1} B L^{-1} L \psi=L^{-1} L L A \psi=\lambda L^{-1} L L \psi
$$

Ces équations peuvent être mises sous forme :

$$
H \phi=\lambda \phi
$$

avec $H=L^{-1} B L^{-1}$ et $\phi=L \psi$. La matrice $H$ est symétrique et tridiagonale, c'est-à-dire $H=H^{t}$, car $B$ est symétrique et tridiagonale et $L$ est diagonal. Désormais, on résout l'équation B-9 pour trouver les valeurs propres $\lambda$ et les vecteurs propres $\phi$. Si $\psi_{k}$ et $\psi_{l}$ sont deux fonctions d'onde associées dans le puits potentiel, elles sont liées l'une à autre par :

$$
\psi_{k}^{T} M \psi_{l}=\left\{\begin{array}{lc}
1 & \text { si } k=l \\
0 & \text { si } k \neq l
\end{array}\right\}
$$

Finalement, dans nos calculs, les niveaux d'énergie et les fonctions d'ondes associées à la matrice réelle $H$ seront obtenues par méthode d'Arnoldi [Lehoucq1998].

On doit noter que cette méthode peut s'appliquer également dans le cas bidimensionnel. En utilisant la même approche de discrétisation pour l'équation de Schrödinger 2D, on pourrait obtenir des équations similaires à (B-7) dans lesquelles $\mathrm{B}$ est également symétrique et $M$ reste diagonale. Par conséquent, après les mêmes opérations matricielles, on obtiendra toujours l'équation B-9. 


\section{Equation de Poisson}

Avant la discrétisation sur l'espace, nous exposons la généralisation de la méthode de Newton pour résoudre l'équation de Poisson non linéaire. La densité des électrons est désormais une fonction du potentiel $\phi(x)$, on peut nommer cette fonction comme : $n(\phi)$. L'équation de Poisson devient donc :

$$
\frac{d}{d x}\left(\varepsilon_{s c} \frac{d \phi}{d x}\right)=\frac{-q\left(N_{d o p}-n(\phi)\right)}{\varepsilon_{0}}
$$

Si on suppose que la solution exacte de cette équation est $\phi^{0}(x)$, pour un profil donné du potentiel d'essai $\phi(x)$, l'objectif est de trouver la fonction de correction $\delta \phi(x)$. On a donc :

$$
\phi^{0}(x)=\phi(x)+\delta \phi(x)
$$

Si on substitue l'équation B-12 dans B-11, on a donc :

$$
\frac{d}{d x}\left(\varepsilon_{s c} \frac{d \phi}{d x}\right)+\frac{d}{d x}\left(\varepsilon_{s c} \frac{d \delta \phi}{d x}\right)=\frac{-q\left(N_{d o p}-n(\phi+\delta \phi)\right)}{\varepsilon_{0}}
$$

Si on définit $n(\phi+\delta \phi)=n(\phi)+\delta n(\phi)$, l'équation B-13 peut se réécrire comme :

$$
-\left(\frac{d}{d x}\left(\varepsilon_{s c} \frac{d \phi}{d x}\right)+\frac{q}{\varepsilon_{0}}\left(N_{d o p}-n(\phi)\right)\right)=\frac{d}{d x}\left(\varepsilon_{s c} \frac{d \delta \phi}{d x}\right)-\frac{q}{\varepsilon_{0}} \delta n(\phi)
$$

Le terme du côté gauche de cette équation représente des erreurs dans l'équation de Poisson pour la fonction d'essai $\phi(x)$. Supposons que $\delta \phi(x)$ est faible et que $\delta n(\phi)$ peut être exprimé par :

$$
\delta n(\phi)=\sum_{k=1}^{m}\left(\delta\left(\psi_{k}^{*} \psi_{k}\right) n_{k}+\psi_{k}^{*} \psi_{k} \delta n_{k}\right)
$$

avec

$$
n_{k}=\frac{m^{*}}{\pi \hbar^{2}} \int_{E_{k}}^{\infty} \frac{1}{1+\exp \left(\frac{E-E_{F}}{k_{B} T}\right)} d E
$$

où $E_{k}$ est l'énergie propre issue de l'équation Schrödinger. Dans l'équation B-15, le premier terme dans la parenthèse (la variation du vecteur propre due au potentiel d'essai et sa correction) est négligeable devant le second terme (la variation de la densité due au potentiel d'essai et sa correction). Il peut être exprimé en fonction de $\delta \phi$ :

$$
\delta n_{k}=n_{k}(\phi+\delta \phi)-n_{k}(\phi)
$$


On obtient finalement la correction sur la densité des électrons par une approximation du premier ordre :

$$
\delta n(\phi)=-\sum_{k=1}^{m} \psi_{k}^{*} \psi_{k} \frac{\partial n_{k}}{\partial E_{k}}\left\langle\psi_{k}|q \delta \phi| \psi_{k}\right\rangle
$$

où $\langle\mid\rangle$ est l'intégrale de bra-ket.

Si on suppose les dopants complètement ionisés et la variation de la fonction d'onde petite par rapport à $\delta \varphi$, l'équation différentielle décrivant l'incrément de potentiel $\delta \phi$ à chaque itération doit satisfaire l'équation :

$$
-\left(\frac{d}{d x}\left(\varepsilon_{s c} \frac{d \phi}{d x}\right)+\frac{q}{\varepsilon_{0}}\left(N_{d o p}(x)-n(x)\right)\right)=\frac{d}{d x}\left(\varepsilon_{s c} \frac{d \delta \phi}{d x}\right)-\frac{q}{\varepsilon_{0}} \sum_{k=1}^{m} \psi_{k}^{*} \psi_{k} \frac{\partial n_{k}}{\partial E_{k}}\left\langle\psi_{k}|q \delta \phi| \psi_{k}\right\rangle
$$

Le terme du coté gauche représente des erreurs par la fonction d'essai du potentiel électrostatique, il devrait égale à zéro si elle est égale à la solution auto-cohérente.

En utilisant la même méthode de discrétisation dans l'équation de Schrödinger, l'équation B-19 peut être exprimée comme :

$$
-e_{i}=C_{i i+1} \delta \phi_{i+1}+\delta \phi_{i}\left(C_{i i}+\frac{q}{\varepsilon_{0}} \sum_{k=1}^{m} \psi_{k_{i}}^{*} \psi_{k_{i}} \frac{\partial n_{k}}{\partial E_{k}}\right)+C_{i i-1} \delta \phi_{i-1}
$$

où,

$$
\begin{gathered}
e_{i}=\sum_{j=1}^{n} C_{i j} \phi_{j}+q\left(N_{d o p}-n_{i}\right) / \varepsilon_{0} \\
C_{i j}=\left\{\begin{array}{ll}
\frac{2 \varepsilon_{i+1 / 2}}{h_{i}\left(h_{i}+h_{i+1}\right)} & \text { si } j=i+1 \\
\frac{2 \varepsilon_{i-1 / 2}}{h_{i}\left(h_{i}+h_{i-1}\right)} & \text { si } j=i-1 \\
-C_{i i+1}-C_{i i-1} & \text { si } j=i \\
0 & \text { sinon }
\end{array}\right\}
\end{gathered}
$$

Si on néglige l'effet de non-parabolicité, on a aussi :

$$
\frac{\partial n_{k}}{\partial E_{k}}=\frac{m^{*}}{\pi \hbar^{2}\left(1+\exp \left(E_{k}-E_{F} / k_{B} T\right)\right)}
$$

Dans l'équation B-20, il y a $n$ équations d'erreur avec $n$ variables inconnues $\left(\delta \phi_{1}, \delta \phi_{2}, \ldots . . \delta \phi_{n}\right)$. Elles pourraient regroupées en une équation matricielle : $C^{\prime} \delta \phi=-\xi$. $C^{\prime}$ est une matrice tridiagonale, non symétrique et avec une dimension $n \times n$. $\delta \phi$ est le vecteur de dimension $n \times 1$ qui contient la correction du potentiel en chaque point de l'espace. Le profil 
du potentiel doit être mis à jour selon ces corrections. $\xi$ est un vecteur de dimension $n \times 1$ qui contient les erreurs dans l'équation de Poisson en chaque point de l'espace. 


\section{Annexe-C :}

\section{Présentation de la simulation particulaire Monte Carlo semi- classique}

\section{C-a L'équation de Boltzmann}

Dans ce paragraphe, nous allons reprendre les grands principes de l'équation de Boltzmann du transport électronique. En physique classique statistique, les quantités caractérisant un système de particules sont déterminées par la fonction de distribution $f(\vec{r}, \vec{p}, t)$ qui donne la probabilité de trouver des particules à la position $\vec{r}$, avec l'impulsion $\vec{p}$ et au temps t. A partir de $f(\vec{r}, \vec{p}, t)$, on définit des probabilités de présence de particules à la position $\vec{r}$ ou ayant l'impulsion $\vec{p}$ par [Reif1965] :

$$
\begin{aligned}
& P(\vec{p})=\int f(\vec{r}, \vec{p}, t) d \vec{r} \\
& P(\vec{r})=\frac{1}{(2 \pi \hbar)^{3}} \int f(\vec{r}, \vec{p}, t) d \vec{p}
\end{aligned}
$$

La valeur moyenne d'une grandeur décrite par la fonction $A(\vec{r}, \vec{p})$ est :

$$
\langle A\rangle=\frac{1}{(2 \pi \hbar)^{3}} \int f(\vec{r}, \vec{p}, t) \cdot A(\vec{r}, \vec{p}) \cdot d \vec{r} d \vec{p}
$$

En l'absence d'interactions entre les particules et avec leur environnement, on aura la même propriété de fonction de distribution pour les particules qui sont dans le volume $d \vec{r} d \vec{p}$ autour $\vec{r}$ et $\vec{p}$ au temps $t$ et celles dans le volume $d \vec{r}^{\prime} d \vec{p}^{\prime}$ autour $\vec{r}^{\prime}$ et $\vec{p}^{\prime}$ au temps $t^{\prime}$ dans l'espace de phases :

$$
f\left(\vec{r}^{\prime}, \vec{p}^{\prime}, t^{\prime}\right) \cdot d \vec{r}^{\prime} d \vec{p}^{\prime}=f(\vec{r}, \vec{p}, t) \cdot d \vec{r} d \vec{p}
$$

où

$$
\begin{aligned}
& \vec{r}^{\prime}=\vec{r}+\dot{\vec{r}} d t \\
& \vec{p}^{\prime}=\vec{p}+\dot{\vec{p}} d t \\
& t^{\prime}=t+d t
\end{aligned}
$$

en utilisant :

$$
d \vec{r}^{\prime} d \vec{p}^{\prime}=|J| d \vec{r} d \vec{p}
$$

où $J=1+\vartheta\left(d t^{2}\right)$. L'approximation $\mathrm{J}=1$ est correcte jusqu'à l'ordre 1 en $d t$. L'équation (C-3) devient alors : 


$$
f(\vec{r}+\dot{\vec{r}} d t, \vec{p}+\dot{\vec{p}} d t, t+d t)=f(\vec{r}, \vec{p}, t)
$$

Cette propriété peut être démontrée en utilisant le théorème de Liouville, en écrivant que la fonction de distribution ne change pas si l'on suit le chemin des particules dans l'espace des phases. L'équation (C-6) peut être exprimée en termes de dérivées partielles [Jacoboni1989] :

$$
\frac{\partial f}{\partial t}+\nabla_{\vec{r}} f \cdot \frac{\partial \vec{r}}{\partial t}+\nabla_{\vec{p}} f \cdot \frac{\partial \vec{p}}{\partial t}=\frac{\partial f}{\partial t}+\nabla_{\vec{r}} f \cdot \frac{\vec{p}}{m}+\nabla_{\vec{p}} f \cdot F^{e x t}=0
$$

où $F^{e x t}$ est la force appliquée.

Cependant, en présence d'interactions, le nombre de particules dans le volume $d \vec{r} d \vec{p}$ peut changer. En effet, des particules ayant une position et une quantité de mouvement n'appartenant pas au volume $d \vec{r} d \vec{p}$ peuvent y entrer après une interaction. Réciproquement, des particules appartenant à ce volume peuvent en sortir. Il faut inclure dans équation (C-7) un terme qui corresponde à l'augmentation nette par unité de temps du nombre de particules dans le volume $d \vec{r} d \vec{p}$ issue de l'interaction. L'équation (C-7) se réécrit comme :

$$
\frac{\partial f}{\partial t}+\nabla_{\vec{r}} f \cdot \frac{\vec{p}}{m}+\nabla_{\vec{p}} f \cdot F^{e x t}=\left(\frac{\partial f}{\partial t}\right)_{\text {coll }}
$$

Dans le traitement semi-classique, on remplace l'impulsion par le vecteur d'onde $(\vec{p}=\hbar \vec{k})$ et le terme de collision est écrit comme un opérateur sur la fonction de distribution $S_{\text {coll }} f(\vec{r}, \vec{p}, t)$. L'équation (C-8) devient donc :

$$
\frac{\partial f}{\partial t}+\nabla_{\vec{r}} f \cdot \frac{\hbar \vec{k}}{m}-\frac{e}{\hbar} \nabla_{\vec{k}} f \cdot E(\vec{r})=S_{\text {coll }} f
$$

Si les particules perdent toutes leurs corrélations les unes avec les autres, le terme de collision peut s'écrire sous la forme [Ferry1997]:

$$
\begin{aligned}
S_{\text {coll }} f & =\sum_{\vec{k}^{\prime}} S\left(\vec{k}, \vec{k}^{\prime}\right) \cdot f(\vec{r}, \vec{k}, t) \cdot\left[1-f\left(\vec{r}, \vec{k}^{\prime}, t\right)\right] \\
& -\sum_{\vec{k}} S\left(\vec{k}^{\prime}, \vec{k}\right) \cdot f\left(\vec{r}, \vec{k}^{\prime}, t\right) \cdot[1-f(\vec{r}, \vec{k}, t)]
\end{aligned}
$$

Le premier terme décrit l'augmentation de $f(\vec{r}, \vec{k}, t)$ due à la transition de l'état initial $\vec{k}$ vers l'état final $\vec{k}^{\prime}$, dont la densité de probabilité par unité de temps est donnée par $S\left(\vec{k}, \vec{k}^{\prime}\right)$ et réciproquement pour le second terme. Le principe d'exclusion de Pauli est pris en compte par le facteur $1-f$ qui limite les transitions aux états d'arrivée non occupés. Dans le traitement quantique des interactions, les densités de probabilité de transition $S\left(\vec{k}, \vec{k}^{\prime}\right)$ sont évaluées en utilisant la règle d'or de Fermi [Mark2000]. Les études sur les propriétés de transport d'un 
point de vue semi-classique consistent à résoudre cette équation de Boltzmann. De façon générale, la résolution de l'équation de Boltzmann se divise en trois types: résolution déterministe directe (ex: expansion des harmoniques sphériques) [Vecchi1998IEEE], résolution simplifiée (ex: modèle dérive-diffusion et modèle hydrodynamique) [Nekovee1992PRB] et résolution statistique (ex : Monte Carlo). Cette dernière est très bien adaptée à la simulation de dispositifs de taille nanométrique en régime statique (DC) [Dollfus1993JAP] et également en régime transitoire (AC) [Dollfus1992EL] reproduisant de façon naturelle les effets de transport fortement hors d'équilibre comme le régime balistique.

\section{C-b Simulateur particulaire MONACO : principe et algorithme}

La méthode Monte Carlo semi-classique est utilisée pour résoudre l'équation de Boltzmann dans un cadre multi-particulaire où chaque porteur est individualisé. Contrairement aux modèles dérive-diffusion ou hydrodynamique, aucune hypothèse sur des grandeurs macroscopiques n'est à faire, les moyennes d'ensemble se construisant à partir des comportements microscopiques de chaque porteur. Les grandeurs physiques relatives au système étudié sont obtenues de façon statistique : avec les caractéristiques dans les espaces réel et réciproque d'un nombre suffisamment important de particules on peut reconstituer au cours du temps l'évolution de la fonction de distribution. Ces comportements des particules sont conditionnés par les différentes collisions (interactions) qu'ils subissent dans leur environnement. La trajectoire des particules est une succession de vols libres entrecoupés d'interactions de différentes natures [Jacoboni1989]. Le principe de base de cette méthode consiste en un tirage au sort de variables aléatoires visant à déterminer : i) la valeur du temps de vol libre ; ii) l'interaction subie à la fin du vol libre ; iii) l'effet de l'interaction sélectionnée (déviation du vecteur d'onde, modification éventuelle de l'énergie du porteur). Pour décrire les lois de probabilité afin d'effectuer les tirages au sort et le mouvement sous le champ de force extérieur durant chaque vol libre, on a aussi besoin de modèles physiques décrivant : i) la relation de dispersion énergie-vecteur d'onde; ii) l'équation du mouvement; iii) les fréquences d'interaction.

Dans MONACO, la relation de dispersion (ou structure de bandes électroniques) est généralement décrite par une approche analytique basée sur l'approximation de masse effective, elle permet d'avoir une relation directe entre le vecteur d'onde et l'énergie. Ce modèle peut se déduire d'approximations analytiques de structures complètes de bandes obtenues par calcul numérique ou de mesures de masse effective aux extrema de bandes. Pour 
le bas de la bande de conduction d'InGaAs, le minimum d'énergie se situe en vallée $\Gamma$ à $\vec{k}=0$ et la relation de dispersion peut être décrite par [Fischetti1988PRB] :

$$
\mathrm{E}(\vec{k})(1+\alpha \cdot \mathrm{E}(\vec{k}))=\frac{\hbar^{2} k^{2}}{2 m^{*}}
$$

où $m^{*}$ est la masse effective qui peut être déduite de une dérivée en bas de bande au voisinage de $\mathrm{k}=0$ :

$$
\frac{1}{m^{*}}=\frac{1}{\hbar^{2}} \frac{\partial^{2} \mathrm{E}(\vec{k})}{\partial k^{2}}
$$

et $\alpha$ le coefficient de non-parabolicité. La surface iso-énergie est de forme sphérique et la masse effective est isotrope. Pour des bandes ellipsoïdales (par exemple vallées $\Delta$ de Si), les porteurs présentent une masse qui dépend de la direction du vecteur d'onde et la relation de dispersion prend une forme du type :

$$
\mathrm{E}(\vec{k})(1+\alpha \cdot \mathrm{E}(\vec{k}))=\frac{\hbar^{2}}{2 m_{0}}\left(\frac{k_{l}^{2}}{m_{l}}+\frac{k_{t}^{2}}{m_{t}}\right)
$$

c'est-à-dire que la masse effective est ici un tenseur composé des masses longitudinale $m_{l}$ et transverse $m_{t}$. La direction longitudinale $l$ est celle de l'axe principal de l'ellipsoïde de révolution que représente une surface iso-énergie et $t$ est associé aux deux directions transverses à $l$. Ce modèle analytique est une bonne approximation pour des énergies allant jusqu'à $2 \mathrm{eV}$ [Kunikiyo94JAP].

Dans une approche classique du transport, on associe le paquet d'ondes de Bloch [Mark2000] à une particule de masse effective $m^{*}$ se déplaçant à la vitesse de groupe $v_{G} \mathrm{du}$ paquet d'ondes centré en $\vec{k}$. La vitesse de groupe associée est également déterminée par la relation de dispersion [Mark2000]:

$$
v_{G}(\vec{k})=\frac{1}{\hbar} \frac{\partial \mathrm{E}(\vec{k})}{\partial \vec{k}}
$$

Cette équation faisant la liaison entre l'espace réel et l'espace réciproque pour l'ensemble de particules individuelles simulées. Si on utilise le modèle (C-11), on a la relation suivante entre la vitesse de groupe et le vecteur d'onde :

$$
\vec{v}_{G}=\frac{\hbar \vec{k}}{m^{*}(1+2 \alpha \cdot \mathrm{E}(\vec{k}))}
$$

Dans MONACO, l'espace réel est découpé en un ensemble de mailles. Dans chacune d'elles, les particules se déplacent sous un champ électrique supposé constant. L'effet des interactions 
se manifeste instantanément dans l'espace réciproque sous la forme d'une déviation du vecteur d'onde et d'une modification de sa norme. Entre deux collisions, la trajectoire de chaque particule soumise à un champ électrique $\vec{E}$ est déterminée par l'équation fondamentale de la dynamique, soit pour une particule d'impulsion $\hbar \vec{k}$ et à la position $\vec{r}$ [Monsef2002Thèse] :

$$
\begin{aligned}
& \dot{\vec{r}}=\vec{v}_{G}(\vec{k})=\frac{1}{\hbar} \frac{\partial \mathrm{E}(\vec{k})}{\partial \vec{k}} \\
& \hbar \dot{\vec{k}}=-q \vec{E}
\end{aligned}
$$

On considère le mouvement dans l'espace réciproque, si $\delta t$ est le temps durant lequel se déplace le porteur, nous aurons, dans le cas d'un électron, le vecteur d'onde $\vec{k}_{f}$ après mouvement au moment de la collision qui est relié au vecteur d'onde $\vec{k}_{i}$ avant vol libre par :

$$
\vec{k}_{f}=\vec{k}_{i}-\frac{\delta t}{\hbar} q \cdot \vec{E}
$$

et ainsi que le mouvement dans l'espace réel est décrit par :

$$
\vec{r}_{f}=\vec{r}_{i}+\vec{v}_{G} \times \delta t
$$

Dans la méthode Monte Carlo, la durée d'un vol libre $t_{v}$ est déterminée par un nombre aléatoire $r$ variant entre 0 et 1 et par la somme totale de toutes les fréquences d'interaction $\lambda_{0}$ à une énergie donnée. Pour simplifier le traitement, on peut considérer $\lambda_{0}$ comme constante en introduisant une interaction fictive, dite de 'self-scattering' [Mark2000], qui est sans effet sur les particules. Le temps de vol libre est alors donné par [Monsef2002Thèse]:

$$
t_{v}=-\frac{\ln r}{\lambda_{0}}
$$

Après chaque vol libre, la particule subit une interaction. On tire encore une fois des nombres au sort pour déterminer le type d'interaction subie et ses effets dans l'espace réciproque [Monsef2002Thèse]. Ces derniers sont typiquement regroupés selon les critères du changement de l'énergie de particule (interaction élastique ou inélastique) et du changement de la direction du vecteur d'onde (interaction isotrope ou anisotrope) [Mark2000]. Selon les conséquences des effets de l'interaction, on calcule le module et la direction du vecteur d'onde final après interaction. On suppose que le processus d'interaction est instantané, ce qui est valide quand la durée d'interaction est très faible comparée à la durée des vols libres. Après l'interaction, on retourne faire un mouvement de vol libre dans les espaces réels et réciproques. Pour déterminer le champ électrostatique comme la source de déplacement du 
vol libre, le simulateur Monte Carlo est couplé de façon auto-cohérente avec la résolution de l'équation de Poisson dans l'espace réel. La contribution de chaque porteur à la concentration électronique résultante $n_{\text {élec }}$ est pondérée par le temps passé dans chaque maille $t_{m}$, telle que $n_{\text {elec }}=n_{\text {élec }}+t_{m} / \delta t$ [Brisset1994Thèse]. La résolution de Poisson doit être effectuée périodiquement pour mettre à jour les valeurs de champ électrique au sein du dispositif. Le pas sur le temps $\delta t$ entre deux résolutions est ajusté par rapport au temps de relaxation diélectrique. Les processus décrits au dessus sont itérés jusqu'à ce qu'atteigne un état stationnaire quand le flux de porteurs devient constant. La simulation Monte Carlo permet ainsi non seulement d'accéder au régime permanent du composant étudié mais aussi à son comportement en transitoire. 


\section{Annexe - D :}

\section{Interactions prises en compte}

\section{Phonons :}

L'interaction de phonon acoustique intra-vallée est traitée comme un processus élastique, avec probabilité isotrope de déviation du vecteur d'onde. La fréquence d'interaction est alors donnée par [Jacoboni1989]:

$$
\lambda_{a c}(E)=\frac{\sqrt{2}}{\pi} \frac{k_{B} T m_{D}^{3 / 2} D_{a c}^{2}}{\hbar \rho v^{2}} \sqrt{E(1+\alpha E)}(1+2 \alpha E)
$$

où $\mathrm{E}$ est l'énergie cinétique des électrons, $m_{D}$ est la masse effective de densité d'états, $v$ est la vitesse moyenne de son, $\rho$ est la densité de masse volumique du matériau et $D_{a c}$ est le potentiel de déformation acoustique.

Dans les matériaux III-V, l'interaction due à la nature polaire des liaisons est généralement dominante. Elle est due à la perturbation des moments dipolaires associés aux liaisons partiellement ioniques entre atomes quand ces derniers ne vibrent pas en phase. Ces phonons optiques polaires (POP) interagissent avec les porteurs selon des processus inélastiques et anisotropes. En supposant le potentiel de perturbation est de type Fröhlich [Nag1972] et la bande est non-parabolique, la fréquence d'interaction intravallée s'écrit comme [Jacoboni1989] et [Bhattacharyya1993JAP] :

$$
\begin{aligned}
\lambda_{P O P}(E)= & \frac{q^{2} w_{p o p} \sqrt{2 m_{D}}}{8 \hbar \pi \varepsilon_{0}}\left(\frac{1}{\kappa_{\infty}}-\frac{1}{\kappa_{s}}\right) \times \\
& \left.\begin{array}{l}
\frac{N_{P O P}}{\sqrt{\gamma(E)}}\left[1+2 \alpha\left(E+\hbar w_{p o p}\right)\right] \ln \left(\frac{\sqrt{\gamma(E)}+\sqrt{\gamma\left(E+\hbar w_{p o p}\right)}}{\sqrt{\gamma(E)}-\sqrt{\gamma\left(E+\hbar w_{p o p}\right)}}\right)+ \\
\frac{N_{P O P}+1}{\sqrt{\gamma(E)}}\left[1+2 \alpha\left(E-\hbar w_{p o p}\right)\right] \ln \left(\frac{\sqrt{\gamma(E)}+\sqrt{\gamma\left(E-\hbar w_{p o p}\right)}}{\sqrt{\gamma(E)}-\sqrt{\gamma\left(E-\hbar w_{p o p}\right)}}\right)
\end{array}\right)
\end{aligned}
$$

où $\kappa_{0}$ et $\kappa_{\infty}$ sont respectivement les constantes diélectriques à faible et haute fréquence. $N_{P O P}$ est le nombre de phonons. $\gamma(E)$ est la relation de dispersion pour la vallée sphérique et non parabolique ( $c f$. Equation C-11). Dans la grande parenthèse, le premier terme représente l'absorption de POP et tandis que le second terme représente l'émission de POP. $\hbar w_{p o p}$ est 
l'énergie associée de POP. On peut noter que le second terme n'a de sens que si $E>\hbar w_{p o p}$ ce qui est bien évidemment nécessaire pour que l'émission ait lieu.

Dans $\mathrm{In}_{0,53} \mathrm{Ga}_{0,47} \mathrm{As}$, les interactions avec les phonons intervallées se produisent entre les vallées non équivalentes : $\Gamma$ - $\mathrm{L}, \Gamma-\mathrm{X}, \mathrm{L}-\mathrm{X}$ ainsi qu'entre les vallées équivalentes : $\mathrm{L}-\mathrm{L}$ et $\mathrm{X}-$ $\mathrm{X}$. La vallée $\Gamma$ se trouve environ à $0,6 \mathrm{eV}$ au dessous de celles en $\mathrm{L}$. Ces transitions intervallées sont généralement traitées par la matrice de transition à l'ordre zéro et la fréquence d'interaction s'écrit alors [Jacoboni1989] :

$$
\begin{aligned}
\lambda_{i v_{0}}(E)= & \frac{Z_{i v}}{\sqrt{2} \pi} \frac{m_{D}^{3 / 2} D_{i v_{0}}^{2}}{\hbar^{2} \rho \hbar w_{i v}}\left(N_{i v}+\frac{1}{2}-\frac{\sigma}{2}\right) \sqrt{E+\sigma \hbar w_{i v}+\Delta E_{i v}} \\
& \times \sqrt{1+\alpha\left(E+\sigma \hbar w_{i v}+\Delta E_{i v}\right)} \times\left(1+2 \alpha\left(E+\sigma \hbar w_{i v}+\Delta E_{i v}\right)\right)
\end{aligned}
$$

où $Z_{i v}$ est le nombre de vallées finales disponibles pour l'interaction. $\Delta E_{i v}$ est la différence entre le bas de bande de conduction dans la vallée finale et initiale. $N_{i v}$ est le nombre de phonon intervallée, donné par la distribution de Bose-Einstein. $\sigma=1$ représente l'absorption de phonon tandis que $\sigma=-1$ représente l'émission de phonon. $D_{i v_{0}}$ est le potentiel de déformation associé.

\section{Impuretés ionisées :}

D'après le modèle de Brooks-Herring, l'interaction des impuretés est traitée via le potentiel Coulombien écranté utilisant une longueur d'écran L qui dépend du moment des porteurs [Takimoto1959JPSJ] :

$$
\frac{1}{L^{2}}=\frac{e^{2} n(\vec{r})}{\varepsilon_{0} \varepsilon_{s c} k_{B} T} F(\xi)=\beta_{D}^{2} F(\xi)
$$

où $\beta_{D}$ est l'inverse de la longueur d'écrantage de Debye-Huckel, $n(\vec{r})$ est la densité de porteur locale, la variable normalisé est définie comme :

$$
\xi^{2}=\frac{\hbar^{2}}{8 e m_{D} k_{B} T} \beta^{2}
$$

où $\vec{\beta}=\vec{k}-\vec{k}^{\prime}$ est le vecteur impliqué dans l'événement de l'interaction de l'état initial $\vec{k}$ à l'état final $\vec{k}^{\prime}$. La fonction d'écrantage peut être exprimée dans l'approximation nondégénérée comme [Joshi1991PRB] et [Hall1962JPCS]: 


$$
F(\xi)=\frac{1}{\xi} \exp \left(-\xi^{2}\right) \int_{0}^{\xi} \exp \left(x^{2}\right) d x
$$

En considérant un angle d'interaction $\theta$ entre $\vec{k}$ et $\vec{k}^{\prime}$, la fréquence d'interaction pour un électron dans l'état $\vec{k}$ ayant une énergie $E$ s'écrit [Jacoboni1989] et [AubryFortuna2005SSE] :

$$
\begin{aligned}
\lambda_{i m p}(\vec{k})= & \frac{Z^{2} e^{4}}{\sqrt{2} \pi \hbar^{4} \varepsilon_{0}^{2} \varepsilon_{s c}^{2}} m_{D}^{3 / 2} N_{i m p}(1+2 \alpha E) \sqrt{E(1+\alpha E)} \\
& \times \int_{-1}^{1} \frac{\mathrm{d}(\cos \theta)}{\left(\beta^{2}+\beta_{D}^{2} F(\xi)\right)^{2}}
\end{aligned}
$$

où $N_{i m p}$ est la concentration des impuretés et $Z$ est le nombre de charge d'impureté. Dans l'intégrale, les termes $\beta$ et $\xi$ dépendent de l'angle $\theta$ par le biais de la relation $\beta^{2}=2 k^{2}(1-\cos \theta)$. L'interaction impureté - électron est intrinsèquement un processus anisotrope, mais pour les calculs de mobilité, elle pourrait être traitée comme un mécanisme isotrope en remplaçant cette fréquence d'interaction par un temps de relaxation du moment $\tau_{\text {imp }}^{-1}$ obtenu en introduisant un facteur $1-\cos \theta$ dans l'intégrale de l'équation (D-7) pour évaluer $\tau_{i m p}^{-1}$ selon une direction [Mark2000]. Grâce à cette approximation isotrope, le taux d'interaction pourra être exprimé en fonction de l'énergie cinétique des porteurs et non pas de leur vecteur d'onde ce qui simplifie fortement l'algorithme [Kosina1997PSS]. En outre, il faut a priori calculer la longueur d'écran en utilisant la densité de porteur locale dans un canal d'inversion $n(\vec{r})$ étant donné sa non-uniformité et sa dépendance par rapport au potentiel appliqué.

\section{Désordre d'alliage :}

L'interaction d'alliage dans $\operatorname{In}_{\mathrm{x}} \mathrm{Ga}_{1-\mathrm{x}} \mathrm{As}$ due au positionnement aléatoire dans le cristal des atomes d'In et As. Dans MONACO, elle est traitée par un modèle standard, c'est-à-dire via une perturbation du potentiel cristallin sous forme de puits d'énergie de hauteur de barrière $U_{\text {all }}$ dans une sphère de volume $V$ centrée sur chaque site d'alliage. Le rayon de cette sphère est arbitrairement choisi comme la distance au plus proche voisin $r_{0}=\sqrt{3} a_{0} / 4$ où $a_{0}$ est le paramètre de maille. Le potentiel d'alliage est considérée égal à $1,5 \mathrm{eV}$, par comparaison avec des données expérimentales sur un dispositif de type HEMT (High 
Electron Mobility Transistor) [Mateos2000IEEE]). Sous 1'hypothèse isotrope, le temps de relaxation d'interaction d'alliage peut s'écrire [Harrison1976PRB] :

$$
\frac{1}{\tau_{\text {all }}(E)}=\frac{3 \pi \sqrt{2} a_{0}^{3}}{64 \hbar^{4}} m_{D}^{3 / 2} x(1-x) U_{\text {all }}^{2}(1+2 \alpha E) \sqrt{E(1+\alpha E)}
$$

\section{Rugosité de surface :}

Dans une couche d'inversion, les propriétés du transport sont fortement affectées par les irrégularités à l'interface oxyde/semi-conducteur et par les distributions de charge fixe dans l'oxyde. L'interaction de la rugosité d'interface peut être traitée en caractérisant les fluctuations de potentiel par deux paramètres, $\Lambda$ et $\Delta$, respectivement la longueur de corrélation et l'amplitude de la rugosité d'interface [Fischetti1993PRB]. Ils dépendent strictement de la technologie mise en jeu. Les interactions coulombiennes avec les charges fixes localisées dans l'oxyde et/ou à l'interface pourraient aussi être prises en compte [Ning1972PRB]. Dans la version 3Dk de MONACO, nous avons choisi d'adopter un traitement plus simple et qui s'est révélé bien adapté au cas des interfaces $\mathrm{SiO}_{2} / \mathrm{Si}$ : traiter les réflexions des porteurs qui frappent l'interface comme une combinaison empirique de réflexions spéculaires et diffusives [Sangiorgi1992IEEE]. Chaque fois qu'un électron atteint à la surface, l'effet diffusive ou spéculaire de la réflexion est déterminée par un nombre aléatoire. Cette méthode a été aussi utilisée pour calibrer la mobilité effective dans l'hétérostructure à base des matériaux III-V [Rodilla2009JAP]. Généralement, la fraction des interactions diffusives $N_{\text {diff }}$ est considérée comme une valeur constante. Dans ce travail, nous avons utilisé une valeur modérée, $18 \%$, qui correspond à une technologie de l'état de l'art assez mature. Par ailleurs, pour un système $\mathrm{SiO}_{2} / \mathrm{Si}$ bien identifié, cette fraction $N_{\text {diff }}$ peut être soigneusement évaluée comme une fonction analytique du champ effectif transverse [Aubry-Fortuna2005SSE], afin de reproduire la courbe de mobilité universelle de Si.

On peut tracer la fréquence d'interaction des électrons en fonction de l'énergie cinétique des électrons pour une densité de dopant $10^{17} \mathrm{~cm}^{-3}$ et à $300 \mathrm{~K}$. Les nomenclatures des interactions sont :

\begin{tabular}{|l|c|}
\hline IMP & Impuretés ionisées \\
\hline $\mathrm{ACO}$ & Phonon Acoustique \\
\hline
\end{tabular}




\begin{tabular}{|c|c|}
\hline POP-E & Phonon Optique Polaire - Emission \\
\hline POP-A & Phonon Optique Polaire - Absorption \\
\hline ONP-A & Phonon Optique Non Polaire - Emission \\
\hline ONP-E & Phonon Optique Non Polaire - Absorption \\
\hline PIE & Piézoélectrique \\
\hline IV0-Г-L-E & Phonon Inter-vallées d'ordre $\mathbf{0}-$ entre $\boldsymbol{\Gamma}$ et $\mathbf{L}-\mathbf{E m i s s i o n}$ \\
\hline IV0-Г-L-A & Phonon Inter-vallées d'ordre $\mathbf{0}-$ entre $\boldsymbol{\Gamma}$ et $\mathbf{L}-\mathbf{A b s o r p t i o n}$ \\
\hline- & Même notation pour les autres interactions phonon inter-vallées \\
\hline ALL & Alliage \\
\hline
\end{tabular}

- Vallée $\Gamma$ :

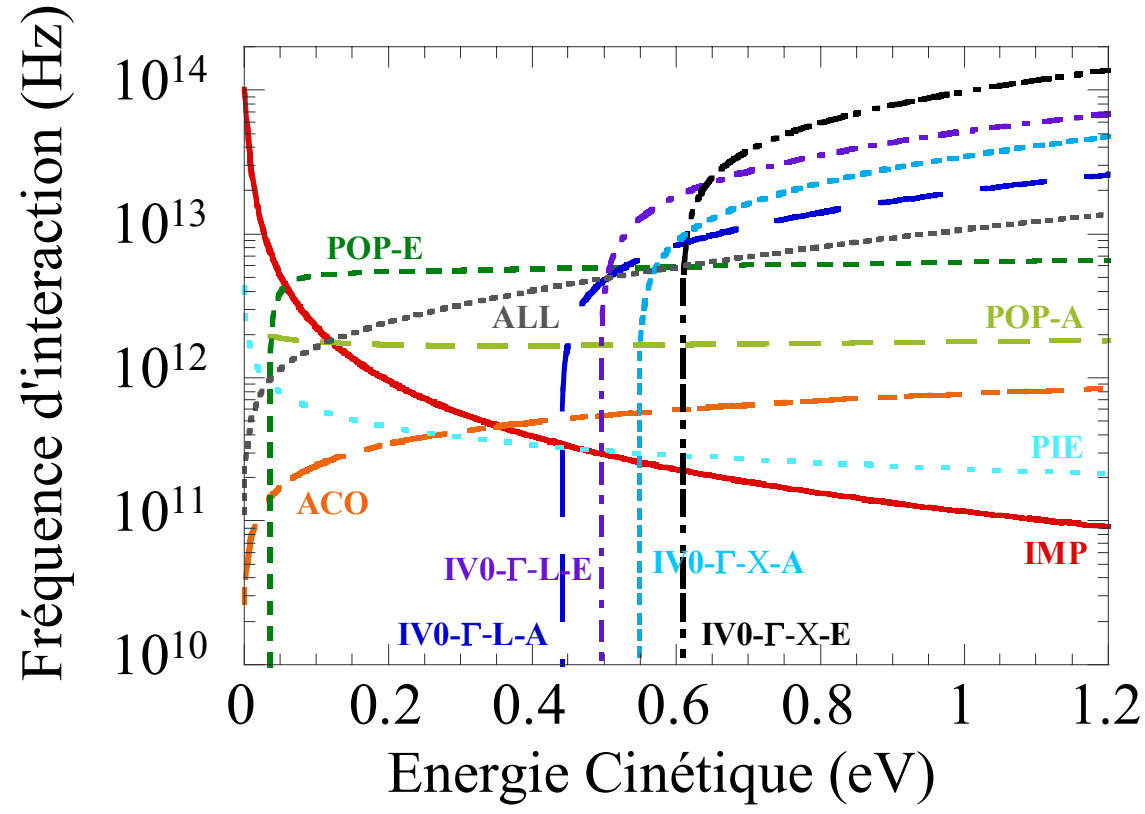

- Vallées L : 


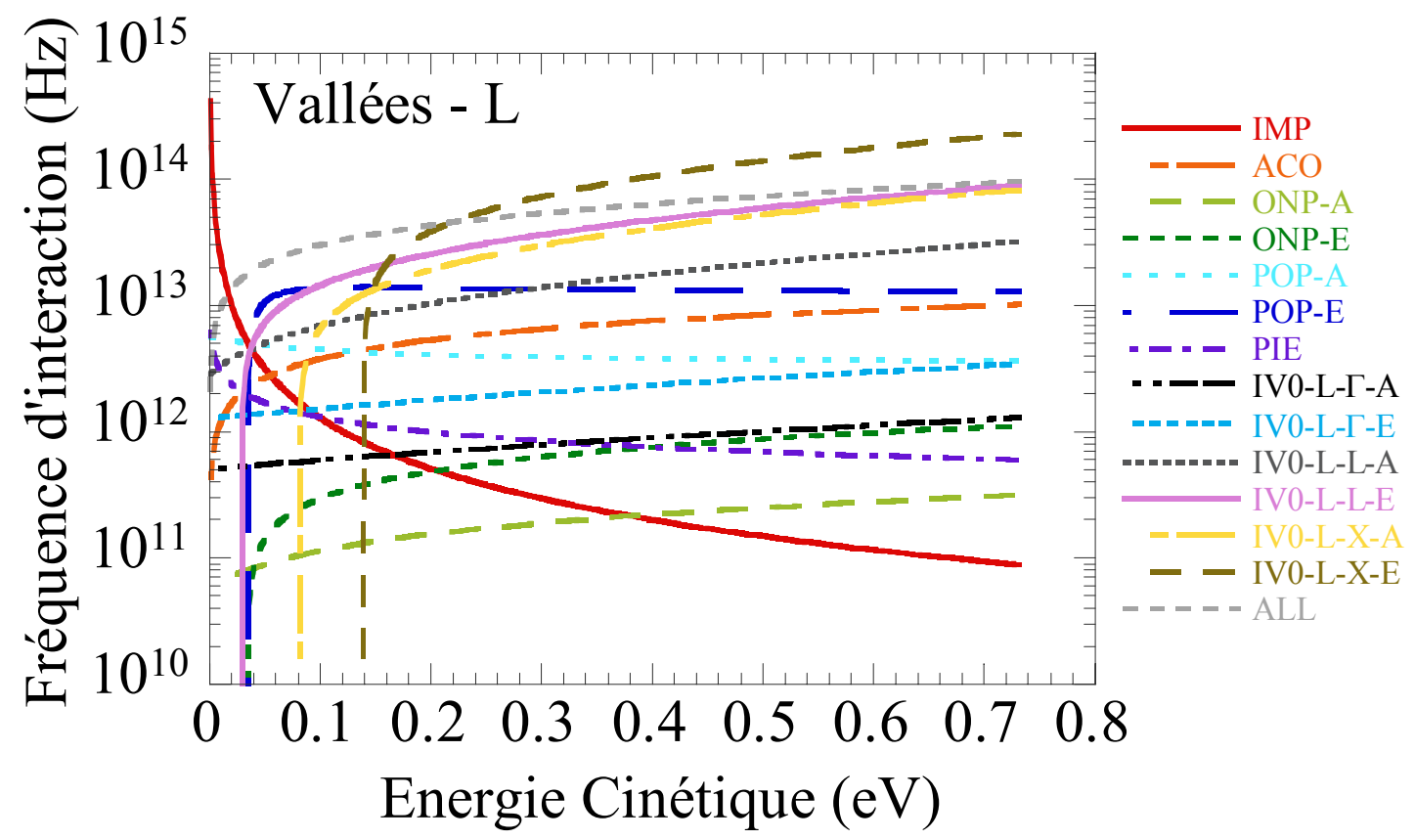

\section{- Vallées X:}

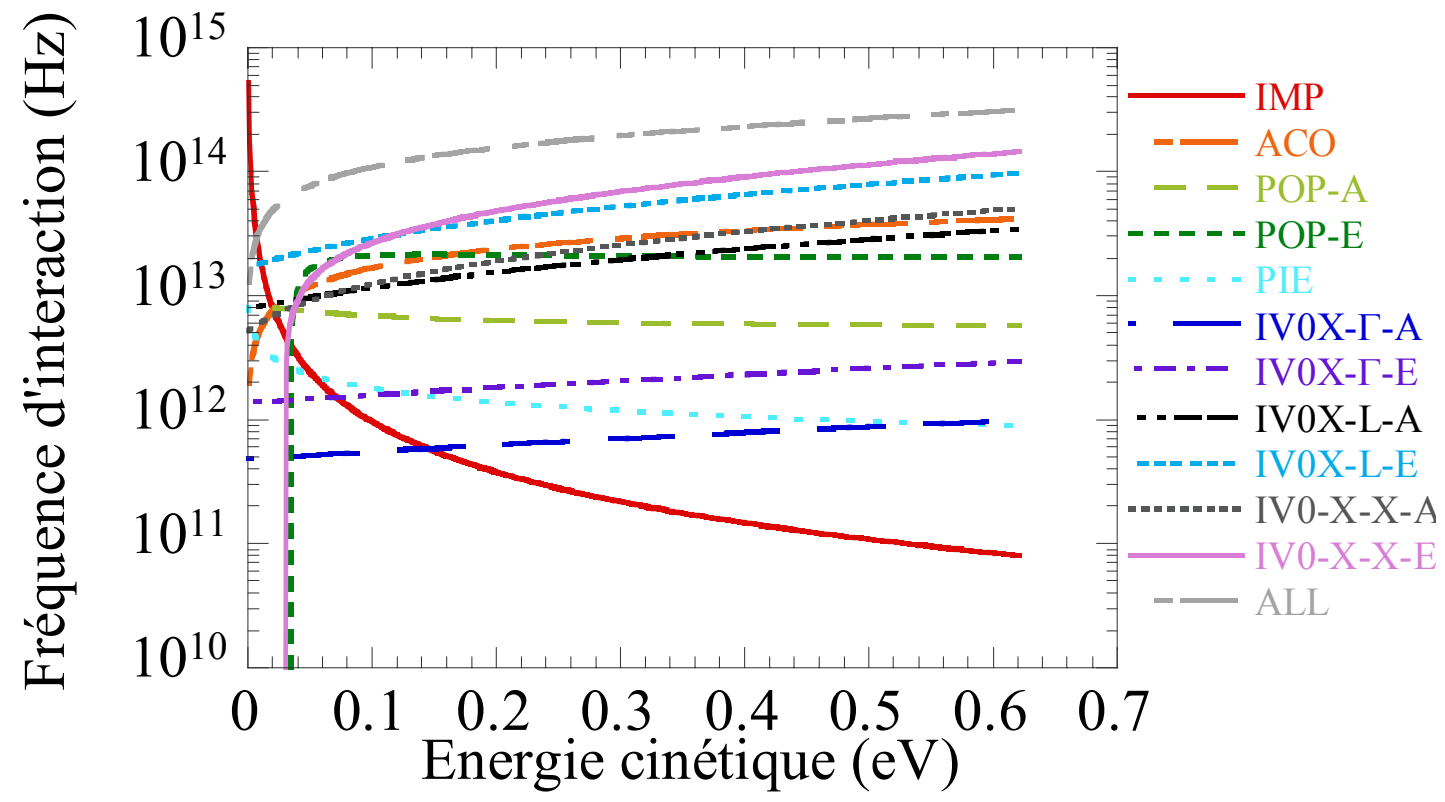




\section{Annexe - E :}

\section{Le modèle physique des contacts}

Dans notre contexte du calcul Monte Carlo, les contacts ohmiques sont considérés comme des régions où l'équilibre thermodynamique est toujours maintenu quand un courant de porteurs les traverse. Ils permettent d'injecter les électrons de manière à ce que la neutralité électrique soit toujours vérifiée dans les régions adjacentes des contacts. Dans notre cas, on simule le mouvement des porteurs à chaque pas de temps, puis on injecte les porteurs nécessaires pour atteindre l'équilibre dans les cellules adjacentes au contact.

Nous maintenons la neutralité de charge seulement dans la cellule adjacente au contact ohmique. Pendant chaque pas du temps $\Delta t$, la concentration des porteurs dans cette cellule, $n_{c t c}$ est calculée par :

$$
n_{c t c}=\frac{1}{V \cdot \Delta x} \sum_{i=0}^{N_{c c c}} \frac{\delta t_{i}}{\Delta t}
$$

où A est la section du contact, $\Delta x$ est la longueur de la cellule, $N_{c t c}$ est le nombre de porteurs qui sont présents dans la cellule pendant le pas du temps. $\delta t_{i}$ est le temps d'un porteur i passe à l'intérieur de la cellule. Si le porteur reste à l'intérieur de la cellule pendant le pas de temps $\delta t_{i}=\Delta t$. Si le porteur sort ou rentre dans la cellule pendant le pas du temps, $\delta t_{i}<\Delta t$. A la fin du pas de temps, $n_{c t c}$ est comparé à la valeur de la densité de dopage dans la cellule $N_{d o p}$. Si $n_{c t c}>N_{d o p}$ aucun porteur n'est injecté. Si $n_{c t c}<N_{d o p}$, on injecte des porteurs. Le porteur est injecté à une position qui correspond au bord de la cellule à côté du contact. Son comportement à l'intérieur du dispositif est simulé pendant un temps égal à $r \Delta t . \mathrm{r}$ est un nombre aléatoire uniformément distribué entre 0 et 1 . La concentration en porteurs dans la cellule adjacente au contact $n_{c t c}$ sera mise à jour en prenant compte la présence de la nouvelle particule. Si $n_{c t c}<N_{d o p}$ est encore vérifiée, un nouveau porteur est injecté de la même manière. Cette procédure continue jusqu'à $n_{c t c}=N_{d o p}$.

Par ailleurs, la dynamique des porteurs injectés dans la cellule adjacente au contact doit être déterminée par une distribution de vitesse $f_{i n j}(\vec{v}), \vec{v}=\left(v_{x}, v_{y}, v_{z}\right)$. Les porteurs injectés suivent typiquement une distribution thermique pondérée par la composante de vitesse $v_{x}$ normale à la surface du contact : 


$$
f_{\text {inj }}(\vec{v})=v_{x} \cdot f_{\text {ther }}(\vec{v}) \quad \text { avec } v_{x}>0
$$

où $f_{\text {ther }}(\vec{v})$ est la distribution thermique à la température du réseau. Dans le cas des contacts non-dégénérés, il correspond à la distribution de type Maxwell - Boltzmann. En outre, on peut définir une fréquence d'injection $\Gamma$, c'est-à-dire le nombre de porteurs qui entrent dans le dispositif par unité de temps :

$$
\Gamma=n_{c t c} \cdot \bar{v}_{+} \cdot S
$$

$n_{c t c}$ est la densité de porteurs dans le contact. S est la section transverse de la cellule du contact, il est normal à la direction de l'injection. Et la vitesse moyenne suivant la direction d'injection $\bar{v}_{+}$vaut:

$$
\bar{v}_{+}=\frac{\int_{0}^{\infty} \int_{-\infty}^{\infty} \int_{-\infty}^{\infty} f_{i n j}(\vec{v}) d v_{x} d v_{y} d v_{z}}{\int_{-\infty}^{\infty} \int_{-\infty}^{\infty} \int_{-\infty}^{\infty} f_{\text {ther }}(\vec{v}) d v_{x} d v_{y} d v_{z}}
$$

Pour les contacts non-dégénérés, la vitesse d'une population se trouve distribuée sur une gaussienne déplacée avec un écart type de l'ordre de $k_{B} T / q$ :

$$
f_{\text {ther }}(\vec{v}) \propto \exp \left(\frac{m \cdot\left(v-v_{0}\right)^{2}}{2 k_{B} T}\right)
$$

Donc $\bar{v}_{+}$est de l'ordre de la vitesse thermique $v_{t h}=\sqrt{2 k_{B} T / \pi m^{*}}$. On peut constater que dans ce cas la vitesse d'injection ne dépendra pas de la condition de polarisation ainsi que de la fréquence d'injection. Le temps entre deux entrées consécutives d'électrons $t_{i n j}$ est généré avec une probabilité par unité de temps :

$$
P\left(t_{i n j}\right)=\Gamma \exp \left(-\Gamma \cdot t_{i n j}\right)
$$

ce qui correspond à une statistique Poissonienne. C'est-à-dire que les électrons sont injectés selon une statistique Poissonienne dans l'espace de temps, avec la fréquence d'injection environ égale à $n_{c t c} v_{t h} S / 2$, et à des instants statistiquement indépendants. Ce schéma d'injection est appliqué à chaque cellule du contact en fonction de la concentration de porteurs $n_{c t c}$. Sur la figure ci-dessous, nous avons reporté la distribution de la vitesse issus de la simulation MC. Seule la composante perpendiculaire aux contacts (c'est-à-dire dans la direction Source - Drain) est tracée. Cette composante de la vitesse est calculée à l'aide du vecteur d'onde et de l'énergie cinétique du porteur $\varepsilon$ : 


$$
v_{x}=\frac{\hbar k_{x}}{m^{*}\left(1+2 \alpha \varepsilon_{k}\right)}
$$

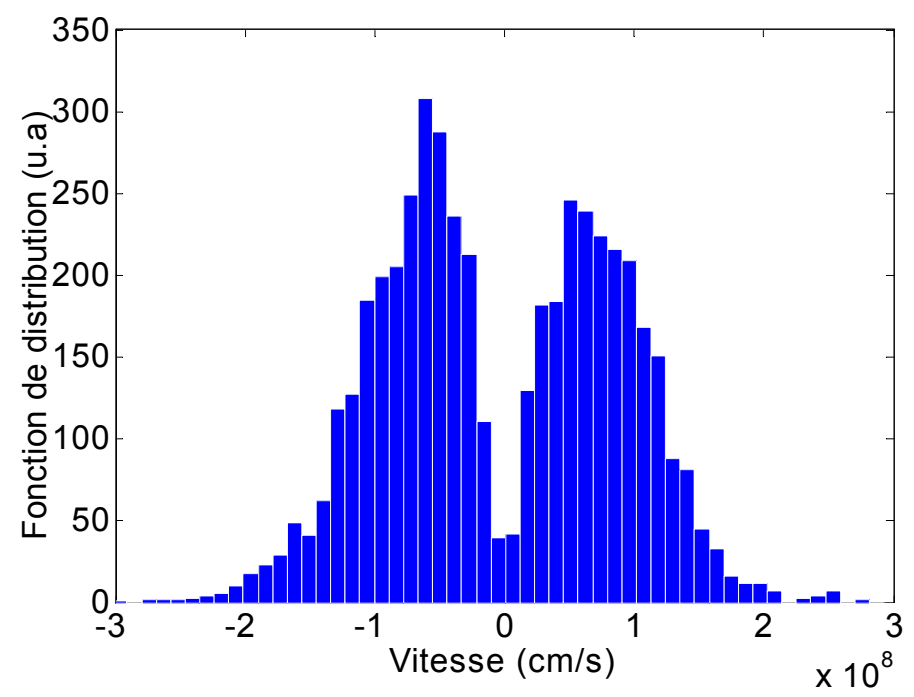

Figure C-1 Fonction de distribution de la vitesse des porteurs injectés par les contacts de source et de drain non-dégénérés

L'amélioration du simulateur, quand les contacts sont complètement dégénérés (réservoir quantique : contact quantique) est en cours d'investigation. Nous présentons ci-après les méthodes utilisées pour cela et leur implémentation dans le simulateur.

Intéressons-nous au tirage au sort des particules injectées aux contacts selon la statistique Fermi-Dirac. Dans ces cas, les lois de probabilité des coordonnées réciproques $k_{x}, k_{y}$ et $k_{z}$ ne sont pas séparables. Mais on aurait besoin d'une distribution suivant une composante privilégiée du vecteur d'onde ou de la vitesse pour éviter une injection de particules de vitesse nulle. Cependant, on suppose que les lois de probabilité pourraient être séparables dans l'espace sphérique $\vec{k}=(k, \theta, \varphi)$, c'est-à-dire que le tirage de $\vec{k}$ sera en coordonnées sphériques avec l'axe principale selon x et l'angle solide élémentaire $d \Omega$. Si on veut tirer $\vec{k}$ pondéré par $k_{x}$, la densité de probabilité est de la forme :

$$
\begin{array}{rlc}
P(\vec{k}) & \propto & k_{x} f(\vec{k}) d \Omega \\
& \propto & k \cdot \cos \theta \cdot f(k) \cdot k^{2} \cdot \sin \theta \cdot d k d \theta d \varphi \\
& \propto & \left(k^{3} f(k) d k\right)(\cos \theta \sin \theta d \theta)(d \varphi) \\
P(\vec{k}) & \propto & (P(k) d k)(P(\theta) d \theta)(P(\varphi) d \varphi)
\end{array}
$$

on peut nommer : 


$$
\begin{aligned}
& P(k) \propto k^{3} f(k) \\
& P(\theta) \propto \cos \theta \sin \theta \\
& P(\varphi)=\text { const }
\end{aligned}
$$

Dans les zones adjacentes du contact, le nombre d'interactions est très élevé, cela induit une distribution isotrope des électrons dans l'espace réciproque, dans ce cas, on pourrait désormais travailler dans l'espace de l'énergie au lieu de l'espace réciproque en supposant: $f(\vec{k})=f(k)=f\left(\varepsilon_{k}\right)$, cette méthode permet de diminuer le bruit associé aux fluctuations des particules. On a donc :

$$
P(k) d k \propto\left(\varepsilon_{k}\left(1+\alpha \varepsilon_{\mathrm{k}}\right)\right)^{3 / 2} f\left(\varepsilon_{k}\right) \frac{\left(1+2 \alpha \varepsilon_{k}\right)}{\sqrt{\varepsilon_{k}\left(1+\alpha \varepsilon_{k}\right)}} d \varepsilon_{k}=P\left(\varepsilon_{k}\right) d \varepsilon_{k}
$$

Si on ignore l'effet de la non-parabolicité, $P\left(\varepsilon_{k}\right) \propto \varepsilon_{k} f\left(\varepsilon_{k}\right)$.

On va tirer les différentes coordonnées à l'aide des trois nombres aléatoires $r_{1}, r_{2}$ et $r_{3}$ qui suivent une loi de probabilité uniforme entre 0 et 1 . On utilise une méthode analytique [Goano1995] pour calculer l'intégrale de Fermi incomplète comme :

$$
I(b, j)=\int_{b}^{\infty} d \varepsilon f\left(\varepsilon_{k}\right) \varepsilon_{k}^{j} \quad \operatorname{avec} I(b, 1)=r_{1}
$$

La borne $\mathrm{b}$ peut déterminée par la méthode de dichotomie telle que $I(b, 1)=r_{1}$ en normalisant pour que $I(0,1)$ soit égale 1 . L'énergie cinétique du porteur correspondra au tirage de la variable aléatoire de la fonction de répartition $I$. Ensuite, le module du vecteur d'onde d'injection sera :

$$
k=\sqrt{\frac{2 m^{*}}{\hbar^{2}}\left(\varepsilon_{k}\left(1+\alpha \varepsilon_{k}\right)\right)}
$$

Pour le tirage sur les angles: $\theta \propto(0, \pi / 2)$ si le contact est à gauche, on a aussi $\int_{0}^{\theta} \sin \theta \cos \theta d \theta \propto 1-\cos (2 \theta)$, on peut normaliser $\operatorname{par} 1-\cos 2 \pi / 2=2$. On cherche donc $\theta$ qui vérifie $(1-\cos 2 \theta) / 2=r_{2}$, on a donc finalement :

$$
\cos \theta=\sqrt{1-r_{2}} \quad \text { et } \quad \sin \theta=\sqrt{1-\cos ^{2} \theta}
$$

Pour $\varphi$, sa densité de probabilité est une constante, on a donc simplement :

$$
\varphi=2 \pi r_{3}
$$

D'après l'observation de nos tests de simulation numérique (sur la Fig. C-2, on illustre la composante de vitesse perpendiculaire aux contacts), la statistique d'injection dans le temps 
suit une distribution binomiale qui est déterminée par la statistique de Fermi-Dirac des électrons. C'est-à-dire que pour un porteur avec un vecteur d'onde $k$ et une énergie cinétique $\varepsilon_{k}$, quand $\varepsilon_{k}-\varepsilon_{F} \ll-k_{B} T, f_{F D}\left(\varepsilon_{k}\right) \approx 1$ et la statistique d'injection de l'état $k$ correspondante devient quasi-uniforme dans le temps par rapport au cas non-dégénéré. Par conséquent, pour un contact quantique, les électrons sont injectés de façon équidistante dans le temps pour tous les vecteurs d'onde jusqu'au vecteur d'onde de Fermi $k_{F}$. Cela peut aussi être interprété par une injection périodique dans le temps avec une vitesse d'injection de l'ordre de la vitesse de Fermi $v_{F}=\hbar k_{F} / m^{*}$. La période est difficile à déterminer analytiquement, elle dépendra principalement de l'énergie cinétique des porteurs qui est obtenue par le tirage au sort selon la distribution de Fermi-Dirac et la densité d'états dans l'espace réciproque et en énergie. En outre, quand $\varepsilon_{k}-\varepsilon_{F} \gg k_{B} T, f_{F D}\left(\varepsilon_{k}\right) \ll 1$, le contact revient à un comportement classique, la statistique d'injection de l'état $k$ correspondante redevient Poissonnienne.

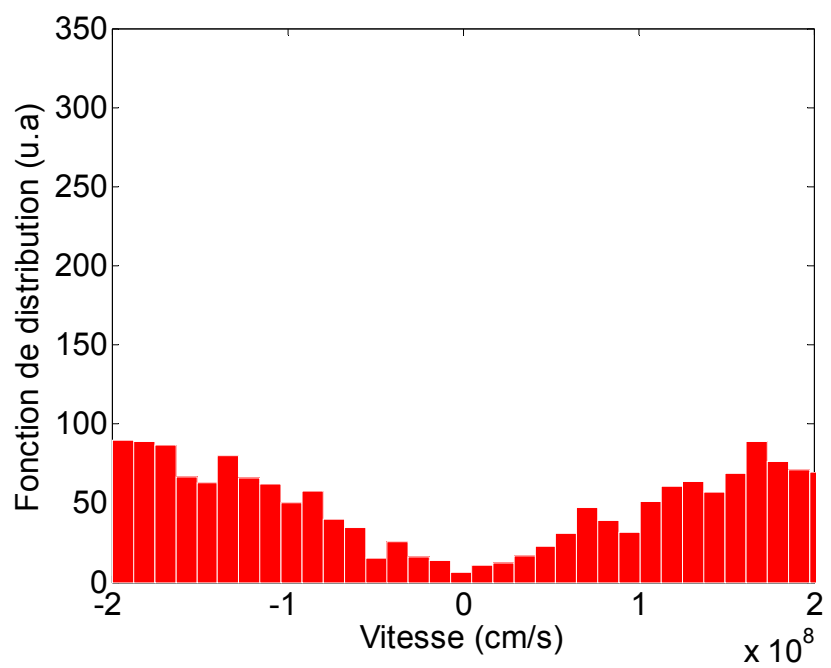

Figure C-2 Fonction de distribution de la vitesse des porteurs injectés par les contacts de source et de drain dégénérés. Le niveau du dopage du caisson est de l'ordre de $2 \times 10^{19} \mathrm{~cm}^{-3}$, le niveau de Fermi

$$
\varepsilon_{F}-E_{C} \approx 20 k_{B} T \text {. }
$$




\section{Annexe - F :}

\section{Calcul du gain unilatéral du composant actif linéaire à l'aide des éléments du modèle petit signal}

Les composants actifs linéaires comme les transistors bipolaires ou à effet de champ peuvent servir comme amplificateurs. Le principe du calcul des amplificateurs en microondes est souvent basé sur les paramètres de réseaux comme les paramètres $\mathrm{S}, \mathrm{Y}$ et de chaine $\mathrm{C}$. Les considérations les plus importantes dans la conception d'un amplificateur sont le gain en puissance, la stabilité, le bruit et le choix du point de polarisation. Dans cette annexe, on s'intéresse seulement à la notion du gain de transfert en puissance. Par définition, le gain de transfert en puissance est le rapport de la puissance délivrée à la charge par la puissance disponible à la source. La puissance disponible à la source est la puissance maximale qu'elle peut délivrer à une charge. Cette puissance s'obtient en fermant le générateur sur une charge qui est égale au complexe conjugué de son impédance interne.

En 1954, S. J. Mason a proposé une technique de contre-réaction sans perte (lossless feedback technique) qui permet de rendre le transistor unilatéral. Dans cette configuration, on définit le gain unilatéral en puissance U ([Mason1954IRE]) du transistor :

$$
U=\frac{\left|y_{21}-y_{12}\right|^{2}}{4 \cdot\left[\mathfrak{R}\left(y_{11}\right) \mathfrak{R}\left(y_{22}\right)-\mathfrak{R}\left(y_{12}\right) \mathfrak{R}\left(y_{21}\right)\right]}
$$

Il représente le maximum du gain de transfert en puissance quand le transistor est unilatéralisé $\left(\mathrm{S}_{12}=0\right)$. La contre-réaction d'un circuit réactif (reactive tuning) pour unilatéraliser le dispositif avec trois terminaux a été proposée par Mason et améliorée par Gupta [Gupta1992IEEE].

Pour une configuration en source commune, le comportement dynamique du transistor intrinsèque peut être décrit par ses éléments du circuit équivalent petit signal (cf. Fig. 23 du Chapitre III) en prenant compte la transcapacité du domaine dipolaire $\mathrm{C}_{\mathrm{DC}}$. On peut calculer les paramètres $\mathrm{Y}$ du transistor intrinsèque en utilisant ses éléments à l'aide d'une configuration d'un circuit en forme de $\mathrm{T}$ :

$$
\begin{gathered}
y_{11}=\Delta\left\{s C_{G S}\left(1 / R_{i}^{2}-s C_{G S} / R_{i}-s^{2} C_{D C}\left(C_{D C}+C_{G S}\right)\right)\right\}+s C_{G D} \\
y_{12}=\frac{-s^{2} C_{D C} C_{G S}}{1 / R_{i}+s\left(C_{D C}+C_{G S}\right)}-s C_{G D}
\end{gathered}
$$




$$
\begin{gathered}
y_{21}=\frac{G_{m}\left(-1 / R_{i}+s C_{D C}\right)-s^{2} C_{D C} C_{G S}}{1 / R_{i}+s\left(C_{D C}+C_{G S}\right)}-s C_{G D} \\
y_{22}=\Delta\left\{\begin{array}{l}
G_{d} / R_{i}^{2}+s\left(C_{D C} / R_{i}^{2}-G_{m} C_{D C} / R_{i}\right) \\
\left.+s^{2}\left(\begin{array}{l}
G_{m} C_{D C}\left(C_{D C}+C_{G S}\right)-G_{d}\left(C_{G S}^{2}+C_{D C}^{2}\right) \\
-2 C_{D C} C_{G S} G_{d}-C_{D C}^{2} / R_{i} \\
-s^{3}\left(C_{G S} C_{D C}\left(C_{D C}+C_{G S}\right)\right)
\end{array}\right)\right\}+s C_{G D}
\end{array}\right\}
\end{gathered}
$$

où $s=j \omega$ et $\Delta=\left(1 / R_{i}^{2}+|s|^{2}\left(C_{G S}+C_{D C}\right)^{2}\right)^{-1}$. En basse fréquence, c'est-à-dire $f<\left(2 \pi R_{i}\left(C_{G S}+C_{D C}\right)\right)^{-1}$ et $\tau \approx R_{i} C_{D C}$ et également $\tau \approx R_{i} C_{G S}$, on peut approcher $G_{m}=G_{m 0} \exp (-s \tau)$ par $G_{m 0}\left(1-s \tau+(s \tau)^{2} / 2\right)$ et $\Delta \approx R_{i}^{2}$. Le numérateur de l'équation D-1 devient alors :

$$
\left|y_{21}-y_{12}\right|^{2} \approx G_{m 0}^{2}\left|\frac{1 / R_{i}+s C_{D C}}{1 / R_{i}+s\left(C_{D C}+C_{G S}\right)}\right|^{2}
$$

tandis que le numérateur de l'équation D-2 peut s'écrire :

$$
\left.\mathfrak{R}\left(y_{11}\right) \Re\left(y_{22}\right)-\mathfrak{R}\left(y_{12}\right) \Re\left(y_{21}\right) \approx s^{2} C_{G S} R_{i}^{3} G_{d}\left\{\begin{array}{l}
1 / R_{i}^{2}\left(C_{D C} G_{m 0} / G_{d}-C_{G S}\right)+ \\
s^{2}\left(C_{G S}\left(C_{D C}+C_{G S}\right)^{2}+\frac{C_{D C} G_{m 0} \tau^{2}}{2 G_{d} R_{i}^{2}}\right)
\end{array}\right)\right\}
$$

On pourra donc approximer l'expression analytique du gain unilatéral, en remplaçant $s$ par $j \omega$, on a donc :

$$
G_{u n i}=\left(\frac{G_{m 0}^{2} / G_{d}}{4 C_{G S} R_{i}\left(C_{G S}-C_{D C} G_{m 0} / G_{d}\right)}\right)\left(\frac{1}{\omega^{2}\left(1-p_{d}^{2} \omega^{2}\right)}\right)
$$

avec un pôle comme :

$$
p_{d}^{2}=\frac{\left(R_{i}^{2} C_{G S}\right)\left(C_{D C}+C_{G S}\right)^{2}+C_{D C} G_{m 0} \tau^{2} / 2 G_{d}}{\left(C_{D C} G_{m 0} / G_{d}-C_{G S}\right)}
$$

A très basse fréquence $\left(f<\left(2 \pi R_{i} C_{G S}\right)^{-1}\right)$, on néglige l'effet non quasi-statique, c'est-à-dire que l'effet de la transcapacité $C_{D C}$ peut être ignoré et on aboutit à l'expression en régime quasi-statique :

$$
G_{u n i}=\frac{G_{m 0}^{2} / G_{d}}{4 C_{G S}^{2} R_{i}} \cdot \frac{1}{\omega^{2}}
$$


La dépendance en fréquence au régime basse fréquence sera déterminée par le terme $1 / \omega^{2}$. On aura donc une pente d'atténuation de $-20 \mathrm{~dB} / \mathrm{dec}$ (soit $-6 \mathrm{~dB} /$ octave). A haute fréquence, l'effet non quasi-statique dû à $C_{D C}$ doit être pris en compte. L'ordre du système est augmenté à cause du terme $1 /\left(1-p_{d}^{2} \omega^{2}\right)$, le comportement de décroissance devient donc de $-40 \mathrm{~dB} /$ décade, c'est-à-dire qu'on ajoute un autre pôle complexe conjugué à une fréquence $f_{p}:$

$$
f_{p}=\frac{1}{2 \pi\left|p_{d}\right|}
$$

Pour les transistors en $R F$, cette fréquence du pôle est typiquement plus faible que $f_{\text {MAX }}$, donc elle peut influencer fortement le comportement du gain $U$. 


\section{Références :}

[Aarts2003DATE] : E. Aarts, R. Roovers. IC Design Challenges for Ambient Intelligence. Proceedings of the Design, Automation and Test in Europe IEEE Conference and Exhibition (DATE'03).

[Ando1982RMP] : T. Ando, A. B. Fowler, F. Stern. Electronic properties of two-dimensional systems. Review of Modern Physics, VOL. 54, pp. 437, 1982.

[Ariel-Altschul1992IEEE] : V. Ariel-Altschul, E. Finkman, G. Bahir. Approximations for Carrier Density in Nonparabolic Semiconductors. IEEE Transactions on Electron Devices, VOL. 39, pp. 1312, 1992.

[Ashkar2010IEEE] : A. Ashkar, Madan, H., Koveshnikov, S., Oktyabrsky, S. Kambhampati, R., Heeg, T., Schlom, D. and Datta, S. Small-Signal Response of Inversion Layers in HighMobility In0.53Ga0.47As MOSFETs Made With Thin High-к Dielectrics. IEEE Transactions on Electron Devices, VOL. 57, pp 742-748, 2010.

[Aubry-Fortuna2005SSE] : V. Aubry-Fortuna, P. Dollfus, S. Galdin-Retailleau. Electron effective mobility in strained-Si/Si $i_{1-x} G e_{x}$ MOS devices using Monte Carlo simulation. SolidState Electronics, VOL. 49, pp. 1320, 2005.

[Babiker1996IEEE] : S. Babiker, A. Asenov, N. Cameron, S. Beaumont, S. Simple approach to include external resistances in the Monte Carlo simulation of MESFETs and HEMTs Electron Devices. IEEE Transactions on Electron Devices, VOL. 43, pp. 2032, 1996.

[Babiker1998IEEE] : S. Babiker, A. Asenov, N. Cameron, S. P. Beaumont, R. B. John. Complete Monte Carlo RF Analysis of "Real" Short-Channel Compound FET's. IEEE Transactions on Electron Devices, VOL. 45, pp. 1644, 1998.

[Balynas 1990APL] : V. Balynas, A. Krotkus, A. Stalnionis, A. T. Gorelionok, N. M. Shmidt, J. A. Tellefsen. Time-resolved, hot-electron conductivity measurement using an electro-optic sampling technique. Applied Physics Letters, VOL. 51, pp. 357, 1990. 
[Barraud2001Thèse] : Sylvain Barraud. Effet d'une répartition discrète et aléatoire des impuretés dans le canal des MOSFET sub-100 nm : Etude théorique par simulation, Thèse de doctorat, Université Paris Sud, 2001

[Bentley2011IEEE] : S. Bentley, M. Holland, X. Li, G. Paterson, H. Zhou, O. Ignatova, D. Macintyre, S. Thoms, A. Asenov, B. Shin, J. Ahn, P. McIntyre, I. Thayne. Electron Mobility in Surface- and Buried-Channel Flatband hbox $\mathrm{In}_{0.53} \mathrm{Ga}_{0.47} \mathrm{As}$ MOSFETs With $\mathrm{ALD} \mathrm{Al}_{2} \mathrm{O}_{3}$ Gate Dielectric. IEEE Electron Device Letters, VOL. 32, pp. 494, 2011.

[Bhattacharyya1993JAP] : K. Bhattacharyya, S. M. Goodnick, J. F. Wager. Monte Carlo simulation of electron transport in alternating-current thin-film electroluminescent devices. Journal of Applied Physics, VOL. 73 , pp. 3390, 1993.

[Bournel2006HDR] : Arnaud Bournel. Composants pour la spintronique, la microélectronique ultime et la nanoélectronique. Habilitation à diriger les recherches, Université Paris-Sud, 2006.

[Bournel2007SSE] : A. Bournel, V. Aubry-Fortuna, J. Saint-Martin, P. Dollfus. Device performance and optimization of decananometer long double gate MOSFET by Monte Carlo simulation. Solid-State Electronics, VOL. 51, pp. 543, 2007.

[Brammertz2007APL] : G. Brammertz, K. Martens, S. Sioncke, A. Delabie, M. Caymax, M. Meuris, M. Heyns, Appl. Phys. Lett. VOL. 91, pp. 133510, 2007.

[Brammertz2009APL] : G. Brammertz, H. C. Lin, M. Caymax, M. Meuris, M. Heyns, and M. Passlack, On the interface state density at $\operatorname{In}_{0.53} \mathrm{Ga}_{0.47} A$ s/oxide interfaces. Appl. Phys. Lett. VOL. 95, pp. 202109, 2009.

[Brammertz2009ECS] : G. Brammertz, H. C. Lin, K. Martens, A.-R. Alian, C. Merckling, J. Penaud, D. Kohen, W. E. Wang, S. Sioncke, A. Delabie, M. Meuris, M. R. Caymax, and M. Heyns, Electrical Properties of III-V/Oxide Interfaces, ECS Trans. VOL. 19, pp. 375, 2009. 
[Brisset1994Thèse] : Christophe Brisset. Etudes théoriques par simulation Monte-Carlo 3D de la sensibilité aux irradiations des technologies CMOS/SOI, Thèse de doctorat, Université Paris Sud, 1994.

[Castagne1971SS] : Castagne, R. and Vapaille, A. Description of the SiO2-Si interface properties by means of very low frequency MOS capacitance measurements Surface Science, VOL 28, pp. 157-193, 1971.

[Chang2008APL] : Y. C. Chang, M. L. Huang, K. Y. Lee, Y. J. Lee, T. D. Lin, M. Hong, J. Kwo, T. S. Lay, C. C. Liao, and K. Y. Cheng, Atomic-layer-deposited HfO2 on In0.53Ga0.47As: Passivation and energy-band parameters Appl. Phys. Lett. VOL. 92, pp. $072901,2008$.

[Chau2005CSIC] : Robert Chau, Suman Datta, and Amlan Majumdar. Opportunities and Challenges of III-V Nanoelectronics for Future High-Speed, Low-Power Logic Applications. IEEE Compound Semiconductor Integrated Circuit Symposium, 2005. CSIC '05.

[Chau2005IEEE] : R. Chau, S. Datta, M. Doczy, B. Doyle, B. Jin, J. Kavalieros, A. Majumdar, M. Metz, M. Radosavljevic. Benchmarking Nanotechnology for High-Performance and LowPower Logic Transistor Applications. IEEE Transactions on Nanotechnology, VOL. 4, pp. 153,2005

[Chau2007Nature]: R. Chau, B. Doyle, S. Datta, J. Kavalieros and K. Zhang, Integrated nanoelectronics for the future, Nature Materials, VOL. 6, pp. 810-812, 2007.

[Chau2008CS]: R. Chau, CS MANTECH Conference, III-V on Silicon for Future High Speed and Ultra-Low Power Digital Applications: Challenges and Opportunities April 14-17, 2008, Chicago, Illinois, USA

[ChauDRC2006]: Chau, R. Challenges and Opportunities of III-V Nanoelectronics for Future Logic Applications Device Research Conference, 2006 64th, pp. 3 -4, 2006.

[Chen1996IEEE]: K. Chen et al. The impact of device scaling and power supply change on CMOS gate performances. IEEE Electron Device Letters, VOL. 17, pp. 202, 1996. 
[Chen2009IEDM]: Chen, J. GPU technology trends and future requirements IEEE International Electron Devices Meeting (IEDM) Tech. Dig., 2009.

[Chevalier1998Thèse] : Chevalier. P., Conception et réalisation de transistors à effet de champ de la filière AlInAs/GaInAs sur le substrat InP. Application à l'amplification faible bruit en ondes millimétriques. Thèse de doctorat, Université Joseph-Fourier-Grenoble 1998.

[Chin1995MJ] : V. W. L. Chin,T. Osotchan, T. L. Tansley,. Electron mobility in $I_{0.5} 3 G a_{0.47} A s$ as a function of concentration and temperature. Microelectronics Journal; VOL. 26, pp. 653, 1995.

[Chiu2008APL] : H. C. Chiu, L. T. Tung, Y. H. Chang, Y. J. Lee, C. C. Chang, J. Kwo, and M. Hong, Achieving a low interfacial density of states in atomic layer deposited $\mathrm{Al}_{2} \mathrm{O}_{3}$ on $I_{0.53} G a_{0.47} A s$ Appl. Phys. Lett. VOL. 93, pp. 202903, 2008.

[Choo2004JAP] : Choo, K. Y. and Ong, D. S. Analytical band Monte Carlo simulation of electron impact ionization in $\operatorname{In}_{0.53} G a_{0.47} A s$ Journal of Applied Physics, VOL. 96, pp. 56495653,2004

[Cohen-Tannoudji1973] : Claude Cohen-Tannoudji, Bernard Diu et Franck Laloë. Mécanique Quantique. Hermann, 1973.

[Connor2009APL] : E. O’Connor, S. Monaghan, R. D. Long, A. O. Mahony, I. M. Povey, K. Cherkaoui, M. E. Pemble, G. Brammertz, M. Heyns, S. B. Newcomb, V. V. Afanas'ev, and P. K. Hurley, Temperature and frequency dependent electrical characterization of HfO2/InxGa1-xAs interfaces using capacitance-voltage and conductance methods. Appl. Phys. Lett. VOL. 94, pp. 102902, 2009.

[Cristoloveanu2010IEEE] : S. Cristoloveanu, N. Rodriguez, F. Gamiz. Why the Universal Mobility Is Not. IEEE Transactions on Electron Devices, VOL. 57, pp. 1327, 2010. 
[Dambrine1988IEEE] : G. Dambrine, A. Cappy, F. Heliodore, E. Playez. A new method for determining the FET small-signal equivalent circuit Microwave Theory and Techniques. IEEE Transactions on Electron Devices, VOL. 36, pp. 1151, 1988.

[Dambrine1998IEEE] : G. Dambrine, J. M. Belquin, F. Danneville, A. Cappy. A new extrinsic equivalent circuit of HEMT's including noise for millimeterwave circuit design. IEEE Trans. Microwave Theory Tech. VOL. 46, pp. 1231, 1998.

[Dambrine1999IEEE] : G. Dambrine, J. P. Raskin, F. Danneville, D. Vanhoenackel Janvier, J.-P. Colinge, A. Cappy. High-frequency four noise parameters of silicon-on-insulator-based technology MOSFET for the design of low-noise RF integrated circuits. IEEE Transactions on Electron Devices, VOL. 46, pp. 1733, 1999.

[Dambrine2005] : G. Dambrine, D. Gloria, P. Scheer, C. Raynaud, F. Danneville, S. Lepilliet, A. Siligaris, G. Pailloncy, B. Martineau, E. Bouhana, R. Valentin. High frequency low noise potentialities of down to $65 \mathrm{~nm}$ technology nodes MOSFETs. Proc. The European Gallium Arsenide and Other Compound Semiconductors Application Symposium 2005 (EGAAS), pp. 97-100, 2005

[Danneville1994IEEE] : F. Danneville, H. Happy, G. Dambrine, J. M. Belquin, A. Cappy. Microscopic noise modeling and macroscopic noise models: How good a connection?. IEEE Transactions on Electron Devices, VOL 41, pp. 779-786, 1994.

[Datta1995] : Supriyo Datta. Electronic transport in mesoscopic system. Cambridge University Press, 1995.

[Datta2007ME]: Datta, S. III-V field-effect transistors for low power digital logic applications Microelectronics Engineering, VOL 84. pp. 2133-2137, 2007.

[De.Visschere1990SSE] : P. De Visschere. The validity of Ramo's theorem. Solid-State Electronics, VOL. 33, pp. 455, 1990.

[Del-Alamo2010AVS] : Del Alamo, J. A., AVS 57th International Symposium \& Exhibition, Albuquerque, New Mexico, 2010. 
[Del-Alamo2011EMRS] : Del Alamo, J. A. III-V CMOS: the key to sub-10 nm electronics? 2011 E-MRS Spring Meeting and Exhibition Symposium P: Interface Engineering for PostCMOS Emerging Channel Materials April 25-29, 2011

[Dennard1974JSSC] : Dennard, R.; Gaensslen, F.; Rideout, V.; Bassous, E. and LeBlanc, A. Design of ion-implanted MOSFET's with very small physical dimensions, IEEE Journal of Solid-State Circuits, VOL. 9, pp. 256 - 268, 1974.

[Dewey2009IEDM] : Dewey, G.; Kotlyar, R.; Pillarisetty, R.; Radosavljevic, M.; Rakshit, T.; Then, H. \& Chau, R. Logic performance evaluation and transport physics of Schottky-gate III 13; V compound semiconductor quantum well field effect transistors for power supply voltages $(V C C)$ ranging from $0.5 v$ to $1.0 \mathrm{~V}$. IEEE International Electron Devices Meeting (IEDM) Tech. Dig., pp. 1-4, 2009.

[Dittrich1999SSE] : R. Dittrich, W. Schroeder. Empirical pseudopotential band structure of $\operatorname{In}_{0.53} G a_{0.47} A s$ and $\operatorname{In}_{0.52} A l_{0.48} A s$. Solid-State Electronics, VOL. 43, pp. 403, 1999.

[Dollfus1992EL] : P. Dollfus, S. Galdin, P. Hesto. Microwave Analysis of AlGaAs/InGaAs HEMT Using Monte-Carlo Simulation. Electronics Letters, VOL. 28, pp. 458, 1992.

[Dollfus1993JAP] : P. Dollfus, C. Bru, P. Hesto. Monte-Carlo Simulation of Pseudomorphic Ingaas/Gaas High Electron-Mobility Transistors - Physical Limitations at Ultrashort Gate Length. Journal of Applied Physics, VOL. 73, pp. 804, 1993.

[Dollfus1999HDR] : Philippe Dollfus. Etudes théoriques de structures pour l'électronique rapide et contribution au développement d'un simulateur particulaire Monte Carlo. Habilitation à diriger des recherches, Université Paris Sud, 1999

[Doornbos2010IEEE] : Doornbos, G. and Passlack, M. Benchmarking of III-V n-MOSFET Maturity and Feasibility for Future CMOS. IEEE Electron Device Letters, VOL. 31, pp 1110 $-1112,2010$. 
[Enge-Herbertl2010JAP] : Engel-Herbert, R.; Hwang, Y. and Stemmer, S. Comparison of methods to quantify interface trap densities at dielectric/III-V semiconductor interfaces Journal of Applied Physics, VOL.108, pp. 12410-1-124101-15. 2010.

[Esseni2005PRB] : D. Esseni, P. Palestri. Linear combination of bulk bands method for investigating the low-dimensional electron gas in nanostructured devices. Phys. Rev. B, VOL. 72, pp. 165342, 2005.

[Ferry 1997] : D. K. Ferry, S. M.Goodnick, J. Bird, Transport in nanostructures, Cambrige. Univ. Press, 1997.

[Fischetti1993PRB] : M. V. Fischetti, S. E. Laux. Monte Carlo study of electron transport in silicon inversion layers. Phys Rev B VOL. 48, pp. 2244, 1993.

[Fischetti2007IEDM] : Fischetti, M., Wangt, L., Yut, B., Sachs, C., Asbeckt, P., Taurt, Y. and Rodwell, M. Simulation of Electron Transport in High-Mobility MOSFETs: Density of States Bottleneck and Source Starvation IEEE International Electron Devices Meeting (IEDM) Tech. Dig., pp. 109 -112, 2007.

[Fischetti2007IEEE] : Fischetti, M., O'Regan, T., Narayanan, S., Sachs, C., Jin, S., Kim, J. and Zhang, Y. Theoretical Study of Some Physical Aspects of Electronic Transport in nMOSFETs at the 10-nm Gate-Length IEEE Transactions on Electron Devices, VOL. 54, pp. $2116-2136,2007$.

[Galdin1992Thèse] : Sylvie Galdin. Etude du transistor bipolaire à double hétérojonction Si/SiGe/Si par simulation Monte Carlo, Thèse de doctorat, Université Paris Sud, 1992 [Goel2007APL] : N. Goel, P. Majhi, W. Tsai, M. Warusawithana, D. G. Schlom, M. B. Santos, J. S. Harris, and Y. Nishi, High-indium-content InGaAs metal-oxide-semiconductor capacitor with amorphous LaAlO3 gate dielectric Appl. Phys. Lett. VOL. 91, pp. 093509 2007.

[Goel2007APL] : N. Goel, P. Majhi, W. Tsai, M. Warusawithana, D. G. Schlom, M. B. Santos, J. S. Harris, and Y. Nishi, High-indium-content InGaAs metal-oxide-semiconductor 
capacitor with amorphous LaAlO3 gate dielectric, Appl. Phys. Lett. VOL. 91, pp. 093509, 2007.

[Goel2008IEDM] : N. Goel, D. Heh, S. Koveshnikov, I. Ok, S. Oktyabrsky, V. Tokranov, R. Kambhampatic, M. Yakimov, Y. Sun, P. Pianetta, C. Gaspe, M. Santos, J. Lee, S. Datta, P. Majhi, W. Tsai. Addressing the gate stack challenge for high mobility $\operatorname{In}_{x} G a_{1-x}$ As channels for NFETs. IEEE International Electron Devices Meeting (IEDM) Tech. Dig., 2008.

[Gonzalez1995IEEE-A] : T. Gonzalez, D. Pardo, L. Varani, and L. Reggiani. Monte Carlo analysis of the behavior and spatial origin of electronic noise in GaAs MESFET's. IEEE Transactions on Electron Devices. VOL. 42, pp. 991, 1995.

[Gonzalez1995IEEE-B] : T. Gonzalez, D. Pardo. Monte Carlo determination of the intrinsic small-signal equivalent circuit of MESFET's. IEEE Transactions on Electron Devices, VOL.42, pp. 605, 1995.

[Greaves1993MOTL] : S. D. Greaves, R. T. Unwin. Accurate noise characterization of short gate length GaAs mesfets and hemts for use in low-noise optical receivers. Microwave and Optical Technology Letters, VOL. 6, pp. 60, 1993.

[Gupta1992IEEE] : M. S. Gupta. Power gain in feedback amplifiers, a classic revisited. IEEE Transactions on Microwave Theory and Techniques, VOL. 40, pp. 864, 1992.

[Hall1962JPCS] : G. L. Hall. Ionized impurity scattering in semiconductors. J. Phys. Chem. Solids, VOL. 23, pp. 1147, 1962.

[Harrison1976PRB] : J. W. Harrison, J. R. Hauser. Alloy scattering in ternary III-V compounds. Physical Review B, VOL.13, pp. 5347, 1976.

[Hasse1999SSE] : M. A. Hasse, N. Robbins, N. Tabatabaie, G. E. Stillmann. Subthreshold electron velocity-field characteristics of GaAs an InGaAs. Solid-State Electronics VOL. 43, pp. 1813, 1999. 
[Hesto1984Thèse] : Patrice Hesto, Simulation Monte Carlo du transport non stationnaire dans les dispositifs submicroniques : importance du phénomène balistique dans GaAs à $77 \mathrm{~K}$. Thèse de doctorat d'Etat, Université Paris Sud, 1984

[Himadri2008IEEE] : Himadri S. Pal, Kurtis D. Cantley, Shaikh Shahid Ahmed, Mark S. Lundstrom. Influence of Bandstructure and Channel Structure on the Inversion Layer Capacitance of Silicon and GaAs MOSFETs. IEEE Transactions on Electron Devices, VOL. 55, pp. 904, 2008.

[Huang2009IEDM] : J. Huang, N. Goel, H. Zhao, C. Y. Kang, K. S. Min, G. Bersuker, S. Oktyabrsky, C. K. Gaspe, M. B. Santos, P. Majhi, P. D. Kirsch, H.-H. Tseng, J. C. Lee, and R. Jammy. InGaAs MOSFET performance and reliability improvement by simultaneous reduction of oxide and interface charge in $A L D(L a) A l O x / Z r O 2$ gate stack. IEEE International Electron Devices Meeting (IEDM) Tech. Dig., 2009.

[Huet2008Thèse] : Karim Huet. Modélisation du transport sous contrainte mécanique dans les transistors sub-65 $\mathrm{nm}$ pour la microélectronique CMOS, Thèse de doctorat, Université Paris-Sud, 2008.

[Hurley2009ECS] : P. K. Hurley, E. O’Connor, S. Monaghan, R. Long, A. O’Mahony, I. M. Povey, K. Cherkaoui, J. MacHale, A. Quinn, G. Brammertz, M. M. Heyns, S. Newcomb, V. V. Afanas'ev, A. Sonnet, R. Galatage, N. Jivani, E. Vogel, R. M. Wallace, and M. Pemble, Structural and Electrical Properties of HfO2/n-InxGa1-xAs structures (x: 0, 0.15, 0.3 and 0.53) ECS Transaction. VOL. 25, pp. 113, 2009.

[Hwang2010APL] : Y. Hwang, R. Engel-Herbert, N. G. Rudawski, and S. Stemmer, Analysis of trap state densities at $\mathrm{HfO}_{2} / \mathrm{In}_{0.53} \mathrm{Ga}_{0.47}$ As interfaces Appl. Phys. Lett. VOL. 96, pp. 102910, 2010 .

[Islam2011SST] : A Islam, K Kalna. Monte Carlo simulations of mobility in doped GaAs using self-consistent Fermi-Dirac statistics. Semicond. Sci. Technol. VOL. 26, pp. 055007, 2011. 
[ITRS] : 2007, 2008 et 2009 Edition International Technology Roadmap for semiconductors ITRS. Disponible sur www.itrs.net/reports

[ITRS2001] : International Technology Roadmap for Semiconductors. San Jose, CA: Semiconductor Industry Association; 2001.

[Iwai2002IEDM] : Iwai, H.; Ohmi, S.; Akama, S.; Ohshima, C.; Kikuchi, A.; Kashiwagi, I.; Taguchi, J.; Yamamoto, H.; Tonotani, J.; Kim, Y.; Ueda, I.; Kuriyama, A. \& Yoshihara, Y. Advanced gate dielectric materials for sub-100 $\mathrm{nm}$ CMOS IEEE International Electron Devices Meeting (IEDM) Tech. Dig., pp. 625 - 629, 2002.

[Iwai2002MR] : Iwai, H. and Ohmi, S. Silicon integrated circuit technology from past to future. Microelectronics Reliability, VOL. 42, pp. 465 - 491, 2002.

[Iwai2009IEDST] : Iwai, H. Technology roadmap for 22nm and beyond Electron Devices and Semiconductor Technology, 2009. IEDST '09. 2nd International Workshop on, pp. 1 -4, 2009.

[Iwai2009MicroEng]: Iwai, H. Roadmap for $22 \mathrm{~nm}$ and beyond. Microelectronic Engineering, VOL. 86, pp. 1520 - 1528, 2009.

[Jacoboni1983RMP] : C. Jacoboni, L. Reggiani. The Monte Carlo method for the solution of charge transport in semiconductors with applications to covalent materials. Rev. Mod. Phys. VOL. 55, pp. 645, 1983.

[Jacoboni1989] : C. Jacoboni, P. Lugli. The Monte Carlo method for semiconductor device simulation. Wien: Springer-Verlag; 1989.

[Jin2007IEEE] : S. Jin, M. V. Fischetti, T.-W. Tang. Modeling of Surface-Roughness Scattering in Ultrathin-Body SOI MOSFETs. IEEE Transactions on Electron Devices, VOL. 54, pp. 2191, 2007.

[Jin2007JAP] : Seonghoon Jin, Massimo V. Fischetti, Ting-Wei Tang,. Modeling of electron mobility in gated silicon nanowires at room temperature: Surface roughness scattering, 
dielectric screening, and band nonparabolicity. Journal of Applied Physics, VOL. 102, pp. $083715,2007$.

[Jin2009IEDM] : Donghyun Jin, Daehyun Kim, Taewoo Kim, J. A. del Alamo. Quantum capacitance in scaled down III-V FETs. IEDM Tech. Dig., 2009.

[Joshi1991PRB] : R. P. Joshi, D. K. Ferry. Effect of multi-ion screening on the electronic transport in doped semiconductors: a molecular dynamics approach. Phys. Rev. B, VOL. 43, pp. 9734, 1991.

[Jungemann1993SSE] : C. Jungemann, A. Emunds, W. L. Engl. Simulation of linear and nonlinear electron transport in homogeneous silicon inversion layers. Solid-State Electron. VOL. 36, pp. 1529, 1993.

[Kakushima2010SST] : K. Kakushima, M. Nakagawa, P. Ahmet, K. Tsutsui, N. Sugii, T. Hattori, H. Iwai. Radio-frequency performance of a sub-100 $\mathrm{nm}$ metal-oxide field-effect transistor with high-k gate dielectric. Semiconductor. Science and Technology, VOL. 25, pp. 045029, 2010.

[Kim1991SSE] : H. Kim, H. S. Min, T. W. Tang, Y. J. Park. An Extended Proof of the RamoShockley Theorem. Solid-State Electronics, VOL. 34, pp. 1251, 1991.

[Kim2007IEEE] : Kim, D. H., Del Alamo, J. A., Lee, J. K. and Seo. K. S. Logic Suitability of 50-nm In0.7Ga0.3As HEMTs for Beyond-CMOS Applications IEEE Electron Device Lettres, VOL. 54, pp. 2606-2613. 2007.

[Kim2008IEDM] : Kim, D. H. and Del Alamo J. A., $30 \mathrm{~nm}$ E-mode InAs PHEMTs for THz and future logic applications. IEEE International Electron Devices Meeting (IEDM) Tech. Dig., 2008.

[Kim2009IEDM] : Kim, D. H., Del Alamo, J. A., Antoniadis, D. and Brar, B., Extraction of virtual-source injection velocity in sub-100 $\mathrm{nm}$ III-V HFETs. IEEE International Electron Devices Meeting (IEDM) Tech. Dig., 2009. 
[Kim2009JAP] : E. J. Kim, E. Chagarov, J. Cagnon, Y. Yuan, A. C. Kummel, P. M. Asbeck, S. Stemmer, K. C. Saraswat, and P. C. McIntyre, Atomically abrupt and unpinned Al2O3/In ${ }_{0.53} G a_{0.47} A s$ interfaces: Experiment and simulation J. Appl. Phys. VOL. 106, pp. 124508-124513, 2009.

[Kim2010APL] : E. J. Kim, L. Wang, P. M. Asbeck, K. C. Saraswat, and P. C. McIntyre, Border traps in $\mathrm{Al}_{2} \mathrm{O}_{3} / \mathrm{In}_{0.53} \mathrm{Ga}_{0.47} \mathrm{As}$ (100) gate stacks and their passivation by hydrogen anneals Appl. Phys. Lett. VOL. 96, pp. 012906, 2010.

[Kim2010IEDM] : Kim, D. H., Del Alamo, J. A., Chen, P., Ha, W., Urteaga, M. and Brar, B. 50-nm E-mode In0.7Ga0.3As PHEMTs on 100-mm InP substrate with fmax > $1 \mathrm{THz}$. IEEE International Electron Devices Meeting (IEDM) Tech. Dig., 2010.

[Kim2010IPRM] : Kim, T. W., Kim, D. K. and Del Alamo, J. A. 'Logic Characteristics of 40 nm thin-channel InAs HEMTs' Indium Phosphide and Related Materials (IPRM), 2010 International Conference on.

[Kosina1997PSS] : H. Kosina. Efficient evaluation of ionized-impurity scattering in Monte Carlo transport calculations. Physica Status Solidi, VOL. 163, pp. 75, 1997.

[Koveshnikov2008APL] : S. Koveshnikov, N. Goel, P. Majhi, H. Wen, M. B. Santos, S. Oktyabrsky, V. Tokranov, R. Kambhampati, R. Moore, F. Zhu, J. Lee, and W. Tsai, $\mathrm{In}_{0.53} \mathrm{Ga}_{0.47} \mathrm{As}$ based metal oxide semiconductor capacitors with atomic layer deposition $\mathrm{ZrO} 2$ gate oxide demonstrating low gate leakage current and equivalent oxide thickness less than 1 nm, Appl. Phys. Lett. VOL. 92, pp. 222904, 2008.

[Kunikiyo94JAP] : T. Kunikiyo, M. Takenaka, Y. Kamakura, M. Yamaji, H. Mizuno, M. Morifuji, K. Taniguchi, C. Hamaguchi. A Monte Carlo simulation of anisotropic electron transport in silicon including full band structure and anisotropic impact-ionization model, Journal of Applied Physics, VOL. 75, pp. 297, 1994.

[Laux1985IEEE] : S. Laux. Techniques for small-signal analysis of semiconductor devices. IEEE Transactions on Electron Devices, VOL. 32, pp. 2028, 1985. 
[Lee1995IEEE] : J. H. Lee, H. S. Yoon, C. S. Park, H. M. Park. Ultra low noise characteristics of AlGaAs/InGaAs/GaAs pseudomorphic HEMTs with wide head T-shaped gate. IEEE Electron Device Letters, VOL. 16, pp. 271, 1995.

[Lee2008APL] : 20K. Y. Lee, Y. J. Lee, P. Chang, M. L. Huang, Y. C. Chang, M. Hong, and J. Kwo, Achieving $1 \mathrm{~nm}$ capacitive effective thickness in atomic layer deposited HfO2 on $I_{0.53} G a_{0.47} A s$, Appl. Phys. Lett. VOL. 92, pp. 252908, 2008.

[Lehoucq1998] R. B. Lehoucq, D. C. Sorensen, C. Yang. ARPACK Users' Guide: Solution of Large-Scale Eigenvalue Problems with Implicitly Restarted Arnoldi Methods, SIAM Publications, Philadelphia, 1998.

[Lim2008IEEE] : T. C. Lim, R. Valentin, G. Dambrine, F. Danneville. MOSFETs RF Noise Optimization via Channel Engineering. IEEE Electron Device Letters, VOL. 29, pp. 118, 2008.

[Lin2009APL] : H. C. Lin, G. Brammertz, K. Martens, G. de Valicourt, L. Negre, W.-E. Wang, W. Tsai, M. Meuris, and M. Heyns, The Fermi-level efficiency method and its applications on high interface trap density oxide-semiconductor interfaces. Appl. Phys. Lett. VOL. 94, pp. 153508, 2009.

[Lin2009MEng]: H.-C. Lin, W.-E. Wang, G. Brammertz, M. Meuris, and M. Heyns, Electrical study of sulfur passivated $\operatorname{In}_{0.53} \mathrm{Ga}_{0.47} \mathrm{As}$ MOS capacitor and transistor with ALD Al2O3 as gate insulator, Microelectronics Engineering. VOL. 86, pp. 1554, 2009.

[Lin2010ECS] : D. Lin, N. Waldron, G. Brammertz, K. Martens, W.-E. Wang, S. Sioncke, A. Delabie, H. Bender, T. Conard, W. H. Tseng, J. C. Lin, K. Temst, A. Vantomme, J. Mitard, M. Caymax, M. Meuris, M. Heyns, and T. Hoffman, Exploring the ALD Al2O3/In0.53Ga0.47As and Al2O3/Ge Interface Properties: A Common Gate Stack Approach for Advanced III-V/Ge CMOS. ECS Transaction. VOL. 28, pp. 173, 2010. 
[Lin2010SSE] : Lin, T., Chiu, H., Chang, P., Chang, Y., Wu, Y., Hong, M. and Kwo, J. Selfaligned inversion-channel In0.75Ga0.25As metal-oxide-semiconductor field-effect-transistors using UHV-Al2O3/Ga2O3(Gd2O3) and ALD-Al2O3 as gate dielectrics. Solid-State Electronics, VOL. 54, pp. 919 - 924, 2010.

[Liu2008IEEE_A] : Yang Liu, N. Neophytou,, G. Klimeck, M. S Lundstrom. Band-Structure Effects on the Performance of III-V Ultrathin-Body SOI MOSFETs. IEEE Transactions on Electron Devices, VOL. 55, pp. 1116, 2008.

[Liu2008IEEE_B] : Yang Liu, N. Neophytou, T. Low, G. Klimeck, M. S. Lundstrom, $A$ Tight-Binding Study of the Ballistic Injection Velocity for Ultrathin-Body SOI MOSFETs. IEEE Transactions on Electron Devices, VOL. 55, pp. 866, 2008.

[Liu2011IEEE] : Yang Liu, M. Luisier, M.S. Lundstrom. Temperature Dependence of the Transconductance in Ballistic III-V QWFETs. IEEE Transactions on Electron Devices, VOL. 58, pp. 1804, 2011.

[López-Villanueva1993PRB] : A. López-Villanueva, I. Melchor, P. Cartujo, J. E. Carceller. Modified Schrödinger equation including nonparabolicity for the study of a two-dimensional electron gas. Phys. Rev. B, VOL. 48, pp. 1626, 1993.

[Lundstrom2000] : M. Lundstrom, Fundamental of carrier transport, 2e édition, Cambridge University Press, 2000.

[Lundstrom2002IEEE] : Mark Lundstrom, Zhibin Ren. Essential physics of carrier transport in nanoscale MOSFETs. IEEE Transactions on Electron Devices, VOL. 49, pp. 133, 2002.

[Luttinger1955PR] : M. Luttinger, W. Kohn. Motion of Electrons and Holes in Perturbed Periodic Fields. Phys. Rev. VOL. 97, pp. 869, 1955.

[Maneglia1996JAP]: Maneglia, Y. and Bauza, D., Extraction of slow oxide trap concentration profiles in metal-oxide-semiconductor transistors using the charge pumping method. Journal of Applied Physics, VOL.79, pp. 4187 - 4192. 1996. 
[Martens2007MicEng] : K. Martens, W. Wang, K. De Keersmaecker, G. Borghs, G. Groeseneken, H. Maes, Microelectron. Eng. VOL. 84, pp. 2146, 2007.

[Martens2008IEEE] : K. Martens, C. Chi On, G. Brammertz, B. De Jaeger, D. Kuzum, M. Meuris, M. Heyns, T. Krishnamohan, K. Saraswat, H. E. Maes, and G. Groeseneken, On the Correct Extraction of Interface Trap Density of MOS Devices With High-Mobility Semiconductor Substrates, IEEE Trans. Electron Devices, VOL. 55, pp. 547, 2008.

[Mason1954IRE] : S. J. Mason. Power gain in feedback amplifiers, IRE Transactions on Circuit Theory, VOL. CT-1, pp. 20, 1954.

[Mateos1997SSE] : J. Mateos, T. Gonzalez, D. Pardo, P. Tadyszak, F. Danneville, A. Cappy. A Noise analysis of 0.1 $\mu \mathrm{m}$ gate MESFETs and HEMTs. Solid-State Electron, VOL. 42, pp. 79, 1997.

[Mateos2000IEEE] : J. Mateos, T. Gonzalez, D. Pardo, V. Hoel, A. Cappy. Monte Carlo simulator for the design optimization of low-noise HEMTs. IEEE Transactions on Electron Devices, VOL. 47, pp. 1950, 2000.

[Mateos2000IEEE] : Javier Mateos, Tomás González, Daniel Pardo, Virginie Hoel, Henri Happy, Alain Cappy. Improved Monte Carlo Algorithm for the Simulation of $\delta$-Doped AlInAs/GaInAs HEMT's. IEEE Transactions on Electron Devices, VOL. 47, pp. 250, 2000.

[Mateos2003IEEE] : J. Mateos, B. Vasallo, D. Pardo, T. Gonzalez, J. Galloo, S. Bollaert, Y. Roelens, A. Cappy. Microscopic modeling of nonlinear transport in ballistic nanodevices Electron Devices, IEEE Transactions on Electron Devices, VOL. 50, pp. 1897, 2003.

[Mateos2004IEEE-A] : J. M. Lopez, T. Gonzalez, D. Pardo, S. Bollaert, T. Parenty, A. Cappy. Design optimization of AlInAs-GaInAs HEMTs for high-frequency applications. IEEE Transactions on Electron Devices, VOL. 51, pp. 521, 2004.

[Mateos2004IEEE-B] : J. Mateos, T. Gonzalez, D. Pardo, S. Bollaert, T. Parenty, A. Cappy. Design optimization of AlInAs-GalnAs HEMTs for low-noise applications. IEEE Transactions on Electron Devices, VOL. 51, pp. 1228, 2004. 
[Mathieu1998] : H. Mathieu, Physique des semiconducteurs et des composants électroniques, 4e édition, Masson, Paris, 1998.

[Millithaler2008APL] : J.-F. Millithaler,L. Reggiani, J. Pousset, L. Varani, C. Palermo, W. Knap, J. Mateos, T. Gonzalez, S. Perez, D. Pardo,. Monte Carlo investigation of terahertz plasma oscillations in ultrathin layers of n-type In ${ }_{0.53} \mathrm{Ga}$ 0.47 As. Applied Physics Letters, VOL. 92, pp. 042113. 2008.

[Mishra1989IEDM] : U.K. Mishra, A.S. Brown, L.M. Jelloian, M. Thompson, L.D. Nguyen, S.E. Rosenbaum, Novel high performance self-aligned 0.15 micron long T-gate AlInAsGaInAs HEMTs IEEE International Electron Devices Meeting (IEDM) Tech. Dig., pp 101$104,1989$.

[MistryIEDM2007] : Mistry, K., Allen, C., Auth, C., Beattie, B., Bergstrom, D., Bost, M., Brazier, M., Buehler, M., Cappellani, A., Chau, R., Choi, C.-H., Ding, G., Fischer, K., A $45 \mathrm{~nm}$ Logic Technology with High-k+Metal Gate Transistors, Strained Silicon, $9 \mathrm{Cu}$ Interconnect Layers, $193 \mathrm{~nm}$ Dry Patterning, and 100\% Pb-free Packaging. IEEE International Electron Devices Meeting (IEDM) Tech. Dig., pp 247-250, 2007.

[Monsef2002Thèse] : Florian Monsef. Etude par simulation Monte Carlo des effets de quantification sur le transport dans les structures à effet de champ. Thèse de doctorat, Université Paris-Sud, 2002.

[Moran2006IEEE] : D. A. J. Moran, H. McLelland, K. Elgaid,, G. Whyte, C. R. Stanley and I. Thayne. 50-nm Self-Aligned and "Standard" T-gate InP pHEMT Comparison: The Influence of Parasitics on Performance at the 50- $\mathrm{nm}$ Node. IEEE Transactions on Electron Devices, VOL 53, pp. $2920-2925,2006$.

[Nag1972] : B. R. Nag. Theory of Electrical Transport in Semiconductors (Pergamon, New York), 1972.

[Natori1994JAP] : Kenji Natori. Ballistic metal-oxide-semiconductor field effet transistor. Journal of Applied Physics, VOL. 76, pp. 4879, 1994. 
[Nekovee1992PRB] : Maziar Nekovee, Bernard J. Geurts, Henk M. J. Boots, Martin F. H. Schuurmans. Failure of extended-moment-equation approaches to describe ballistic transport

[Nicollian1982] : E. H. Nicollian and J. R. Brews, MOS (Metal Oxide Semiconductor) Physics and Technology Wiley, New York, 1982.

[Ning1972PRB] : T. H. Ning, C. T. Sah, Phys. Rev. B, VOL. 6, pp. 4605, 1972.

[Nonner2004BJ] : W. Nonner, A. Peyser, D. Gillespiey, B. Eisenbergy. Relating Microscopic Charge Movement to Macroscopic Currents: The Ramo-Shockley Theorem Applied to Ion Channels. Biophysical Journal, VOL. 87, pp. 3716, 2004.

[Nougier2001] : Jean-Pierre Nougier. Méthode De Calculs Numériques - Volume 2, Fonctions Équations Aux Dérivées.

[O’Regan2010JAP] : T. P. O'Regan, M. V. Fischetti, B. Soree, S. Jin, W. Magnus, M. Meuris. Calculation of the electron mobility in III-V inversion layers with high-k dielectrics. Journal of Applied Physics, VOL. 108, pp. 103705, 2010.

[Obradovis2006APL] : Obradovic, B.; Kotlyar, R.; Heinz, F.; Matagne, P.; Rakshit, T.; Giles, M. D.; Stettler, M. A. and Nikonov, D. E. Analysis of graphene nanoribbons as a channel material for field-effect transistors Applied Physics Letters, VOL. 88, pp. 142102, 2006.

[Ono1995IEEE] : M. Ono, M. Saito, T. Yoshitomi, C. Fiegna, T. Ohguro, H.S. Momose, H. Iwai, A study on hot carrier effects on N-MOSFETs under high substrate impurity concentration. IEEE Trans. Electron. Dev. VOL. 42, pp. 1510, 1995.

[O'Regan2011APL] : T. P. O'Regan, P. K. Hurley. Calculation of the capacitance-voltage characteristic of GaAs, $\operatorname{In}_{0.53} G a_{0.47} A s$, and InAs metal-oxide-semiconductor structures. Applied Physics Letters, VOL. 99, pp. 163502, 2011.

[Pacelli1999IEEE] : Pacelli, A. Spinelli, A. and Perron, L. Carrier quantization at flat bands in MOS devices, IEEE Transactions on Electron Devices, VOL. 46, pp. 383 -387, 1999. 
[Pailloncy2004SSE] : G. Pailloncy, B. Iniguez, G. Dambrine, J. P. Raskin, F. Danneville. Noise modeling in fully depleted SOI MOSFETs. Solid-State Electronics, VOL. 48, pp. 813, 2004.

[Passlack2002IEEE] : Passlack, M., Droopad, R., Yu, Z., Medendorp, N., Braddock, D., Wang, X., Ma, T. and Buyuklimanli, T. Self-Aligned GaAs p-Channel Enhancement Mode MOS Heterostructure Field-Effect Transistor IEEE Electron Device Letter, VOL. 23, pp.508, 2002.

[Passlack2006IEEE] : Passlack, M.; Rajagopalan, K.; Abrokwah, J. \& Droopad, R. Implantfree high-mobility flatband MOSFET: principles of operation IEEE Transactions on Electron Devices, VOL. 53, pp. 2454 -2459, 2006.

[Passlack2007IEDM] : Passlack, M., Zurcher, P., Rajagopalan, K., Droopad, R., Abrokwah, J., Tutt, M., Park, Y.-B., Johnson, E., Hartin, O., Zlotnicka, A., Fejes, P., Hill, R., Moran, D., Li, X., Zhou, H., Macintyre, D., Thorns, S., Asenov, A., Kalna, K. and Thayne, I. High Mobility III-V MOSFETS For RF and Digital Applications IEEE International Electron Devices Meeting (IEDM) Tech. Dig., pp. 621 -624, 2008.

[Pavlidis1999]: MESFET: What's Best and Why ? (Comparaison des performances hyperfréquences entre les HBT, PM-HEMT et MESFET) Compound Semiconductor. Magazine No. 5 (June 1999) ; ou sur http://www.gaasman-tech.org/Digests/1999/PDF/42.pdf

[Pavlidis2006] : Pavlidis, D., 'Wide and Narrow-Bandgap Semiconductor Materials'. thema Forschung, VOL. 2, pp. 38-41, 2006.

[Pearsall1982] : T. P. Pearsall, GaInAsP Alloy Semiconductors. John Wiley and Sons. 1982. [Pellegrini1986PRB] : B. Pellegrini. Electric charge motion, induced current, energy balance, and noise. Physical Review B, VOL. 34, pp. 5921, 1986.

[Pérez2008JAP] : S. Pérez, T. González, D. Pardo, J. Mateos. Terahertz Gunn-like oscillations in InGaAs/InAlAs planar diodes. Journal of Applied Physics, VOL. 103, pp. 094516, 2008. 
[Pop2010NanoRes] : Pop, E. Energy Dissipation and Transport in Nanoscale Devices. Nano Res, VOL. 3, pp. 147-169, 2010.

[Pospieszalski1989IEEE] : M. W. Pospieszalski. Modeling of noise parameters of MESFET'S and MODFET's and their frequency and temperature dependence. IEEE Transctions on Microwave Theory Tech. VOL. 37, pp. 1340, 1989.

[Pucel1975AEEP] : R. A. Pucel, H. A. Haus. Signal and noise properties of gallium arsenide microwave field effect transistors. In Advances in Electronics and Electron Physics, New York: Academic, VOL. 38, pp. 195, 1975.

[Querlioz2008Thèse] : Damien Querlioz. Phénomènes quantiques et décohérence dans les nano-dispositifs semiconducteurs : étude par une approche Wigner Monte Carlo, Thèse de doctorat, Université Paris Sud, 2008

[Radosavljevic2009IEDM] : Radosavljevic, M., Chu-Kung, B., Corcoran, S., Dewey, G., Hudait, M.K., Fastenau, J.M., Kavalieros, J., Liu, W.K., Lubyshev, D., Metz, M., Millard, K., Mukherjee, N., Rachmady, W., Shah, U., Chau, R., Advanced high-K gate dielectric for highperformance short-channel In0.7Ga0.3As quantum well field effect transistors on silicon substrate for low power logic applications. IEEE International Electron Devices Meeting (IEDM) Tech. Dig., 2009.

[Rahman2003IEEE] : A. Rahman, J. Guo, S. Datta, M. Lundstrom, Theory of ballistic nanotransistors. IEEE Transactions on Electron Devices, VOL. 50, pp. 1853, 2003.

[Ramayya2007IEEE] : B. Ramayya, D. Vasileska, S. M. Goodnick, I. Knezevic, IEEE Transactions on Nanotechnology, VOL. 6, pp. 1132007.

[Ramo1939IRE] : S. Ramo. Currents induced by electron motion. Proc. IRE VOL. 27, pp. 584, 1939.

[Reif1965] : F. Reif, Fundamentals of Statistical and Thermal Physics. McGraw-Hill, 1965. 
[Ren2001Thèse] : Z. Ren. Nanoscale MOSFETs: Physics simulation and design. Thèse de doctorat, Purdue University 2001.

[Rengel2001SST] : R. Rengel, J. Mateos, M. J. Martín. Monte Carlo analysis of dynamic and noise performance of submicron MOSFETs at RF and microwave frequencies. Semiconductor Science and Technology, VOL. 16, pp. 939, 2001.

[Rengel2006IEEE] : R. Rengel, M. Martin, T. Gonzalez, J. Mateos, D. Pardo, G. Dambrine, J.-P. Raskin, F. Danneville, F. A microscopic interpretation of the RF noise performance of fabricated FDSOI MOSFETs. IEEE Transactions on Electron Devices, VOL 53, pp. 5232006.

[Rengel2011IEEE] : R. Rengel, M. J. Martin, F. Danneville. Microscopic Modeling of RF Noise in Laterally Asymmetric Channel MOSFETs. IEEE Electron Device Letters, VOL 32, pp. 72, 2011.

[Robertson2011APL] : Lin, L. and Robertson, J. Defect states at III-V semiconductor oxide interfaces Applied Physics Letters, VOL 98, pp. 082903, 2011.

[Robertsona2006JAP] : J. Robertson, B. Falabretti. Band offsets of high K gate oxides on III-V semiconductors. Journal of Applied Physics, VOL. 100, pp. 014111, 2006.

[Rodilla2009JAP] : H. Rodilla, T. Gonzalez, D. Pardo, J. Mateos. High-mobility heterostructures based on InAs and InSb: A Monte Carlo study. Journal of Applied Physics VOL. 105, pp. 113705, 2009.

[Rollett1962IRE] : J. M. Rollett. Stability and power gain invariants of linear two port. IRE Transactions on Circuit Theory CT-9, 29, 1962.

[Rothe1956IRE] : H. Rothe, W. Dahlke. Theory of Noisy Fourpoles. Proceedings of the IRE VOL.44, pp. 811, 1956.

[Sabatini2009Thèse] : G. Sabatini. Etude Monte Carlo des matériaux et composants électroniques à haute mobilité pour les applications TeraHertz, Thèse de doctorat, Université Montpellier 2, 2009. 
[Sabnis1979IEDM] : A. G. Sabnis, J. T. Clemens. Characterization of the electron mobility in the inverted $<100>$ Si surface. IEDM Tech Dig, pp. 18-21, 1979.

[Saint-Martin2004IEEE] : J. Saint-Martin, A. Bournel, P. Dollfus. On the ballistic transport in nanometer-scaled DG MOSFETs. IEEE Transactions on Electron Devices, VOL. 51, pp. $1148,2004$.

[SaintMartin2005Thèse] : J. Saint-Martin. Etude par simulation Monte Carlo d'architectures de MOSFET ultracourts à grille multiple sur SOI, Thèse de doctorat, Université Paris-Sud, 2005

[Sakurai2003ISSCC]: Sakurai, T. Perspectives on power-aware electronics Solid-State Circuits Conference, 2003. Digest of Technical Papers. ISSCC. 2003 IEEE International, VOL. 1, pp. $26-29,2003$.

[Sangiorgi1992IEEE] : E. Sangiorgi, M. R. Pinto. A semi-empirical model of surface scattering for Monte Carlo simulation of silicon n-MOSFETs. IEEE Transactions on Electron Devices, VOL. 39, pp. 356, 1992.

[Schroder2003JAP] : D. K. Schroder, J. A. Babcock. Negative bias temperature instability: road to cross in deep submicron silicon semiconductor manufacturing. J. Appl Phys, VOL. 94, pp. 1-18, 2003.

[Schwierz2001MR] : Schwierz F. and Liou J.J. 'Semiconductor devices for RF applications: evolution and current status' Microelectronics Reliability, VOL. 41, pp.145-168. 2001.

[Schwierz2003SSE] : Schwierz F. and Liou J.J. 'RF MOSFET: recent advances, current status and future trends' Solid-State Electronics, VOL. 47, pp. 1881-1895, 2003.

[Schwierz2007SSE] : Schwierz F. and Liou J.J. 'RF transistors: Recent developments and roadmap toward terahertz applications' Solid-State Electronics, VOL. 51, pp. 1079-1091, 2007 
[Shinichi2008IEEE] : Shinichi T., Toshifumi I., Tsutomu T., Toshinori N., Shu N. CarrierTransport-Enhanced Channel CMOS for Improved Power Consumption and Performance IEEE Transac. Electron Devices, VOL. 55, pp. 21, 2008.

[Shockley1938JAP] : W. Shockley. Current to conductors induced be a moving point charge. Journal of Applied Physics, VOL. 9, pp. 635, 1938.

[Shockley1952PR] : W. Shockley, W. T. Read, Jr. Statistics of the Recombinations of Holes and Electrons. Physical Review, VOL. 87, pp. 835, 1952.

[Singisetti2009IEEE] : Singisetti, U.; Wistey, M.; Burek, G.; Baraskar, A.; Thibeault, B.; Gossard, A.; Rodwell, M.; Shin, B.; Kim, E.; McIntyre, P.; Yu, B.; Yuan, Y.; Wang, D.; Taur, Y.; Asbeck, P. and Lee, Y.-J. InGaAs Channel MOSFETs With Self-Aligned InAs Source/Drain Formed by MEE Regrowth IEEE Electron Device Letters, VOL. 30, pp. 1128 1130, 2009.

[Skotnicki1994IEDM] : T. Skotnicki et al. A New Analog/Digital CAD Model for Subhalfmicron MOSFETs. IEDM Technical Digest, 1994. pp. 165-168.

[Skotnicki1998IEEE] T. Skotnicki, G. Merckel, T. Pedron The voltage-doping transformation: a new approach to the modeling of MOSFET short-channel effects. IEEE Electron Device Letters. VOL. 9, pp. 109, 1998.

[Skotnicki2003] T. Skotnicki, F. Boeuf. Physique des dispositifs pour circuits intégrés silicium, sous la direction de J. Gautier, Hermes Science, EGEM 2003.

[Sonnet2011APL] : A. M. Sonnet,R. V. Galatage, P. K. Hurley, E. Pelucchi, K. K. Thomas, A. Gocalinska, J. Huang, N. Goel, G. Bersuker, W. P. Kirk, C. L. Hinkle, R. M. Wallace, E. M. Vogel. On the calculation of effective electric field in $\operatorname{In}_{0.53} G a_{0.47} A s$ surface channel metaloxide-semiconductor field-effect-transistors. Applied Physics Letters, VOL. 98, pp. 193501, 2011.

[Spicer1980JVST] : W. E. Spicer, I. Lindau, P. Skeath, and C. Y. Su, Unified defect model and beyond J. Vac. Sci. Technol. VOL. 17, pp. 1019, 1980. 
[Steer1986IEEE] : M. Steer, R. Trew. High-frequency limits of millimeter-wave. IEEE Electron Device Letters, VOL. 7, pp. 640, 1986.

[Sun1980IEEE] : S. C. Sun, J. D. Plummer. Electron mobility in inversion and accumulation layers on thermally oxidized silicon surfaces. IEEE Transactions on Electron Devices, VOL. 27, pp. 1497, 1980.

[Sun2008DRC] : Y. Sun, E. W. Kiewra, J. P. de Souza, J. J. Bucchignano, K. E. Fogel, D. E. Sadana, G. G. Shahidi. High Performance Long- and Short-Channel In ${ }_{0.7} \mathrm{Ga}_{0.3} \mathrm{As}$-channel MOSFETs. Device Research Conference, p. 41, 2008.

[Sun2008IEDM] : Y. Sun, E. W. Kiewra, J. P. de Souza, J. J. Bucchignano, K. E. Fogel, D. K. Sadana and G. G. Shahidi. Scaling of In0.7Ga0.3As Buried-Channel MOSFETs. IEEE International Electron Devices Meeting (IEDM) Tech. Dig., 2008.

[Sze1981] : Sze, S.M. Physics of semiconductor devices. 2nd ed. New York: Wiley; 1981.

[Takagi1994IEEE] : S. Takagi, A. Toriumi, M. Iwase, H. Tango. On the universality of inversion layer mobility in Si MOSFETs: Part I: Effects of substrate impurity concentration. IEEE Transactions on Electron Devices, VOL. 41, pp. 2357, 1994.

[Takimoto1959JPSJ] : N. Takimoto. On the screening of impurity potential by conduction electrons. J. Phys. Soc. Jpn. VOL. 4, pp. 1142, 1959.

[Tang2010SSE] : Y. Tang, L. Zhang, Y. Wang. Accurate small signal modeling and extraction of silicon MOSFET for RF IC application. Solid-State Electronics, VOL. 54, pp. $1312,2010$.

[Taur1998CUP] : Taur, Y. et Ning, T. H. «Fundamentals of modern VLSI devices », Cambridge University Press. 1998. 
[Thayne2009ECS] : I.G. Thayne, R.J.W. Hill, M.C. Holland, X. Li, H.Zhou, D.S. Macintyre, S. Thoms, K. Kalna, C.R. Stanley, A. Asenov, R.Droopad, M. Passlackc. Review of Current Status of III-V MOSFETs, ECS Transactions, VOL. 19, pp. 275-286, 2009.

[Thobel1990APL] : J.-L. Thobel, L. Baudry, A. Cappy, P. Bourel, R. Fauquembergue. Electron transport properties of strained InxGa1-xAs. Applied Physics Letters, VOL. 56, pp. $346,1990$.

[VanderZiel1986] : A. Van der Ziel. Noise in Solid State Devices and Circuits, New York: Wiley, 1986.

[Varghese2008IEDM] : Varghese, D., Xuan, Y., Wu, Y.Q., Shen, T., Ye, P.D., Alam, M.A., Multi-probe interface characterization of $\mathrm{In}_{0.65} \mathrm{Ga}_{0.35} \mathrm{As}_{\mathrm{A}} \mathrm{Al}_{2} \mathrm{O}_{3} \mathrm{MOSFET}$. IEEE International Electron Devices Meeting (IEDM) Tech. Dig., 2008.

[Vecchi1998IEEE] : Maria Cristina Vecchi and Massimo Rudan. Modeling Electron and Hole Transport with Full-Band Structure Effects by Means of the Spherical-Harmonics Expansion of the BTE. IEEE Transactions on Electron Devices, VOL.45, pp. 230, 1998.

[Vurgaftman2001JAP] I. Vurgaftman, J. R. Meyer, L. R. Ram-Mohan. Band parameters for III-V compound semiconductors and their alloys. Journal of Applied Physics, VOL. 89, pp. $5815,2001$.

[Waldron2007IEDM] : N. Waldron, D.-H. Kim, and J. A. del Alamo, "90 $\mathrm{nm}$ self-aligned enhancement-mode InGaAs HEMT for logic applications" IEEE International Electron Devices Meeting (IEDM) Tech. Dig., pp. 633-636, 2007.

[Waldron2010IEEE] : Waldron, N., Kim, D.-H. and Del-Alamo, J. A. Self-Aligned InGaAs HEMT Architecture for Logic Applications IEEE Transactions on Electron Devices, VOL. 57, pp. 297-304, 2010.

[Xuan2007APL] : Y. Xuan and P. D. Ye. Substrate engineering for high-performance surface-channel III-V metal-oxide-semiconductor field-effect transistors. Applied Physics Letters, VOL 91, pp. 232107, 2007. 
[Xuan2007IEDM] : Y. Xuan, Y. Q. Wu, T. Shen, T. Yang, P. D. Ye. High-Performance Surface Channel In-Rich In $_{0.75} G a_{0.25}$ As MOSFETs with ALD High-K as Gate Dielectric IEDM 2007.

[Xuan2007IEEE] : Y. Xuan, Y. Q. Wu, T. Shen, T. Yang, P. D. Ye. High-Performance Surface Channel In-Rich In0.75Ga0.25As MOSFETs with ALD High-K as Gate Dielectric. IEEE International Electron Devices Meeting (IEDM) Tech. Dig., 2007.

[Xue2011APL] : F. Xue, H. Zhao, Y.-T. Chen, Y. Wang, F. Zhou, J. C. Lee. High-k InGaAs metal-oxide-semiconductor field-effect-transistors with various barrier layer materials. Applied Physics Letter, VOL. 99, pp. 033507, 2011.

[Yan1992IEEE] : Ran-Hong Yan, Abbas Ourmazd, Kwing F. Lee. Scaling the Si MOSFET: From bulk to SOI to bulk. IEEE Transactions on Electron Devices, VOL. 39, pp. 1704, 1992.

[Ye2008JVST] : P. D. Ye, Main determinants for III-V metal-oxide-semiconductor fieldeffect transistors J. Vac. Sci. Technol. VOL. 26, pp. 697, 2008.

[Zhao2009APL] : H. Zhao, J. Huang, Y. T. Chen, J. H. Yum, Y. Z. Wang, F. Zhou, F. Xue, and J. C. Lee, Effects of gate-first and gate-last process on interface quality of $\operatorname{In}_{0.53} \mathrm{Ga}_{0.47} A s$ metal-oxide-semiconductor capacitors using atomic-layer-deposited Al2O3 and HfO2 oxides Appl. Phys. Lett. VOL. 95, pp. 253501, 2009. 


\section{ETAT DES PUBLICATIONS}

\section{Articles dans des revues avec comité de lecture (acceptés)}

M. Shi, J. Saint-Martin, A. Bournel, D. Querlioz, P. Dollfus, J. J. Mo, N. Wichmann, L. Desplanque, X. Wallart, F. Danneville, S. Bollaert "Numerical and experimental assessment of charge control in III-V nano-MOSFET under low supply voltage", accepté pour publication dans Journal of Nanoscience and Nanotechnology, à paraître.

M. Shi, J. Saint-Martin, A. Bournel, P. Dollfus "Numerical simulation of III-V FET architectures for high frequency and low consumption applications", Microelectronic Engineering VOL. 88, pp. $354-359$ (2011). doi: 10.1016/j.mee.2010.10.026

M. Shi, J. Saint-Martin, A. Bournel, H. Maher, M. Renvoise, P. Dollfus "Monte Carlo simulation of III-V material-based MOSFET for high frequency and ultra-low consumption applications" Journal of Nanoscience and Nanotechnology VOL. 10, pp. 7015-7019 (2010). doi: $10.1166 /$ jnn.2010.2931

\section{Communications dans des conférences internationales avec actes (acceptées)}

M. Shi, J. Saint-Martin, A. Bournel, D. Querlioz, P. Dollfus, J. J. Mo, N. Wichmann, L. Desplanque, X. Wallart, F. Danneville, S. Bollaert "Assessment of III-V MOSFET architectures for low power applications using static and dynamic numerical simulation" (oral) 37th IEEE International SOI Conference, Arizona, USA, 3-6 octobre, 2011, IEEE Proceedings SOI Conf., pp. 158 (2011)

M. Shi, J. Saint-Martin, A. Bournel, P. Dollfus "Optimization of III-V FETS architecture for high frequency and low consumption applications" (oral) 40th European Solid-State Device Research Conference (ESSDERC), Séville, Espagne, 14-16 septembre 2010, Proceedings of ESSDERC, pp. 424 (2010) 
M. Shi, J. Saint-Martin, A. Bournel, P. Dollfus "Schrödinger-Poisson and Monte Carlo analysis of III-V MOSFETs for high frequency and low consumption applications" (oral) International Conference on Simulation of Semiconductor Processes and Devices (SISPAD), Bologne, Italie, 6-8 septembre 2010, Proceedings of SISPAD, pp. 83 (2010)

\section{Communications dans des conférences internationales sans actes}

M. Shi, J. Saint-Martin, A. Bournel, D. Querlioz, P. Dollfus, J. J. Mo, N. Wichmann, L. Desplanque, X. Wallart, F. Danneville, S. Bollaert "Numerical assessment of III-V nanoMOSFET for high frequency performance under low supply voltage" (oral) International Conference on Nanoscience \& Technology (ChinaNANO 2011), Pékin, Chine, 7-9 septembre 2011.

M. Shi, J. Saint-Martin, A. Bournel, P. Dollfus "Numerical simulation of III-V FET architectures for high frequency and low consumption applications" (oral) E MRS 2010 Spring Meeting Symposium "Post-Si CMOS electronic devices: the role of Ge and III-V materials", Strasbourg, France, 7-11 juin 2010.

M. Shi, J. Saint-Martin, A. Bournel, H. Maher, M. Renvoise, P. Dollfus "Monte Carlo simulation of III-V material-based MOSFET for high frequency and ultra-low consumption applications" (The Best Poster Awards of PhD. student) International Conference on Nanoscience \& Technology (ChinaNANO 2009), Pékin, Chine, 1-3 septembre 2009.

\section{Communications nationales avec ou sans actes}

M. Shi, J. Saint-Martin, A. Bournel, P. Dollfus "Simulation de transistors à effet de champ en matériaux III-V pour une électronique haute fréquence et ultra basse consommation" (poster) 13èmes Journées Nationales du Réseau Doctoral en Microélectronique, Montpellier, France, 7-9 juin 2010 ; in Actes des JNRDM, pp. 48-51, 2010 
M. Shi, J. Saint-Martin, A. Bournel, P. Dollfus "Simulation Monte Carlo de MOSFET à base de matériaux à faible bande interdite pour une électronique haute fréquence ultra basse consommation" (poster), 12èmes Journées Nationales du Réseau Doctoral en Microélectronique, Lyon, France, 18-20 mai 2009 ; in Actes des JNRDM, SimP6, 4 pages, 2009

M. Shi, J. Saint-Martin, A. Bournel, P. Dollfus "Simulation Monte Carlo de MOSFET à base de matériaux à faible bande interdite pour une électronique haute fréquence ultra basse consommation" (poster), Journées thématiques "De la modélisation des nanodispositifs aux circuits innovants" des GdR Nanoélectronique et SoC-SiP, Talence, France, 9-11 septembre 2009.

\section{Autres}

M. Shi "Schrödinger-Poisson and Monte Carlo analysis of III-V FETs for high frequency and low consumption applications", séminaire doctorant IEF, Orsay, France, 2 juin 2010. 